ION TRANSPORT IN POLYMER ELECTROLYTES

Jie Shi

A Thesis Submitted for the Degree of PhD

at the

University of St Andrews

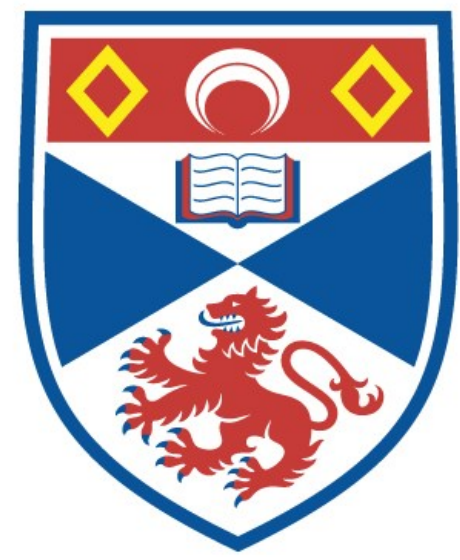

1993

Full metadata for this item is available in

St Andrews Research Repository

at:

http://research-repository.st-andrews.ac.uk/

Please use this identifier to cite or link to this item:

http://hdl.handle.net/10023/15522

This item is protected by original copyright 


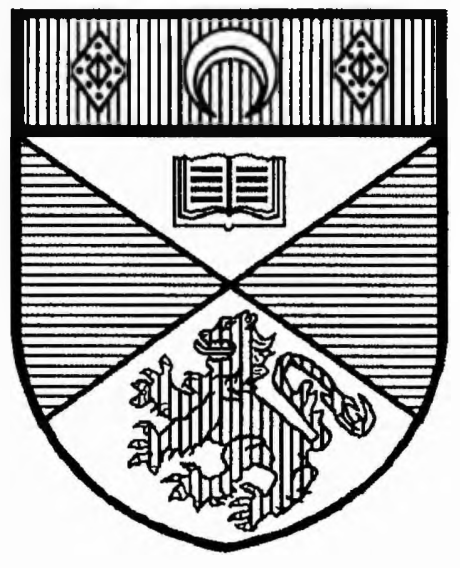

\section{ION TRANSPORT IN POLYMER ELECTROLYTES}

A thesis presented for the degree of

Doctor of Philosophy

in the Faculty of Science of the University of St Andrews

by Jie Shi, B.Sc., M.Sc.

Department of Chemistry

St Andrews

January 1993

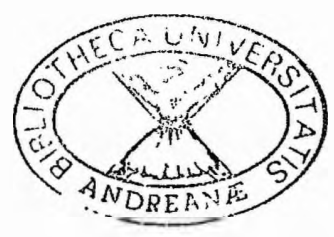


ProQuest Number: 10170729

All rights reserved

INFORMATION TO ALL USERS

The quality of this reproduction is dependent upon the quality of the copy submitted.

In the unlikely event that the author did not send a complete manuscript and there are missing pages, these will be noted. Also, if material had to be removed, a note will indicate the deletion.

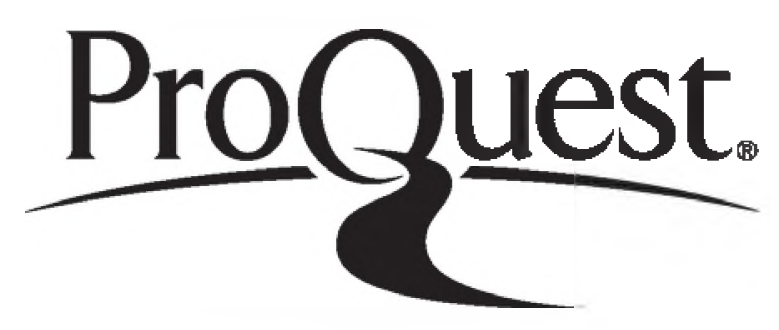

ProQuest 10170729

Published by ProQuest LLC (2017). Copyright of the Dissertation is held by the Author.

All rights reserved.

This work is protected against unauthorized copying under Title 17, United States Code Microform Edition (c) ProQuest LLC.

ProQuest LLC.

789 East Eisenhower Parkway

P.O. Box 1346

Ann Arbor, Ml $48106-1346$ 
Sw $B 2^{9^{8}}$ 


\section{CERTIFICATION}

I hereby certify that Jie Shi, B.Sc, M.Sc, has spent twelve terms of research work under my supervision, and that he has fulfilled the conditions of the Resolution and Regulations appropriate to the degree of Doctor of Philosophy.

January 1993

Signed:

C. A. Vincent

Director of Research

\section{LIBRARY DECLARATION}

In submitting this thesis to the University of St Andrews I understand that I am giving permission for it to be made available for use in accordance with the regulations of the University Library for the time being in force, subject to any copyright vested in the work not being affected thereby. I also understand that the title and abstract will be published, and that a copy of the work may be made and supplied to any bona fide library or research worker.

Signed: 


\section{Acknowledgements}

I would like to thank my supervisor, Professor Colin Vincent, for his guidance and patience throughout the three years of this project.

Dr. David Tunstall has guided me in the use of solid state NMR to study polymer electrolytes.

Thanks also go to Drs Peter Bruce, Fiona Gray, Martin Hardgrave and Sam Arumugam for helpful discussions.

Much of the experimental work could not have been carried out without the use of excellent equipment made by James Rennie and the Departmental Mechanical Workshop. All the atomic absorption analyses were helped by Sylvia Smith.

Without my wife Nan's firm support and great love, I cannot imagine that I could finish this thesis. My parents have given me a lot of encouragement, though they are geographically far from me.

Finally, I would like to thank all colleagues in the CEMS and the Department for their patience and kindness. 


\section{ABSTRACT}

The ion-polymer and ion-ion interactions in polymer electrolytes based on high molecular weight, amorphous methoxy-linked PEO (PMEO) and lithium salts have been investigated by conductivity measurement, magic-angle spinning NMR (mas NMR) and pulsed field gradient NMR (pfgNMR) techniques. In the very dilute salt concentration region, ion pairing effects are dominant in these polymer electrolytes. Ion association is found to increase with temperature and salt concentration. Ion transport for these electrolytes is controlled both by segmental motion of the polymer and activation process, in which the former is important for the dilute concentration samples while the latter is important for the concentrated samples. The mass transport process in polymer electrolytes based on a zinc salt has been investigated by steady state dc polarisation and Hittorf techniques. Zinc ion constituents in these electrolytes are mobile with a limiting current fraction of about 0.2 at $80^{\circ} \mathrm{C}$, and the transference number measured by the Hittorf method is less than 0.1 . The main species in these electrolytes are proposed to be neutral mobile triples. The electrode-electrolyte interfaces in polymer electrolytes based on calcium and magnesium salts have been studied. Dc polarisation experiments for these polymer electrolytes were carried out using two electrode cells with the metal anode and mercury film amalgam cathode. The results of dc polarisation experiments suggest that calcium species are mobile in high molecular weight electrolytes, while magnesium species are immobile. The influence of the molecular weight of the polymer on the dynamics of cation constituents has been studied based on the experimental results of dc polarisation and pfg NMR, and on the theoretical analyses of the reptation theory and the Rouse model. It is found that the transport of the gravity centre of the polymer will influence the ion transport in polymer electrolytes based on PEO in a manner described by the Rouse model when the molecular weight of PEO is less than 3200 . 


\section{TABLE OF CONTENTS}

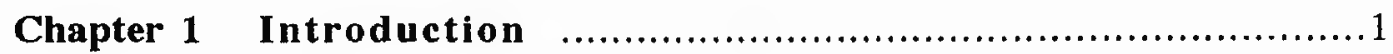

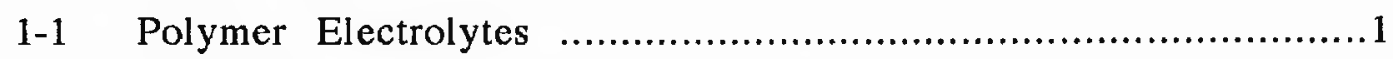

1-1-1 Ion-Polymer and Ion-Ion Interactions............................1

1-1-2 Structure and Morphology ..................................... 3

1-1-3 Ion Transport ............................................. 4

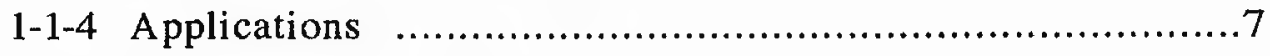

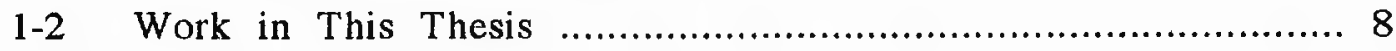

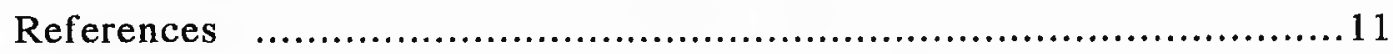

Chapter 2 Dynamic Models and Theories for Ionic Conduction

in Polymer Electrolytes ................................. 19

2-1 Macroscopic Models ............................................20

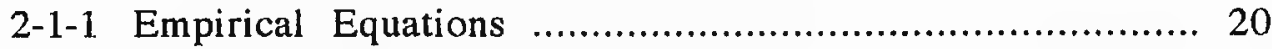

2-1-2 Free Volume Model .........................................21

2-1-3 Configurational Entropy Model .................................23

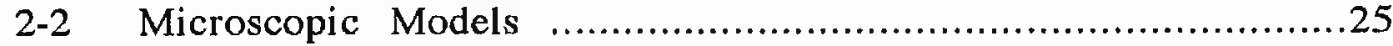

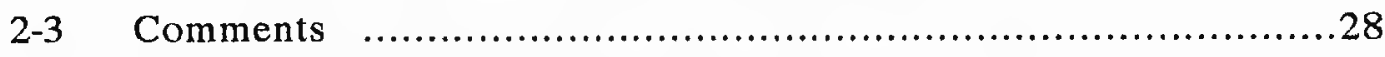

2-3-1 Comments on the Macroscopic Models ...........................28

2-3-2 Comments on the Microscopic Models ............................. 29

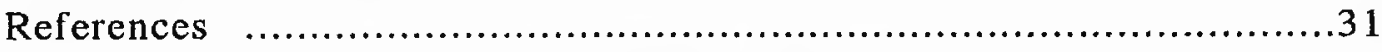

Chapter 3 Experimental Techniques: Preparation,

Characterisation and Electrochemical

Investigation of Polymer Electrolytes ......................32

3-1 Chemical Samples ................................................ 32

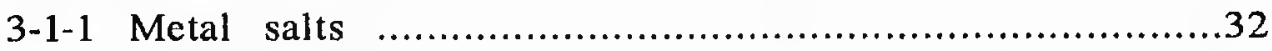

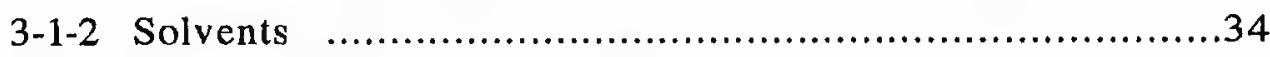




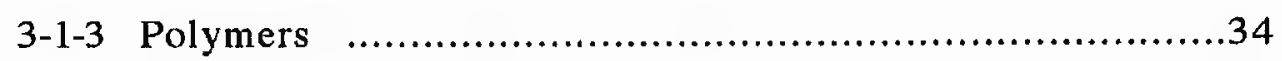

3-1-4 Metals ........................................................ 35

3-2 Preparation, Modification and Purification of Polymer Host ...................35

3-2-1 Preparation of Methoxy-linked Poly(ethylene oxide) .................35

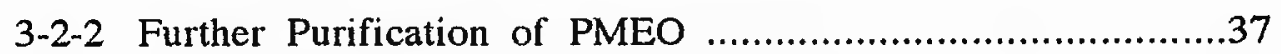

3-2-3 Methylation of Poly(ethylene oxide) ..................................39

3-3 Preparation of Polymer Electrolytes .....................................40

3-3-1 Solvent-Casting Methods ....................................40

3-3-2 Solvent-Free Methods .........................................41

3-3-2-1 Grinding / Hot Pressing Method ...........................41

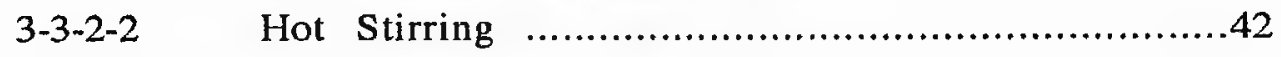

3-4 Cells and Electrodes for Electrochemical Measurements on

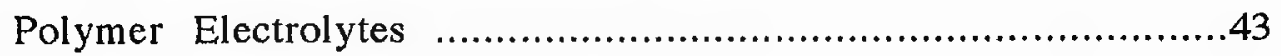

3-4-1 Two Electrode Cells ............................................44

3-4-2 Three Electrode Cells ............................................46

3-5 Direct Current Measurements .............................................47

3-5-1 De Polarisation of Two Electrode Cells ...........................47

3-5-1-1 Principle of Steady State Method ............................ 47

3-5-1-2 Experimental Details ...................................48

3-5-2 De Polarisation of Hittorf Cell ....................................50

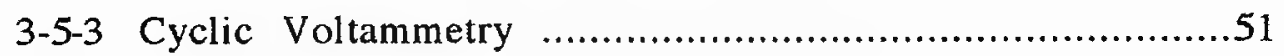

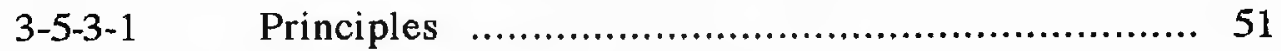

3-5-3-2 Experimental Details .................................52

3-6 Alternating Current Measurements .....................................53

3-6-1 Principles of Ac Impedance Spectroscopy ............................54

3-6-2 Ac Impedance Spectroscopy of Systems

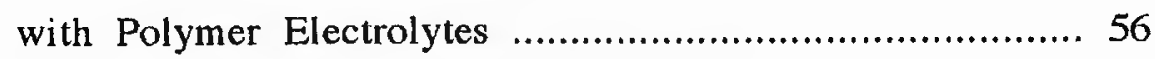

3-6-2-1 Blocking Electrodes ................................. 56

3-6-2-2 Non-Blocking Electrodes .................................57 
3-6-3 Experimental Details ...................................................59

3-6-3-1 Conductivity Measurements ……............................59

3-6-3-2 Electrode-Electrolyte Interfaces ….............................60

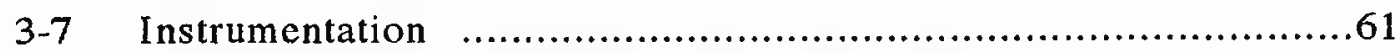

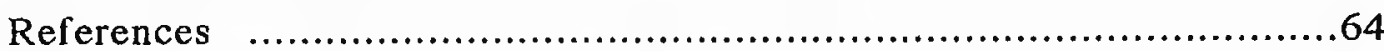

Chapter 4 Experimental Techniques for Solid State

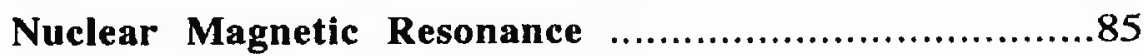

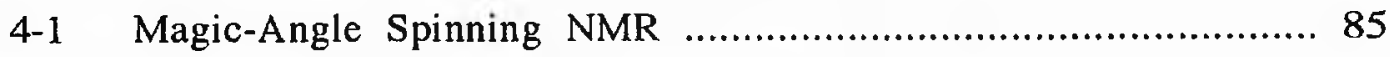

4-1-1 Introduction ….................................................... 85

4-1-2 Principle of Magic-Angle Spinning ……..............................87

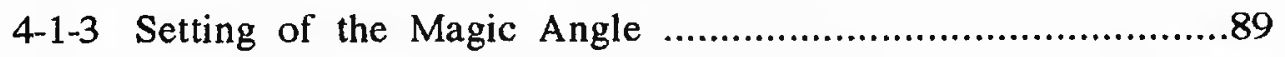

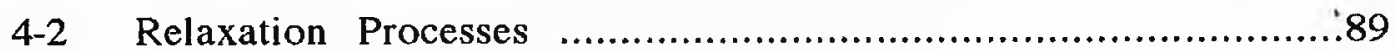

4-2-1 Spin-Lattice Relaxation and Spin-Spin Relaxation .......................89

4-2-2 Relation of $T_{1}$ and $T_{2}$ to Molecular Motion ............................ 90

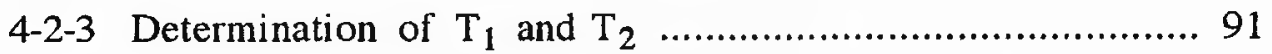

4-3 Field Gradient NMR and Measurements of Diffusion Coefficient ..............96

4-3-1 Diffusion in a Steady Field Gradient ...................................... 96

4-3-2 Pulsed Field Gradient NMR (pfg NMR) ...............................97

4-3-3 Gradient Calibration ....................................................99

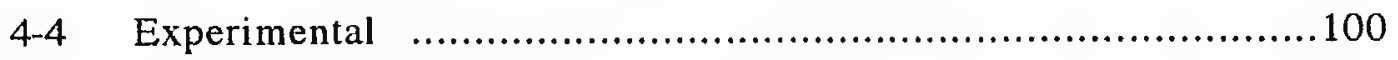

4-4-1 MAS NMR of Polymer Electrolytes .................................. 100

4-4-2 Measurements of $T_{1}$ and $T_{2}$ of Polymer Electrolytes ................ 101

4-4-3 Measurements of Diffusion Coefficient of Polymer Electrolytes

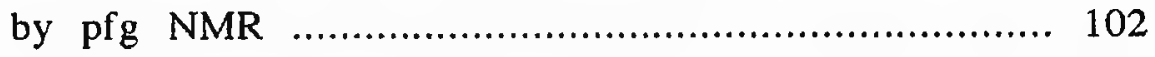

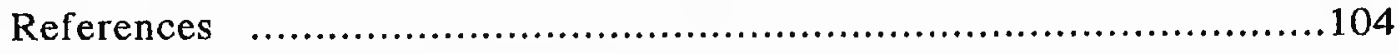




\section{Chapter 5 Ionic Conduction and Interaction in Polymer Electrolytes}

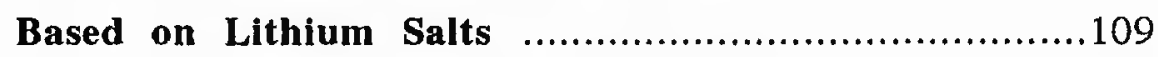

5-1 Conducting Species and Ion Transport Investigated

by Conductivity measurement ........................................ 110

5-1-1 Relationship Between Salt Concentration and Conductivity ........ 110

5-1-2 Relationship Between Conductivity and Temperature .............. 115

5-1-3 Deviation From the VTF Behaviour ...............................117

5-1-4 Conductive Behaviour in Polymer Electrolytes ......................119

5-1-5 Conductivity Measurement of PMEO-LiPF 6 Electrolytes ...........125

5-2 Species in Polymer Electrolytes Investigated by Magic-Angle

Spinning NMR

5-2-1 Origin of NMR Spectra in the Polymer Electrolytes

Containing Lithium Salts

5-2-2 Assignment of ${ }^{7} \mathrm{Li}$ Mas NMR spectra of Polymer Electrolytes .... 129

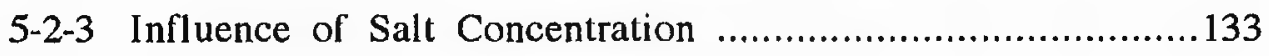

5-2-4 Influence of Temperature ........................................135

5-3 Mass Transport in Polymer Electrolytes Investigated by Pulsed

Field Gradient NMR .............................................136

5-3-1 Diffusion of 31P Nuclei ...................................... 136

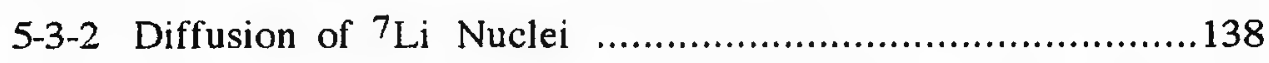

5-3-3 Relationship Between Conductivity and Diffusion Coefficients .....141

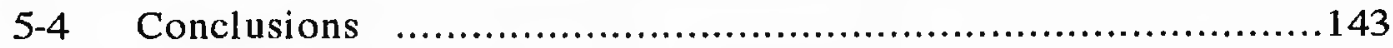

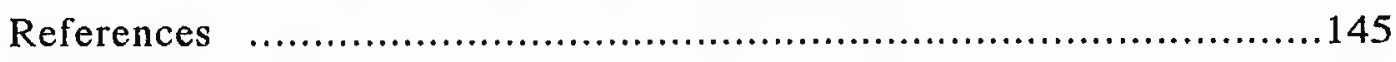

Chapter 6 Mass Transport in Polymer Electrolytes

Based on a Zine Salt ................................... 184

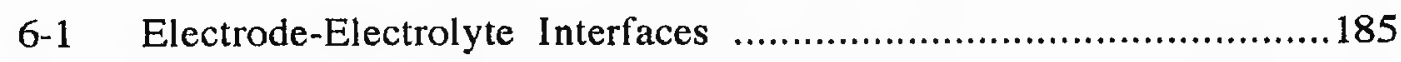

6-1-1 Passivating Layer at the Electrode-Electrolyte Interface ..............185

6-1-2 Treatment of the Electrode-Electrolyte Interface ...................187 
6-2 De Polarisation and Steady State Current Flow in

Symmetric Zinc Cells ...................................................188

6-2-1 Steady State Current Flow in Solid Electrolyte Cells ................ 188

6-2-2 De Polarisation of PMEO-Zn( $\left(\mathrm{CF}_{3} \mathrm{SO}_{3}\right)_{2}$ Electrolytes .............. 194

6-3 Measurement of Ionic Transference Number by the Hittorf Method .........198

6-3-1 Introduction to the Hittorf Method ................................ 198

6-3-2 Experimental .................................................201

6-3-3 Determination of the Ionic Transference Number .................. 203

6-3-4 Experimental Results ........................................206

6-3-5 Transference Number in PEO- $\mathrm{Zn}\left(\mathrm{CF}_{3} \mathrm{SO}_{3}\right)_{2}$ Electrolyte ............208

6-4 Conductivity of $\mathrm{PMEO}-\mathrm{Zn}\left(\mathrm{CF}_{3} \mathrm{SO}_{3}\right)_{2}$ Electrolyte .....................210

6-4-1 Phase Behaviour ..............................................210

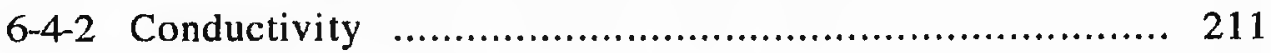

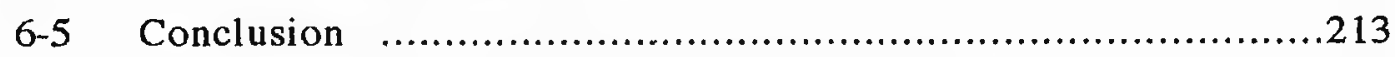

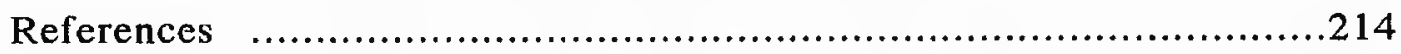

Chapter 7 Conductivity and Transport Properties in Polymer

Electrolytes Based on Calcium and Magnesium Salts....239

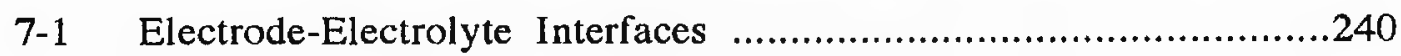

7-1-1 Electrochemical Reaction on $\mathrm{Ca}$ and $\mathrm{Mg}$ Electrodes .................240

7-1-2 Electrochemical Reactions on Mercury Film Electrodes .............242

7-1-3 The Electrode-Electrolyte Interfaces Under Dc Polarisation ..........244

7-2 De Polarisation of $\mathrm{M} / \mathrm{PEO}-\mathrm{M}\left(\mathrm{CF}_{3} \mathrm{SO}_{3}\right)_{2} / \mathrm{Hg}(\mathrm{M})$

Two Electrode Cells .....................................................246

7-2-1 Dc Polarisation of $\mathrm{Ca} / \mathrm{PEO}-\mathrm{Ca}\left(\mathrm{CF}_{3} \mathrm{SO}_{3}\right)_{2} / \mathrm{Hg}(\mathrm{Ca})$ Cells ........ 246

7-2-2 Dc Polarisation of $\mathrm{Mg} / \mathrm{PEO}-\mathrm{Mg}\left(\mathrm{CF}_{3} \mathrm{SO}_{3}\right)_{2} / \mathrm{Hg}(\mathrm{Mg})$ Cells ........250

7-3 Explanation of the Mobilities of Calcium and Magnesium Species

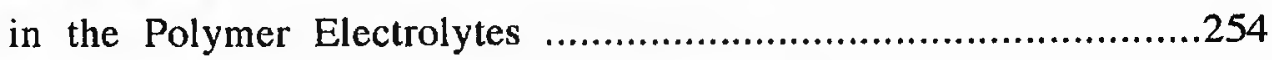


7-3-1 Interaction Between Cations and Polymer Host:

the Hard-Soft Acid-Base Principle

7-3-2 Kinetics of Exchange of Bound Water Molecules in

Aqueous Solutions

7-4 Conductivity and Phases in $\mathrm{PEO}-\mathrm{Ca}\left(\mathrm{CF}_{3} \mathrm{SO}_{3}\right)_{2}$ and

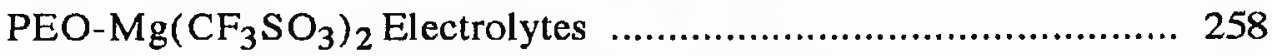

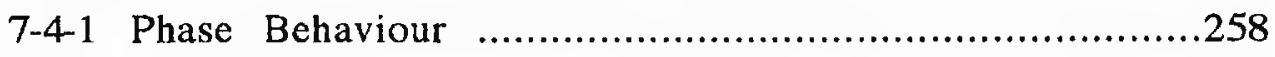

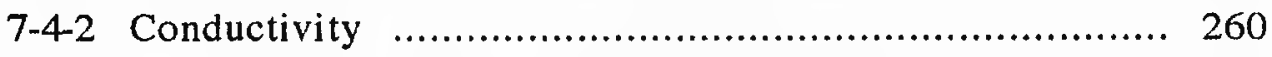

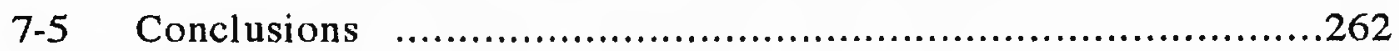

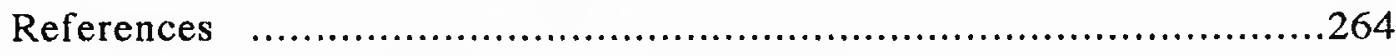

Chapter 8 The Influence of the Molecular Weight of Polymer on the Ionic Conduction in Polymer Electrolytes .................299

8-1 Dynamics of Linear Polymer Solutions and Melts ........................ 300

8-1-1 Dynamics of Simple Liquids or Solutions ........................300

8-1-2 Dynamics of Unentangled Polymer Liquids and melts

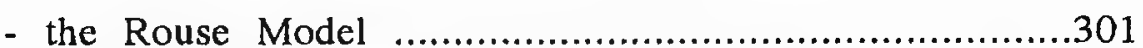

8-1-3 Dynamics of Entangled Polymer Melts

- the Reptation Theory ............................................305

8-2 Dynamics of Non-linear Polymer Solutions and Melts.......................310

8-2-1 Dynamics of Polymer Liquids and Melts with Branched Chains ....310

8-2-2 Dynamics of Branched Polymer in Linear Polymer Melts ............312

8-3 Dynamics of Polymer Chain Diffusion in Polymer Electrolytes ..............315

8-3-1 Models for the Diffusion of Polymer Chain in

Polymer Electrolytes............................................315

8-3-2 Relaxation Modes of Polymer Chains and Ionic Conduction in

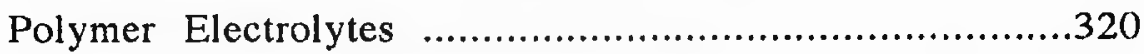

8-4 Relationship Between the Molecular Weight of Polymer and

the Cationic Conductivity in Polymer Electrolytes 
8-4-1 Polymer Electrolytes with Cations Tightly Bound by

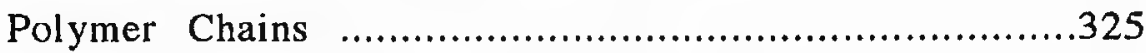

8-4-2 Polymer Electrolytes with Loosely Coordinated Cations .............327

8-5 Experimental Results and Discussion ..................................334

8-5-1 Influence of the Molecular Weight of PEO on Ionic Conduction in PEO- $\mathrm{Mg}\left(\mathrm{CF}_{3} \mathrm{SO}_{3}\right)_{2}$ Electrolytes ..............................335

8-5-2 Influence of the Molecular Weight of PEO on Ionic Conduction in

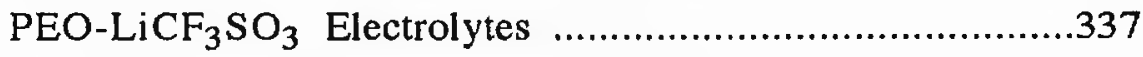

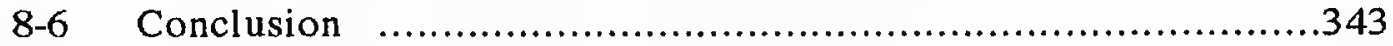

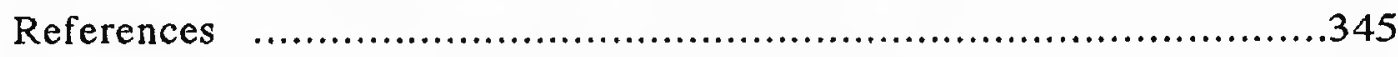

Chapter 9 Ion Transport in Polymer Electrolytes:

General Conclusion

9-1 Ion Association and Ion Transport in High Molecular Weight Polymer Electrolytes Based on Lithium Salts 360

9-2 Ion Transport in Polymer Electrolytes Based on Divalent Salts 361

9-3 Influence of the Molecular Weight of Polymer on the Cationic Transport in Polymer Electrolytes .363 
To Nan, the love of my life,

To my parents, with all my love. 


\section{CHAPTER 1}

\section{INTRODUCTION}

\section{1-1 Polymer Electrolytes}

Ionically conducting phases based on the dissolution of salts in suitable ioncoordinating polymers have been termed polymer electrolytes [1].

It had been found that some alkali metal salts were able to dissolve in polyethers, such as poly(ethylene oxide), PEO, and poly(propylene oxide), PPO to form complexes as early as $1966[2,3]$. However, the ionic conduction of these complexes was not recognised until 1973 [4]. After it was reported that these complexes might act as practical electrolytes [5], extensive studies ensued.

\section{1-1-1 Ion-Polymer and Ion-Ion Interactions}

Generally, the first prerequisite for a suitable polymer host is that the polymer has polar atoms, eg. $O, N, S$ etc. which are able to coordinate metal ions, in their backbone or side chains. In the absence of d-orbital involvement, the order of stability for the coordinating bond between metal ions and polar groups is: $\mathrm{O}>\mathrm{NR}>\mathrm{NH}>\mathrm{S}$. The second requirement is that the segments of the polymer host are very mobile and the third that the polymer has a suitable configuration which allows the cations to coordinate with the polar atoms in the chains. For example, some metal salts can dissolve in polymers such as $\left(\mathrm{CH}_{2} \mathrm{CH}_{2} \mathrm{O}\right)_{n},\left(\mathrm{CH}_{2} \mathrm{CH}_{3} \mathrm{CHO}\right)_{n},\left(\mathrm{CH}_{2} \mathrm{CH}_{2} \mathrm{NH}\right)_{\mathbf{n}}$ and $\left(\mathrm{CH}_{2} \mathrm{CH}_{2} \mathrm{~S}\right)_{\mathbf{n}}$. However, the conductivity of the electrolytes based on $\left(\mathrm{CH}_{2} \mathrm{CH}_{2} \mathrm{~S}\right)_{n}$ is very low, due to its high glass transition temperature. There is no evidence that $\left(\mathrm{CH}_{2} \mathrm{O}\right)_{\mathrm{n}}$. $\left(\mathrm{CH}_{2} \mathrm{CH}_{2} \mathrm{CH}_{2} \mathrm{CH}_{2} \mathrm{O}\right)_{n}$ can solvate salts, although there are oxygen atoms in the 
backbone chain. This may result from the fact that the configurations of these polymers do not favour the coordination between metal ions and more than one polar atom. Poly(ethylene oxide), poly(propylene oxide) and polymers containing PEO or PPO segments have been shown to be able to dissolve salts well, and polymer electrolytes based on these hosts have displayed good ionic conductivity and have been extensively studied.

It has been found that many metal salts based on monovalent or multivalent cations can dissolve in PEO [6] (Table 1-1). The enthalpy of solvation of metal salts in a polymer primarily arises from the ion-polymer interactions, either electrostatic interaction or formation of coordinate bonds by sharing of a lone pair of electrons on a coordinating atom in the polymer. Because the polymer hosts are aprotic solvents with low dielectric constant, anion stabilisation energies in polymer electrolytes are negligible. Thus, the most suitable anions for polymer electrolytes are large, soft ions, such as $\mathrm{ClO}_{4}^{-}, \mathrm{BF}_{4}^{-}$etc. In addition, some proton-conducting polymer electrolytes have been reported [7].

In aprotic solvents with low dielectric constants, ion association is generally observed. In polymer electrolytes, strong ion-ion interactions lead to the formation of ion pairs, ion clusters. For example, in i.r. and Raman spectroscopic studies $[8,9]$, "free ions", ion pairs and ion clusters have been observed. The investigation of ion-ion interactions is very important, because they can change the properties of carriers, not only in the concentration and charge, but also the mobilities and even mechanisms for ionic conduction. The techniques of ten employed to investigate the ion-polymer and ionion interactions are conductivity measurements and spectroscopic methods including vibrational spectroscopy, nuclear magnetic resonance etc. 


\section{1-1-2 Structure and Morphology}

High molecular weight PEO is a semicrystalline material whose crystal structure is based on TTGTT $\bar{G}$ helix [10], which melts at about $60^{\circ} \mathrm{C}$. When salts are dissolved in PEO, a multi-phase system is often formed, containing, for example, crystalline complexes with fixed stoichiometry, crystalline host polymer and amorphous phases. The existence of phases and the phase transition temperatures have been recorded by thermal analysis and $\mathrm{X}$-ray diffraction techniques, leading eventually to the constitution of phase diagrams for many polymer electrolytes. The phase diagram of $\mathrm{PEO}-\mathrm{LiClO}_{4}$ electrolyte is shown in Figure 1-1 [11]. The crystal structures of PEO-salt complexes have been derived [12] by the X-ray diffraction techniques. A crystal structure for PEO$\mathrm{NaClO}_{4}[13]$ is illustrated in Figure 1-2.

Currently, many studies of structure and morphology are focused on the polymer electrolytes based on multivalent salts. Due to the complexities of the multivalent cations, especially those of transition metal ions, understanding is still limited [14].

Because in the crystalline region the segmental motion which controls the ion transport in polymer electrolytes is constrained, ionic conduction mainly occurs in the amorphous region [15]. Thus, understanding of the amorphous region is very important. Although the investigation of structures of complexes proves to be more difficult in the amorphous than in the crystalline region, the structures of solvated species in the amorphous region have been observed for some systems by vibrational spectroscopy [16], extended X-ray absorption fine structure spectroscopy $[17,18]$ and nuclear magnetic resonance [19] techniques.

In order to depress the crystallisation of polymer electrolytes and thus increase the ionic conductivity, much work has been focused on the development of polyme: 
hosts which have lower glass transition temperatures and which are amorphous at ambient temperature, which has led to forming amorphous salt-polymer complexes in many systems. For example, introducing irregularity into the segment sequences leads to the formation of amorphous polymer, such as methoxy-linked poly (ethylene oxide) [20]; crosslinking short chain amorphous PEO leads to amorphous polymer networks $[21,22]$ as does choosing a flexible backbone with short PEO which leads to amorphous comb-like polymers $[23,24]$ etc. Polymer electrolytes based on such polymer hosts have displayed relatively high ionic conductivity at ambient temperatures.

In addition to the development of polymer hosts, some efforts have been devoted to choosing suitable salts, or additives to give polymer electrolytes high conductivity either by depressing the crystallisation and / or by increasing the mobility of segments. For example, the ionic conductivity of polymer electrolytes based on PEO and salts with $\mathrm{N}\left(\mathrm{SO}_{2} \mathrm{CF}_{3}\right)_{2}{ }^{-}$anions is high, due to depressing the crystallisation of the electrolyte [25]; it has also been found that the crystallisation of electrolytes was depressed by adding $\mathrm{Al}_{2} \mathrm{O}_{3}$ [26]; the ionic conductivity has been increased drastically by adding low molecular weight plasticizers, such as propylene carbonate, crown ether and low molecular weight PEG etc.[27,28], which not only effectively depressed the crystallisation of the polymer electrolytes but also reduced the glass transition temperatures.

\section{1-1-3 Ion Transport}

In conventional electrolyte solutions, solvated ions which have fast or slow solvent exchange rates can move in an electric field. The diffusion coefficients of these ions are inversely proportional to the viscosity of the solution. In the inorganic solid state ionic conductors, the carrier ions basically hop between vacant sites in a host lattice. 
In polymer electrolytes, cations are coordinated by polar atoms in the polymer chains. Thus, the solvated ions cannot undergo macroscopic motion with their solvation sheath as in the conventional liquid electrolyte solutions. Segmental motion is very fast in polymer systems, but this is a short range motion. In polymer electrolytes, if the exchange rate of solvation sheath for cations is fast, long range ion transport can occur associated with the segmental motion of the host polymer, together with occasional jumps between polymer strands. It has been well accepted that ionic conduction in polymer electrolytes is basically controlled by the segmental motion.

The mobility of segments is related to the characteristics of polymer hosts and the ion-polymer interactions. Usually, it has been found that the lower is the glass transition temperature of the polymer host, the higher will be the mobility of the segments [29]. Dissolved salt in polymer electrolyte has been found to cause "transient crosslinking" [30], which leads to increasing the glass transition temperature, and hence reduces the mobility of segments.

The ion conductive behaviour in the amorphous region of a polymer electrolyte has been found to be described by empirical equations of the Vogel-Tamman-Fulcher (VTF) type [31]: $\sigma=\sigma_{0}\left(-B / T-T_{0}\right)$, where $\sigma_{0}, B$ and $T_{0}$ are fitting constants and $T$ is the temperature. Many models have been proposed so far to explain such behaviour. These models can be basically classified as macroscopic, such as the free volume model, or microscopic, such as the dynamic bond percolation model (DBP model). The former models are based on pseudo-thermodynamic arguments, and the latter are based on descriptions at the atomic level. Although these models can usually describe the conductive behaviour quite well, they still have some disadvantages as discussed in Chapter 2. 
Ion transport has been investigated by many techniques, which basically can be classified into two categories: (i) electrochemical methods and (ii) spectroscopic methods. The first type methods include:

- direct current technique, by dc polarisation of a symmetric two electrode cell $[32,33]$; Hittorf and Tubadt methods to measure the transference numbers of ions [34,35]; cyclic voltammetry and chronopotentiometry at microelectrodes to measure the diffusion coefficient of the cation in some electrolytes systems [36],

- ac techniques $[37,38]$.

The second type methods include

- vibrational spectroscopy: the concentration of species, and polymer-ion interactions in polymer electrolytes have been investigated by Raman spectroscopy [39], and infrared spectroscopy [40],

- nuclear magnetic resonance spectroscopy: the segmental motion of polymer can be investigated by ${ }^{1} \mathrm{H}-\mathrm{NMR}$, for example, by measuring $T_{1}$ and $T_{2}$, the correlation time for the segmental motion has been derived $[41,42]$; the motion of some ions, such as ${ }^{7} \mathrm{Li}$ ions and ${ }^{19} \mathrm{~F}$ ions can also be investigated by the above method; the diffusion coefficient of NMR active ions or species can be measured by pulsed field gradient NMR $[43,44]$,

- Brillouin scattering and dielectric relaxation spectroscopy: these two methods have been employed to investigate the segmental motion of polymer electrolytes. by which the ion transport process have been discussed $[45,46]$.

So far, the understanding of the transport properties of polymer electrolytes based on multivalent salts has been much less than the monovalent salts systems. This is 
not only because the complexities in the preparation, structure and morphology, but also the limitation of research techniques. For example, common electrochemical methods cannot readily be applied to the studies of polymer electrolytes based on calcium and magnesium salts, due to the existence of very resistive passivating layers on calcium or magnesium electrodes.

\section{1-1-4 Applications}

Polymer electrolytes have attracted considerable attention since they were recognised over a decade ago, because of their potential applications. Polymer electrolytes have many advantages over the conventional electrolyte solutions and inorganic solid state ionics in terms of their application to batteries.

First of all, polymer solvents can give a large electrochemical window. Simple polyethers are particularly stable, because there are only strong $\mathrm{C}-\mathrm{O}, \mathrm{C}-\mathrm{H}$ and $\mathrm{C}-\mathrm{C}$ bonds. For $\mathrm{PEO}-\mathrm{LiClO}_{4}$ electrolytes, an electrochemical window more than $4 \mathrm{~V}$ has been reported, and for $\mathrm{PEO}-\mathrm{LiCF}_{3} \mathrm{SO}_{3}$ electrolytes, a window of $3.8 \mathrm{~V}$ has been reported [47]. Secondly, unlike inorganic solid state ionic conductors, polymer electrolytes are compliant in nature and are able to accommodate volume changes associated with the cell cycling without progressive deterioration of interfacial contacts. Thirdly, because polymer chains cannot undergo macroscopic motion, there is no coinsertion of solvent in reversible intercalation cathodes. The drawback of the relatively low conductivity of polymer electrolytes can be compensated by preparing very thin polymer electrolytes films with larger area.

Prototype polymer electrolyte lithium batteries have been prepared. Current

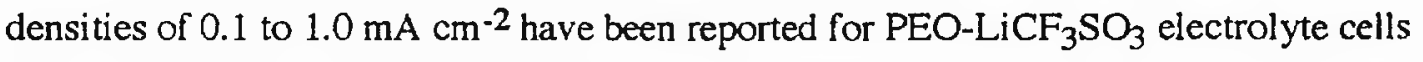
with $\mathrm{Li}$ anode and $\mathrm{V}_{6} \mathrm{O}_{13}$ or $\mathrm{TiS}_{2}$ as cathode $[48,49]$, and energy efficiencies and specific power of 70 to $80 \%$ and 100 to $300 \mathrm{~W} \mathrm{dm}^{-3}$ respectively. 
In addition to their application to batteries, polymer electrolytes can also be used in other electric devices. Polymer electrolytes with $\mathrm{Li}^{+}, \mathrm{Na}^{+}$or $\mathrm{H}^{+}$conduction have been applied to electrochromic devices [50]. Polymer electrolytes have also been applied to the fuel cells at ambient temperature [51]. The prospect of applications in addition to these may also include (i) modified electrodes / sensors, (ii) solid state reference electrode systems, (iii) supercapacitors, (iv) thermoelectric generators, (v) high vacuum electrochemistry and (vi) electrochemistry switching [52].

\section{1-2 Work in This Thesis}

Five years ago, Vincent [1] suggested that research in the field of polymer electrolytes could be divided into the following areas:

(i) experimental and theoretical investigation directed towards an understanding of the basic ion-polymer and ion-ion interactions involved in the formation of polymer electrolytes,

(ii) synthesis of new polymers in order to form polymer electrolytes with optimised electrical, chemical and mechanical properties, together with a search for new polymer / electrolyte combinations,

(iii) characterisation of the ion transport process and the development of the models for its description ,

(iv)studies of electrochemical cells based on polymer electrolytes, and development of practical devices based on them. 
In this thesis, research is focused mainly on the investigation of ion-ion, ionpolymer interactions and ion transport mechanisms in polymer electrolytes based on monovalent and divalent salts. The following are included:

(1) Investigation of the ion-ion, ion-polymer interactions and ion transport in polymer electrolytes based on amorphous methoxy-linked PEO (PMEO) and lithium salts. The dynamics of ion transport has been studied by conductivity measurements of the polymer electrolytes with the salt concentration down to $7 \times 10^{-4} \mathrm{~mol} \mathrm{dm}^{-3}$. Models for the description of ion transport have been examined.

(2) Ion-ion and ion-polymer interactions have been investigated by solid state NMR spectroscopy.

(3) Ion diffusion has been studied by pulsed field gradient NMR, and a mechanism for ion transport has been proposed.

(4) Polymer electrolytes based on PMEO and $\mathrm{Zn}\left(\mathrm{CF}_{3} \mathrm{SO}_{3}\right)_{2}$ salt have been characterised. Ion transport has been investigated by dc polarisation and Hittorf methods.

(5) Polymer electrolytes based on $\mathrm{PEO}$ and $\mathrm{Ca}\left(\mathrm{CF}_{3} \mathrm{SO}_{3}\right)_{2}$ and $\mathrm{Mg}\left(\mathrm{CF}_{3} \mathrm{SO}_{3}\right)_{2}$ salts have been characterised. The electrode-electrolyte interfaces have been investigated by electrochemical methods, in order to develop a suitable electrode for dc experiments with two electrode cells. The ion transport was subsequently investigated by the dc polarisation technique. The results suggest that calcium species in polymer electrolytes based on the high molecular weight PEO may be mobile, while the magnesium species may be immobile. When the molecular weight of polymer is low enough, magnesium species may be mobile. 
(6) The influence of the molecular weight of the polymer on ion transport has been studied theoretically and experimentally. When the molecular weight of polymer is very high, there are no significant macroscopic (centre of gravity) motion of polymer chains, because they are constrained by entanglements. When the molecular weight of polymer is lower than a critical value, significant diffusion of the polymer chains may be expected as described by the Rouse model. By dc polarisation of $\mathrm{PEO}-\mathrm{Mg}\left(\mathrm{CF}_{3} \mathrm{SO}_{3}\right)_{2}$, $\mathrm{PEO}-\mathrm{LiCF}_{3} \mathrm{SO}_{3}$ electrolytes with different molecular weight of PEO, theoretical predictions have been examined and confirmed, and the critical value of the molecular weight of polymer has been determined. This has been further supported using the ${ }^{7} \mathrm{Li}$ pulsed field gradient NMR technique.

(7) In addition to the above work, description of the preparation of high purity amorphous polymer host and polymer electrolytes is also included.

(8) Some background for ion transport, electrochemical and nuclear magnetic resonance techniques is presented. 


\section{References}

[1] C. A. Vincent, Pro. Solid State Chem., 17, 145 (1987).

[2] R. D. Lundberg, J. Polym. Sci., Part A, 4, 1563 (1966).

[3] J. Moacanin, E. F. Cuddihy, J. Polym. Sci., C, 14, 313 (1966).

[4] D. E. Fenton, J. M. Parker and P. V. Wright, Polymer, 14, 589 (1973).

[5] M. B. Armand, J. M. Chabagno and M. Duclot, Second Intenational Coference on Solid Electrolytes, St. Andrews, 1978, paper 6.5.

[6] M. B. Armand, Adv. Mater, 2, 281 (1990).

[7] F. Defendini, M. B. Armand, W. Gorecki and C. Berthier, Proceedings, Electrochemical Society Meeting, San Diego, 1986, page 893.

[8] R. Frech, J. Manning, D. Teeters and B. E. Black, Solid St. Ionics, 28-30, 954 (1988).

[9] S. Schantz, J. Sandahl, L. Borjesson, L. M. Torell and J. R. Stevens, Solid St. Ionics, 28-30, 1047 (1988).

[10] Y. Takahashi, H. Tadokoro, Makromol., 6, 672 (1973).

[11] C. D. Robitaille and D. Fauteux, J. Electrochem. Soc. 133, 315 (1986). 
[12] Y. Chatani and S. Okamura, Polymer, 28, 1815 (1987).

[13] P. Lightfoot, M. A. Mehta and P. G. Bruce, J. Mater. Chem., 2, 379 (1992).

[14] G. C. Farrington and R. G. Linford, in Polymer Electrolyte Reviews 2, eds. J. R. MacCallum and C. A. Vincent, Elsevier, London, 1989, page 255.

[15] C. Berthier, W. Gorecki, M. Minier, M. B. Armand, J. M. Chabagno and P. Rigaud, Solid St. Ionics, 11, 91 (1983).

[16] R. Dupon, B. L. Papke, M. A. Ratner, D. H. Whitemore and D. F. Shriver, J. Amer. Chem. Soc., 104, 6247 (1982).

[17] C. R. A. Catlow, A. V. Chadwick, G. N. Greaves, L. M. Moroney and M. R. Worboys, Solid St. Ionics, 9 / 10, 1107 (1983).

[18] K. Andrews, M. Cole, R. J. Latham, R. G. Linford, H. M. Williams and B. R. Dobson, Solid St. Ionics, 28-30, 929 (1988).

[19] S. G. Greenbaum, Solid St. Ionics, 15, 259 (1985).

[20] J. R. Craven, R. H. Mobbs, C. Booth and J. R. M. Giles, Makromol. Chem., Rapid Commun., 7, 81 (1986).

[21] A. Killis, J. F. LeNest, A. Gandini and H. Cheredame, J. Polym. Sci., Polym., Phys. Ed., 19, 1073 (1981). 
[22] M. Watanabe, K. Sanui, N. Ogata, F. Inoues, T. Kobayashi and Z. Ohataki, Polym. J., 16, 711 (1984).

[23] J. M. G. Cowie and A. C. S. Martin, Polymer, 28, 627 (1987).

[24] P. M. Blonsky, D. F. Shriver, P. E. Austin and H. R. Allcock, J. Amer. Chem. Soc., 106, 6854 (1984).

[25] M. B. Armand, M. Gauthier and R. Andreani, in Second International Symposium on Polymer Eelectrolytes, ed. B. Scrosati, Elsevier, London, 1990, page 187.

[26] F. Croce, F. Bonimo, S. Panero and B. Scrosati, Phil. Mag. B 59, 161 (1989).

[27] G. Nagasubramanian and S. Di Stefano, J. Electrochem. Soc., 137, 3830 (1990).

[28] J. Kelly, J. R. Owen and B. C. H. Steele, J. Electroanal. Chem. Interfacial Electrochem., 168, 467 (1984).

[29] J. D. Ferry, Viscoelastic Properties of Polymers, Wiley, New York, 1970, 2nd ed.

[30] H. Cheradame and J. F. Le Nest, in Polymer Electrolyte Reviews 1, eds. J. R. MacCallum and C. A. Vincent, Elsevier, London, 1987, page 103.

[31] M. B. Armand, J. M. Chabagno and M. J. Duclot, in Fast-Ion Transport in Solids, eds. P. Vashita, J. N. Mundy and G. Shenoy, North-Holland, Amsterdam, 1979, page 131.

[32] P. G. Bruce and C. A. Vincent, J. Electroanal. Chem., 225, 1 (1987). 
[33] M. Watanabe, S. Nagano, K. Sanui and N. Ogata, Solid St. Ionics, 28-30, 911 (1988).

[34] M. Leveque, J. F. Le Nest, A. Gandini and H. Cheradame, J. Power Sources, 14,27 (1985).

[35] P. G. Bruce, M. T. Hardgrave and C. A. Vincent, Solid St. Ionics, 53-56, 1087 (1992).

[36] H.S. Choe and G. C. Farrington, Solid St. Ionics, 57, 35 (1992).

[37] P. R. Sorensen and T. Jacobsen, Electrochim. Acta, 27, 1671 (1982).

[38] F. M. Gray, Solid St. Ionics, 40 / 41, 637 (1990).

[39] B. L. Papke, M. A. Ratner and D. F. Shriver, J. Phys. Chem. Solids, 42, 493 (1981).

[40] D. Teeters and R. Frech, Solid St. Ionics, $18 / 19,271$ (1986).

[41] W. Gorecki, R. Andreani, C. Berthier, M. B. Armand, M. Mali, J. Roos and D. Brinkmann, Solid St. Ionics, 18 / 19, 295 (1986).

[42] D. P Tunstall, A. S. Tomlin, J. R. MacCallum and C. A. Vincent, J. Phys. C.. 21, 1039 (1988).

[43] M. Minier, C. Berthier and G. Gorecki, J. Phys. (Paris), 45, 739 (1984). 
[44] S. Bhattacharja, S. W. Smoot and D. H. Whitmore, Solid St. Ionics, 18 / 19 , 306 (1986).

[45] J. Sandahl, S. Schantz, L. M. Torell and R. Frech, Solid St. Ionics, 28-30, 958 (1988).

[46] F. M. Gray, C. A. Vincent and M. Kent, Solid St. Ionics, 28-30, 936 (1988).

[47] M. B. Armand, M. J. Duclot and P. Rigaud, Solid St. Ionics, 3 / 4, 429 (1981).

[48] A. Hooper and J. M. North, Sold St. Ionics, 9/10, 1161 (1983).

[49] M. Gauthier, D. Fauteux, G. Vassort, A. Belanger, M. Duval, P. Ricoux, J. M. Chabagno, D. Muller, P. Rigaud, M. B. Armand and D. Deroo, J. Electrochem. Soc, 132, 1333 (1985).

[50] O. Bohnke, C. Bohnke and S. Amal, Mater. Sci. Eng., B3, 197 (1989).

[51] M. S. Wilson and S. Gottesfeld, J. Appl. Electrochem., 22, 1 (1992).

[52] C. A. Vincent, Chem. in Britain, 391 (1989) 


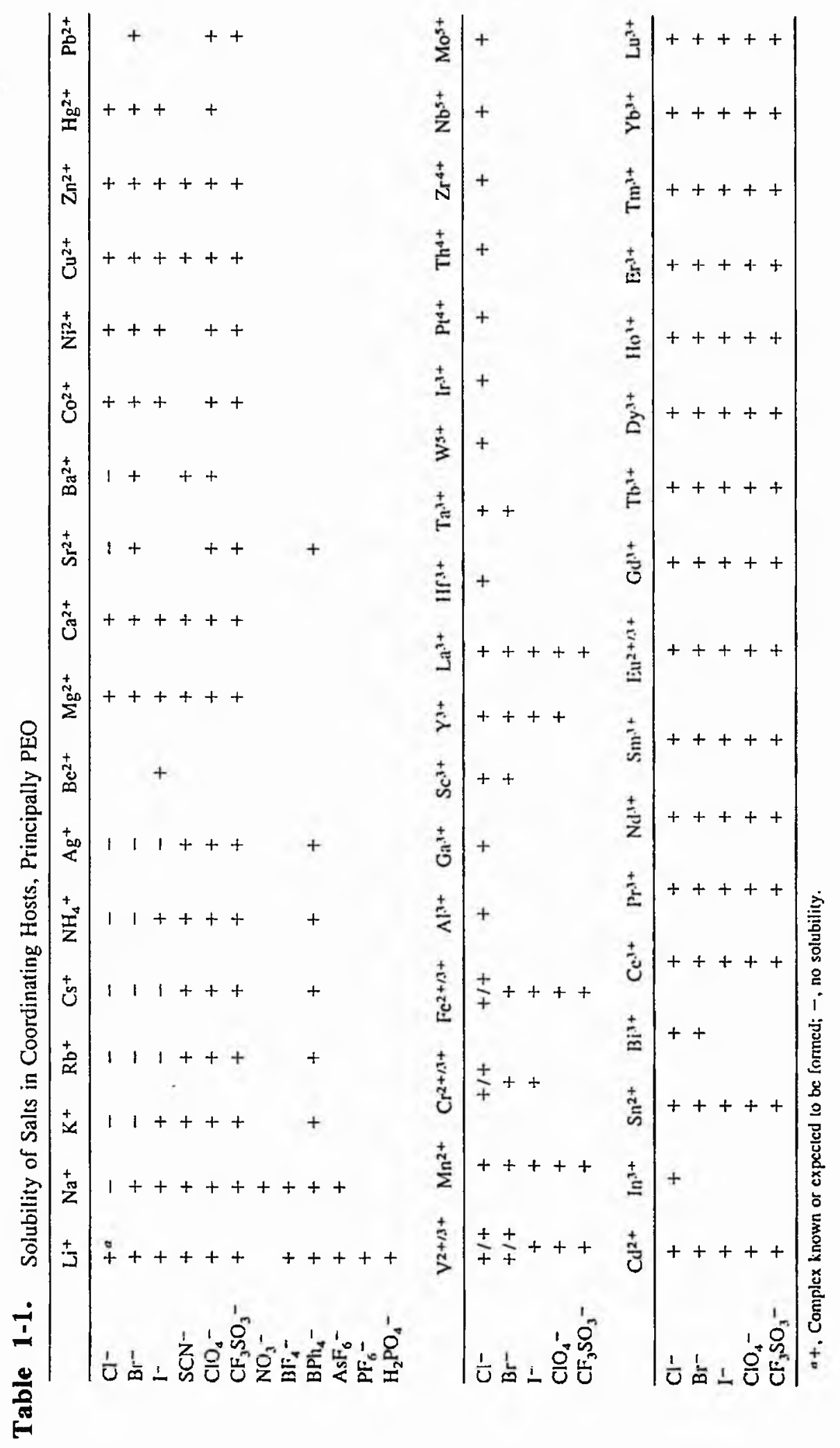




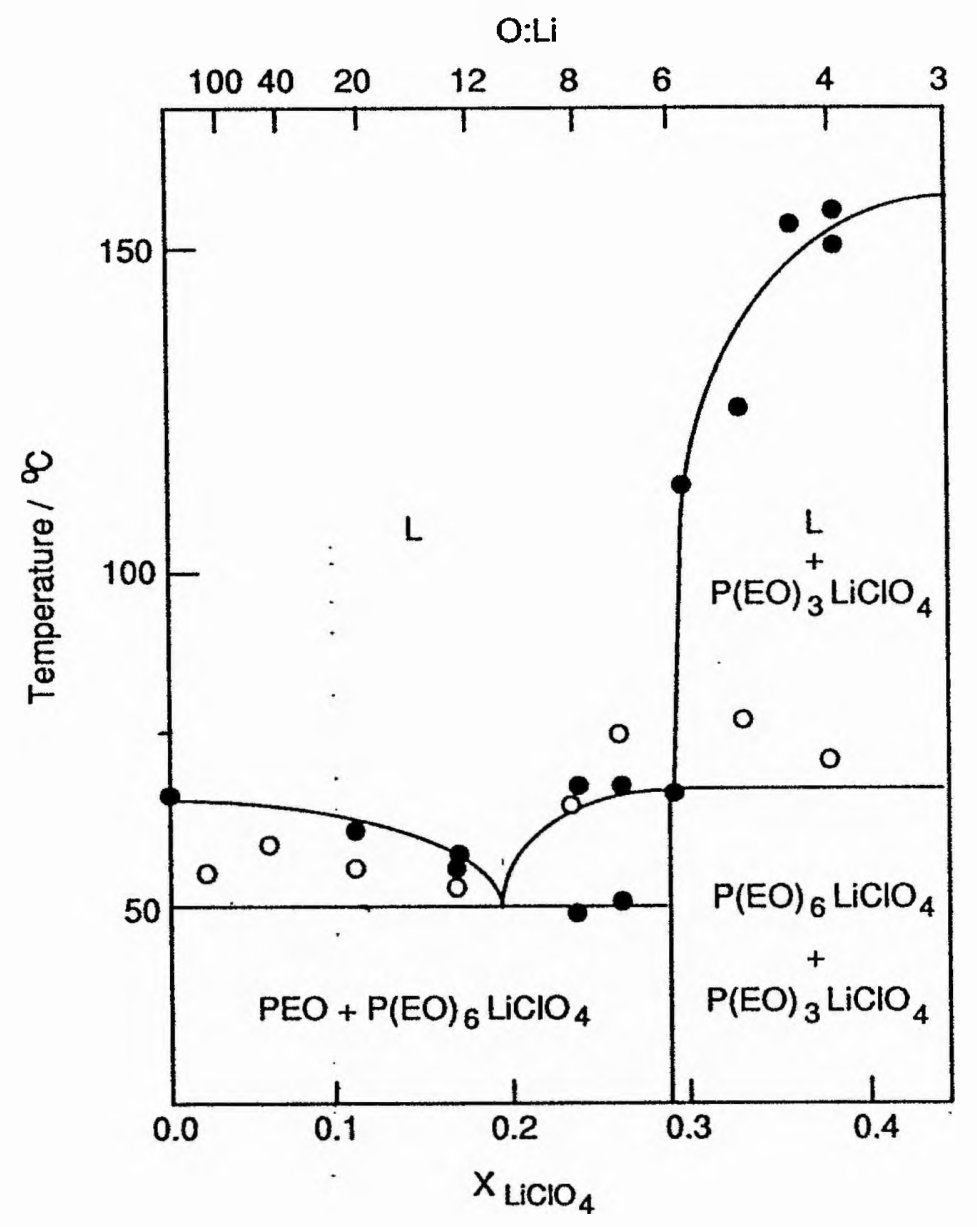

Figure 1-1 Phase diagram of $\mathrm{PEO}-\mathrm{LiClO}_{4}$ electrolyte. 

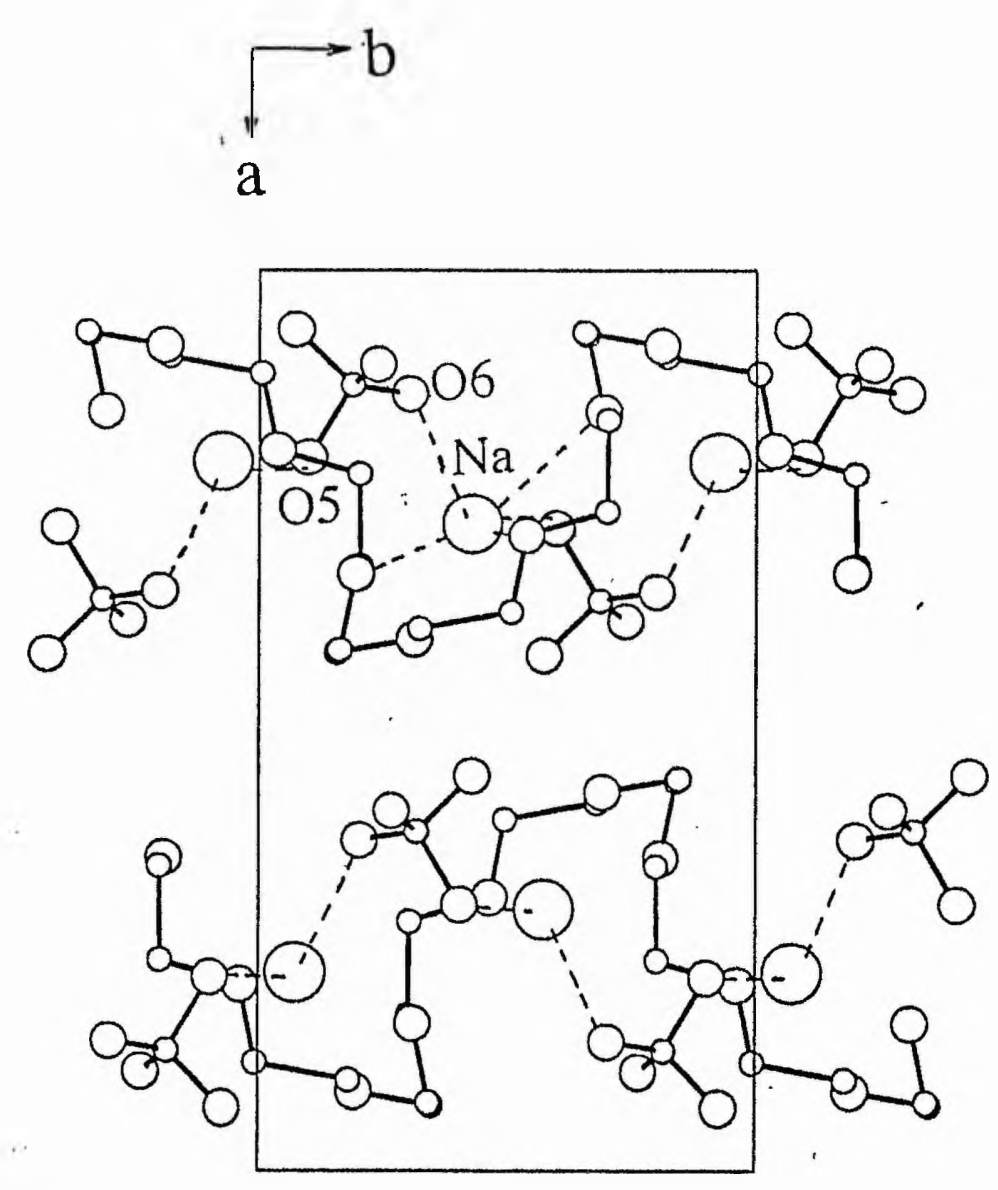

Figure 1-2 Crystal structure of $\mathrm{PEO}-\mathrm{NaClO}_{4}$ electrolyte. 


\section{CHAPTER 2}

\section{DYNAMIC MODELS AND THEORIES FOR IONIC CONDUCTION IN POLYMER ELECTROLYTES}

Unlike conventional inorganic solid state ionic conductors, solid polymer electrolytes display liquid-like behaviour on the microscopic scale, due to the shortrange segmental motion of the polymer. On the macroscopic scale, the entanglements between the polymer chains restrict their long-range motion, and these materials may behave as solid materials. The liquid-like segmental motion is believed to control the ionic conduction in polymer electrolytes based on high molecular weight polymers.

The models which have been proposed so far to describe the dynamics of ionic conduction in polymer electrolytes can be classified into two categories, according to the level of properties concerned. One type, based on the observation of macroscopic properties, such as fluidity, are known as macroscopic models. Another type, based on molecular properties, such as the ion hopping rate, segmental renewal time etc., are called microscopic models. 


\section{2-1 Macroscopic Models}

\section{2-1-1 Empirical Equations}

It has been found that the temperature dependence of the ionic conductivity can often be fitted to the Vogel-Tammann-Fulcher (VTF) equation [1] and the WilliamsLandel-Ferry (WLF) equation [2], for amorphous polymer electrolytes at a temperature above the glass transition temperatures, $\mathrm{T}_{\mathrm{g}}$.

The VTF equation was originally developed to describe the viscosity properties of supercooled liquids. The temperature dependence of viscosity can be written as [3]:

$$
\eta=C \exp \left[\frac{B}{T-T_{0}^{\prime}}\right]
$$

where $C \propto T^{1 / 2}, B$ and $T_{o}$ are fitted parameters. By combining the Stokes-Einstein equation (2-1-2) and the Nernst-Einstein equation (2-1-3), and assuming the number and charge of carriers to be invariant with changing temperature, the VTF equation (2-14) for the conductivity can be deduced.

$$
D=\frac{k T}{6 \pi R \eta}
$$

where $\mathrm{D}$ is the diffusion coefficient, $\mathrm{k}$ is the Boltzmann constant, $\mathrm{T}$ is the temperature, and $R$ the radius of molecules.

$$
\sigma=\frac{\mathrm{nq}^{2}}{\mathrm{kT}} \mathrm{D}
$$

where $\mathrm{n}$ is the number in unit volume and $\mathrm{q}$ is the charge of carriers. 


$$
\sigma=\sigma_{\mathrm{o}} \exp \left[\frac{-\mathrm{B}}{\mathrm{T}-\mathrm{T}_{\mathrm{o}}}\right]
$$

where $B$ and $T_{0}$ have the same meanings as before, $\sigma_{0}$ is a fitted parameter which may contain a term in $\mathrm{T}^{1 / 2}$.

Williams, Landel and Ferry [4] considered the relaxation processes in glassforming materials. They found that the shift factor $\alpha_{\mathrm{T}}$, the ratio of any mechanical relaxation process at temperature $T$ to its value at some reference temperature $T_{s}$, can be expressed by an equation, now known as WLF equation:

$$
\begin{aligned}
\log \alpha_{T} & =\text { const. }+\log \left[\frac{\eta(T)}{\eta\left(T_{S}\right)}\right] \\
& =\frac{-C_{1}\left(T-T_{S}\right)}{C_{2}+T-T_{S}}
\end{aligned}
$$

where $C_{1}, C_{2}$ are constant, $T_{s}$ is a reference temperature which is usually $50 \mathrm{~K}$ higher than $T_{g}$. Empirically, it is found: $C_{2} \approx 102 \mathrm{~K}, C_{1} \approx 8.9$. The WLF equation (2-1-5) can be converted into the $\operatorname{VTF}(2-1-1)$ and $(2-1-4)$ by choosing $C_{2}=T_{s}-T_{0}$.

\section{2-1-2 Free Volume Model}

The free volume model of ionic conduction in polymer electrolytes considers the dynamic properties of the polymer rather than those of ions themselves. The basic assumption proposed by Cohen and Turnbull [5] is that the diffusion of molecules is not activated but occurs as a result of the redistribution of free volume. The free volume per molecule $v_{\mathrm{f}}$ is defined as: $v_{f}=\bar{v}-v_{0}$, where $\bar{v}$ is the average volume per molecule in the sample and $v_{0}$ is the van der Waals volume. By assuming that transport can creur only if the free volume of the molecules studied exceeds some critical value $v^{* *}$. the 
diffusion coefficient can be written as:

$$
\mathrm{D}=\int_{v^{*}}^{\infty} \mathrm{D}_{(v)} \mathrm{P}_{(v)} \mathrm{dv}
$$

where $D_{(v)}$ is the diffusion coefficient for a molecule in a cage of free volume $v$, and $P_{(v)}$ is the probability of finding the free volume $v$ between $v$ and $v+d v . P_{(v)}$ can be derived by maximizing the number of ways of distributing the free volume. Eventually, the viscosity can be written as:

$$
\eta=A^{\prime} \exp \left(\frac{\gamma v *}{v_{f}}\right)
$$

where $\gamma$ is a Lagrange parameter, $\mathrm{A}^{\prime}$ is a constant. By assuming that the free volume is linearly expanded with temperature, the above equation may converted to the VTF form:

$$
\eta=A^{\prime} \exp \left(\frac{B}{T-T_{0}}\right)
$$

where $B=\gamma v^{*} / \alpha \bar{v}$ ( $\alpha$ is the thermal expansivity) and $T_{0}$ is a temperature at which the free volume vanishes. The WLF equation can also derived from equation (2-1-8), by taking: $T_{s}=C_{2}+T_{0}$. As before, the VTF equation for conductivity (2-1-4) can be derived.

In this model, transport is controlled by the availability of free volume in the system. Hence, ionic conduction is also assumed to be controlled by the redistribution of free volume (hereafter called the "free volume process") in polymer electrolytes. 


\section{2-1-3 Configuration Entropy Model}

Although the free volume model can describe many properties of polymer electrolytes quite successfully, there are still some weakness. One of them is that the model ignores the kinetic effects associated with polymers. This difficulty can be more or less overcome by applying the configurational entropy model developed by Gibbs and coworkers [6] to describe transport-related properties of polymers.

Note that again this model considers the properties of polymer rather those of ions in the polymer electrolyte. Mass transport in this model is assumed to be controlled by group co-operative rearrangement of the polymer chains. The average probability of a rearrangement is given as:

$$
\bar{W}=A \exp \left(\frac{-\Delta \mu s_{c}{ }^{*}}{k T S_{c}}\right)
$$

where $\mathrm{A}$ is a constant, $\mathrm{s}_{\mathrm{c}}{ }^{*}$ is the minimum configurational entropy required for rearrangement, of ten taken as: $s_{c}{ }^{*}=k \ln 2, s_{c}$ is the configurational entropy at the temperature $T$, and $\Delta \mu$ is the free energy barrier per mole which impedes the rearrangement. The configurational entropy $S_{c}$ becomes very small at the glass transition temperature $T_{g}$, because of the great increase in the segmental relaxation times. The configurational entropy will eventually vanish at a temperature $T_{0}$ which is usually $50 \mathrm{~K}$ lower than $\mathrm{T}_{\mathrm{g}}$.

By relating $\bar{W}$ to the inverse relaxation time, and assuming that the heat capacity difference between the liquid and glass state $\Delta C_{p}$ is temperature independent, a relationship of the WLF form can be deduced:

$$
\log \alpha_{T}=\frac{-a_{1}\left(T-T_{s}\right)}{a_{2}+T-T_{s}}
$$


where $a_{1}$ and $a_{2}$ are defined as:

$$
\begin{aligned}
& a_{1}=\frac{2.303 \Delta \mu \mathrm{s}_{\mathrm{c}}{ }^{*}}{\Delta \mathrm{C}_{\mathrm{p}} \mathrm{k} \mathrm{T}_{\mathrm{s}} \ln \left(\frac{\mathrm{T}_{\mathrm{s}}}{\mathrm{T}_{\mathrm{o}}}\right)} \\
& \mathrm{a}_{2}=\frac{\mathrm{T}_{\mathrm{s}} \ln \left(\frac{\mathrm{T}_{\mathrm{s}}}{\mathrm{T}_{\mathrm{o}}}\right)}{1+\ln \left(\frac{\mathrm{T}_{\mathrm{s}}}{\mathrm{T}_{\mathrm{o}}}\right)}
\end{aligned}
$$

$a_{2}$ is slightly temperature dependent. If $\ln \left(T / T_{0}\right) \approx\left(T-T_{0}\right) / T_{0}$, and $T / T_{0} \approx 1, z$ VTF form of equation can be deduced from equation (2-1-10):

$$
\bar{W}=A \exp \left(\frac{-K_{\sigma}}{T-T_{0}}\right)
$$

where $\mathrm{K}_{\sigma}=\frac{\Delta \mu \mathrm{s}_{\mathrm{c}}{ }^{*}}{\mathrm{k} \Delta \mathrm{C}_{\mathrm{P}}}$. By assuming $\Delta \mathrm{C}_{\mathrm{P}}=\mathrm{B}_{1} / \mathrm{T}\left(\mathrm{B}_{1}\right.$ is a constant $)$, and using the relationship: $\eta_{(T)} \propto \bar{W}^{-1}$, the VTF equation for viscosity can be deduced:

$$
\eta=A \exp \left(\frac{K_{\sigma}}{T-T_{0}}\right)
$$

where $K_{\sigma}=\Delta \mu s_{c} * T_{o} / k B_{1}, A$ is a constant. Again, the VTF equation (2-1-4) for the conductivity can be derived.

In the configurational entropy model, the ionic conduction in polyme: 
electrolytes is related to the change of free energy of configurational transition of the polymer host required to permit the motion of the ions.

\section{2-2 Microscopic Models}

The macroscopic models can describe the temperature dependence of some transport properties, such as conductivity, viscosity and diffusivity etc.in polymer electrolytes reasonably well in a broad temperature range, but they lack any mechanistic interpretation. An alternative model, called the dynamic bond percolation (DBP) theory was developed by Ratner and coworkers $[7,8]$ to describe the ionic conduction in polymer electrolytes by considering microscopic properties.

For ionic motion in an immobile framework, the motion of carriers is defined as:

$$
\dot{P}_{i}=\sum_{j}\left(P_{j} W_{j i}-P_{i} W_{i j}\right)
$$

where $P_{i}$ is the probability of finding the carrier at site $i$ at time $t$, and $W_{j i}$ is the rate at which the carrier hops from site $\mathrm{j}$ to site $\mathrm{i}$. It is assumed that the hopping rate of a carrier from site $\mathrm{i}$ to a neighbouring vacant site $\mathrm{j}$ is $\mathrm{w}$, and that to the other sites is zero, i.e.,

$$
W_{i j}= \begin{cases}W & \text { probability }=\mathrm{f} \\ 0 & \text { probability }=1-\mathrm{f}\end{cases}
$$

where $f$ represents the fraction of "bonds" or pathways for motion between sites, which are open, while (1- $)$ is the fraction which are closed.

Due to the liquid-like segmental motion in polymer electrolytes, the fixed lattice 
concept is no longer valid at a temperature above the glass transition temperature $T_{g}$. Thus, in addition to the hopping rate $w$ and fraction of available bonds $f$, a new parameter $\lambda$, a characteristic change rate between opening and closing bonds, is introduced. $\lambda=1 / \tau_{T}$, where $\tau_{T}$ is the renewal time, which is a characteristic parameter for segmental motion of the polymer.

From this model, the following principal results can be derived:

(i) for the observation time $t_{o b s} \gg \tau_{T} \gg 1 / W$, at any value $f>0$, the system will be diffusive;

(ii) if the renewal time is very fast, i.e., $1 / W \gg>\tau_{\mathrm{T}}$, the motion corresponding to hopping in a homogeneous system, with an effective hopping rate of $\mathrm{w} \mathrm{x}$; when the renewal rate is very slow, $1 / w \ll \tau_{\mathrm{T}}$ then the observed motion is simply that of the static bond percolation model, and the system is no longer diffusive;

(iii) the diffusion coefficient of a system undergoing dynamic percolation, $D_{\text {dy }}(\omega)$ is related to the diffusion coefficient of static percolation $D_{s t}$ by formula:

$$
D_{\text {dy }}(\omega)=D_{s t}(\omega-i \lambda)
$$

where $\omega$ is the frequency. Thus, the spectroscopic behaviour and other frequencydependent properties, such as conductivity and dielectric relaxation etc. can be described by the DBP model.

For the motion of cations, the hopping rate w between neighbouring sites may include the the rate of changing the coordination shell by breaking some bonds and forming some new bonds. Thus, if the hopping rate is very low, there may be no cationic conduction observed in polymer electrolytes.

In the DBP model, the segmental motion of polymer is characterised by the 
renewal time $\tau_{\mathrm{r}}$ It can be related to the free volume model by

$$
\tau_{r}=\left(\frac{a_{s}}{\mu_{s}}\right)\left[\exp \gamma\left(\frac{v_{s}^{*}}{v_{f}}\right)-1\right\}
$$

where $a_{s}, \mu_{s}$ and $v_{s}^{*}$ are the space, the velocity and the critical free volume of segments respectively, and $\gamma$ is an overlap factor for the free volume.

The parameter $f$ can be also related to the availability of critical free volume for transport by

$$
f=\exp \left(-\gamma^{*} / v_{\mathrm{f}}\right)
$$

Thus, $f$ increases with increasing free volume in the polymer electrolytes.

Because the renewal time can be related to the free volume model, if the renewal process is controlling, the transport of ions can be related to the free volume model. The diffusion coefficient is:

$$
D \propto a_{s} \mu_{s} \exp \left[-\gamma v^{*} / \alpha \bar{v}\left(T-T_{o}\right)\right]
$$

where $\bar{v}$ is the mean segment volume, $\alpha$ is the thermal expansitivity. By assuming that the Nernst-Einstein equation holds, the VTF-form equation (2-1-4) for conductivity can once again be derived. 


\section{2-3 Comments}

\section{2-3-1 Comments on the Macroscopic Models}

In the macroscopic models, the ionic conduction is assumed to be completely controlled by the segmental motion of polymer host. It has been found that these models can apparently describe the transport properties of ions very well in the temperature range above $T_{\mathbf{g}}$. In addition, they can easily and clearly relate the ionic conduction to the motion of polymer segments.

They, however, still have some weakness, due to their assuming that the transport of ions is the same as that of the polymer host. The conductivity of polymer electrolytes can be expressed by a general formula:

$$
\sigma=\sum_{i} n_{i} q_{i} \mu_{i}
$$

where $n_{i}$ is the number and $q_{i}$ is the charge of carrier $i, \mu_{i}$ is the mobility of carrier $i$. It is obvious that the conductivity is not only directly proportional to the mobility of carriers but also to the concentration of carriers. For strong electrolytes, because the concentration of carriers is invariant with temperature, the temperature dependence of conductivity completely arises from the temperature dependence of mobility. Under these circumstances, the assumption in the macroscopic models may be acceptable. In polymer electrolytes, however, because the dielectric constant of the polymer host is rather low, ion association may take place. Because the association equilibria may be shifted by changing temperature, the ionic conduction is also controlled by the generation of carriers. In addition, for electrolytes with high salt concentration, the transport of ions may differ from that in the dilute cases where ion transport is exclusively controlled by the segmental motion. Under this circumstance, ion transport process may be controlled both by an activated process and by the segmental motion as discussed in Chapter 5. Secondly, at temperatures below $T_{g}$, the observed conductance 
cannot be explained by these models, because the segmental motion is frozen out. Thirdly, the lability of cation-polymer bonds is not reflected in these models. Therefore, other forms of ion motion (e.g. hopping, bond exchanging) may have to be considered in addition to motion associated with the segmental motion of the polymer. Fourthly, while for high molecular weight polymer, the transport properties of polymer may be well described by the macroscopic models, under some circumstances, the transport properties are no longer exclusively dominated by the segmental motion. For example, when the molecular weight of polymer host is reduced, the conductivity is influenced by other modes of polymer motion such as reptation as described in Chapter 8. Finally; because the macroscopic models are based on pseudo-thermodynamic arguments, they cannot describe frequency-dependent transport properties (e.g. dielectric or spectroscopic behaviour).

\section{2-3-2 Comments on the Microscopic Models}

In the microscopic model (DBP model), three parameters, $w, \lambda$ and $f$, are used to describe the ionic conduction in polymer electrolytes. This model considers the ion motion, i.e. the contribution of jonic conduction arising from the ion hopping, exchanging of the coordination sheath etc., is included in the model, and frequencydependent transport properties can be described. In addition, this model can be related to the macroscopic model by assuming the segmental renewal process to be dominant in the system.

The DBP model however still has some drawbacks. Firstly, this model is developed from the bond percolation model in which a lattice is essential. Although in the DBP model a renewal process for this lattice is introduced, a lattice is still necessar: In amorphous polymer electrolytes, due to the liquid-like behaviour for segmental motion, it seems not to be very reasonable to assume the existence of a lattice. Secondly, the hopping process may be more plausible for cations than for anions. 
because anions interact very weakly with the polymer. Therefore, the overall ionic conductivity which arises from the transport of both cations and anions may not be well described by the hopping process. In addition, although the hopping process is assumed to relate to the exchange of cation-polymer bonds, there is no quantitative relationship available between them. Thirdly, the real physical meaning of the parameter $f$ is not very clear in the DBP model, though it is assumed that $\mathrm{f}$ can be related to the critical free volume for transport in the polymer electrolytes. Finally, information on the correlation of these three parameters to the measurable properties is so far not well developed. 


\section{References}

[1] M. B. Armand, J. M. Chabagno and M. J. Duclot, in Fast-Ion Transport in Solids, ed. P. Vashishta, J. N. Mundy and G. Shenoy, North-Holland, Amsterdam, 1979, page 131 .

[2] A. Killis, J. F. Le Nest, H. Cheradame and A. Gandini, Makromol. Chem., 183, 2835 (1982).

[3] H. Vogel, Phys. Z., 22, 645 (1921).

[4] M. L. Williams, R. F. Landel and J. D. Ferry, J. Amer. Chem. Soc., 77, 3701 (1955).

[5] M. H. Cohen and D. Turnbull, J. Chem. Phys., 31, 1164 (1959).

[6] J. H. Gibbs and E. A. Di Marzio, J. Chem. Phys., 28, 373 (1958).

[7] S. D. Druger, A. Nitzan and M. A. Ratner, J. Chem. Phys., 79, 3133 (1983).

[8] M. A. Ratner, in Polymer Electrolyte Reviews 1, eds. J. R. MacCallum and C. A. Vincent, Elsevier, London, 1987, page 173. 


\section{CHAPTER 3}

\section{EXPERIMENTAL TECHNIQUES: PREPARATION, CHARACTERISATION AND ELECTROCHEMICAL INVESTIGATION OF POLYMER ELECTROLYTES}

This chapter is one of two chapters describing the experimental techniques employed in this work and some of the principles of these techniques. In this chapter, the techniques and procedures for treatment of chemicals, and the preparation, modification, purification and characterisation of polymers and polymer electrolytes are described in detail. The electrochemical measurements, experimental procedures and design of electrochemical cells are also described in this chapter. Finally, a description of equipment and instrumentation employed is included.

\section{3-1 Chemical Samples}

\section{3-1-1 Metal Salts}

(1) Lithium perchlorate (Aldrich Chemical Co. Ltd.)was dried under vacuum at $150^{\circ} \mathrm{C}$ for 48 hours.

(2) Lithium trifluoromethane sulphonate (Aldrich Chemical Co. Ltd.) was dried under vacuum at $150^{\circ} \mathrm{C}$ for 48 hours.

(3) Lithium tetrafluoroborate $98 \%$ (Aldrich Chemical Co. Ltd.) was dried under vacuum at $100^{\circ} \mathrm{C}$ for 48 hours. 
(4) Lithium thiocyanate (Aldrich Chemical Co. Ltd.) was dried under vacuum at $120^{\circ} \mathrm{C}$ for 48 hours.

(5) Lithium hexafluorophosphate $98 \%$ (Aldrich Chemical Co. Ltd.) was dried under vacuum at $80^{\circ} \mathrm{C}$ for 48 hours.

(6) Magnesium trifluoromethane sulphonate (Aldrich Chemical Co. Ltd.) was dried under vacuum at $250^{\circ} \mathrm{C}$ for 72 hours.

(7) Zinc trifluoromethane sulphonate (Aldrich Chemical Co. Ltd.) was dried under vacuum at $80^{\circ} \mathrm{C}$ for 24 hours.

(8) Calcium trifluoromethane sulphonate was prepared in this laboratory. Calcium carbonate $(\mathrm{BDH})$ and concentrated trifluoromethane sulphonic acid( $98 \%$, Aldrich Chemical Co. Ltd.) were mixed with a mole ratio of about 1: 2 (calcium carbonate was actually slightly excessive) under magnetic stirring. After reaction for about 24 hours, distilled water was added to dilute the solution, which was then filtered by a sintered funnel under reduced pressure. The water was removed by a rotary evaporator. The salt produced was pre-dried at $80^{\circ} \mathrm{C}$, and was further dried under vacuum at $180^{\circ} \mathrm{C}$ for 48 hours.

All the above treated salts were stored in an argon-filled glovebox.

(9) Potassium hydroxide (Aldrich Chemical Co. Ltd.) was used as received. 


\section{3-1-2 Solvents}

(1) Acetonitrile (Aldrich Chemical Co. Ltd.) with water content of less $0.005 \%$ was stored in argon and used as received.

(2) Methylene chloride (Aldrich Chemical Co. Ltd.) had purity for HPLC use, and was used as received.

(3) Anhydrous ethanol (water $<0.005 \%$ ), toluene and heptane (Aldrich Chemical Co. Ltd.) were used as received.

\section{3-1-3 Polymers}

(1) Poly(ethylene glycol) 400 (Union Carbide Co. Ltd.) was dried under vacuum for 8 hours prior to synthesis of methoxy-linked PEO.

(2) Poly(ethylene glycol) 1000, 1450, 3350 and 8000 (Union Carbide Co. Ltd.) and poly(ethylene oxide) 4000,6000,10000, 20000 and $4 \times 10^{6}$ (BDH Ltd.) were dried under vacuum at $50^{\circ} \mathrm{C}$ for 72 hours.

(3) Poly(ethylene oxide) 400 dimethyl ether (Fluka Chemical Co. Ltd.) was firstly dried under vacuum for 8 hours, and then was further dried by storing over molecular sieves for a week.

(4) Poly (ethylene oxide) 1000 dimethyl ether and poly(ethylene oxide) 2000 dimethyl ether (Fluka Chemical Co.Ltd.) were dried under vacuum at $50^{\circ} \mathrm{C}$ for 72 hours.

All the above dried polymers were stored in an argon-filled glovebox. 


\section{3-1-4 Metals}

(1) Lithium rod (Aldrich Chemical Co. Ltd.) was cut to disc for use.

(2) Mercury, triple distilled 99.99\% (Aldrich Chemical Co. Ltd.) was used as received.

(3) Zinc foil $99.9 \%$, calcium shot $99.9 \%$, magnesium foil $99.9 \%$ and gold disc $99.99 \%$ (Goodfellow Metals Ltd.): zinc and magnesium foils were freshly scraped before use. Calcium shot were pressed into discs which were freshly scraped prior to use. Gold discs were firstly treated with concentrated nitric acid and then washed with distilled water, and finally dried.

\section{3-2 Preparation, Modification and Purification of Polymer} Host

\section{3-2-1 Preparation of Methoxy-linked Poly(ethylene oxide)}

Poly(ethylene glycol) 400 was vacuum dried with magnetic stirring for 8 hours before use. Potassium hydroxide was freshly ground into a fine powder before use.

The preparation of methoxy-linked poly(ethylene oxide), PMEO, followed the method proposed by Craven and coworkers [1] and can be expressed by:

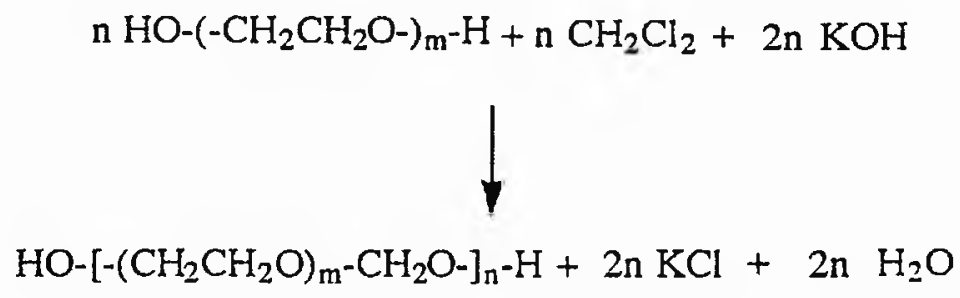

The molecular weight of PMEO is strongly influenced by the moisture level 12 the system. the amount of methylene chloride added and fineness of the $\mathrm{KOH}$ powder. Methylene chloride is not only used as reactant but also used as solvent for the reacturn. 
and exceeds the moles of PEO(400). Potassium hydroxide is used as catalyst, but also acts as reactant

In a dry $250 \mathrm{ml}$ three-neck flask, with mechanical stirring and under the protection of argon or nitrogen atmosphere (Figure 3-1), 40 grams of $\mathrm{KOH}$ was firstly added followed by $20 \mathrm{ml}$ methylene chloride. After mixed for about 2 minutes, $20 \mathrm{ml}$ $\mathrm{PEO}(400)$ was quickly added into the flask under vigorous mechanical stirring. The system became very hot and viscous, and was cooled in a water bath for the first 30 minutes. More methylene chloride was then slowly added from an equal pressure funnel into the flask to keep the system from becoming over-viscous. This process was very critical, since if the amount of methylene chloride was insufficient, a non-soluble polymer was produced; if the amount of methylene chloride was too much, a low molecular weight polymer was formed. The total amount of methylene chloride added was approximately $120 \mathrm{ml}$. After reaction for about 5 hours, a further $100 \mathrm{ml}$ of methylene chloride was added into the flask to terminate the reaction. The mixture was then transferred to a $500 \mathrm{ml}$ flask, and $200 \mathrm{ml}$ methylene chloride was added to dissolve the product. The mixture was left overnight.

The mixture was filtered through a sintered funnel twice under reduced pressure to give a clear solution. The solvent was removed on a rotary evaporator under vacuum at about $60^{\circ} \mathrm{C}$. A soft rubbery solid, slightly yellow in colour, was eventually obtained.

The above polymer was purified to remove low molecular weight products. Typically, 5 grams of the raw polymer was dissolved in $100 \mathrm{ml}$ of toluene under magnetic stirring. After complete dissolution, about $27 \mathrm{ml}$ heptane was added to the solution to form a turbid mixture, where only the high molecular weight polymer precipitated out. The mixture was heated at $70-80^{\circ} \mathrm{C}$ with magnetic stiring till the precipitate was dissolved once again. The mixture was then allowed to cool down, and 
the high molecular weight polymer re-precipitated. The precipitate and solution was separated by decanting the solution. The solvent was removed by a rotary evaporator under vacuum at about $80^{\circ} \mathrm{C}$. A colourless soft solid was obtained.

In order to further dry the polymer, it was dissolved in methylene chloride again to give a clear solution. Then this solution was rotary evaporated under vacuum at about $60^{\circ} \mathrm{C}$. The polymer was then vacuum dried at $80^{\circ} \mathrm{C}$ for 12 hours. This polymer was ready for preparation of polymer electrolytes, except for those with very low salt concentration.

The polymer treated by the above procedures was dissolved in THF and characterised by gel permeation chromatography (GPC). The GPC was calibrated by linear polystyrene. The molecular weight in weight average $\left(\overline{\mathrm{M}}_{\mathrm{w}}\right)$ and in number average $\left(\bar{M}_{n}\right)$ was 150,700 and 92,000 respectively, and the polydisperse index $\left(\bar{M}_{w} / \bar{M}_{n}\right)$ was 1.63 (Table 3-1).

A DSC trace of the polymer is showed in Figure 3-2. The glass transition temperature, $\mathrm{T}_{\mathrm{g}}$, of the polymer was $-57.1^{\circ} \mathrm{C}$. There were some crystalline phases melting in the temperature range of $-5^{\circ} \mathrm{C}$ to $0^{\circ} \mathrm{C}$. Above $10^{\circ} \mathrm{C}$, the polymer was completely amorphous.

\section{3-2-2 Further Purification of PMEO}

There were still some impurities in PMEO prepared by the above treatment. The conductivity of it was in the order of $10^{-7} \mathrm{Scm}^{-1}$ (Figure 3-3). To study polymer electrolytes with very low salt concentration, say $10^{-3} \mathrm{~mol} \mathrm{dm}^{-3}$, the conductivity of the salt-free polymer was in the range of the electrolyte. Thus, the polymer for preparation of those polymer electrolytes was further purified using the following methods. 


\section{(1) Filtration}

1 gram of polymer was dissolved in approximately $50 \mathrm{ml}$ of distilled water. The aqueous solution was filtered by a Sartorius Ultrasart Cell 50 which was able to remove residual ionic impurities and polymer fraction $<10,000$. The filtered solution was firstly distilled under reduced pressure to remove water. 5 drops of trimethyl orthoformate was then added into the polymer overnight, whereby residual water reacted with trimethyl orthoformate to form volatile substances [2]. The polymer was then further dried under vacuum at $80^{\circ} \mathrm{C}$ for 8 hours. The conductivity of the purified polymer was $1.1 \times 10^{-8}$ $\mathrm{Scm}^{-1}$ at $25^{\circ} \mathrm{C}$.

After the purification, the polydisperse index was reduced from 2.02 to 1.34 . However, the molecular weight of the polymer was also reduced from 127,200 to 44,900 for $\bar{M}_{n}$. This indicated that the polymer depolymerised during the purification to some extent, which might be caused by standing in dilute aqueous solution for long time during the purification. (The filtration of $50 \mathrm{ml}$ solution took about 7 days).

Therefore, although the filtration method could remove ionic and low molecular weight impurities in the polymer, it was not chosen in recent work, due to the depolymerisation of polymer.

\section{(2) Dialvsis}

The polymer aqueous solution ( 1 gram PMEO in $50 \mathrm{ml}$ distilled water) was poured into a dialysis tube (Sigma, D-0655 Dialysis Tubing). The tube was immersed in 2 litres of distilled water contained in a flask under magnetic stirring at the room temperature. The water was changed completely three times, each change occurring after two hours dialysis time. The dialysed solution was firstly distilled under reduced pressure to remove water. To remove residual water, the polymer was dissolved in dry pure acetonitrile. Then solvent was evaporated under vacuum. The polymer was finally 
dried under vacuum at $80^{\circ} \mathrm{C}$ for 8 hours. The conductivity of the purified polymer was $1.2 \times 10^{-8} \mathrm{Scm}^{-1}$ at $25^{\circ} \mathrm{C}$ (Figure 3-3).

After the purification, the molecular weight of the polymer increased slightly (from 92,100 to 100,600 for $\bar{M}_{n}$ ), while the polydisperse index was slightly decreased (from 1.63 to 1.60 ).

\section{3-2-3 Methylation of Poly(ethylene oxide)}

In order to eliminate the influence of $\mathrm{OH}$ end-groups on the solvation of salt, these were methylated for certain investigations. The methylation method most commonly used was reaction with dimethyl sulphate in the presence of sodium hydroxide aqueous solution [3].

$50 \mathrm{~g}$ of poly(ethylene oxide) 1450 was dissolved in $100 \mathrm{ml}$ of methylene chloride in a flat bottom flask. Then $\mathrm{NaOH}$ aqueous solution $(6.9 \mathrm{~g} \mathrm{NaOH}$ in $20 \mathrm{ml}$ $\mathrm{H}_{2} \mathrm{O}$ ) was added to the solution, followed by $17 \mathrm{ml}$ of dimethyl sulphate. After stirring by a magnetic bar for 24 hours at room temperature, the organic and aqueous phases were separated. The organic phase was washed with distilled water twice to remove the residual salt. The organic phase was dried by $\mathrm{CaSO}_{4}$ for 12 hours. The solvent was removed on a rotary evaporator. The methylated polymer was then dried under vacuum at $60^{\circ} \mathrm{C}$ for 24 hours.

FTIR analysis showed that the $\mathrm{OH}$ band of $3450 \mathrm{~cm}^{-1}$ [4] almost disappeared after methylation (Figure 3-4), indicating that the $\mathrm{OH}$ end-groups were effectively methylated by this method.

Poly(ethylene oxide) 3350 was also methylated by the same procedures as the above. 


\section{3-3 Preparation of Polymer Electrolytes}

\section{3-3-1 Solvent-Casting Methods}

Solvent-casting is the conventional method for the preparation of polymer electrolytes [5]. Although this method is limited sometimes by finding a suitable solvent to dissolve the salt and by the difficulty in removing all of the solvent at the end of the preparation, it is widely used, especially in the cases where polymer hosts are rubbery or wax-like, because it is very difficult to use other techniques, eg. grinding / hot pressing technique. In addition, in the preparation of polymer electrolytes of low salt concentration, the solvent-casting method has the advantage of ensuring a more even distribution of the salt. Therefore, polymer electrolytes based on PMEO and relatively low molecular weight PEO (say $\mathrm{M}_{\mathrm{W}}$ of $\mathrm{PEO}<20,000$ ) and metal salts, such as $\mathrm{LiClO}_{4}$, $\mathrm{LiPF}_{6}, \mathrm{Ca}\left(\mathrm{CF}_{3} \mathrm{SO}_{3}\right)_{2}$ and $\mathrm{Zn}\left(\mathrm{CF}_{3} \mathrm{SO}_{3}\right)_{2}$, were prepared by conventional solvent-casting methods.

Polymer and salt were weighed separately in an argon-filled glovebox, and were dissolved in acetonitrile to form $5 \mathrm{wt} \%$ solutions. Both solutions were then mixed under magnetic stirring overnight to ensure homogeneity. The resulting solution was cast on a glass plate. The solvent was evaporated under reduced pressure. The electrolyte was finally heated at $80-90^{\circ} \mathrm{C}$ under vacuum for 8 hours.

For those polymer electrolytes with very dilute salt concentration, eg. less than $0.3 \mathrm{~mol} \mathrm{dm}^{-3}$, due to the small amount of salt involved, the conventional solventcasting method was slightly modified. The salt contained in a DSC aluminium sample pan was accurately weighed on a six-figure microbalance (Sartorius Microbalance) sitting in the glovebox. This was added to the weighed PMEO in a glass flask with a magnetic bar. Then the mixture was dissolved in acetonitrile to form $5 \mathrm{wt} \%$ solution. All the above operations were carried out in the argon-filled glovebox. The solution was 
subjected to magnetic stirring for 12 hours. After the magnetic bar and the aluminium pan had been removed, the solvent was evaporated under reduced pressure, and the electrolyte was heated under vacuum at $80-90^{\circ} \mathrm{C}$ for 8 hours.

In the FTIR spectra of the electrolytes prepared by this method, the $\mathrm{OH}$ band of $3450 \mathrm{~cm}^{-1}$ was not observed(Figure $3-5$ ). This indicated that in the polymer electrolytes prepared by solvent-casting there was no significant moisture.

PMEO-Mg( $\left.\mathrm{CF}_{3} \mathrm{SO}_{3}\right)_{2}$ electrolytes were also prepared by solvent-casting method. Because $\mathrm{Mg}\left(\mathrm{CF}_{3} \mathrm{SO}_{3}\right)_{2}$ could not be dissolved in acetonitrile, it was firstly dissolved in anhydrous ethanol to form $5 \mathrm{wt} \%$ solution. This solution was then mixed with the polymer acetonitrile solution ( 5 wt \%). The electrolytes were prepared by the same procedures as described above. FTIR of this electrolyte showed the $3450 \mathrm{~cm}^{-1}$ band (Figure 3-6), which indicated that there was still a significant amount of ethanol in the electrolyte. This might be explained to the very strong interaction between magnesium cations and ethanol. Thus, solvent-free polymer electrolytes based on magnesium salt could not be prepared by conventional solvent-casting methods.

\section{3-3-2 Solvent-Free Methods}

\section{3-3-2-1 Grinding / Hot Pressing Method}

Polymer electrolytes based on semi-crystalline PEO and metal salts were prepared by the grinding / hot pressing method developed in this laboratory [6]. The main advantage of this method is that low molecular weight solvent is not involved. In addition, the preparation procedures are short, and thick electrolyte films may be produced easily. However, the grinding procedure does not always produce uniform electrolytes with low salt concentration [7] (eg. EO / M > 100). Polymer electrolytes based on semi-crystalline PEO and metal salts, such as $\mathrm{LiClO}_{4}, \mathrm{LiCF}_{3} \mathrm{SO}_{3}$, $\mathrm{Ca}\left(\mathrm{CF}_{3} \mathrm{SO}_{3}\right)_{2}, \mathrm{Mg}\left(\mathrm{CF}_{3} \mathrm{SO}_{3}\right)_{2}$ and $\mathrm{Zn}\left(\mathrm{CF}_{3} \mathrm{SO}_{3}\right)_{2}$ etc. were prepared by this method. 
Weighed PEO and salt were sealed by a rubber bung in a steel test-tube containing about fifty steel ball-bearings of diameter $5 \mathrm{~mm}$, in an argon-filled glovebox. The tube was shaken at liquid nitrogen temperatures for $15-20$ minutes to mix and grind the PEO and the salt powders. After grinding, the electrolyte was taken out from the tube in the glovebox.

To produce an electrolyte disc of $13 \mathrm{~mm}$ diameter, the required amount of produced electrolyte was firstly pressed in a die to 5 tonnes at room temperature for several seconds. Then the pressure was released. The die was enclosed in an aluminium jacket and heated at $120-130^{\circ} \mathrm{C}$ for 3 hours. The die was then allowed to cool down to $70^{\circ} \mathrm{C}$, before being pressed to $1-2$ tonnes, and finally allowed to cool under this pressure overnight. Electrolyte discs with the thickness of $200 \mu \mathrm{m}$ to $1 \mathrm{~mm}$ were prepared by this procedure.

$\mathrm{X}$-ray powder diffraction was used to characterise the polymer electrolytes based on $\mathrm{Zn}\left(\mathrm{CF}_{3} \mathrm{SO}_{3}\right)_{2}$ and $\mathrm{Mg}\left(\mathrm{CF}_{3} \mathrm{SO}_{3}\right)_{2}$ prepared by this method. The X-ray diffraction patterns of $\mathrm{P}(\mathrm{EO})_{4} \mathrm{Zn}\left(\mathrm{CF}_{3} \mathrm{SO}_{3}\right)_{2}$ and $\mathrm{P}(\mathrm{EO})_{6} \mathrm{Zn}\left(\mathrm{CF}_{3} \mathrm{SO}_{3}\right)_{2}$ are shown in Figure 3-7. together with the patterns of crystalline $\mathrm{PEO}$ and $\mathrm{Zn}\left(\mathrm{CF}_{3} \mathrm{SO}_{3}\right)_{2}$. The $\mathrm{X}$-ray diffraction patterns of $\mathrm{PEO}-\mathrm{Mg}\left(\mathrm{CF}_{3} \mathrm{SO}_{3}\right)_{2}$ are discussed in detail in Chapter 7. These results indicated that polymer electrolytes were produced by this method. It has also been reported that polymer electrolytes based on lithium salt [6] and $\left.\mathrm{Ca}_{(\mathrm{CF}} \mathrm{SO}_{3}\right)_{2}$ [8] may te prepared by the grinding / hot pressing method.

\section{3-3-2-2 Hot Stirring}

Because polymer electrolytes based on war-like low molecular weight PEO would be viscous liquids at the hot pressing temperature $\left(120^{\circ} \mathrm{C}\right)$. a modified methoi was employed. 
Electrolytes based on $\mathrm{PEO}(1000), \mathrm{PEO}(2000)$ dimethyl ethers, methylated $\mathrm{PEO}(1450)$ and $\mathrm{PEO}(3350)$, and $\mathrm{PEO}(4000), \mathrm{PEO}(6000)$ and $\mathrm{PEO}(8000)$ were prepared by adding the required amount of polymer and salt in a glass bottle with a magnetic bar, and sealing in an argon-filled glovebox. The mixture was stirred at $90^{\circ} \mathrm{C}$, at which temperature the polymer was in the form of a melt, for 5 days. A homogeneous melt was eventually obtained.

Electrolytes based on $\operatorname{PEO}(10,000)$ and $\operatorname{PEO}(20,000)$ were prepared by firstly grinding the polymer and salt at liquid nitrogen temperature for 15 minutes in a steel test-tube with ball-bearings as described in the above, in order to mix the polymer and salt well, because these polymers were quite hard. Then, the mixture was stirred by a magnetic stirrer at $90^{\circ} \mathrm{C}$, at which temperature the polymer was again in the form of a melt, for 5 days.

$\mathrm{PEO}(400)$ dimethyl ether- $\mathrm{Mg}\left(\mathrm{CF}_{3} \mathrm{SO}_{3}\right)_{2}$ electrolyte was prepared by dissolving the salt in PEO directly followed by magnetic stirring for 48 hours at ambient temperature.

\section{3-4 Cells and Electrodes for Electrochemical Measurements on Polymer Electrolytes}

Two types of cells were used for electrochemical measurements on polymer electrolytes. The first were two electrode cells with blocking or non-blocking electrodes. These were used for the measurements of conductivity and dc polarisation current. The second were three electrode cells which were used to investigate the electrode-electrolvte interfaces. 


\section{3-4-1 Two Electrode Cells}

(1) Cells with blocking electrodes

These cells had two stainless steel electrodes with the polymer electrolyte sandwiched between them. There were three versions of these cells, depending on the state of the electrolyte.

CELL 1 (Figure 3-8) consisted of two stainless steel electrodes and a Teflon spacer used to keep a constant volume between the electrodes. It was suitable for the measurement of conductivities of polymer electrolytes based on PMEO which were soft and rubberylike.

CELL 2 (Figure 3-9) was similar to CELL 1, but was well sealed, making it suitable for the measurement of conductivities of liquid or molten polymer electrolytes.

CELL 3 (Figure 3-8) was developed from CELL $l$ by removing the Teflon spacer and was used for the measurement of conductivities of polymer films based on PEO of the molecular weight of $4 \times 10^{6}$.

(2) Cells with non-blocking electrodes

These cells consisted of two non-blocking electrodes, and polymer electrolytes. The two non-blocking electrodes were either identical or non-identical, depending on the systems studied.

CELL 4 (Figure 3-10) consisted of two lithium electrodes with constant separation, which was used to study dc polarisation of polymer electrolytes based on PMEO, low molecular weight PEO and lithium salts, because it was well sealed. A section of lithium rod was firstly pressed into the groove in the stainless steel base, and then its surface 
was scraped by a knife in an argon-filled glovebox.

CELL 5 (Figure 3-11) consisted of two zinc electrodes separated by a Teflon spacer, which was used to study dc polarisation of polymer electrolytes based on PMEO and zinc salt. The zinc electrode was polished by fine emery paper and then cleaned by tissue. After each experiment, the electrode was washed by acetonitrile in a drybox and then dried under vacuum.

CELL 6 (Figure 3-12) consisted of a metal electrode (Ca or Mg) and a mercury film electrode, with constant separation. This cell was used to study the dc polarisation of polymer electrolytes based on PMEO and low molecular weight PEO and calcium or magnesium salts.

Magnesium electrodes were prepared from magnesium foil, and were scraped by a knife before using. Calcium electrodes were prepared by pressing calcium shot into a rebate cut in the stainless steel. The electrode surface was scraped mechanically before use. After each experiment, they were washed by acetone and then dried under vacuum.

Mercury film electrode was prepared by immersing a gold disc into triple distilled mercury for 2-3 minutes. After each experiment, it was treated by concentrated nitric acid, heated to boiling for a few minutes.

CELL 7 (Figure 3-13) consisted of two electrodes, either identical or non-identical, which was used to study the dc polarisation of polymer electrolytes based on PEO of the molecular weight of $4 \times 10^{6}$.

CELL 8 (Figure 3-14) was used for the Hittorf method of measuring the transference number of zinc. It was constructed from a zinc metal anode and lead metal cathode. The 
long distance between these two electrodes allowed the concentration gradient only to be established in the electrode compartments. The three splits on the cover allowed the electrolyte to be cut into four pieces, i.e. the anode compartment, cathode compartment, middle anode and middle cathode compartments, after the electrolysis.

\section{3-4-2 Three Electrode Cells}

Three electrode cells were used to investigate the electrode-electrolyte interfaces by cyclic voltammetry and ac impedance spectroscopy.

CELL 9 (Figure 3-11) had zinc working, reference and counter electrodes. The reference electrode was inserted into the centre of the counter electrode disc and was separated from it by a thin Teflon film. The working electrode was a disc, separated by a Teflon spacer from the counter electrode. This cell was used to investigate the $\mathrm{Zn} /$ $\mathrm{PMEO}-\mathrm{Zn}\left(\mathrm{CF}_{3} \mathrm{SO}_{3}\right)_{2}$ interfaces by ac impedance spectroscopy.

CELL 10 (Figure 3-15): The working and counter electrodes were mercury film or metal $\mathrm{M}(\mathrm{M}=\mathrm{Ca}$ or $\mathrm{Mg})$ electrodes. The reference electrode was always the metal $\mathrm{M}$, which was prepared by cutting the metal foils into narrow strips. These three electrodes were separated by paper pads (Watermann glass fibre paper). This cell was used to investigate $\mathrm{M} / \mathrm{PEO}(400)$ dimethyl ether $-\mathrm{M}\left(\mathrm{CF}_{3} \mathrm{SO}_{3}\right)_{2}$ and $\mathrm{Hg}(\mathrm{M}) / \mathrm{PEO}(400)$ dimethyl ether - $\mathrm{M}\left(\mathrm{CF}_{3} \mathrm{SO}_{3}\right)_{2}$ interfaces by cyclic voltammetry and ac impedance spectroscopy. 


\section{3-5 Direct Current Measurements}

\section{3-5-1 Dc Polarisation of Two Electrode Cells}

\section{3-5-1-1 Principle of Steady State Method}

The steady state dc polarisation technique was widely applied in the studies of this thesis. Detailed discussion of the application of this method can be found in the relevant chapters. In this section, the principle of the steady state method is briefly presented.

Consider that a fully dissociated polymer electrolyte PEO-MX is sandwiched between two electrodes $M$ which are non-blocking towards $M^{+} / M$. It is assumed that the electrode reactions are significantly faster than the mass transport in the electrolyte bulk and that no kinetic polarisation of the electrodes occurs. When a de potential is applied, the cations $\mathrm{M}^{+}$migrate from the anode area to the cathode area, and the anions migrate in the opposite direction (Figure 3-16). The current will decrease with time untill a steady state is reached where a stable concentration gradient across the electrolyte is built up and gives a invariant steady state current (Figure 3-17).

At steady state, the potential distribution within the cell is:

$$
\Delta V=\Delta E+\Delta \phi
$$

where $\Delta V$ is the applied potential after interfacial resistance correction, $\Delta E$ is the Nernst potential difference between the two electrodes and $\Delta \phi$ the potential difference across the electrolyte. When the applied potential is not over $20 \mathrm{mV}$ [9], the following relationship exists:

$$
\Delta V=2 \Delta E=2 \Delta \phi=\frac{2 R T}{F} \ln \left(\frac{C_{a}}{C_{c}}\right)
$$

where $C_{a}$ and $C_{c}$ are the salt concentration at anode and cathode respectively. Thus, the cationic conductivity can be derived by 


$$
\sigma_{+}=I_{s} / \Delta \phi=2 I_{s} / \Delta V
$$

where $I_{s}$ is the steady state current, providing that the cell constant is unity.

For the electrolyte with mobile associated species such as ion pairs, the conductivity derived from the steady state current is the "effective conductivity" which is different from the cationic conductivity [9].

\section{3-5-1-2 Experimental Details}

(1) De polarisation of two electrode cells with identical non-blocking electrodes

(a) Polymer electrolytes with lithium salts

Polarisation of $\mathrm{PMEO}-\mathrm{LiPF}_{6}$ electrolytes and $\mathrm{PEO}-\mathrm{LiCF}_{3} \mathrm{SO}_{3}$ electrolytes, except with the highest molecular weight PEO, was carried out in CELL 4. The cell used for the latter was CELL 7. Before assembling the cell, a section of lithium was freshly cut from a lithium rod to produce a disc with a fresh surface. The assembled cell was heated at $80^{\circ} \mathrm{C}$ for 30 minutes to allow a good mechanical electrode-electrolyte contact. The cell was then cooled down.

Before applying the polarisation potential, the resistance $R_{i o}$ arising from the electrode-electrolyte interface and the conductivity of the electrolyte were measured by ac impedance spectroscopy as described in section 3-6-3. The polarisation voltage was supplied by a Solartron / Schlumberger 1286 Electrochemical Interface (ECI). The flowing current was also recorded by the $1286 \mathrm{ECI}$. The applied potential was usually $10-20 \mathrm{mV}$, depending on the voltage drop by the interfacial resistance. The applied voltage $\Delta V$ was corrected by the voltage drop in the electrode-electrolyte interface, i.e.

$$
\begin{aligned}
& \text { for initial polarisation: } \Delta V=\Delta V_{\text {app }}-I_{o} R_{\text {io }} \\
& \text { for reaching steady state: } \Delta V=\Delta V_{\text {app }}-I_{s} R_{\text {is }}
\end{aligned}
$$

where $\Delta V_{\text {app }}$ is the voltage supplied by the instrument, $I_{o}$ and $I_{s}$ are the initial and steady state currents, $R_{i o}$ and $R_{\text {is }}$ are the interfacial resistances at initial and steady state. 
Basically, the potential applied across the electrolyte was ensured to be less than $20 \mathrm{mV}$.

The achievement of steady state was judged by that the current measured varied less than $0.5 \%$ for 30 minutes. After reaching steady state, ac impedance spectrum was measured immediately when the polarisation was stopped, in order to obtain the interfacial resistance $R_{i s}$ after reaching the steady state. The typical ac impedance spectra before and after the polarisation are shown in Figure 3-18.

(b) Polymer electrolytes with zinc salt

Polarisation of PMEO- $\mathrm{Zn}\left(\mathrm{CF}_{3} \mathrm{SO}_{3}\right)_{2}$ electrolytes was carried out in CELL. 5. Before assembling the cell, the zinc electrodes were freshly scraped. The assembled cell was heated at $80^{\circ} \mathrm{C}$ for 30 minutes to allow a good mechanical electrode-electrolyte contact.

The cell was initially "electrochemical polished" by applied $\pm 0.5 \mathrm{~V}$ potential alternatively for 3 times as described in Chapter 6 . It was then left for 12 hours to allow to equilibrate. Cells treated by this method were ready for dc polarisation. The processes and conditions for dc polarisation were the same as those described above.

(2) Dc polarisation of two electrode cells with non-identical electrodes

Dc polarisation of PMEO-Ca( $\left.\mathrm{CF}_{3} \mathrm{SO}_{3}\right)_{2}$ and $\mathrm{PEO}(\mathrm{Mw})-\mathrm{Mg}\left(\mathrm{CF}_{3} \mathrm{SO}_{3}\right)_{2}$ electrolytes were carried out by using CELL 6 with the mercury film electrode as the cathode and metal ( $\mathrm{Ca}$ or $\mathrm{Mg}$ ) as the anode, except for those based on PEO (4 x 106). CELL 7 was used for the latter systems. The metal electrode was freshly scrapped and the mercury film electrode was freshly deposited. Such cells did not have zero open circuit voltages as discussed in Chapter 7. The values of applied voltages supplied by a $1286 \mathrm{ECI}$ were $0.5 \mathrm{~V}$ for the calcium system and $0.1 \mathrm{~V}$ for the magnesium system. After applied the polarisation potential for 5 minutes, the ac impedance spectrum was 
measured under polarisation. Other details of the polarisation method were the same as for the identical electrodes case.

All the operations for the above dc polarisation were carried out in an argonfilled Braun glovebox with the oxygen and water level less of $1 \mathrm{ppm}$. Temperature was controlled to within $\pm 0.5^{\circ} \mathrm{C}$ by placing the cells in a Buchi TO-51 oven.

\section{3-5-2 Dc Polarisation of Hittorf Cell}

(1) Introduction of the Method

The detailed discussion of the principles of the Hittorf method is presented in Chapter 6. Here, the basic idea of this method is briefly mentioned. In a two electrode cell: $M$ / PEO-MX $/ \mathrm{M}$ where $M$ represents the non-blocking electrode towards $\mathrm{M}^{2+}$, when a potential difference is applied across the cell, the mobile ionic species M(II) and $X$ - in the electrolyte will move under applied potential, i.e. the cationic species move from the anode area towards the cathode area, while the anionic species move from the cathode area towards the anode area under the electric field. Meantime, the cationic species are reduced at the cathode and at the same time the cationic species will be continuously produced at the anode by oxidation of the metal, which leads to a change of salt concentration in the electrolyte near the electrodes.

If the change of salt concentration is limited only to the regions near the electrodes, the change of the concentration of salt $\mathrm{MX}_{2}$ in the cathode and the anode regions is: $|\Delta \mathrm{m}|=(\mathrm{Q} / \mathrm{zF})\left(1-\mathrm{T}_{+}\right)$, where $\mathrm{Q}$ is the charge passed, $\mathrm{z}$ is the number of charge on the ions and $T_{+}$is the transference number of cation-constituents. By measuring change of concentration of the salt in the electrode region, the transference number can be derived.

The Hittorf method requires that the change of salt concentration is limited to the 
electrode region only. Thus, the distance between the two electrodes is long and the polarisation should be carried out for a period chosen so that the middle part of the electrolyte is not affected by any concentration change.

(2) Electrolysis

PEO- $\mathrm{Zn}\left(\mathrm{CF}_{3} \mathrm{SO}_{3}\right)_{2}$ electrolyte was mounted between the zinc metal anode and the lead cathode (CELL 8 ). The cell was heated at $120^{\circ} \mathrm{C}$ for 8 hours to produce thermally equilibrated polymer electrolyte. Then a constant current of $4 \mu \mathrm{A}$ was applied across the cell at $120^{\circ} \mathrm{C}$, and the the potential across the cell was monitored. After the current had flowed for about 65 hours, electrolysis was terminated. The electrolyte was then immediately cut into four pieces which were ready for weighing, digestion and analysis. Further details of this experiment will be presented in section 3-7 and Chapter 6.

\section{3-5-3 Cyclic Voltammetry}

\section{3-5-3-1 Principles}

(1) Reversible reactions

Consider a three electrode cell with a working, reference and counter electrodes. The following electrode reaction is assumed to take place reversibly in the cell:

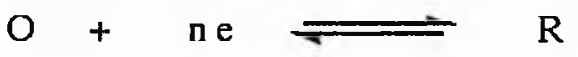

where $O$ and $R$ represent the oxidised and reduced species respectively. It is assumed that the electrolyte solution initially contains $O$. When a linear potential sweep with a rate of $v$ is applied, as soon as a potential where $O$ is reduced is reached, the surface concentration of $O$ decreases from its bulk value, and a concentration gradient is set up in the region next to the electrode, known as the diffusion layer. If the sweep rate is relatively fast, the diffusion layer does not have sufficient time to relax to its equilibrium state which gives rise to a non-linear concentration gradient, and the surface 
concentration of the reactant decreases progressively. As a result a current proportional to the value of this gradient at the electrode surface flows in the external circuit. Once the surface concentration of the reactant reaches to zero, the concentration gradient starts to decrease due to the relaxation effect, and the current flowing thus also decreases. When the sweep is reversed, there is a significant concentration of $R$ present near the electrode, and it continues to be formed on the reverse sweep until the potential approaches the equilibrium potential $\mathrm{E}_{\mathrm{e}}$. A typical cyclic voltammogram is shown in Figure 3-19, in which the separation of the peaks of the anionic and cathodic process is (59/n) $\mathrm{mV}$ ( where $\mathrm{n}$ is the number of electrons) and the position of the peak is independent of the scan rate $v$. The absolute values of the peak current $i_{a}$ and $i_{c}$ which are proportional to the square root of the scan rate are the same.

\section{(2) Irreversible Reactions}

In irreversible systems, the electron transfer rates are low. At low potential sweep rates the rate of electron transfer may be faster than the mass transfer, and a reversible cyclic voltammogram may be recorded. As the sweep rate is increased, however, the rate of mass transport increases and becomes comparable to the rate of electron transfer, the peak separation increases (Figure 3-19) to values greater than $(59 / \mathrm{n}) \mathrm{mV}$, and the peak current increases with the square root of the scan rate. For a totally irreversible system, there is no reverse peak.

\section{3-5-3-2 Experimental Details}

Cyclic voltammetry was used to investigate electrochemical behaviour of $\mathrm{M-}$ species in polymer electrolytes at $M$ electrodes or the mercury electrode in $\mathrm{PEO}(400)$ dimethyl ether- $\mathrm{M}\left(\mathrm{CF}_{3} \mathrm{SO}_{3}\right)_{2}[\mathrm{M}=\mathrm{Ca}$ and $\mathrm{Mg}]$ based systems.

For the investigation of $\mathrm{M}$ electrodes, CELL 10 with $\mathrm{M}$ metal as the working electrode and reference electrode and mercury film electrode as the counter electrode was 
used. $\mathrm{M}$ electrodes were freshly scraped and the mercury electrode was freshly deposited before use. The potential was supplied by an AMEL 567 Function Generator I HI-TEK PP RI Waveform Generator. The cyclic voltammogram was recorded by a LINSEIS LY $18100 X Y$ recorder. The range of potential was usually from $-2 \mathrm{~V}$ to $+2 \mathrm{~V}$, and the scan rate $10 \mathrm{mVs}^{-1}$. The first cycle was always in the anodic direction starting from $0 \mathrm{mV}$.

For the investigation of the mercury film electrode, CELL 10 was also used. The working electrode here was the mercury film electrode and the reference and counter electrodes were $M$ metal. Other details were the same as above, except that the first cycle was always in the cathodic direction starting from the equilibrium potential which was about $0.6 \mathrm{~V}$ for the calcium system and about $1.2 \mathrm{~V}$ for the magnesium system. Experiments were carried out at room temperature. In the above measurements, the iR drop was not compensated. All the operations were carried out in a Braun argon-filled glovebox with water and oxygen level less $1 \mathrm{ppm}$.

\section{3-6 Alternating Current Measurements}

Ac techniques are often employed to study the properties of electrochemical systems. This method has some advantages over dc techniques in the study of polymer electrolytes: bulk electrolyte properties can be investigated by a simple two electrode cell with blocking electrode, and information is provided about polarisation phenomena occurring within the cell, in addition to the long-range migration of ions. In this section, the principle of ac impedance measurements is briefly presented and the experimental techniques are described. 


\section{3-6-1 Principles of Ac Impedance Spectroscopy}

When an ac signal with a phase angle $\theta$ (Figure 3-20) is applied across an electrochemical cell, eg. a two electrode cell, generally, both the impedance of the cell and the phase angle $\theta$ are functions of the applied frequency. The impedance of the cell $Z$ can be expressed by a complex form: $Z=Z^{\prime}-j Z^{n}$, where $Z^{\prime}$ and $Z^{n}$ are the real and imaginary components and $\mathrm{j}$ is a complex number $\sqrt{-1}$. Thus, a complex impedance plot can be derived by plotting the complex impedance on the complex plane as a function of the signal frequency.

The ac impedance of a electrochemical cell can be interpreted by finding the equivalent circuit which models the impedance data and extracting values for the individual components which may then be related to the fundamental electrical properties of the cell. The following are the simplest circuits consisting of the individual components.

(1) Resistor: The voltage applied across a resistor is always in phase with the current i.e. $\theta=0$, and the impedance is independent of frequency with magnitude equal to the resistance. Thus, the impedance of a resistor is a point on the real axis as shown in the following schematic diagram.

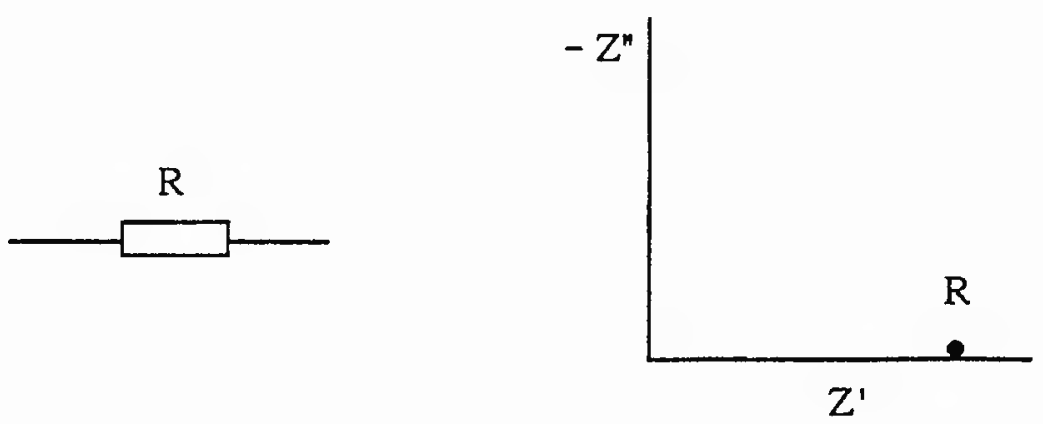

(2) Capacitor: The voltage applied lags behind the current by $90^{\circ}$, i.e. $\theta=-\pi / 2$, and the the impedance is frequency dependent. The magnitude of the impedance is: $|Z|=1$ I $\omega \mathrm{C}$, where $\mathrm{C}$ is the capacitance. Thus, the impedance of a capacitor defines a vertical 
spike coincident with the imaginary axis as shown in the following schematic diagram.

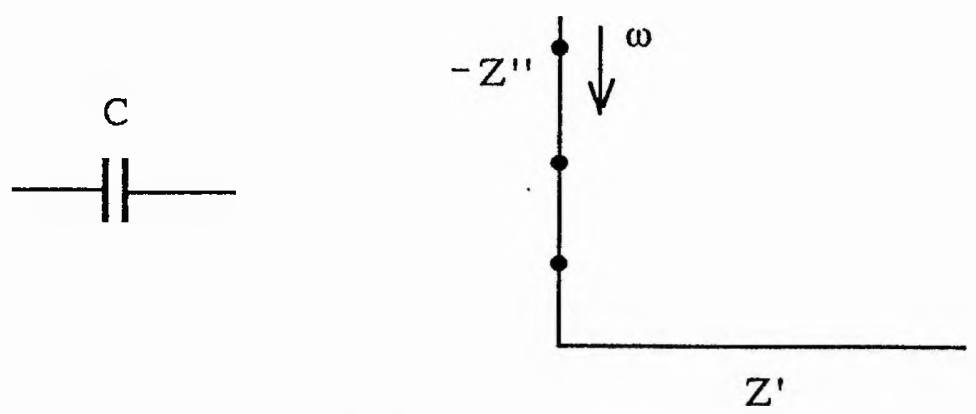

(3) Combination of resistors and capacitors in series: The impedance of the system is the sum of the impedance of the individual impedance. Thus, $Z=Z_{1}+Z_{2}+Z_{3}+\cdots$. For the combination of a single resistor and a capacitor in series, the total impedance of the system is: $Z=R-j / \omega C$. In the complex impedance plane this defines a vertical spike displaced a distance $\mathrm{R}$ along the real axis. Thus, it can be schematically represented as:
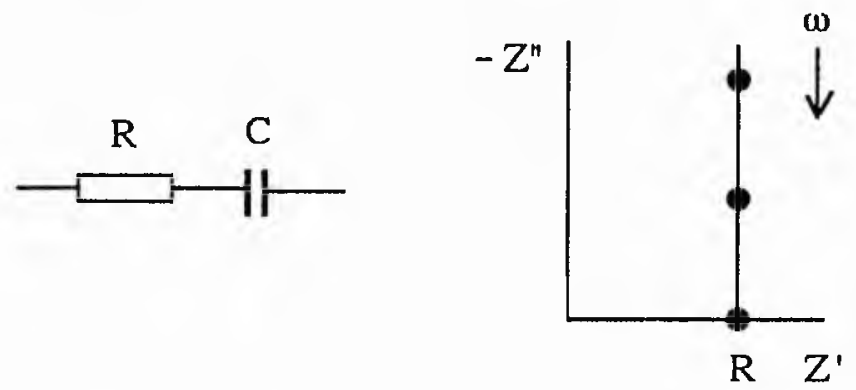

(4) Combination of resistors and capacitors in parallel: The impedances are not additive in this case, but the complex admittances are additive. Thus:

$$
\mathrm{Y}=1 / \mathrm{Z}=\mathrm{Y}_{1}+\mathrm{Y}_{2}+\mathrm{Y}_{3}+\cdots=1 / \mathrm{Z}_{1}+1 / \mathrm{Z}_{2}+1 / \mathrm{Z}_{3}+\cdots
$$

For the combination of a single resistor and a capacitor in parallel, the total admittance is:

$$
Y=\frac{1}{R}+j \omega C
$$

and the total impedance is:

$$
Z=R\left[\frac{1}{1+(\omega C R)^{2}}\right]-j R\left[\frac{\omega C R}{1+(\omega C R)^{2}}\right]
$$


This defines a semicircle in the complex impedance plane with a diameter $\mathrm{R}$ extending along the real axis from the origin. At the frequency corresponding to the maximum of the semicircle, $\omega_{\max }$, the magnitude of the impedance of the resistor and capacitor are equal, i.e. $\omega_{\max } \mathrm{RC}=1$. The complex impedance plot can be schematically represented as:

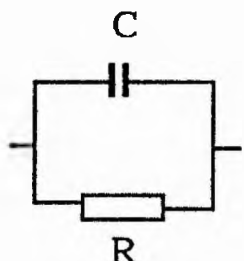

$\mathrm{R}$

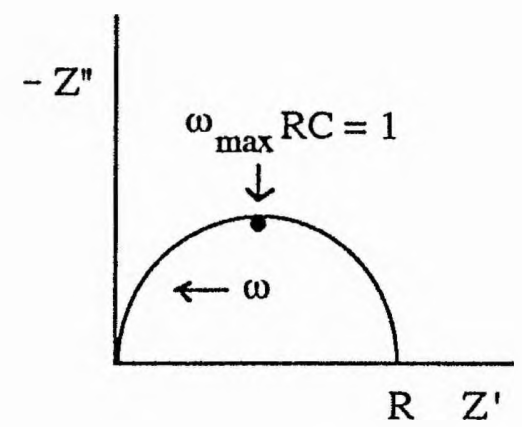

\section{3-6-2 Ac Impedance Spectroscopy of Systems with Polymer Electrolytes}

In a two electrode cell containing a polymer electrolyte in which both cations and anions are mobile, the following simple cases are briefly discussed.

\section{3-6-2-1 Blocking Electrodes}

If the electrodes in a two electrode cell are blocking towards both the cations and anions in the polymer electrode, eg. a PEO- $\mathrm{LiClO}_{4}$ electrolyte sandwiched between two platinum electrodes, the equivalent circuit and the complex impedance plot can be schematically represented as:
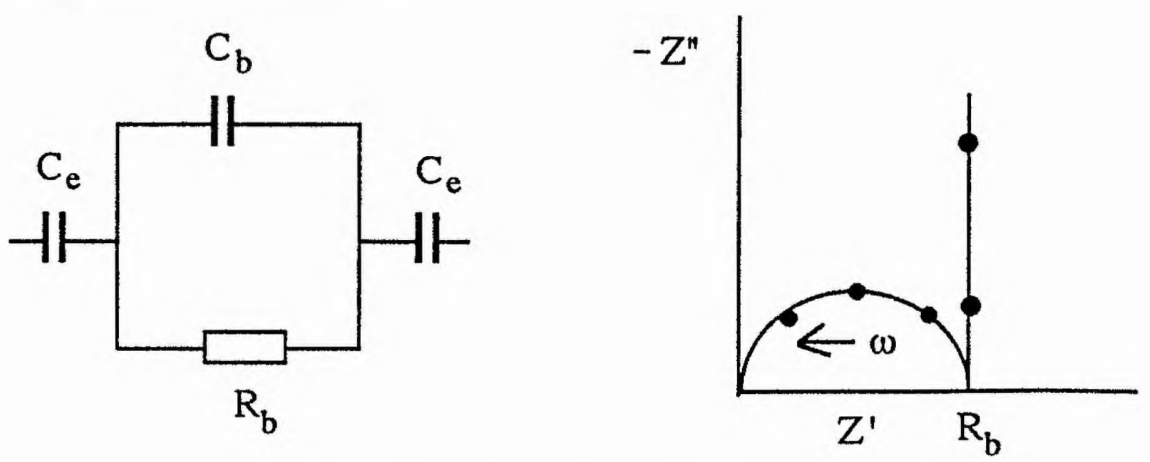

When an ac signal applied, the electrodes become alternatively positively and negatively charged and the alternating electric field across the electrolyte gives rise to back and 
forth migration of the ions. Meantime the there will be dielectric polarisation in the electrolyte bulk. The migration of the ions is represented by a resistor $R_{b}$, and the dielectric polarisation of the electrolyte bulk is represented by a capacitor $C_{b}$. The polarisation of ions at the electrodes is represented by a capacitor $\mathrm{C}_{\mathrm{e}}$.

In the above complex impedance plot, the semicircle part comes from the the response of the polymer electrolyte bulk, while the vertical spike arises from the electrodes. By analysis of the semicircle, the conductivity and capacitance can be derived, if the cell dimensions are known.

\section{3-6-2-2 Non-Blocking Electrodes}

If the electrodes are non-blocking towards the cations and blocking towards the anions in a two electrode cell containing polymer electrolyte, eg. $\mathrm{Li} / \mathrm{PEO}-\mathrm{LiClO}_{4} / \mathrm{Li}$ cell, the equivalent circuit and the complex impedance plot can be represented as:
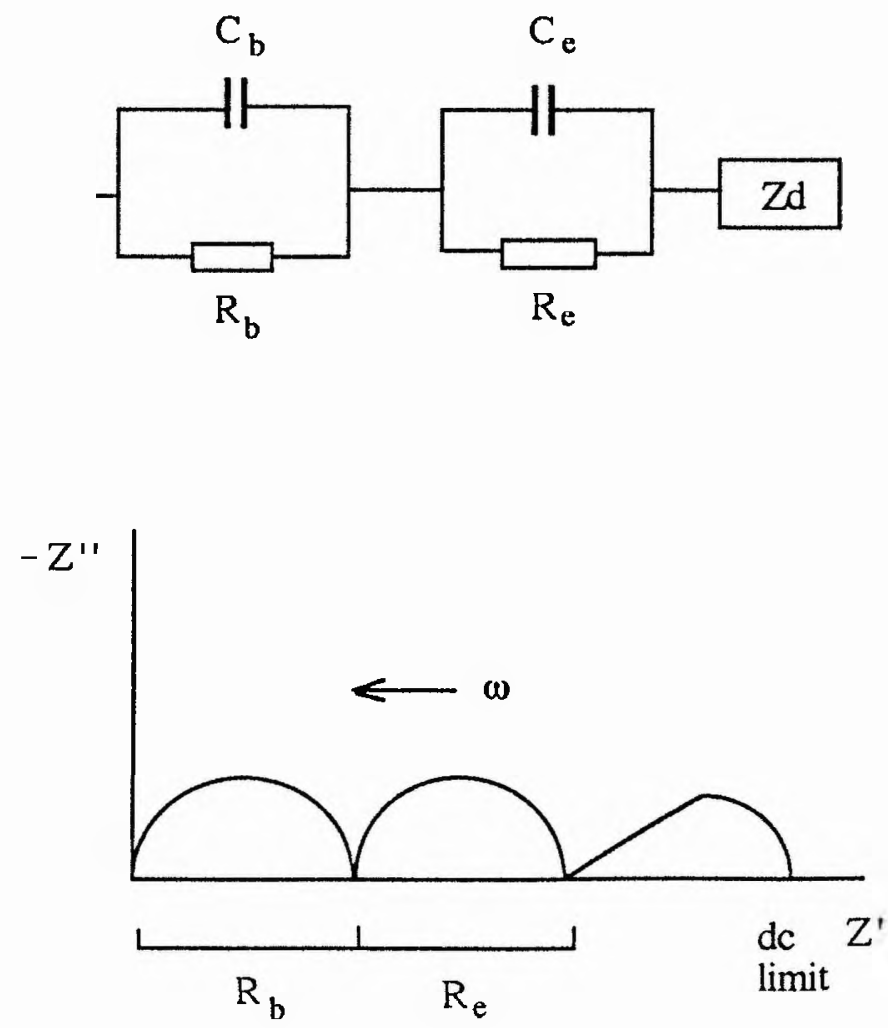
When an ac signal is applied across the cell, in addition to the back and forth migration of cations and anions, there is charge transfer process at the electrodes, i.e. cations are reduced or metals are oxidized alternatively at the electrode. The high frequency semicircle in the complex impedance plot arises from the response of the bulk electrolyte as discussed above. The electrode reaction is represented by a resistor $R_{e}$ in the equivalent circuit, and $\mathrm{C}_{\mathrm{e}}$ is associated with the charging and discharging of the electrode-electrolyte double layer. In the complex impedance plot, this process is attributed to the intermediate frequency semicircle. The lowest frequency part in the complex impedance plot can be attributed to the diffusion of ions in the electrolyte. This is represented by $\mathrm{Zd}$ in the equivalent circuit. The lowest frequency part which is usually shown a straight line inclined at an angle of $45^{\circ}$ to the $\mathrm{x}$-axis is described as a Warburg impedance. This arises from the diffusion controlled process for the electroactive species in the electrolyte bulk or the electrode at very low frequency. The equivalent circuit for the latter can also represented as:

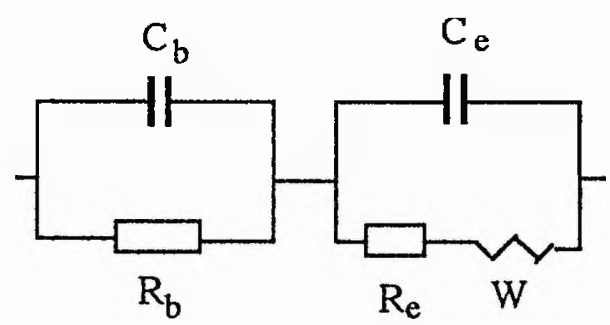

where $\mathrm{W}$ is the diffusion-controlled impedance.

By analysis of the semicircle corresponding to the electrode-electrolyte interface, the impedance of the electrode reaction can be estimated. If $R_{e}$ is large, the electrode reaction is slow. The interfacial capacitance is of ten in the order of magnitude of 20-50 $\mu \mathrm{F} \mathrm{cm}-2$. The diffusion coefficient of the electroactive species can be derived by analysis of the Warburg impedance. For the electrode-electrolyte interface with surface layer, the interfacial capacitance is usually in the order of magnitude of $0.1 \mu \mathrm{F} \mathrm{cm}^{-2}$ or less in a 
cell with solid state electrolyte [10].

\section{3-6-3 Experimental Details}

In recent work, conductivities of polymer electrolytes and the electrodeelectrolyte interfaces have been studied by the ac impedance techniques.

\section{3-6-3-1 Conductivity Measurements}

The cell (CELL 1 ) for the measurement of conductivities of $\mathrm{PMEO}-\mathrm{LiClO}_{4}$, PMEO-LiPF 6 , PMEO-LiSCN, PMEO-LiCF $\mathrm{SO}_{3}, \mathrm{PMEO}-\mathrm{Ca}\left(\mathrm{CF}_{3} \mathrm{SO}_{3}\right)_{2}$ and PMEO$\mathrm{Zn}\left(\mathrm{CF}_{3} \mathrm{SO}_{3}\right)_{2}$ electrolytes was mounted by sandwiching the electrolyte film between two stainless steel electrodes with a fixed distance apart. The cell for the measurements of conductivities of $\mathrm{PEO}(1500)-\mathrm{Mg}\left(\mathrm{CF}_{3} \mathrm{SO}_{3}\right)_{2}, \quad \mathrm{PEO}(3350)-\mathrm{Mg}\left(\mathrm{CF}_{3} \mathrm{SO}_{3}\right)=$. $\mathrm{PEO}(6000)-\mathrm{Mg}\left(\mathrm{CF}_{3} \mathrm{SO}_{3}\right)_{2}$ and $\mathrm{PEO}(20000)-\mathrm{Mg}\left(\mathrm{CF}_{3} \mathrm{SO}_{3}\right)_{2}$ electrolytes was $\mathrm{CELL} 2$ with a constant separation between the two electrodes. CELL3 was used for the conductivity measurement of $\mathrm{PEO}\left(4 \times 10^{6}\right)-\mathrm{Ca}\left(\mathrm{CF}_{3} \mathrm{SO}_{3}\right)_{2}, \mathrm{PEO}\left(4 \times 10^{6}\right)$ $\mathrm{Mg}\left(\mathrm{CF}_{3} \mathrm{SO}_{3}\right)_{2}$ and $\mathrm{PEO}\left(4 \times 10^{6}\right)-\mathrm{Zn}\left(\mathrm{CF}_{3} \mathrm{SO}_{3}\right)_{2}$ electrolyte films with typical thickness of $300 \mu \mathrm{m}$.

Before measurements, the cell was heated at $80^{\circ} \mathrm{C}$ for 60 minutes to ensure gont electrode-electrolyte contact. The cell was then left at room temperature for 12 hours The temperature of the cell was controlled within $\pm 0.5^{\circ} \mathrm{C}$ by a Buchi TO-51 oven, and was allowed to equilibrate for at least 60 minutes at each temperature studied. The $\approx$ impedance spectra of the cell were measured by using variable frequency analysis in complex plane by Solartron / Schlumberger 1250 Frequency Response Analyser and 1286 Electrochemical Interface, controlled by a Zenith computer. The frequency was decreased logarithmically from $65 \mathrm{kHz}$ to $1 \mathrm{~Hz}$, with either the number of points per decade or the total number of points being specified. An integration time of 200 cycles was used. A typical complex impedance plot is shown in Figure 3-21. The electrolyte 
bulk resistance was extracted from the semicircle corresponding to the bulk in the complex impedance plot. The conductivity of the electrolyte, $\sigma$, was calculated from the bulk resistance $R_{b}$, i.e. $\sigma=L /\left(A R_{b}\right)$, where $L$ and $A$ represent the distance between electrodes and area of the electrode respectively, and L/A is the cell constant.

The conductivities of the above electrolytes were also measured by using a Wayne-Kerr Autobalance Universal Bridge B641 with the same cells at the same temperatures. The conductivities measured by the ac impedance spectroscopy and the ac bridge are the same within the experimental error ( For the former, it is within $\pm 0.5 \%$ and for the latter, it is within $\pm 0.01 \%$ ).

\section{3-6-3-2 Electrode-Electrolyte Interfaces}

The electrode-electrolyte interface was investigated by the ac impedance technique.

(1) $\mathrm{M}$ ( where $\mathrm{M}=\mathrm{Ca}$ and $\mathrm{Mg}$ ) / $\mathrm{PEO}(400)$ dimethyl ether $-\mathrm{M}\left(\mathrm{CF}_{3} \mathrm{SO}_{3}\right)_{2}$ and $\mathrm{Hg}(\mathrm{M})$ / PEO(400) dimethyl ether- $\mathrm{M}\left(\mathrm{CF}_{3} \mathrm{SO}_{3}\right)_{2}$ interfaces

A three electrode cell ( CELL 10) with the freshly scraped calcium metal (or Mg metal )working electrode, $\mathrm{Ca}$ (or $\mathrm{Mg}$ ) reference electrode and the freshly deposited mercury film electrode as the counter electrode. These three electrodes were separated by two pieces of glass fibre paper (Watermann paper).

The desired polarisation potential was supplied by a Solartron / Schlumberger 1286 Electrochemical Interface (ECI). The variable frequency ac signal which was superimpose on the polarisation potential was supplied by a 1250 Frequency Response Analyser (FRA) which was controlled by a Zenith computer. The frequency was decreased logarithmically from $65 \mathrm{kHz}$ to $0.1 \mathrm{~Hz}$. The ac impedance spectrum was measured directly without iR compensation. The experiment was carried out at room temperature. 


\section{(2) $\mathrm{Zn} / \mathrm{PMEO}-\mathrm{Zn}\left(\mathrm{CF}_{3} \mathrm{SO}_{3}\right)_{2}$ interfaces}

The three electrode cell for this investigation was CELL 9 with zinc metal as the working, reference and counter electrodes. The electrolyte was sandwiched between two zinc discs. Before assembling the cell, zinc electrodes were freshly scraped. After assembling the cell, the cell was heated at $80^{\circ} \mathrm{C}$ for 30 minutes to improve the electrodeelectrolyte contact. The cell was then cooled to room temperature.

As in the above investigation, the polarisation potential was supplied by a 1286 $\mathrm{ECI}$, and the ac signal was supplied by a 1250 FRA. The measurement was carried out at room temperature, and the frequency was decreased logarithmically from $65 \mathrm{kHz}$ to $0.1 \mathrm{~Hz}$. The $\mathrm{iR}$ drop between the working and reference electrodes was not compensated.

All operations were carried out in a Braun argon-filled glovebox with water and oxygen level of less $1 \mathrm{ppm}$.

\section{3-7 Instrumentation}

(1) Differential scanning calorimetry (DSC)

Glass transition temperatures and phase transition temperatures of polymer electrolytes were measured by differential scanning calorimetry. Measurements were carried out on a Perkin-Elmer DSC 7 under nitrogen flow. The sample was cooled by liquid nitrogen and the instrument was calibrated using $n$-hexane and $n$-dodecane for the measurement of glass transition temperature. The temperature range was typically $-70-$ $10^{\circ} \mathrm{C}$ and with the heating rate for these measurement of $10^{\circ} \mathrm{C} \mathrm{min}^{-1}$. For the investigation of phase transitions, the instrument was calibrated using standard zinc and 
indium samples. The temperature range was typically $20-150^{\circ} \mathrm{C}$ and with the heating rate of $20^{\circ} \mathrm{C} \mathrm{min}^{-1}$.

(2) Fourier transformed infrared spectroscopy (FTIR)

$\mathrm{OH}$ end-groups in PEO and the moisture content were qualitatively analysed by FTIR using a Perkin-Elmer 1710 Infrared Fourier Transform Spectrometer. Liquid samples, eg. $\mathrm{PEO}(400)$ dimethyl ether- $\mathrm{Ca}\left(\mathrm{CF}_{3} \mathrm{SO}_{3}\right)_{2}$, were injected into a constant volume liquid cell with $\mathrm{KBr}$ plates, and the cell was then sealed. Samples based on low molecular weight PEO or PMEO, eg. methylated PEO (1450), were heated to melt or soften, and a thin layer of the melt or the softened sample was spread between two $\mathrm{KBr}$ plates. The gap between the two salt plates was then sealed by Teflon tape. The sampling procedures were carried out in a Braun argon-filled glovebox. The wavenumber range recorded was typically from $800 \mathrm{~cm}^{-1}$ to $4200 \mathrm{~cm}^{-1}$.

(3) X-ray powder diffraction

The sample powder prepared by grinding was put into a Lindemann tube with diameter of $0.5 \mathrm{~mm}$. The length of sample in the tube was about $2 \mathrm{~cm}$. The open end of the tube was sealed by epoxy resin in an argon-filled glovebox. To ensure a homogeneous sample, it was heated at $120-130^{\circ} \mathrm{C}$ for 4 days, and then kept at $60^{\circ} \mathrm{C}$ for a further 4 days to allow better crystallisation of the sample. Finally, this sample was left at the room temperature for 4 days. This sample was then analysed by a Stoe STAD1/P X-ray powder diffractometer operating with $\mathrm{Cu} \mathrm{K}_{\alpha 1}$ radiation $(\lambda=1.54056$ $\AA$ ). The temperature variation was carried out by using a furnace attached to the machine. At each temperature the sample was held for 30 minutes to allow the temperature to equilibrate before recording the diffraction pattern.

(4) A tomic absorption analysis

The zinc content of aqueous solutions prepared by the digestion of polymer 
electrolytes by concentrated sulphuric acid and perchloric acid was analysed using a Pye Unicam PU 9000 AAS. The gas used was an air / acetylene mixture with a gas flow rate of $0.81 \mathrm{~min}^{-1}$. The operating wavelength was $213.9 \mathrm{~nm}$. The concentration of zinc required for the analysis was 0.5 to $2 \mathrm{ppm}$. The machine was calibrated by the standard zinc solutions with the zinc content of $0.50,1.00$ and $2.00 \mathrm{ppm}$. 


\section{References}

[1] J. R. Craven, R. H. Mobbis, C, Booth and J. R. M. Giles, Makromol. Chem. Rapid Comm., 7, 81(1986).

[2] F. M. Gray, Solid St. Ionics, 40 / 41, 637 (1990).

[3] A. Merz, Angew. Chem. Int. Ed., 12, 846 (1973).

[4] B. G. Zupancic and M. Sopcic, Synthesis, 123 (1979).

[5] M. B. Armand, J. M. Chabagno and M. J. Duclot, Fast-Ion Transport in Solids, ed. P. Vashishta, J. M. Mundy and G. Shenoy, Elsevier, New York, 1979, page131.

[6] F. M. Gray, J. R. MacCallum and C. A. Vincent, Solid St. Ionics, 18/19, 252 (1986).

[7] M. T. Hardgrave, PhD thesis University of St. Andrews 1990.

[8] M. A. Mehta, private communication.

[9] P. G. Bruce and C. A. Vincent, J. Electroanal. Chem., 225, 1 (1987).

[10] P. G. Bruce, Polymer Electrolyte Reviews 1, ed. J. R. MacCallum and C. A. Vincent, Elsevier, London, 1987, page 237. 
Table 3-1. Molecular weight of PMEO measured by GPC

\begin{tabular}{lllll}
\hline & without purification & purified by dialysis* & \multicolumn{2}{l}{ purified by filtration } \\
before & after \\
\hline$\overline{\mathrm{M}}_{\mathrm{n}}$ & 92000 & 100600 & 127200 & 44900 \\
$\overline{\mathrm{M}}_{\mathrm{w}}$ & 150700 & 161000 & 258000 & 60000 \\
$\overline{\mathrm{M}}_{\mathrm{w}} / \overline{\mathrm{M}}_{\mathrm{n}}$ & 1.63 & 1.60 & 2.02 & 1.34 \\
\hline
\end{tabular}

* the data before dialysis are the same as those without purification. 


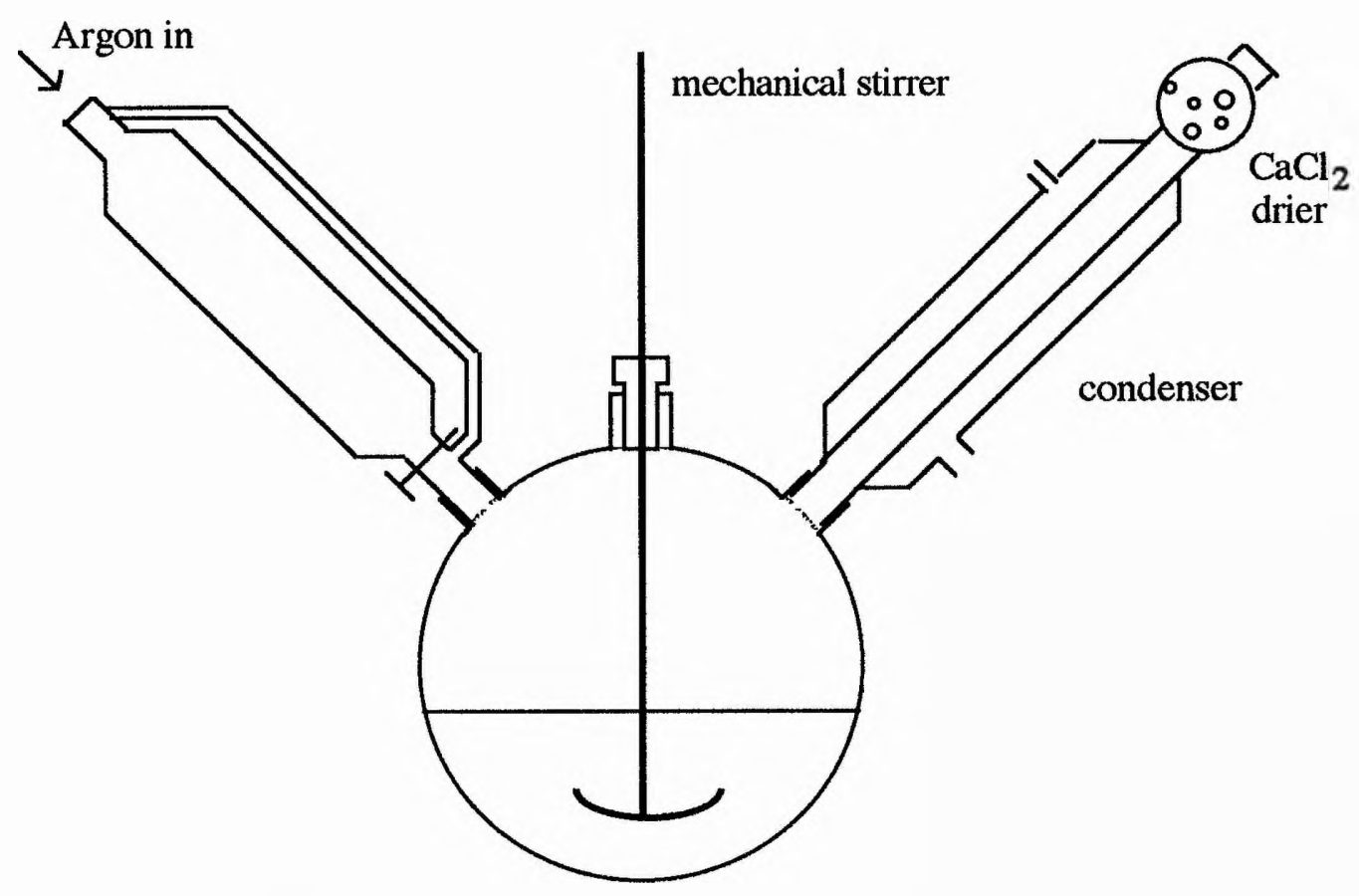

Figure 3-1 Schematic illustration of the apparatus for the synthesis of PMEO. 

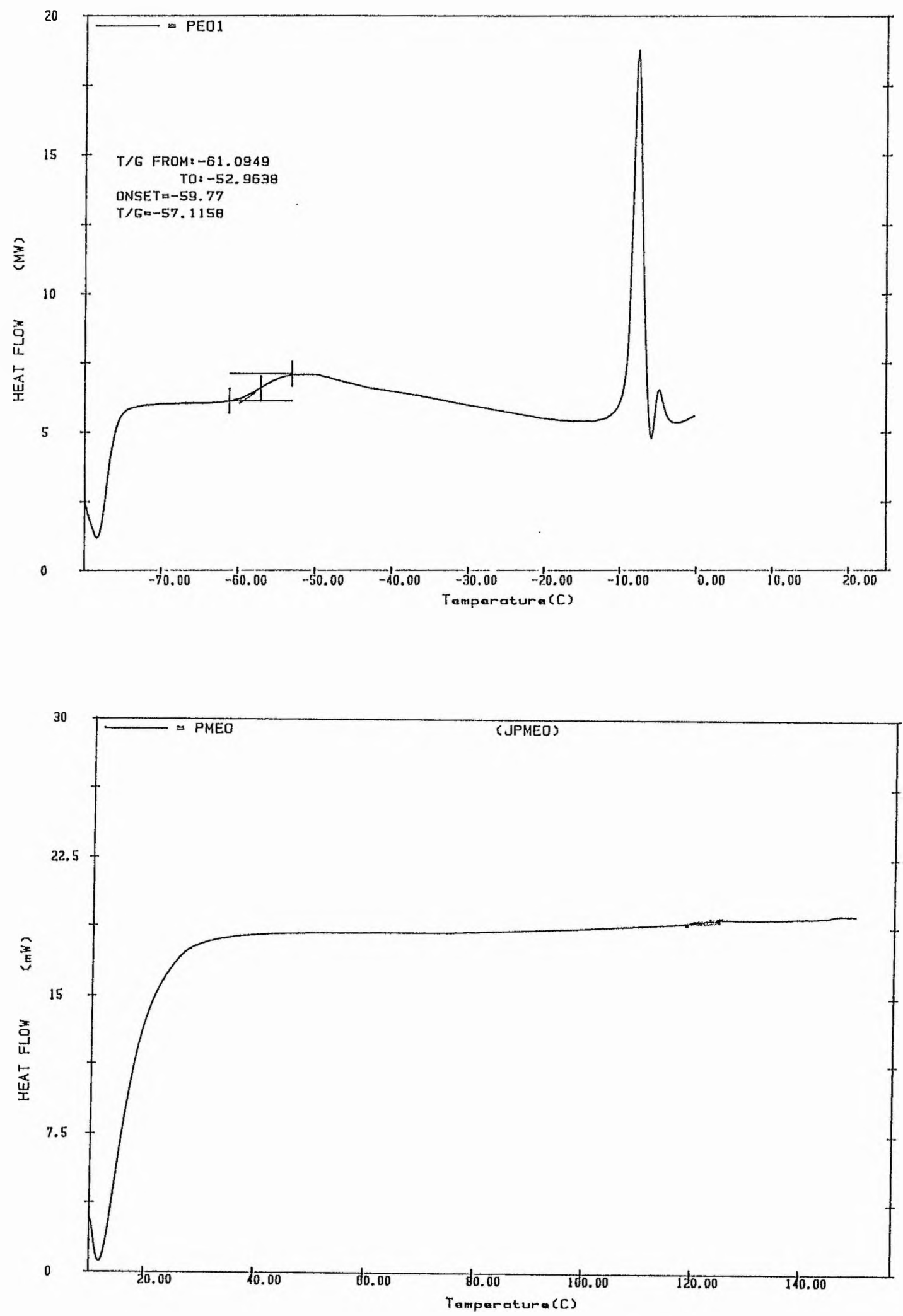

Figure 3-2 DSC trace of PMEO. 


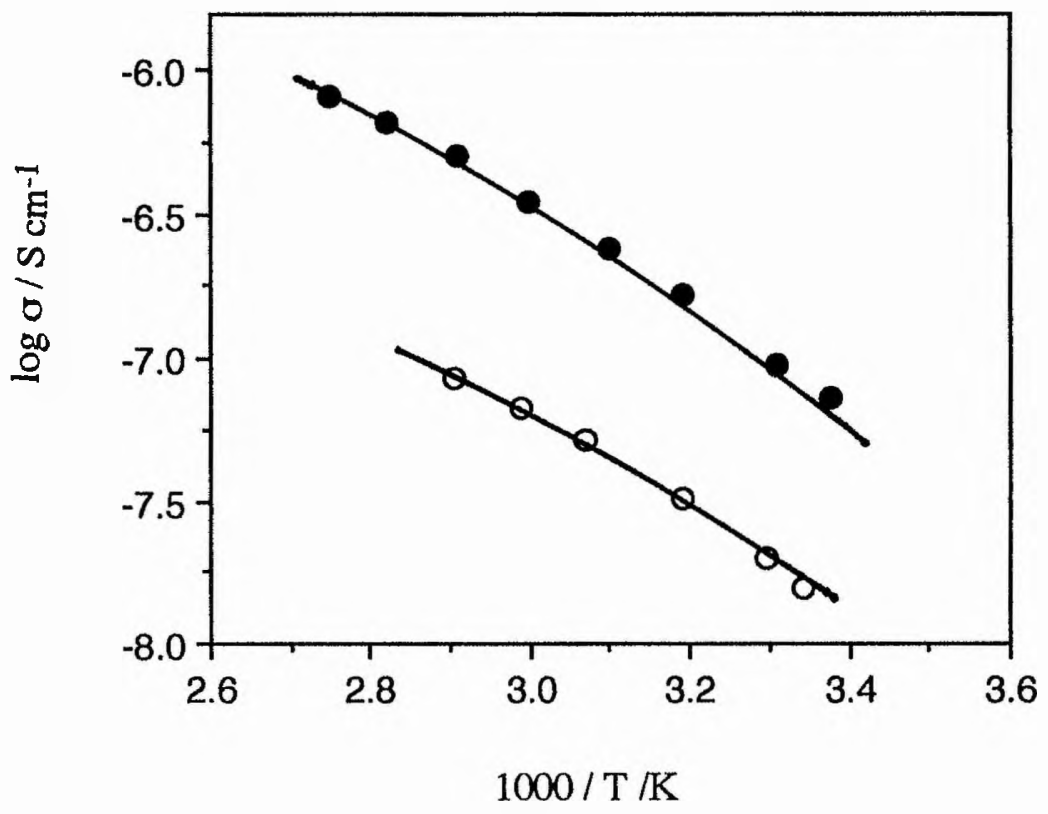

Figure 3-3 Temperature dependence of conductivity for the salt free PMEO (O) and dialysed PMEO $(\mathrm{O})$. 

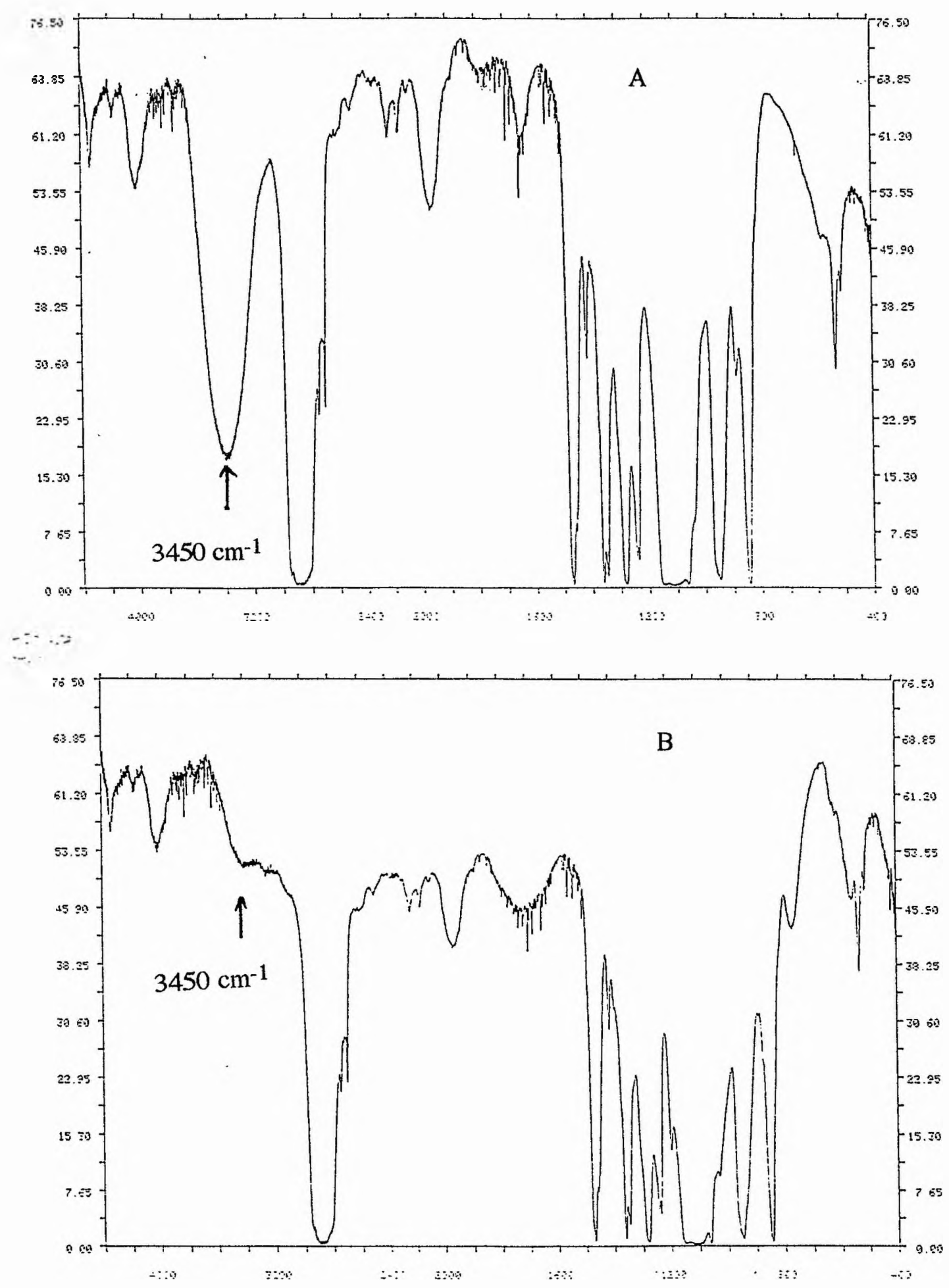

Figure 3-4 FTIR of PEO1450 before (A) and after (B) methylation. 


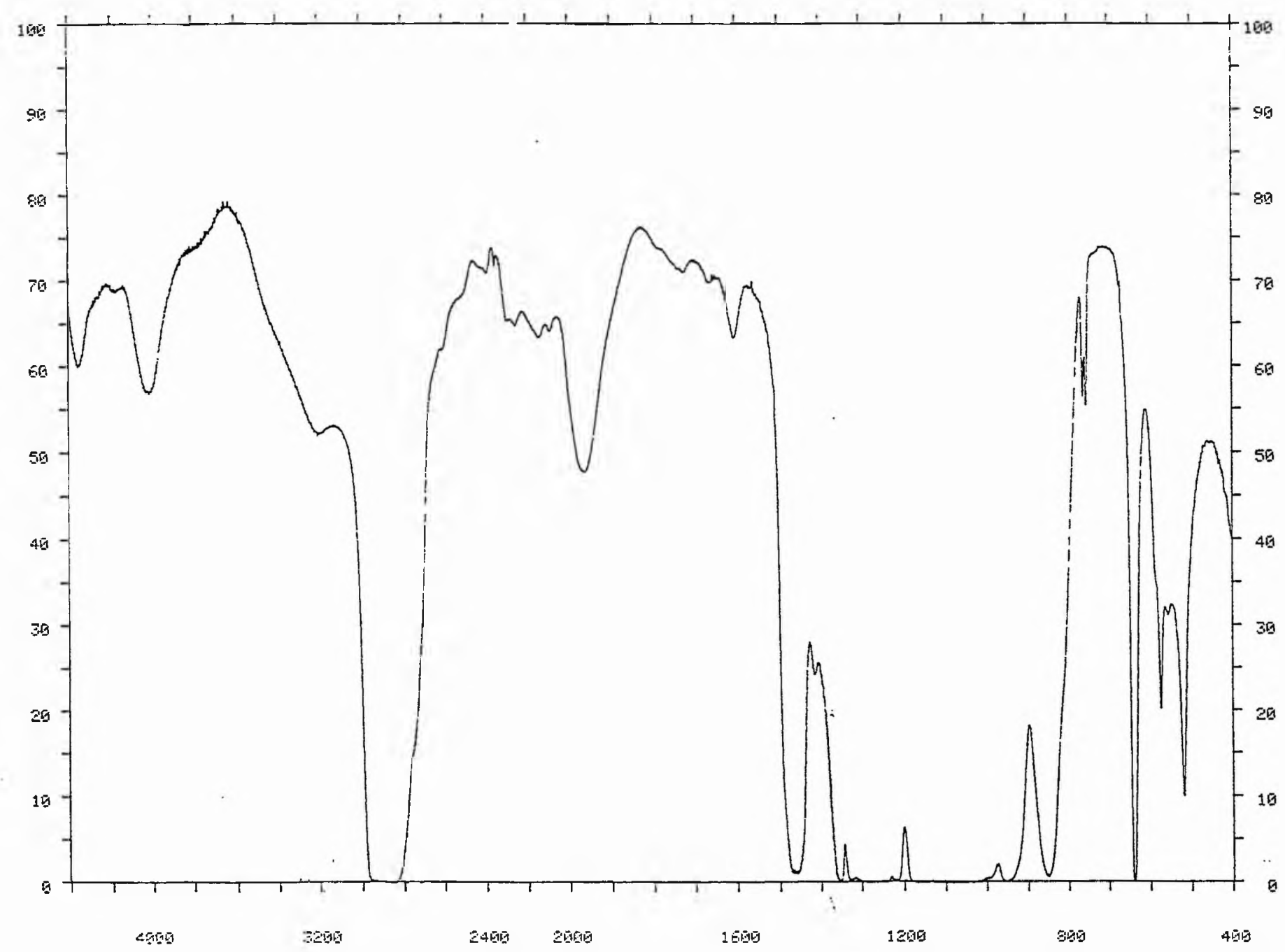

Figure 3-5 FTIR of PMEO-Zn( $\left(\mathrm{CF}_{3} \mathrm{SO}_{3}\right)_{2}$ (70:1) electrolyte preparated by solvent casting. 


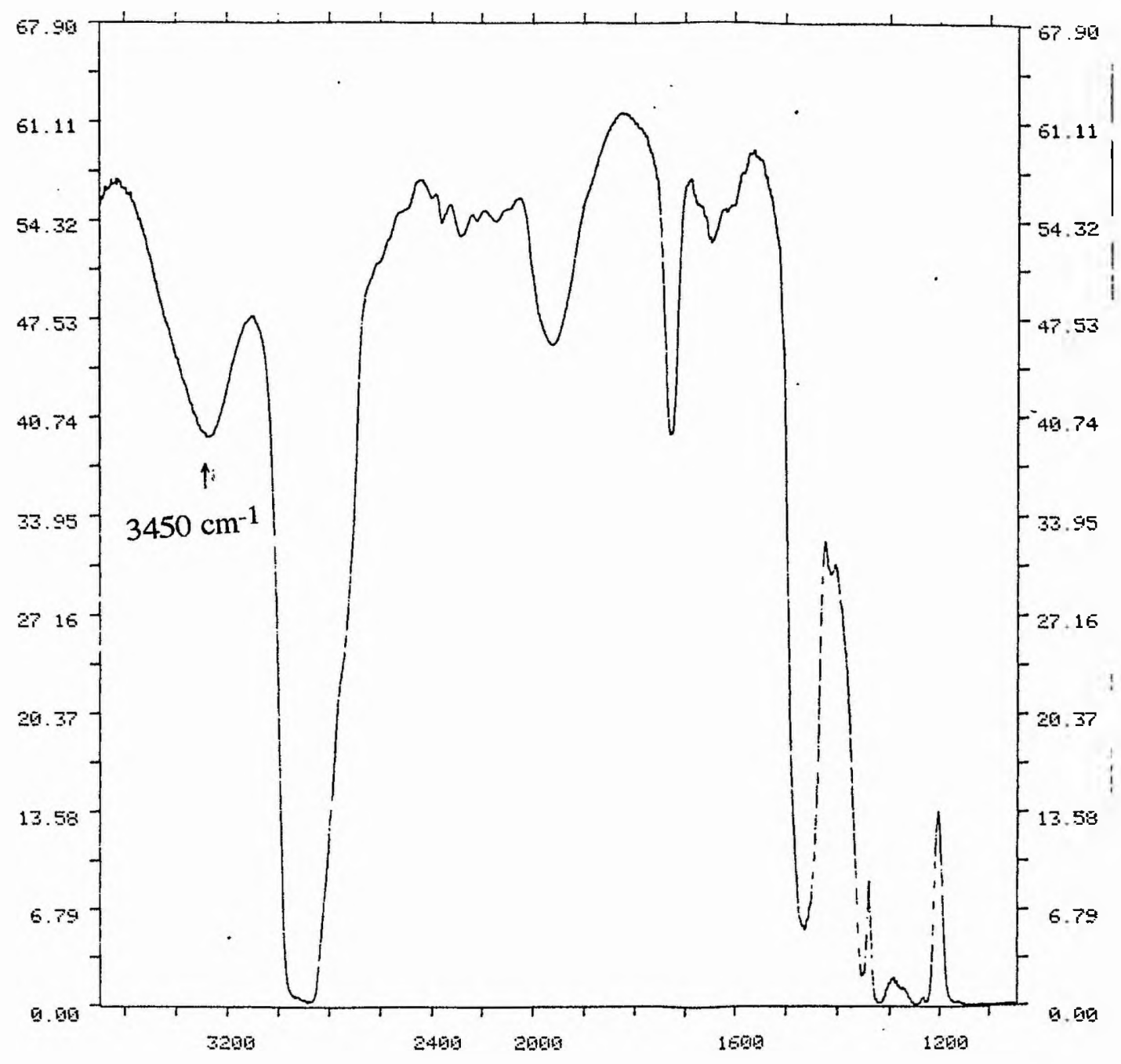

Figure 3-6 FTIR of PMEO-Mg( $\left.\mathrm{CF}_{3} \mathrm{SO}_{3}\right)_{2}$ (20:1) electrolyte preparated by solvent casting. 


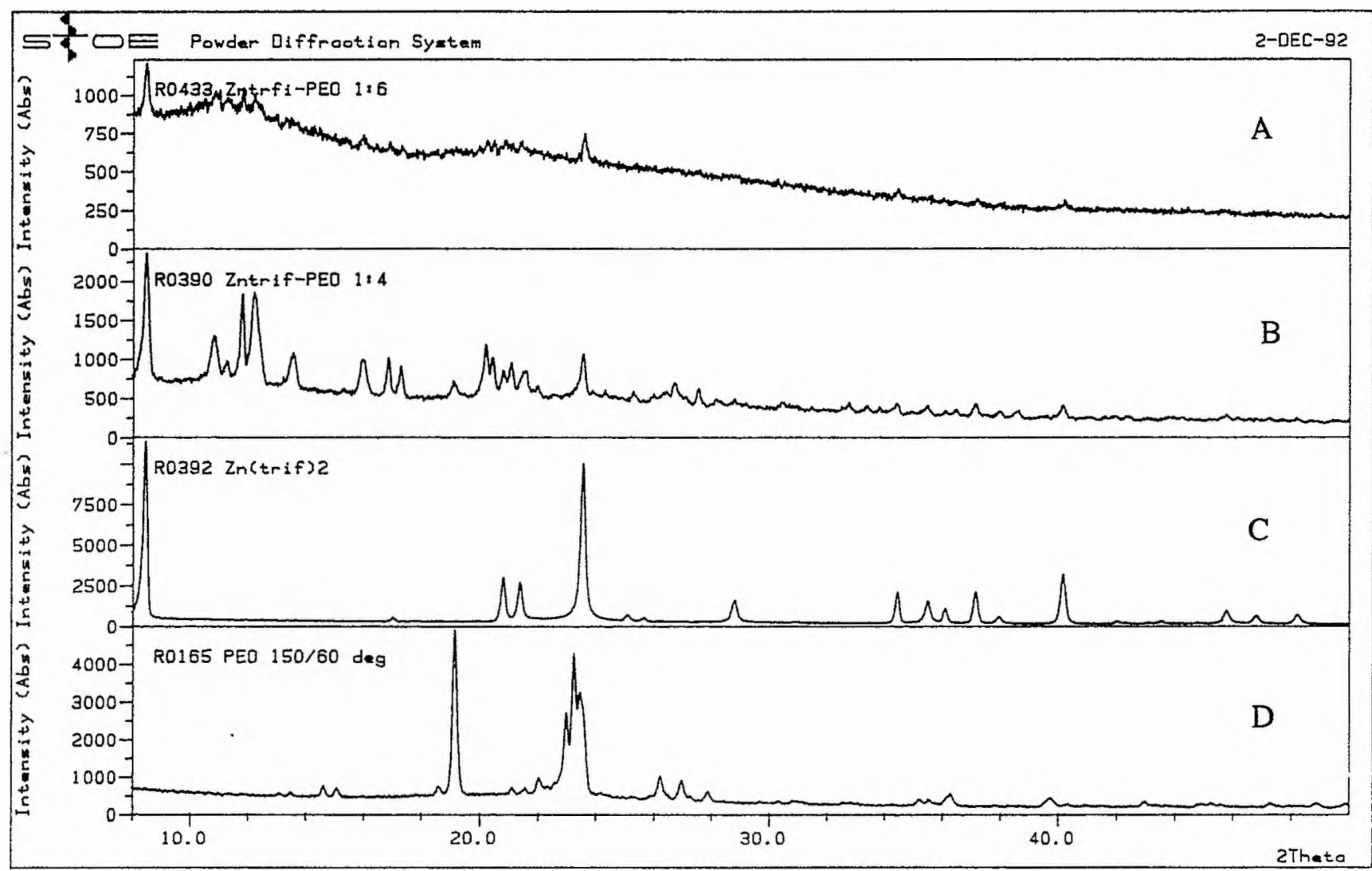

Figure 3-7 X-ray diffraction pattern of $\mathrm{PEO}-\mathrm{Zn}\left(\mathrm{CF}_{3} \mathrm{SO}_{3}\right)_{2}$ at room temperature: (A) $\mathrm{EO} / \mathrm{Zn}=6$, (B) $\mathrm{EO} / \mathrm{Zn}=4,(\mathrm{C}) \mathrm{Zn}\left(\mathrm{CF}_{3} \mathrm{SO}_{3}\right)_{2}$ salt and (D) PEO. 


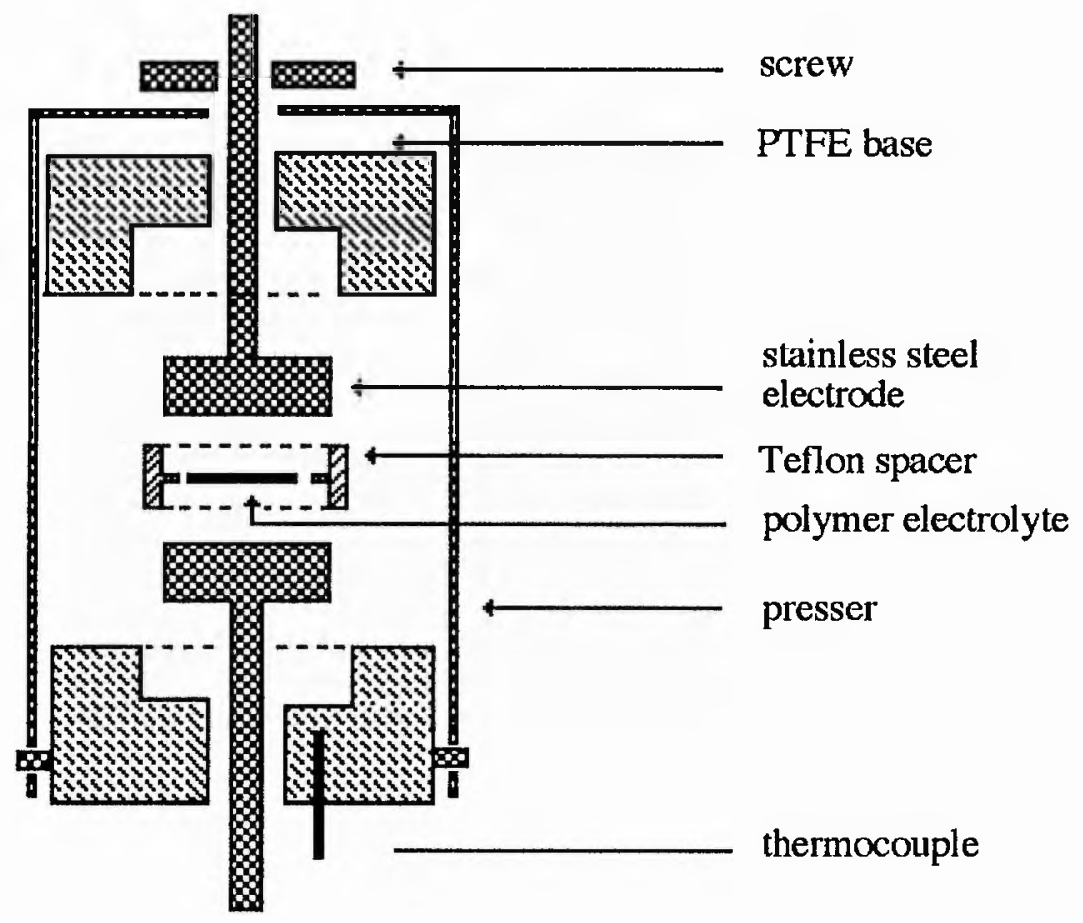

CELL $1 \& C E L L 3$

Figure 3-8 Two electrode cell for conductivity measurement of soft polymer electrolytes $C E L L 1 . C E L L 3$ is constructed by removing the Teflon spacer. 


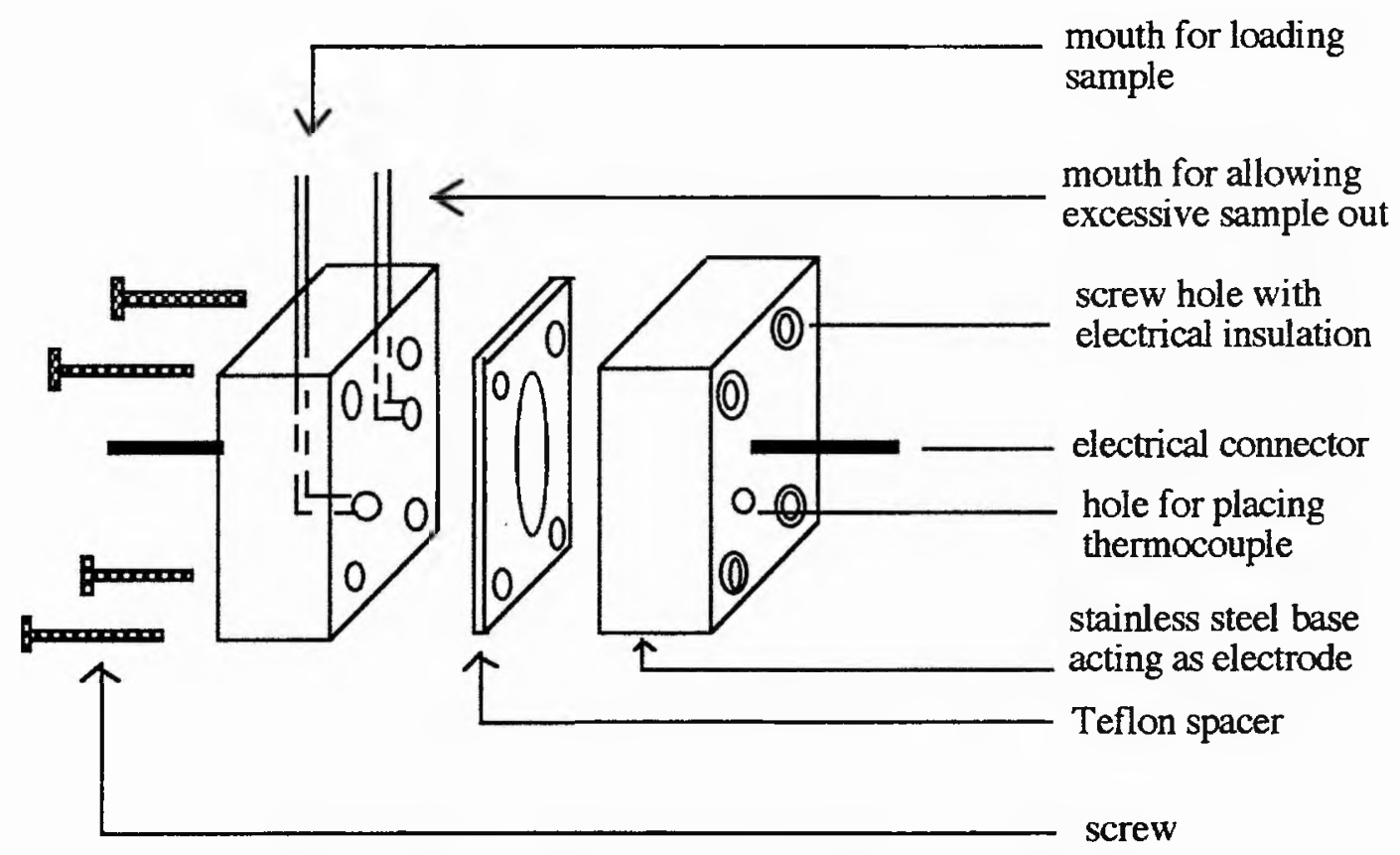

CELL 2

Figure 3-9 Two electrode cell for conductivity measurement of liquid polymer electrolytes. 


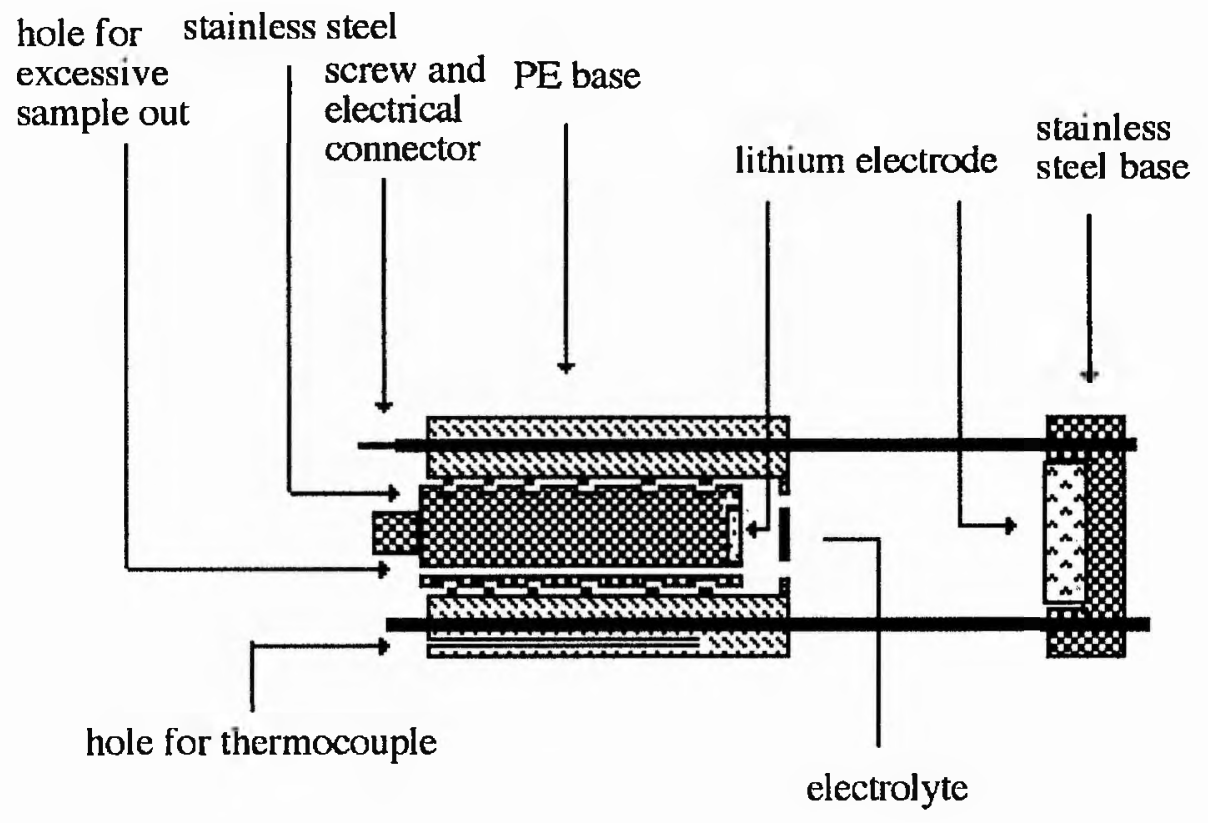

CELL 4

Figure 3-10 Two electrode cell with lithium electrodes for dc polarisation of soft and liquid polymer electrolytes based on lithium salts. 


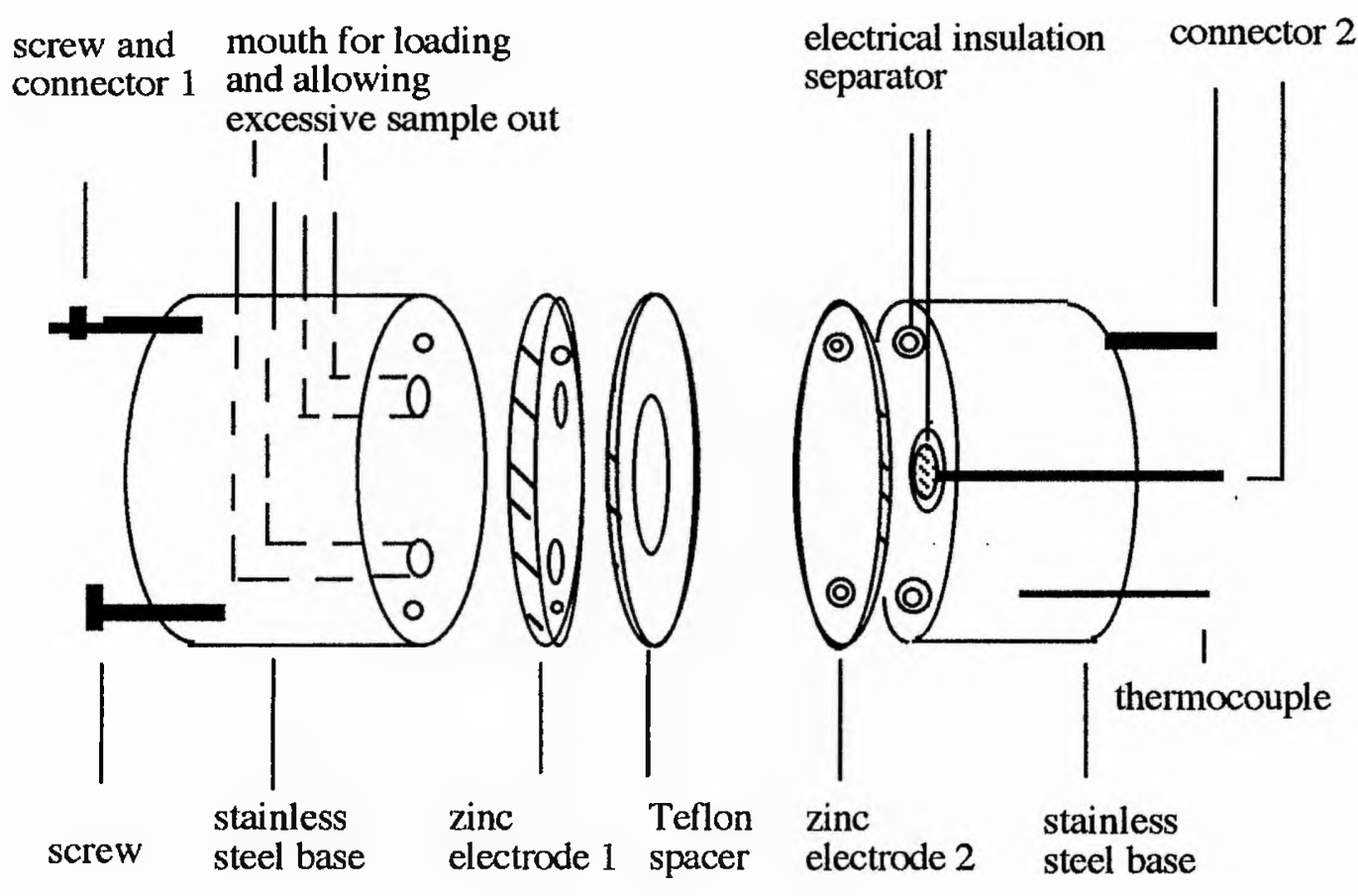

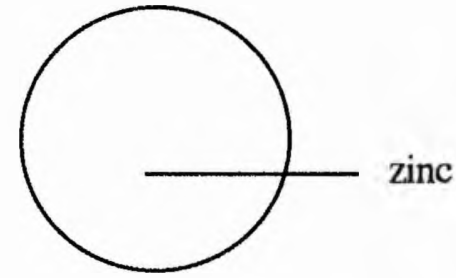

zinc electrode 2 for two electrode cell

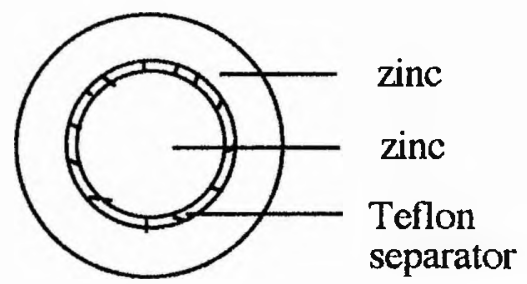

zinc electrode 2 for three electrode cell

CELL 5 \& CELL 9

Figure 3-11 Zinc two electrode and three electrode cell for liquid and soft polymer electrolytes based on zinc.salts. 


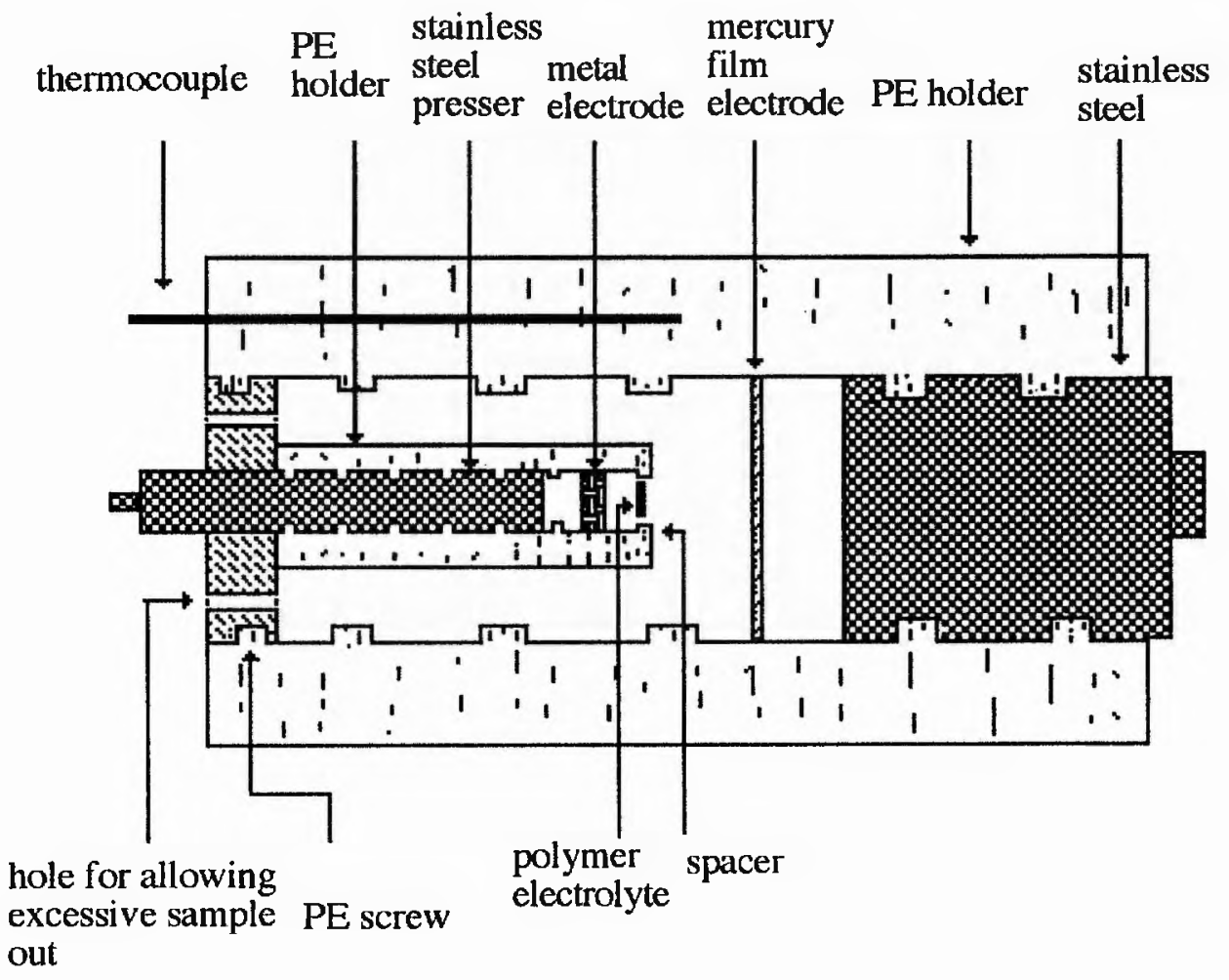

CELL 6

Figure 3-12 Two electrode cell for dc polarisation of soft and liquid polymer electrolytes based on calcium and magnesium salts. 


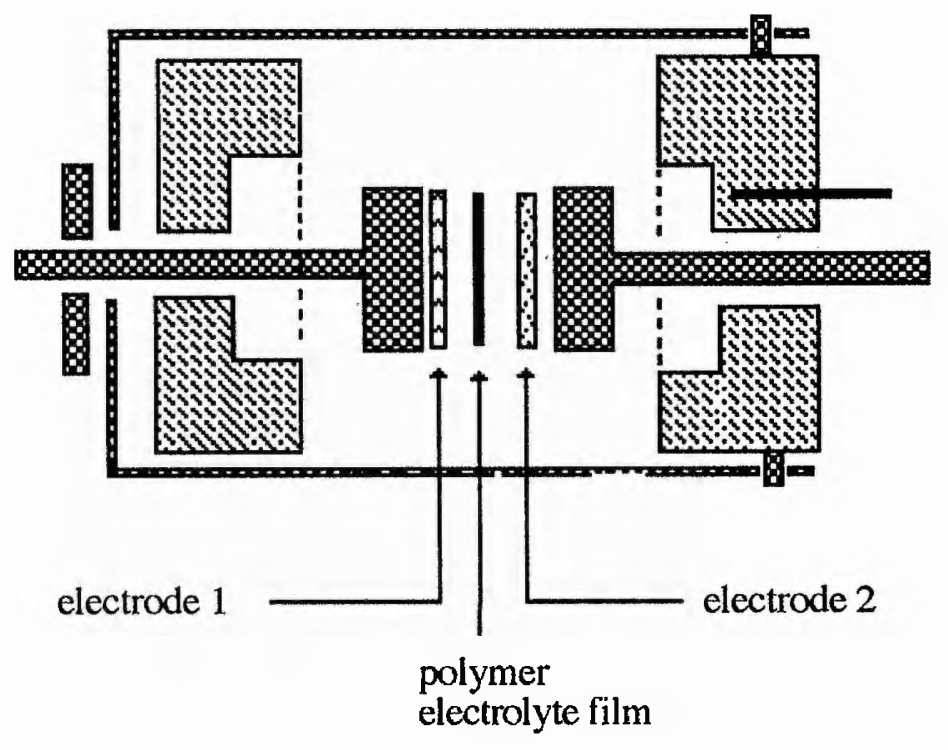

CELL 7

Figure 3-13 Two electrode cell for dc polarisation of polymer electrolytes based on PEO $4 \times 10^{6}$. 

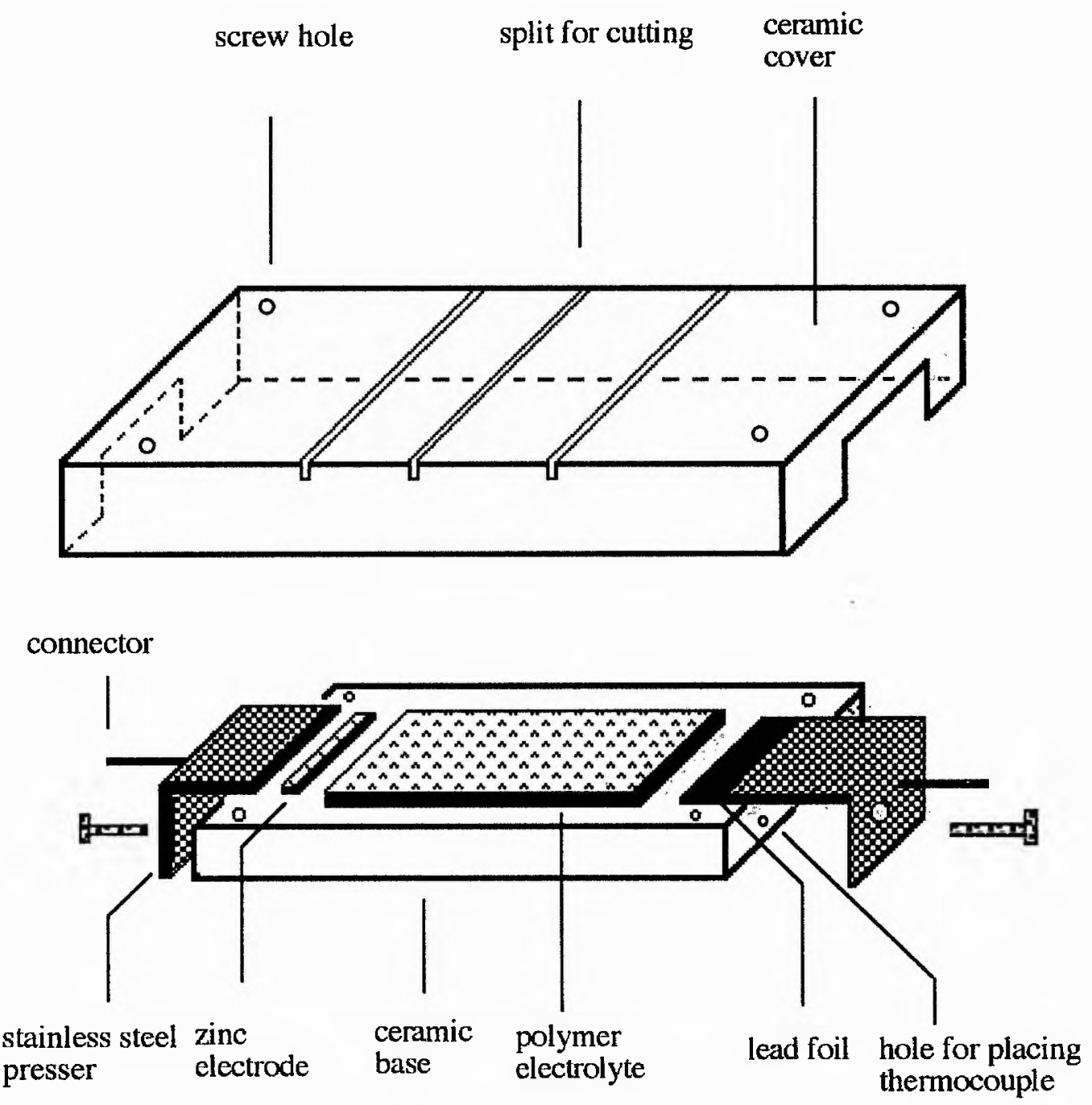

\section{CELL 8}

Figure 3-14 Schematic illustration of the Hittorf cell for determining the ionic transference number of $\mathrm{PEO}-\mathrm{Zn}\left(\mathrm{CF}_{3} \mathrm{SO}_{3}\right)_{2}$ electrolytes. 


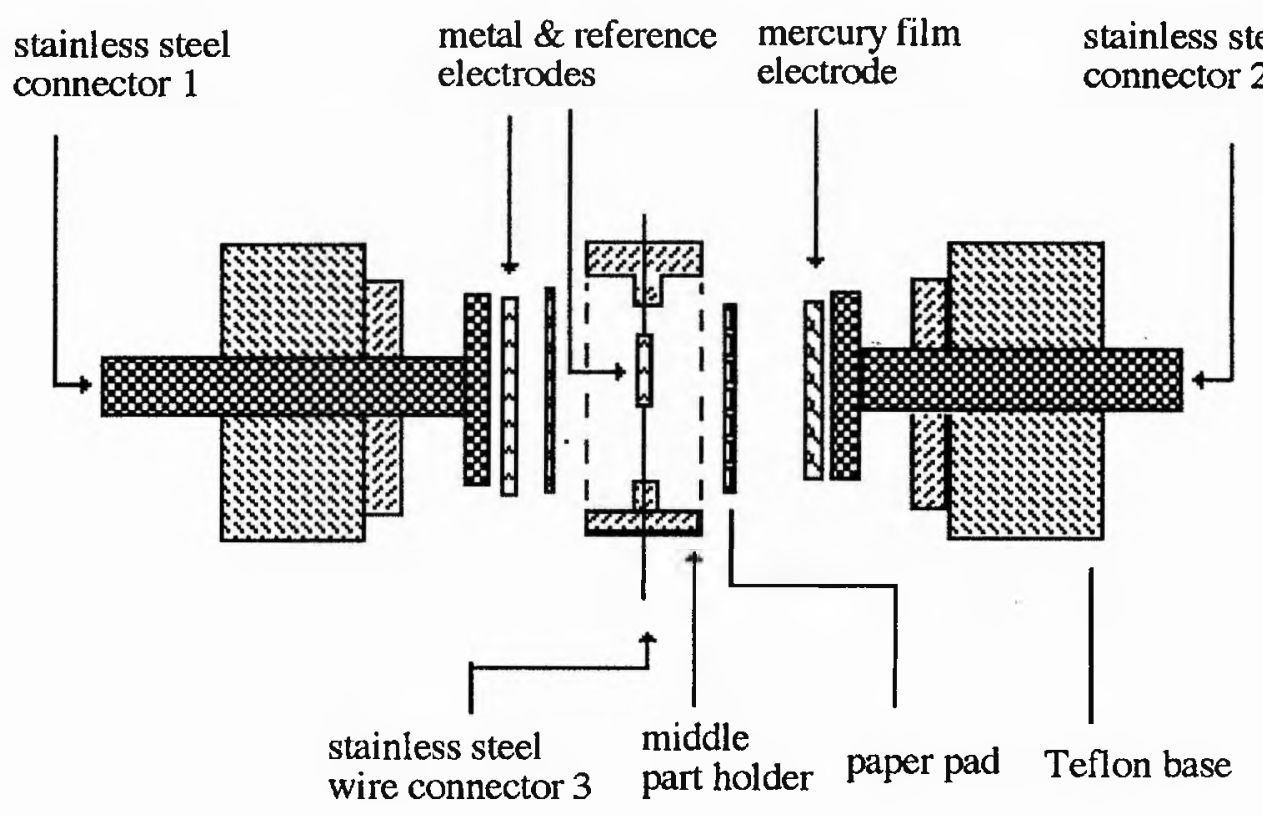

CELL 10

Figure 3-15 Three electrode cell for cyclic voltammetry and ac impedance study of polymer electrolytes based on PEO(400) dimethyl ether and calcium or magnesium salts at room temperature. 


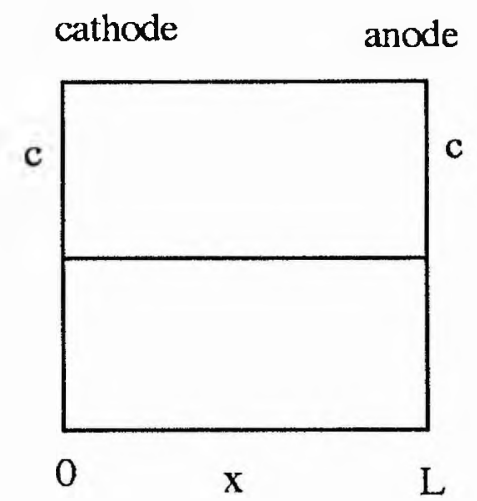

A

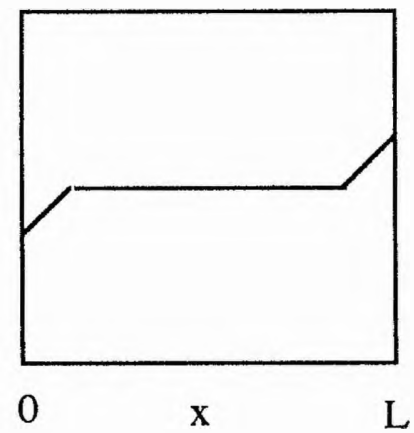

B

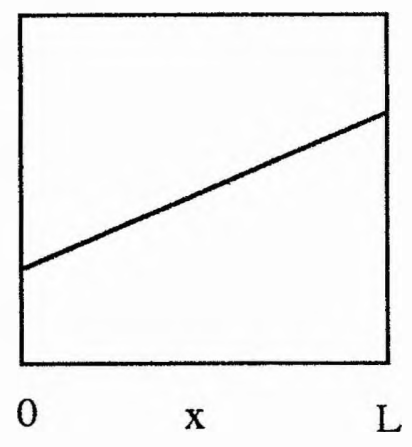

C

Figure 3-16 The change of the salt concentration within a polymer electrolyte for dc polarisation of a two electrode cell at $t=0(A), t>0$ before reaching steady state (B) and steady state $(\mathrm{C})$.

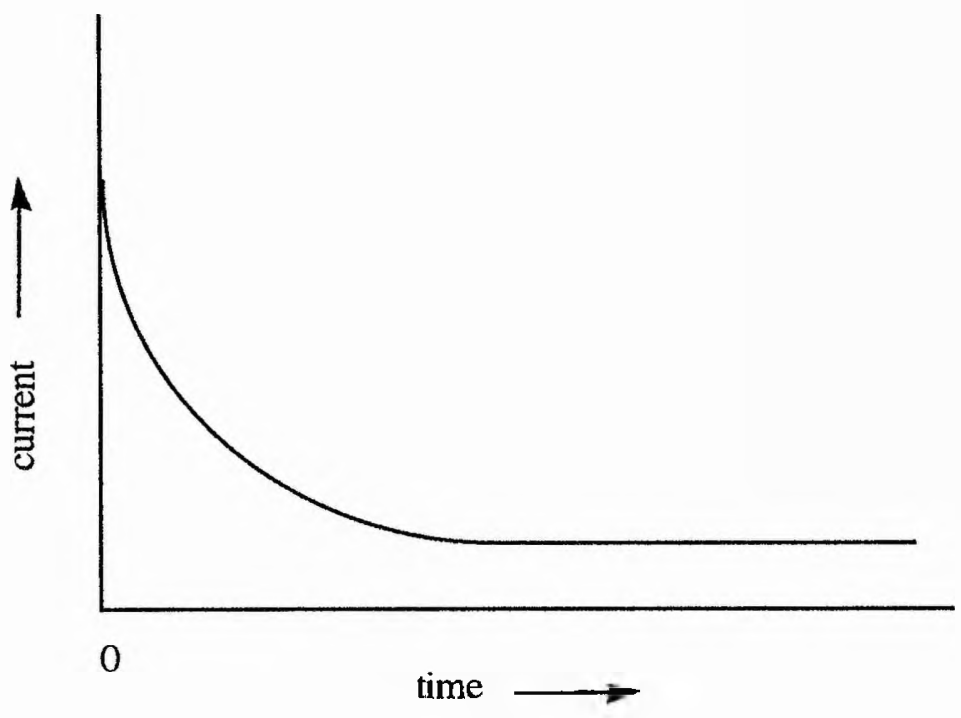

Figure 3-17 Schematic representation of plot of the current against time for dc polarisation of a two electrode cell with polymer electrolyte 
File: LP1005.DAT

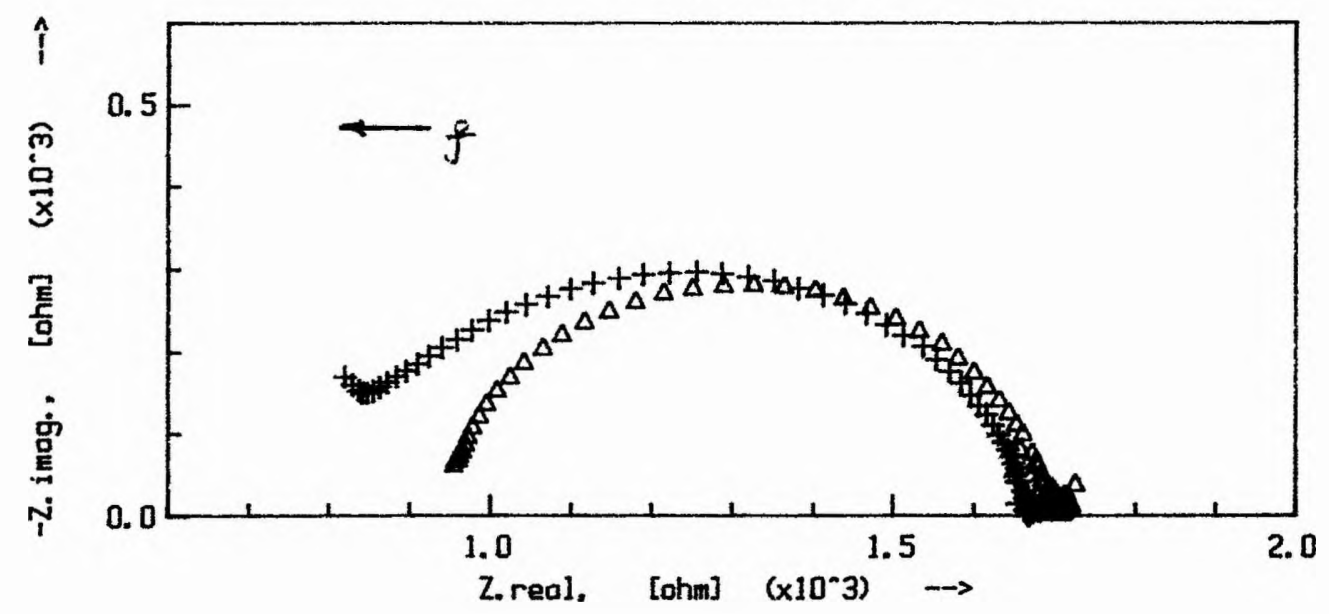

Figure 3-18 Ac impedance spectra for Li / PMEO-LiPF 6 (20:1) / Li cell before (+) and after $(\Delta)$ dc polarisation with $10 \mathrm{mV}$ applied voltage for 15 hours at $60^{\circ} \mathrm{C}$. 


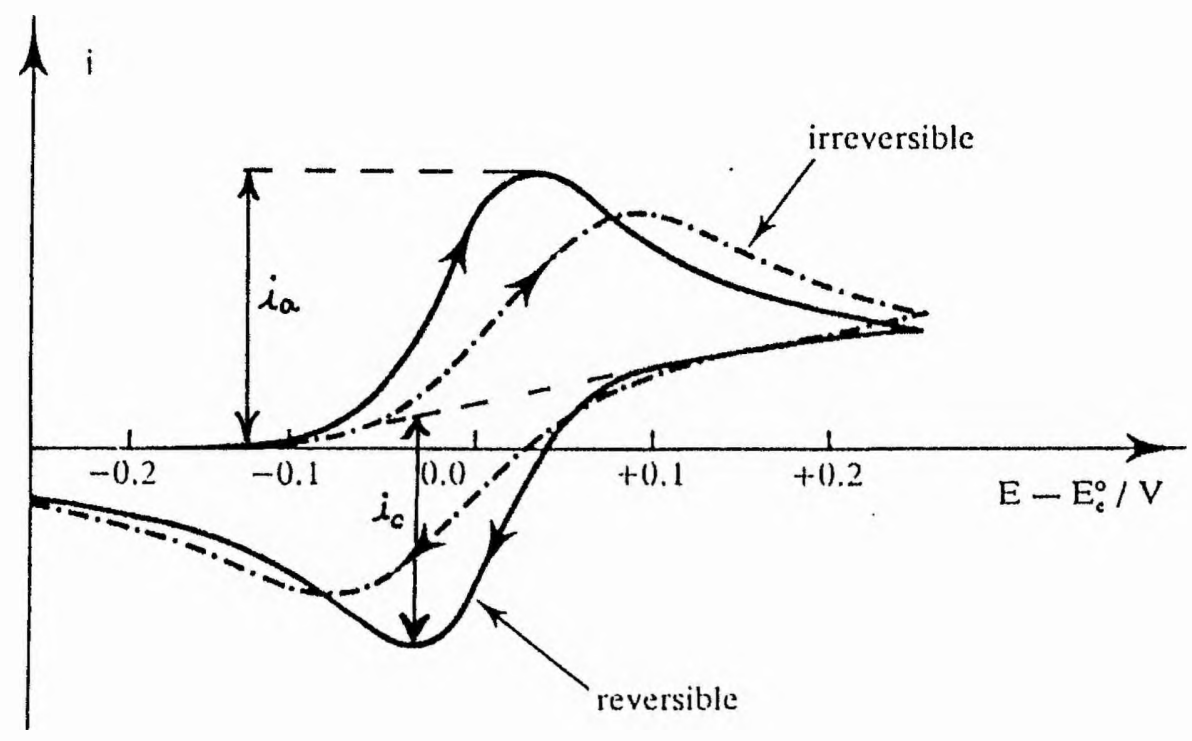

Figure 3-19 Schematic illustration of cyclic voltammgram for reversible and irreversible systems.

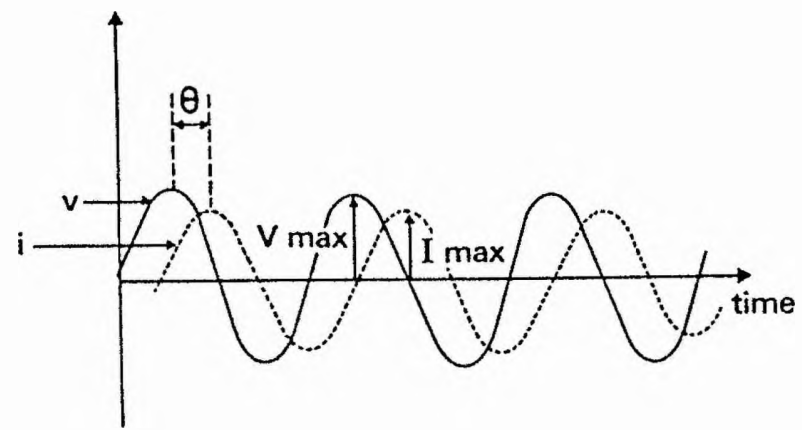

Figure 3-20 Schematic illustration of an ac signal applied to a cell. 


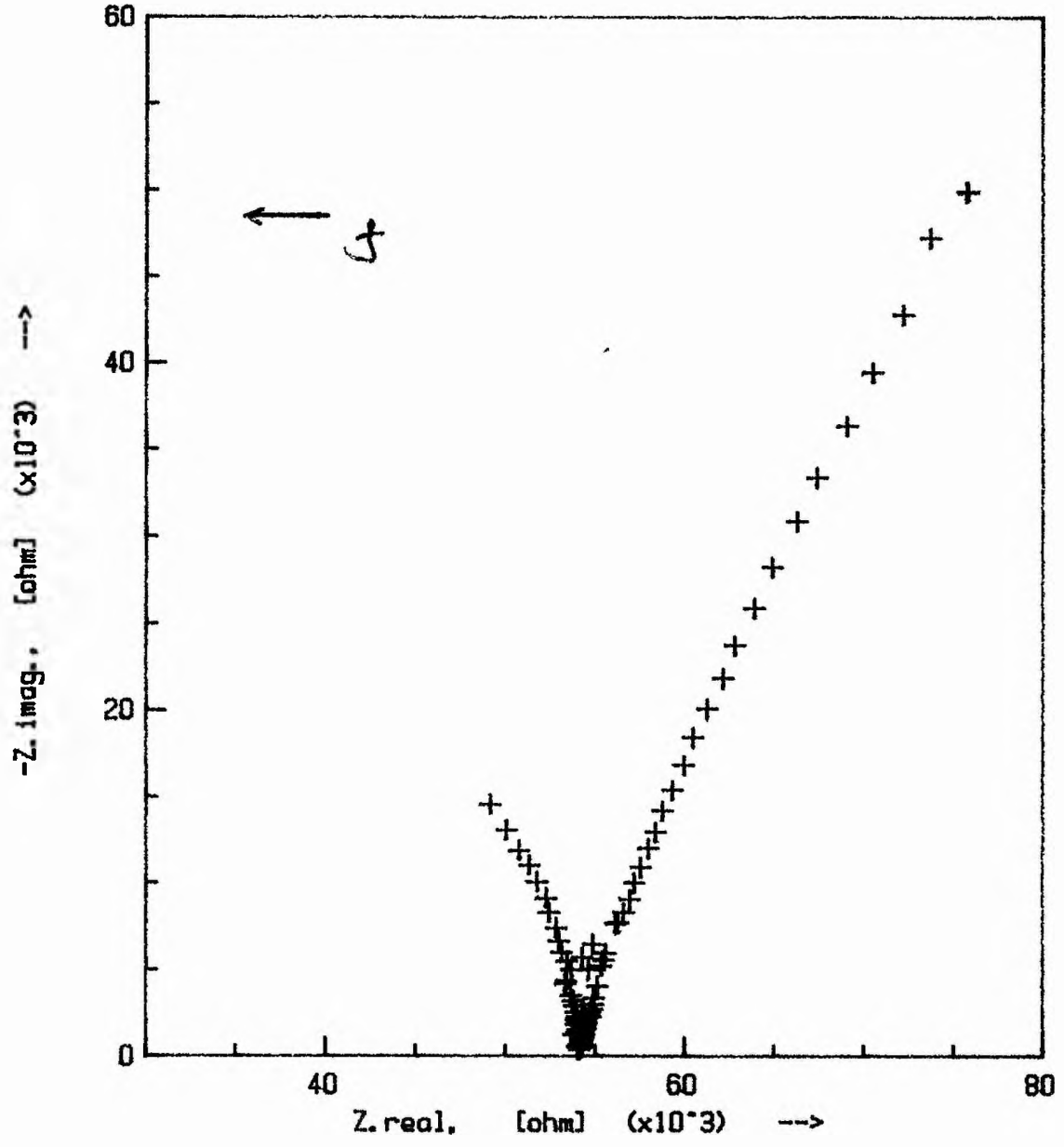

Figure 3-21 Ac impedance spectrum of a cell with $\mathrm{PMEO}^{-\mathrm{LiClO}_{4}}$ (salt concentration of $0.003 \mathrm{~mol} \mathrm{dm}^{-3}$ ) and two stainless steel electrodes at $88^{\circ} \mathrm{C}$. 


\section{CHAPTER 4}

\section{EXPERIMENTAL TECHNIQUES FOR SOLID STATE NUCLEAR MAGNETIC RESONANCE}

Solid state NMR has been used in the investigation of polymer electrolytes since the 1980's [1], and has principally involved measurements of the spin-lattice relaxation time $T_{1}$ and the spin-spin relaxation time $T_{2}$, and the diffusion coefficients of cations and anions. In this thesis, the species existing in polymer electrolytes have been investigated by magic-angle spinning NMR, and the diffusion coefficients of these species have been studied by pulsed field gradient NMR. In this chapter, the principle of solid state NMR, in particular mas NMR and pfg NMR is briefly presented, and the experimental techniques used are described.

\section{4-1 Magic-Angle Spinning NMR}

\section{4-1-1 Introduction}

High resolution NMR of solution samples has been well studied and has proved to be a very powerful technique for probing the chemical environment of the nucleus in molecules and for offering useful information about the dynamics of molecules in solutions. Due to the characteristics of solids, solution NMR technique cannot be directly applied to solid systems.

The main interactions involving a nucleus with a magnetic moment which may occur in the solid state are expressed as follows. 
(i) Zeeman interaction: This occurs for all nuclei with odd atomic mass or odd atomic number between the magnetic moment of the nucleus and the applied field $\mathrm{H}_{\mathrm{o}}$. The Hamil tonian may be written as:

$$
\mathbf{H}_{\mathrm{z}}=-\boldsymbol{\gamma} \boldsymbol{h} \mathbf{H}_{\mathrm{O}} \mathbf{I}_{\mathrm{Z}}
$$

where $\gamma$ is the gyromagnetic ratio, $h$ is a constant and $H_{0}$ and $I_{z}$ are the applied magnetic field and the spin moment respectively.

(ii) Direct dipole-dipole interactions: This arises from the dipole-dipole interactions between nuclei. For a solid containing a single type of spin, I, of gyromagnetic ratio $\gamma$, the interaction may be written as:

$$
H_{D}=\frac{\gamma^{2} h^{2}}{r_{i j}^{3}} \vec{I} \cdot \hat{D} \cdot \vec{I}
$$

where $\boldsymbol{r}_{\mathbf{i j}}$ is the internuclear distance and $\hat{D}$ is the dipolar coupling tensor.

(iii) Chemical shift interaction: This is due to the shielding effect on the nucleus of the fields produced by the surrounding electrons and can be expressed as:

$$
\mathbf{H}_{\mathrm{CS}}=\gamma \mathrm{h} \overrightarrow{\mathbf{I}} \cdot \hat{\boldsymbol{\sigma}} \cdot \overrightarrow{\mathbf{H}}
$$

where $\hat{\mathbf{a}}$ is the chemical shift tensor. The interaction is sensitive to the geometry and identity of other atoms surrounding a particular nucleus and will usually be the most useful chemical diagnostic measurable in NMR studies.

(iv) Spin-spin coupling interaction: This arises from the interaction between a pair of spins I and $S$, and can be expressed as:

$$
\mathbf{H}_{\mathrm{sS}}=\overrightarrow{\mathrm{I}} \cdot \hat{\mathrm{J}} \cdot \overrightarrow{\mathrm{S}}
$$

where $\mathrm{f}$ is the spin-spin coupling tensor. 
(v) Quadrupolar interaction: This occurs only when I $>1 / 2$ and arises from the interaction of the nuclear electric quadrupolar moment eQ with the non-spherically symmetrical field gradient around the nucleus. It can be described as:

$$
\mathbf{H}_{\mathrm{Q}}=\overrightarrow{\mathbf{I}} \cdot \hat{\mathbf{Q}} \cdot \overrightarrow{\mathbf{I}}
$$

where $\mathbf{Q}$ is the quadrupolar interaction tensor.

In a solution, because nuclei are separated by solvent molecules, the fast and essentially isotropic motions of the molecules give an averaging of the interactions of the nuclei both with other nuclei and with electric field gradients if present. Thus in solution NMR discrete average values are found for chemical shifts and scalar coupling. For dipolar and quadrupolar coupling, average values are exactly zero and these effects are not observed at all in solution NMR.

In the solid state, because the nucleus is trapped in a solid matrix, the above interactions will be anisotropic which will complicate the analysis of solid state NMR spectra. For example, the linewidth of the spectrum is very broad, anisotropic chemical shifts are observed and quadrupolar splitting is sometimes found. Some specific techniques are employed in solid state NMR to solve these problems. One of them is the magic-angle spinning which often is used in the study of chemical environment in solids.

\section{4-1-2 Principle of Magic-Angle Spinning}

Consider a solid sample to be rotated about an axis, $R$, inclined at angle $\theta$ to the applied magnetic field $H_{0}$ (Figure 4-1). When the angle $\theta$ is $54^{\circ} 44^{\prime}$, the term $\left(3 \cos ^{2} \theta-1\right)$ will be zero, which results in an averaging of some of the interactions discussed in the above section. This angle, $\theta=54^{\circ} 44^{\prime}$, is called the magic angle. 
The dipolar interaction between two identical nuclei can be expressed as:

$$
\mathbf{H}_{\mathrm{D}}=-\frac{1}{2}\left(3 \cos ^{2} \theta_{\mathrm{ij}}-1\right) \sum_{\mathrm{i}<\mathrm{j}} \mathrm{g}_{\mathrm{N}}^{2} \beta_{\mathrm{N}}^{2} \frac{1}{\mathbf{r}_{\mathrm{ij}}^{3}}\left(1-3 \cos ^{2} \gamma_{\mathrm{ij}}\right)
$$

where $\theta$ and $\gamma$ are defined in Figure 4-1, $g_{N}$ is the nuclear $g$ factor and $\beta_{N}$ is the Bohr magneton. Because the magic-angle spinning causes the term $\left(3 \cos ^{2} \theta-1\right)$ to be equal to zero, the dipolar interaction term vanishes. Therefore, the dipolar broadened line is narrowed by the magic-angle spinning.

Magic-angle spinning can not only average homonuclear and heteronuclear dipolar interactions, but also average the chemical shifts and the spin-spin coupling to their isotropic values. For $I=1 / 2$ nuclei, the isotropic chemical shifts obtained by mas NMR in the solid state may be different from the isotropic values in solution. However, it is found that they are generally very similar to the solution values, and the chemical shift - molecular structure relationship developed from solution studies may be used for structure determinations in the solid state.

For quadrupolar nuclei with integer spins, eg. ${ }^{2} \mathrm{H}$ with $\mathrm{I}=1$, magic-angle spinning can narrow the spectra dramatically (for ${ }^{2} \mathrm{H}$, it is narrowed about a factor of $10^{3}$ ). For quadrupolar nuclei with non-integral spins, eg. ${ }^{23} \mathrm{Na}$ with $\mathrm{I}=3 / 2$, the central transition between $\mathrm{m}=1 / 2$ and $\mathrm{m}=-1 / 2$ levels is unaffected by the quadrupolar interaction to first order (Figure 4-2). The effects of the second order quadrupolar interaction can however be reduced, but not completely removed by the magic angle spinning. Therefore, the pattern of the central line is narrowed and changed, but its centre remains shifted. Quadrupolar effects, unlike chemical shifts which are proportional to the field strength, are inversely proportional to the field strength. For those nuclei with small quadrupolar interaction, high-resolution NMR spectra can be 
derived by rapid magic-angle spinning at relatively high field. For example, ${ }^{11_{\mathrm{B}}}$ mas NMR was successfully applied to investigate the structure of borosilicate glass [2].

When magic-angle spinning rates (spinning frequencies) are smaller than the chemical shift anisotropy, spinning sidebands separated by the spinning frequency symmetrically placed about the isotropic average peak will be shown in the spectrum.

\section{4-1-3 Setting of the Magic Angle}

For maximum resolution, the angle must be set as accurately as possible, a line broadening of about $2.5 \%$ of the static linewidth being introduced for every degree the spinning axis deviates from $54^{\circ} 44^{\prime}$. Most of ten this is done by setting the angle on an easily observable reference sample which has a large shift anisotropy, and then changing to the sample of interest without changing the angle.

The quadrupolar nucleus ${ }^{79} \mathrm{Br}$, in crystal $\mathrm{KBr}$ is of ten used to set the magic angle. Because the crystal symmetry of $\mathrm{KBr}$ is cubic, a sharp absorption is observed for the central $(m=1 / 2) \leftrightarrow(m=-1 / 2)$ transition. Spinning sidebands separated by the spinning frequency are clearly observed in the spectrum and these are very sensitive to the exact angle of the spinning axis. This method allows the angle to be set within $0.1^{\circ}$ of the magic angle [3].

\section{4-2 Relaxation Processes}

\section{4-2-1 Spin-Lattice Relaxation and Spin-Spin Relaxation}

When a particle with $I=1 / 2$ and magnetic moment $\mu$ is placed in a static magnetic field $\mathrm{H}_{0}$, two energy states with separation of $\Delta \mathrm{E}=2 \mu \mathrm{H}$ are produced. If a group of spin -1/2 nuclei are placed in a magnetic field, they will distribute themselves between the two states according to the Boltzmann relation, so that the ratio of the number of spins in the upper energy state to that in the lower energy state is 
$\exp (-\Delta E / k T)$ at equilibrium. As a result, there will be more spins in the lower energy state than in the upper state, and the population difference gives rise to a macroscopic magnetization. The magnitude of the magnetization can be described by Curie's Law. The process of growth towards the equilibrium magnetization is defined as the spinlattice relaxation. The time for the magnetization to recover $63 \%$, i.e. $\left(1-\mathrm{e}^{-1}\right)$ of the equilibrium value, is defined as the spin-lattice relaxation time, $T_{1}$.

Spin-spin relaxation, also called transverse relaxation, is a process in which the magnetization in the $x-y$ plane, perpendicular to the static field, decays. The time constant for the decaying of magnetization to $1 / \mathrm{e}$ of its equilibrium value is defined as the spin-spin relaxation time, $\mathrm{T}_{2}$.

The $\mathrm{T}_{1}$ relaxation process involves exchange of the absorbed if energy from the perturbed nuclei to the surroundings. The $T_{2}$ process however, does not involve any exchange of energy with the world outside the spin system; it involves only the direct interaction of spins.

\section{4-2-2 Relation of $T_{1}$ and $T_{2}$ to Molecular Motion}

The molecular motion in a sample of spin $-1 / 2$ nuclei can be correlated to the spin relaxation processes. A simple and of ten employed equation is BPP theory (Bloembergen, Purcell and Pound):

$$
\frac{1}{T_{1}} \propto \frac{B_{l o c} \tau_{c}}{1+\omega_{o}^{2} \tau_{c}^{2}}
$$

where $\mathrm{B}_{\text {loc }}$ is that part of the local dipolar field modulated by the local motion, $\omega_{0}$ corresponds to the frequency, and $\tau_{c}$ is a correlation time for the motion. For spin-spin relaxation time, the following relation exists: 


$$
\frac{1}{\mathrm{~T}_{2}} \propto \tau_{\mathrm{c}}
$$

The temperature dependence of the correlation time $\tau_{\mathrm{c}}$ depends on the mechanism of the relaxation process of the molecular motion. For an activated relaxation, it can be described by an Amhenius equation:

$$
\tau_{c}=\tau_{0} \exp \left(E_{a} / k T\right)
$$

where $\tau_{0}$ is a constant, $E_{a}$ is the activation energy, $k$ is the Boltzmann constant and $T$ is the temperature. For polymer system, it is of ten described by the VTF equation:

$$
\tau_{c}=\tau_{o} \exp \left[B /\left(T-T_{o}\right)\right]
$$

where $\tau_{0}, B$ and $T_{o}$ are constants, $T$ is the temperature. The relationship between $T_{1}, T_{2}$ and the temperature can be plotted as in Figure 4-3. There are several characteristic temperatures in the plot. (i) A minimum occurs for $T_{1}$, at which $\omega_{0} \tau_{c} \approx 1$. This enables a good estimate to be made of the correlation time describing the motion at the temperature corresponding to the minimum. (ii) At lower temperatures or in the limit of zero motion where $\tau_{c}$ is very large and spin-spin interactions are very strong, $T_{2}$ reaches a value which is sometimes known as the 'rigid lattice' value, common to most solids. When temperature increases, the molecular motion increases, i.e. $\tau_{c}$ decreases, and $T_{2}$ increases. Because there are distriutions of relaxation times in polymers, the BPP equation (4-2-1) is often invalid in correlation of the relaxation times of polymer segments.

\section{4-2-3 Determination of $T_{1}$ and $T_{2}$}

(1) Determination of $T_{1}$

Double pulse sequences [4] are often used in the determination of spin-lattice time $T_{I}$. There are two popular sequences for this method. 
(i) $\pi-\delta-\pi / 2$ sequence

This sequence is a inversion-recovery sequence. The first $\pi$ pulse inverts the magnetization by $180^{\circ}$. Then the magnetization recovers from $-\mathrm{M}_{\mathrm{o}}$ to its equilibrium value $M_{0}$. After recovering for a time $\delta, a \pi / 2$ pulse is applied to tip the magnetization into the $x-y$ plane producing a free induction decay (fid) which can be observed by the detector coil. The schematic representation of the $\pi-\delta-\pi / 2$ pulse sequence is expressed as:

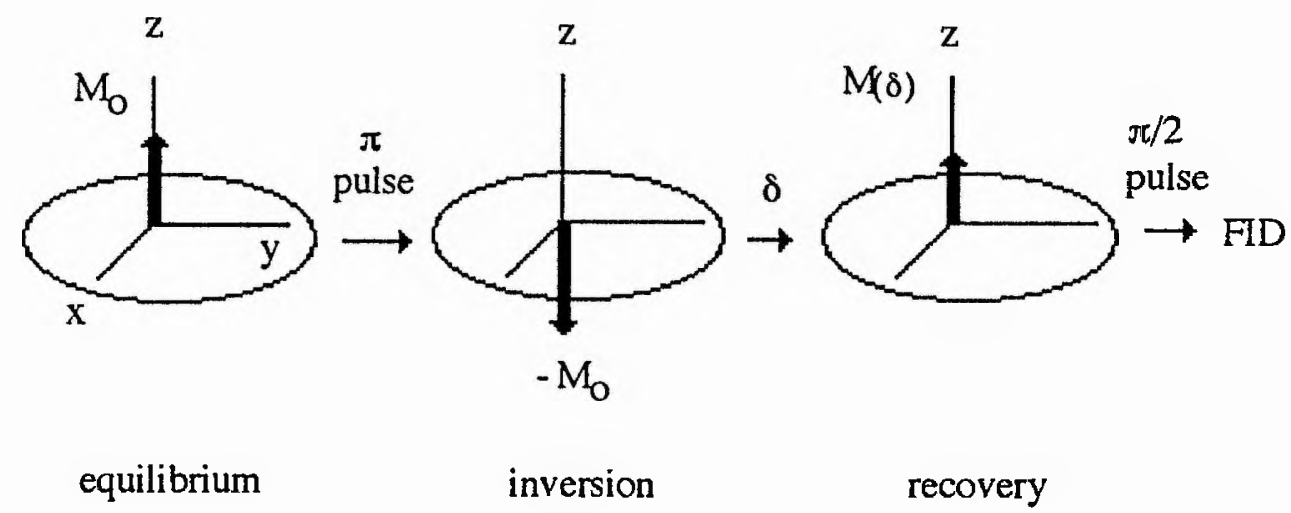

The magnetization after waiting for a period of $\delta$ is given by

$$
\frac{M_{(\delta)}}{M_{O}}=1-2 \exp \left(-\frac{\delta}{T_{1}}\right)
$$

Thus, $T_{1}$ can be derived from the above equation, providing that $\delta, M_{0}$ and $M_{(\delta)}$ are known.

(ii) $\pi / 2-\delta-\pi / 2$ sequence

This sequence is called saturation recovery sequence. The first $\pi / 2$ pulse is to allow the low and high energy levels be equally populated giving rise to zero macroscopic magnetization, i.e. the saturation of magnetization. After the magnetization 
has recovered towards the equilibrium value for a period of time $\delta$, another $\pi / 2$ pulse is applied to tip the magnetization into $x-y$ plane and produce a free induction decay. The schematic representation of the $\pi / 2-\delta-\pi / 2$ pulse sequence is expressed as:

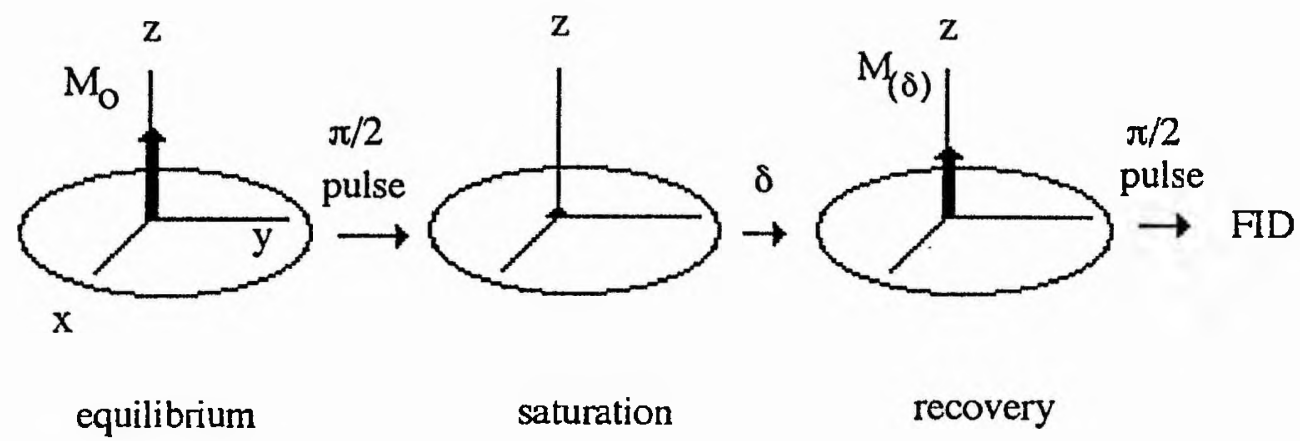

The magnetization after time $\delta$ is given by

$$
\frac{M_{(\delta)}}{M_{O}}=1-\exp \left(-\frac{\delta}{T_{1}}\right)
$$

$T_{1}$ can be derived from the above equation, because $M_{0}, M_{(\delta)}$ and $\delta$ are measurable parameters.

For the $\pi / 2-\delta-\pi / 2$ sequence, it is possible to use an arbitrarily short delay between the two pulses. For the $\pi-\delta-\pi / 2$ sequence, the two pulses must be separated by several $T_{1}$ 's. This is because in the $\pi / 2-\delta-\pi / 2$ sequence the $\mathrm{z}$-component of the magnetization is zero after the first $\pi / 2$ pulse regardless of the state of the magnetization immediately before the pulse, so that nothing can be gained by waiting between the pulse pairs. The $\pi-\delta-\pi / 2$ sequence, however, requires a long period between the two pulses to let the fully inverted magnetization completely recover. Therefore, the 
$\pi / 2-\delta-\pi / 2$ sequence is much more efficient than the $\pi-\delta-\pi / 2$ sequence in the case where $T_{1}$ is longer than $T_{2}$.

(2) Determination of $\mathrm{T}_{2}$

There are a number of methods for determining $T_{2}$ [4]. Here, two common methods are presented.

(i) Analysis of the free induction decay or linewidth of spectrum

When a $\pi / 2$ pulse is applied to tip the magnetization $90^{\circ}$ to the $x-y$ plane, the following fid will be observed:

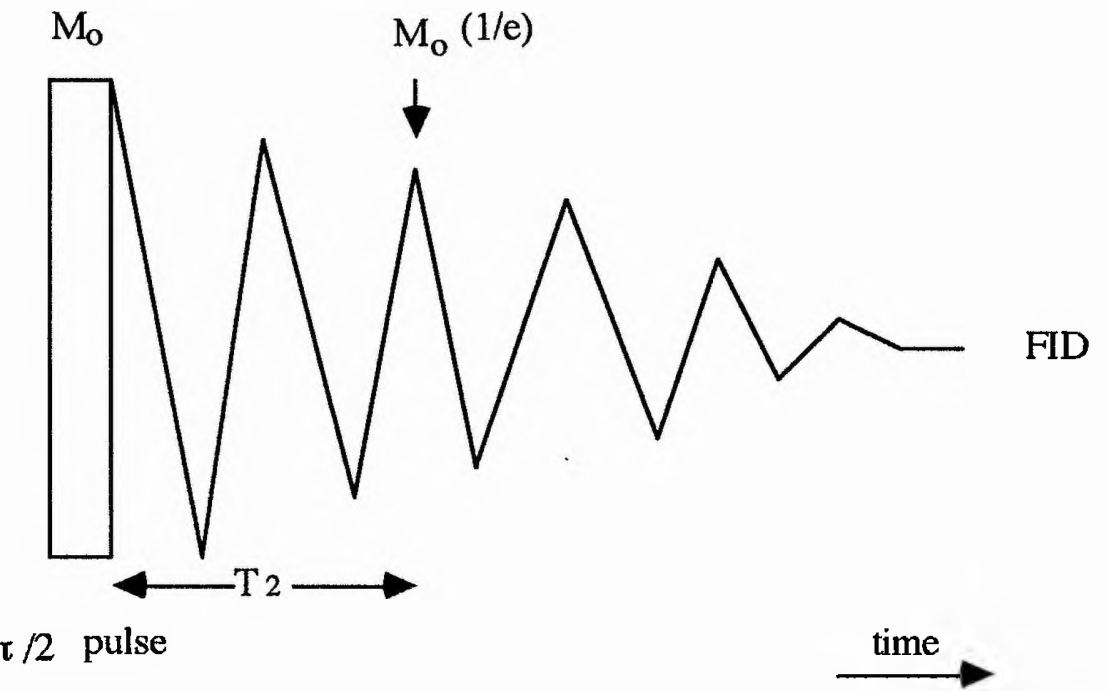

The magnetization can be related to $\mathrm{T}_{2}$ by

$$
M_{(t)}=M_{o} \exp \left(-t / T_{2}\right)
$$

where $t$ is decay time. The time for decaying to $M_{0}(1 / e)$ is $T_{2}$. 
If the above fid is Fourier transformed, $\mathrm{T}_{2}$ can be correlated to the half height linewidth. For a Gaussian line, $W_{1 / 2}=3.34 /\left(2 \pi T_{2}\right)$, and for a Lorenzian line, $W_{1 / 2}=1 /\left(\pi T_{2}\right)$, where $W_{1 / 2}$ is the half height linewidth. Thus, $T_{2}$ can be derived from the half height linewidth of a Fourier transformed spectrum.

\section{(ii) Spin-echo method}

The spin-echo method is based on the $\pi / 2-\delta-\pi$ pulse sequence. The first pulse $(\pi / 2)$ tips the equilibrium magnetization into the $x-y$ plane where inhomogeneities in the applied field $\mathrm{H}_{\mathrm{o}}$ dephase the magnetization at a rate faster than that governed by the true spin-spin relaxation process. After a period of time of $\delta$, a second pulse $(\pi)$ is applied to flip all spin vectors $180^{\circ}$ into the $-x-y$ plane and the magnetization rephases at $2 \delta$ giving a spin echo. The pulse sequence can be represented as follows:

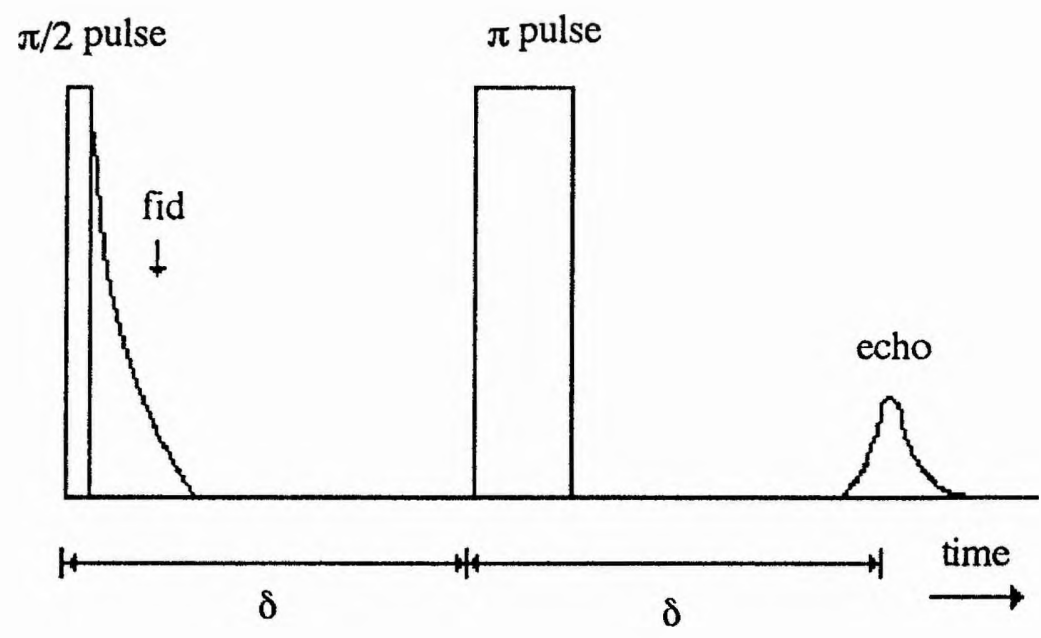

The amplitude of the echo at $2 \delta$ is given by:

$$
M_{(2 \delta)}=M_{0} \exp \left(-2 \delta / T_{2}\right)
$$


where $M_{0}$ is the initial amplitude of the magnetization. From the above equation, $T_{2}$ can be derived by measuring $\mathrm{M}_{0}, \mathrm{M}_{(2 \delta)}$ and $\delta$.

\section{4-3 Field Gradient NMR and Measurements of Diffusion Coefficient}

\section{4-3-1 Diffusion in a Steady Field Gradient}

Measurement of the self-diffusion coefficient is very important in the study of transport properties. The diffusion coefficient may be measured by tracer diffusion, NMR or electrochemical methods. In many cases, the NMR method is superior to the tracer diffusion method. For example, the diffusion coefficient of tracer may not correspond to that of the major chemical species, and furthermore, the NMR techniques do not contaminate the sample, the measured value is not disturbed by isotope effects, and the method is not limited to the availability of suitable isotopes. Of course, the NMR method is limited to suitable NMR nuclei, and to a restricted range of the diffusion coefficients.

The diffusion coefficient of nucleus of interest can be measured by the spin-echo technique. In the spin-echo experiment as discussed above, when a $\pi / 2-\tau-\pi$ pulse sequence with a field gradient $G$ is employed, an echo will be produced. Diffusion in an applied field gradient serves to diminish the amount of magnetization recoverable by echo formation. When a sample experiences a distribution of applied fields, the magnetization disperses because different regions of the sample give rise to magnetization components with different Larmor frequencies. A $\pi$ pulse can refocus those various components only if the nuclei do not migrate to a region with a different field during the course of the experiment. Thus, the amplitude of the echo is:

$$
M_{(\tau)}=M_{0} \exp \left[-\left(2 \tau / T_{2}+2 \gamma^{2} D G^{2} \tau^{3} / 3\right)\right]
$$


where $M_{o}$ is the amplitude of magnetization without field gradient, $\gamma$ is the gyromagnetic ratio, $G$ is the strength of field gradient and $\tau$ is the time between $\pi / 2$ and $\pi$ pulses. Because $M_{(\tau)}, M_{0}$ and $\tau$ are measurable parameters in the experiment, the diffusion coefficient $D$ can be calculated from the above equation if $G$ and $T_{2}$ are known.

\section{4-3-2 Pulsed Field Gradient NMR ( pfg NMR)}

The above continuous field gradient method is restricted to the measurement of high diffusion coefficients, usually $D>10^{-7} \mathrm{~cm}^{2} \mathrm{~s}^{-1}$, because in order to focus the magnetization properly in the $x-y$ plane, the field gradient $G$ cannot be very large, usually $\mathrm{G}<30 \mathrm{G} \mathrm{cm}^{-1}$. By using a pulsed field gradient however, the gradient diffusion measurement can be extended to much lower $D$, say $D<10^{-8} \mathrm{~cm}^{2} \mathrm{~s}^{-1}$. Because the field gradient is off when the rf pulses are on, it is easier to focus all the magnetization and thus a high field gradient can be applied.

(1) Stejskal and Tanner sequence

A common pulse field gradient sequence due to Stejskal and Tanner [5] is shown as:

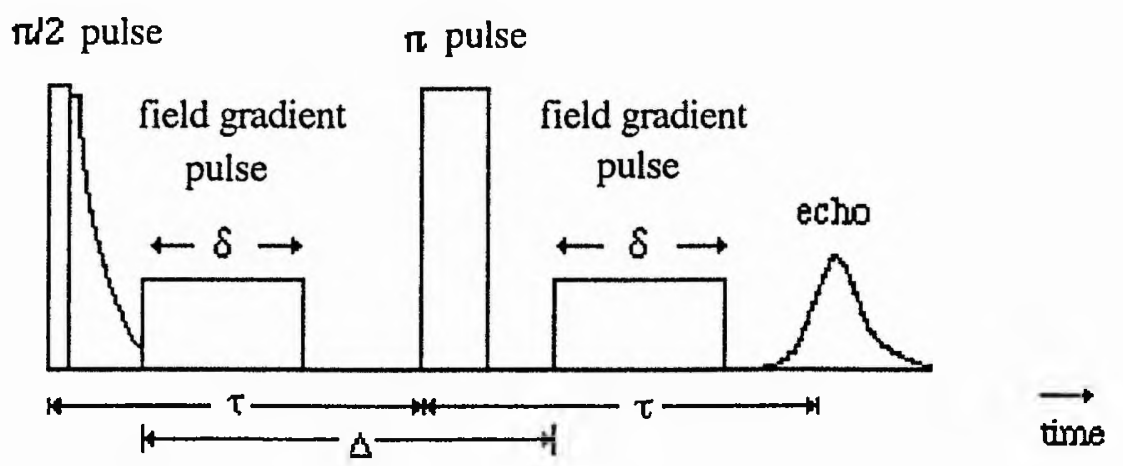

If there is no dc field gradient and identical width field gradient pulses of duration $\delta$ are used, the echo amplitude is given by 


$$
\ln \left[\mathrm{M}_{(\tau)} / \mathrm{M}_{0}\right]=-\gamma^{2} \mathrm{D} \delta^{2} \mathrm{G}^{2}(\Delta-\delta / 3)
$$

where $\delta$ is the duration of the field gradient pulse and $\Delta$ is the time difference between the two field gradient pulses. The meanings of other symbols are the same as above. In this equation, $M_{0}, M_{(\tau)}, \delta, \Delta$ and $G$ are measurable parameters, and thus the diffusion coefficient $\mathrm{D}$ can be calculated. In a real experiment, $\delta$ is often varied, and by plotting In $\left[M_{(\tau)} / M_{o}\right]$ against $\gamma^{2} \delta^{2} G^{2}(\Delta-\delta / 3), D$ can be derived from the slope of the straight line.

(2) Stimulated echo method

The situation can occur in which it is not possible to measure the diffusion coefficient by the above method due to very slow diffusion or to a short $T_{2}$, or both. In systems where $T_{1} \gg T_{2}$, the stimulated echo sequence can be used instead of the $\pi / 2-\delta-\pi-\delta$ sequence because it is limited by $T_{1}$ rather than $T_{2}$. The sequence is expressed as:

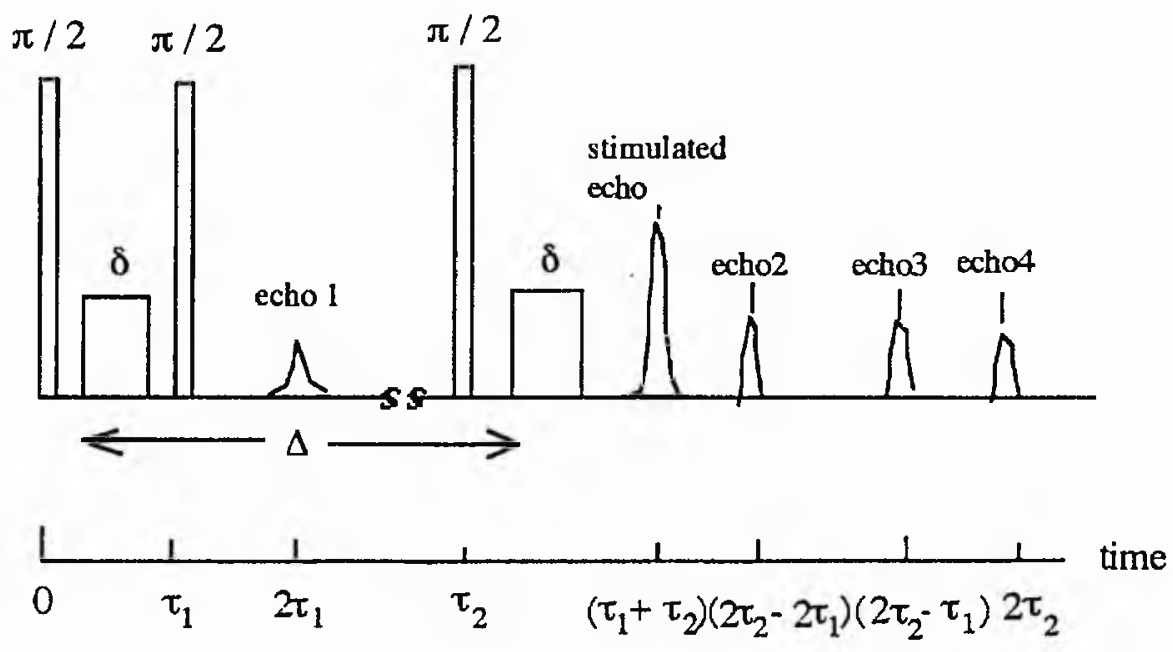

A three pulse sequence of $\pi / 2$ pulses, all with the same phase, can yield five echoes as shown in the above sequence [6]. The echo occurring at $\left(\tau_{1}+\tau_{2}\right)$ is called the stimulated 
echo. If there is no dc field gradient, the amplitude of the stimulated echo can also be expressed by equation (4-2-10). In the experiment, either $\delta$ or $\Delta$ can be varied, and by plotting $\ln \left[M_{(\tau)} / M_{0}\right]$ against $\gamma^{2} \delta^{2} G^{2}(\Delta-\delta / 3)$, D can be derived from the slope of the straight line. Generally, $\Delta$ can be varied from $50 \mathrm{~ms}$ to several hundred milliseconds, which permits measurement of $D$ of the systems with very long $T_{1}$.

\section{4-3-3 Gradient Calibration}

As discussed in the above sections, the diffusion coefficient of a nucleus can be determined by field gradient NMR, and especially by pfg NMR in slow diffusion systems. The precision of the determination of $D$ is directly related to the precision of the determination of times $\delta$ and $\Delta$ and of the gradient $G$. The former can be set directly by the spectrometer. The latter can be calibrated either using a sample whose D is known or by analysis of the Fourier transformed echo. The field gradient can be calculated from the following equation for the ${ }^{1} \mathrm{H}$ nucleus:

$$
G=\frac{2 \pi \Delta H}{\gamma_{H} L}
$$

where $\gamma_{\mathrm{H}}$ is the gyromagnetic ratio of ${ }^{1} \mathrm{H}, \mathrm{L}$ is the length of sample and $\Delta \mathrm{H}$ is the width of the Fourier transformed (FT) echo. In calibrating the pulsed gradient, the echo can be obtained without applying any gradient when the if pulses are on. The width of the FT echo can be determined as shown:

FT echo

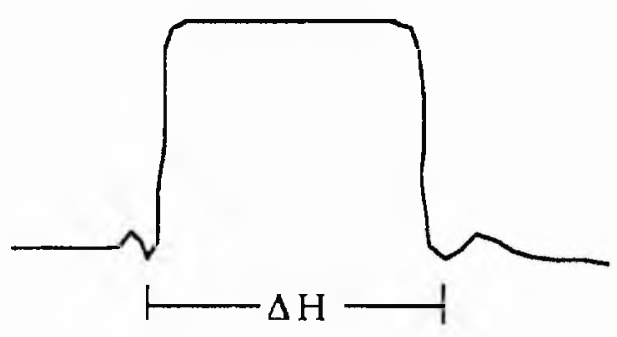




\section{4-4 Experimental}

\section{4-4-1 MAS NMR of Polymer Electrolytes}

(1) Sampling

Polymer electrolytes for mas NMR measurements were packed in a $4 \mathrm{~mm}$ diameter $\mathrm{ZrO}_{2}$ cylindrical rotor (Bruker, $\mathrm{CXP} / \mathrm{MSL}$ ) in an argon-filled Braun glovebox. Polymer electrolytes based on PMEO were cut into small pieces and packed piece by piece under pressure, because they were quite soft and rubbery-like. The rotor was then sealed by a cap.

(2) NMR measurements

The NMR measurement was carried out on a Bruker MSL 500 NMR Spectrometer with MSL probe. ${ }^{7} \mathrm{Li}$ mas NMR was carried out at the frequency of 194.366 MHz, and 11B mas NMR was carried out at the frequency of $160.402 \mathrm{MHz}$

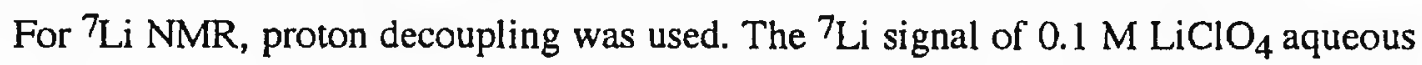
solution was set to be $0 \mathrm{ppm}$, and the $\mathrm{BF}_{3}-\mathrm{Et}_{2} \mathrm{O}$ complex was used as external reference for the ${ }^{11} \mathrm{~B}$ chemical shifts. The spinning rate was usually $5 \mathrm{kHz}$, except where specifically noted.

Temperature variation was carried out by the VT equipment attached to the spectrometer. At each temperature, the sample was allowed to equilibrate for 10 minutes. ${ }^{7} \mathrm{Li}$ and ${ }^{11} \mathrm{~B}$ mas NMR spectra of polymer electrolytes are shown in Chapter 5. 


\section{4-4-2 Measurements of $T_{1}$ and $T_{2}$ of Polymer Electrolytes}

(1) $\mathrm{T}_{1}$

The $\mathrm{T}_{1}$ of ${ }^{7} \mathrm{Li}$ in PMEO-LiPF 6 electrolytes was measured for the purpose of pfg NMR. The sample was packed in a $5 \mathrm{~mm}$ diameter thin-wall glass tube with sample height of $5 \mathrm{~mm}$. The top of the tube was sealed by a cap and Teflon tape. A $\pi / 2-\delta-\pi / 2$ pulse sequence was used. The experiment was carried out in the Bruker MSL 500 spectrometer with a Doty Scientific pfg probe operating at $194.366 \mathrm{MHz}$. The length of $\pi / 2$ pulse was $7.7 \mu \mathrm{s}$, and the $\delta$ was varied from $100 \mathrm{~ms}$ to $5 \mathrm{~s}$. The amplitude of the Fourier transformed spectrum was measured by varying $\delta$. The amplitude of the spectrum with $\delta=60 \mathrm{~s}$ was used as initial value. By plotting $\ln [M(60)-M(\delta)]$ versus $\delta$, a straight line with slope $-A$ was obtained as shown in Figure 4-4. Thus, $T_{1}=A^{-1}$. The $\mathrm{T}_{1}$ of ${ }^{7} \mathrm{Li}$ for those polymer electrolytes was typically $3-5 \mathrm{~s}$.

$\mathrm{T}_{1}$ of ${ }^{31} \mathrm{P}$ in $\mathrm{PMEO}-\mathrm{LiPF}_{6}$ was also measured by the same method, except that the frequency was $202.4 \mathrm{MHz}$. The $\mathrm{T}_{1}$ of $31 \mathrm{P}$ for those polymer electrolytes was typically $16-18 \mathrm{~s}$.

(2) $\mathrm{T}_{2}$

$\mathrm{T}_{2}$ of ${ }^{1} \mathrm{H}$ in $\mathrm{PMEO}-\mathrm{LiClO}_{4}$ electrolytes was measured by the method of linewidth analysis. The sample was packed in a $4 \mathrm{~mm}$ rotor as described for the MAS experiment. The experiment was carried out with the MSL probe at the frequency of ${ }^{1} \mathrm{H}$ without spinning. The temperature variation was carried out as for the MAS case. $T_{2}$ was calculated from $T_{2}=\left(\pi W_{1 ; 2}\right)^{-1}$.

The $\mathrm{T}_{2}$ of ${ }^{7 \mathrm{Li}}$ and ${ }^{31} \mathrm{P}$ in $\mathrm{PMEO}^{-\mathrm{LiPF}_{6}}$ was also measured for the purpose of the pfg NMR experiment. The experiment was the same as above, except that the sample was packed in a $5 \mathrm{~mm}$ glass tube and the probe was the Doty Scientific pfg probe. 
Frequencies of $194.366 \mathrm{MHz}$ for ${ }^{7} \mathrm{Li}$ and $202.4 \mathrm{MHz}$ for ${ }^{31} \mathrm{P}$ were used. Typical $\mathrm{T}_{2}$ for ${ }^{7} \mathrm{Li}$ was $8-10 \mathrm{~ms}$ and that for ${ }^{31} \mathrm{P}$ was $3-5 \mathrm{~ms}$.

\section{4-4-3 Measurements of Diffusion Coefficient of Polymer Electrolytes by pfg NMR}

\section{(1) Sampling}

The sample was packed in a thin-wall glass tube with $5 \mathrm{~mm}$ diameter and 12.5 $\mathrm{cm}$ length. The actual sample length was about $5 \mathrm{~mm}$. The open end of the tube was sealed by a plastic cap and further sealed by Teflon tape.

\section{(2) Calibration of the field gradient strength}

The field gradient strength was calibrated by $1 \mathrm{H}$ nucleus of pure glycerin which was packed in a $5 \mathrm{~mm}$ thin-wall glass tube with a height of $5-7 \mathrm{~mm}$. The sample was measured using a ruler and the centre of the sample was carefully set in the centre of the coil. The Fourier transformed echo was produced when rf was on at the frequency of ${ }^{1} \mathrm{H}$ and the field gradient pulse was off. The width of the line was measured as discussed above, and $\mathrm{G}$ was calculated according to equation (4-2-11).

(3) Measurements

The sample tube was located by a holder, which fixed the distance between the centre of the sample and the end of the holder at about $5.25 \mathrm{~cm}$. The current for the pulse gradient coil was supplied by a Techron 7570 Power Supply Amplifier. The temperature was controlled by the Doty Scientific temperature controller. The temperature range studied was typically $30-90^{\circ} \mathrm{C}$. The sample was allowed to equilibrate at each temperature for 15 minutes. 
(i) Diffusion of $7 \mathrm{Li}$

The diffusion coefficient of $7 \mathrm{Li}$ nuclei was measured by the stimulated pulse sequence at a frequency of $194.366 \mathrm{MHz}$. The pulse length of the $\pi / 2$ pulse was typically $7.7 \mu \mathrm{s}$. $\Delta$ was fixed, while $\delta$ was varied. $\Delta$ was typically $200-300 \mathrm{~ms}$, and $\delta$ varied from $500 \mu \mathrm{s}$ to $6 \mathrm{~ms}$. The delay time between two sequences was $5 \mathrm{~s}$. The field gradient was $292.7 \mathrm{G} \mathrm{cm}^{-1}$.

(ii) Diffusion of $31 \mathrm{P}$

The diffusion coefficient of ${ }^{31} \mathrm{P}$ nuclei in the polymer electrolytes was also measured by the stimulated pulse sequence at a frequency of $202.4 \mathrm{MHz}$. Due to short $\mathrm{T}_{2}$ (about $4 \mathrm{~ms}$ ) and very long $\mathrm{T}_{1}$ (about $16-18 \mathrm{~s}$ ), $\delta$ was fixed at $1 \mathrm{~ms}$ and $\Delta$ was varied from $200 \mathrm{~ms}$ to $900 \mathrm{~ms}$. The length of $\pi / 2$ pulse was typically $14 \mu \mathrm{s}$, and the delay time between two sequence was $18 \mathrm{~s}$ and $\mathrm{G}$ was $292.7 \mathrm{G} \mathrm{cm}^{-1}$.

By plotting $\ln M_{(\tau)}$ against $\gamma^{2} \delta^{2} G^{2}(\Delta-\delta / 3)$, D was derived from the slope of the straight line. Figure $4-5$ and Figure $4-6$ are typical plots for ${ }^{7} \mathrm{Li}$ and ${ }^{31} \mathrm{P}$. 


\section{References}

[1] C. Berthier, W. Gorecki, M. Minier, M. B. Armand, J. M. Chabagno and P. Rigaud, Solid State Ionics, 11, 91(1983).

[2] S. Schramn and E. Oldfield, J. Chem.Soc. Chem. Commun. 980 (1982).

[3] J. S. Frye and G. E. Maciel, J. Magnetic Resonance, 48, 125 (1982).

[4] E. Fukushima, S. B. W. Roeder, Experimental Pulse NMR: A Nuts and Bolts Approach, Addision-Wesley Publishing Co. Inc., London 1981.

[5] E. O. Stejskal and J. E. Tanner, J. Chem. Phys. 42, 288 (1965).

[6] J. E. Tanner, J. Chem. Phys. 52, 2533 (1970). 


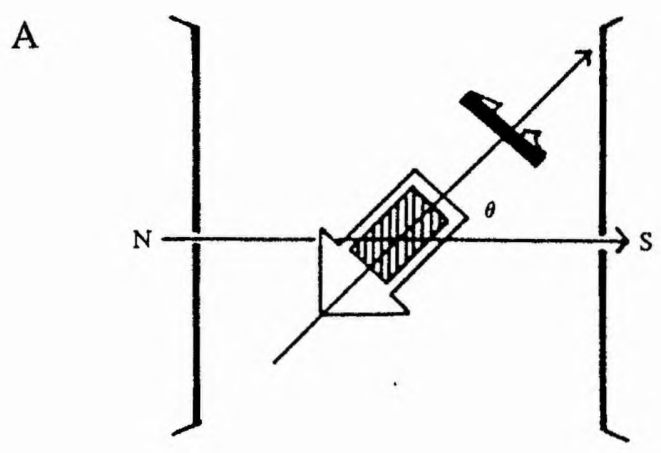

B

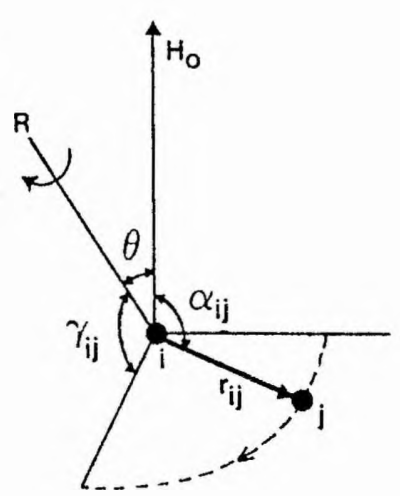

C

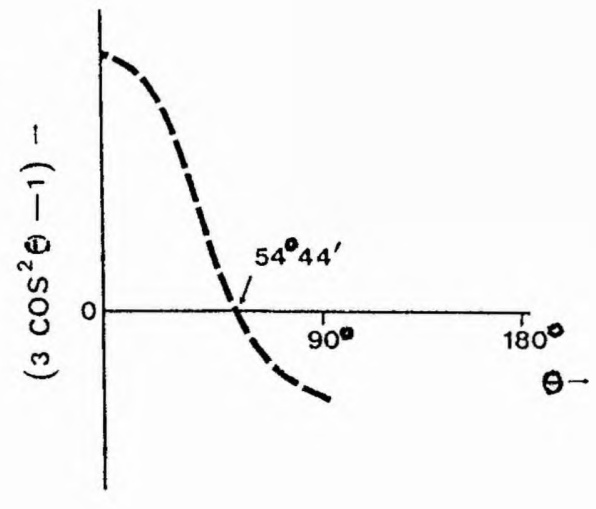

Figure 4-1 Schematic representation of magic angle spinning: (A) a sample spinning in the magnetic field, $(\mathrm{B})$ representation in a coordinate and (C) angle $\theta$ dependence of $\left(3 \cos ^{2} \theta-1\right)$ term. 


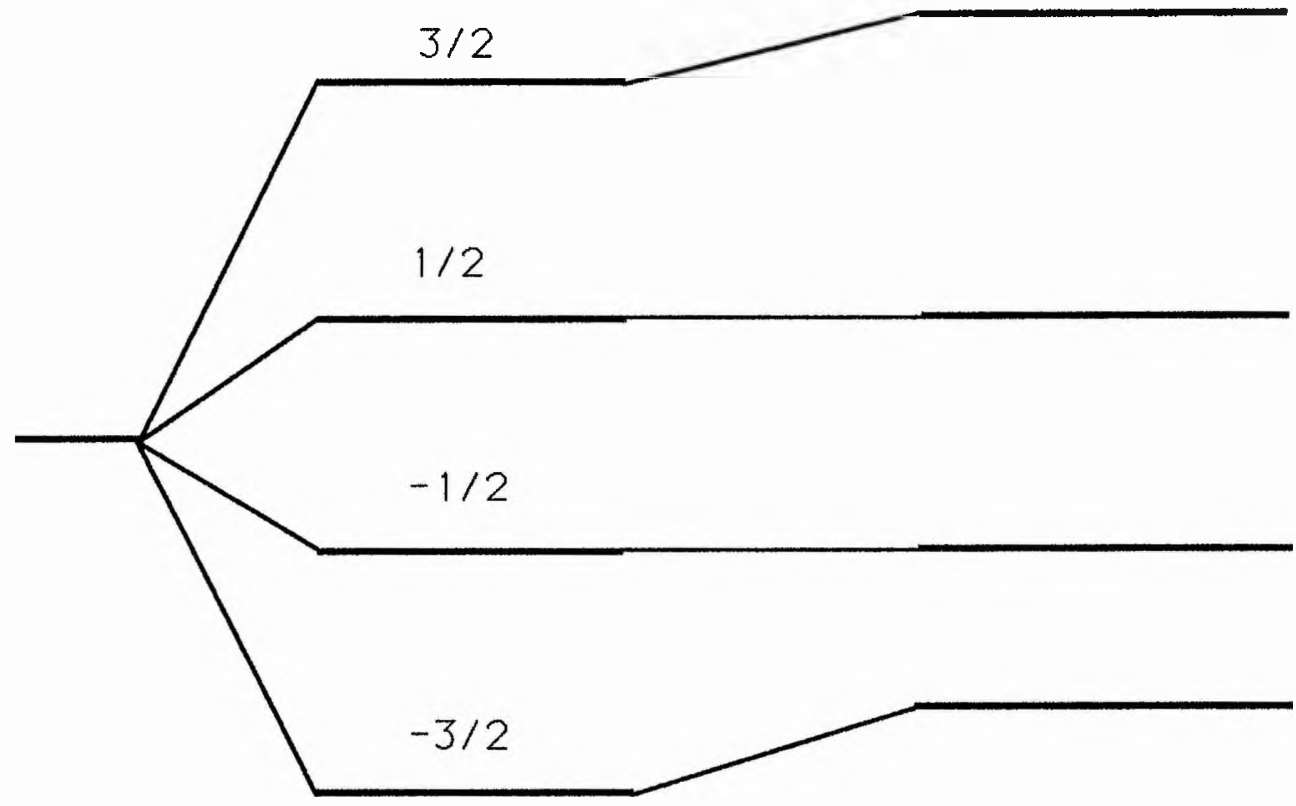

$\begin{array}{ll}\text { no quadrupolar } & \text { first order quadrupolar } \\ \text { interaction } & \text { interaction }\end{array}$

Figure 4-2 The Zeeman energy level for a $I=3 / 2$ nucleus with and without first order quadrupolar interaction.

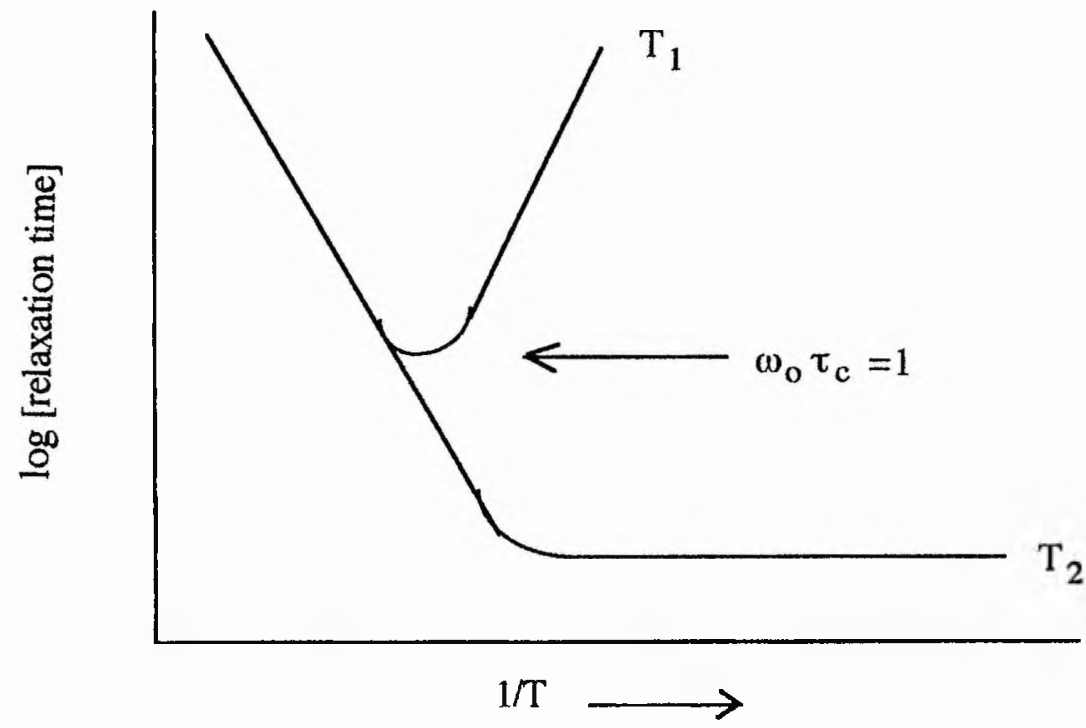

Figure 4-3 Temperature dependence of $T_{1}$ and $T_{2}$. 


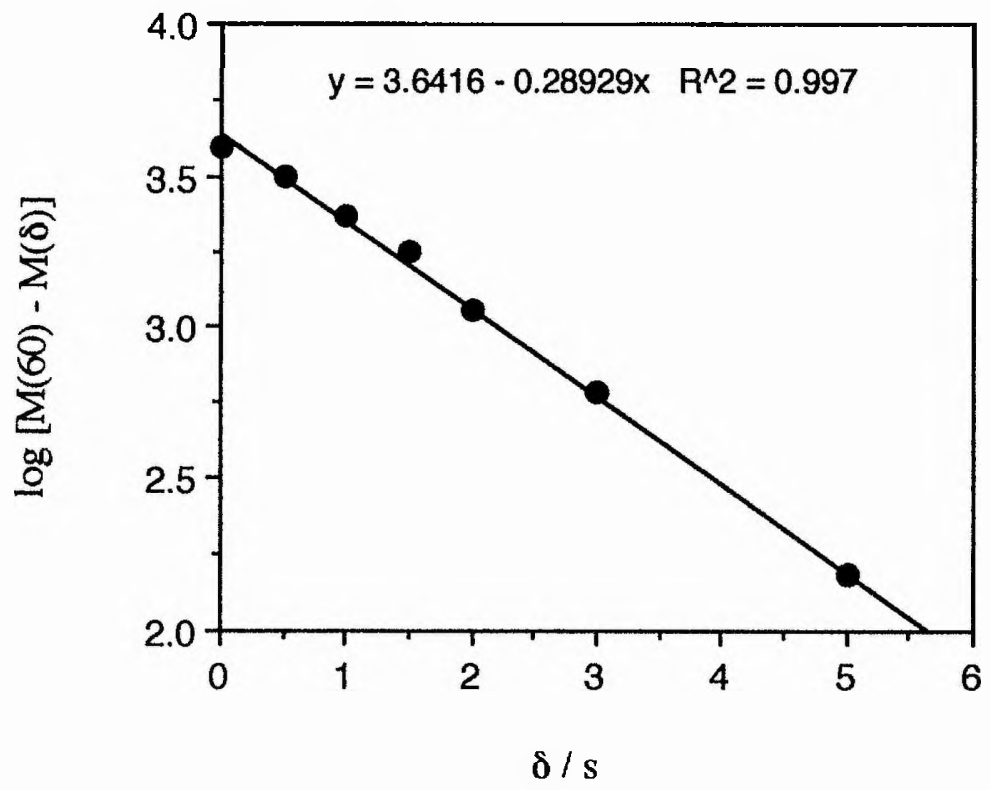

Figure 4-4 $\log [\mathrm{M}(60)-\mathrm{M}(\delta)]$ versus time for $\mathrm{PMEO}^{-\mathrm{LiPF}_{6}}$ (20:1) electrolyte at $80^{\circ} \mathrm{C}$.

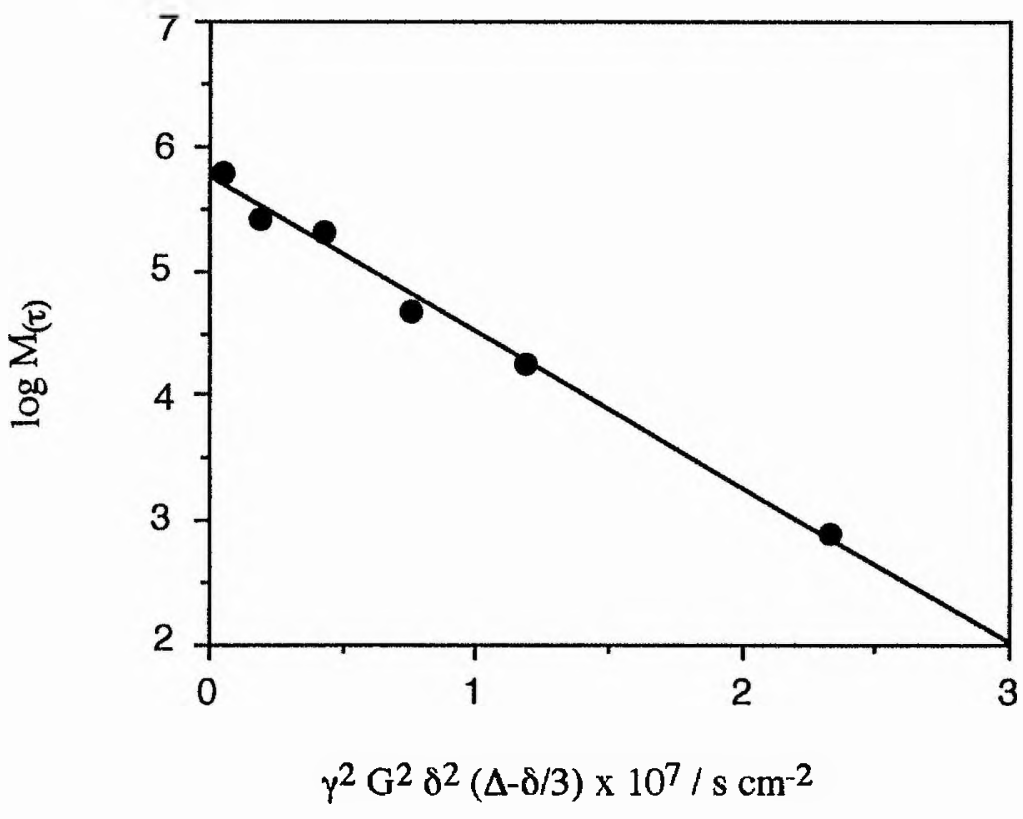

Figure 4-5 Typical plot of $\ln [\mathrm{M}(\tau)]$ against $\gamma^{2} \mathrm{G}^{2} \delta^{2}(\Delta-\delta / 3)$ for ${ }^{7} \mathrm{Li}$, measured for

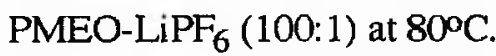




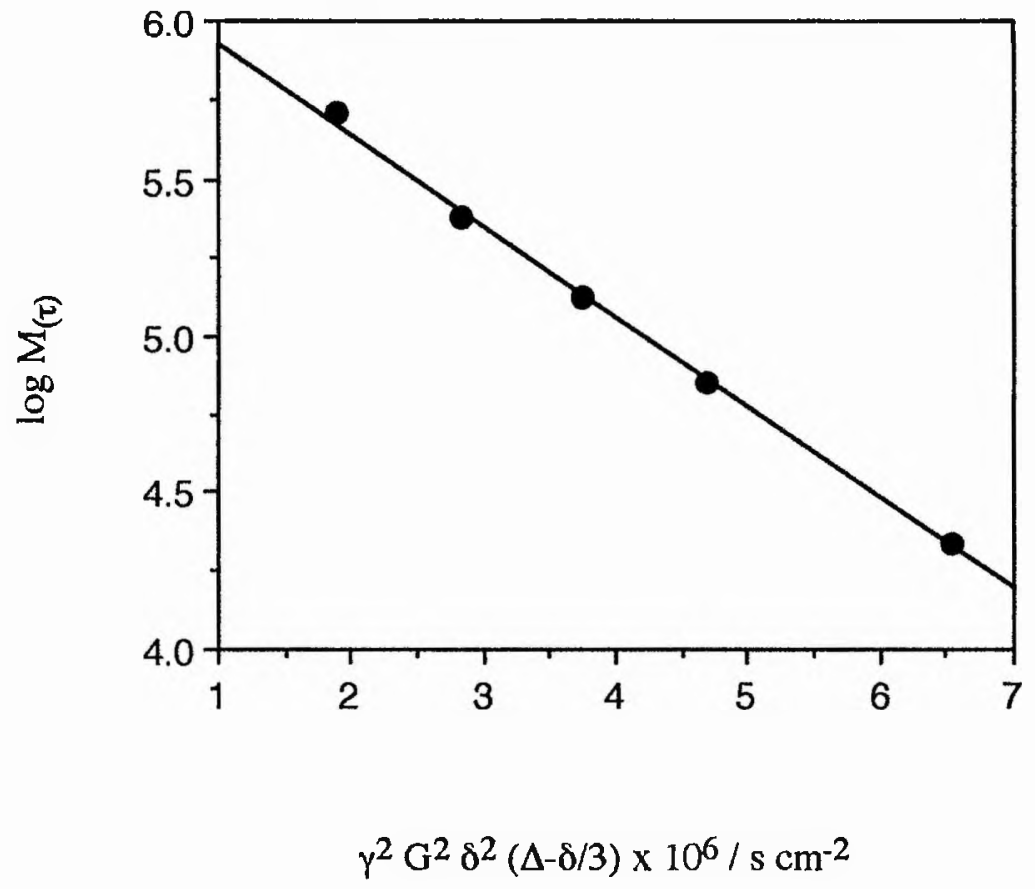

Figure 4-6 Typical plot of In $\left[\mathrm{M}_{(\tau)}\right]$ against $\gamma^{2} \mathrm{G}^{2} \delta^{2}(\Delta-\delta / 3)$ for ${ }^{31} \mathrm{P}$, measured for PMEO-LiPF $6(50: 1)$ at $60^{\circ} \mathrm{C}$. 


\section{CHAPTER 5}

\section{IONIC CONDUCTION AND INTERACTION IN POLYMER ELECTROLYTES BASED ON LITHIUM SALTS}

In studies of polymer electrolytes, an understanding of the mechanism of ion conduction and of the species present in the system is very important. So far, a number of theoretical models describing ion conducting behaviour in polymer electrolytes have been proposed [1]. However, all of these have some disadvantages (see Chapter 2). It is therefore of interest to examine these models in the light of the experimental results and to make them more applicable to polymer electrolyte systems.

In this chapter, the ion conducting behaviour of the polymer electrolytes based on amorphous PMEO and lithium salts is reported, and the nature of the conducting species present in these polymer electrolytes is suggested. The conductivity data have been fitted to equations for describing conductive behaviour, eg. the VTF equation. Both magic-angle spinning NMR and pulsed field gradient NMR have been employed to investigate the species and ion diffusion in these polymer electrolytes. In the light of the analysis of the experimental results, the ion conducting mechanism in these polymer electrolytes is discussed. 


\section{5-1 Conducting Species and Ion Transport Investigated by Conductivity Measurement}

In the last decade, extensive measurements of polymer electrolyte conductivity have been made [2], in order to characterise polymer electrolytes and give information about the conductive species and the mechanisms for ion transport.

Conductivity can be generally related to the concentrations and mobilities of carriers in a homogeneous electrolyte by

$$
\sigma=\sum_{i} n_{i} q_{i} \mu_{i}
$$

where $n_{i}$ is the number, $q_{i}$ the charge and $\mu_{i}$ the mobility of carrier $i$.

\section{5-1-1 Relationship Between Salt Concentration and Conductivity}

For a strong electrolyte $\mathrm{MX}$ in an electrolyte solution, the isothermal conductivity will increase linearly with increasing salt concentration providing the mobilities of the jons are invariant with the concentration: i.e. the molar conductivity, $\Lambda$ $=\sigma / \mathrm{c}$, where $\mathrm{c}$ is the salt concentration, is constant under these conditions.

The conductivity of $\mathrm{PMEO}-\mathrm{LiClO}_{4}$ electrolytes increases with increasing salt concentration (Figure 5-1). At first sight, this may suggest that the increase of salt concentration leads to an increase of number of carriers, thus leading to a conductivity increase. By plotting the molar conductivity* against concentration (Figure 5-2). however, the molar conductivity is found not to be invariant as in the strong electrolyte constant mobility case. There are three regions in the plot of $\Lambda$ against $\mathrm{c}$ : the first region

\footnotetext{
* In the very dilute region, the molar conductivity was calculated by first subtracing the background conductivity of the pure PMIEO.
} 
is the concentration up to about $0.01 \mathrm{~mol} \mathrm{dm}^{-3}$, where $\Lambda$ decreases rapidly with increasing salt concentration to reach a minimum; the second region extends to about 0.1 mol dm${ }^{-3}$, where $\Lambda$ increases to reach a flat maximum with increasing salt concentration; the third region is the concentration above $0.1 \mathrm{~mol} \mathrm{dm}^{-3}$, where $\Lambda$ again decreases with increasing salt concentration.

In conventional liquid electrolyte systems, it is well known that the mobilities of ions will be reduced when the salt concentration is very high, due to an increase in the viscosity of the solution [3]. In polymer electrolytes, when the salt concentration is high, the mobility of polymer segments will be reduced to a great extent due to the "transient crosslinking" effect of the salt [4]. The measurements of the glass transition temperature $T_{g}$ (Table 5-1) have shown that the $T_{g}$ values of the electrolytes increase with increasing salt concentration in the region above $0.1 \mathrm{~mol} \mathrm{dm}^{-3}$, while they are invariant on changing the salt concentration in the region below $0.1 \mathrm{~mol} \mathrm{dm}^{-3}$. Generally, the glass transition temperature is related to the segmental motion of the polymer (see Chapter 2), i.e. the higher $\mathrm{T}_{\mathrm{g}}$, the less mobile the segment will be. Therefore, in the first and second regions the mobility of polymer segments is invariant. and there is no significant transient crosslinking effect of the salt. At a constant reduced temperature, $T=T_{g}+k$ ( $k$ is a constant), where the mobility of polymer segment is invariant, the molar conductivity $\Lambda$ in the third region increases with increasing salt concentration (Figure 5-3). Hence, the decrease of $\Lambda$ with increasing salt concentration in the third region may be mainly attributed to the transient crosslinking effect of the salt.

Because the permittivity of PEO is low [5] $(\varepsilon \approx 5)$, there may be ion association present in the polymer electrolytes. In the first region, the rapid decrease of $\Lambda$ with increasing salt concentration may be attributed to the formation of neutral ion pairs which make no contribution to the ion conductance. 
When the salt concentration is increased further, there may be further ion association taking place in the electrolytes to form mobile ion triples [6] which can act as carriers. The equilibria can be written as:

$$
\begin{aligned}
& \mathrm{Li}^{+}+\mathrm{ClO}_{4}^{-} \rightleftharpoons \mathrm{KiClO}_{4}{ }^{\circ} \\
& \mathrm{LiClO}_{4}{ }^{\mathrm{O}}+\mathrm{ClO}_{4} \rightleftharpoons \stackrel{\mathrm{K}_{\mathrm{T} 1}}{\rightleftharpoons} \mathrm{Li}\left(\mathrm{ClO}_{4}\right)_{2}^{-} \\
& \mathrm{LiClO}_{4}{ }^{\circ}+\mathrm{Li}^{+} \stackrel{\mathrm{K}_{\mathrm{T} 2}}{\rightleftharpoons} \mathrm{Li}_{2} \mathrm{ClO}_{4}{ }^{+}
\end{aligned}
$$

where $\mathrm{K}_{\mathrm{p}}, \mathrm{K}_{\mathrm{T} 1}$ and $\mathrm{K}_{\mathrm{T} 2}$ are the single and triple ion equilibrium constants respectively. By assuming that $\mathrm{K}_{\mathrm{T} 1}=\mathrm{K}_{\mathrm{T} 2}=\mathrm{K}_{\mathrm{T}}$, and that the dominant species in the electrolyte solution are non-conducting ion pairs, i.e. $\alpha_{1}$ and $\alpha_{T} \ll 1$, where $\alpha_{i}$ and $\alpha_{T}$ are the free ion and triple ion ratios in the electrolyte, the following equation can be given [7]:

$$
\Lambda c^{1 / 2}=\frac{\Lambda_{\mathrm{o}}^{\mathrm{T}} \mathrm{K}_{\mathrm{T}}}{\mathrm{K}_{\mathrm{P}}^{1 / 2}} \mathrm{c}+\frac{\Lambda_{\mathrm{o}}^{!}}{\mathrm{K}_{\mathrm{P}}^{1 / 2}}
$$

where $\Lambda_{0}^{!}$is the sum of the limiting molar conductance of free ions and $\Lambda_{0}^{T}$ represents that for triple ions. The above equation predicts that there will be a linear relationship between $\Lambda \mathrm{c}^{1 / 2}$ and $\mathrm{c}$, if the electrolyte solution is dominated by ion pairs with some triple ions.

In an electrolyte dominated by neutral ion pairs, the molar conductivity can be increased in the following ways: (i) formation of mobile charged triples by the further association of ion pairs with free ions and / or (ii) redissociation of ion puirs to form fre: 
ions. The former can be described by equation (5-1-5). The latter is caused by the increase of the permittivity of the electrolyte due to the formation of ion pair dipoles which increases the polarisation of the electrolyte. A high permittivity destabilizes the ion pairs and thus reduces the association constant $\mathrm{K}_{\mathrm{p}}$. It has been reported that a significant deviation of equation (5-1-5) would be observed if a change in the permittivity of an electrolyte with salt concentration was significant [8]. Figure 5-4 shows that the relationship between $\Lambda c^{1 / 2}$ and $c$ in the concentration region above 0.01 mol $\mathrm{dm}^{-3}$ up to $0.2 \mathrm{~mol} \mathrm{dm}^{-3}$ is linear, suggesting that, in the concentration region above $0.01 \mathrm{~mol} \mathrm{dm}^{-3}$, the increase of the molar conductivity is likely to arise from the formation of mobile triple ions.

Figure 5-5 shows the relationship between the molar conductivity of PMEO$\mathrm{LiCF}_{3} \mathrm{SO}_{3}$ electrolytes and the salt concentration. This is no significant difference between that of $\mathrm{PMEO}-\mathrm{LiClO}_{4}$ electrolytes. Thus, it may be also concluded that in the region up to $0.1 \mathrm{~mol} \mathrm{dm}^{-3}$, the dominant species are again non-conductive ion pairs. When the concentration is high, the transient crosslinking effect of the salt is significant and the molar conductivity is reduced.

As a comparison, PMEO-LiSCN electrolytes have also been investigated by conductivity measurements. Figure 5-6 shows the temperature dependence of the conductivity. The conductivity of PMEO-LiSCN electrolyte is approximately one order magnitude lower than that of $\mathrm{PMEO}^{-\mathrm{LiClO}_{4}}$ electrolyte at the same salt concentration. For the SCN- anion, due to its higher ion-dipole stabilisation energy relative to that of the $\mathrm{ClO}_{4}^{-}$anion and the larger lattice energy of $\mathrm{LiSCN}$ in comparison to $\mathrm{LiClO}_{4}$, it is expected that the interaction between cations and anions in the LiSCN system will be stronger than that in the $\mathrm{LiClO}_{4}$ system. Thus, the proportion of non-conducting ion pairs in PMEO-LiSCN may be larger than that in PMEO-LiClO $\mathrm{P}_{4}$ electrolyte, leading to a lower conductivity. 
It is of interest to note that in the plot of molar conductivity against salt concentration (Figure 5-7), the molar conductivity increases slightly after the minimum, and there is no clear maximum observed up to $0.2 \mathrm{~mol} \mathrm{dm}^{-3}$, similar to the observation in the liquid PPO-LiSCN system [9]. This may be caused by several factors. One factor to be considered is the dissociation of ion pairs. Both the formation of ion triples and dissociation of ion pairs can make the molar conductivity increase, as long as the mobilities of ions are relatively invariant with increasing concentration. Because of the stronger interactions between $\mathrm{Li}^{+}$and $\mathrm{SCN}^{-}$, the ion pairs are more difficult to dissociate, which will lead to a smaller increase in the molar conductivity. Another possible factor may be that in PMEO-LiSCN electrolyte it may be difficult for the ion pairs to further associate to form ion triples or larger ion clusters as in $\mathrm{PMEO}-\mathrm{LiClO}_{4}$ electrolyte. This will lead to much less increase of the molar conductivity after the minimum. If some of the transient crosslinking effect of the salt arises from the formation of ion clusters in addition to from the interchain coordination of the cations, this effect may also be reduced in this electrolyte.

By plotting $\Lambda \mathrm{c}^{1 / 2}$ against $\mathrm{c}$ (Figure 5-4) for PMEO-LiSCN electrolyte, a straight line is given in the concentration region above $0.01 \mathrm{~mol} \mathrm{dm}^{-3}$ up to $0.2 \mathrm{~mol} \mathrm{dm}^{-3}$. This may imply that the dissociation of ion pairs is not important here. The slope of the straight line is much smaller than that of PMEO- $\mathrm{LiClO}_{4}$ electrolytes. This will be

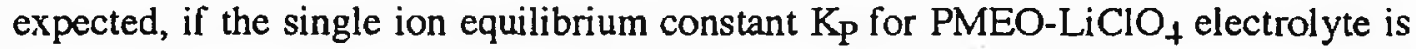
smaller than that for PMEO-LiSCN electrolyte, while the triple ion equilibrium constant $\mathrm{K}_{\mathrm{T}}$ for the former is larger than that for the latter. Thus, this suggests that the second factor in the above analysis may be more important in the PMEO-LiSCN electrolyte system. 


\section{5-1-2 Relationship Between Conductivity and Temperature}

The conductive behaviour as a function of temperature of the polymer electrolytes with crystalline phases can often be described by the Arrhenius equation $(5-1-6)$

$$
\sigma=\sigma_{o} \exp \left(-E_{a} / R T\right)
$$

where $\sigma_{0}$ is a pre-exponential parameter, $E_{a}$ is the activation energy, $R$ is the gas constant and $\mathrm{T}$ is the absolute temperature. In contrast, the temperature dependence of the conductivity of totally amorphous polymer electrolytes can usually be described by the VTF (Vogel-Tamman-Fulcher) equation (5-1-7):

$$
\sigma=\sigma_{0} \exp \left[-B /\left(T-T_{0}\right)\right]
$$

where $\sigma_{0}, B$ and $T_{0}$ are fitted parameters. $T_{0}$ is usually related to the glass transition temperature of the polymer electrolyte, $T_{g}$, by $T_{0} \approx T_{g}-50 K . B$ may be assigned different meanings in different models. The conductive behaviour of $\mathrm{PMEO}^{-\mathrm{LiClO}_{4}}$ electrolytes, which are completely amorphous above room temperature, shows a nonArrhenius relationship (Figure 5-1).

In order to examine whether the VTF equation can adequately describe the

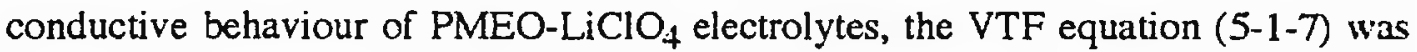
fitted to the conductivity data on computer, using the 'Minitab' program [10]. This involved choosing an initial estimate for the $T_{0}$ parameter after inputting the conductivity data with the corresponding temperatures, and then increasing this value while monitoring a statistical analysis parameter known as 'residual sum of squares' RSS, for the data fit at each $T_{0}$ value. When the RSS term was at a minimum value, the best fitted 
$T_{o}$ and $B$ values were given. The fitted $T_{0}, \sigma_{o}$ and $B$ are listed in Table 5-1. Some typical log $\sigma$ versus $1000 /\left(\mathrm{T}-\mathrm{T}_{\mathrm{o}}\right.$ ) plots are shown in Figure 5-8.

The $\mathrm{T}_{\mathrm{o}}$ for the electrolytes in the concentration region below $0.1 \mathrm{~mol} \mathrm{dm}-3$ was found to be remarkably invariant, with a value of $217 \mathrm{~K}$ which was quite close to the glass transition temperature of the electrolytes (Table 5-1). It is of interest to note however, that in the concentration region above $0.1 \mathrm{~mol} \mathrm{dm}^{-3}$, the fitted $\mathrm{T}_{\mathrm{o}}$ decreased with increasing salt concentration, rather than increasing as $\mathrm{T}_{\mathbf{g}}$ does, although the plot of $\log \sigma$ versus $1000 /\left(T-T_{0}\right)$ could be linearized by the fitted $T_{0}$. Because $T_{0}$ is a temperature where the free volume or configurational entropy vanishes, usually $50 \mathrm{~K}$ lower than $T_{g}$, it should show the same tendency as $T_{g}$ with changing salt concentration. In the present case, the fitted $T_{0}$ is not consistent with its physical meaning in the free volume and configurational entropy models. Reasons for this will be discussed in detail in the following section.

The fitted $\sigma_{0}$ increased with increasing salt concentration over the concentration range examined. There was no significant change for the fitted $\mathrm{B}$ in the concentration region below $0.1 \mathrm{~mol} \mathrm{dm}^{-3}$, and above this region, it increased with increasing the sait concentration. As discussed in Chapter 2, in the free volume model, B may be related to the critical free volume $v^{*}$ for transport and to the expansivity of free volume by

$$
\mathrm{B}=\gamma v^{*} /\left(\frac{\partial v_{\mathrm{f}}}{\partial \mathrm{T}}\right)
$$

where $v_{f}$ is the free volume. From this equation, if the expansivity of the free volume is taken as effectively constant, B should be proportional to the critical free volume. Because segmental motion is assumed not to take place unless the free volume excecds a critical value, the larger $B$ the more free volume is needed for segmental motion. In 
the configurational entropy model, $\mathrm{B}$ is correlated to the the free energy barrier $\Delta \mu$ which opposes the configurational rearrangement by the expression:

$$
\mathrm{B} \triangleq \frac{\Delta \mu \mathrm{s}_{\mathrm{c}}{ }^{*}}{\mathrm{k} \Delta \mathrm{C}_{\mathrm{P}}}
$$

where $\mathrm{k}$ is the Bolzmann constant, $\Delta \mathrm{C}_{\mathrm{P}}$ is the heat capacity difference between the liquid and glass state (assumed to be temperature independent ), $\mathrm{s}_{\mathrm{c}}{ }^{*}$ is the minimum configurational entropy required for rearrangement and of ten taken as $s_{\mathrm{C}}{ }^{*}=\mathrm{k} \ln 2$. Thus, it is seen from equation (5-1-9) that the larger $B$, the larger the free energy barrier will be. Therefore, although $\mathrm{B}$ has different meanings in different models and cannot be simply related to the barrier for the segmental motion, it reflects to some extent the difficulties to be overcome for segmental motion in the polymer electrolyte. In the region below $0.1 \mathrm{~mol} \mathrm{dm}^{-3}$, the almost invariant $B$ values may again indicate that the mobilities of the ions are invariant.

\section{5-1-3 Deviation From the VTF Behaviour}

In the macroscopic models (the free volume model and configurational entropy model), $T_{0}$ as mentioned above, is a temperature at which the free volume or the configurational entropy vanishes. The glass transition temperature $T_{g}$ is a temperature at which the behaviour of an amorphous polymer undergoes a transition from liquid-like to glass-like, i.e. segmental motion of the polymer is significantly restricted below $T_{g} . T_{0}$ thus should show a similar tendency to that of $\mathrm{T}_{\mathbf{g}}$ on changing salt concentration in a polymer electrolyte in terms of the segmental motion, but $T_{0}$ is usually found to be $50 \mathrm{~K}$ lower than $\mathrm{T}_{\mathrm{g}}$.

In order to further examine the behaviour of $T_{0}$ with changing salt concentration, the spin-spin relaxation time, $\mathrm{T}_{2}$, of protons in the polymer electrolyte was measured by 
IH solid state NMR with changing temperature (Figure 5-9). As discussed in Chapter 4, at very low temperature or in the limit of zero motion at which the correlation time $\tau_{c}$ is very large and common to most solids. Thus, the temperature for the onset of $T_{2}$ may correspond to the beginning of the motion of the protons, eg. the rotation and vibration of $\mathrm{C}-\mathrm{H}$ bonds, and furthermore, may correspond to $\mathrm{T}_{\mathrm{o}}$ in the macroscopic models. The temperatures for the onset of $T_{2}$ are listed in Table 5-1. It is seen that they are close to the fitted $\mathrm{T}_{\mathrm{o}}$ values in the dilute region. In the region above $0.1 \mathrm{~mol} \mathrm{dm}^{-3}$, they increase slightly, instead of decreasing as do the $T_{0}$ values, and are significantly lower than the $T_{g}$ values.

The different tendency between $T_{0}$ and $T_{g}$, or onset temperature of $T_{2}$ with changing salt concentration in the polymer electrolyte suggests that the VTF equation (5-1-7) cannot describe the conductive behaviour properly in the concentration region

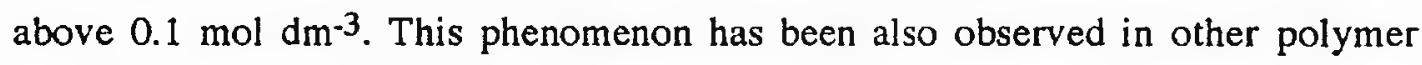
electrolyte systems $[11,12]$.

What factors may cause the conductive behaviours of amorphous polymer electrolytes to deviate from the VTF equation ? The following, at least, must be considered:

(i) There is significant macroscopic motion of the polymer chains. Under these circumstances, the ionic conduction arises not only from the segmental motion but also from the diffusion of the chains. This can be neglected in polymer electrolytes based on high molecular weight polymer hosts, because the diffusion coefficient of the polymer chains is constrained by the entanglements, and the diffusion coefficient of the chain is inversely proportional to the square of the molecular weight (see Chapter 8).

(ii) Ions move in a 'Coulomb fluid', i.e. the principal arriers are free ions formed by the dissociation of ion pairs as a result of electrostatic interactions in a Coulombic trap. 
The ionic conduction in this instance is in fact an activated process, and can be described by the Arrhenius equation (5-1-6). This will be important in electrolytes with very high salt concentrations. In an electrolyte with salt concentration around 0.1 to $0.2 \mathrm{~mol} \mathrm{dm}^{-3}$, this may not be important.

(iii) Ion association is crucial in the electrolyte, and the association equilibria can be shifted by changing temperature. Under these circumstances, in equation (5-1-1), both the mobilities of ions and the concentrations will be changed with changing temperature. Thus, it may be not enough that only the mobilities of ions, or more exactly, the the segmental motion of the polymer are considered. This may be more plausible in the present electrolyte system because, as discussed above, neutral ion pairs are very important.

(iv) In an electrolyte, if ion association is dominant and those associated species are too large to undergo translational motion, the ionic conduction may be carried out by an activation mechanism other than the free volume mechanism, or by the combination of the two mechanisms, eg. an exchange mechanism as discussed later. This may also be plausible in the present electrolyte system.

\section{5-1-4 Conductive Behaviour in Polymer Electrolytes}

For $\mathrm{PMEO}-\mathrm{LiClO}_{4}$ electrolytes, in the relatively low salt concentration region, i.e. less than $0.25 \mathrm{~mol} \mathrm{dm}^{-3}$, the dominant species are assumed to be non-conductive ion pairs. Carriers thus have to be generated for ionic conduction either by further association to form charged ion triples or by dissociation to form free ions. These processes are actually activated processes. Moreover, in the high concentration region, where the mobilities of ion pairs, larger ion clusters and segments of the polymer are significantly reduced by the transient crosslinking effect of the salt, the mechanism of ion transport may not be the same as in the dilute case. As will be discussed later, such a 
mechanism is likely to be an activated process. Therefore, when ionic conduction is considered in a polymer electrolyte, carrier generation and / or ion activation process have to be considered in addition to the mobilities of ions in equation (5-1-1).

In order to consider these processes, Cheradame [13] following the earlier work of Miyamoto and Shibayama [14] proposed a modified VTF equation by superimposing an Arrhenius term:

$$
\sigma \propto \exp \left[-\frac{\gamma v^{*}}{v_{f}}-\frac{\Delta E}{R T}\right]
$$

where $\gamma, v^{*}, v_{f}, R$ and $T$ have their meanings as before. $\Delta E$ is an activation energy, and is taken as

$$
\Delta E=E_{a}+E_{b}+W / \varepsilon
$$

where $\varepsilon$ is the dielectric constant of the solvent, $W$ is the dissociation energy of the salt, $E_{a}$ is the potential energy barrier for displacement of the polymer segment, and $E_{b}$ is not defined, but is presumably the activation energy required by the ions to jump.

This equation, although containing a contribution associated with carrier generation, does not considers the different processes which may be involved in generating the carriers, eg. further association to form triple ions, or the ion activation process. It has been reported that this equation could not explain the observation in spectroscopic studies, where ion association was found to increase with increasing temperature which corresponds to a negative activation energy rather than a positive activation energy as predicted by the equation [15]. 
Because ion generation and ion activation are activated processes, and the segmental motion of polymer is a free volume or configurational entropy controlled process, we propose an equation with a similar form as equation (5-1-10), but having different meanings of the terms to fit the conductivity data:

$$
\sigma=\sigma_{o} \exp \left[\frac{-B}{T-T_{0}}-\frac{\Delta E}{R T}\right]
$$

where $\sigma_{0}, B, T_{0}, R$ and $T$ have their meanings as in the VTF equation (5-1-7). $\triangle E$ is an activation energy for the carrier generation and/ or activation. In this equation, the first exponential term represents the segmental motion where temperature dependence is described by the VTF equation, and the second exponential term represents the carrier generation and / or activation process. If $B /\left(T-T_{0}\right) \gg \Delta E / R T$, the ionic conduction is controlled by the free volume process. If $B /\left(T-T_{0}\right) \ll \Delta E / R T$, it is controlled by an activated process. If $B /\left(T-T_{0}\right) \approx \Delta E / R T$, the ionic conduction is controlled by both the free volume process and the activated process.

The conductivity data were fitted by equation (5-1-12) using the 'Minitab' program. The equation was converted to: $\ln \sigma=-\frac{B}{T-T_{0}}-\frac{\Delta E}{T}+\ln \sigma_{o}$, where $B$, $\Delta E$ and $\ln \sigma_{0}$ were taken as constants, and $1 /\left(T-T_{0}\right)$ and $1 / T$ as variables. This was fitted by a linear least square equation. After inputting the conductivity data and the corresponding temperatures, an initial estimated value of $T_{0}$ was given, and this initial value was then increased while monitoring a statistical analysis parameter RSS for the data fit at each $T_{0}$ value. When the RSS term was at a minimum value, the best fitted $T_{0}$ and $B, \Delta E$ values were given. The fitted $T_{0}, \sigma_{0}, B$ and $\Delta E$ which are represented by $B_{1}=B, T_{01}=T_{0}, \sigma_{0}{ }^{*}=\sigma_{0}$ and $B_{2}=\Delta E / R$, in order to distinguish from the parameters fitted by the VTF equation, are listed in Table 5-1. At first sight, it is seen that, over the entire concentration range, the fitted $T_{o l}$ values are only slightly lower 
than the onset temperatures of $\mathrm{T}_{2}$ of the protons, and show a similar tendency with changing salt concentration. This suggests that the fitted $\mathrm{T}_{\mathrm{ol}}$ is closer to its real physical meanings in the free volume model or the configurational entropy model than $T_{\mathrm{o}}$ in the VTF fit.

In the very dilute concentration region up to $0.01 \mathrm{~mol} \mathrm{dm}^{-3}$, there is no significant difference between $B$ and $T_{0}$, fitted by the VTF equation (5-1-7), and $B_{1}$ and $T_{o 1}$, fitted by the modified VTF equation $(5-1-12)$. The fitted $B_{2}$ is negative, since in this concentration region, free ions associate to form non-conducting ion pairs, and as reported previously [15] the ion pairing effect gradually increases with increasing temperature, leading to a decrease of the number of carriers with increasing temperature. Hence, the "activation" energy has a negative value. Because $\mathrm{B}_{2}$ is much smaller than $B_{1}$, i.e. the influence of the Arrhenius term is not significant, the same values as $B$ and $T_{0}$ in the VTF equation fit are thus given. Therefore, in this concentration region, the ionic conduction is mainly a free volume controlled process.

In the region of the salt concentration from 0.01 to $0.1 \mathrm{~mol} \mathrm{dm}^{-3}$, the fitted $B_{1}$ and $T_{o 1}$ are again not much different from those fitted by the VTF equation. The fitted $B_{2}$, however, has a positive value. The reason for the very close agreement of $B_{1}$ and $T_{01}$ values with the VTF fitted parameters $B$ and $T_{0}$ may be the same as in the very dilute region, i.e. $B_{2}$ is very small in comparison with $B_{1}$. This is also implies that the free volume process is dominant in the region. Here, the question that now arises is: why does $\mathrm{B}_{2}$ have a positive value and what does it mean ? It may be argued that in the concentration region of 0.01 to $0.1 \mathrm{~mol} \mathrm{dm}^{-3}$, there may exist both the dissociation process and further association processes for ion pairs. It has been observed that the permittivity of the electrolyte increases with increasing salt concentration [16]. This effect may prefer the dissociation process, because the higher permittivity can stabilize the free ions as discussed above. If this effect in this concentration region is very 
important, it will be observed that the activation energy for dissociation may decrease with increasing salt concentration. Furthermore, because the spectroscopic studies [15] and mas NMR studies (see next section) suggest that ion association increases with increasing temperature, the value of $\mathrm{B}_{2}$ should be negative, as in the very dilute region. However, the fitted activation energy does not change significantly with increasing salt concentration, and has a positive value. This implies that it is further ion association to give rise to higher ionic conductivity, i.e. the formation of ion triples, as discussed above.

In the concentration region above $0.1 \mathrm{~mol} \mathrm{dm}^{-3}$, the fitted $B_{1}$ and $T_{o 1}$ differ significantly from the $B$ and $T_{0}$ fitted by the VTF equation. The fitted $T_{o 1}$ values increase slightly and are very close to the onset temperatures of $\mathrm{T}_{2}$ of the proton. Also, the values of $B_{1}$ increase with increasing salt concentration, but not so steeply as $B$ in the VTF equation. The increase of $B_{1}$ as discussed above, is due to the transient crosslinking effect of the salt. In the VTF equation fit, because there is no consideration of the contribution of an Arrhenius term, the B value may be overestimated, and a steep change is given. A less steep change of $B_{1}$ may also be anticipated, if the ionic conduction in the region is less controlled by the segmental motion. The activation energy in this concentration region varies slightly with increasing salt concentration, and is much higher than that in the lower concentration region. This may indicate that the ion activation process begins to become important in this region. It is therefore suggested that the ionic conduction in this concentration region may be controlled both by the free volume / configurational entropy model and by an activated process. The proposed ion transport mechanism dominated by the activation process in the relatively high concentration region $[17,18]$ is:

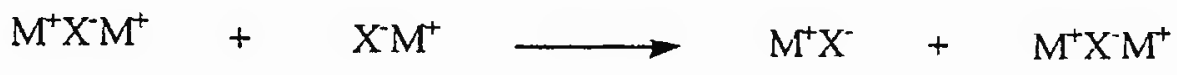


This mechanism will be further supported by examination of the results of the pulsed field gradient NMR experiments in the following section.

The conductivity data of PMEO-LiSCN electrolytes were also fitted by the VTF equation (5-1-7) and the modified VTF equation (5-1-12). The fitted parameters are listed in Table 5-2. $\mathrm{T}_{\mathrm{o}}$ fitted by the VTF equation was found to change quite irregularly with increasing salt concentration. The fitted $B$ was much higher than that in PMEO$\mathrm{LiClO}_{4}$ electrolyte, and was invariant with changing salt concentration. This is difficult to understand according to the previous discussion, because the only difference between PMEO-LiSCN and PMEO-LiClO 4 electrolytes is in the anions. The segmental motion of the polymer may not be so different between these two electrolyte systems in the low salt concentration region where the transient crosslinking effect of the salt is not significant. This may again indicate the deviation from the VTF behaviour, because of the dominant non-conducting ion pairs in the system.

The fitted $\mathrm{T}_{\mathrm{ol}}$ in the concentration region studied is similar to that in the PMEO$\mathrm{LiClO}_{4}$ system, except those in the very dilute region which are apparently somewhat lower than those in the latter system, and may be attributed to relatively large errors in the conductivity data arising from the very low conductivity of the electrolyte in the ver: dilute region which is close to the background conductivity of the pure polymer (at $25^{\circ} \mathrm{C}$, the conductivity of electrolyte with LiSCN concentration of $2.36 \times 10^{-3} \mathrm{~mol} \mathrm{dm}^{-3}$ is $7.89 \times 10^{-8} \mathrm{~S} \mathrm{~cm}^{-1}$, while that of the pure polymer is $1.21 \times 10^{-8} \mathrm{~S} \mathrm{~cm}^{-1}$ ). A similar value of $\mathrm{T}_{\mathrm{ol}}$ in this salt concentration region is expected to that in $\mathrm{PMEO}-\mathrm{LiClO}_{4}$ and PMEO-LiSCN systems, because the the segmental motion of polymer should not be significantly affected by the nature of the anion in the low salt concentration region.

The fitted $B_{1}$ in the very dilute region is very close to $B$ fitted by the VTF equation. This is again similar to the situation described for the P.EO- $\mathrm{LiClO}_{4}$ system. 
The slightly higher $B_{1}$ value may also be attributed to the larger errors in the conductivity data. In the concentration region up to $0.19 \mathrm{~mol} \mathrm{dm}^{-3}$, fitted $B_{1}$ values are significantly lower than the VTF fitted B values, and are very close to those of PMEO$\mathrm{LiClO}_{4}$ system in the concentration region below $0.1 \mathrm{~mol} \mathrm{dm}^{-3}$. This is again expected behaviour, due to the similar mobility of segments of the polymer in these two electrolyte system in the concentration region without significant transient crosslinking effect of the salt.

The $\mathrm{B}_{2}$ in the very dilute concentration region also has a negative value, which is larger than that in the $\mathrm{LiClO}_{4}$ system. This may be explained if it is assumed that cations and anions form ion pairs more easily in the PMEO-LiSCN system. In the concentration region higher than that corresponding to the minimum in the plot of $\Lambda$ versus $c_{\text {, the }} B_{2}$ is significantly higher than that in the $\mathrm{PMEO}-\mathrm{LiClO}_{4}$ electrolyte. This may indicate that the carrier generation / activation process is more difficult than in the $\mathrm{LiClO}_{4}$ system. which agrees well with the analysis in the above section, i.e. ion pairs may not readily form ion triples or larger clusters in the PMEO-LiSCN system, and also with the observation that the ionic conductivity is much lower than that of the PMEO-LiClO. electrolyte.

In conclusion, for PMEO-LiSCN electrolyte, the modified VTF equation (5-1-12) fit supports the conclusion that the ion pairing effect is more severe, and that the carrier generation / activation process is more difficult than in the $\mathrm{LiClO}_{4}$ system.

\section{5-1-5 Conductivity Measurement of PMEO-LiPF 6 Electrolytes}

$\mathrm{PMEO} \mathrm{LiPF}_{6}$ electrolytes are suitable electrolytes for the measurement of the diffusion coefficients of cation- and anion-based species by pulsed field gradient NMR. because ${ }^{7} \mathrm{Li}$ and ${ }^{31} \mathrm{P}$ nuclei are NMR active. Before the NMR experiment, the ion 
transport of these polymer electrolytes with the salt content of EO / Li ratio from 100:1 to 20: 1 was investigated by conductivity measurement and dc polarisation.

The conductive behaviour is obviously non-Arrhenius (Figure 5-10). Thus, the conductivity data were then fitted by the VTF equation (5-1-7). The fitted $T_{0}, B, \sigma_{0}$ are listed in Table 5-3a. For the electrolyte with lower salt content ( $\mathrm{EO} / \mathrm{Li}=100$ ), the fitted $T_{o}$ and $B$ are similar to the fitted $T_{o l}$ and $B_{1}$ of the $P M E O-\mathrm{LiClO}_{4}$ electrolytes. This may indicate that at this salt concentration, the ionic conduction is mainly controlled by the segmental motion of the polymer. When the salt content increases, the fitted $T_{0}$ decreases instead of increasing, and the fitted $B$ is very high. This is similar to the observation of the VTF equation fit in the $\mathrm{PMEO}-\mathrm{LiClO}_{4}$ system in the higher concentration region which indicates the deviation from the VTF behaviour. Thus, for the higher salt content in PMEO-LiPF 6 electrolytes, their conductive behaviour may also be controlled by the activation process.

The steady state current of PMEO-LiPF 6 was measured by dc polarisation of the symmetric cell: $\mathrm{Li} / \mathrm{PMEO}-\mathrm{LiPF}_{6} / \mathrm{Li}$ with a small dc potential $(10 \mathrm{mV})$ at $80^{\circ} \mathrm{C}$. The limiting current fraction $F_{+}$'s are listed in Table 5-3b, calculated from

$$
F_{+}=\frac{I_{s}}{I_{0}} \frac{\Delta V-I_{o} R_{i 0}}{\Delta V-I_{s} R_{i s}}
$$

where $I_{o}$ and $I_{s}$ are the initial and steady state currents respectively, $\Delta V$ is the applied potential, and $R_{i o}$ and $R_{i s}$ are the initial and steady state interfacial resistances. The limiting current fractions are very small in comparison with the polymer electrolytes based on $\mathrm{LiClO}_{4}$ and $\mathrm{LiCF}_{3} \mathrm{SO}_{3}$ [19] (and also Chapter 8). This indicates that the mobilities of ion pairs or cationic species are much smaller than those of anions or anionic species. 


\section{5-2 Species in Polymer Electrolytes Investigated by Magic- Angle Spinning NMR}

The studies of conductivity in the above section have shown that ion-associated species may exist in the polymer electrolytes studied, $\mathrm{PMEO}-\mathrm{LiClO}_{4}, \mathrm{PMEO}-\mathrm{LiSCN}$ and PMEO-LiPF6. Solid state NMR as discussed in Chapter 4 allows investigation of the local structure of ${ }^{7} \mathrm{Li},{ }^{23} \mathrm{Na}$ etc. nuclei. Since the 1980's [20-22], solid state NMR has been used to investigate the ion species in polymer electrolytes. However, only limited information has been obtained from these earlier studies, due both to the complexity of the polymer electrolytes studied and limitations of the resolution of the spectrometers employed. In this section, the species in the polymer electrolytes are discussed, in the light of the analysis of the results mainly of $7 \mathrm{Li}$ mas NMR.

\section{5-2-1 Origin of NMR Spectra in the Polymer Electrolytes Containing Lithium Salts}

The ${ }^{7} \mathrm{Li}$ mas NMR of PMEO- $\mathrm{LiClO}_{4}$ with salt concentration of $1.0 \mathrm{~mol} \mathrm{dm}^{-3}$ is shown in Figure 5-11a. There are basically three peaks with chemical shifts of -0.71 , -1.27 and -1.68 ppm and with a linewidth of about $30 \mathrm{~Hz}$ for the most intense line. Similar spectra were also observed for $\mathrm{PEO}\left(4 \times 10^{6}\right)-\mathrm{LiClO}_{4}$ with an $\mathrm{EO} / \mathrm{Li}$ ratio of 20 at $80^{\circ} \mathrm{C}$ ( Figure 5-12), where it was totally amorphous.

In the magic-angle spinning spectrum, spinning side-bands can sometimes be observed. However, in polymer electrolytes, the peaks observed do not arise from the spinning side-bands, because the differences of chemical shift between these peaks are 
not more than $1 \mathrm{ppm}$ for ${ }^{7} \mathrm{Li}$, i.e. less than $200 \mathrm{~Hz}$, while the spinning side-bands should be separated by the spinning rate, i.e. $5 \mathrm{kHz}$.

For a quadrupolar nucleus with $\mathrm{I}=3 / 2$ there should theoretically be three peaks observed which correspond to $(m=-3 / 2) \leftrightarrow(m=-1 / 2),(m=-1 / 2) \leftrightarrow(m=1 / 2)$ and $(m=1 / 2) \leftrightarrow(m=3 / 2)$ transitions in an electric field gradient. The central transmission of $(\mathrm{m}=-1 / 2) \leftrightarrow(\mathrm{m}=1 / 2)$ is only affected by the second order quadrupolar interactions but by the first order quadrupolar interactions. In most cases [23], however, what can be observed in solid state NMR spectrum is the central line pattern rather than the satellite lines. For polycrystalline powder especially with random orientation of nuclei, the first order quadrupolar interactions can be averaged, and only the central line pattern is observed.

For amorphous polymer electrolytes based on lithium salts, although ${ }^{7} \mathrm{Li}$ is a quadrupolar nucleus with $I=3 / 2$, the satellite lines arising from the first order quadrupolar interaction are unlikely to be observed, due to the random distribution and orientation of nuclei in the amorphous system. This is further supported by the spectra recorded at high temperature $\left(90^{\circ} \mathrm{C}\right.$, Figure 5-11b), in which the three-line pattern is also observed. There is no significant change of the pattern except for a slight difference in the ratio of the peaks. At this temperature, the conductivity measurement has suggested that the ions are very mobile, which may lead to further averaging of the first order quadrupolar effect. If the first order quadrupolar effect has given rise to satellite lines, it may eventually give rise to a significant change of the line pattern. Furthermore, the second order quadrupolar interaction is less likely to be observed in the spectra, not only because the ${ }^{7} \mathrm{Li}$ nucleus has a small quadrupolar coupling constant, leading to small quadrupolar interactions, but because the magic-angle spinning can reduce the second order quadrupolar interactions. In addition, because the second order quadrupolar interactions are inversely proportional to the field strength, the second order quadrupolar 
interactions are thus significantly reduced by the strong field applied by the NMR spectrometer.

For PMEO-LiBF 4 electrolyte with a salt concentration of $1.0 \mathrm{~mol} \mathrm{dm}^{-3}$, both ${ }^{11} \mathrm{~B}$ and ${ }^{7} \mathrm{Li}$ mas NMR spectra show a three-line pattern (Figure 5-13 and 5-14a). For the ${ }^{7} \mathrm{Li}$ case as discussed above, only the central line corresponding to $(m=-1 / 2) \leftrightarrow$ $(\mathrm{m}=1 / 2)$ transition can be observed. For ${ }^{11} \mathrm{~B}$ case, the satellite lines arising from the quadrupolar interactions of ${ }^{11} \mathrm{~B}$ nuclei with $\mathrm{I}=3 / 2$ again cannot be observed due to the same reasons suggested for the ${ }^{7} \mathrm{Li}$.

\section{5-2-2 Assignment of ${ }^{7} \mathrm{Li}$ Mas NMR Spectra of Polymer Electrolytes}

In order to understand what kind of species may give rise to the different chemical shifts observed for the ${ }^{7} \mathrm{Li}$ nucleus in the polymer electrolyte, lithium-polymer host and lithium-anion interactions must first be considered. In terms of the polymer host, PMEO is basically similar to PEO in every aspect except that PMEO segments with about $9 \mathrm{EO}$ units are linked by $-\mathrm{CH}_{2} \mathrm{O}$ - groups rather than $-\mathrm{CH}_{2} \mathrm{CH}_{2} \mathrm{O}$ - groups. The similar line pattern of ${ }^{7} \mathrm{Li}$ mas NMR spectra for the polymer electrolytes based on PMEO (Figure 5-11a) and PEO (Figure 5-12) indicates that there are no significant differences between the interactions of $\mathrm{Li}^{+}-\mathrm{PMEO}$ and $\mathrm{Li}^{+}-\mathrm{PEO}$. Therefore, the chemical shifts in ${ }^{7} \mathrm{Li}$ mas NMR spectra do not arise from the different interactions of lithium cations with $-\mathrm{CH}_{2} \mathrm{O}$ - and with $-\mathrm{CH}_{2} \mathrm{CH}_{2} \mathrm{O}$ - groups in PMEO.

The ${ }^{7} \mathrm{Li}$ mas NMR spectra for PMEO-LiBF 4 , PMEO-Lil and PMEO-LiSCN with the salt concentrations of $1.0 \mathrm{~mol} \mathrm{dm}^{-3}$ electrolytes are shown in Figure 5-14a, 5-14b and 5-14c respectively. For PMEO-LiSCN electrolyte, both the line shape and the peak position are different from those of PMEO- $\mathrm{LiClO}_{4}$. For the others, the peak position differs from $\mathrm{PMEO}-\mathrm{LiClO}_{4}$, but the line shape does not differ significantly. As discussed in the above section, the interactions between cations and anions are 
significantly different in $\mathrm{PMEO}^{-\mathrm{LiClO}_{4}}$ and $\mathrm{PMEO}-\mathrm{LiSCN}$ electrolytes, implying that the different chemical environment in the electrolytes may arise from the different interactions between lithium nuclei and anions.

In the ${ }^{7} \mathrm{Li}$ mas $\mathrm{NMR}$ spectrum of $\mathrm{PMEO}-\mathrm{LiClO}_{4}$ electrolytes with a salt concentration of $0.1 \mathrm{~mol} \mathrm{dm}^{-3}$, there are only two peaks which can be observed (Figure 5-15a), i.e. the one with the lowest chemical shift disappears. As discussed in the conductivity measurement, when the salt concentration increases in the polymer electrolytes, the ion associations may increase. Thus, the missing line in the low salt concentration electrolyte may correspond to the lithium nuclei with strong ion association, i.e. ion clusters. ${ }^{7} \mathrm{Li}$ NMR spectra have also been recorded for liquid $\mathrm{PEO}(400)$ dimethyl ether- $\mathrm{LiClO}_{4}$ electrolytes. For that with a salt concentration of $1.0 \mathrm{~mol} \mathrm{dm}^{-3}$, the spectrum (Figure 5-16b) shows two peaks clearly. The chemical shifts are $-1.37 \mathrm{ppm}$ and $-1.69 \mathrm{ppm}$ respectively which are close to the chemical shifts of the two peaks at lower chemical shifts in the $\mathrm{PMEO} \mathrm{LiClO}_{4}$ electrolyte. The spectrum of the $\mathrm{PEO}(400)$ dimethyl ether with the salt concentration of $0.1 \mathrm{~mol} \mathrm{dm}^{-3}$ clearly shows just a single peak with chemical shift of $-1.36 \mathrm{ppm}$ (Figure 5-16a). Because it has been reported that the end-capped $\mathrm{PEO}(400)-\mathrm{LiClO}_{4}$ electrolyte was dominated by ion pairs and ion clusters (eg. triple ions) when the salt concentration was high [24], the two peaks observed may be attributed to ion pairs and ion clusters. When the salt concentration is rather low, the ion pairs are the dominant species in the $\mathrm{PEO}(400)$ dimethyl ether- $\mathrm{LiClO}_{4}$ electrolyte. The single peak with a chemical shift of $-1.36 \mathrm{ppm}$ observed in the low concentration sample may thus be attributed to ion pairs. The one with chemical shift of -1.69 ppm appeared in $\mathrm{PEO}(400)$ dimethyl ether- $\mathrm{LiClO}_{4}$ electrolyte, in addition to the $-1.35 \mathrm{ppm}$ peak which may be assigned to ion pairs as discussed above, may thus be attributed to ion clusters. 
In light of the analysis of $\mathrm{PEO}(400)$ dimethyl ether- $\mathrm{LiClO}_{4}$ electrolytes together with the observation of the change of the peak with salt concentration in PMEO- $\mathrm{LiClO}_{4}$ electrolytes, it is suggested that the peak with high chemical shift may be assigned to "free ions", the middle peak to ion pairs and the one with the lowest chemical shift to ion clusters.

It has been reported that ${ }^{6} \mathrm{Li}$ and ${ }^{7} \mathrm{Li}$ mas $\mathrm{NMR}$ of ionically conductive nonoxide chalcogenide glasses [25] showed that the higher the covalency of Li-S bond, the more positive ${ }^{6} \mathrm{Li}$ and $7 \mathrm{Li}$ chemical shifts were observed. It was also found that the chemical shifts of ${ }^{7} \mathrm{Li}$ mas NMR of lithium salts decrease with decreasing covalency of $\mathrm{Li}$-anion bond [26]. For example, the ${ }^{7} \mathrm{Li}$ chemical shifts decrease in the order: $\mathrm{Li}_{2} \mathrm{~S}>$ $\mathrm{LiOH}>\mathrm{LiIO}_{3}>\mathrm{LiNO}_{3}$. It is generally believed that in polymer electrolytes, the coordinating bond is formed by partially sharing of a lone pair of electrons on a coordinating atom in the polymer [27]. Thus, "free ions" are those lithium species which are coordinated by oxygen atoms, in which lithium ion and oxygen atom share a lone pair of electrons from the oxygen atom and thus shows some characteristics of covalency. For ion pairs and ion clusters, on the other hand, the lithium ions interact not only with oxygen atoms in the polymer chains but also with anions, which leads to an increase in the contribution of ionic bond. Thus, the above assignment also seems to be consistent to the order of the chemical shifts, i.e. the free ions have the highest chemical shift and the ion clusters show the lowest chemical shift.

It is interesting to note that ${ }^{11} \mathrm{~B}$ mas $\mathrm{NMR}$ of the $\mathrm{PMEO}-\mathrm{LiBF}_{4}$ electrolyte with a salt concentration of $1.0 \mathrm{~mol} \mathrm{dm}^{-3}$ also shows a three-line pattern (Figure 5-13). In polymer electrolytes, it is usually believed that there are very weak interactions between anions and polymer host. Thus, it is likely that these peaks arise from the different interactions between anions and cations. In Raman spectroscopy studies of polymer electrolytes, three types of Raman bands of anions corresponding to "free ions". icn 
pairs and ion clusters have been reported [18]. Because in PMEO-LiBF 4 , as in PMEO$\mathrm{LiClO}_{4}$, the ${ }^{7} \mathrm{Li}$ mas NMR spectrum shows a three-line pattern, the three-line pattern of 11B mas NMR spectrum may also be attributed to the "free ions", ion pairs and ion clusters. By comparing the relative intensity of each peak with that of ${ }^{7} \mathrm{Li} N M R$

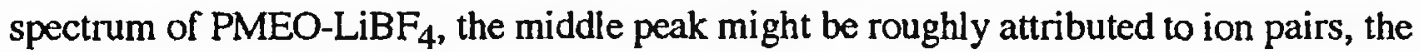
one with high chemical shift to the free ions and the one with lowest chemical shift to the ion clusters.

There may be two types of ion pairs to be considered in an electrolyte, i.e. contact ion pairs and solvent separated ion pairs. It has been found that in a solution based on a relatively low permittivity solvent, the solvent separated ion pairs are formed in dilute concentration and the contact ion pairs are formed in relatively high concentration [28]. For example, in the methoxy-poly ethylene-glycol $400 /$ lithium picrate electrolyte, it was found that contact ion pairs were dominant when the salt concentration was as high as $0.002 \mathrm{M}$, while it was also reported that in solid polymer electrolytes the solvent separated ion pairs were more important than the contact ion pairs in the medium salt concentration [29]. It is unlikely that the line assigned to ion pairs may arise from solvent separated ion pairs for the following reasons: (i) If in the above NMR spectra, the line arises from ion pairs are solvent separated, these ion pairs may be difficult to distinguish from the free ions, especially in ${ }^{11} \mathrm{~B}$ mas $\mathrm{NMR}$, because the interaction between ${ }^{11} \mathrm{~B}$ and ${ }^{7} \mathrm{Li}$ is through fluorine atoms. (ii) If the lines assigned to ion pairs in the electrolytes based on liquid PEO 400 dimethyl ether may arise from contact ion pairs as reported in ref [29], since the positions of these lines are not much different from those shown in the solid samples, the line may thus arise from the contact ion pairs in electrolytes based on PMEO. According to this analysis, it should be noted that the line assigned to the "free ions" may not only arise from free ions, but also arise from the solvent separated ion pairs. 


\section{5-2-3 Influence of Salt Concentration}

It has been shown that when the salt concentration increases in the region of above $0.1 \mathrm{~mol} \mathrm{dm}^{-3}$, the glass transition temperature (Table 5-1) increases, indicating a significant transient crosslinking effect of the salt. In the ${ }^{7} \mathrm{Li}$ NMR spectra of PMEO$\mathrm{LiClO}_{4}$ electrolytes, the linewidth increases with increasing salt concentration from $0.5 \mathrm{~mol} \mathrm{dm}^{-3}$ to $4.0 \mathrm{~mol} \mathrm{dm}^{-3}$ (Table $5-4$ ). For the $4.0 \mathrm{~mol} \mathrm{dm}^{-3}$ sample, only a broad line is observed (Figure 5-15c), indicating that the motion of lithium nuclei is significantly reduced by the transient crosslinking effect of the salt, in agreement with the above discussion.

The linewidth of the ${ }^{7} \mathrm{Li}$ mas NMR of $\mathrm{PMEO}^{-\mathrm{LiClO}_{4}}$ with a salt concentration of $0.1 \mathrm{~mol} \mathrm{dm}^{-3}$ is, however, extraordinarily large (Table 5-4) in comparison with those at higher salt concentrations, especially for the peak with the chemical shift of $-0.5 \mathrm{ppm}$. The linewidth of $0.2 \mathrm{~mol} \mathrm{dm}^{-3}$ sample (Figure 5-15b) is comparable to that of the $1.0 \mathrm{~mol} \mathrm{dm}-3$ sample. This cannot be explained by the transient crosslinking effect of the salt.

The factors affecting the linewidth of a NMR spectrum may include the motion of the nuclei, the exchange rate of the nuclei between sites etc. Motion of the nuclei will lead to narrowing of the line. Fast exchange rates between sites will also give rise to narrow lines. Relatively slow exchange will give rise to broad lines, with extremely slow exchange rates giving rise to separated peaks. In the low concentration region, because the transient crosslinking effect may be not so important as in the higher concentration cases, the motion of the nuclei is not significantly reduced by this effect. The motion factor for broadening the line need not be considered. For lithium nuclei in polymer electrolytes, there must inevitably exist some kind of exchange between different coordinating sites or species. In dilute solution, the smaller number of sites or species may cause relatively slower exchange rates, leading to broadening of the line. In 
concentrated solution however, because the large number of sites or species may enable fast exchange between those sites, the motion factor for broadening the line becomes important. This also implies that the real situation of lithium species in the polymer electrolytes may be more complicated than those observed in the NMR due to the existence of exchange, and what can be observed in the NMR may be only part of the lithium species. In particular, it should be pointed out that there may exist many forms of ion clusters in the electrolyte, eg. $\mathrm{Li}_{2} \mathrm{ClO}_{4}{ }^{+}, \mathrm{Li}\left(\mathrm{ClO}_{4}\right)_{2}{ }^{-}$etc. The line assigned to the ion clusters may only reflect some of these ion clusters. For some clusters, because the mobilities of the lithium nuclei are largely reduced by the transient crosslinking effect, the line would be too broad to be well defined in the spectrum.

In order to obtain the ratio of the peaks, the spectrum was deconvoluted using the GLINFIT program [30] in the computer affiliated to Bruker MSL 500 NMR spectrometer, which permits resolution of an experimental lineshape into a sum of Lorentzian or Gaussian lines and gives line positions, widths and intergrals. By minimizing the root mean square (RMS) error, the best fitted spectrum can be given.

For the ${ }^{7} \mathrm{Li}$ mas NMR spectrum of $\mathrm{PMEO}-\mathrm{LiClO}_{4}$ with salt concentration from $0.2 \mathrm{~mol} \mathrm{dm}^{-3}$ to $2.0 \mathrm{~mol} \mathrm{dm}^{-3}$, the spectrum was deconvoluted into three Lorentzian lines with the RMS error less than 6.5\% (Table 5-5). A typical example for the $1.0 \mathrm{~mol}$ $\mathrm{dm}^{-3}$ electrolyte is shown in Figure 5-17. For $0.1 \mathrm{~mol} \mathrm{dm}^{-3}$ sample, the spectrum was deconvoluted into two Lorentzian lines. Because of the very broad line, the fitted spectrum is not so good as that for the higher concentration samples, and the RMS error was $11.33 \%$.

With increasing salt concentration, the ratio of the line assigned to the "free ions" decreases, while both the ratio of ion pairs and ion clusters increase. When the salt concentration is $0.1 \mathrm{~mol} \mathrm{dm}^{-3}$, the ratio of the line assigned to the "free ions" is very" 
high, indicating that the important species observed in ${ }^{7} \mathrm{Li}$ NMR may be free ions and / or solvent separated ion pairs. For the $0.2 \mathrm{~mol} \mathrm{dm}^{-3}$ sample, the ratio of the lines assigned to free ions and ion pairs are comparable. When the sample is more concentrated, the ratio of the line assigned to the ion pairs is high, indicating that the ion pairs may become important. For the $2.0 \mathrm{~mol} \mathrm{dm}^{-3}$ sample, the ratio of the line corresponding to ion clusters is significant, indicating that the ion clusters may be significant.

If the exchange and a variety of the species are present, the direct correlation of the ratios of deconvoluted lines to the actual concentration of species in the polymer electrolyte should be taken cautiously, because as pointed out above, only a proportion of certain species may be clearly defined in the NMR spectra. As a qualitative comparison however, the deconvolution results are largely consistent with the interpretation of the conductivity measurement.

\section{5-2-4 Influence of Temperature}

The influence of temperature on the ${ }^{7} \mathrm{Li}$ mas NMR spectrum was investigated by carrying out the experiment at three different temperatures, viz $297 \mathrm{~K}, 333 \mathrm{~K}$ and $363 \mathrm{~K}$ for the PMEO- $\mathrm{LiClO}_{4}$ electrolyte with salt concentration of $0.5 \mathrm{~mol} \mathrm{dm}^{-3}$ and $2.0 \mathrm{~mol}$ $\mathrm{dm}^{-3}$ (Figure 5-18 and Figure 5-19).

When the temperature was increased, the line narrowed, due to the increase of the mobility of the lithium nuclei. The linewidth of the $2.0 \mathrm{~mol} \mathrm{dm}^{-3}$ sample was $71 \mathrm{~Hz}$ at $297 \mathrm{~K}, 33 \mathrm{~Hz}$ at $333 \mathrm{~K}$ and $26 \mathrm{~Hz}$ at $363 \mathrm{~K}$. For the $0.5 \mathrm{~mol} \mathrm{dm}^{-3}$ sample, the linewidth was $27 \mathrm{~Hz}$ at $297 \mathrm{~K}, 21 \mathrm{~Hz}$ at $333 \mathrm{~K}$ and $19 \mathrm{~Hz}$ at $363 \mathrm{~K}$. The effect of temperature on the linewidth was more significant for the $2.0 \mathrm{~mol} \mathrm{dm}^{-3}$ sample than for 
the $0.5 \mathrm{~mol} \mathrm{dm}-3$ sample. This is probably because the transient crosslinking effect is more pronounced in the former than in the latter.

When the temperature was increased, the ratio of the line assigned to the ion pairs increased relative to that assigned to free ions (Table 5-6). This is consistent with the observation in vibrational spectroscopy [15] that the ratio of ion pairs increased with increasing temperature, suggesting that the ion association increases with temperature. It is interesting to note that the relative ratio of the line assigned to ion clusters does not change significantly with temperature, which again agrees with the observation in the vibrational spectroscopy study.

\section{5-3 Mass Transport in Polymer Electrolytes Investigated by Pulsed Field Gradient NMR}

As discussed in Chapter 4, the pulsed field gradient NMR technique can provide information on the diffusion of the NMR-active nuclei. Thus investigation of a polymer electrolyte system by this technique may give further insight into ion transport. However, so far there have only been few reports of the use of the pfg NMR techniques to investigate polymer electrolyte systems $[31,32]$. In this section, the diffusion coefficients of species containing the cation nuclei and the anion nuclei are investigated by pfg NMR technique, in the polymer electrolyte based on PMEO and LiPF 6 .

\section{5-3-1 Diffusion of ${ }^{31} \mathrm{P}$ Nuclei}

Due to a very long $T_{1}$ of the ${ }^{31} \mathrm{P}$ nucleus and the coupling with ${ }^{19} \mathrm{~F}$ nuclei in $\mathrm{PF}_{6}{ }^{-}$group, the experiment to measure the diffusion coefficient of ${ }^{31} \mathrm{P}$ nuclei in dilute electrolytes proved to be very difficult and time consuming. Thus, the measurement was 
confined to electrolytes with EO/Li ratio of 50:1 and 20:1. The temperature dependence of the diffusion coefficient of $31 \mathrm{P}$ nuclei is shown in Figure 5-20.

As discussed above, there may exist free ions, ion pairs and ion clusters in the electrolytes based on PMEO and lithium salts. The diffusion coefficient of ${ }^{31} \mathrm{P}$ nuclei is therefore a mean value of these species, since pfg NMR provides a mean diffusion coefficient of all the species containing the probing nuclei, i.e.

$$
D=\sum_{i} \alpha_{i} D_{i}
$$

where $\alpha_{i}$ is the fraction and $D_{i}$ is the diffusion coefficient of species $i$. It is believed that in polymer electrolytes, there are no significant interactions between anions and polymer host [33]. The diffusion coefficient of anions should be larger than cations and cation constituents bound by polymer chains. On the other hand, if the mean value of the diffusion coefficient arises mainly from the diffusion of associated species, eg. ion pairs, the value should not much differ from that of the ${ }^{7} \mathrm{Li}$ nuclei. In the present case, however, the diffusion coefficient of the ${ }^{31} \mathrm{P}$ nuclei is almost one order of magnitude higher than the ${ }^{7} \mathrm{Li}$ (Figure 5-21a). Further, in the current fraction measurements, it has shown that $F_{+}$values are very small (Table 5-3b). These facts may suggest that the contribution of the anions to the diffusion coefficient is important. As an approximation, the mean value of the diffusion coefficient of anion constituents is taken as that for the anions. The diffusion coefficient of anions can then be described by the Stokes-Einstein equation:

$$
D=\frac{k T}{6 \pi \eta_{(r)} r}
$$

where $\mathrm{k}$ is the Boltzmann constant, $\mathrm{T}$ is the temperature, $\eta_{(\mathrm{r})}$ is the microscopic viscosity of the solution and $\mathrm{r}$ is the radius of the anion. Because the relevant viscosily of the polymer electrolyte is the microscopic viscosity which is dominated by the 
segmental motion of polymer, and can be described by the VTF equation, the diffusion coefficient of anions can thus be also described by the VTF equation. The curved relationship between $\log \mathrm{D}$ and $1000 / \mathrm{T}$ is expected according to the above analysis.

\section{5-3-2 Diffusion of ${ }^{7} \mathrm{Li}$ Nuclei}

As discussed above, the diffusion coefficient obtained is a mean value for all the species containing ${ }^{7} \mathrm{Li}$. The temperature dependence of the diffusion coefficient of ${ }^{7} \mathrm{Li}$ nuclei for PMEO-LiPF 6 electrolytes with the EO/Li ratio of 100:1, 50:1, 30:1 and 20:1 is shown in Figure 5-21a, 5-21b. It is of interest to note that the plot $\log \mathrm{D}$ versus 1000/T shows a linear relationship for the samples with EO/Li ratio of 30:1 and 20:1, while for the samples with EO/Li ratio of 100:1 and 50:1 it is curved.

In the dynamic bond percolation theory proposed by Ratner and coworkers [1], the motion of cations is determined by two factors, providing there are enough available sites. One is the renewal time $\lambda$, which is related to the segmental motion of the polymer. And the other is the hopping rate, $w$, of cations between neighbour vacant sites. The former factor can be related to the free volume model (see Chapter 2), and the latter is an activated process. Thus, if the hopping rate of ions is very fast, the motion of solvated cations is dominated by the segmental motion of the polymer, which is usually described by the VTF equation (5-1-7). If the hopping process is rate-determining, the motion of the cations may display an activated behaviour, which can be described either by the Arrhenius equation (5-1-6) or the modified VTF equation (5-1-12).

In $\mathrm{PMEO}-\mathrm{LiPF}_{6}$ electrolytes, the motion of ${ }^{7} \mathrm{Li}$ species may be controlled as follows:

(i) Segmental motion dominated: If other processes are negligible in comparison with the process controlled by the segmental motion of the polymer, whatever the forms of 
the ${ }^{7} \mathrm{Li}$ species are as long as they are solvated by the polymer host, i.e. completely or partially coordinated with the oxygen atoms in the polymer host chains, the motion of the species will be an non-activated process, and can be described by the VTF equation. For the samples with the EO/Li ratio of 100:1 and 50:1, the curved relationship between $\log \mathrm{D}$ and $1000 / \mathrm{T}$ may correspond to such a non-activated process, where the motion of ${ }^{7} \mathrm{Li}$ species is dominated by the segmental motion of the polymer.

(ii) Activated motion:: In polymer electrolytes with relatively high salt concentration, ion association leads to the formation of ion pairs and ion clusters. Due to the transient crosslinking effect of the salt and the relatively large cluster size, it may not be possible for clusters to diffuse as easily as free ions. It has been proposed that when the salt concentration is high, a transition may occur, such as:

$\mathrm{M}^{+} \mathrm{XM}^{+}+\mathrm{X}^{-} \mathrm{M}^{+} \longrightarrow \mathrm{M}^{+} \mathrm{X}^{-}+\mathrm{M}^{+} \mathrm{X}^{-} \mathrm{M}^{+}$

The transition of the cations can be described by the Arrhenius equation (5-1-6) or the modified VTF equation (5-1-12), instead of the VTF equation (5-1-7). If salt concentration is very high, the interionic distance will be small. Under this condition, $\mathrm{M}^{+}$ions may hop between neighbouring sites, leading to an Arrhenius behaviour. For samples with the EO/Li ratio above $30: 1$, the linear relationship between $\log D$ and $1000 / T$ may correspond to these processes.

The effect of above two types of mechanisms on the diffusion of $7 \mathrm{Li}$ species depends on the species in the electrolyte. When the salt concentration is low, the former mechanism is more likely. When the salt concentration is high and associated species are important, the motion of ${ }^{7} \mathrm{Li}$ nuclei may be dominated by the activated process. The segmental dominated motion and activation dominated motion may co-exist in an 
electrolyte system when the salt concentration is in the moderate region, as discussed in Section 5-1.

It is interesting to note that the diffusion coefficients of ${ }^{7} \mathrm{Li}$ nuclei for the samples with the EO/Li ratio of 50:1,30:1 and 20:1 are quite similar. As noted in the first section of this chapter, when the salt concentration is increased, the glass transition temperature $\mathrm{Tg}$ increases, which indicates that the mobility of the segment is reduced by the transient crosslinking effect of the salt. In PMEO-LiPF, because the diffusion coefficient of ${ }^{31} \mathrm{P}$ is dominated by the local viscosity, i.e. the segmental motion of the polymer, the decrease of the diffusion coefficient of $31 \mathrm{P}$ species implies that the segmental motion of the polymer is indeed reduced by increasing the salt concentration. Thus, the invariance of the diffusion coefficient of the ${ }^{7} \mathrm{Li}$ nuclei for the high salt concentration samples may indicate that the motion of the lithium nuclei is much less dependent on the segmental motion of the polymer, and is likely to be the case for the activated transition mechanism. This is consistent to the observation of the slight change of $\mathrm{B}_{1}$ in the relatively high concentration region for $\mathrm{PMEO}-\mathrm{LiClO}_{4}$ electrolytes in Section 5-1.

For anions, segmental motion controlled behaviour rather than the activated process (5-3-3) has been observed, i.e. the following process does not appear to be significant:

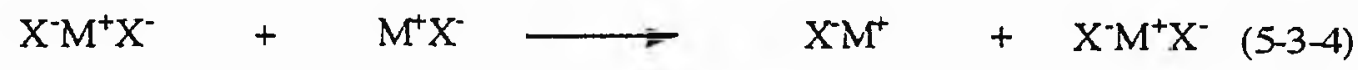

This may be explained by (i) the competitive segmental motion dominated mechanism for anion-based species being very much faster than that observed for the activated lithium transition mechanism, (ii) the larger size of the $\mathrm{PF}_{6}-$ in comparison with $\mathrm{Li}^{+}$may make an activated anion transition of this sort less probable. 
Why are the curved lines, rather than straight lines, observed for the sample with high salt concentration in the plot of $\log \sigma$ versus 1000/T (Figure 5-10) ? The diffusion coefficient of anions is much higher than that of cations and cation constituents (almost an order of magnitude higher), and as discussed in the following section, the conductivity of the electrolyte is largely determined by the transport of anions. Thus, what can be observed in the conductivity measurement is the sum of the ionic conductivities but with a dominant influence of the anions.

\section{5-3-3 Relationship Between Conductivity and Diffusion Coefficients}

For a fully dissociated electrolyte, transport of ions due to an electric field or a concentration gradient can be related by the Nernst-Einstein relation:

$$
\sigma_{i}=z_{i} c_{i} F^{2} D_{i} / R T
$$

where $D_{i}$ is the diffusion coefficient, $z_{i}$ is the charge, $c_{i}$ is the concentration and $\sigma_{i}$ the conductivity of ion $\mathrm{i}, \mathrm{R}$ is the gas constant, $\mathrm{F}$ is Faraday's constant and $\mathrm{T}$ the absolute temperature.

For a 1:1 strong electrolyte, the conductivity of the electrolyte can be related to the diffusion coefficients of cations and anions by

$$
\sigma=c F^{2}\left(D_{+}+D_{-}\right) / R T
$$

where $D_{+}$and $D_{-}$are the diffusion coefficients of cations and anions respectively. From this equation, the transport number can be calculated by

$$
t_{-}=\frac{D_{+}}{D_{+}+D_{-}}
$$


The Nernst-Einstein relationship fails, however, for a number of reasons. First, and of minor importance in the present study, the electrophoretic and relaxation field effects operate in a different manner for diffusion and migration and hence the absolute mobilities of ions in diffusion vary differently with concentration from their mobilities in electrolytic conduction. A much more important limitation of the Nernst-Einstein equation relates to the requirement that at the concentration of interest, the species involved in diffusion must also be responsible for electrical conduction. When the diffusion coefficients measured by pfg NMR, radiotracer diffusion etc. are related to the conductivity by the Nernst-Einstein equation, it is required that there are no mobile neutral species in the system, because the diffusion coefficient is the mean value for all the species with the nucleus of interest irrespective of charge.

In the $\mathrm{PMEO}-\mathrm{LiPF}_{6}$ electrolyte there may exist free ions, i.e., $\mathrm{Li}^{+}, \mathrm{PF}_{6}^{-}$, neutral ion pairs and ion clusters (eg. ion triples, $\mathrm{Li}_{2} \mathrm{PF}_{6}{ }^{+}, \mathrm{Li}\left(\mathrm{PF}_{6}\right)_{2}^{-}$) etc. Thus when the Nernst-Einstein equation is employed to relate the conductivity and the diffusion coefficients of ${ }^{7} \mathrm{Li}$ and ${ }^{31} \mathrm{P}$ nuclei measured by pfg NMR, deviations from the NernstEinstein equation may be expected.

In Table 5-7, the experimental and calculated conductivities are listed for two samples at three temperatures, where the salt concentration in the sample is directly used for the calculation of the conductivity by the Nernst-Einstein equation. The calculated conductivities are much higher than the experimental data. This deviation arises not only from the change of the concentration of charged species due to ion association, but also from the use of the mean diffusion coefficient to replace the individual diffusion coefficients of the charged species. If the ratio of species are invariant with varying temperature, according to equation (5-3-5), the slope of $\log D$ versus $\log (\sigma T)$ should be unity. However, the slopes of $\log$ D vs. $\log ($ OT) deviate significanty from unity and change with different salt concentrations (the slope is 2.64 for the sample with the EO/Li 
ratio of 50:1, and for the sample with EO/Li of 20:1, it is 2.10) (Figure 5-22). This may arise from changing the equilibria of ion association with varying temperature as shown in the mas NMR measurement. This is because, the change of the ion association equilibria will lead to a change in the concentration (or ratio) of the species in the electrolyte, leading to a change in the mean value of the diffusion coefficient with temperature due to the different mobilities of each species.

\section{5-4 Conclusions}

(1) In polymer electrolytes based on $\mathrm{PMEO}$ and $\mathrm{LiClO}_{4}, \mathrm{LiCF}_{3} \mathrm{SO}_{3}$ or $\mathrm{LiSCN}$, the ion pairing effect is dominant in the very dilute concentration region. In the medium salt concentration region, the increase of the molar conductivity may arise mainly from the formation of triple ions. In the relatively high concentration region, the decrease of the molar conductivity may arise from the transient crosslinking effect of the salt.

(2) Ionic conduction of these polymer electrolytes is basically controlled by the segmental motion of the polymer and can be described by the VTF equation in the low and medium concentration region. In the relatively high concentration region an activated process is important for the ion transport, and the ionic conduction can be described by a modified VTF equation.

(3) ${ }^{7} \mathrm{Li}$ mas $\mathrm{NMR}$ of $\mathrm{PMEO}-\mathrm{LiClO}_{4}$ electrolytes shows three-line pattern spectra. The existence of "free ions", ion pairs and ion clusters allows these spectra to be interpreted.

(4) In conductivity and mas NMR measurement, it is found that ion association in PMEO-LiClO 4 electrolyte increases with increasing temperature and salt concentration. 
(5) The diffusion coefficient of the anion constituents measured by the pfg NMR experiment on PMEO-LiPF 6 electrolytes is almost an order of magnitude higher than that of the cation constituents. The results of the pfg NMR experiment support the theory that in low salt concentration region, transport of cationic species is controlled by segmental motion of the polymer, but is controlled by an activated process in the relatively high salt concentration region. The transport of anionic species, however, may be mainly controlled by the segmental motion of the polymer in the studied concentration range. 


\section{References}

[1] M. A. Ratner, in Polymer Electrolyte Reviews -1, ed. J. R. MacCallum and C. A. Vincent, Elsevier, London, 1987, page 173.

[2] Polymer Electrolyte Reviews 1 \& 2, ed. J. R. MacCallum and C. A. Vincent, Elsevier, London, 1987 \& 1989.

[3] P. G. Hall, G. R. Davies, I. M. Ward and J. E. McIntyre, Polymer Commun. 27, 100 (1986).

[4] H. Cheradame and J. F. LeNest, in Polymer Electrolyte Reviews -1, ed. J. R. MacCallum and C.A. Vincent, Elsevier, London, 1989, page 103.

[5] M. B. Armand, in Polymer Electrolyte Reviews -1, ed. J. R. MacCallum and C.A. Vincent, Elsevier, London, 1989, page 1.

[6] F. M Gray, Solid St. Ionics, 40 / 41, 637 (1990).

[7] R. M. Fuoss and C. A. Krause, J. Amer. Chem. Soc. 55, 2387 (1933).

[8] M. Delsignore, H. Farber and S. Petrucci, J. Phys. Chem. 89, 4968 (1985).

[9] G. G. Cameron, J. L. Harvie, M. D. Ingram and G. A. Sorrie, Br. Polym. J. 20, 199 (1988).

[10] Minitab Reference Manual, Minitab Inc., 1985. 
[11] K. J. Advamic, F. J. Owens, S. G. Greenbaum, M. C. Wintersgill and J. J. Fontanella, in Second International Symposium on Polymer Electrolytes, ed. B. Scrosati, Elsevier, London, 1990, page 61.

[12] I Albinsson, B. -E. Mellander and J. R. Stevens, J. Chem. Phys. 96, 681 (1992).

[13] H. Cheradame, in IPUAC Macromolecules, ed. H. Benoit and P. Rempp, Pergamon, New York, 1982, page 351.

[14] T. Miyamoto and K. Shibayama, J. Appl. Phys. 44, 5372 (1973).

[15] M. Kakihana, S. Schantz, B. -E Mellander and L. M. Torell, in Second International Symposium on Polymer Electrolytes, ed. B. Scrosati, Elsevier, London, 1990, page 23.

[16] L. D. Pettit and S. Bruckenstein, J. Amer. Chem. Soc. 88, 4783 (1966).

[17] C. A. Vincent, Prog. Solid St. Chem. 17, 145 (1987).

[18] D. Teeters and R. Frech, Solid St. Ionics, 18 / 19, 271(1986).

[19] P. G. Bruce, M. T. Hardgrave and C. A. Vincent, Electrochim. Acta, 37, 1517 (1992).

[20] M. C. Wintersgill, J. J. Fontanella, S. G. Greenbaum and K. J. Adamic, Br. Polym. J., 20. 195 (1988). 
[21] R. Spindler and D. F. Shriver, J. Amer. Chem. Soc., 110, 3036 (1988).

[22] J. F. O'Gara, G. Nazri and M. MacArthur, Solid St. Ionics, 47, 87 (1991).

[23] C. A. Fyfe, in Solid State NMR For Chemists, C. F. C Press, Guelph, 1983.

[24] J. R. MacCallum, A. S. Tomlin and C. A. Vincent, Eur. Polym. J. 22, 787 (1986).

[25] H. Eckert, Z. Zhang and J. H. Kennedy, Mat. Res. Soc. Symp. Proc., 135, 259 (1989).

[26] D. P. Tunstall, private communication.

[27] C. A. Vincent, in Electrochemical Science and Technology of Polymers 2, ed. R. Linford, Elsevier, London, 1990.

[28] M. Nicolas and R. Relch, J. Phys. Chem. 85, 2843 (1981).

[29] W. Y. Xu, J. Smid and M. VanBeylen, Solid St. Ionics, 57, 133 (1992).

[30] A. D. Bain, ABACUS Program-Description, Catalog No. ABA078.

[31] S. Bhattacharja, S. W. Smout and D. H. Whitemore, Solid St. Ionics, $18 / 19$, 306 (1986).

[32] W. Gorecki, P. Donoso, C. Berthier, M. Mali, J. Roos, D. Brinkmann and M.B. Armand, Solid St. Ionics, 28 / 30, 1018 (1988). 
[33] C. A. Vincent, in Polymer Electrolyte Reviews -1, ed. J. R. MacCallum and C. A. Vincent, Elsevier, London, 1987, page 23. 


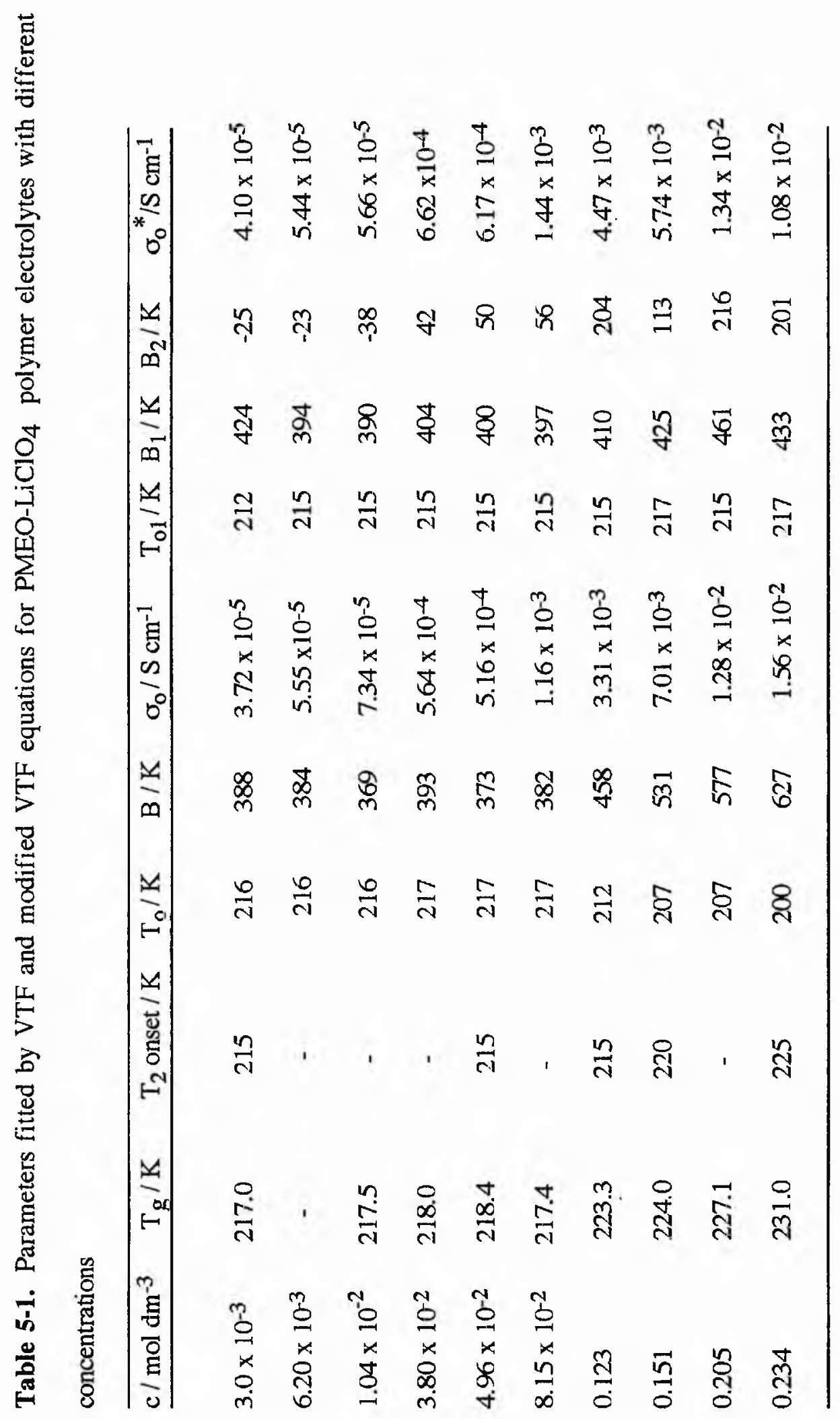


Table 5-2 Paramenters fitted by VTF and modified VTF equations for PMEO-LiSCN electrolytes

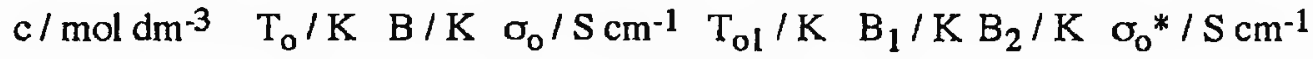
$\begin{array}{llllllll}2.36 \times 10^{-3} & 200 & 539 & 1.67 \times 10^{-5} & 200 & 549 & -66.3 & 1.51 \times 10^{-5}\end{array}$

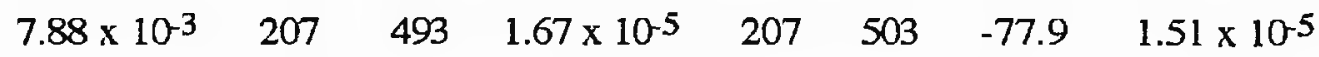
$\begin{array}{llllllll}1.36 \times 10^{-2} & 190 & 695 & 4.68 \times 10^{-5} & 210 & 397 & 834 & 1.12 \times 10^{-4}\end{array}$

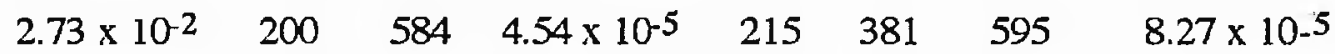

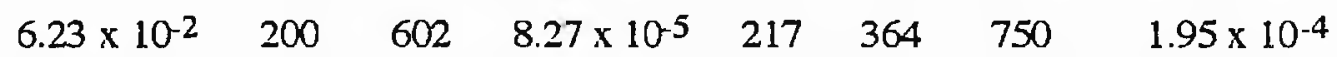

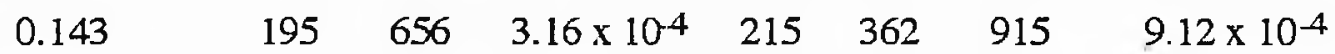

$\begin{array}{llllllll}0.195 & 205 & 603 & 5.16 \times 10^{-4} & 215 & 439 & 582 & 1.09 \times 10^{-3}\end{array}$

Table 5-3a Parameters fitted by VTF equation for PMEO-LiPF 6 electrolytes

\begin{tabular}{lllll}
\hline EO/Li & $20: 1$ & $30: 1$ & $50: 1$ & $100: 1$ \\
$\mathrm{~T}_{\mathrm{o}} / \mathrm{K}$ & 201 & 199 & 200 & 215 \\
$\mathrm{~B} / \mathrm{K}$ & 886 & 801 & 714 & 457 \\
$\mathrm{\sigma}_{\mathrm{o}} / \mathrm{S} \mathrm{cm} \mathrm{cm}^{-1}$ & $4.41 \times 10^{-2}$ & $1.14 \times 10^{-2}$ & $1.05 \times 10^{-2}$ & $1.26 \times 10^{-3}$ \\
\hline
\end{tabular}


Table 5-3b Data for de polarisation of $\mathrm{Li} / \mathrm{PMEO}^{\mathrm{L}} \mathrm{LiPF}_{6} / \mathrm{Li}$ cell

\begin{tabular}{lccccccc}
\hline EO/Li & $\mathrm{T} /{ }^{\circ} \mathrm{C}$ & $\Delta \mathrm{V}_{\mathrm{a}} / \mathrm{mV}$ & $\mathrm{R}_{\mathrm{io}} / \mathrm{k} \Omega$ & $\mathrm{R}_{\mathrm{is}} / \mathrm{k} \Omega$ & $\mathrm{I}_{\mathrm{o}} / \mu \mathrm{A}$ & $\mathrm{I}_{\mathrm{s}} / \mu \mathrm{A}$ & $\mathrm{F}_{+}$ \\
\hline $20: 1$ & 80 & 7.07 & 0.12 & 0.18 & 24.00 & 4.15 & 0.13 \\
$20: 1$ & 60 & 5.31 & 0.82 & 0.78 & 5.72 & 2.16 & 0.19 \\
$30: 1$ & 80 & 6.88 & 0.13 & 0.09 & 24.8 & 4.55 & 0.13 \\
$30: 1$ & 60 & 5.78 & 0.48 & 0.50 & 8.79 & 1.94 & 0.14 \\
$50: 1$ & 80 & 6.09 & 0.22 & 0.31 & 17.78 & 3.09 & 0.12 \\
$50: 1$ & 60 & 2.89 & 1.83 & 1.93 & 3.94 & 1.17 & 0.11 \\
$100: 1$ & 80 & 2.88 & 1.23 & 0.91 & 5.79 & 1.53 & 0.09 \\
\hline
\end{tabular}

$\Delta \mathrm{V}_{\mathrm{a}}$ is corrected by the interfacial drop.

Table 5-4 Linewidth of ${ }^{7} \mathrm{Li}$ mas NMR spectrum with different salt concentration at $298 \mathrm{~K}$

\begin{tabular}{lllllll}
\hline $\mathrm{c} / \mathrm{dm}^{-3}$ & 0.1 & 0.2 & 0.5 & 1.0 & 2.0 & 4.0 \\
linewidth $/ \mathrm{Hz}$ & $114^{*}$ & 30 & 27 & 30 & 71 & 143 \\
\hline
\end{tabular}

* For $0.1 \mathrm{~mol} \mathrm{dm}^{-3}$ sample, the linewidth of the line with highest chemical shift is estimated to be $572 \mathrm{~Hz}$. 
Table 5-5. Data obtained by deconvolution of ${ }^{7} \mathrm{Li}$ mas NMR spectrum with different salt concentrations at $298 \mathrm{~K}$

\begin{tabular}{lccccccc}
\hline $\mathrm{c} / \mathrm{mol} \mathrm{dm}^{-3}$ & \multicolumn{3}{c}{ linewidth $/ \mathrm{Hz}$} & \multicolumn{3}{c}{ ratio/\% } & RMS/\% \\
& A & B & C & A & B & C & \\
\hline 0.10 & 819 & 100 & - & 70.9 & 29.1 & - & 11.33 \\
0.20 & 130 & 27 & 26 & 43.2 & 54.3 & 2.5 & 5.47 \\
0.50 & 134 & 31 & 35 & 35.8 & 60.8 & 3.3 & 5.91 \\
1.0 & 126 & 31 & 53 & 32.9 & 55.6 & 11.4 & 6.38 \\
2.0 & 101 & 66 & 128 & 7.7 & 76.1 & 16.2 & 4.67 \\
\hline
\end{tabular}

A, B and C represent lines corresponding to "free ions", ion pairs and ion clusters respectively in spectra.

Table 5-6 Data obtained by deconvolution of ${ }^{7} \mathrm{Li}$ mas NMR spectrum with different temperatures for the PMEO-LiClO4 electrolyte with salt concentration of $0.5 \mathrm{~mol} \mathrm{dm}^{-3}$

\begin{tabular}{lccccccc}
\hline T/K & \multicolumn{3}{c}{ width $/ \mathrm{Hz}$} & \multicolumn{3}{c}{ ratio \% } & RMS\% \\
& A & B & C & A & B & C & \\
\hline 298 & 151 & 26 & 26 & 35.8 & 60.8 & 3.3 & 5.91 \\
333 & 98 & 21 & 10 & 21.4 & 76.3 & 2.3 & 6.02 \\
363 & 57 & 21 & 14 & 2.9 & 93.6 & 3.4 & 5.04 \\
\hline
\end{tabular}

Table 5-7. Experimental and calculated conductivities of $\mathrm{PMEO}^{-\mathrm{LiPF}_{6}}$ electrolytes with $\mathrm{EO} / \mathrm{Li}$ ratios of $50: 1$ and $20: 1$ at different temperatures

\begin{tabular}{ccccc}
\hline $\mathrm{T} /{ }^{\circ} \mathrm{C}$ & $\sigma_{\mathrm{exp}} / \mathrm{S} \mathrm{cm}^{-1}$ & $\sigma_{\mathrm{cal}} / \mathrm{S} \mathrm{cm}^{-1}$ & $\sigma_{\text {exp }} / \mathrm{S} \mathrm{cm}^{-1}$ & $\sigma_{\mathrm{cal}} / \mathrm{S} \mathrm{cm}^{-1}$ \\
$50: 1$ & $50: 1$ & $20: 1$ & $20: 1$ \\
\hline 40 & $1.88 \times 10^{-5}$ & $2.48 \times 10^{-4}$ & $1.40 \times 10^{-5}$ & $1.92 \times 10^{-4}$ \\
60 & $4.89 \times 10^{-5}$ & $3.28 \times 10^{-4}$ & $4.90 \times 10^{-5}$ & $3.53 \times 10^{-4}$ \\
80 & $1.04 \times 10^{-4}$ & $4.11 \times 10^{-4}$ & $1.33 \times 10^{-4}$ & $5.08 \times 10^{-4}$ \\
\hline
\end{tabular}




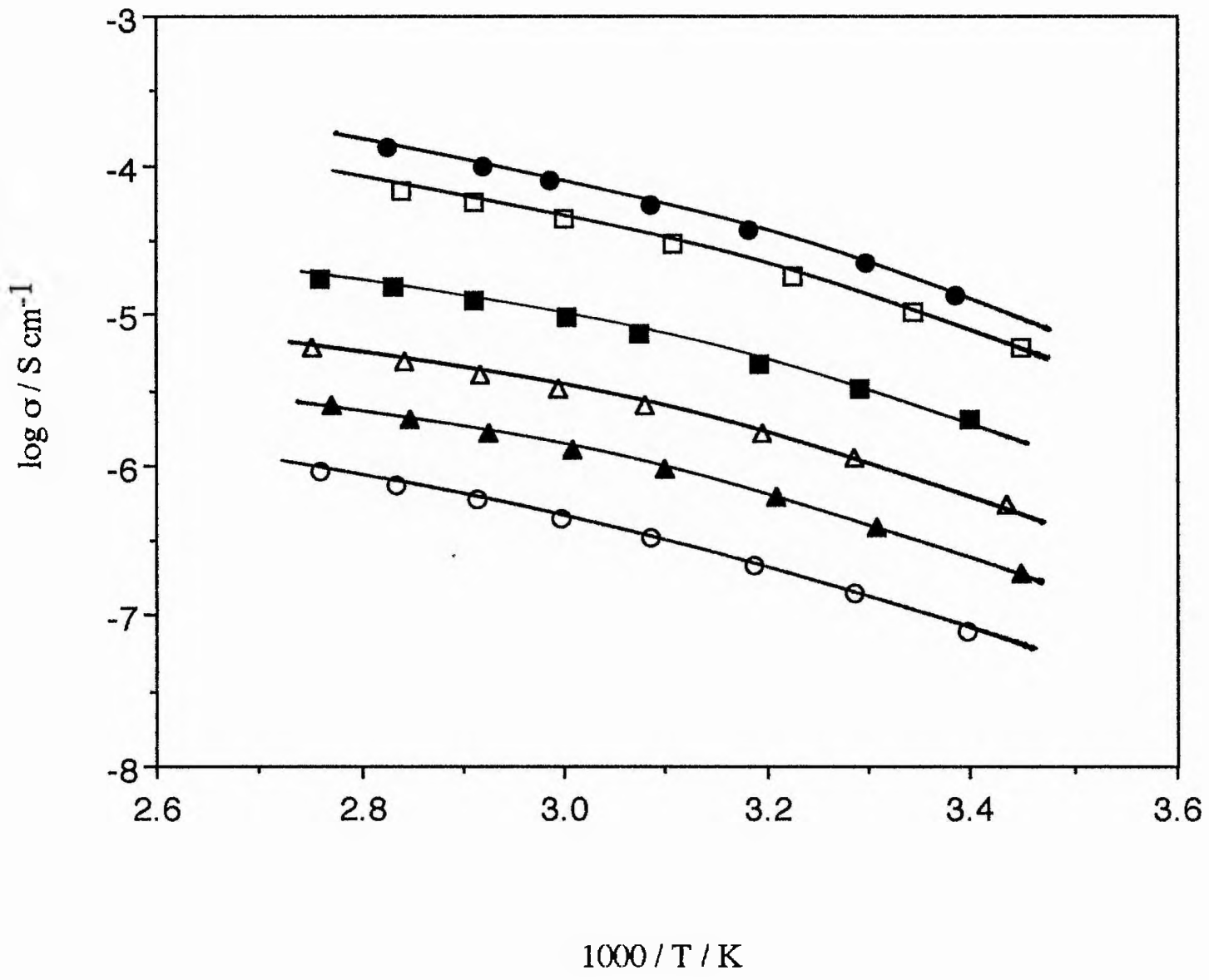

Figure 5-1 Dependence of conductivity on reciprocal absolute temperature for PMEO$\mathrm{LiClO}_{4}$ electrolytes with concentrations: (O) $8.0 \times 10^{-4} \mathrm{~mol} \mathrm{dm}^{-3} ;$ (A) $3.0 \times 10^{-3} \mathrm{~mol}$ $\mathrm{dm}^{-3} ;(\Delta) 1.04 \times 10^{-2} \mathrm{~mol} \mathrm{dm}^{-3} ;(\square) 4.96 \times 10^{-2} \mathrm{~mol} \mathrm{dm}-3$; (口) $8.15 \times 10^{-2} \mathrm{moldm}^{-3}$; (•) $0.123 \mathrm{~mol} \mathrm{dm}^{-3}$. 


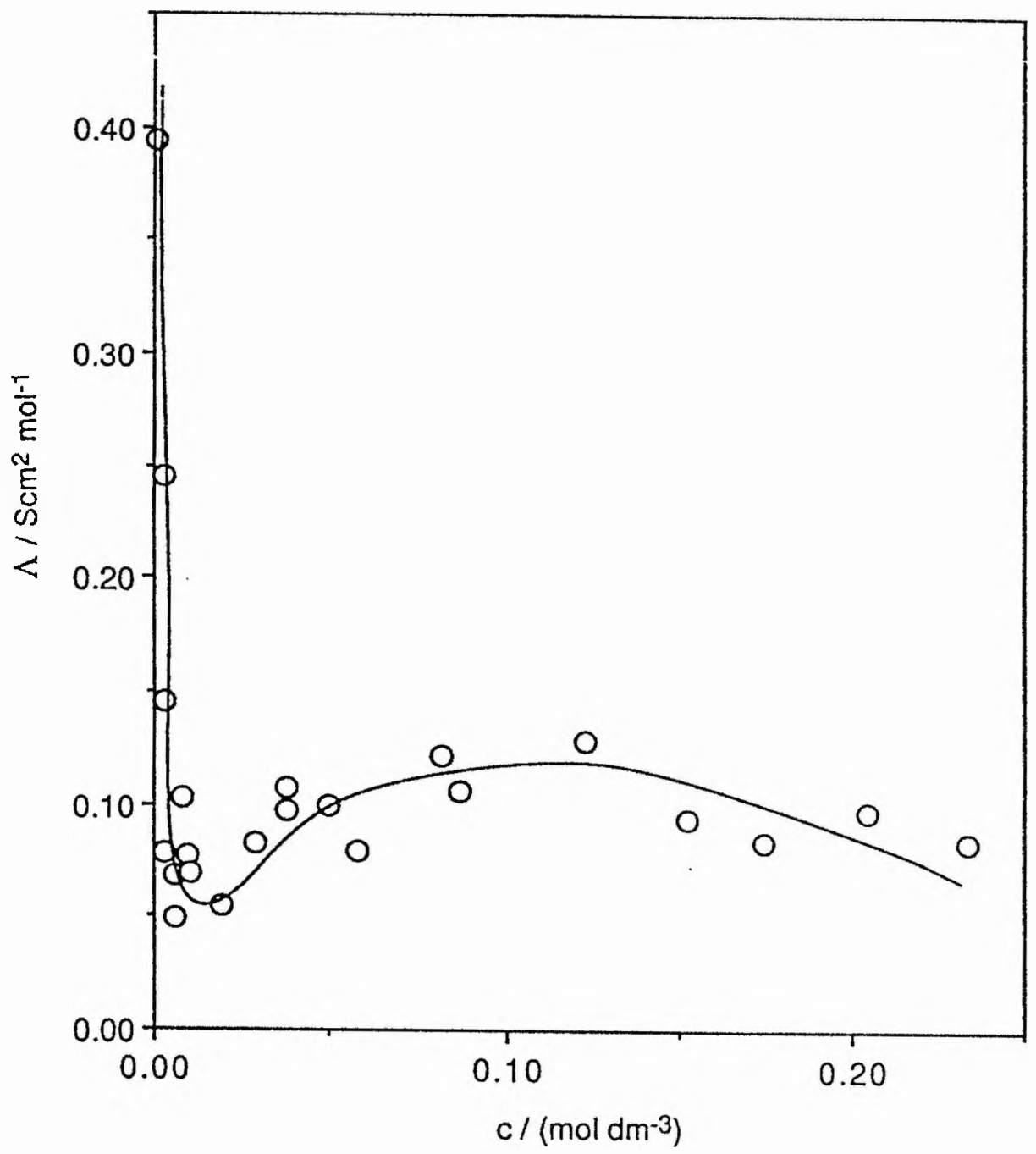

Figure 5-2 Molar conductivity as a function of salt concentration for PMEO-LiClO electrolytes at $25^{\circ} \mathrm{C}$. 


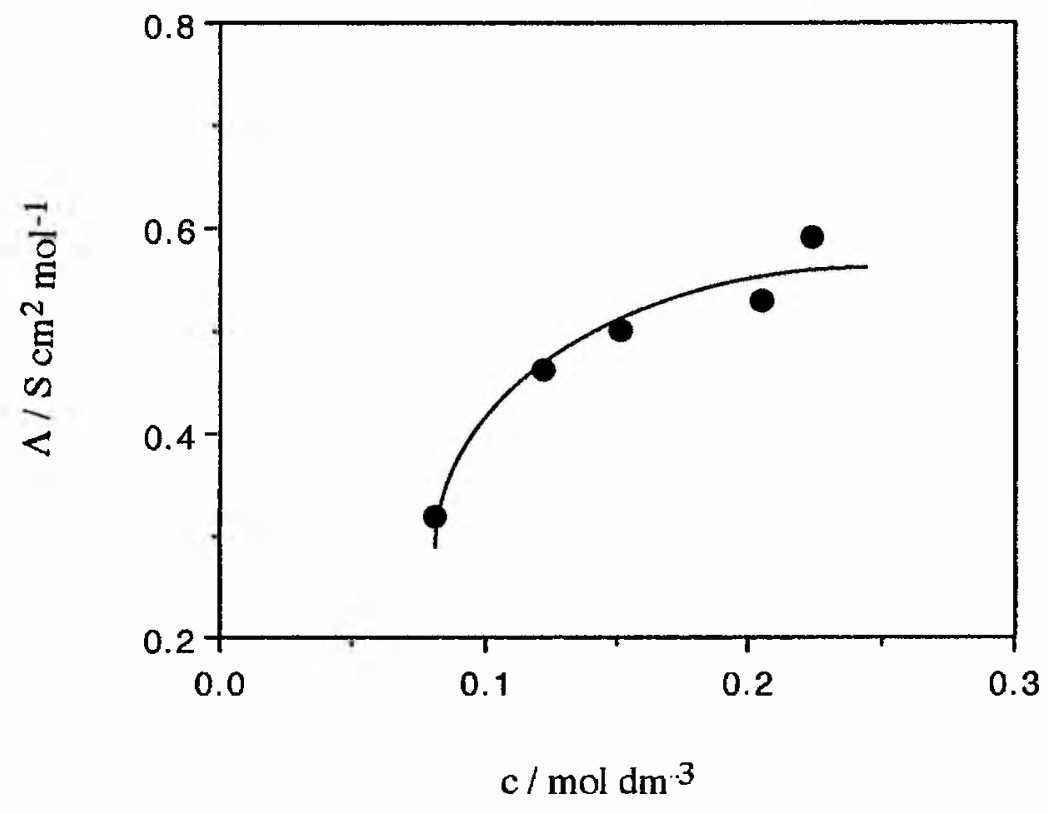

Figure 5-3 Variation in molar conductivity with salt concentration at a reduced temperature $\mathrm{T}=\mathrm{T}_{\mathrm{g}}+100 \mathrm{~K}$ for $\mathrm{PMEO}-\mathrm{LiClO}_{4}$ electrolytes.

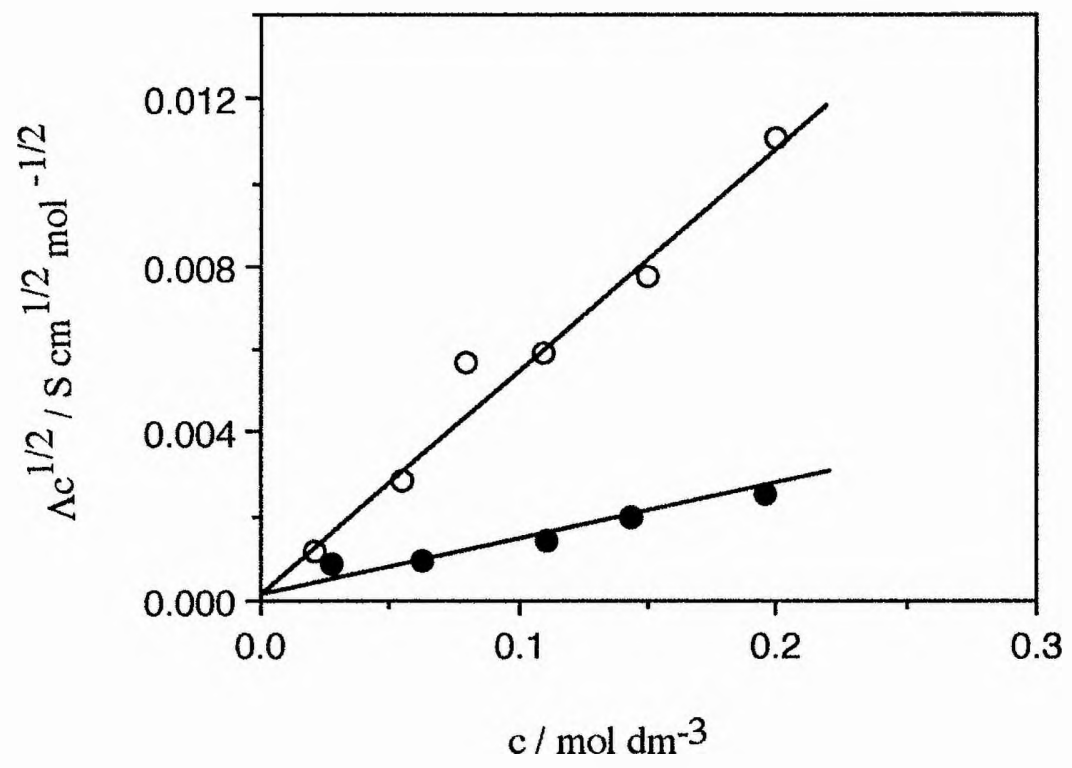

Figure 5-4 $\Lambda \mathrm{c}^{1 / 2}$ versus concentration $\mathrm{c}$ of $\mathrm{PMEO}-\mathrm{LiClO}_{4}(\mathrm{O})$ and PMEO-LiSCN (๑) electrolytes at $25^{\circ} \mathrm{C}$. 


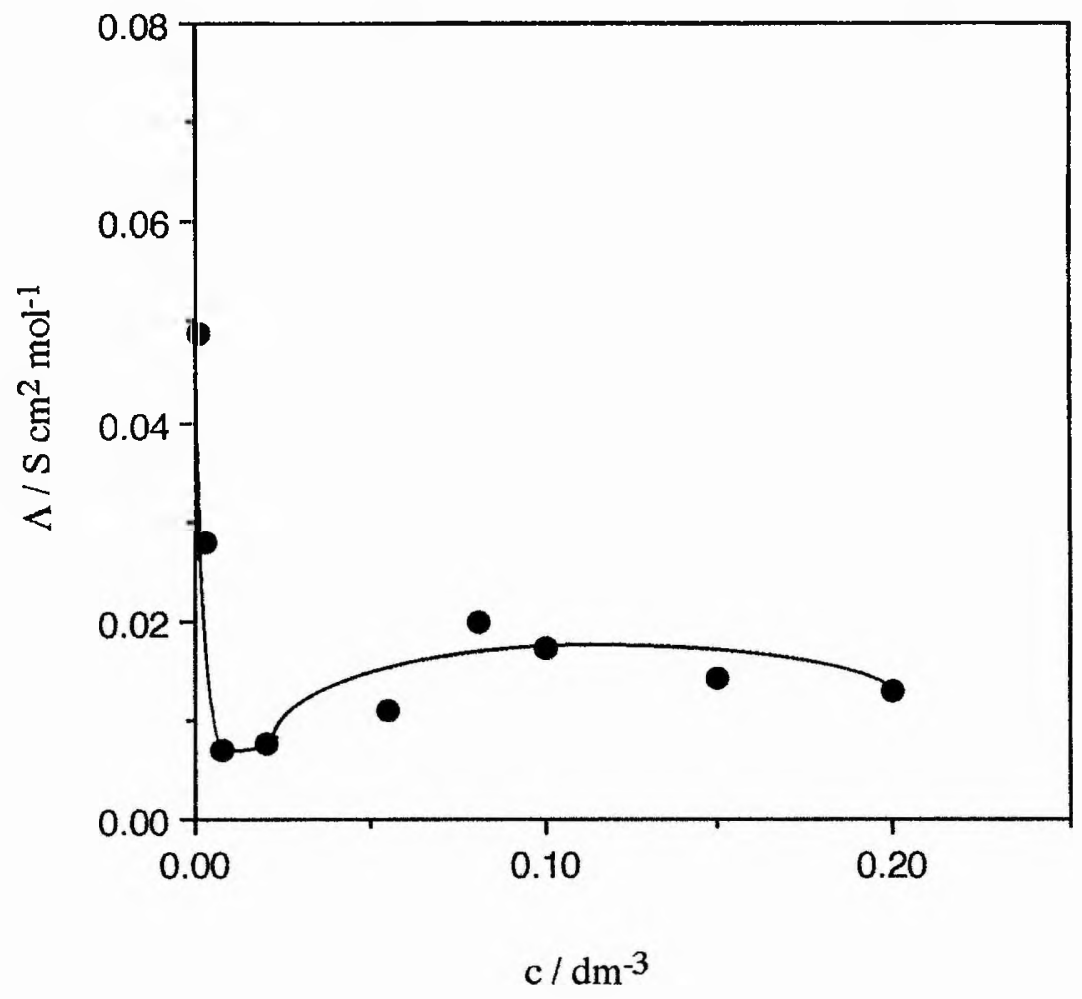

Figure 5-5 Molar conductivity $\Lambda$ versus concentration c of $\mathrm{PMEO}-\mathrm{LiCF}_{3} \mathrm{SO}_{3}$ electrolytes at $25^{\circ} \mathrm{C}$. 


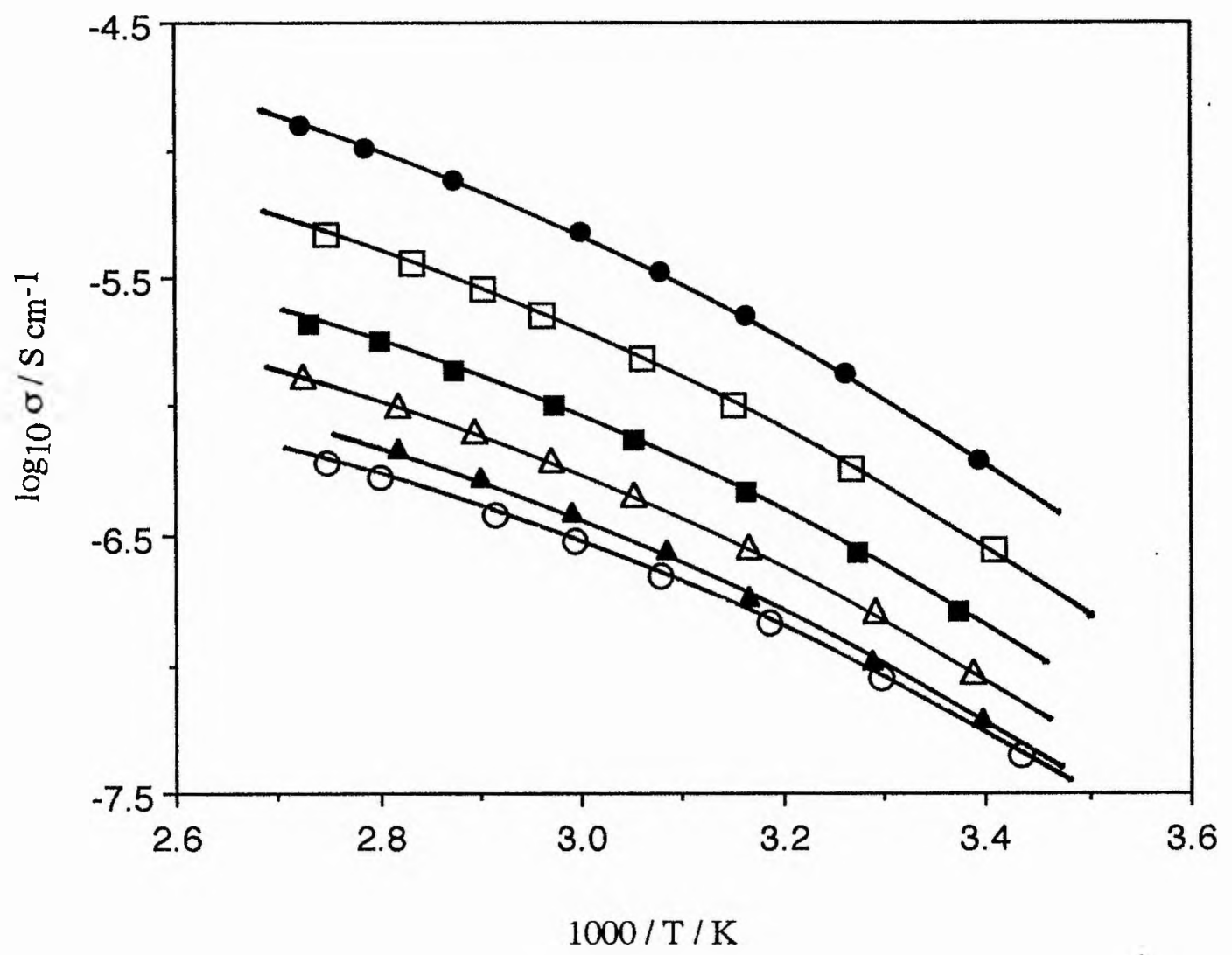

Figure 5-6 Typical temperature dependence of conductivity of PMEO-LiSCN electrolytes: (O) $0.002 \mathrm{~mol} \mathrm{dm}^{-3},(\mathbf{\Delta}) 0.013 \mathrm{~mol} \mathrm{dm}^{-3},(\Delta) 0.027 \mathrm{~mol} \mathrm{dm}^{-3}$, (口) $0.062 \mathrm{~mol} \mathrm{dm}^{-3}$, (ㅁ) $0.112 \mathrm{~mol} \mathrm{dm}^{-3}$, (•) $0.191 \mathrm{~mol} \mathrm{dm}^{-3}$. 


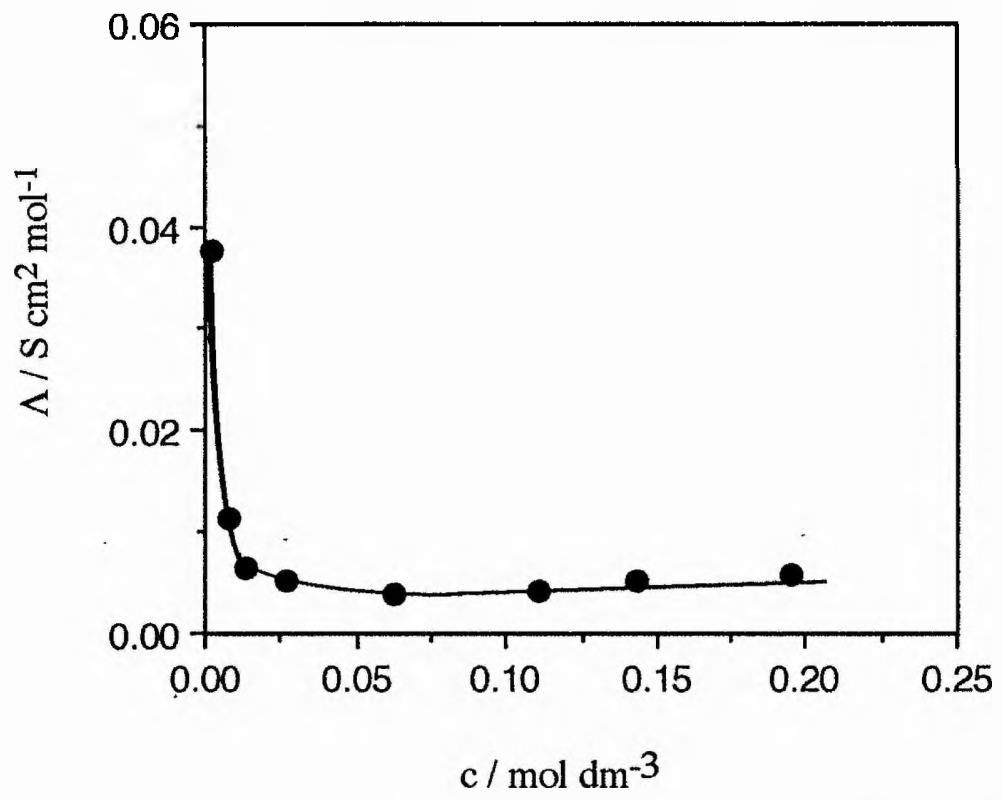

Figure 5-7 Molar conductivity $\Lambda$ versus concentration $\mathrm{c}$ of PMEO-LiSCN electrolytes at $25^{\circ} \mathrm{C}$.

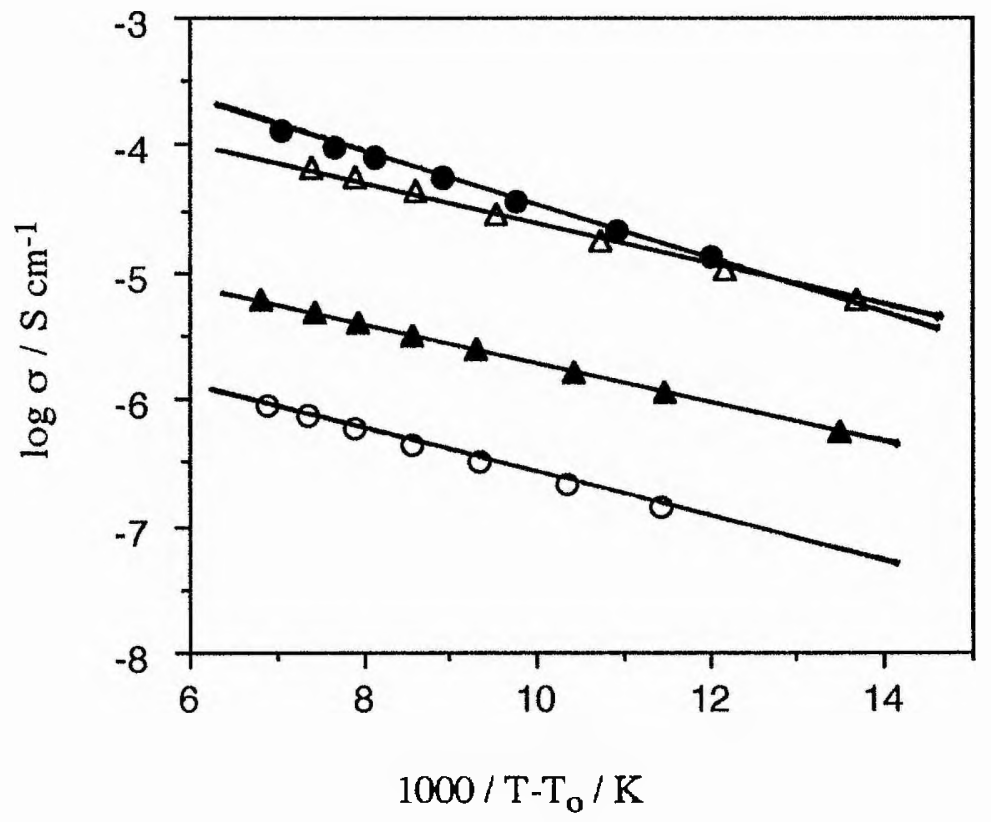

Figure 5-8 Typical plots of log $\sigma$ against $1000 / \mathrm{T}-\mathrm{T}_{\mathrm{o}}$ for $\mathrm{PMEO}-\mathrm{LiClO}_{4}$ electrolytes:

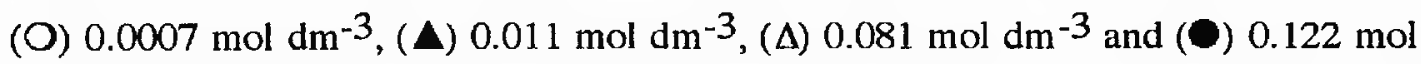
$\mathrm{dm}^{-3}$. 


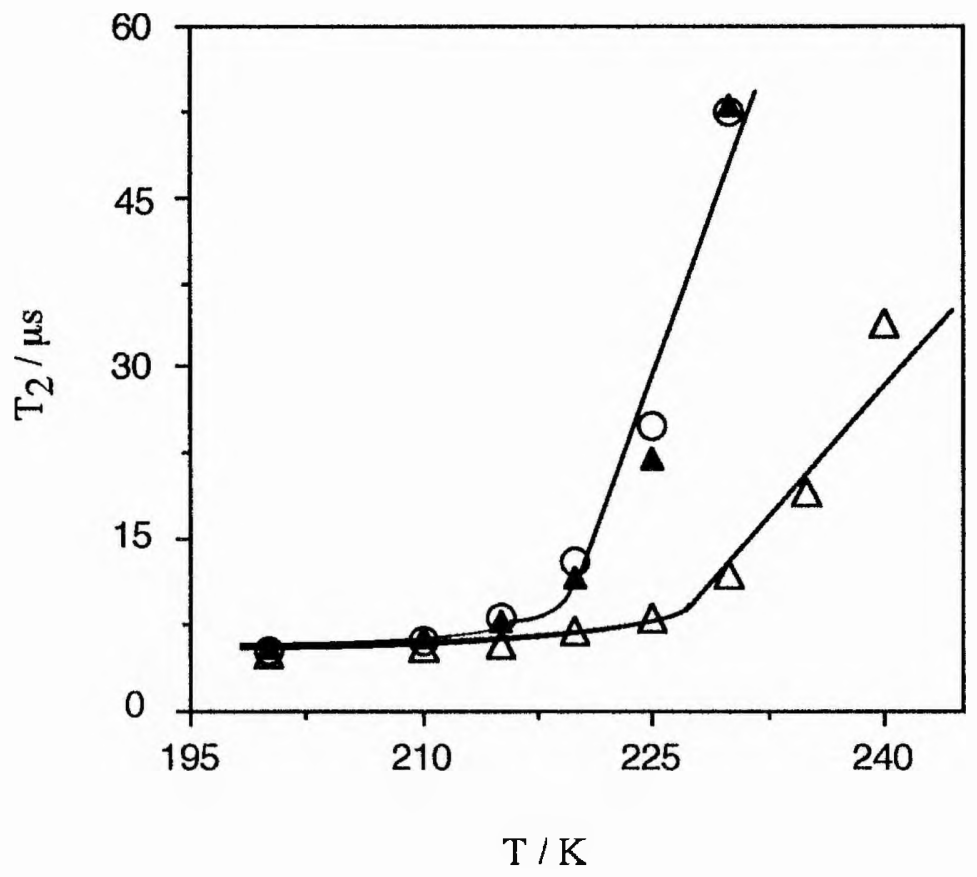

Figure 5-9 Typical plots of temperature aganist the spin-spin relaxation time $\mathrm{T}_{2}$ for PMEO-LiClO 4 electrolytes: (O) pure PMEO, ( $\Delta$ ) $0.050 \mathrm{~mol} \mathrm{dm}^{-3}$ and ( $\Delta$ ) $0.234 \mathrm{~mol}$ $\mathrm{dm}^{-3}$. 


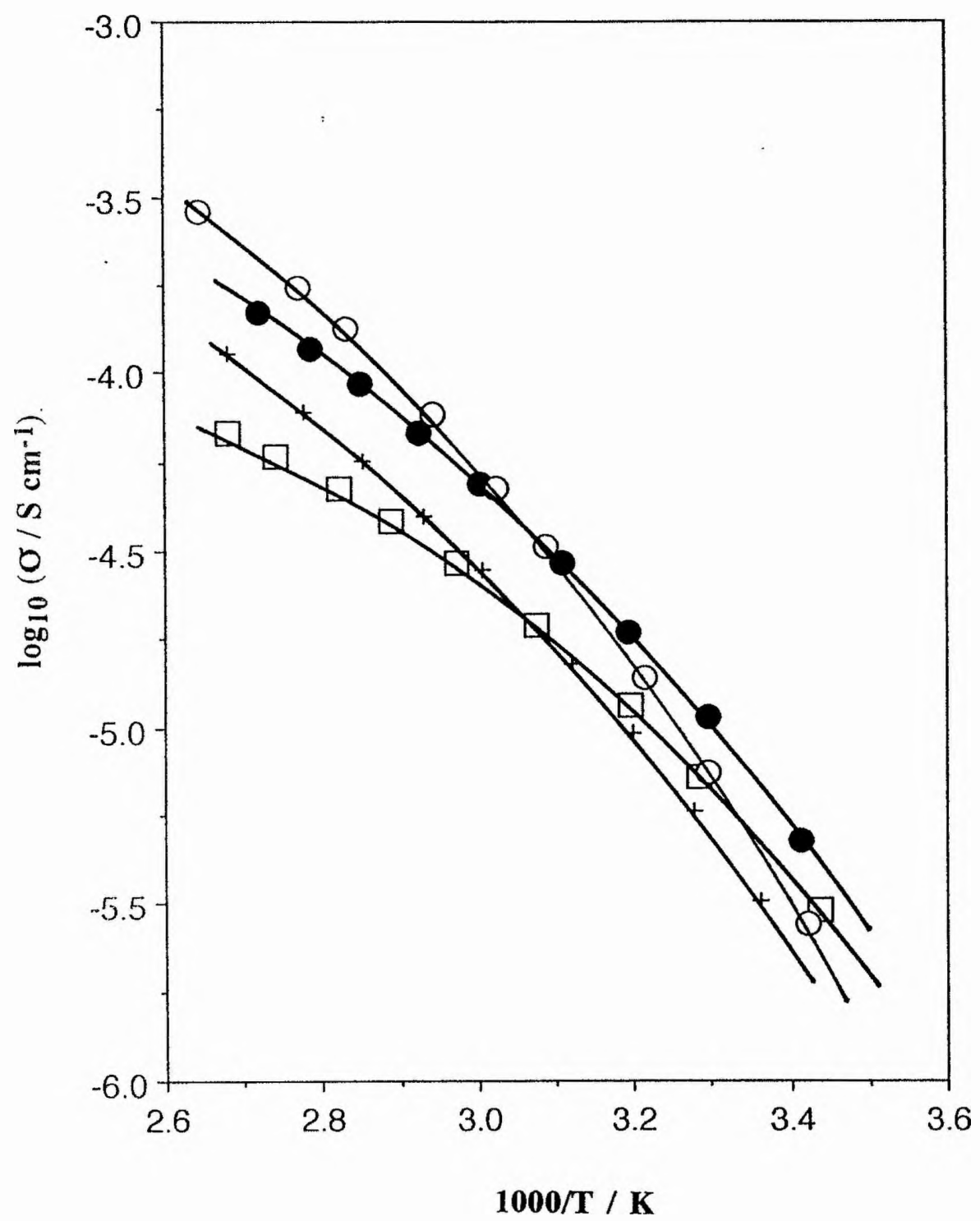

Figure 5-10 Dependence of conductivity on reciprocal absolute temperature for PMEO-LiPF 6 electrolytes with EO/Li ratios of 100:1 (口), 50:1 (•), 30:1 (+) and 20:1 (O) 


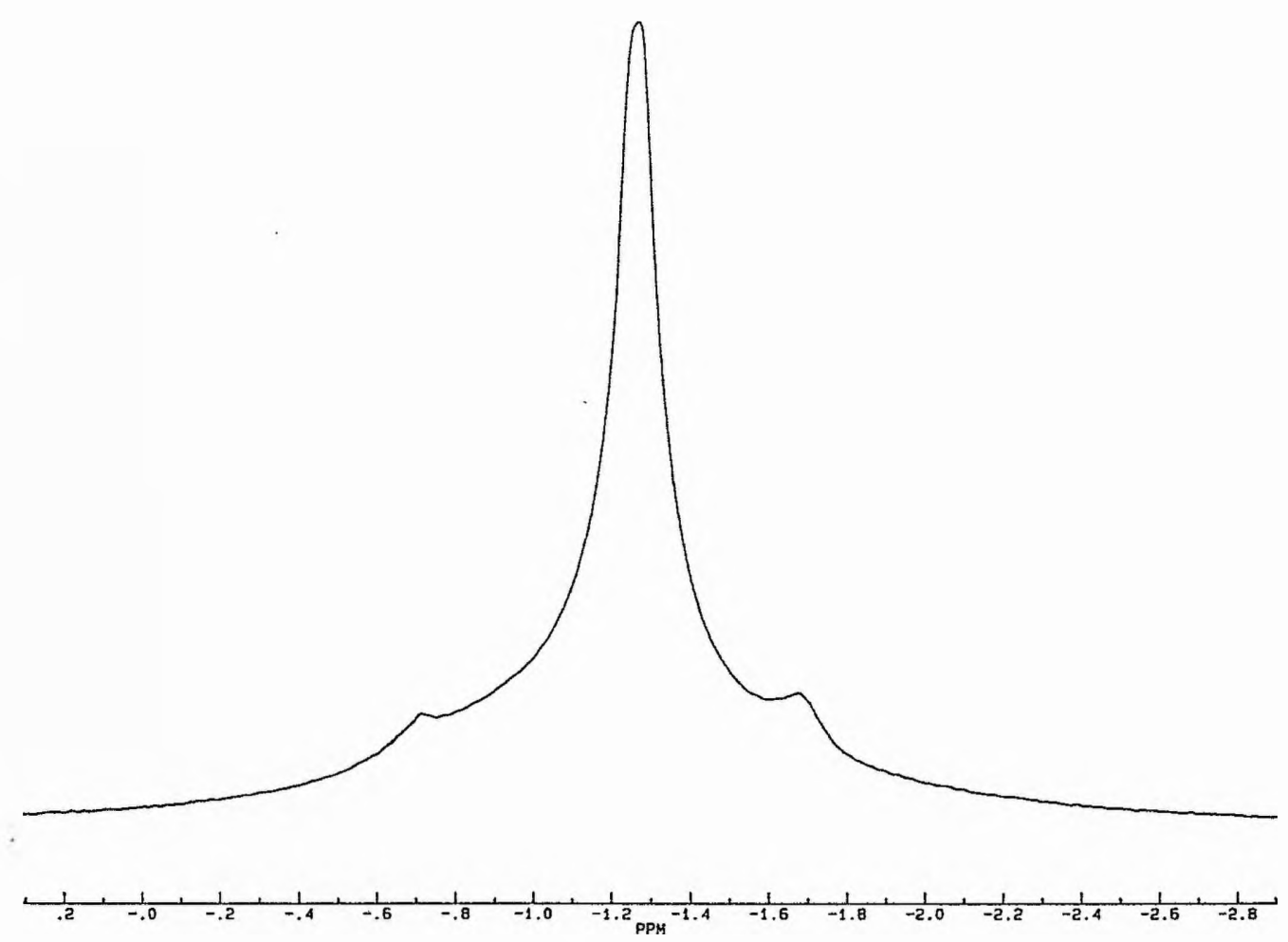

Figure 5-11a ${ }^{7} \mathrm{Li}$ mas NMR spectrum for $\mathrm{PMEO}-\mathrm{LiClO}_{4}$ electrolyte with salt concentration of $1.0 \mathrm{~mol} \mathrm{dm}^{-3}$ and spinning rate of $5 \mathrm{kHz}$ at $25^{\circ} \mathrm{C}$. 


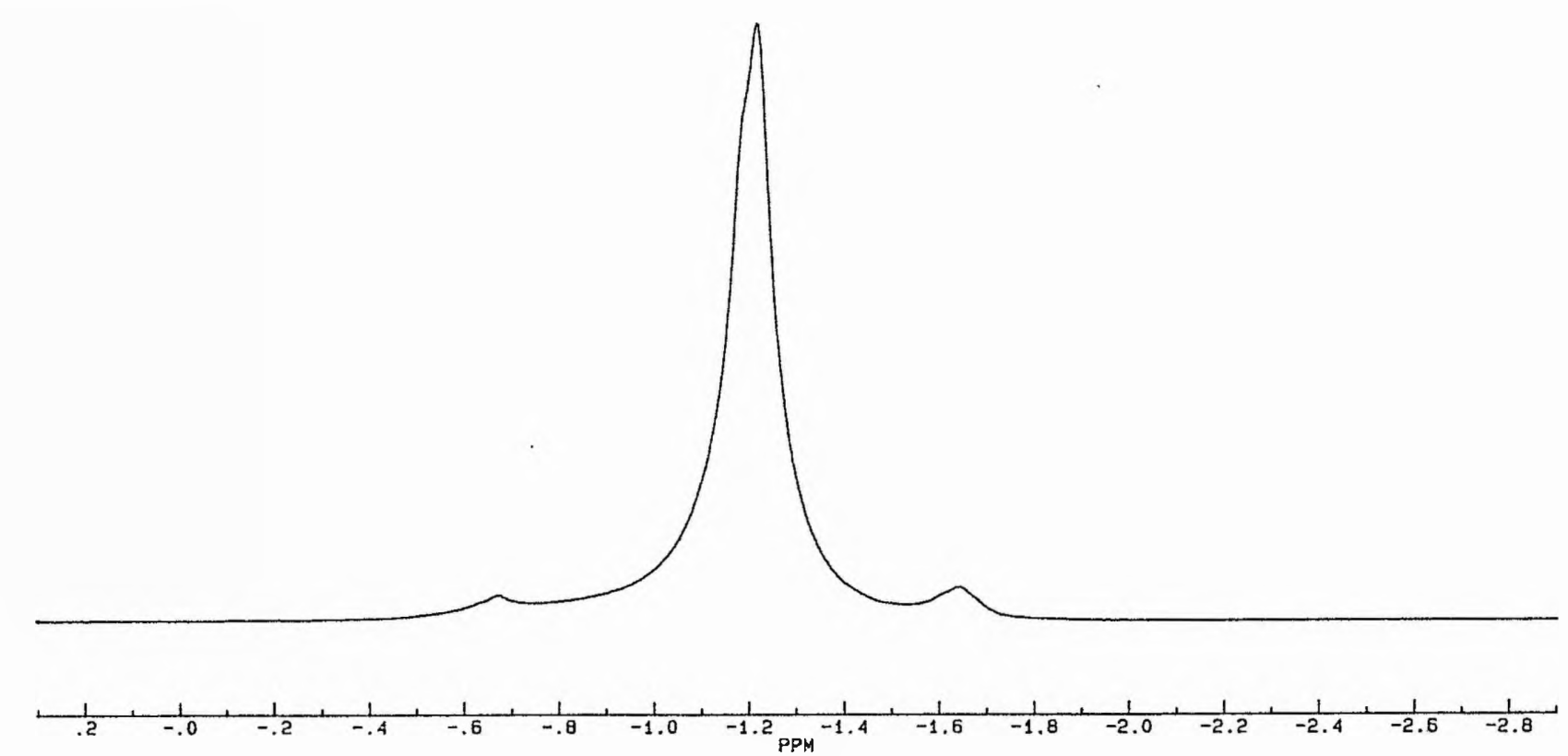

Figure 5-11b ${ }^{7} \mathrm{Li}$ mas NMR spectrum for $\mathrm{PMEO}-\mathrm{LiClO}_{4}$ electrolyte with salt concentration of $1.0 \mathrm{~mol} \mathrm{dm}^{-3}$ and spinning rate of $5 \mathrm{kHz}$ at $90^{\circ} \mathrm{C}$. 


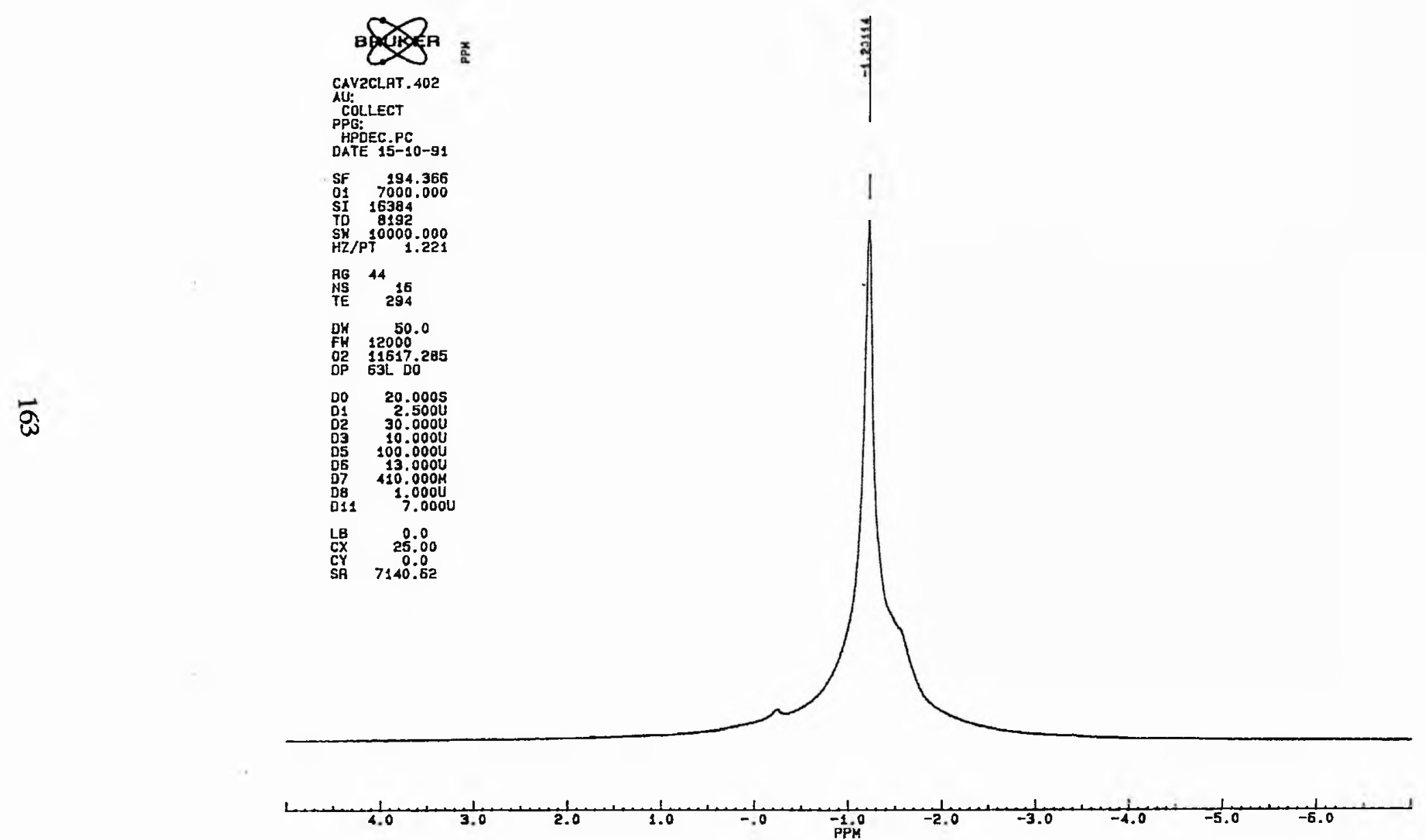

Figure 5-12 ${ }^{7} \mathrm{Li}$ mas NMR spectrum for $\mathrm{PEO}-\mathrm{LiClO}_{4}$ electrolyte with $\mathrm{EO} / \mathrm{Li}=20: 1$ and spinning rate of $5 \mathrm{kHz}$ at $80^{\circ} \mathrm{C}$. 


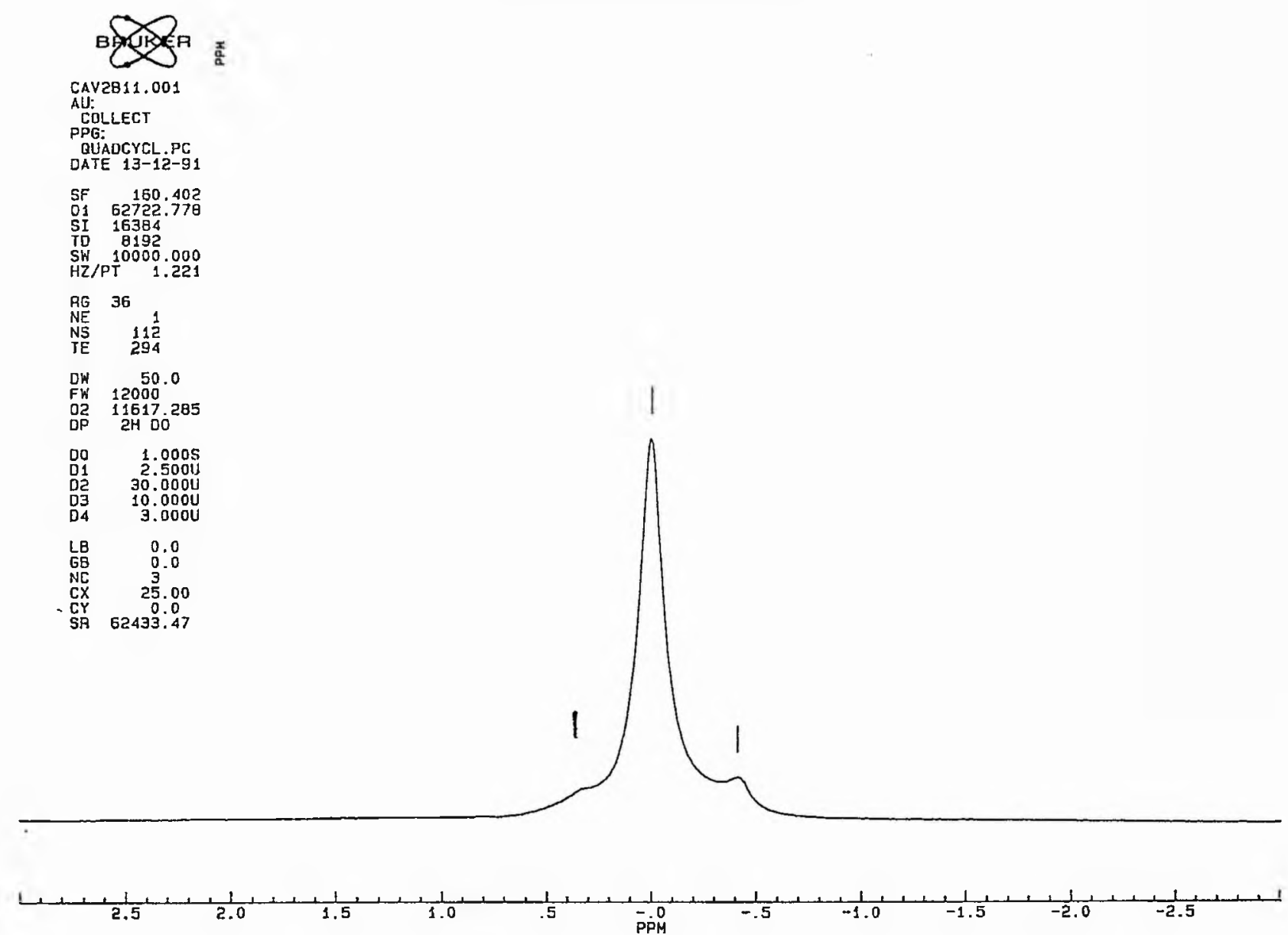

Figure 5-13 ${ }^{11} \mathrm{~B}$ mas NMR spectrum for $\mathrm{PMEO}-\mathrm{LiBF}_{4}$ electrolyte with salt concentration of $1.0 \mathrm{~mol} \mathrm{dm}^{-3}$ and spinning rate of $5 \mathrm{kHz}$ at $25^{\circ} \mathrm{C}$. 


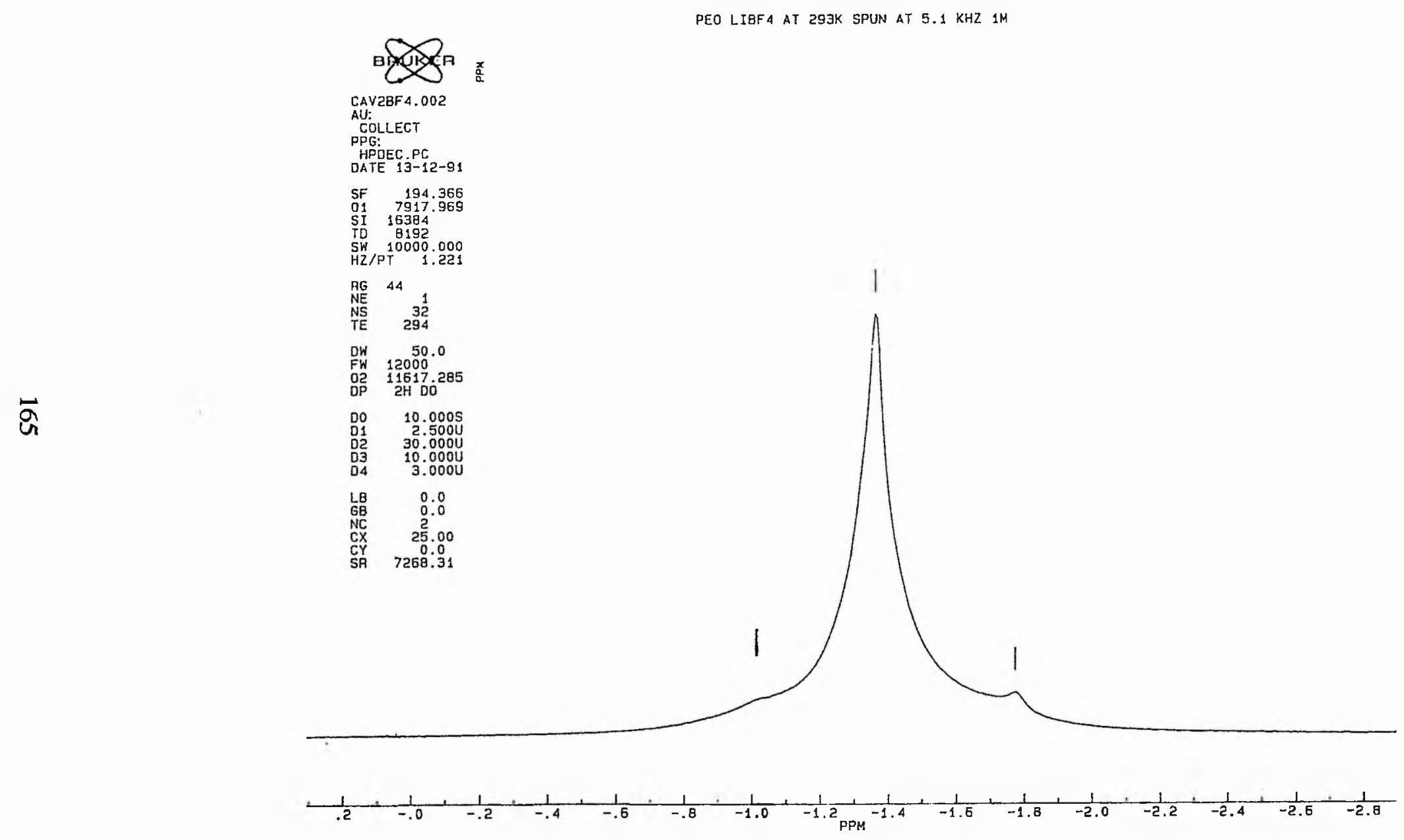

Figure 5-14a ${ }^{7} \mathrm{Li}$ mas NMR spectrum for $\mathrm{PMEO}-\mathrm{LiBF}_{4}$ electrolyte with salt concentration of $1.0 \mathrm{~mol} \mathrm{dm}^{-3}$ and spinning rate of $5 \mathrm{kHz}$ at $25^{\circ} \mathrm{C}$. 


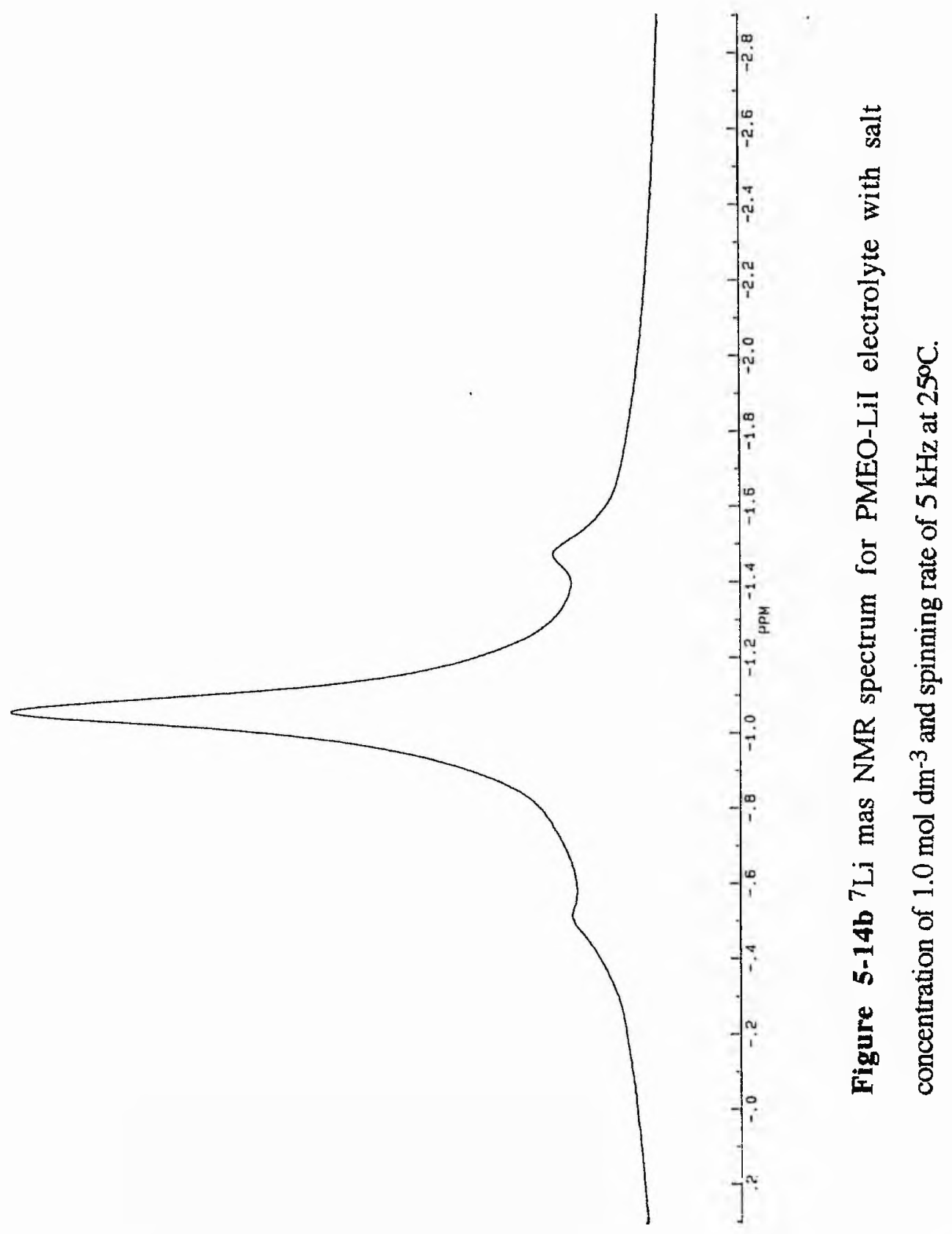




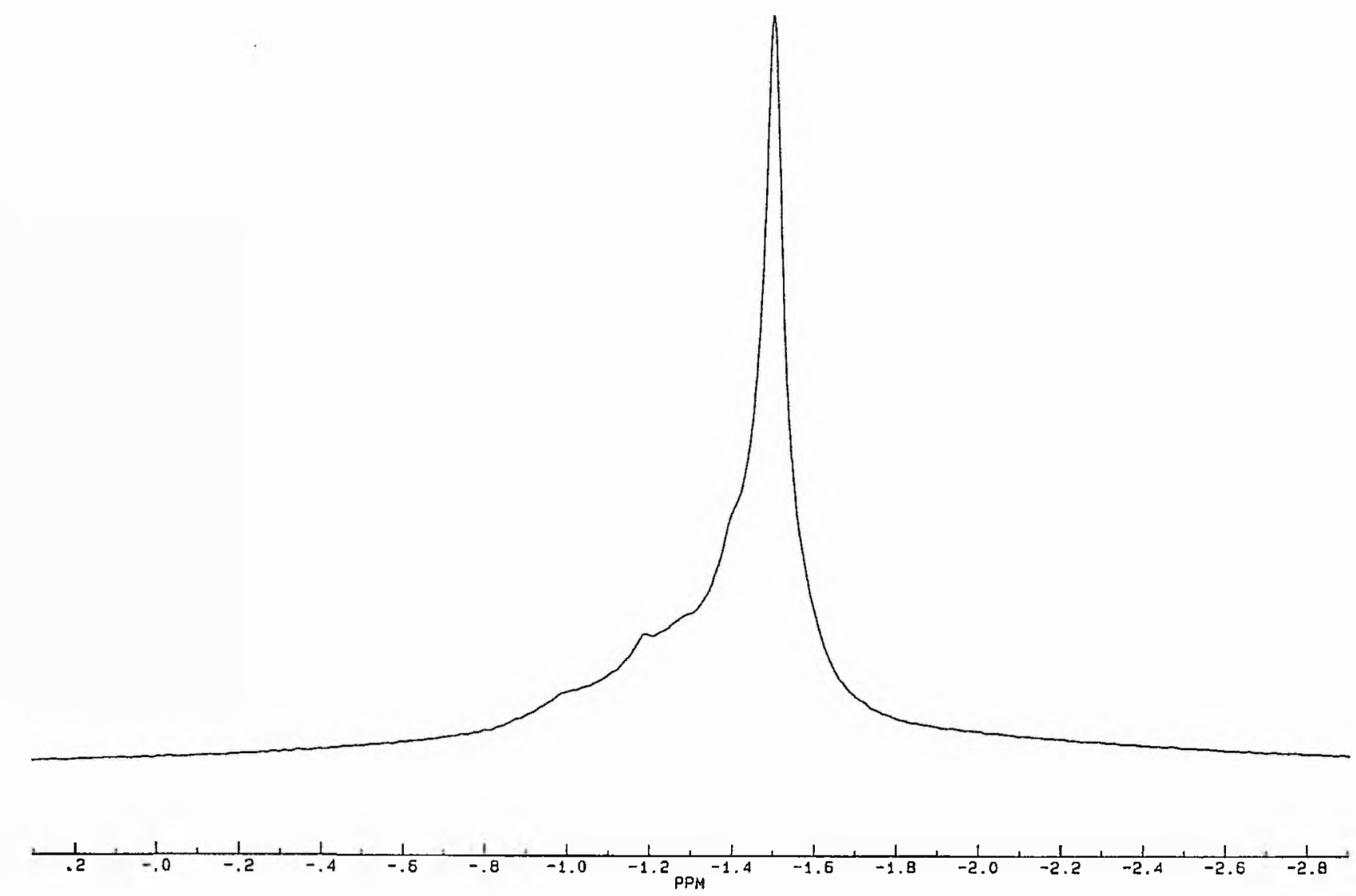

Figure $5-14 c^{7} \mathrm{Li}$ mas NMR spectrum for PMEO-LiSCN electrolyte with salt concentration of $1.0 \mathrm{~mol} \mathrm{dm}-3$ and spinning rate of $5 \mathrm{kHz}$ at $25^{\circ} \mathrm{C}$. 


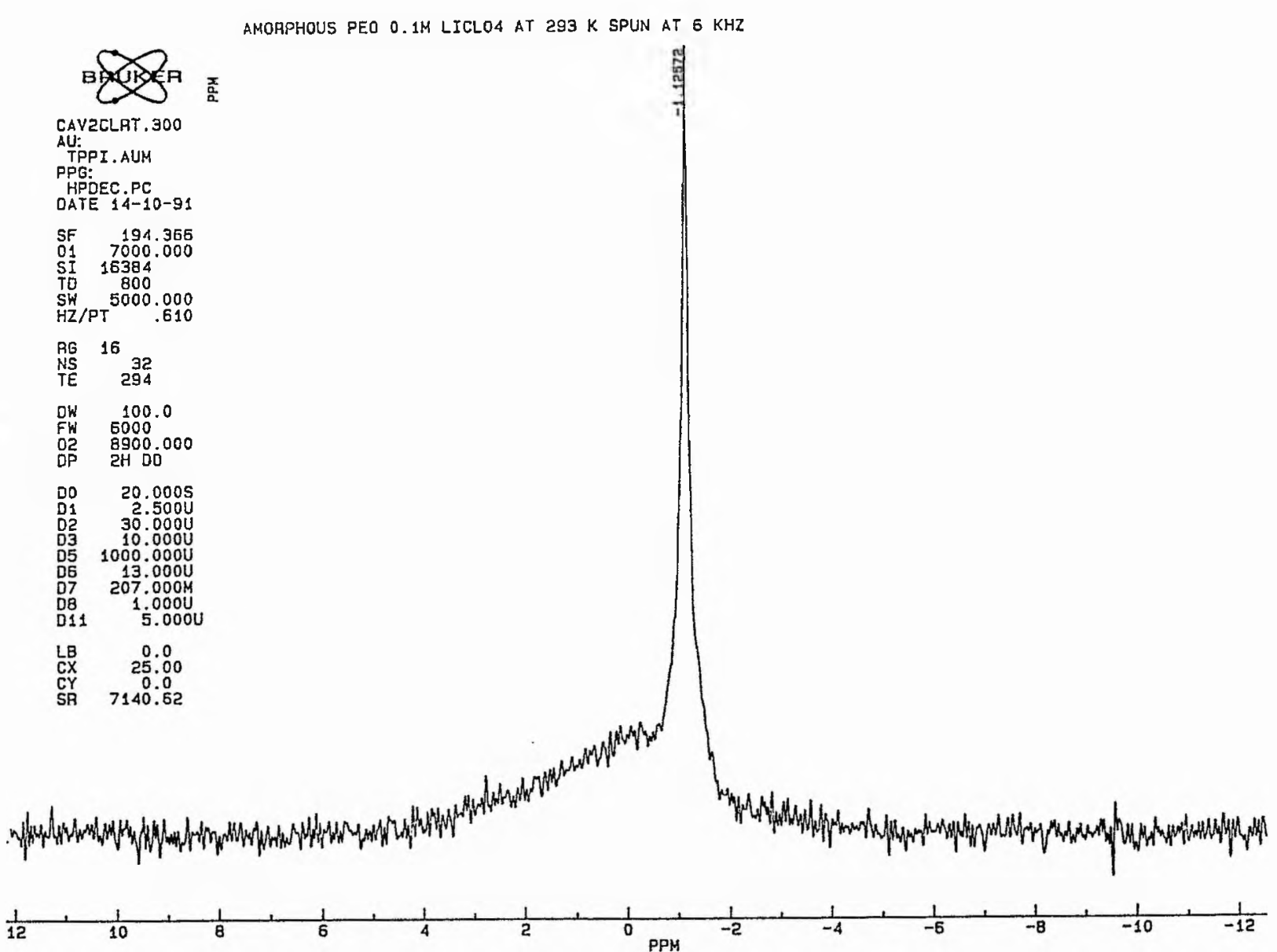

Figure 5-15a ${ }^{7} \mathrm{Li}$ mas NMR spectrum for $\mathrm{PMEO}-\mathrm{LiClO}_{4}$ electrolyte with salt concentration of $0.1 \mathrm{~mol} \mathrm{dm}^{-3}$ and spinning rate of $5 \mathrm{kHz}$ at $25^{\circ} \mathrm{C}$. 


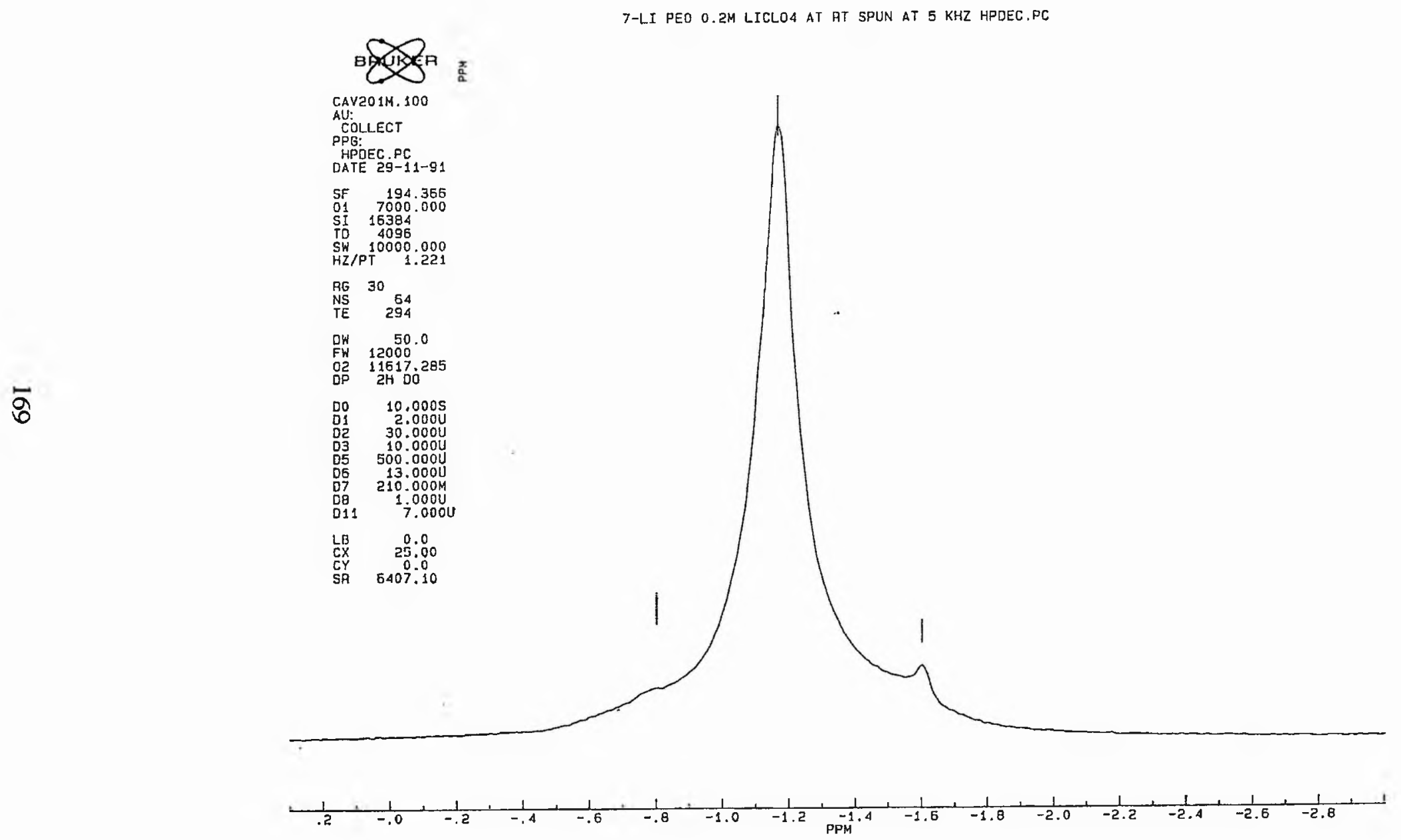

Figure 5-15b ${ }^{7} \mathrm{Li}$ mas NMR spectrum for $\mathrm{PMEO}^{-\mathrm{LiClO}_{4}}$ electrolyte with salt concentration of $0.2 \mathrm{~mol} \mathrm{dm}^{-3}$ and spinning rate of $5 \mathrm{kHz}$ at $25^{\circ} \mathrm{C}$. 


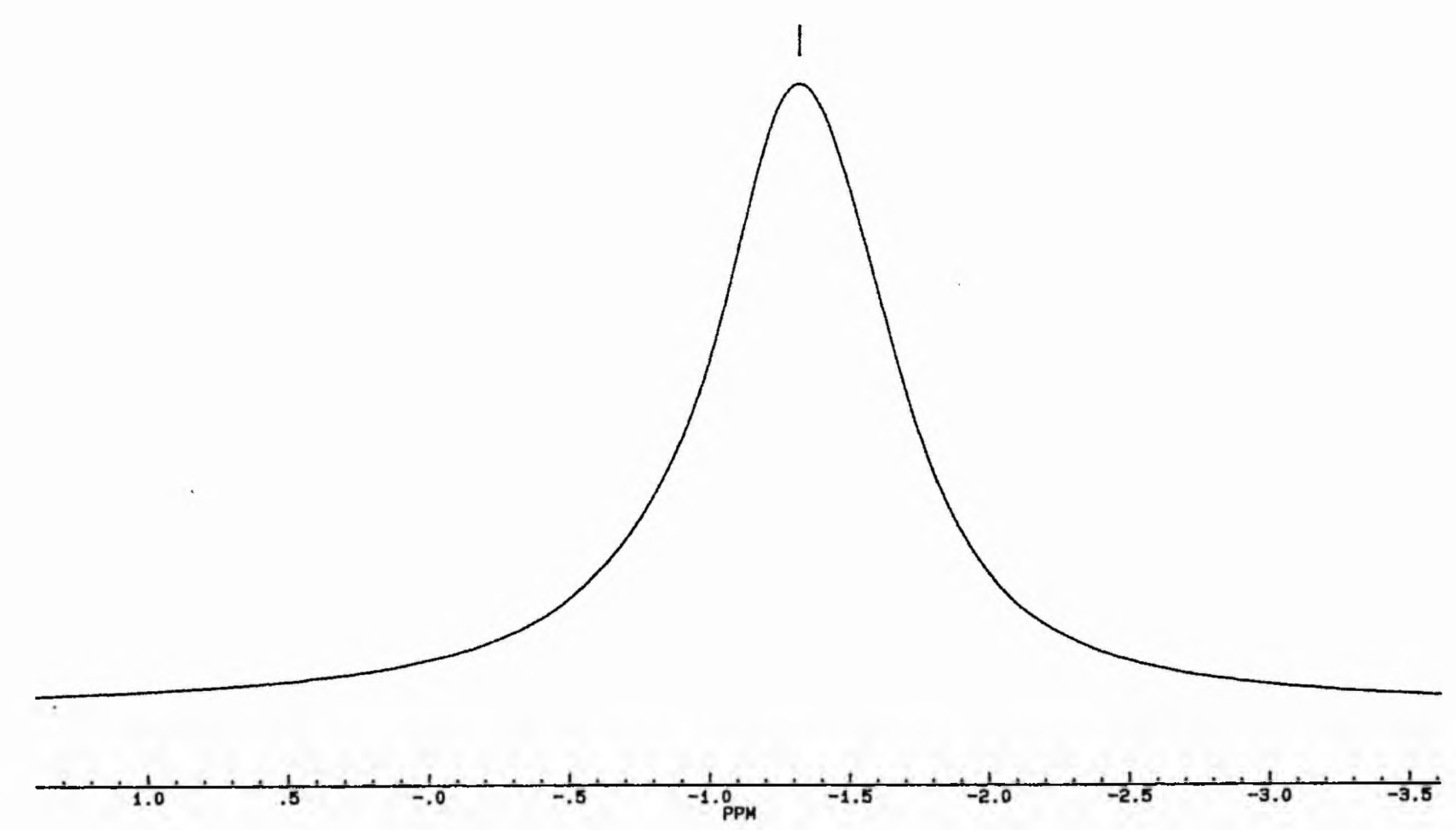

Figure 5-15c ${ }^{7} \mathrm{Li}$ mas NMR spectrum for $\mathrm{PMEO}-\mathrm{LiClO}_{4}$ electrolyte with salt concentration of $4.0 \mathrm{~mol} \mathrm{dm}^{-3}$ and spinning rate of $5 \mathrm{kHz}$ at $25^{\circ} \mathrm{C}$. 


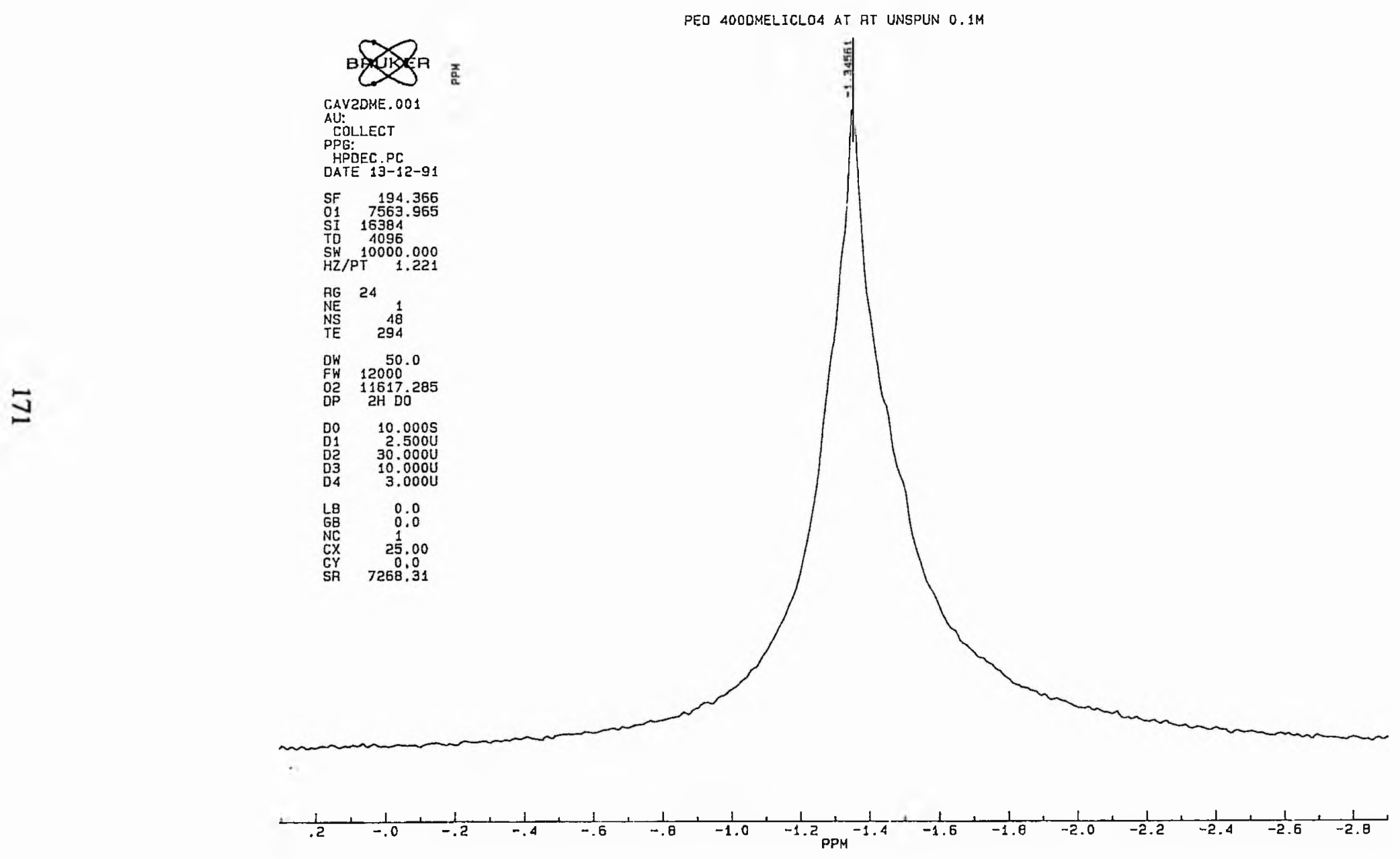

Figure 5-16a ${ }^{7} \mathrm{Li}$ NMR spectrum for $\mathrm{PEO}(400)$ dimethyl ether $-\mathrm{LiClO}_{4}$ electrolyte with salt concentration of $0.1 \mathrm{~mol} \mathrm{dm}^{-3}$ without spinning at $25^{\circ} \mathrm{C}$. 
PEO 400OME LICLO4 AT 293K UNSPUN IM

ล

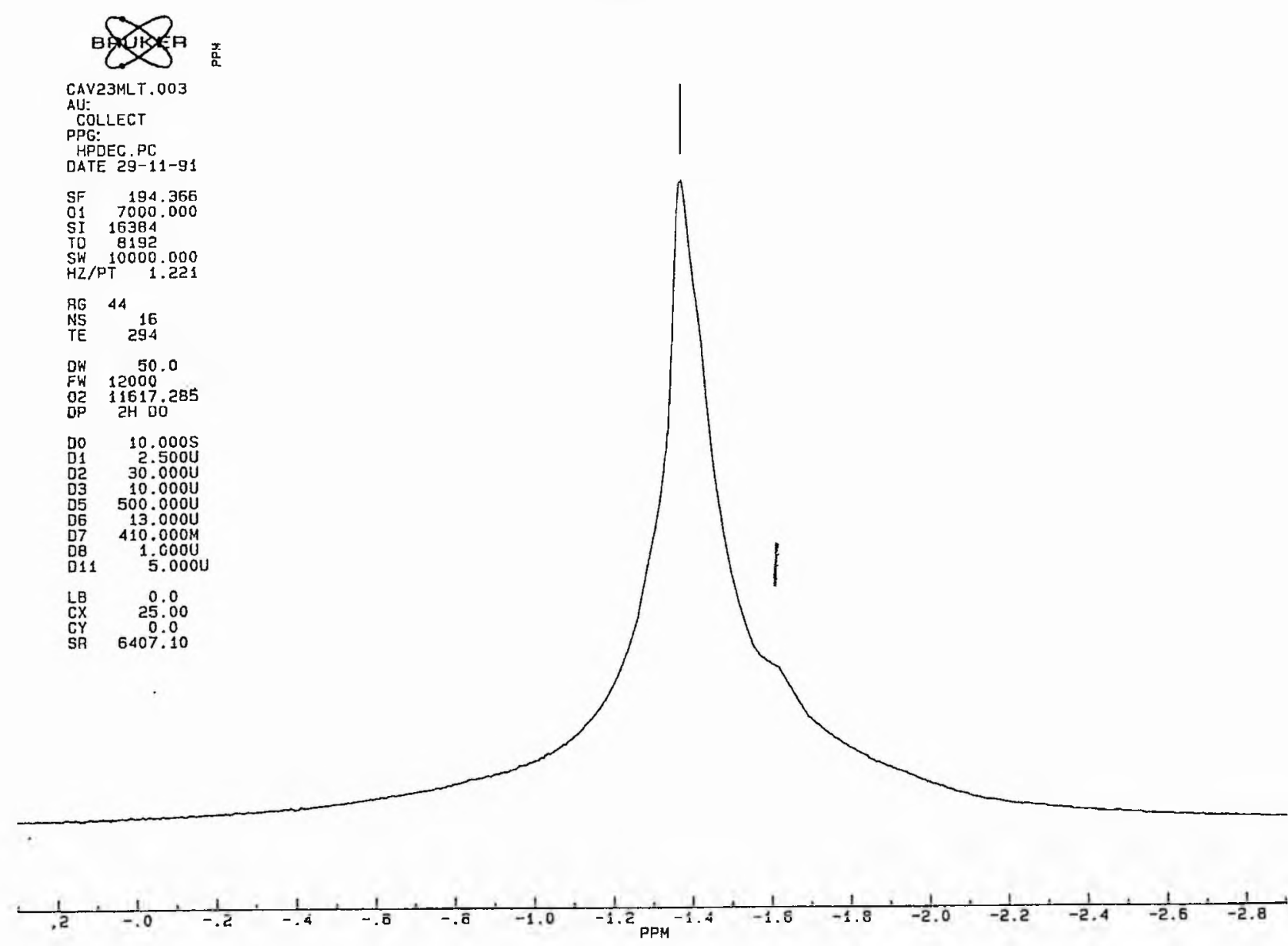

Figure 5-16b ${ }^{7} \mathrm{Li} \mathrm{NMR}$ spectrum for $\mathrm{PEO}(400)$ dimethyl ether $-\mathrm{LiClO}_{4}$ electrolyte with salt concentration of $1.0 \mathrm{~mol} \mathrm{dm}^{-3}$ without spinning at $25^{\circ} \mathrm{C}$. 


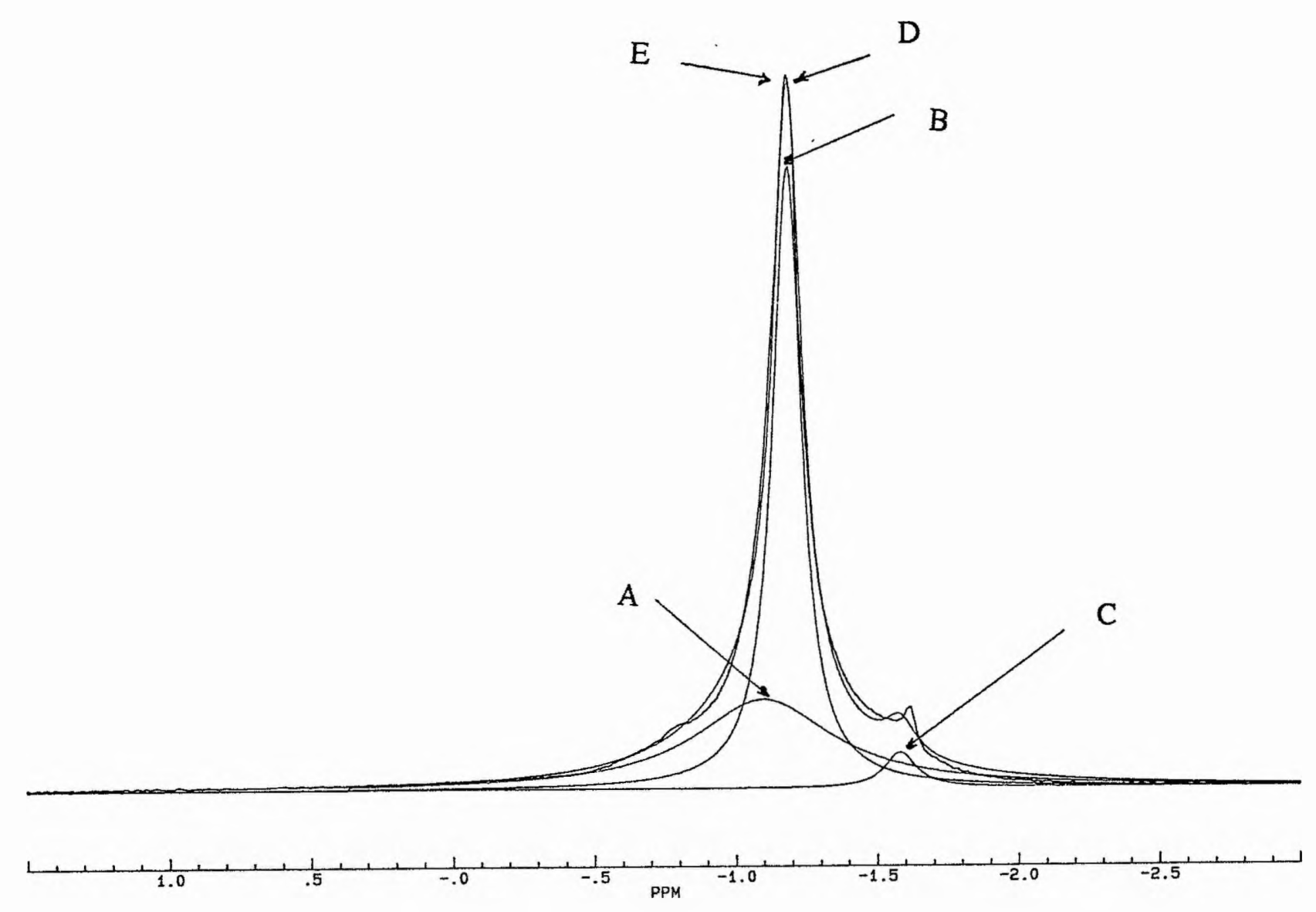

Figure 5-17 Fitted ${ }^{7} \mathrm{Li}$ mas NMR spectrum for $\mathrm{PMEO}-\mathrm{LiClO}_{4}$ electrolyte with salt concentration of $1.0 \mathrm{~mol} \mathrm{dm}^{-3}$ and spinning rate of $5 \mathrm{kHz}$ at $25^{\circ} \mathrm{C}$ : (A) line assigned to "free ions"; (B) line assigned to ion pairs; (C) line assigned to ion clusters; (D) fitted line and $(E)$ experimental line. 


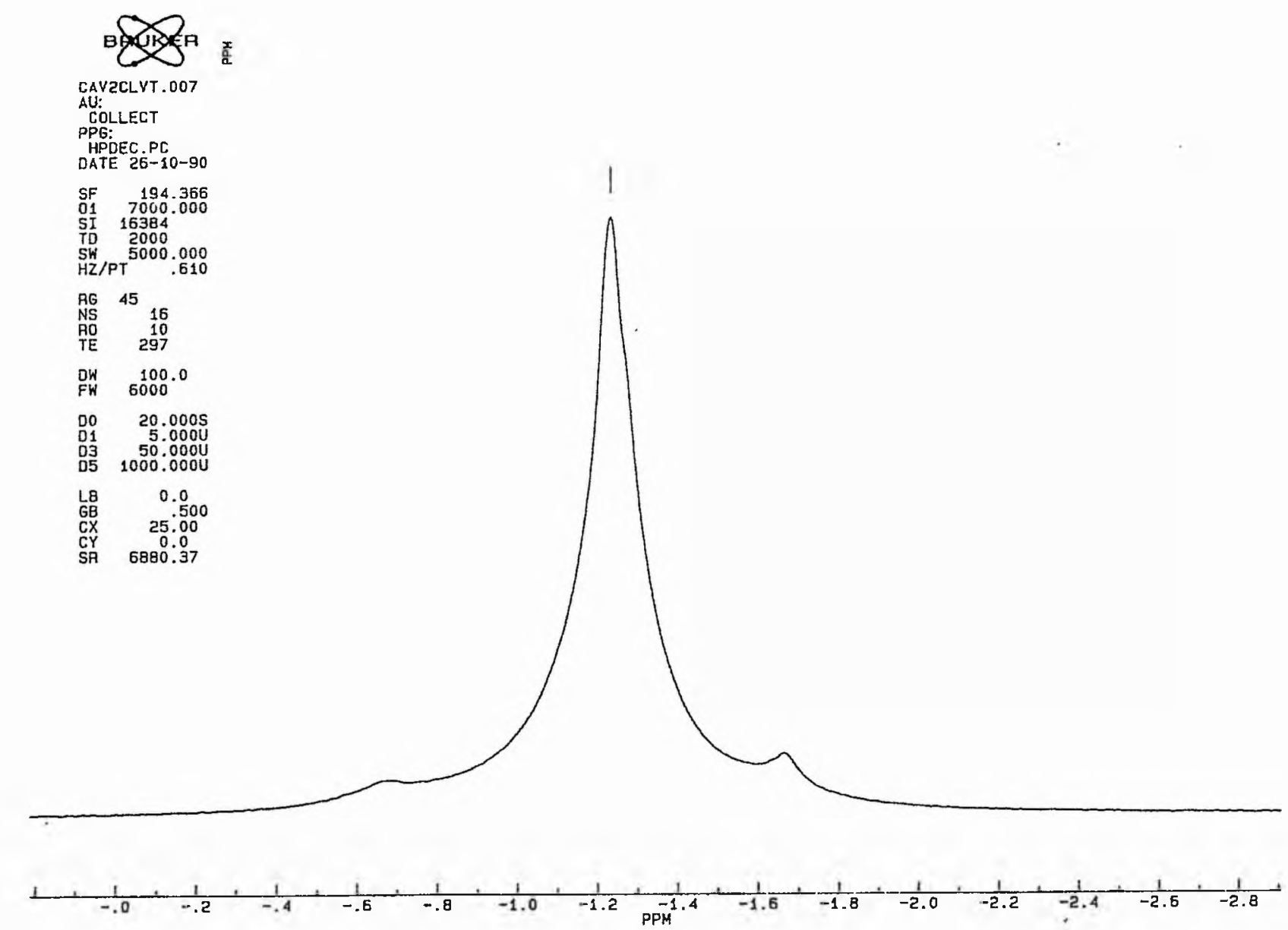

Figure 5-18a ${ }^{7} \mathrm{Li}$ mas NMR spectrum for $\mathrm{PMEO}-\mathrm{LiClO}_{4}$ electrolyte with salt concentration of $0.5 \mathrm{~mol} \mathrm{dm}^{-3}$ and spinning rate of $5 \mathrm{kHz}$ at $25^{\circ} \mathrm{C}$. 


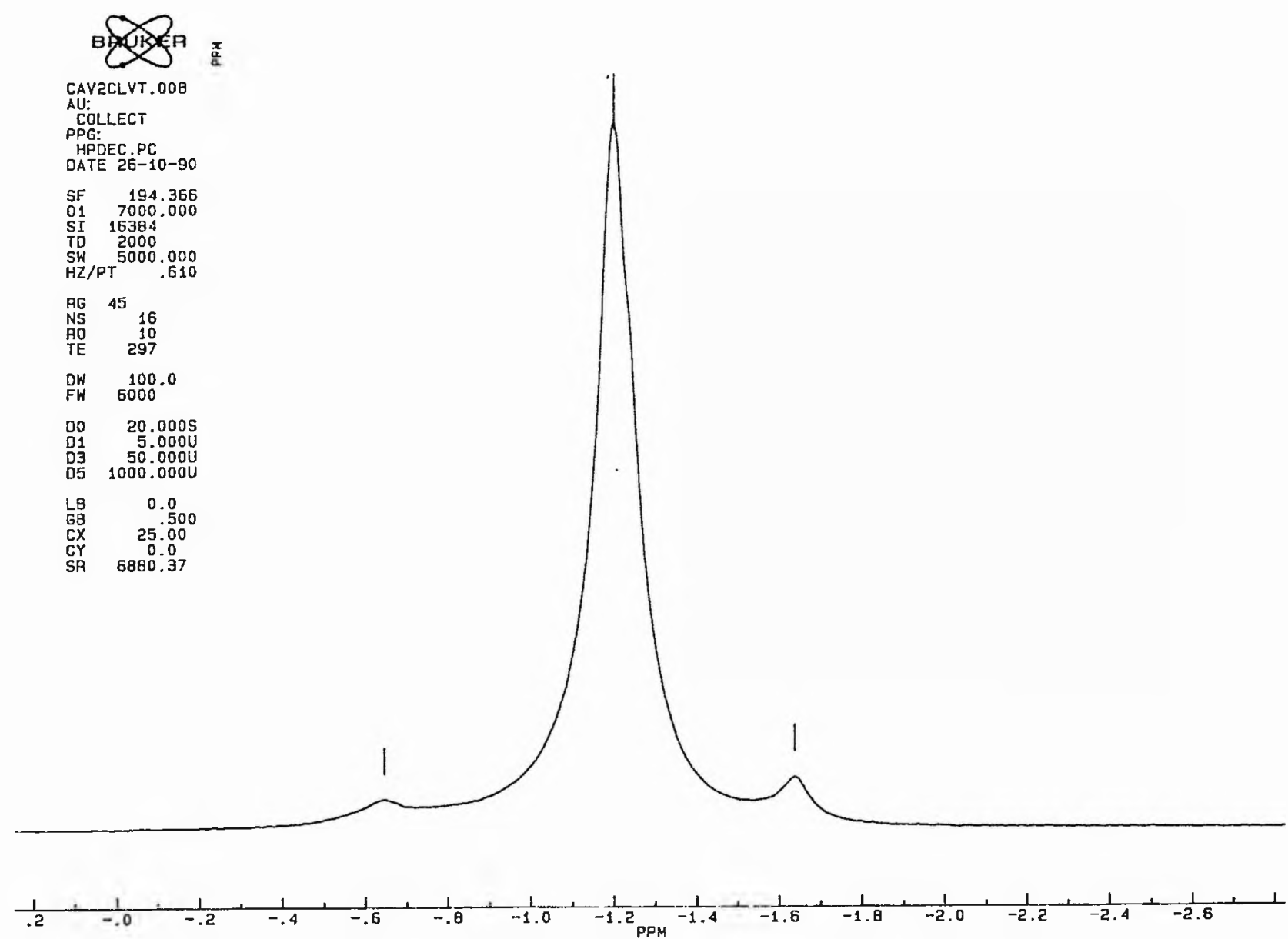

Figure 5-18b ${ }^{7} \mathrm{Li}$ mas NMR spectrum for $\mathrm{PMEO}-\mathrm{LiClO}_{4}$ electrolyte with salt concentration of $0.5 \mathrm{~mol} \mathrm{dm}-3$ and spinning rate of $5 \mathrm{kHz}$ at $60^{\circ} \mathrm{C}$. 


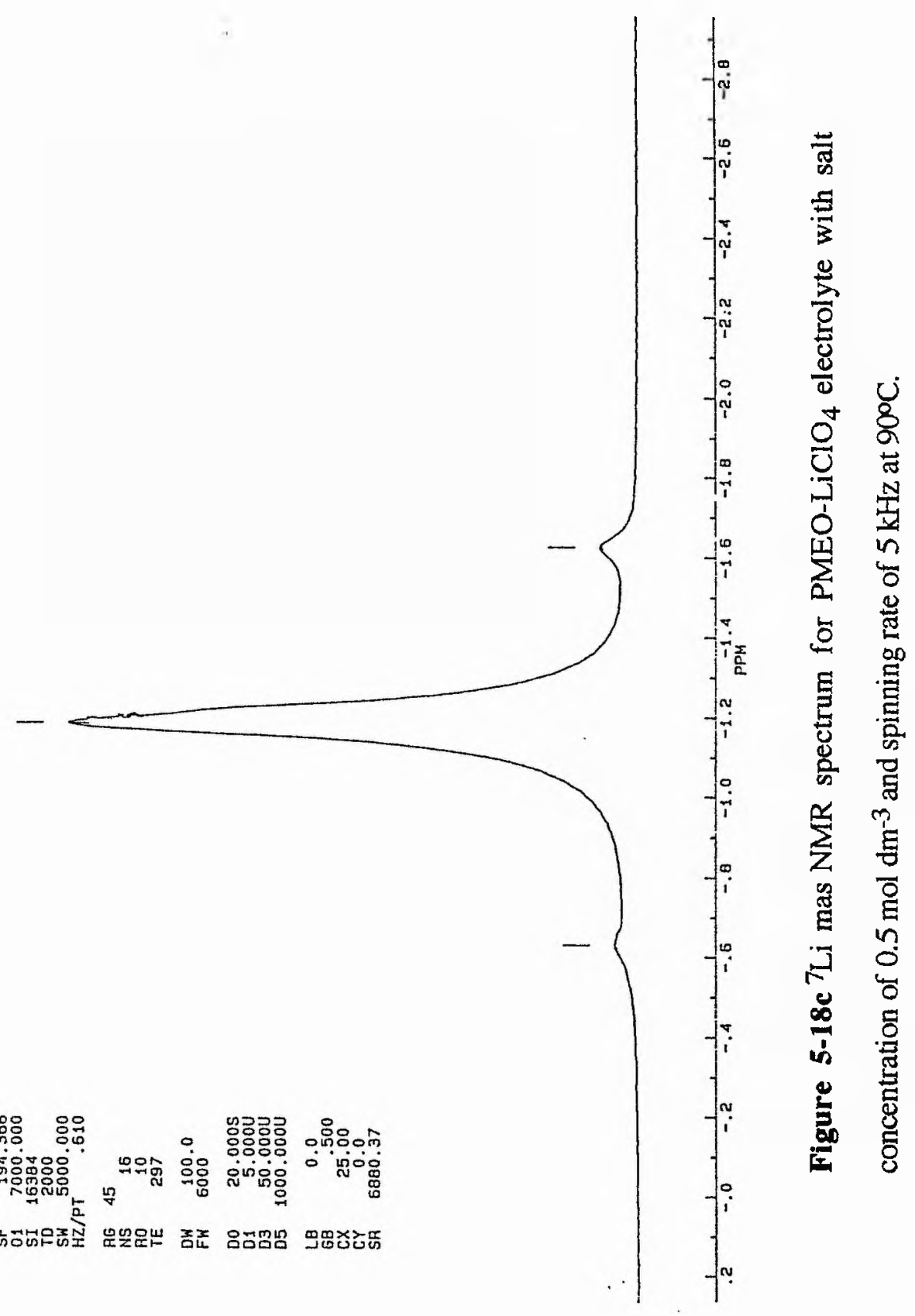




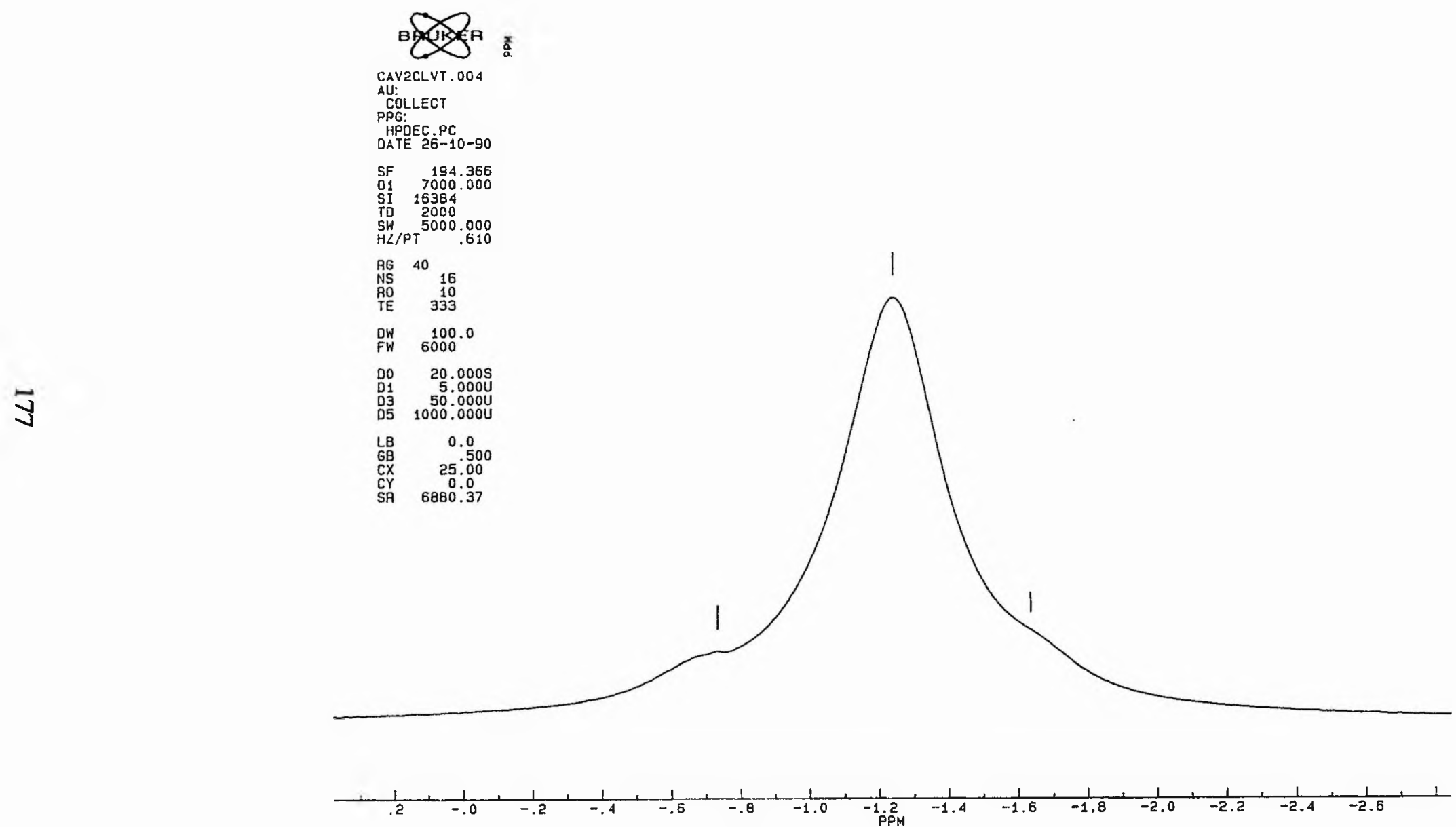

Figure 5-19a ${ }^{7} \mathrm{Li}$ mas NMR spectrum for $\mathrm{PMEO}-\mathrm{LiClO}_{4}$ electrolyte with salt concentration of $2.0 \mathrm{~mol} \mathrm{dm}^{-3}$ and spinning rate of $5 \mathrm{kHz}$ at $25^{\circ} \mathrm{C}$. 


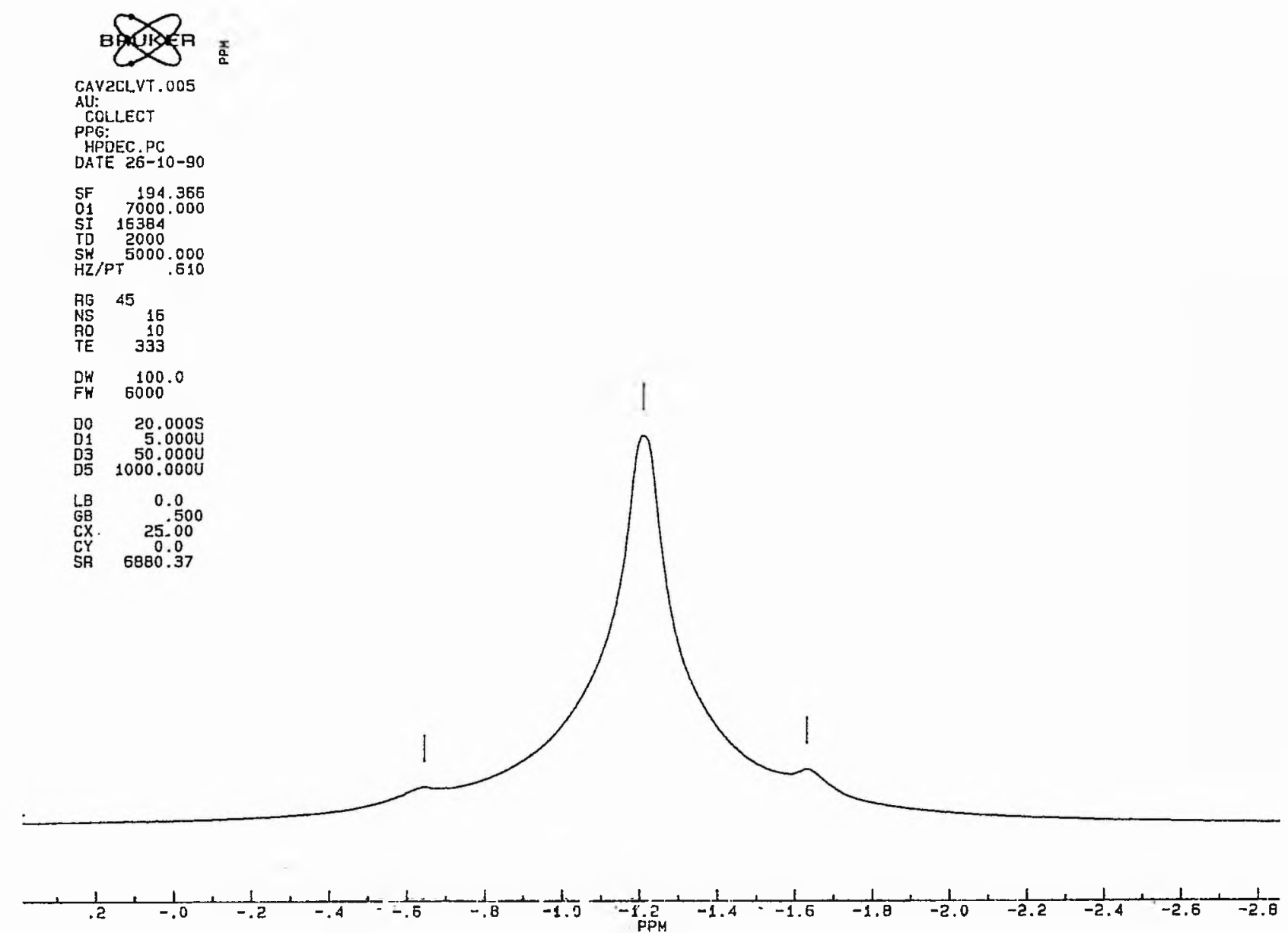

Figure 5-19b ${ }^{7} \mathrm{Li}$ mas NMR spectrum for $\mathrm{PMEO}-\mathrm{LiClO}_{4}$ electrolyte with salt concentration of $2.0 \mathrm{~mol} \mathrm{dm}^{-3}$ and spinning rate of $5 \mathrm{kHz}$ at $60{ }^{\circ} \mathrm{C}$. 


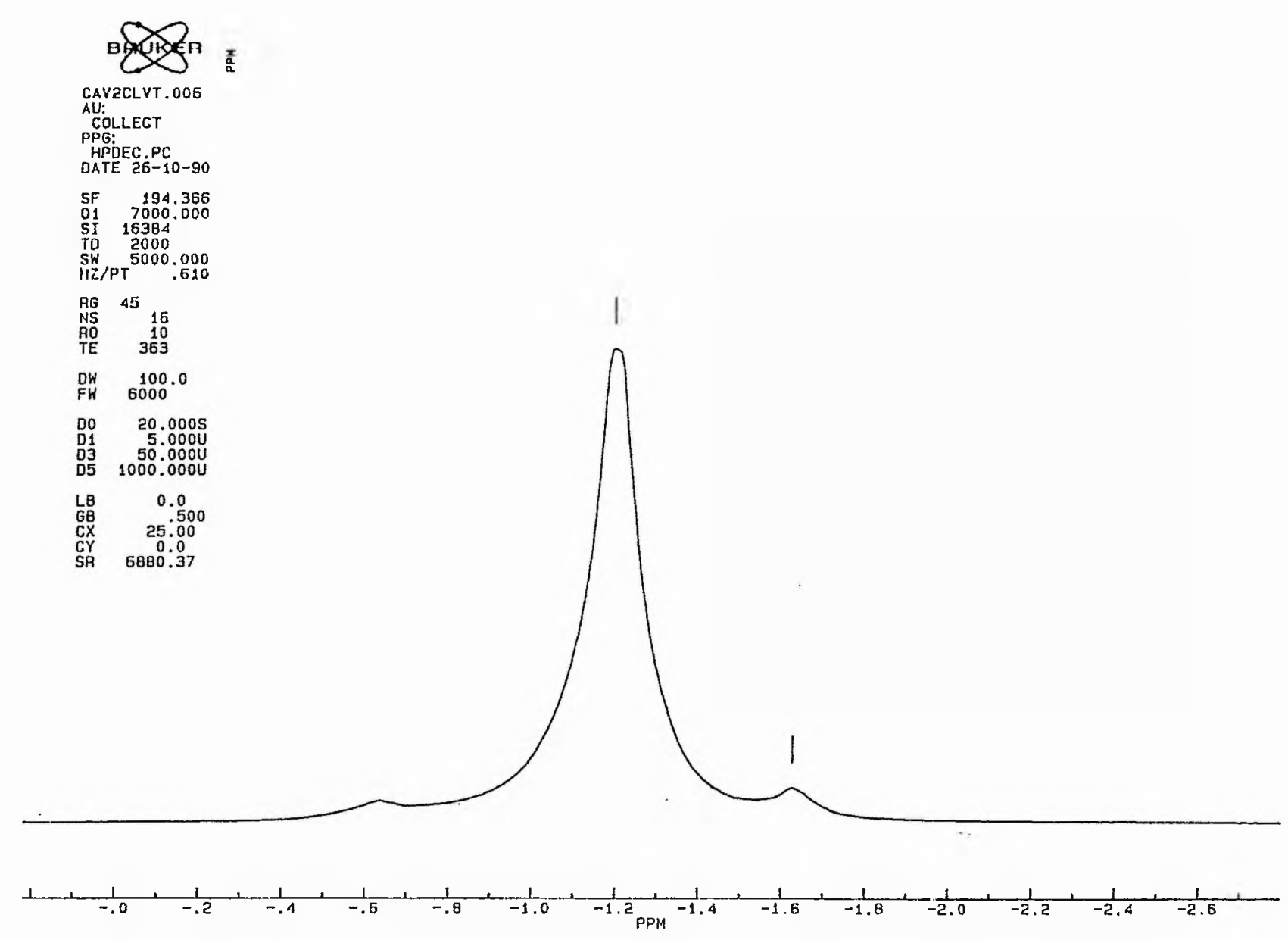

Figure 5-19c ${ }^{7} \mathrm{Li}$ mas NMR spectrum for $\mathrm{PMEO}-\mathrm{LiClO}_{4}$ electrolyte with salt concentration of $2.0 \mathrm{~mol} \mathrm{dm}^{-3}$ and spinning rate of $5 \mathrm{kHz}$ at $90{ }^{\circ} \mathrm{C}$. 


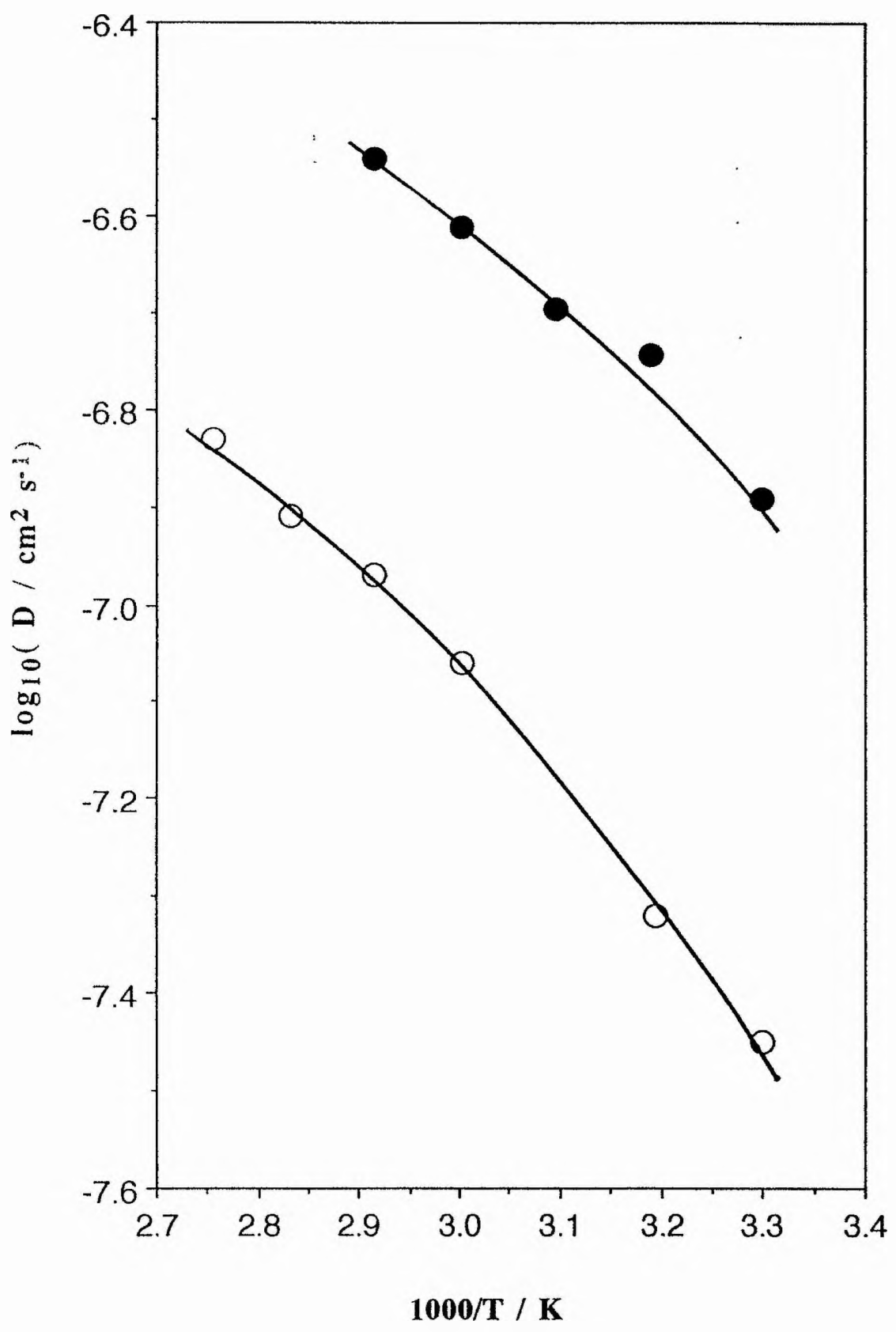

Figure 5-20 Diffusion coefficient of ${ }^{31} \mathrm{P}$ as a function of temperature for PMEO$\mathrm{LiPF}_{6}$ electrolytes with $\mathrm{EO} / \mathrm{Li}$ of 50:1 (๑) and 20:1 (O). 


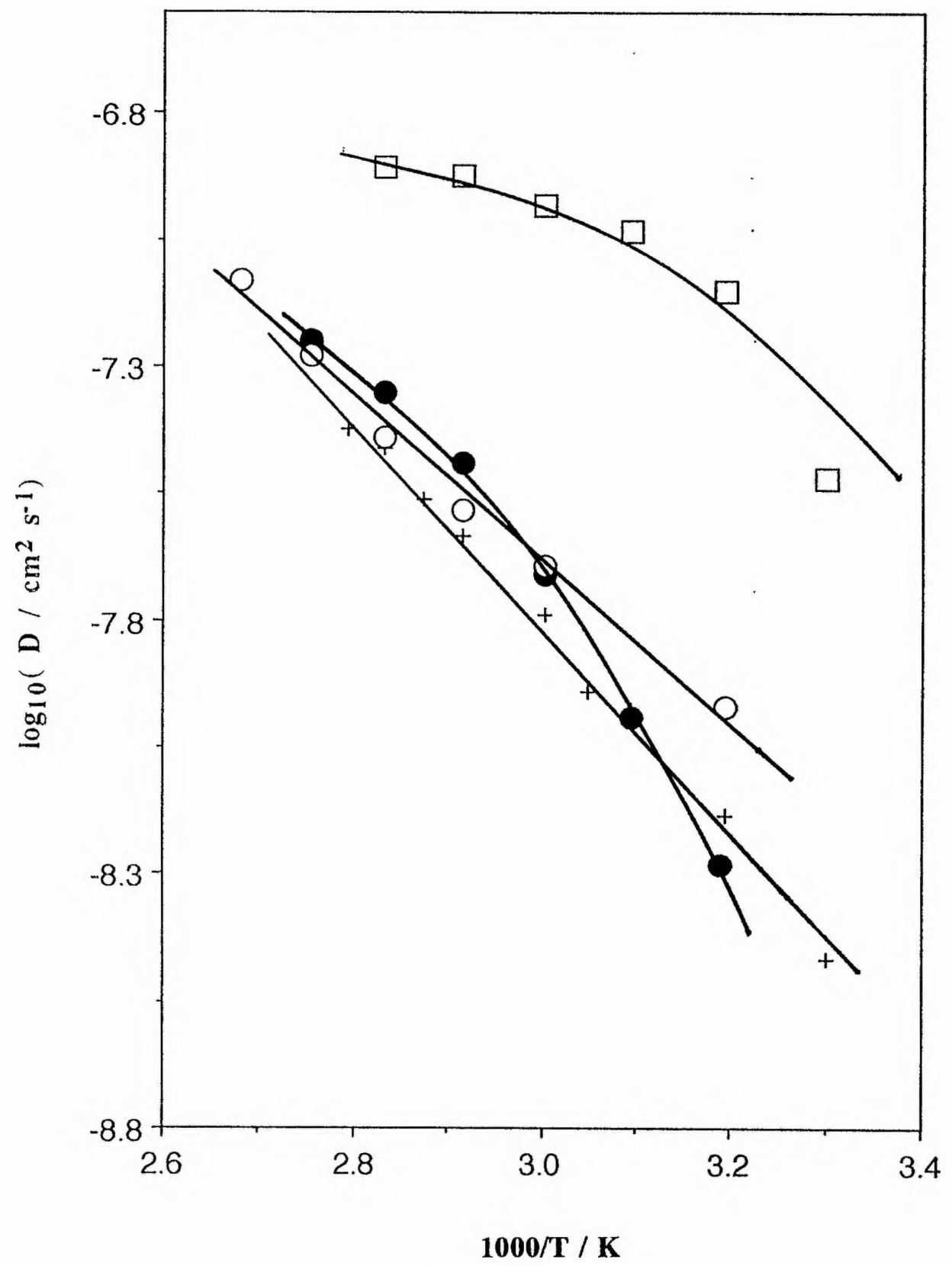

Figure 5-21a Diffusion coefficient of ${ }^{7} \mathrm{Li}$ as a function of temperature for PMEO$\mathrm{LiPF}_{6}$ electrolytes with EO/Li of 100:1(口), 50:1(O), 30:1 (t) and 20:1(O). 


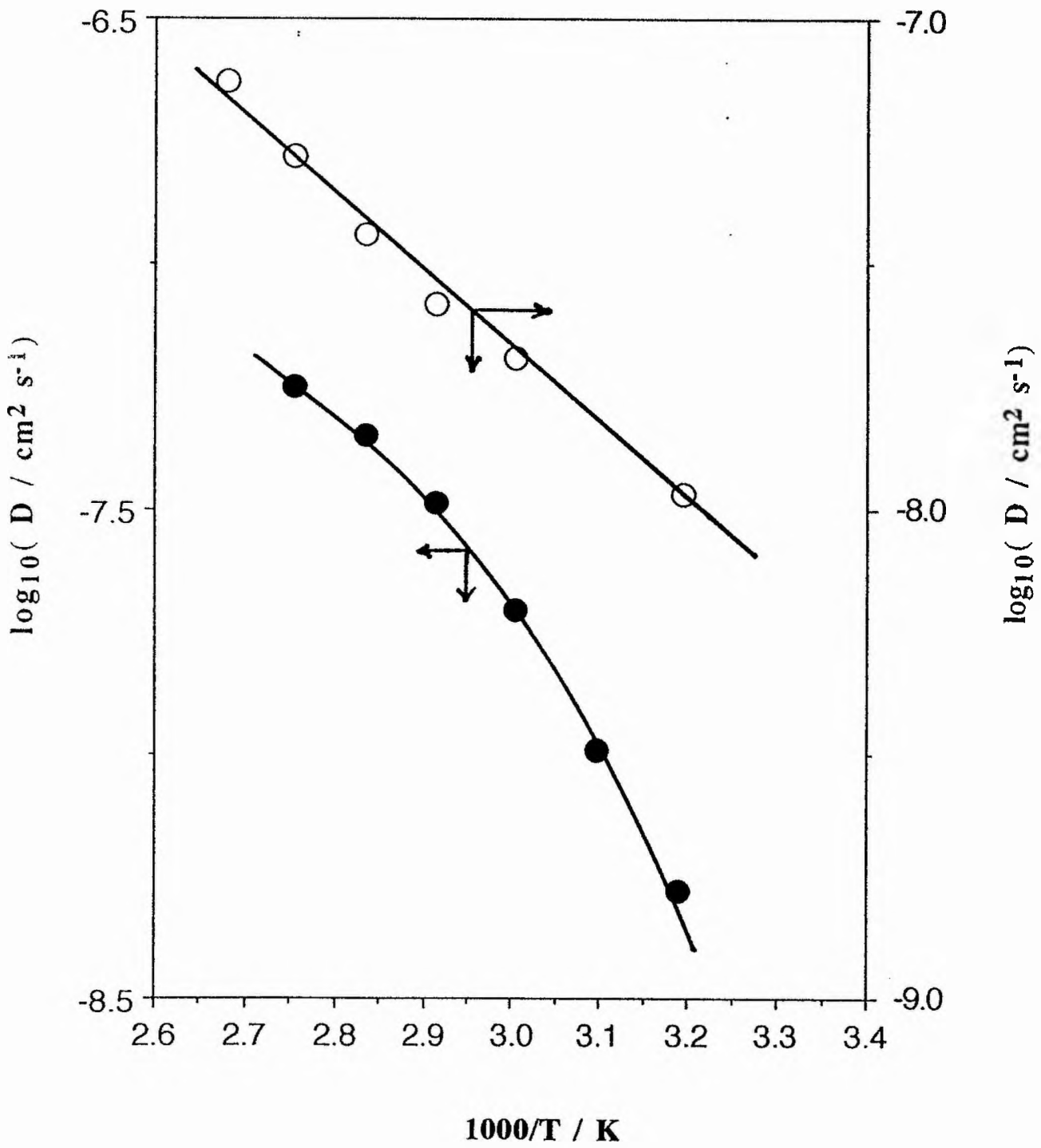

Figure 5-21b Diffusion coefficient of ${ }^{7} \mathrm{Li}$ for PMEO-LiPF 6 electrolytes with $\mathrm{EO} / \mathrm{Li}$ of 50:1 (O) and 20:1 (O) plotted to show different forms of temperature dependence. 


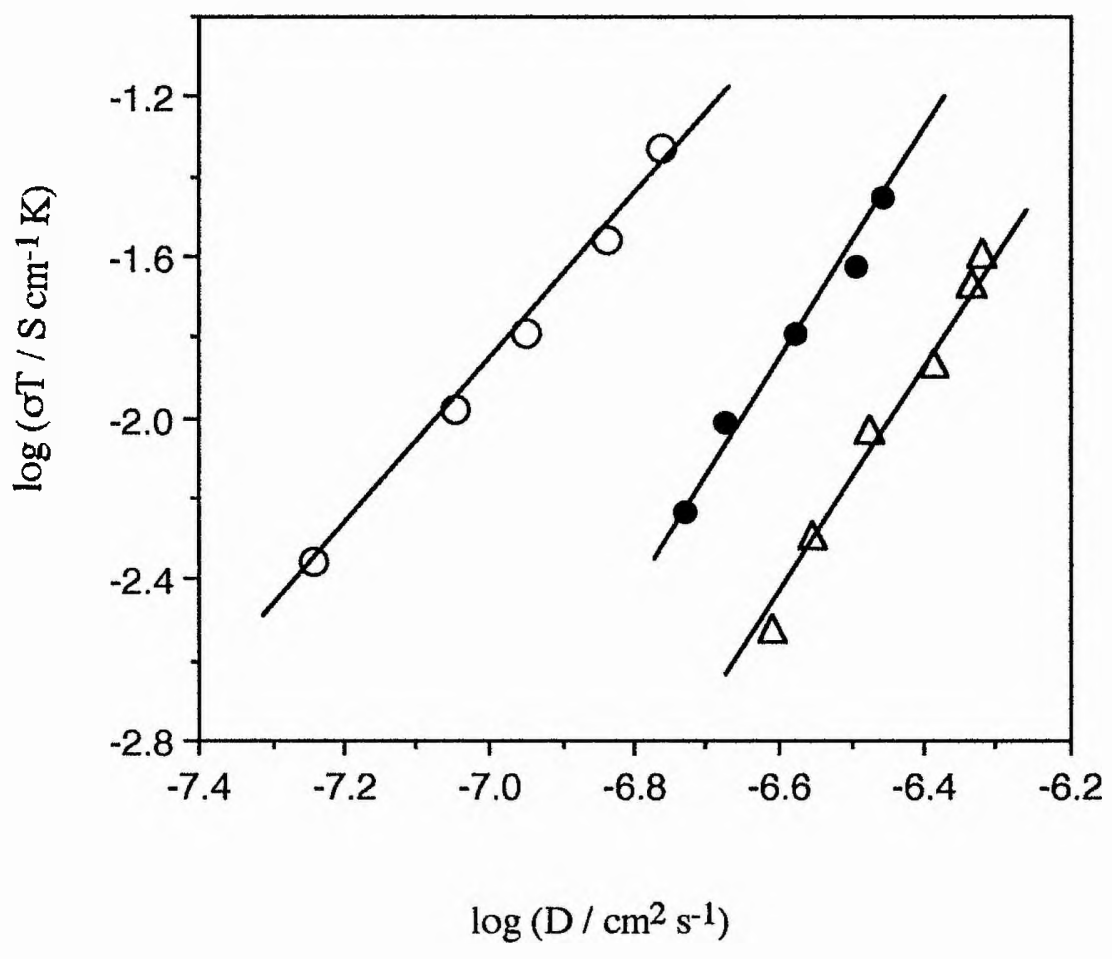

Figure 5-22 Plot of $\log \mathrm{D}$ versus $\log (\sigma \mathrm{T})$ for $\mathrm{PMEO}-\mathrm{LiPF}_{6}$ electrolytes with $\mathrm{EO} / \mathrm{Li}$ ratio of 50:1 $(\bullet), 30: 1(\Delta)$ and $20: 1(0)$. 


\section{CHAPTER 6}

\section{MASS TRANSPORT IN POLYMER ELECTROLYTES BASED ON A ZINC SALT}

The understanding of the ionic conduction in the polymer electrolytes based on multivalent salts is much less than for those based on monovalent salts. Both the interest of fundamental understanding and the possibility of potential applications encourage study of polymer electrolytes based on multivalent salts.

Much attention has been given to zinc-ion based polymer electrolytes, because of the existence of useful structural data from EXAFS studies [1-3]. There is, however, considerable confusion in the literature over the question of the mobility of the zinc. Using various systems and criteria, different authors have characterised zinc as being either "immobile" or "very highly mobile". The reasons for this are related to the complexity of divalent ion based polymer electrolytes. First, different conditions and methods for preparation may result in different moisture levels in polymer electrolytes, which influence the interaction between the zinc ions and the polymer chains, if the zinc is partially hydrated. Second, semicrystalline PEO-based electrolytes containing zinc ions are usually complex mixtures of several phases. Third, the zinc may be present both as simple cation solvated by the polymer or as a "complex" such as $\mathrm{ZnX}$, $\mathrm{ZnX}$. $\mathrm{ZnX}_{3}{ }^{-}$, etc. These complexes may or may not be mobile. In addition, zinc is known to form passivating layers which make the interpretation of dc measurements in cells with zinc electrodes difficult. 
In this chapter, mass transport in polymer electrolytes based on amorphous methoxy-linked poly(ethylene oxide) and zinc trifluoromethane sulphonate has been investigated using two electrode cells with electrodes which are non-blocking towards zinc. In addition, the conductivities and Hittorf transference numbers of these electrolytes have been investigated.

\section{6-1 Electrode-Electrolyte Interfaces}

When the dc polarisation method is employed to study mass transport in a polymer electrolyte PEO-MX $X_{n}$, the electrodes must be non-blocking, i.e. the electrode reactions for reduction of $\mathrm{M}^{\mathrm{n}+}$ to $\mathrm{M}_{(\mathrm{s})}$ and oxidation of $\mathrm{M}_{(\mathrm{s})}$ to $\mathrm{M}^{\mathrm{n}+}$ must be fast, and passivating layers should be absent.

Two and three electrode cells with zinc metal as working, reference and counter electrodes were used to investigate the interfaces between PMEO- $\mathrm{Zn}\left(\mathrm{CF}_{3} \mathrm{SO}_{3}\right)_{2}$ electrolyte and zinc electrodes. Because zinc metal can be easily oxidised in air, zinc electrodes were freshly scraped in the glovebox immediately before cell assembly.

\section{6-1-1 Passivating Layer at the Electrode-Electrolyte Interface}

For a freshly assembled two electrode cell, the ac impedance spectrum is shown in Figure 6-1, with the high frequency semicircle attributed to the bulk electrolyte (resistance $R_{e}$ ) and the second semicircle attributed to the electrolyte-electrode interface (resistance $\mathrm{R}_{\mathbf{i}}$ ).

The interfacial resistance increased significantly with time until it achieved a stable value after 24 hours (Figure 6-1). The interfacial capacitance decreased simultaneously. The initial interfacial capacity was $5 \mu \mathrm{F} \mathrm{cm}^{-2}$ which is quite close to the 
expected value for the electrical double layer. After the cell had been assembled for 24 hours, the interfacial capacity was $0.3 \mu \mathrm{F} \mathrm{cm}^{-2}$ which is typical value for a thin surface layer at the electrode-electrolyte interface [4], suggesting that a passivating layer formed on the zinc electrode. In $\mathrm{Li} / \mathrm{PEO}-\mathrm{LiCF}_{3} \mathrm{SO}_{3} / \mathrm{Li}$ system [5], the interfacial resistance $\mathrm{R}_{\mathbf{i}}$ was also found to increase with time, but not so rapidly as in the zinc cell, and the passivating layer formed on the lithium electrode was not so resistive as that on the zinc electrode. Thus the influence of the passivating layer on dc polarisation is more severe in the case of zinc.

The capacitance $\mathrm{C}$ and thickness $\mathrm{L}$ of the passivating layer can be correlated by equation (6-1-1), by assuming that the layer acts as a parallel plate capacitor between the electrode and electrolyte. Thus:

$$
C=\varepsilon_{o} \varepsilon \frac{A}{L}
$$

where $C$ is capacitance, $\varepsilon_{O}$ and $\varepsilon$ are the permittivities of free space and the passivating layer, and $A$ is the area of the layer. Because the interfacial resistance $R_{i}$ is proportional to L/A, if the increase of the interfacial resistance arises from the formation of surface layer, the product of $\mathrm{CR}_{\mathrm{i}}$ will be a constant. Figure 6-2 shows the linear relationship between $C$ and $1 / R_{i}$ measured during the period from 1 hour to 10 hours after cell assembly. This strongly suggests that a surface layer indeed forms on the zinc electrode.

The mechanism of the formation of the passivating layer between a lithium electrode and an electrolyte based on a lithium salt involves the reactions between lithium and impurities in the electrolyte or possibly products from decomposed anions. to form a layer based on mixed lithium oxide / lithium fluoride [6]. For instance, lithium reacts with any residual water and substances with $\mathrm{OH}$ groups, which inevitably exist in the system under normal experimental conditions. Although zinc metal is not so active 
as lithium, it is considered possible for zinc to react with those impurities to form a resistive passivating layer [7].

The influence of this layer must be considered when the technique of dc polarisation of a $\mathrm{Zn} /$ polymer electrolyte based on zinc (II) salt / $\mathrm{Zn}$ cell is used to study the transport properties of zinc. For example, if the interfacial resistance was very large, a very small steady state current might be observed (Figure 6-3), from which it might be incorrectly concluded that zinc species in the polymer electrolyte based on high molecular weight PEO were immobile.

\section{6-1-2 Treatment of the Electrode-Electrolyte Interface}

A passivating electrode can often be activated by electropolishing, or electrochemical etching [8]. In a certain polarisation potential region, the passivation can be eliminated either by reducing or further oxidising the layer, or by its physical breakdown.

In order to investigate the effect of dc polarisation on the passivating layer between a zinc electrode and a polymer electrolyte, a three electrode cell with zinc working, reference and counter electrodes was employed. When anodic de potentials were applied to the zinc electrode, the interfacial resistance decreased remarkably with increasing potential up to $0.5 \mathrm{~V}$ after which the interfacial resistance changed only slightly (Figure 6-4a). Meantime, the current increased significantly (Figure 6-4b). This may indicate that the electrode can be activated by anodic polarisation with a potential of about $0.5 \mathrm{~V}$, caused probably by the physical breakdown of the passivating layer.

The ac impedance spectra for the zinc electrode before and after anodic polarisation for 5 hours at $0.5 \mathrm{~V}$ are shown in Figure 6-5a. The interfacial resistance was reduced by the polarisation from $1.12 \times 10^{6} \Omega$ to $8.61 \times 10^{+} \Omega$, while the 
interfacial capacitance increased from $1.83 \mu \mathrm{F} \mathrm{cm}^{-2}$ to $3.46 \mu \mathrm{F} \mathrm{cm}^{-2}$. In an alternative treatment, the electrode was subjected to alternating positive and negative potentials with a 1 hour interval as in "electropolishing". The interfacial resistance was reduced more effectively (Figure 6-5b) to $1.41 \times 10^{4} \Omega$, and the interfacial capacitance was increased to $5.73 \mu \mathrm{F} \mathrm{cm}^{-2}$. By increasing the number of electropolishing cycles, the interfacial resistance was further slightly reduced to $1.33 \times 10^{4} \Omega$ (Figure $6-5 \mathrm{~b}$ ), and the interfacial capacitance increased to $16.6 \mu \mathrm{F} \mathrm{cm}^{-2}$.

The electrode-electrolyte interfacial resistance in $\mathrm{Zn} / \mathrm{PMEO}-\mathrm{Zn}\left(\mathrm{CF}_{3} \mathrm{SO}_{3}\right)_{2} / \mathrm{Zn}$ cells could therefore be effectively reduced by applying dc polarisation with $\pm 0.5 \mathrm{~V}$ potentials successively for three cycles of 1.0, 1.5 and 2.0 hours. After the above treatment, the interface was quite stable with only slightly changes with time (Figure 6-6). This type of treatment was used in the subsequent study of dc polarisation of the two electrode cell.

\section{6-2 De Polarisation and Steady State Current Flow in Symmetric Zinc Cells}

\section{6-2-1 Steady State Current Flow in Solid Electrolyte Cells}

The steady state current method has been widely used to study transport in polymer electrolytes. The theory has been discussed by Bruce, Hardgrave and Vincent $[9,10]$. Here, the application of this theory to polymer electrolytes based on zinc(II) salts is briefly discussed.

If a divalent metal salt $\mathrm{MX}_{2}$, say $\mathrm{Zn}\left(\mathrm{CF}_{3} \mathrm{SO}_{3}\right)_{2}$, is dissolved in high molecular weight amorphous PMEO, ion association may take place to form species such as $\mathrm{MLX}^{+}$, $\mathrm{MX}_{2}, \mathrm{MX}_{3}{ }^{-}$etc. because of the low permittivity. Here, the two simplest cases are 
considered: (i) fully dissociated polymer electrolyte, i.e. only $\mathrm{M}^{2+}$ and $\mathrm{X}^{-}$exist in system and (ii) associated polymer electrolyte with only $\mathrm{MX}^{+}, \mathrm{MX}_{2}$ and $\mathrm{X}^{-}$species present.

1. Fully dissociated polymer electrolyte

Consider a symmetric cell: $\mathrm{M} / \mathrm{PEO}-\mathrm{MX}_{2} / \mathrm{M}$ with fast electrode kinetics, in which the electrodes are non-blocking to $\mathrm{M}^{2+}$ (and $\mathrm{MX}^{+}$in the latter case) and blocking to $\mathrm{X}^{-}$. The cell, with a distance of $\mathrm{L}$ between the two electrodes and an electrode area of $A$, is symmetric with respect to the $\mathrm{x}$ axis and the diffusion and migration of ions are taken to occur parallel to this axis.

At steady state where the current is invariant with time, the diffusion of anions from anode to cathode balances their migration from cathode to anode:

$$
\text { FD. } \frac{d C}{d x}=\frac{F^{2}}{R T} \text { D. C. } \frac{d \phi}{d x}
$$

where $D_{-}$and $C_{-}$are the diffusion coefficient and concentration of anions respectively; and $F, R$ and $T$ have their conventional meanings. The relationship between the salt concentration $\mathrm{C}$ and cation and anion concentrations, $\mathrm{C}_{+}$and $\mathrm{C}_{-}$, is: $2 \mathrm{C}=2 \mathrm{C}_{+}=\mathrm{C}_{-}$. Thus, the potential difference across the electrolyte can be obtained by integration of equation (6-2-1) to give:

$$
\Delta \phi=\frac{R T}{F} \ln \left(\frac{C_{a}}{C_{c}}\right)
$$

where $C_{a}$ and $C_{c}$ are the salt concentrations at anode and cathode. The Nernst potential difference $\Delta E$ resulting from the salt concentration difference between anode and cathode can be expressed as: 


$$
\Delta E=\frac{R T}{2 F} \ln \left(\frac{C_{a}}{C_{c}}\right)
$$

The total potential difference acting over the electrolyte at steady state is : $\Delta \mathrm{V}=\Delta \mathrm{E}+\Delta \phi$. Thus, the relationship between $\Delta V$ and concentrations at anode and cathode can be expressed as:

$$
\Delta V=\frac{3 R T}{2 F} \ln \left(\frac{C_{a}}{C_{c}}\right)
$$

The steady state current $\mathrm{I}_{s}^{+}$can be expressed in following equation:

$$
I_{s}^{+}=-2 A F D_{+} \frac{d C}{d x}-\frac{4 A F^{2}}{R T} D_{+} C \frac{d \phi}{d x}
$$

where $D_{+}$is diffusion coefficient of cations. By noting that $\frac{d C}{d x}=\frac{\left(C_{c}-C_{a}\right)}{L}$ and combining equation (6-2-1), steady state current $\mathrm{I}_{s}^{+}$can also be correlated to $\mathrm{C}_{\mathrm{a}}$ and $\mathrm{C}_{\tau}$

$$
I_{s}^{+}=\frac{6 A F D_{+}}{L}\left(C_{c}-C_{a}\right)
$$

For small concentration difference between anode and cathode, the following approximation is applicable:

$$
C_{a}-C_{c}=\frac{1}{2}\left(C_{a}+C_{c}\right) \ln \left(\frac{C_{a}}{C_{c}}\right)
$$

By noting that $C_{o}=\left(C_{a}+C_{c}\right) / 2$, where $C_{o}$ is the initial salt concentration, $-I_{s}^{+} / \Delta V$ :an be then obtained: 


$$
-I_{s}^{+} / \Delta V=\frac{4 A F^{2}}{L R T} D_{+} C_{o}
$$

Therefore, the cationic conductivity $\sigma_{+}$which is the conductivity at steady state $\sigma_{S}$ can be obtained as follows:

$$
\sigma_{+}=\sigma_{s}=\frac{-I_{s}^{+} L}{\Delta V A}=\frac{4 F^{2}}{R T} D_{+} C_{o}
$$

Also the initial conductivity is given by:

$$
\sigma_{o}=\sigma_{+}+\sigma_{-}=\frac{2 F^{2} C_{o}}{R T}\left(2 D_{+}+D_{-}\right)
$$

Therefore, the relationship between cationic transport number 4 and steady state current ratio $\mathrm{I}_{\mathrm{s}}^{+} / \mathrm{I}_{\mathrm{o}}$ can be deduced from steady state current.

$$
\frac{I_{s}^{+}}{I_{o}}=\frac{\sigma_{s}}{\sigma_{0}}=\frac{2 D_{+}}{2 D_{+}+D_{-}}=t_{+}
$$

After correction of the voltage drop at the electrode-electrolyte interface, the equation being often employed for calculation of $t_{+}$from dc polarisation experimental results can be obtained [11].

$$
t_{+}=\frac{I_{s}\left(\Delta V_{a}-I_{o} R_{\text {io }}\right)}{I_{0}\left(\Delta V_{a}-I_{s} R_{\text {is }}\right)}
$$

where $I_{s}$ and $I_{o}$ are steady state current and initial current respectively: $\Delta \mathrm{V}_{\mathrm{a}}$ is applied de potential, $R_{i s}$ and $R_{\text {io }}$ are electrode-electrolyte interfacial resistances at steady state and 
initial state.

2. Associated polymer electrolyte

Assume that there is only one equilibrium in the system, i.e., $\mathrm{MX}^{+}+\mathrm{X}^{-}=$ $\mathrm{MX}_{2}$, and that the concentration of $\mathrm{M}^{2+}$ is negligible. At steady state, following equation can be set up for anions:

$$
\text { FD. } \frac{d C}{d x}+F D_{o} \frac{d C_{N}}{d x}=\frac{F^{2}}{R T} \text { D. C. } \frac{d \phi}{d x}
$$

where $D_{-}$and $D_{0}$ are the diffusion coefficients for $X^{-}$and $M X_{2}$ species respectively, and $\mathrm{C}_{\mathrm{N}}$ is the concentration of $\mathrm{MX} \mathrm{X}_{2}$. The steady state current results from the diffusion and migration of $\mathrm{MX}^{+}$and the diffusion of $\mathrm{MX}_{2}$. Thus,

$$
I_{s}=-A F\left(D_{+} \frac{d C_{+}}{d x}+D_{o} \frac{d C_{N}}{d x}+\frac{F}{R T} D_{+} C_{+} \frac{d \phi}{d x}\right)
$$

where $\mathrm{D}_{+}$is the diffusion coefficient of $\mathrm{MX}^{+}$species.

By noting that $\left[\mathrm{MX}_{2}\right]=\mathrm{K}[\mathrm{MX}+][\mathrm{X}]$, i.e., $\mathrm{C}_{\mathrm{N}}=\mathrm{KC}$, and integrating of equation (6-2-13), $\Delta \phi$ can be obtained.

$$
\Delta \phi=\frac{R T}{F}\left[\ln \left(\frac{C_{a}}{C_{c}}\right)+\frac{2 K D_{o}}{D_{.}}\left(C_{a}-C_{c}\right)\right]
$$

The Nernst potential $\Delta \mathrm{E}$ can be expressed as the equation (6-2-3). Therefore, the total potential difference across the cell is:

$$
\Delta V=\frac{2 R T}{F}\left[\frac{3}{4} \ln \left(\frac{C_{a}}{C_{c}}\right)+\frac{K D_{o}}{D .}\left(C_{a}-C_{c}\right)\right]
$$


From equation (6-2-13) and (6-2-14), steady state current can be expressed as:

$$
I_{s}=\frac{-F A}{L}\left(C_{a}-C_{c}\right)\left[\frac{K D_{o}\left(D_{+}+D_{-}\right)}{D_{-}}\left(C_{a}+C_{c}\right)+2 D_{+}\right]
$$

By using the same condition as in (6-2-7) and $C_{0}=(C a+C c) / 2$, the effective conductivity $\sigma_{\text {eff }}$ which is the conductivity of electrolyte at steady state can be expressed as:

$$
\sigma_{\text {eff }}=\frac{-I_{s} L}{\Delta V A}=\frac{F^{2} D_{+} C_{o}}{R T}\left[1+\frac{1+\frac{4 K D_{o} C_{o}}{D_{+}}}{3+\frac{4 K D_{o} C_{o}}{D .}}\right]
$$

In this case, $\left(I_{s} / I_{0}\right)$ appearing in equation (6-2-11) and (6-2-12) is no longer the ion transport number, which is called the current fraction. The limiting current fraction, $F_{+}$, is defined to be the current fraction for $\Delta \mathrm{V} \rightarrow 0$.

Here, two extreme cases are considered. First, when $\mathrm{MX}_{2}$ species are immobile, i.e. $\mathrm{D}_{\mathrm{o}}$ is very small, $\mathrm{KDoCo} / \mathrm{D}_{-} \ll 1$, and $(6-2-18)$ becomes:

$$
\sigma_{\text {eff }}=\frac{4 F^{2}}{3 R T} D_{+} C_{0}
$$

This is the same result as (6-2-9), except for the difference in the coefficient arising from the difference in charge number between $\mathrm{M}^{2+}$ and $\mathrm{MX}^{+}$. Thus the steady state current results from the migration and diffusion of $\mathrm{MX}^{+}$species. Second, when $\mathrm{MX}_{2}$ species are very mobile and dominant in the system, i.e. $\mathrm{D}_{\mathrm{o}}$ is large and $\mathrm{KDoCo} / \mathrm{D}->>$ $1,(6-2-18)$ is simplified to: 


$$
\sigma_{e f f}=\frac{F^{2} C_{o}}{R T}\left(D_{+}+D_{-}\right)
$$

Here, the effective conductivity is larger than that in the former case.

\section{6-2-2 De Polarisation of PMEO-Zn( $\left(\mathrm{CF}_{3} \mathrm{SO}_{3}\right)_{2}$ Electrolytes}

The typical current-time curve for a two electrode $\mathrm{Zn} / \mathrm{PMEO}-\mathrm{Zn}\left(\mathrm{CF}_{3} \mathrm{SO}_{3}\right)_{2}$ $(\mathrm{EO} / \mathrm{Zn}=20) / \mathrm{Zn}$ cell with pre-treated electrodes at $80^{\circ} \mathrm{C}$ is shown in Figure 6-7. Typical ac impedance spectra before polarisation and after reaching steady state are shown in Figure 6-8. Figure 6-9 shows the relationship between the steady state current $I_{s}$ and corrected applied potential difference $\Delta V$. When the applied voltage was low, the relationship between $I_{s}$ and $\Delta V$ was linear as expected. For fully dissociated polymer electrolytes containing only free ions, the relationship between concentration of salt at the electrodes and the applied potential difference can be obtained from (6-2-4).

$$
\mathrm{C}_{\mathrm{c}}=\frac{2 \mathrm{C}_{\mathrm{o}}}{1+\exp \left(\frac{2 \mathrm{~F} \Delta \mathrm{V}}{3 \mathrm{RT}}\right)} ; \quad \mathrm{C}_{\mathrm{a}}=\frac{2 \mathrm{C}_{\mathrm{o}}}{1+\exp \left(\frac{-2 \mathrm{~F} \Delta \mathrm{V}}{3 \mathrm{RT}}\right)}
$$

It was found [9] that when the applied potential difference was less $20 \mathrm{mV}$, the error in approximation equation (6-2-7) would be small, but that when the potential difference across the electrolyte was higher than about $30 \mathrm{mV}$, the error would increase very. rapidly. In the present case, when $\Delta \mathrm{V}<20 \mathrm{mV}$, the error was less than $1 \%$, and when $\Delta V=60 \mathrm{mV}$, the error should reach about $20 \%$. This predicts that the linear $I_{s}$ vs $\Delta V$ relationship should hold up to $20 \mathrm{mV}$ for a fully dissociated polymer electrolyte. It is of interest to notice that the linear $I_{s}$ vs $\Delta V$ relationship in Figure 6-9 held up to $60 \mathrm{mV}$. 
Two common factors can lead to extension of linear range of of the plot of $\Delta \mathrm{V}$ versus Is. First, if mobile neutral species $\mathrm{MX}_{2}$ are present, the linear range would be extended [10]. Second, in real fully dissociated electrolytes based on MX salt, the steady state potential can be expressed as $[11,12]$ :

$$
\begin{gathered}
\Delta V=(1+G) \frac{d \ln a_{ \pm}}{d \ln C} \frac{R T}{F} \ln \left(\frac{C_{a}}{C_{c}}\right) \\
I_{s}^{+}=-(1+G) \frac{d \ln a_{ \pm}}{d \ln C} F D_{+}\left(C_{a}-C_{c}\right)
\end{gathered}
$$

where $\mathrm{G}$ depends on the coupling of the fluxes and is related to the cross coefficient in the irreversible thermodynamics equations, $a_{ \pm}$is the mean ionic activity and (dln $a_{ \pm} / d \ln C$ ) is the thermodynamic enhancement factor. For dilute electrolytes, $G$ and (dln $a_{ \pm} / d \ln C$ ) both tend to unity. At higher ion concentrations, however, they exceed unity, leading to broader linear $I_{s}$ vs $\Delta V$ region. This is also applicable to the present case.

The limiting current fractions for PMEO- $\mathrm{Zn}\left(\mathrm{CF}_{3} \mathrm{SO}_{3}\right)_{2}$ electrolytes with different concentrations and at different temperatures are listed in Table 6-1 together with the applied potentials, bulk and interfacial resistances, initial and steady state currents etc. The limiting current fractions were less sensitive to changing salt concentration than for $\mathrm{PEO}-\mathrm{LiCF}_{3} \mathrm{SO}_{3}$ electrolytes [10]. This may imply that the conductive behaviour of both for cations and anions may not be significantly changed by concentration in the region studied.

The limiting current fractions are significantly smaller than those for PEO$\mathrm{LiCF}_{3} \mathrm{SO}_{3}$ electrolytes measured at $120^{\circ} \mathrm{C}$ with the same concentrations [11]. This is 
not unexpected. First, if it is assumed that there are only free ions in the electrolyte, because zinc is a divalent ion, it may be expected that the cation-polymer interaction will be stronger than that in a system with lithium cations, i.e. the zinc cations may be bound more tightly by the polymer chains than the lithium cations, leading to smaller mobilities and eventually to a smaller limiting current fraction. Second, if there is ion association leading to formation of neutral mobile species, because of the size of the zinc species, the mobility of the neutral species in zinc electrolyte will be smaller than that of ion pairs in the lithium system, thus also giving rise to a smaller limiting current fraction.

It was observed that the limiting current fractions decreased significantly with increasing temperature. For polymer electrolyte based on lithium salts, it has been found [10] that their limiting current fractions increased with increasing temperature; this has been explained by suggesting that the ionic association increases with temperature and ion pairs are more mobile than free cations. In the associated polymer electrolytes, as discussed above, the steady state current is controlled by the diffusion and migration of all mobile species. If a change of temperature influences the equilibria between these species, the steady state transport will be influenced. While there may be many species arising from the ion associations in the electrolyte, two simple cases can be considered. (i) $\mathrm{ZnX}+$ species are dominant in the electrolyte. Basically, there may be two principal equilibria existing in the system, i.e.

(a) $\quad \mathrm{ZnX}^{+}+\mathrm{X}^{-} \rightleftharpoons \mathrm{ZnX}_{2}$

(b) $\quad \mathrm{ZnX}^{+} \rightleftharpoons \mathrm{Zn}^{2+}+\mathrm{X}^{-}$

If $\mathrm{ZnX}_{2}$ species are mobile, effective conductivity is increased as shown by equation (6-2-18). Thus, if increase of temperature shifts equilibrium (a) from the left-hand side to the right-hand side, the limiting current fractions will increase with increasing 
temperature. In polymer electrolytes based on alkali metal salts, it has been suggested that the mobility of ion pairs is larger than that of free cations[13], because free cations are more tightly bound on polymer chains. If mobilities of associated ions are larger than that of free cations in this case, the limiting current fractions will decrease with increasing temperature when the increase of temperature favours equilibrium (b) shifting from the left-hand side to the right-hand side. Therefore, in the case with many $\mathrm{ZnX}{ }^{+}$ species, the limiting current fraction will be reduced by increasing temperature, if the increase of temperature favours dissociation.

(ii) Mobile $\mathrm{ZnX}_{2}$ species are dominant in the electrolyte. There may again be two principal equilibria in the system, i.e.

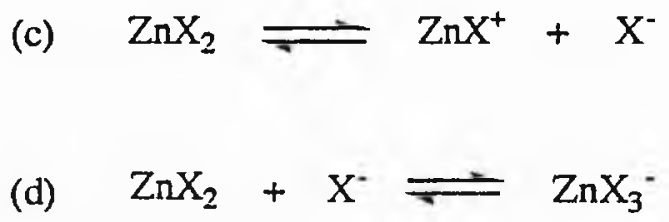

Because the steady state current flow is increased by the existence of the mobile neutral species, the consequence of shifting equilibrium (c) from the left-hand side to the right-hand side is to reduce the quantity of $\mathrm{ZnX}_{2}$ species in the system, and this may reduce the limiting current fractions with increasing temperature. If an increase of temperature shifts equilibrium (d) to the right-hand side, the steady current may again be reduced, leading to reduction of the limiting current fractions. Because $\mathrm{ZnX}_{3}$ - cannot be oxidised at the anode, the transport of $\mathrm{ZnX}_{3}{ }^{-}$species at steady state must be sustained by transport of the species produced by the association / dissociation equilibria.

Therefore, decrease of the limiting current fractions with increasing temperature may suggest that mobile $\mathrm{ZnX}_{2}$ species are dominant in the electrolyte, though there may be some $\mathrm{ZnX}^{+}$and $\mathrm{ZnX}_{3}$ - species in addition. 


\section{6-3 Measurement of Ionic Transference Number by the Hittorf Method}

Ionic transference numbers can be determined from dc polarisation measurements on symmetric cells only in the absence of mobile neutral species. In this section, the results of the application of the Hittorf method to the study of polymer electrolyte based on zinc salts is discussed. This method enables the real ionic transference numbers in PEO- $\mathrm{Zn}\left(\mathrm{CF}_{3} \mathrm{SO}_{3}\right)_{2}$ electrolyte to be determined.

\section{6-3-1 Introduction to the Hittorf Method}

(1). Ion-constituent transference number

In an electrolyte solution, especially when based on a solvent with low dielectric constant, there may exist many kinds of species which are with or without charge. For example, zinc (II) may be present in the form of $\mathrm{Zn}^{2+}, \mathrm{ZnX}^{+}, \mathrm{ZnX}_{2}$ and $\mathrm{ZnX}_{3}$ - etc in polymer electrolytes based on a $\mathrm{ZnX}_{2}$ salt. When chemical analytic methods (e.g. atomic absorption) are used to determine the concentration of zinc, what can be obtained is the total concentration of zinc(II) species (i.e. zinc ion-constituent) existing in the analyzed samples rather than just the concentration of individual species such as $\mathrm{Zn}^{2+}$, $\mathrm{ZnX}+$ etc. Thus, it is the transfer of ion-constituents rather than that of ions that can be measured when an electric current flows through the electrolyte.

Spiro [14] gave the definition of ion-constituent transference number, $T_{R}$, of a cation or anion constituent $\mathrm{R}$ as the net number of faradays carried by that constituent in the direction of the cathode or anode, respectively, across a reference plane fixed with respect to the solvent, when one faraday of electricity passes across the plane. Obviously, $T_{R}$ is a dimensionless parameter and the sum of $T_{R}$ equals unity, i.e. 


$$
\sum_{R} T_{R}=1
$$

$T_{R}$, however, can be not only positive or zero but also negative. For example, in a concentrated aqueous solution of $\mathrm{CdI}_{2}$ salt a majority of $\mathrm{Cd}(\mathrm{II})$ is present in the form of $\mathrm{CdI}_{3}{ }^{-}$and $\mathrm{CdI}_{4}{ }^{2-}$, which results in the net transfer of $\mathrm{Cd}(\mathrm{II})$ ion-constituent toward anode, and thus the transference number of $\mathrm{Cd}$ (II) ion-constituent is negative [14].

The ion-constituent transference number $T_{R}$ can be correlated to ionic transport number $t_{i}$ by following equation.

$$
T_{R}=\sum_{i}\left(z_{R} / z_{i}\right) N_{R / i} t_{i}
$$

where $z_{R}$ and $z_{i}$ are charge number of ion - constituent $R$ and ion $i$, and $N_{R / i}$ are moles of ion - constituent $\mathrm{R}$ in each mole of ion $\mathrm{i}$.

In the following discussion, the term "the ionic transference number" means the ion-constituent transference number. For example, the cationic transference number in $\mathrm{PEO}-\mathrm{Zn}\left(\mathrm{CF}_{3} \mathrm{SO}_{3}\right)_{2}$ electrolyte actually means the zinc(II) ion-constituent transference number.

\section{(2). Principle of the Hittorf method}

Consider the cell illustrated in Figure 6-10 containing an electrolyte with solute $\mathrm{MX}_{2}$. The cell may formally be separated into five compartments, i.e., EA, EC for anode and cathode compartments respectively, MA, MM and MC for anode middle, middle middle and cathode middle compartments respectively. At the anode, metal $M$ is oxidized to $M($ II) and at the cathode, M(II) ions are reduced to $M$ metal. 
Supposing the $M(I I)$ ion-constituent transference number is $T_{+}$, the changes of $\mathrm{M}(\mathrm{II})$ ion-constitutes concentration when $\mathrm{Q}$ coulombs electric charge passed through the cell are expressed as follows.

In the anode compartment: $\quad \mathrm{Q} / 2 \mathrm{~F}$ moles of $\mathrm{M}(\mathrm{II})$ ions are produced by oxidizing $\mathrm{M}$ metal and $(\mathrm{Q} / 2 \mathrm{~F}) \mathrm{T}_{+}$moles of $\mathrm{M}(\mathrm{II})$ ion-constituents migrate out. Thus, the change of moles of $\mathrm{M}(\mathrm{II})$ ion-constituents in the anode compartment is:

$$
\Delta \mathrm{m}=\frac{\mathrm{Q}}{2 \mathrm{~F}}\left(1-\mathrm{T}_{+}\right)
$$

In the cathode compartment: $\mathrm{Q} / 2 \mathrm{~F}$ moles of $\mathrm{M}(\mathrm{II})$ ion - constituents are reduced to metal, and $(\mathrm{Q} / 2 \mathrm{~F}) \mathrm{T}_{+}$moles of $\mathrm{M}(\mathrm{II})$ ion-constituents migrate in. Thus the change of moles of M(II) ion-constituents in the cathode compartment is:

$$
|\Delta \mathrm{m}|=\frac{\mathrm{Q}}{2 \mathrm{~F}}\left(1-\mathrm{T}_{+}\right)
$$

Therefore, the $M(I I)$ ion-constituent transference number, based on the change of number of moles of M(II) ion-constituents in the anode or / and cathode compartments is:

$$
\mathrm{T}_{+}=1-\frac{2 \mathrm{~F}}{\mathrm{Q}}|\Delta \mathrm{m}|
$$

In the middle compartments: Because the number of moles of $\mathrm{M}(\mathrm{II})$ ion-constituents migrating in and out are equal in these compartments, there should be no net change of concentration of M(II) ion-constitutes.

The Hittorf method is a non-steady state method. In order to ensure the change of concentration of $\mathrm{M}(\mathrm{II})$ ion-constituents is limited only to the anode and cathode compartments, the distance between these two electrodes is usually designed to be large, while the polarisation time is kept relatively short. The middle compartments are always 
analyzed to ensure that no concentration change has occurred. The accuracy of the experiment depends completely on the accuracy of analysis of the concentration of $M(I I)$ ion-constituent in each compartment, especially in anode and cathode compartments.

The Hittorf method has been widely applied to the study of ionic transport properties of liquid electrolytes [15], because, after passing electric current through the electrolyte, the separation of each compartment of liquid electrolyte is straightforward. Cameron and corworkers [16] have applied the Hittorf method to studying the liquid polymer electrolytes based on sodium salts. Recently, Bruce and coworkers [17] have applied the Hittorf method to the study of solid PEO-LiX electrolytes by designing a suitable polarisation cell. This cell was described in Chapter 3.

\section{6-3-2 Experimental}

(1) Polarisation of the cell

Polarisation of a Hittorf cell with PEO-Zn( $\left(\mathrm{CF}_{3} \mathrm{SO}_{3}\right)_{2}$ electrolytes and zinc anode and lead cathode was carried out at $120^{\circ} \mathrm{C}$ with a constant current of $4 \mu \mathrm{A}$ for 65 hours, as described in Chapter $3(3-5-2)$. The quantity of charge passed through the electrolyte can usually be accurately determined by recording the time and current precisely. In this experiment, the current was set with accuracy of $\pm 0.05 \mu \mathrm{A}$, and the time was recorded by a watch with accuracy of $\pm 0.5 \mathrm{~s}$.

(2) Sample weighing

After passing electric current through the polymer electrolyte, it was cut into four pieces, i.e., A, MA, MC and C for anode, middle anode, middle cathode and cathode compartments respectively. Each compartment was about $60 \mathrm{mg}$, which was divided to 6 pieces. The maximum weight that a 6 -figure micro-balance could weigh was about 15 mg. Each piece was weighed on the micro-balance in a glovebox with an error of $\pm 0.001 \mathrm{mg}$. Thus, the error for weighing a whole compartment was about $\pm 0.006 \mathrm{mg}$. 
(3) Digestion of polymer electrolyte

The weighed polymer electrolytes, A, MA, MC and stock sample S which was cut from the same electrolyte disc as used for the electrolysis were put in Teflon bombs. In each bomb, $1 \mathrm{~cm}^{3}$ of concentrated percholoric acid (Aldrich) and $6 \mathrm{~cm}^{3}$ of concentrated sulphuric acid were subsequently added and the cap of the bomb was then tightly sealed. The bombs were placed in a microwave oven to be heated with medium power level for 0.5 minute at first, then for another 0.5 minute, and finally for 2 minutes. After heating, the bombs were allowed to cool down for 2 hours before the tops were opened. The transparent solutions produced were diluted by distilled water to $100 \mathrm{~cm}^{3}$. Then, about $10 \mathrm{~cm}^{3}$ of the solution were further diluted into $100 \mathrm{~cm}^{3}$ to give a zinc content in the range of $0.5-2 \mathrm{ppm}$, ready for analysis by atomic absorption spectroscopy.

(4) Separation of zinc dendrites in the cathodic compartment

Any zinc dendrites which had formed on the cathode and were attached to the electrolyte in the cathode compartment were separated by filtration. The weighed cathode compartment was dissolved in anhydrous acetonitrile. The solution was then filtered through a quantitative filter paper $(7.0 \mathrm{~cm}$, Watermann). The zinc dendrites were left on the filter paper, which was washed five times by acetonitrile.

The filtered electrolyte solution was put in a $100 \mathrm{~cm}^{3}$ round bottom flask. The solvent was removed under a reduced pressure. Solid polymer electrolyte was then obtained. This electrolyte was further dried under vacuum at $80^{\circ} \mathrm{C}$ for 8 hours. The flask with the polymer electrolyte was then transferred into a glovebox, where the polymer electrolyte was weighed by the 6-figure micro-balance. This polymer electrolyte was digested as described above. 
The filter paper with the zinc dendrites was dried and then soaked in $2 \mathrm{M}$ $\mathrm{H}_{2} \mathrm{SO}_{4}$. This was again filtered to remove the paper. The filtered solution was diluted to $100 \mathrm{~cm}^{3}$, and then was ready to the $\mathrm{Zn} \mathrm{AAS} \mathrm{analysis.}$

(5) Zn atomic absorption analysis

The experimental conditions have been described in Chapter 3 (3-7). The accuracy of Zn-AAS for the spectrometer used in this study was about $\pm 0.01 \mathrm{ppm}$. Because the digested polymer electrolyte was typically diluted to form $100 \mathrm{~cm}^{3}$ solution, and $10 \mathrm{~cm}^{3}$ of it was diluted to $100 \mathrm{~cm}^{3}$ for $\mathrm{Zn}$-AAS analysis, the total error in the analysis of the zinc content in a whole compartment was approximately $\pm 0.01 \mathrm{mg}$.

\section{6-3-3 Determination of the Ionic Transference Number}

When changes of the concentration of salt in anode or cathode compartments are determined, ionic transference number can be calculated from equation (6-3-5). For the polymer electrolyte in the anode and cathode compartments, the EO/M ratio was changed due to the change of salt concentration. Thus, equation (6-3-5) must be modified before calculating for the ionic transference number of a polymer electrolyte.

Suppose that the anode compartment with a weight of $\mathrm{W}$ grams (with $\mathrm{EO} / \mathrm{Zn}=$ $\mathrm{R}$ ) is digested by acid, and is then diluted to $100 \mathrm{~cm}^{3}$ aqueous solution $\mathrm{A}$. $\mathrm{L} \mathrm{cm}^{3}$ of the solution $\mathrm{A}$ is further diluted to $100 \mathrm{~cm}^{3}$. The zinc content measured by AAS is $\mathrm{m}$ ppm after electrolysis. The quantity of charge $Q=I t$, where $I$ is the current and $t$ is the time for electrolysis. Thus, the salt concentration after polarisation is:

$$
M_{1}=\frac{m}{65.38 \mathrm{~L}} \times 10^{-2} \quad \text { mole }
$$

where 65.38 is the atomic weight of zinc. The weight $P_{w}$ of pure PEO in the polymer electrolyte of anode compartment is: 


$$
P_{W}=W-\frac{363.5 \mathrm{~m}}{65.38 \mathrm{~L}} \times 10^{-2} \quad \mathrm{~g}
$$

where 363.5 is the molecular weight of $\mathrm{Zn}\left(\mathrm{CF}_{3} \mathrm{SO}_{3}\right)_{2}$ salt. Thus, the zinc content, in the polymer electrolyte before polarisation is:

$$
\mathrm{M}_{2}=\frac{\mathrm{P}_{\mathrm{W}}}{44.05 \mathrm{R}} \quad \text { mole }
$$

where 44.05 is the formula weight of EO unit. The change of zinc content is:

$$
\Delta M=M_{1}-M_{2}=\frac{10^{-2} m}{65.38 L}-\frac{P_{w}}{44.05 R} \quad \text { mole }
$$

The anionic transference number according to equation (6-3-5) is:

$$
\mathrm{T}_{.}=\frac{2 \Delta \mathrm{MF}}{\mathrm{Q}}=\frac{2 \mathrm{~F}}{\mathrm{It}}\left[\frac{10^{-2} \mathrm{~m}}{65.38 \mathrm{~L}}-\frac{1}{44.05 \mathrm{R}}\left(\mathrm{W}-\frac{363.5 \times 10^{-2} \mathrm{~m}}{65.38 \mathrm{~L}}\right)\right]
$$

Although zinc can easily forms alloys with lead metal which was used as cathode, some zinc metal dendrites were still found to form in the cathode compartment. This caused an apparent increase in zinc content in the cathode compartment, rather than the decrease expected due to reduction of zinc cations in the electrolyte after polarisation (Figure 6-11). In order to get rid of the contamination of the cathode compartment by zinc dendrites, polymer electrolyte and zinc dendrites were separated as described above. The calculation of ionic transference number is not much different from equation (6-3-6), except that the weight $W$ of cathode compartment is now not solely the weight $\mathrm{W}_{\mathrm{p}}$ of polymer electrolyte. If the zinc dendrite content measured by AAS is $\mathrm{n}$ ppm, and it was prepared to $100 \mathrm{~cm}^{3}$ solution of which $1 \mathrm{~cm}^{3}$ was diluted again to $100 \mathrm{~cm}^{3}$, the weight of polymer electrolyte is: 


$$
W_{P}=W-\frac{n \times 10^{-2}}{1} \quad g
$$

where $\mathrm{W}$ is the gross weight of the cathode compartment, $\mathrm{W}_{\mathrm{p}}$ is the net weight of polymer electrolyte in the cathode compartment, and $10^{-2} n / 1$ is the weight of zinc dendrites. Because only part of the polymer electrolyte was taken for AAS analysis, the formula for the calculation of the anionic transference is the same as (6-3-6) except for replacing $\mathrm{W}$ by $\mathrm{W}_{\mathrm{p}}$, but the AAS measured zinc content must be converted to the that in the whole polymer electrolyte in the cathode compartment, i.e. $m$ in equation (6-3-6) should be replaced by $m^{\prime} W_{p} / W^{\prime}$, where $m$ ' is the zinc content measured by AAS in the $\mathrm{W}^{\prime}$ polymer electrolyte, and $\mathrm{W}^{\prime}$ is the weight of polymer electrolyte taken for AAS analysis. Thus, the equation for the calculation of $T$. based on the cathode compartment is:

$$
T_{-}=\frac{-2 \mathrm{~F}}{\mathrm{It}}\left[\frac{10^{-2} \mathrm{~m}^{\prime} \mathrm{W}_{\mathrm{P}}}{65.38 \mathrm{LW}^{\prime}}-\frac{1}{44.05 \mathrm{R}}\left(\mathrm{W}_{\mathrm{P}}-\frac{363.5 \times 10^{-2} \mathrm{~m}^{\prime} \mathrm{W}_{\mathrm{P}}}{65.38 \mathrm{LW}^{\prime}}\right)\right]
$$

In order to check the filtration method for separating the zinc dendrites, zinc powder ( 100 mesh, $99.999 \%$, Aldrich) was mixed with polymer electrolyte. This mixture was then dissolved in anhydrous acetonitrile, which was separated by filtering through a $7.0 \mathrm{~cm}$ quantitative filter paper (Watermann). The preparation of zinc solution and evaporation of the solvent, digestion of polymer are the same as described above.

The weight of zinc powder added into the electrolyte was $3.316 \mathrm{mg}$, which was prepared into $100 \mathrm{~cm}^{3}$ solution. $5 \mathrm{~cm}^{3}$ of this solution was further diluted into $100 \mathrm{~cm}^{3}$ for AAS. The weight of the polymer electrolyte $\left[\mathrm{PEO}-\mathrm{Zn}\left(\mathrm{CF}_{3} \mathrm{SO}_{3}\right)_{2}\right.$ with $\mathrm{EO} / \mathrm{Zn}$ of 50.05] was $68.594 \mathrm{mg}$. The weight of the electrolyte for digestion was $32.114 \mathrm{mg}$. The content of zinc powder left on the filter paper measured by Zn AAS without calibration 
was similar to the actual content within the error range $\pm 0.02 \mathrm{mg}$. The zinc content in the polymer electrolyte measured by AAS was also similar to the actual content within the error range of $\pm 0.01 \mathrm{ppm}$, after being determined by the calibration curves as outlined below. All the results are listed in Table 6-2. This indicates that separation of zinc powder from the polymer electrolyte can be successfully carried out by filtration.

\section{6-3-4 Experimental Results}

(1) Zn AAS of the stock polymer electrolyte sample

Theoretically, after the polymer host has been digested, the zinc content given by the $\mathrm{Zn}$-AAS analysis should be the same as the actual content in the polymer electrolyte within experimental error. However, the zinc content measured by the AAS analysis was about $75 \%$ of the actual value in the stock polymer electrolyte (Table 6-3). It has been found that $\mathrm{Zn}$-AAS measurement on cationic surfactant containing 8-10 ethoxy units [18] had interference caused by the formation of ion association complexes in the system. In the present case, if the polymer was digested into very short chains which may be like the cationic surfactant, the zinc content measured by the AAS analysis may be also be affected by the interference. The lower zinc content measured by the AAS may be partially attributed to this factor.

By diluting the solution prepared from the stock sample, a straight line can be derived by plotting the zinc content in the solution measured by the AAS analysis against the actual content (Figure 6-12). By using this straight line as a calibration curve, the zinc content measured can be calibrated to the actual content with the error of $\pm 0.02 \mathrm{ppm}$. This was repeated for five stock samples and a calibration curve was produced. The results are listed in Table 6-3.

(2) Zinc content in the middle compartments

The zinc contents in the middle compartments, either MA or MC for three 
experiments are listed in Table 6-4a. They were no changes before and after the electrolysis, which indicated that changes of the salt concentration were indeed limited in the electrode compartments.

(3) Analysis of the anode compartment

The zinc content in the anode compartment was increased after the electrolysis (Figure 6-11).This is expected according to equation (6-3-3), if $T_{+}<1$. The AAS data and calibrated data for a Hittorf experiment are listed in Table 6-4. The AAS data in four experiments were determined by corresponding calibration curves $1,2,3$ and 4 respectively (Figure 6-12) with the calibration equation: $y=0.0299+1.245 \mathrm{x}$ for curve $1, y=0.0705+1.220 x$ for curve $2, y=0.1006+1.203 x$ for curve 3 and $y=0.0778+1.224 x$ for curve 4 .

The anionic transference $T$. was calculated by equation $(6-3-6)$, where $\mathrm{Q}=0.90 \mathrm{C}, \mathrm{L}=8 \mathrm{ml}, \mathrm{W}=87.451 \mathrm{mg}, \mathrm{R}=50.05$, and $\mathrm{m}$ was taken from by the above calibration equations. The calculated $T$. for each experiment is: $0.95,1.00,0.92$ and 0.92 (Table 6-4b). The mean value of $T_{-}$is 0.95 with a standard deviation of 0.038 .

(4) Analysis of the cathode compartment

According equation (6-3-4), if $T_{+}<1$, the content of zinc in the cathode compartment after the electrolysis should be reduced. However, if the cathode compartment was directly digested without separation of the zinc dendrites, the content of zinc does not decrease at all (Figure 6-11).

The zinc dendrites in the cathode compartment were separated by the above method and the zinc content in the electrolyte in the cathode compartment was analysed. The results are listed in Table 6-4c. 
The weight of the polymer electrolyte in the cathode compartment, $\mathrm{W}_{\mathrm{p}}$, can be calculated by equation (6-3-7), where $\mathrm{W}=67.299 \mathrm{mg}, \mathrm{l}=15 \mathrm{ml}, \mathrm{n}$ is listed in Table 6-7. The anionic transference can be calculated by modified equation (6-3-8), where $\mathrm{m}^{\prime}$ is listed in Table 6-4c, $\mathrm{W}^{\prime}=21.212 \mathrm{mg}, \mathrm{R}=50.05, \mathrm{~L}=20 \mathrm{ml}$ and $\mathrm{Q}=\mathrm{It}=0.90 \mathrm{C}$.

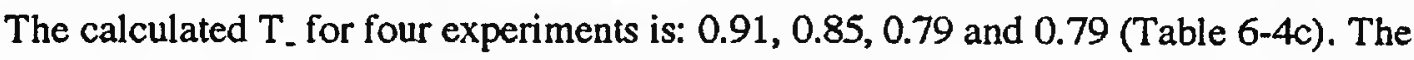
mean value of $T_{-}$is 0.84 with a standard deviation of 0.062 .

\section{6-3.5 Transference Number in $\mathrm{PEO}-\mathrm{Zn}\left(\mathrm{CF}_{3} \mathrm{SO}_{3}\right)_{2}$ Electrolyte}

The mean anionic transference number determined based on analysing the anode and cathode compartments is: 0.95 and 0.84 respectively. From equation (6-3-3) and (6-3-4), the transference number based on analysing the anode and cathode compartments should be the same. The following factors may influence the accuracy of the transference number determined, and account for the significant difference in the two determinations.

For the analysis based on the anode compartment, if there is any passivating layer at the zinc anode which can be physically broken down during the electrolysis as discussed in the section $6-1$, the zinc content will be overestimated, because the passivating layer can produce zinc ions in the anode compartment without a faradaic process. This leads to overestimating the change in zinc content in this compartment. In consequence, the anionic transference number may be overestimated. In the present case, because the zinc electrode was freshly scraped in the glovebox before the electrolysis, and the area of the electrode was quite small (less than $0.1 \mathrm{~cm}^{2}$ ), the zinc ions produced by breaking down the passivating layer may not be significant, and the error in the transference number based on analysing the anode compartment may not be significant.

For the analysis based on the cathode compartment, there are likely to be more 
factors influencing the determination of the transference number. First, if there are some impurities such as $\mathrm{H}_{2} \mathrm{O}$ in the electrolyte, these may be reduced at the cathode, eg. $\mathrm{H}^{+}$is reduced into $\mathrm{H}_{2}$, which may lead to less change of the zinc content in the compartment and a smaller anionic transference number. This factor may not be too important in the present case, because the polymer electrolyte was made by the grinding / hot pressing method in the glovebox. Second, if the zinc dendrites are not well separated from the electrolyte, the change of the zinc content in this compartment will be underestimated, which will again lead to a smaller anionic transference number. This factor may have to be considered here. Although it was shown that zinc powder ( 100 mesh) can be separated from the electrolyte satisfactorily, if some of the zinc dendrites are too fine, they may get through the filter paper. Third, if some electrolyte solution is lost during the separation of the dendrites, the change of the zinc content may be apparently too high, which may lead to an overestimated anionic transference number. The apparently smaller anionic transference number based on the analysis of the cathode compartment may be attributed to the first two factors.

From the above analysis, it is suggested that the anionic transference number based on the analysis of the anode compartment, i.e. $T_{\text {. }}=0.95$, is more reliable.

At first sight, the near-unity value of the anionic transference number may be taken to indicate that the zinc species are immobile in the electrolyte. This conclusion is probably oversimplified, because it does not confirm with the results of the limiting current fraction measured by the steady state current method, in which the current fraction is about 0.2 . In fact, there may be many reasons leading to very small cationic transference numbers in the polymer electrolytes.

(i) Because the ion constituent transference number is related to the ionic transport numbers by formula (6-3-2), if in addition to the cationic zinc species, such as $\mathrm{Zn}^{2+}$ and 
$\mathrm{ZnX}+$ etc., there are enough mobile zinc species with negative charge, eg. $\mathrm{ZnX}_{3}{ }^{-}$, in the electrolyte, the cationic transference number $T_{+}$may be very small, or even zero and negative. However, at steady state, because $\mathrm{ZnX}_{3}$ - species move towards the anode and cannot be oxidised on the anode, the existence of many stable $\mathrm{ZnX} \mathrm{X}_{3}^{-}$species will reduce the steady state transport, leading to small limiting current fraction. This case is not consistent with the results of the limiting current fraction.

(ii) If mobile neutral $\mathrm{ZnX}_{2}$ are the dominant species in the electrolyte, the cationic transference number will be very small, because of the zero charge of the $\mathrm{ZnX} \mathrm{X}_{2}$ species. The mobile uncharged $\mathrm{ZnX} \mathrm{X}_{2}$ species can contribute to the steady state transport, and may lead to a significant limiting current fraction. Thus, this case is more plausible.

It may therefore be concluded that, in $\mathrm{PEO}-\mathrm{Zn}\left(\mathrm{CF}_{3} \mathrm{SO}_{3}\right)_{2}$ electrolyte, $\mathrm{ZnX} 2$ species may be the principal mobile zinc-containing species; in addition there may also exist some $\mathrm{ZnX}+\mathrm{ZnX}_{3}-$ species due to the existence of association / dissociation equilibria. This seems to agree with the conclusions of the molecular dynamics simulation and EXAFS of $\mathrm{PEO}-\mathrm{ZnBr}_{2}$ electrolyte $[1,19]$.

\section{6-4 Conductivity of PMEO- $\mathrm{Zn}\left(\mathrm{CF}_{3} \mathrm{SO}_{3}\right)_{2}$ Electrolyte}

\section{6-4-1 Phase Behaviour}

The DSC trace of $\mathrm{PMEO}-\mathrm{Zn}\left(\mathrm{CF}_{3} \mathrm{SO}_{3}\right)_{2}$ with $\mathrm{EO} / \mathrm{Zn}$ ratio of $20: 1$ is shown in Figure $6-13 \mathrm{a}$. There are no peaks in the temperature range from $30^{\circ} \mathrm{C}$ to $150^{\circ} \mathrm{C}$. This indicates that the 20:1 PMEO-Zn( $\left(\mathrm{CF}_{3} \mathrm{SO}_{3}\right)_{2}$ electrolyte, like the 20:1 PMEO-LiClO 4 electrolyte, is totally amorphous at temperatures above room temperature.

The DSC trace of $\mathrm{PEO}\left(4 \times 10^{6}\right)-\mathrm{Zn}\left(\mathrm{CF}_{3} \mathrm{SO}_{3}\right)_{2}$ with $\mathrm{EO} / \mathrm{Zn}$ ratio of $20: 1$ is also 
shown in Figure 6-13b. There is an endothermic peak at $72.2^{\circ} \mathrm{C}$, which may correspond to the melting point of crystalline PEO. It seems that no crystalline complexes are formed in this electrolyte. This is consistent to the results of X-ray diffraction as shown in Figure 3-7 (Chapter 3), where the patterns of the crystalline complexes have been shown for $\mathrm{PEO}-\mathrm{Zn}\left(\mathrm{CF}_{3} \mathrm{SO}_{3}\right)_{2}$ with the EO/Zn ratio of $4: 1$, and their intensities have been drastically reduced in the sample with the EO/Zn ratio of $6: 1$.

\section{6-4-2 Conductivity}

The temperature dependence of conductivity for electrolytes based on PMEO or $\mathrm{PEO}$ and $\mathrm{Zn}\left(\mathrm{CF}_{3} \mathrm{SO}_{3}\right)_{2}$ salt is shown in Figure 6-14. The salt concentration dependence of conductivity for PMEO-Zn( $\left(\mathrm{CF}_{3} \mathrm{SO}_{3}\right)_{2}$ electrolytes at $30^{\circ} \mathrm{C}$ and $60^{\circ} \mathrm{C}$ is shown in Figure 6-15. There is a maximum at an EO/Zn ratio of $50: 1$, which is very close to that

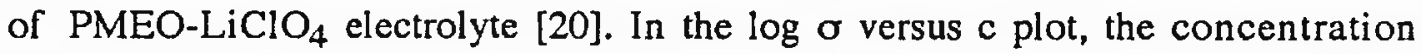
dependence of the conductivity is stronger at low temperature than at high temperature. This may be explained by suggesting that the transient crosslinking effect of the salt which usually increases with salt concentration may be less important at high temperature, because the segmental motion is already fast. The conductivity of the electrolyte with the $\mathrm{EO} / \mathrm{Zn}$ ratio of $50: 1$ is comparable with that of $\mathrm{PMEO}^{-\mathrm{LiClO}_{4}}$ with an EO/Li ratio of 50:1 [20]. On one hand, because zinc ion is divalent, and each zinc ion interacts with two anions, if the salt is fully dissociated, the conductivity of the electrolyte may be higher than that with lithium salt, providing that the transient crosslinking effect is similar. On the other hand, the transient crosslinking effect in zinc system may be more significant than in the lithium system, leading to smaller mobilities of ions, and eventually leading to a decrease in the conductivity. These two contras: factors may consequently give rise a conductivity in the zinc system which is comparable with that in the lithium electrolyte.

The ionic conduction of PMEO- $\mathrm{Zn}\left(\mathrm{CF}_{3} \mathrm{SO}_{3}\right)_{2}$ electrolytes displays a non- 
Arrhenius behaviour. This is because the electrolytes are totally amorphous in the temperature range studied. Usually, the conduction in such samples can be described by the VTF equation

$$
\sigma=\sigma_{0} \exp \left[-\mathrm{B} /\left(\mathrm{T}-\mathrm{T}_{\mathrm{o}}\right)\right]
$$

where $\sigma_{0}, B$ and $T_{0}$ are fitted constants. Thus, the conductivity data of PMEO$\mathrm{Zn}\left(\mathrm{CF}_{3} \mathrm{SO}_{3}\right)_{2}$ electrolytes were fitted by the VTF equation as described in Chapter 5. The fitted parameters are listed in Table 6-5.

The fitted $T_{0}$ and $B$ for the electrolyte with the lowest salt concentration $(\mathrm{EO} / \mathrm{Zn}=100)$ are similar to those for $\mathrm{PMEO}-\mathrm{LiClO}_{4}$ with low salt concentration $(<0.1$ mol dm${ }^{-3}$ ). This may imply that the mechanism for ion transport in the zinc electrolyte is the same as in the lithium electrolyte with low salt concentration. When the concentration zinc salt increased, the $T_{0}$ changes irregularly, which may imply deviation from VTF behaviour due to the change of mechanism for ion transport as seen in the lithium system.

For PEO- $\mathrm{Zn}\left(\mathrm{CF}_{3} \mathrm{SO}_{3}\right)_{2}$ with a EO/Zn ratio of $50: 1$, there is a "knee point" at around $71^{\circ} \mathrm{C}$ in the plot of $\log \sigma$ against $1000 / \mathrm{T}$, which may correspond to the eutetic point of the electrolyte. In the temperature region below this point, an Arrhenius-like behaviour is noted. In the temperature region above this point, a curved line is found, which may correspond to the VTF behaviour. As expected, the conductivity at the temperature region above the "knee point" is comparable to that of PMEO- $\mathrm{Zn}\left(\mathrm{CF}_{3} \mathrm{SO}_{3}\right)_{2}$ with the EO/Zn of $50: 1$. 


\section{6-5 Conclusion}

There are passivating layers on the zinc metal electrodes in the $\mathrm{Zn} / \mathrm{PMEO}$ $\mathrm{Zn}\left(\mathrm{CF}_{3} \mathrm{SO}_{3}\right)_{2} / \mathrm{Zn}$ cell, which can block the current flow. The passivating layer can be reduced by "electropolishing", i.e. dc polarisation by application of successive $\pm 0.5 \mathrm{~V}$ potentials.

PMEO-Zn( $\left(\mathrm{CF}_{3} \mathrm{SO}_{3}\right)_{2}$ electrolytes are totally amorphous above room temperature. The maximum conductivity appears at an EO/Zn ratio of $50: 1$, which is comparable with that of $\mathrm{PMEO}-\mathrm{LiClO}_{4}$ with an EO/Li ratio of 50:1. The ionic conducting behaviour of these electrolytes is non-Arrhenius and can be fitted to the VTF equation.

De polarisation of $\mathrm{Zn} / \mathrm{PMEO}-\mathrm{Zn}\left(\mathrm{CF}_{3} \mathrm{SO}_{3}\right)_{2} / \mathrm{Zn}$ cell shows that zinc species are mobile in the system. The current fractions have been found to be insensitive to changes of the salt concentration in the electrolytes for all samples examined, but, decrease with increasing temperature. The current fraction of $\mathrm{PMEO}-\mathrm{Zn}\left(\mathrm{CF}_{3} \mathrm{SO}_{3}\right)_{2}$ electrolyte is 0.3 at $30^{\circ} \mathrm{C}$ and 0.2 at about $80^{\circ} \mathrm{C}$.

The anionic transference number determined by the Hittorf method in PEO$\mathrm{Zn}\left(\mathrm{CF}_{3} \mathrm{SO}_{3}\right)_{2}$ electrolytes is: 0.95 with a standard deviation of 0.038 , based on the analysis of the anode compartment, 0.84 with a standard deviation of 0.062 , based on the analysis. The former value may be somewhat more reliable. The main zinc species in the polymer electrolytes are assigned to be mobile uncharged triples, $\mathrm{Zn}\left(\mathrm{CF}_{3} \mathrm{SO}_{3}\right)_{2}$, while there may also exist some $\mathrm{Zn}\left(\mathrm{CF}_{3} \mathrm{SO}_{3}\right)^{+}$and $\mathrm{Zn}\left(\mathrm{CF}_{3} \mathrm{SO}_{3}\right)_{3}^{-}$species. 


\section{References}

[1] M. Cole, R. J. Latham, R. G. Linford, W. S. Schlindwein and M. H. Sheldon, Material Research Soc. 1988 Fall Meeting, paper M61.

[2] P. M. Blonsky, P. F. Shriver, P. Austin and H. R. Allcock, Solid St. Ionics, 18/19, 258 (1986).

[3] H. Yang and G. C. Farrington, J. Electrochem. Soc., 139, 1646 (1992).

[4] P. G. Bruce, in Polymer Electrolyte Review 1, ed. J. R. MacCallum and C. A. Vincent, Elsevier, London, 1987, page 237.

[5] D. Fauteux, Solid St. Ionics, 17, 133 (1985).

[6] D. Fauteux, J. Electrochem. Soc., 135, 2231 (1988).

[7] S. Biallozor and E. T. Bandura, Electrochim. Acta, 32, 891 (1987).

[8] J. M. West, Electrodeposition and Corrosion Processes, 2nd ed. van Nostrand Reinhold Co., London, 1970.

[9] P. G. Bruce and C. A. Vincent, J. Electroanal. Chem., 225, 1 (1987).

[10] M. T. Hardgrave, PhD thesis, University of St. Andrews, 1990. 
[11] P. G. Bruce, M. T. Hardgrave and C. A. Vincent, Electrochim. Acta, 37, 1517 (1992).

[12] P. G. Bruce, Synthetic Metals, 45, 267 (1991).

[13] G. G. Cameron and M. D. Ingram, in Polymer Electrolyte Review 2, ed. J. R. MacCallum and C. A. Vincent, Elsevier, London, 1989.

[14] M. Spiro, in Physical Methods of Chemistry Part II A, Eelectrochemical Methods, ed. A. Weissberger and B. W. Rossiter, Wiley-Interscience, New York, 1971.

[15] G. Jones and M. Dole, J. Amer, Chem. Chem. Soc., 51, 1073 (1929).

[16] G. G. Cameron, M. D. Ingram and J. L. Harvie, Disc. Farad. Soc. 88, 55 (1989).

[17] P. G. Bruce, M. T. Hardgrave and C. A. Vincent, Solid St. Ionics, 53-56, 1087 (1992).

[18] S. S. Hassan, Organic Analysis Using Atomic Absorption Spectrometry, Ellis Horwood Ltd. Chichester, 1984.

[19] L. Xie and G. C. Farrington, Solid St. Ionics, 53-56, 1054 (1992).

[20] F. Gray, Solid St. Ionics, 40 / 41, 637 (1990). 
Table 6-1a. Polarisation data for $\mathrm{Zn} / \mathrm{PMEO}-\mathrm{Zn}\left(\mathrm{CF}_{3} \mathrm{SO}_{3}\right)_{2}(\mathrm{EO} / \mathrm{Zn}=10) / \mathrm{Zn}$ cell with different potentials at $84^{\circ} \mathrm{C}$

\begin{tabular}{llllll}
\hline$\Delta \mathrm{Va} / \mathrm{mV}$ & $\mathrm{Iob} / \mu \mathrm{A}$ & $\mathrm{Is} / \mu \mathrm{A}$ & Rio $/ \mathrm{k} \Omega$ & $\mathrm{Ris} / \mathrm{k} \Omega$ & $\mathrm{F}_{+}$ \\
\hline 18.6 & 5.28 & 1.24 & 0.92 & 1.09 & 0.19 \\
21.5 & 6.34 & 1.84 & 2.30 & 4.60 & 0.20 \\
32.7 & 9.24 & 2.26 & 1.64 & 3.21 & 0.19 \\
36.5 & 11.80 & 2.99 & 1.09 & 1.17 & 0.19 \\
41.8 & 5.28 & 3.39 & 7.18 & 2.41 & 0.19 \\
51.3 & 13.76 & 3.02 & 0.74 & 1.23 & 0.19 \\
61.7 & 13.16 & 3.13 & 2.66 & 2.65 & 0.14 \\
71.8 & 16.26 & 4.44 & 2.53 & 1.84 & 0.15 \\
90.6 & 18.18 & 3.44 & 2.18 & 2.72 & 0.13 \\
112.0 & 32.40 & 3.85 & 0.92 & 1.99 & 0.10 \\
\hline
\end{tabular}

a: corrected for interfacial losses;

b: calculated from ac impedance and Ohm's Law.

Table 6-1b. Polarisation data for $\mathrm{Zn} / \mathrm{PMEO}-\mathrm{Zn}\left(\mathrm{CF}_{3} \mathrm{SO}_{3}\right)_{2}(\mathrm{EO} / \mathrm{Zn}=10) / \mathrm{Zn}$ cell at different temperatures

\begin{tabular}{lllllll}
\hline $\mathrm{T} /{ }^{\circ} \mathrm{C} \Delta \mathrm{V} / \mathrm{mV}$ & $\mathrm{I}_{\mathrm{o}} / \mu \mathrm{A}$ & $\mathrm{I}_{\mathrm{s}} / \mu \mathrm{A}$ & $\mathrm{R}_{\mathrm{io}} / \mathrm{k} \Omega$ & $\mathrm{R}_{\mathrm{is}} / \mathrm{k} \Omega$ & $\mathrm{F}_{+}$ \\
\hline 30 & 13.0 & 1.38 & 0.70 & 12.3 & 15.3 & 0.34 \\
63 & 20.6 & 2.17 & 0.86 & 8.06 & 10.9 & 0.24 \\
84 & 18.6 & 5.28 & 1.24 & 0.92 & 1.09 & 0.19 \\
\hline
\end{tabular}


Table 6-1c. Polarisation data for $\mathrm{Zn} / \mathrm{PMEO}-\mathrm{Zn}\left(\mathrm{CF}_{3} \mathrm{SO}_{3}\right)_{2} / \mathrm{Zn}$ cell with different salt concentrations at $84^{\circ} \mathrm{C}$

\begin{tabular}{lllllll}
\hline EOLi & $\Delta V / \mathrm{mV}$ & $\mathrm{I}_{\mathrm{o}} / \mu \mathrm{A}$ & $\mathrm{I}_{\mathrm{s}} / \mu \mathrm{A}$ & $\mathrm{R}_{\mathrm{io}} / \mathrm{k} \Omega$ & $\mathrm{R}_{\mathrm{is}} / \mathrm{k} \Omega$ & $\mathrm{F}_{+}$ \\
\hline $10: 1$ & 32.7 & 9.24 & 2.26 & 1.64 & 3.21 & 0.19 \\
$20: 1$ & 19.7 & 34.3 & 7.35 & 0.31 & 0.43 & 0.15 \\
$50: 1$ & 27.1 & 23.5 & 15.6 & 0.95 & 0.18 & 0.19 \\
$50: 1^{\mathrm{a}}$ & 18.6 & 27.4 & 7.80 & 0.05 & 0 & 0.26 \\
\hline
\end{tabular}

a: data for $\mathrm{PEO}-\mathrm{Zn}\left(\mathrm{CF}_{3} \mathrm{SO}_{3}\right)_{2}$ electrolyte at $120^{\circ} \mathrm{C}$.

Table 6-2a. Zinc content after separation of zinc powder and stock PEO$\mathrm{Zn}\left(\mathrm{CF}_{3} \mathrm{SO}_{3}\right)_{2}$ electrolyte

\begin{tabular}{lclll}
\hline Sample & real Zn in solution /ppm & AAS / ppm & AAS / ppm & AAS /ppm \\
\hline electrolyte & 1.63 & $\begin{array}{l}1.28 \\
1.62^{\mathrm{b}}\end{array}$ & $\begin{array}{l}1.26 \\
1.61^{\mathrm{c}}\end{array}$ & $\begin{array}{l}1.27 \\
1.63^{\mathrm{d}}\end{array}$ \\
\hline zinc powder & & 1.65 & 1.68 & 1.65 \\
\hline
\end{tabular}

a: $32.114 \mathrm{mg}$ separated electrolyte diluted to $100 \mathrm{~cm}^{3}$, and $20 \mathrm{~cm}^{3}$ of the solution was further diluted to $100 \mathrm{~cm}^{3}$ for $\mathrm{AAS}, \mathrm{EO} / \mathrm{Zn}=50.05$;

b: calibrated by calibration curve 1;

c: calibrated by calibration curve 2 ;

d: calibrated by calibration curve 3 ;

e: $3.316 \mathrm{mg}$ zinc was separated and prepared to $100 \mathrm{~cm}^{3}$ solution, and $5 \mathrm{~cm}^{3}$ of this solution was further diluted to $100 \mathrm{~cm}^{3}$ for AAS. 
Table 6-3a. AAS results for calibration curves for $\mathrm{PEO}-\mathrm{Zn}\left(\mathrm{CF}_{3} \mathrm{SO}_{3}\right)_{2}$ electrolyte*

\begin{tabular}{clllll}
\hline solution Zn content / ppm & AAS 1/ppm & AAS 2 /ppm & AAS 3 /ppm & AAS4 /ppm \\
\hline 1 & 0.75 & 0.58 & 0.56 & 0.55 & 0.55 \\
2 & 0.93 & 0.73 & 0.71 & 0.71 & 0.72 \\
3 & 1.40 & 1.05 & 1.05 & 1.02 & 1.02 \\
4 & 1.50 & 1.16 & 1.18 & 1.18 & 1.19 \\
5 & 1.87 & 1.49 & 1.48 & 1.49 & 1.47 \\
\hline
\end{tabular}

*: $\mathrm{EO} / \mathrm{Zn}=50.05 ; 73.466 \mathrm{mg}$ electrolyte was digested and prepared to $100 \mathrm{~cm}^{3}$ solution. $4 \mathrm{~cm}^{3}, 5 \mathrm{~cm}^{3}, 7 \mathrm{~cm}^{3}, 8 \mathrm{~cm}^{3}$ and $10 \mathrm{~cm}^{3}$ of the solution were further diluted to $100 \mathrm{~cm}^{3}$ to prepare solution No. $1,2,3,4$ and 5 for AAS.

Table 6-3b. AAS results of stock PEO- $\mathrm{Zn}\left(\mathrm{CF}_{3} \mathrm{SO}_{3}\right)_{2}$ electrolyte

\begin{tabular}{lllll}
\hline samplea & weight $/ \mathrm{mg}$ & zinc $/ \mathrm{ppm}$ & AAS $/ \mathrm{ppm}$ & calibrated AAS $/ \mathrm{ppm}$ \\
\hline 1 & 34.347 & 0.87 & 0.66 & 0.85 \\
2 & 64.401 & 1.63 & 1.27 & 1.61 \\
3 & 85.868 & 2.19 & 1.74 & 2.20 \\
4 & 51.521 & 1.31 & 1.03 & 1.31 \\
5 & 42.934 & 1.09 & 0.84 & 1.08 \\
\hline
\end{tabular}

a: $E O / \mathrm{Zn}=50.05$, digested sample was diluted to $100 \mathrm{~cm}^{3}$, and $10 \mathrm{~cm}^{3}$ of the solution was furter diluted to $100 \mathrm{~cm}^{3}$ for AAS;

b: calibrated by calibration curve 1 . 
Table 6-4 a. Result based on the analysis of the middel compartments of PEO$\mathrm{Zn}\left(\mathrm{CF}_{3} \mathrm{SO}_{3}\right)_{2}$ electrolyte* for Hittorf experiment

Middlle anode compartment: $Q=0.90 \mathrm{C}, \mathrm{T}=120^{\circ} \mathrm{C}$, weight $\mathrm{W}=42.934 \mathrm{mg}$, $\mathrm{R}=\mathrm{EO} / \mathrm{Zn}=50.05$

\begin{tabular}{lllll}
\hline experiment No. & 1 & 2 & 3 & 4 \\
\hline real $\mathrm{Zn}$ in solution /ppm & 1.09 & 1.09 & 1.09 & 1.09 \\
\hline ZnAAS / ppm & 0.84 & 0.85 & 0.83 & 0.84 \\
\hline calibrated $\mathrm{Zn} / \mathrm{ppm}$ & 1.08 & 1.11 & 1.10 & 1.10 \\
\hline
\end{tabular}

Middle cathode compartment: $Q=0.90 \mathrm{C}, \mathrm{T}=120^{\circ} \mathrm{C}$, weight $\mathrm{W}=51.521 \mathrm{mg}$, $\mathrm{R}=\mathrm{EO} / \mathrm{Zn}=50.05$

\begin{tabular}{lllll}
\hline experiment No. & 1 & 2 & 3 & 4 \\
\hline real $\mathrm{Zn}$ in solution /ppm & 1.31 & 1.31 & 1.31 & 1.31 \\
\hline $\mathrm{ZnAAS} / \mathrm{ppm}$ & 1.03 & 1.01 & 1.04 & 1.00 \\
\hline calibrated $\mathrm{Zn} / \mathrm{ppm}$ & 1.31 & 1.30 & 1.35 & 1.30 \\
\hline
\end{tabular}

* the digested sample was diluted to $100 \mathrm{~cm}^{3}$, and $10 \mathrm{~cm}^{3}$ of the solution was further diluted to $100 \mathrm{~cm}^{3}$ for $\mathrm{Zn}$ AAS; AAS results in experiment $1,2,3$ and 4 were calibrated by calibration curve $1,2,3$ and 4 respectively. 
Table 6-4b. Results based on the analysis of the anode compartment of PEO$\mathrm{Zn}\left(\mathrm{CF}_{3} \mathrm{SO}_{3}\right)_{2}$ electrolytea for Hittorf experiment.

Anode compartment: $Q=0.90 \mathrm{C}, \mathrm{T}=120^{\circ} \mathrm{C}$, weight $\mathrm{W}=87.451 \mathrm{mg}$, $\mathrm{R}=\mathrm{EO} / \mathrm{Zn}=50.05$

\begin{tabular}{lllll} 
experiment No. & 1 & 2 & 3 & 4 \\
\hline AAS result / ppm & 1.57 & 1.57 & 1.56 & 1.55 \\
\hline calibrated $\mathrm{Zn}^{\mathrm{b}} \mathrm{m} / \mathrm{ppm}$ & 1.98 & 1.99 & 1.97 & 1.97 \\
\hline $\mathrm{T} \mathrm{c}$ & 0.95 & 1.00 & 0.92 & 0.92 \\
\hline
\end{tabular}

a: the electrolyte was digested and prepared to $100 \mathrm{~cm}^{3}$ solution, and $8 \mathrm{~cm}^{3}$ of this solution was further diluted to $100 \mathrm{~cm}^{3}$ for $A A S$, i.e. $L=8$ in equation $(6-3-6)$;

b: AAS results for No. 1, 2, 3 and 4 experiments were calibrated by calibration curve $1,2,3$ and 4 respectively;

c: T. was calculated according equation (6-3-6). 
Table 6-4c. Results based on the analysis of the cathode compartment of PEO$\mathrm{Zn}\left(\mathrm{CF}_{3} \mathrm{SO}_{3}\right)_{2}$ electrolyte ${ }^{\mathrm{a}}$ for Hittorf experiment

Cathode compartment: $\mathrm{Q}=0.90 \mathrm{C}, \mathrm{T}=120^{\circ} \mathrm{C}$, weight $\mathrm{W}=67.299 \mathrm{mg}$, $\mathrm{R}=\mathrm{EO} / \mathrm{Zn}=50.05$, weight of the polymer electrolyte for digestion $\mathrm{W}^{\prime}=21.212 \mathrm{mg}$

\begin{tabular}{lcccc}
\hline $\begin{array}{l}\text { experiment } \\
\text { No. }\end{array}$ & Zn AAS / ppm & calibrated $\mathrm{Zn}^{\mathrm{b}} \mathrm{m}^{\prime} / \mathrm{ppm}$ & Zn & Zndrites \\
& & & & \\
1 & 0.72 & 0.93 & 0.30 & 0.91 \\
2 & 0.71 & 0.94 & 0.30 & 0.85 \\
3 & 0.71 & 0.95 & 0.30 & 0.79 \\
4 & 0.71 & 0.95 & 0.30 & 0.79 \\
\hline
\end{tabular}

a: the digested polymer electrolyte was prepared to $100 \mathrm{~cm}^{3}$ solution, $20 \mathrm{~cm}^{3}$ of this solution was further diluted to $100 \mathrm{~cm}^{3}$ for AAS, i.e. $L=20 ; 15 \mathrm{~cm}^{3}$ of $100 \mathrm{~cm}^{3}$ the separated zinc dendrites solution was prepared to $100 \mathrm{~cm}^{3}$ for AAS, i.e. $1=15$;

b: the AAS results of No. 1,2,3 and 4 were calibrated by calibration curve 1, 2, 3 and 4 respectively;

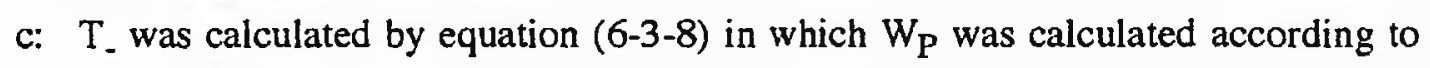
formular (6-3-7). 
Table 6-5. Results for the VTF equation fitting for $\mathrm{PMEO}-\mathrm{Zn}\left(\mathrm{CF}_{3} \mathrm{SO}_{3}\right)_{2}$ electrolytes

\begin{tabular}{llll}
\hline$E O / \mathrm{Zn}$ & $\mathrm{T}_{\mathrm{o}} / \mathrm{K}$ & $\mathrm{B} / \mathrm{K}$ & $\sigma_{\mathrm{o}} / \mathrm{S} \mathrm{cm}^{-1}$ \\
\hline $100: 1$ & 215 & 427 & $7.85 \times 10^{-4}$ \\
$70: 1$ & 235 & 285 & $2.56 \times 10^{-4}$ \\
$50: 1$ & 200 & 662 & $8.31 \times 10^{-3}$ \\
$30: 1$ & 220 & 474 & $4.67 \times 10^{-4}$ \\
$20: 1$ & 208 & 839 & $5.57 \times 10^{-3}$ \\
$10: 1$ & 220 & 1282 & 0.31 \\
\hline
\end{tabular}




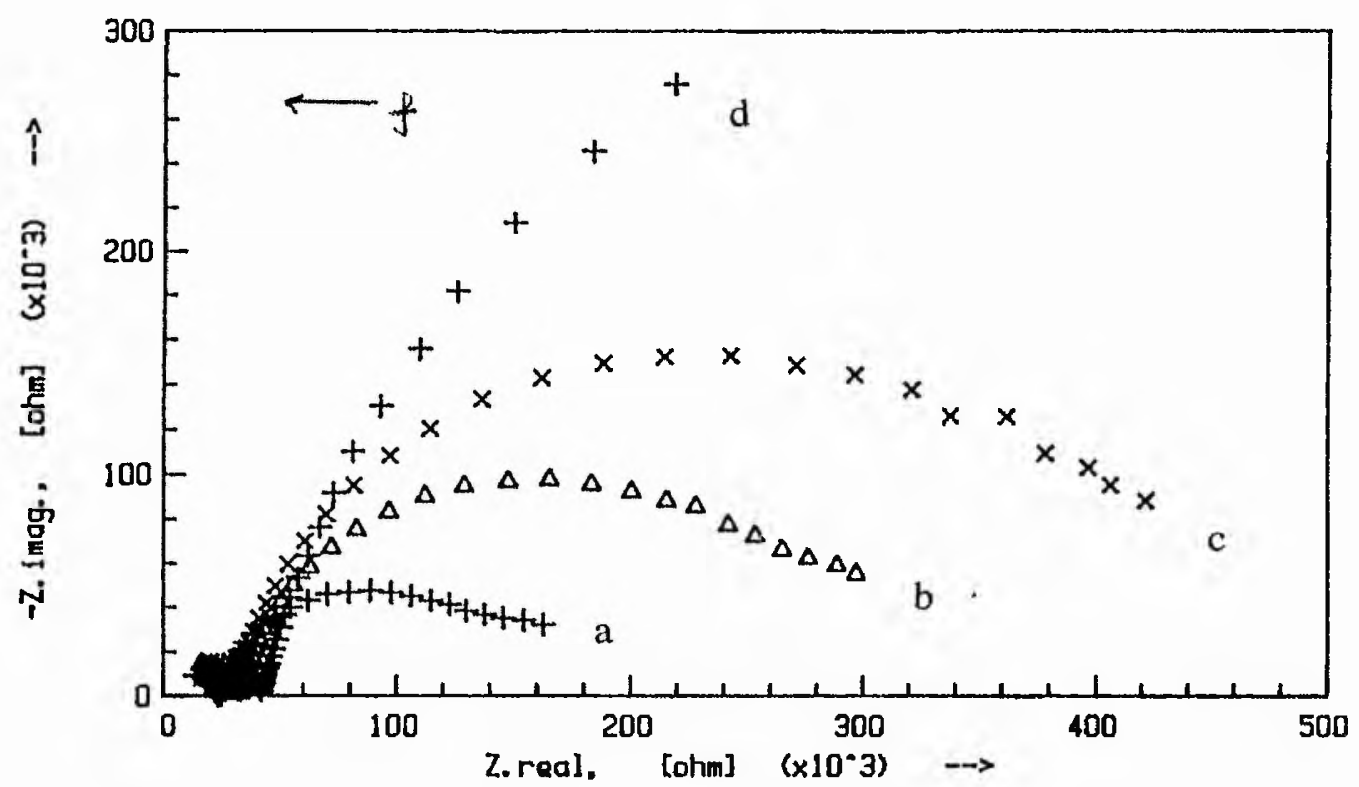

Figure 6-1 Ac impedance spectra for $\mathrm{Zn} / \mathrm{PMEO}-\mathrm{Zn}\left(\mathrm{CF}_{3} \mathrm{SO}_{3}\right)_{2}(20: 1)$ interface at $30^{\circ} \mathrm{C}$ after cell assembly for $\mathrm{O} \mathrm{hr}(\mathrm{a}), 0.5 \mathrm{hr}$ (b), $5 \mathrm{hrs}$ (c) and $24 \mathrm{hrs}(\mathrm{d})$. 


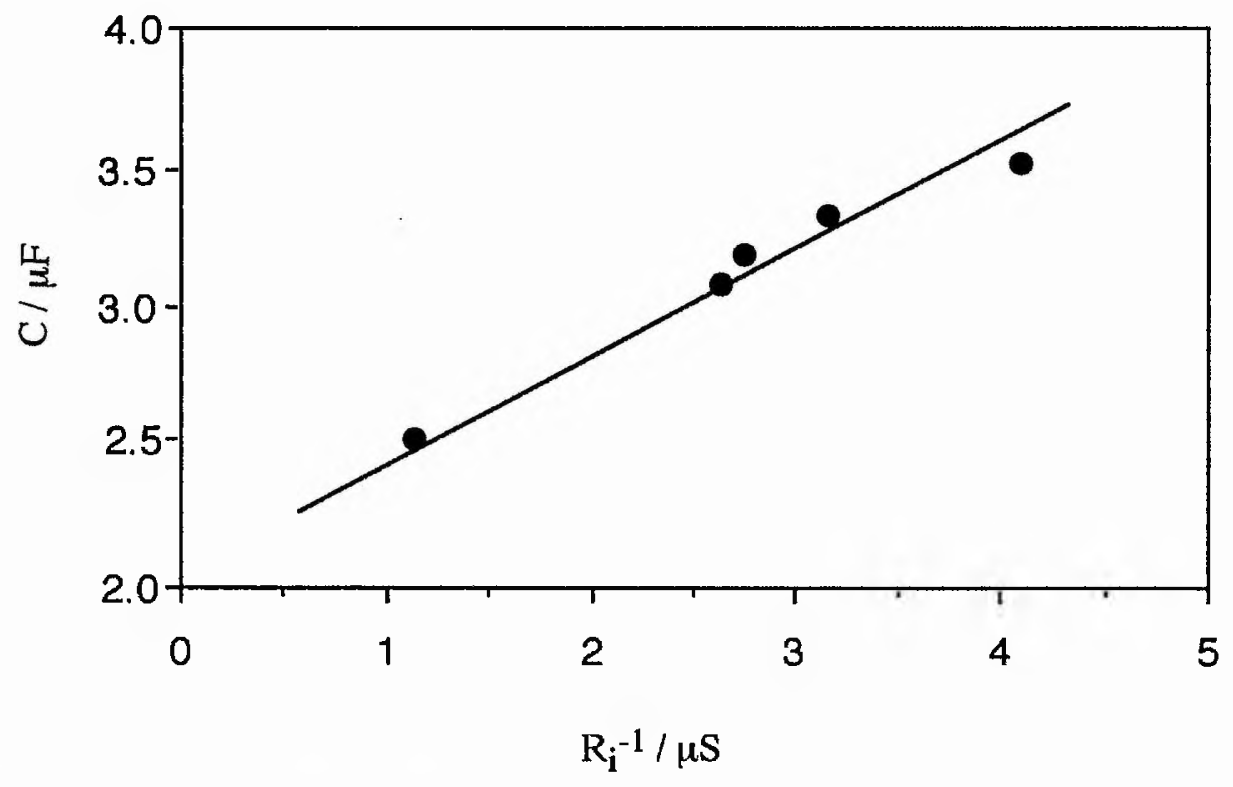

Figure 6-2 Relationship between the capacitance and the reciprocal of the resistance of the passivating layer on the zinc electrode.

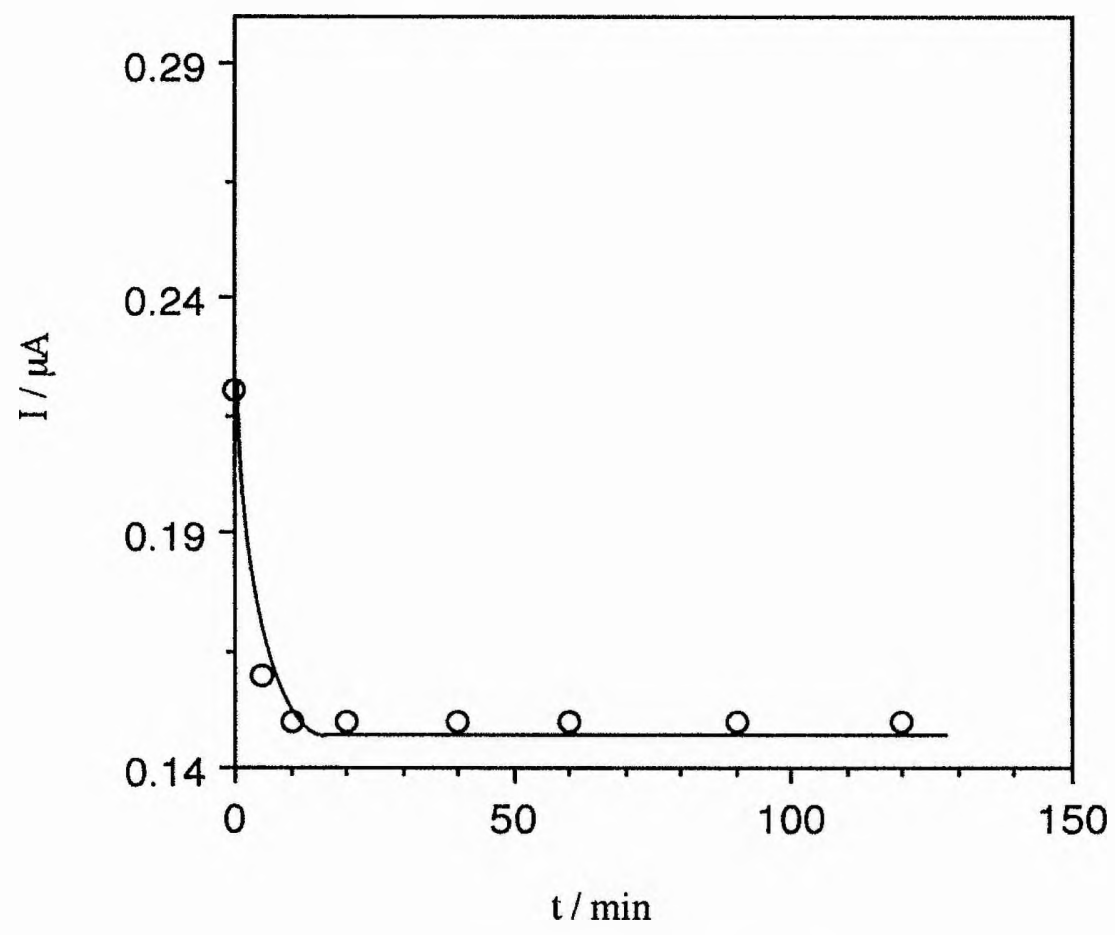

Figure 6-3 I-t curve for dc polarisation of $\mathrm{Zn} / \mathrm{PMEO}-\mathrm{Zn}\left(\mathrm{CF}_{3} \mathrm{SO}_{3}\right)_{2}(20: 1) / \mathrm{Zn}$ cell with $0.1 \mathrm{~V}$ applied voltage at $30^{\circ} \mathrm{C}$ without pre-treatment of the electrodes. 


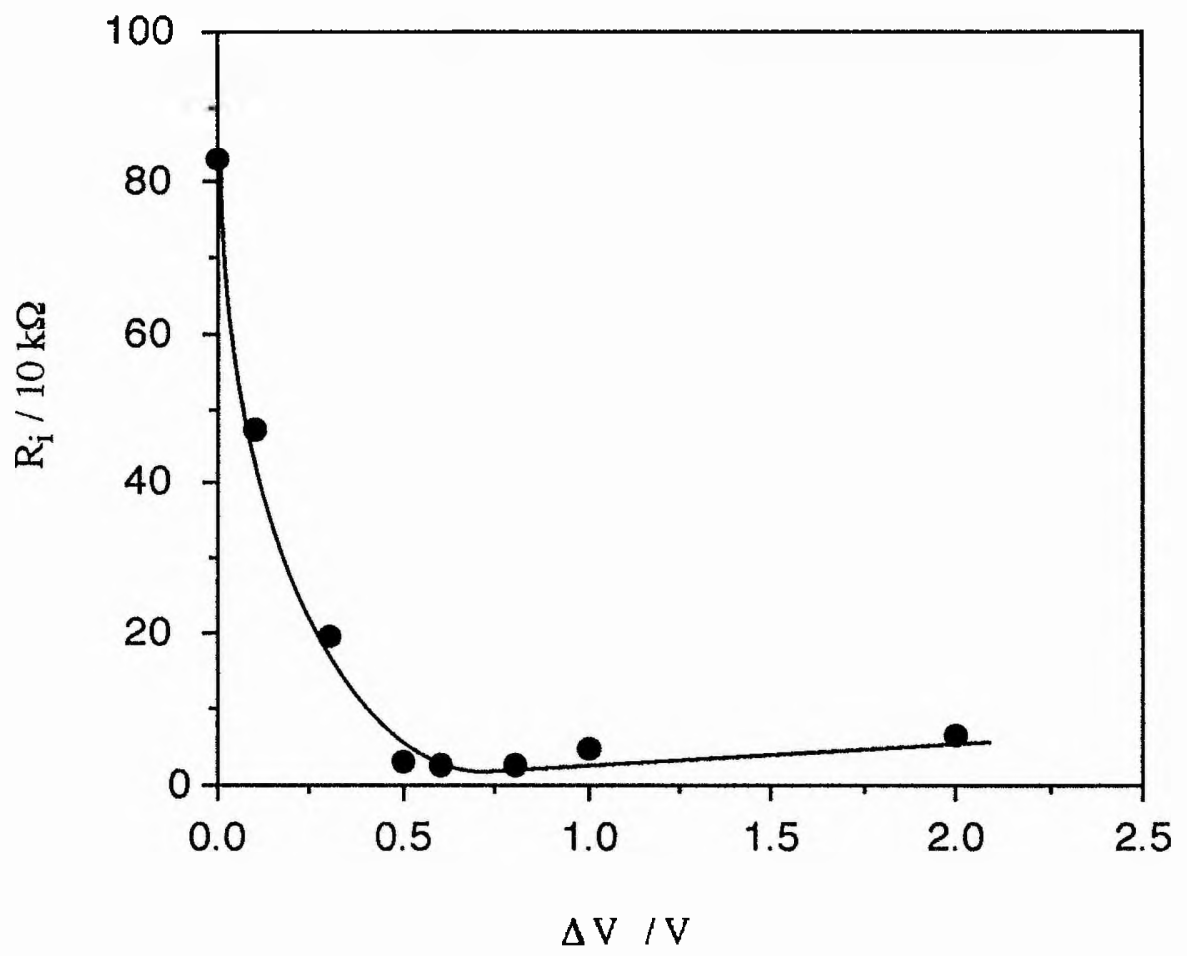

Figure 6-4a Variation in the interfacial resistance with the applied anodic potential for the $\mathrm{Zn} / \mathrm{PMEO}-\mathrm{Zn}\left(\mathrm{CF}_{3} \mathrm{SO}_{3}\right)_{2}$ (20:1) interface at $30^{\circ} \mathrm{C}$.

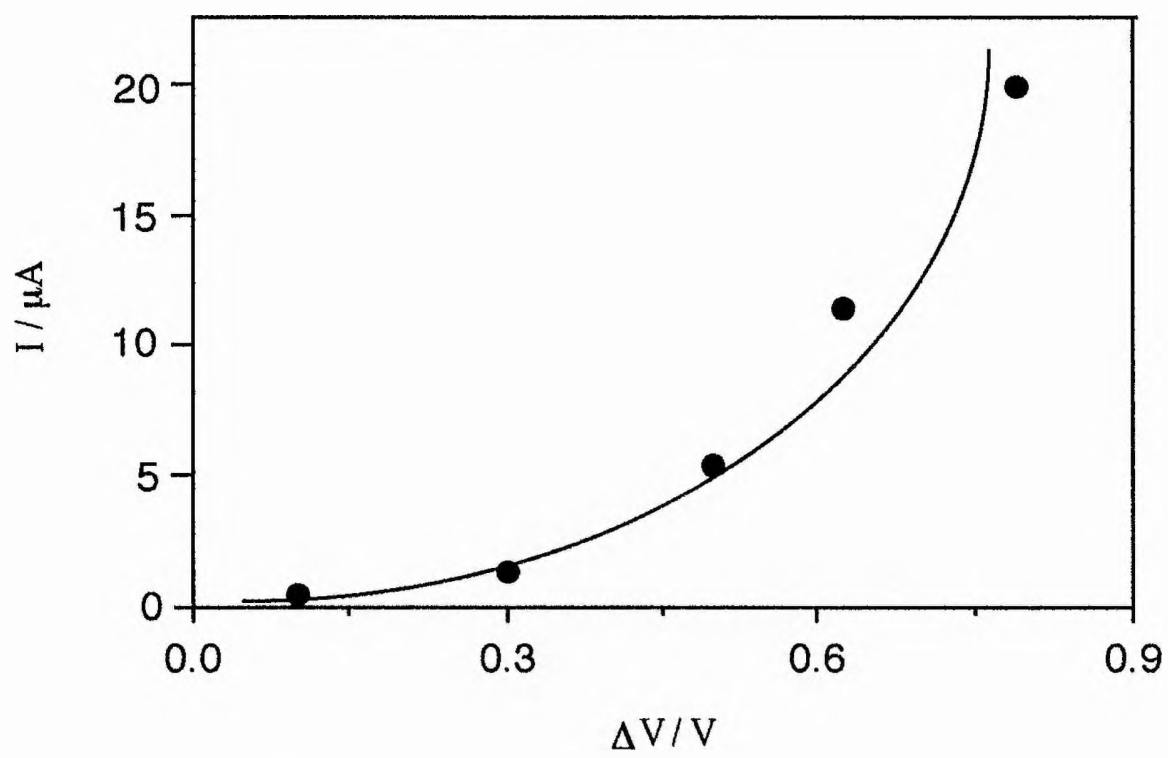

Figure 6-4b Variation of polarisation current with the applied anodic potential for the $\mathrm{Zn} / \mathrm{PMEO}-\mathrm{Zn}\left(\mathrm{CF}_{3} \mathrm{SO}_{3}\right)_{2}(20: 1)$ interface at $30^{\circ} \mathrm{C}$. 


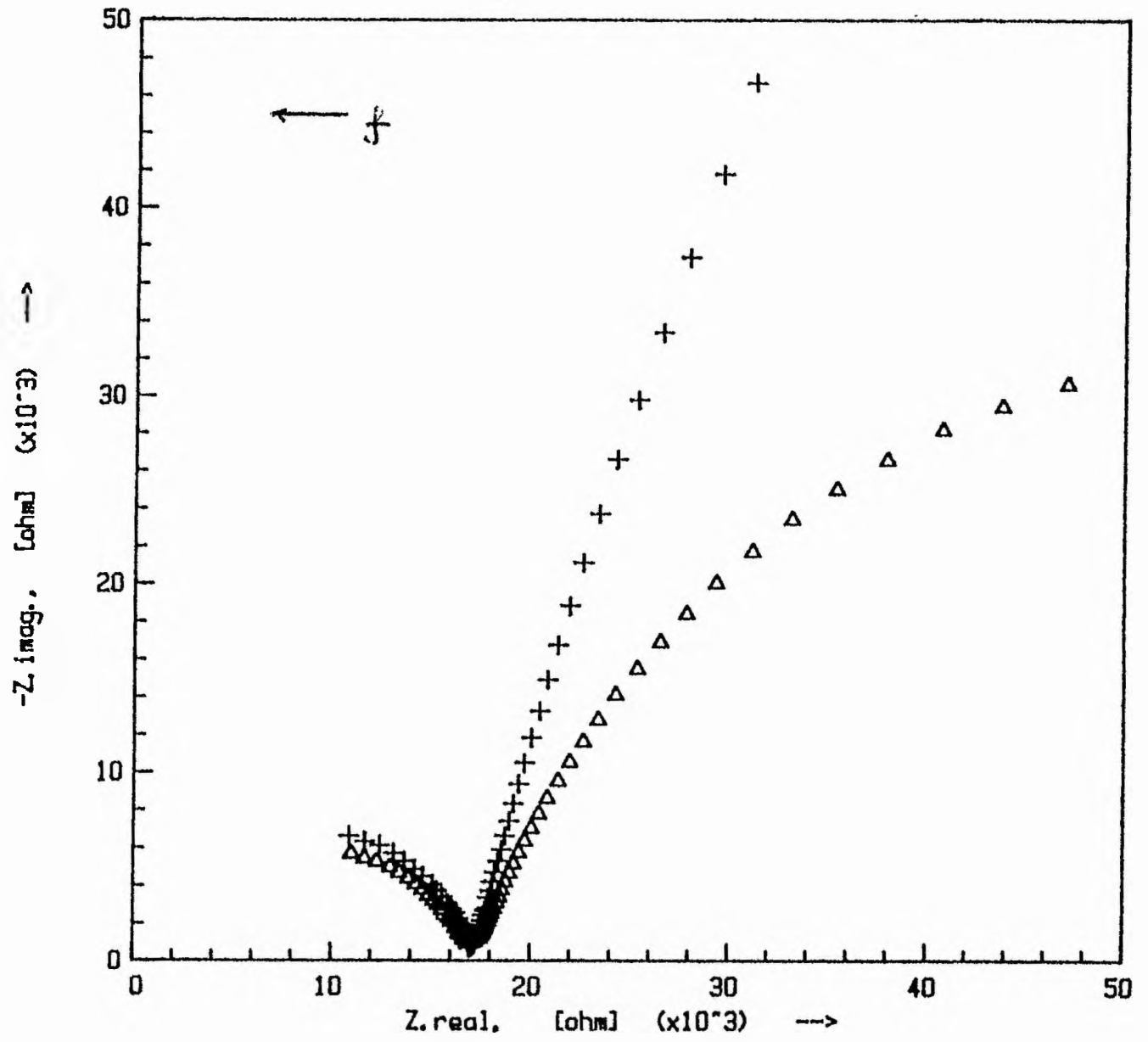

Figure 6-5a Ac impedance spectra for $\mathrm{Zn} / \mathrm{PMEO}-\mathrm{Zn}\left(\mathrm{CF}_{3} \mathrm{SO}_{3}\right)_{2}(20: 1)$ interface at $30^{\circ} \mathrm{C}(+)$ before polarisation and $(\Delta)$ after $0.5 \mathrm{~V}$ anodic polarisation for $5 \mathrm{hrs}$. 


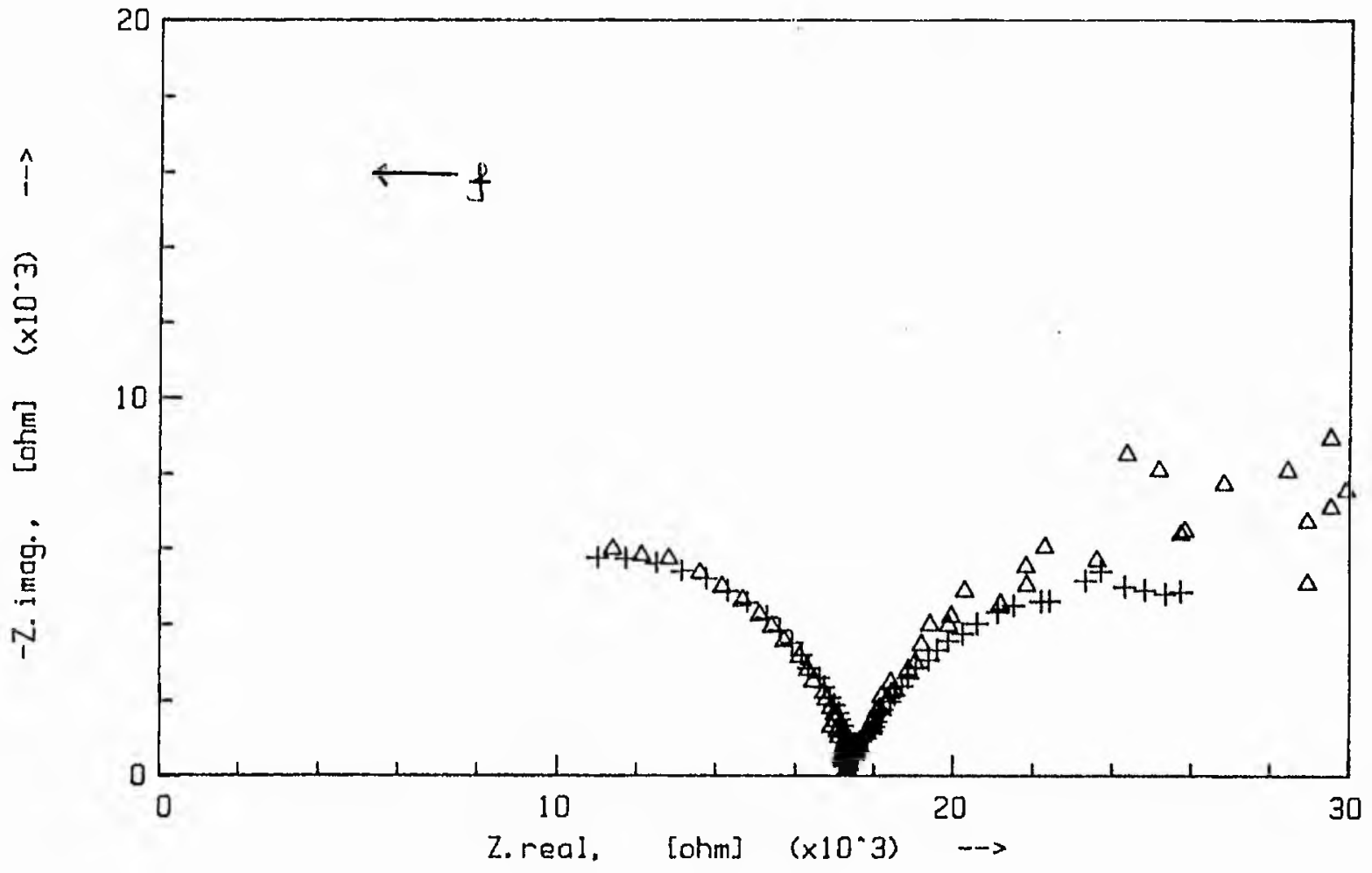

Figure 6-5b Ac impedance spectra for $\mathrm{Zn} / \mathrm{PMEO}-\mathrm{Zn}\left(\mathrm{CF}_{3} \mathrm{SO}_{3}\right)_{2}(20: 1)$ interface at $30^{\circ} \mathrm{C}(\Delta)$ after polarisation with $\pm 0.5 \mathrm{~V}$ potentials for a cycle with 1 hour interval, $(+)$ after polarisation with $\pm 0.5 \mathrm{~V}$ potentials for 3 cycles with 1 hour intervals. 


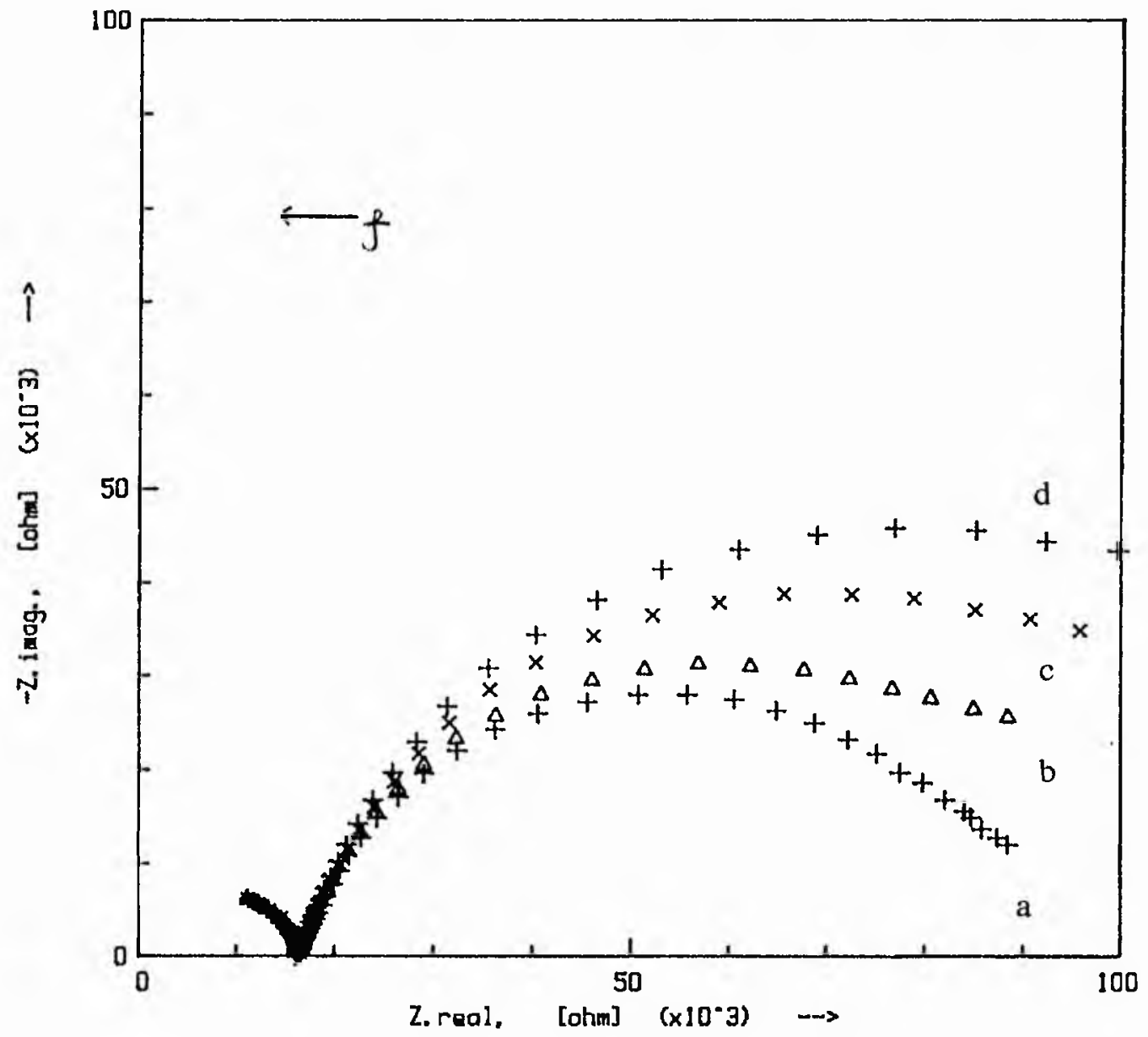

Figure 6-6 Ac impedance spectra for $\mathrm{Zn} / \mathrm{PMEO}-\mathrm{Zn}\left(\mathrm{CF}_{3} \mathrm{SO}_{3}\right)_{2}(20: 1) / \mathrm{Zn}$ cell at $30^{\circ} \mathrm{C}$ after stopping polarisation with $\pm 0.5 \mathrm{~V}$ potentials for 3 cycles with $1 \mathrm{hr}, 1.5 \mathrm{hr}$ and $2 \mathrm{hrs}$ intervals for (a) $0 \mathrm{hr}$, (b) $0.5 \mathrm{hr}$, (c) $5 \mathrm{hrs}$ and (d) $24 \mathrm{hrs}$. 


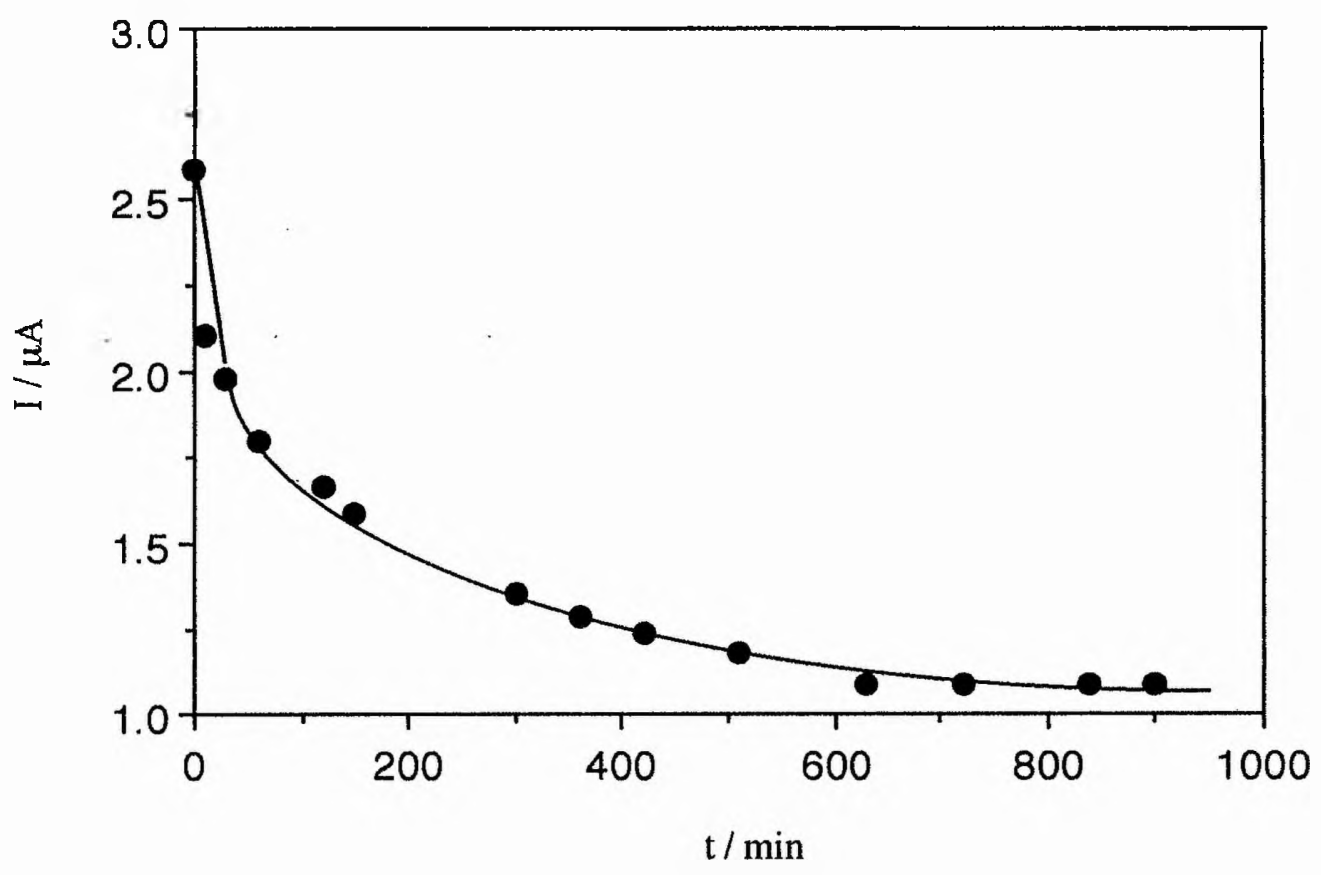

Figure 6-7 I-t curve for dc polarisation of $\mathrm{Zn} / \mathrm{PMEO}-\mathrm{Zn}\left(\mathrm{CF}_{3} \mathrm{SO}_{3}\right)_{2}(20: 1) / \mathrm{Zn}$ cell with applied voltage of $50 \mathrm{mV}$ at $84^{\circ} \mathrm{C}$. 


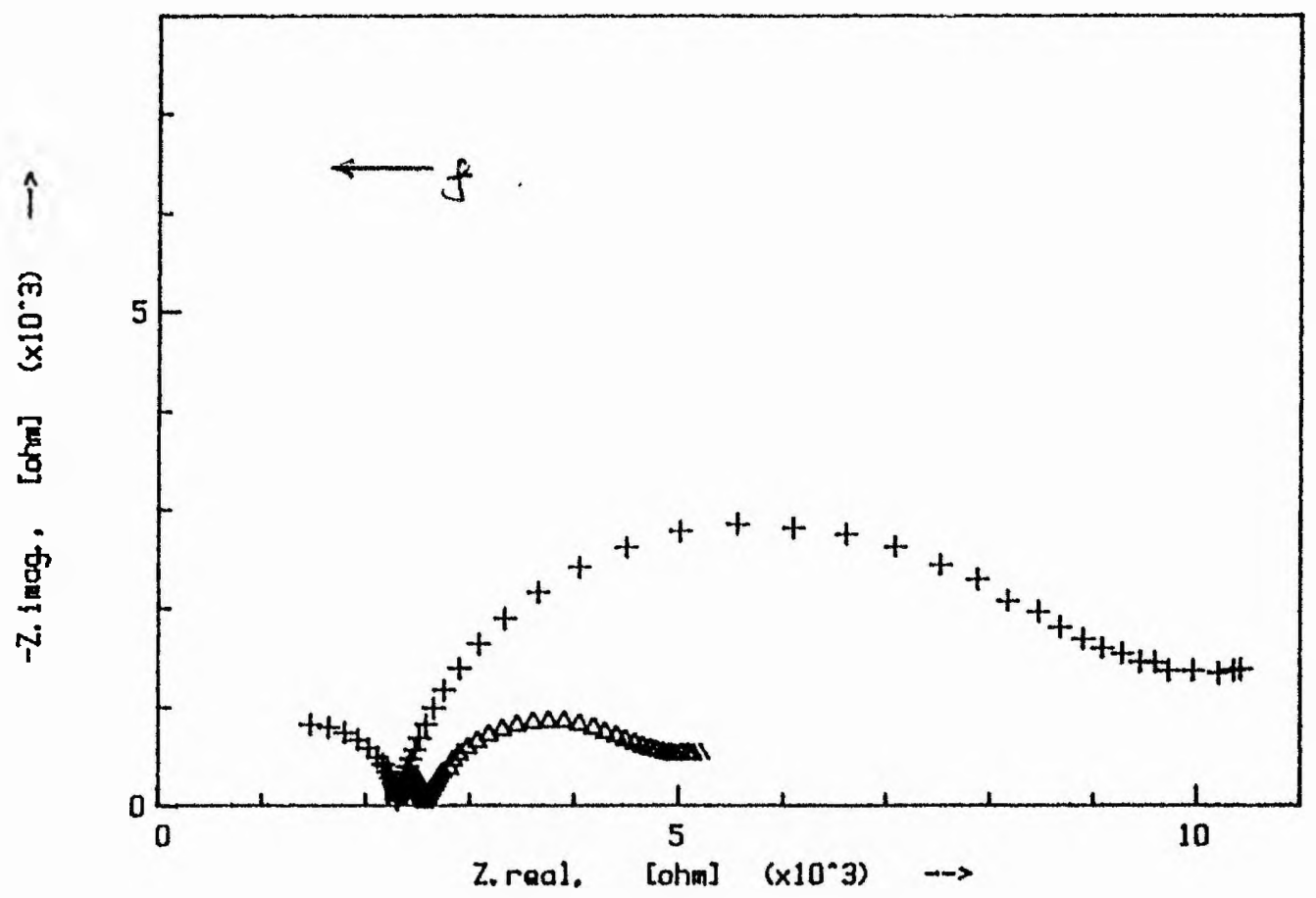

Figure 6-8 Ac impedance spectra for $\mathrm{Zn} / \mathrm{PMEO}-\mathrm{Zn}\left(\mathrm{CF}_{3} \mathrm{SO}_{3}\right)_{2}(10: 1) / \mathrm{Zn}$ cell at $84^{\circ} \mathrm{C}$ before $(+)$ and after $(\Delta)$ dc polarisation with $50 \mathrm{mV}$ applied voltage. 


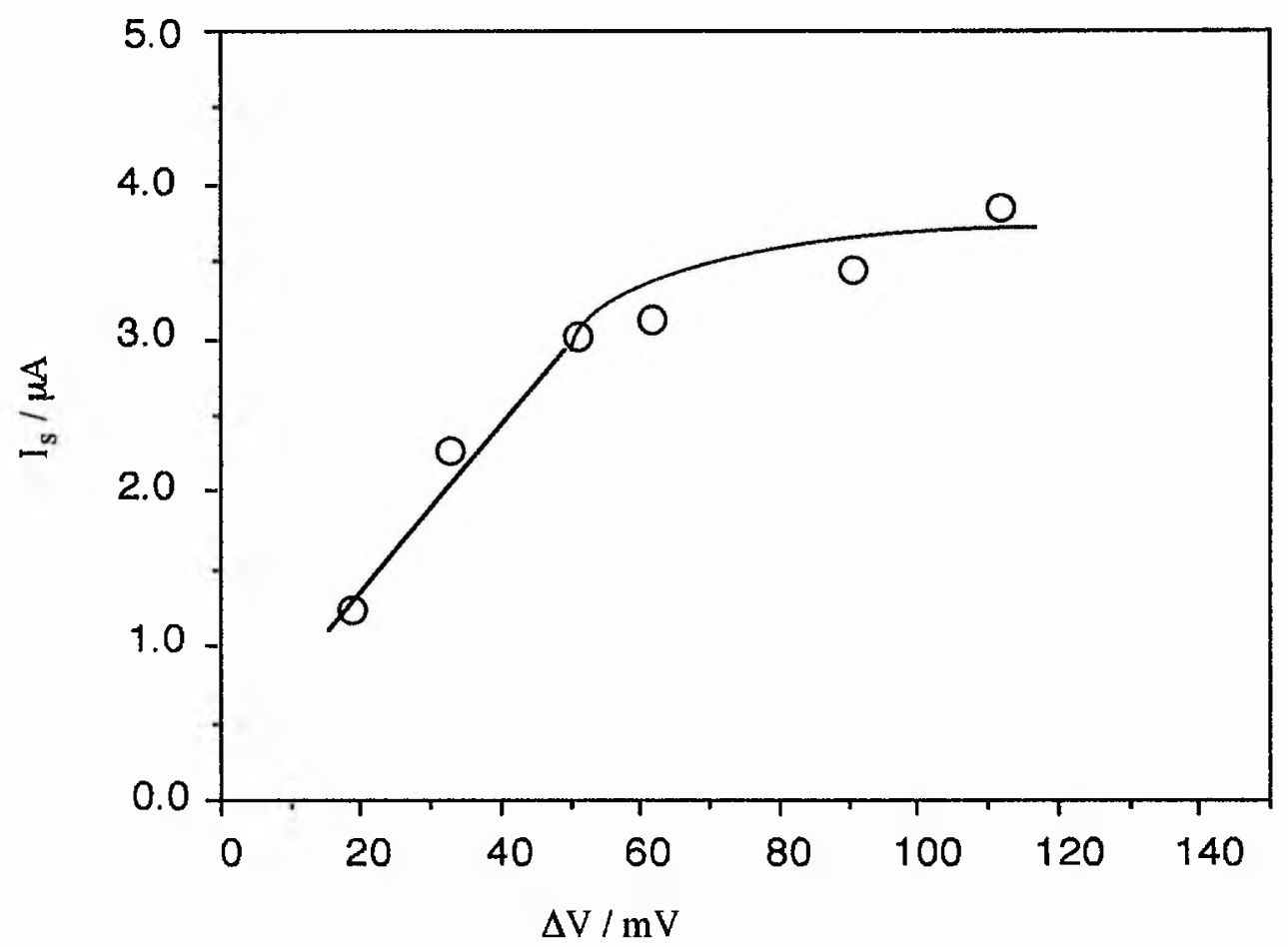

Figure 6-9 Plot of steady state current $\mathrm{I}_{\mathrm{s}}$ against polarisation potential $\Delta \mathrm{V}$ for $\mathrm{Zn} /$ $\mathrm{PMEO}-\mathrm{Zn}\left(\mathrm{CF}_{3} \mathrm{SO}_{3}\right)_{2}(\mathrm{EO} / \mathrm{Zn}=10) / \mathrm{Zn}$ cell at $84^{\circ} \mathrm{C}$.

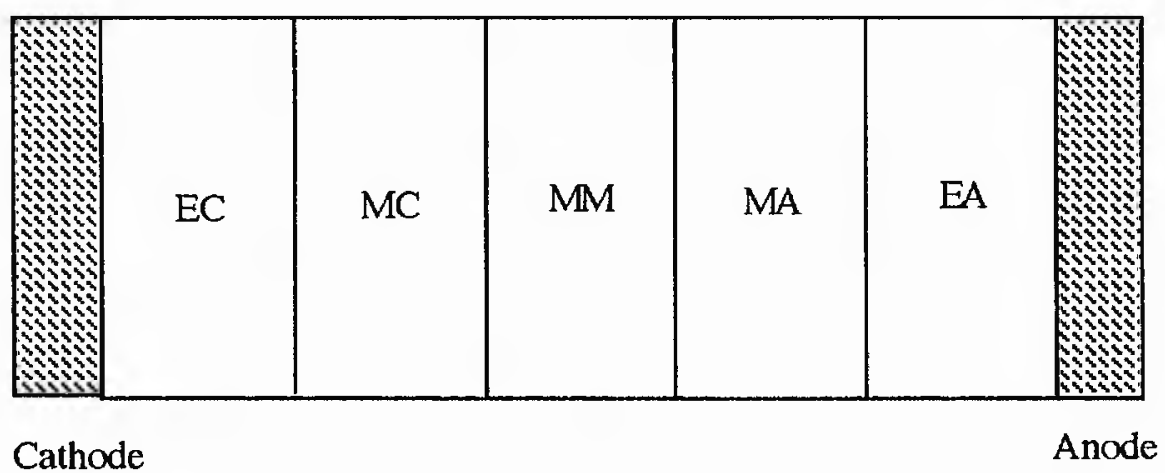

Figure 6-10 Schematic representation of a Hittorf cell. 


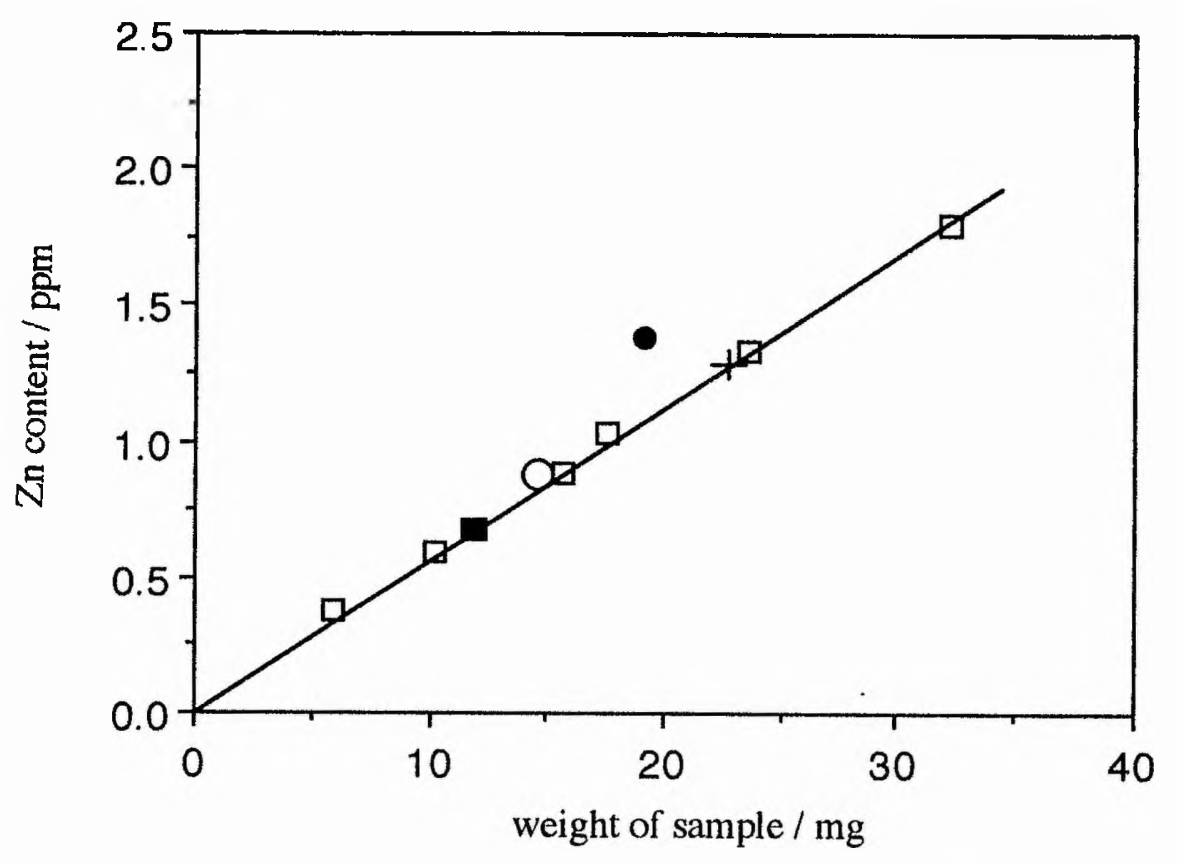

Figure 6-11 Zinc content measured by $\mathrm{Zn}-\mathrm{AAS}(\square)$ in stock $\mathrm{PEO}-\mathrm{Zn}\left(\mathrm{CF}_{3} \mathrm{SO}_{3}\right)_{2}$ electrolyte (+) in MA ( $\square$ ) in MC (O) in A and (O) in C containing zinc dendrites. 
Calibration curve 1

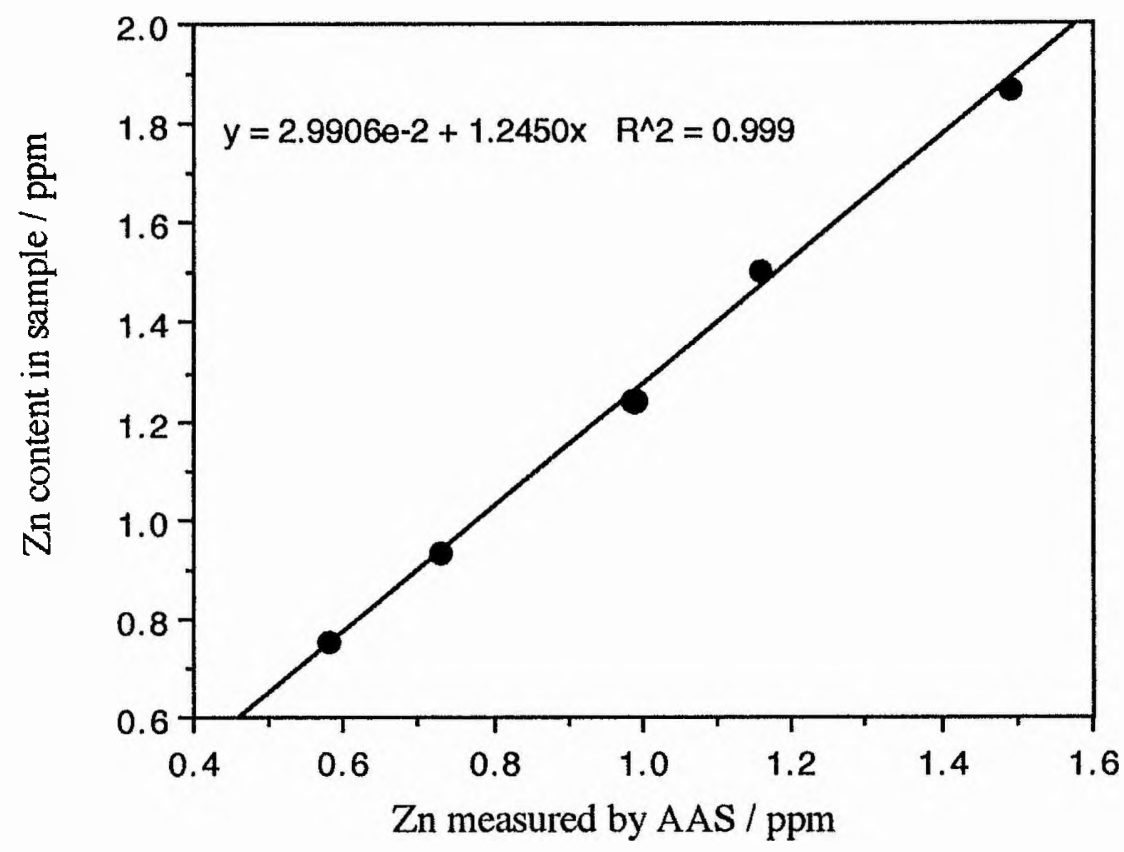

Calibration curve 2

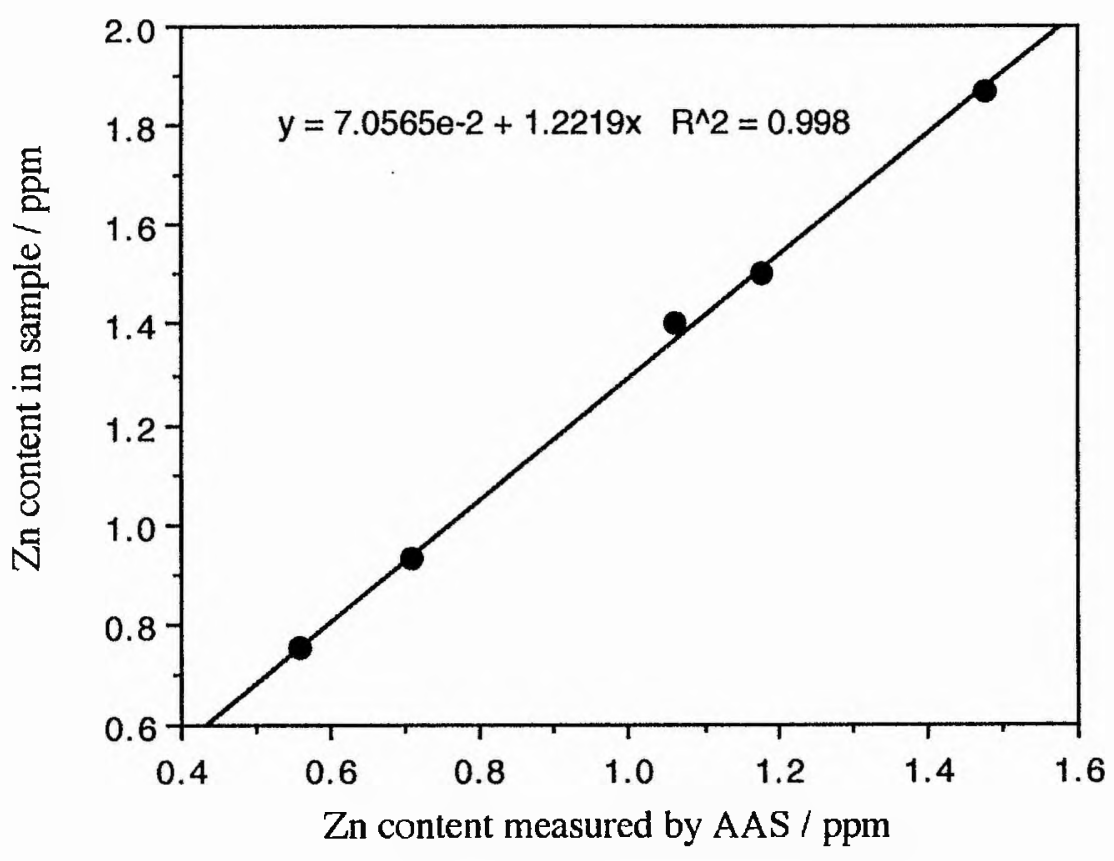



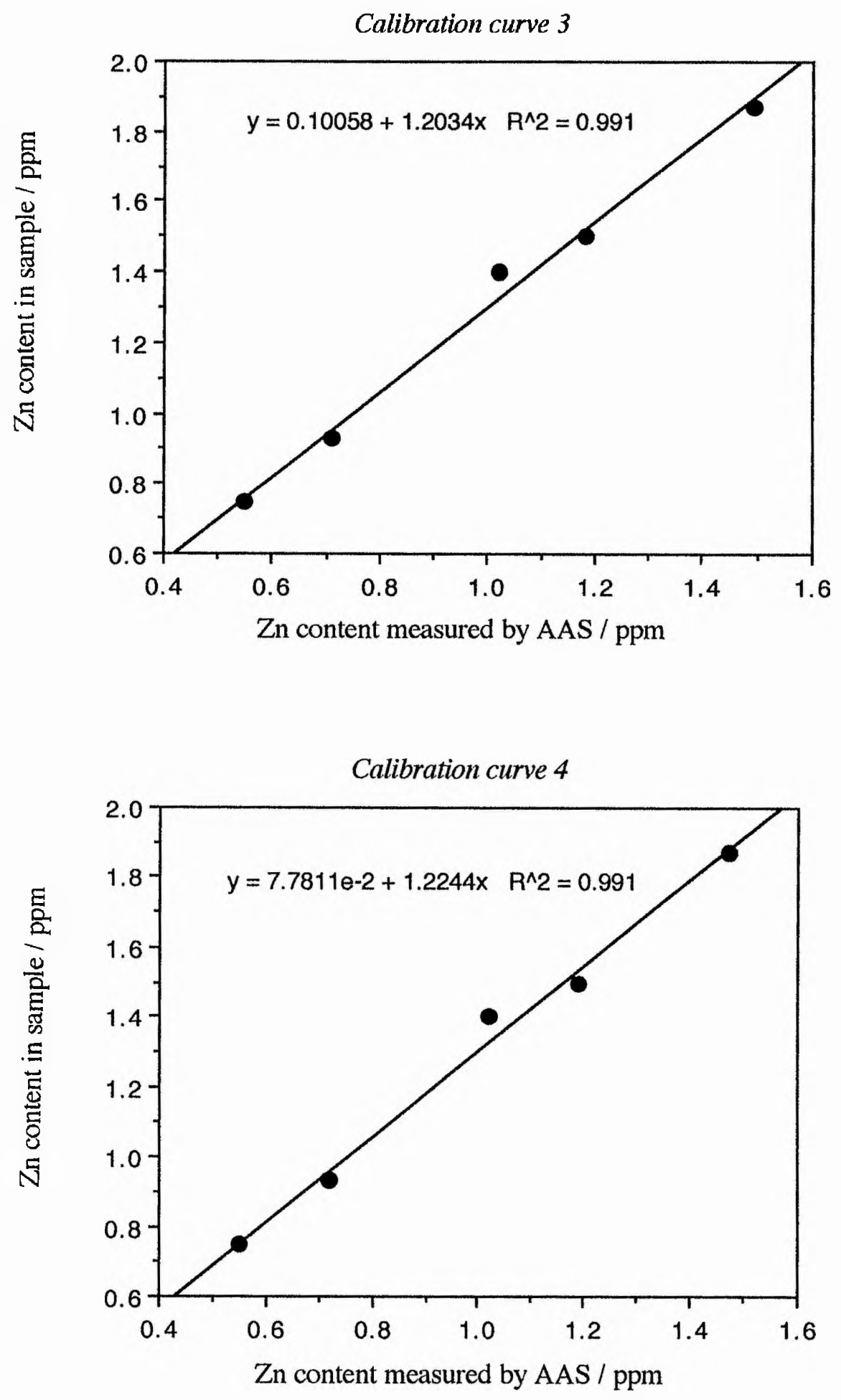

Figure 6-12 Calibration curves for calibrating zinc content measured by AAS. 


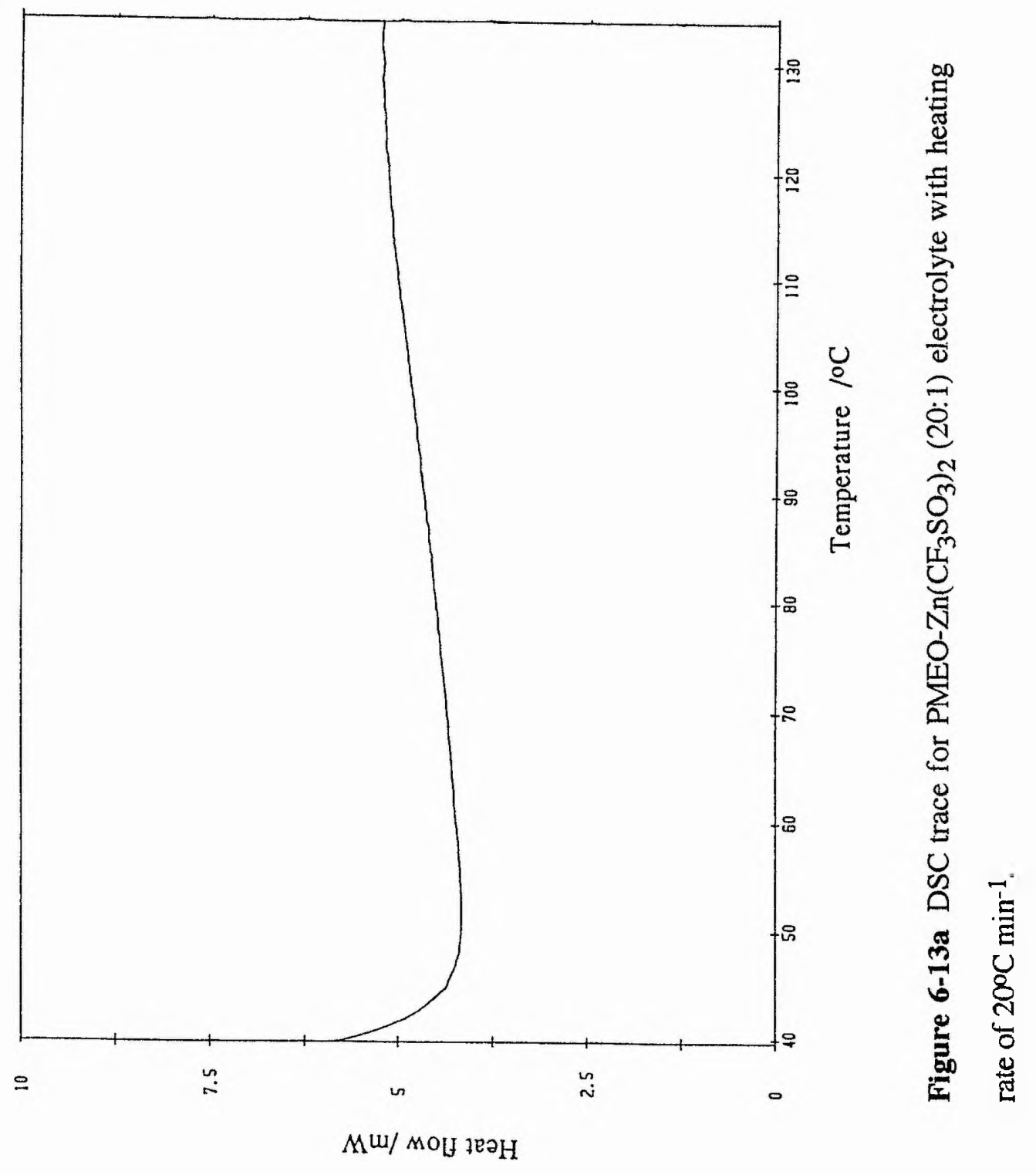




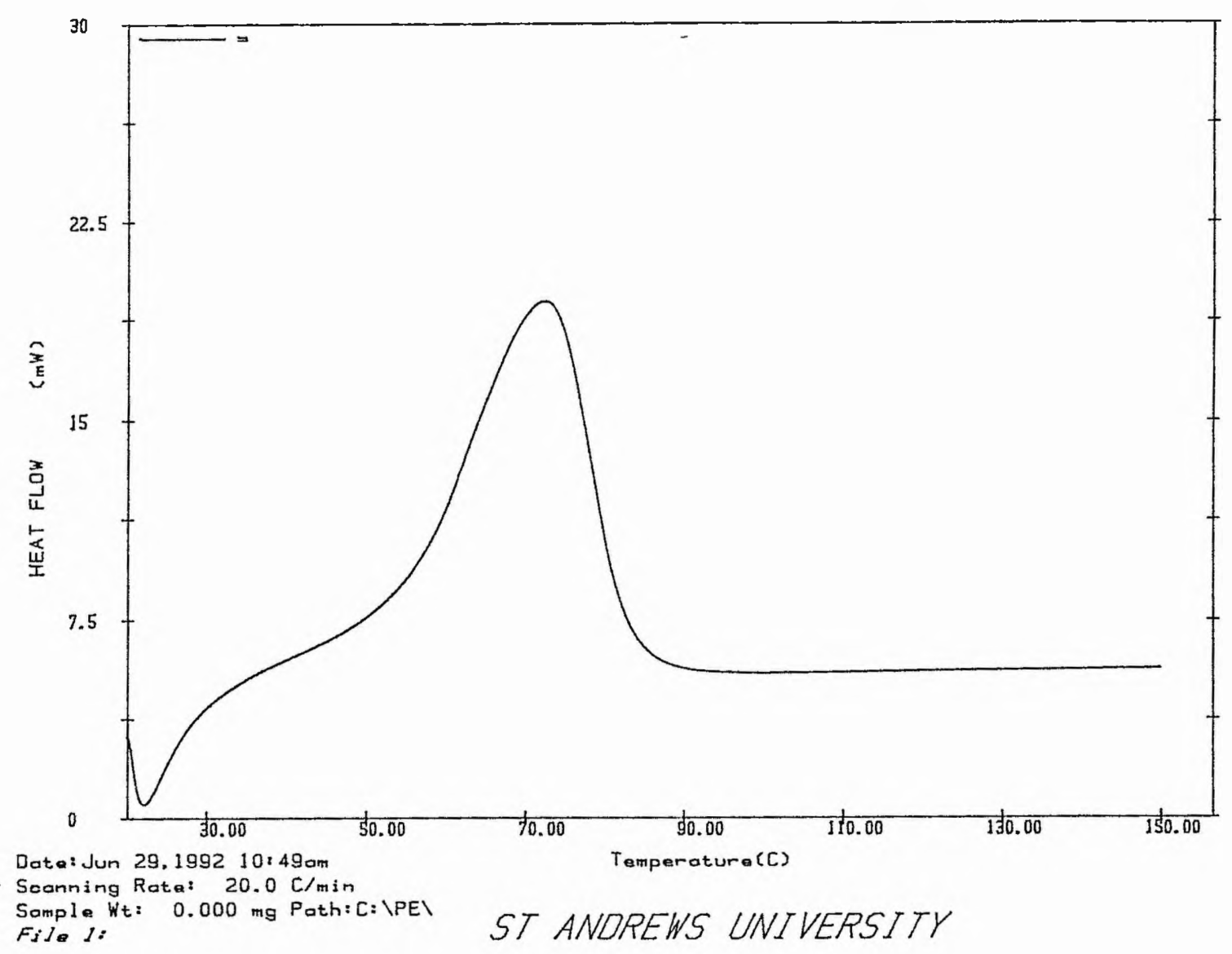

Figure 6-13b DSC trace for $\mathrm{PEO}-\mathrm{Zn}\left(\mathrm{CF}_{3} \mathrm{SO}_{3}\right)_{2}(20: 1)$ electrolyte with heating rate of $20^{\circ} \mathrm{C} \mathrm{min}^{-1}$. 


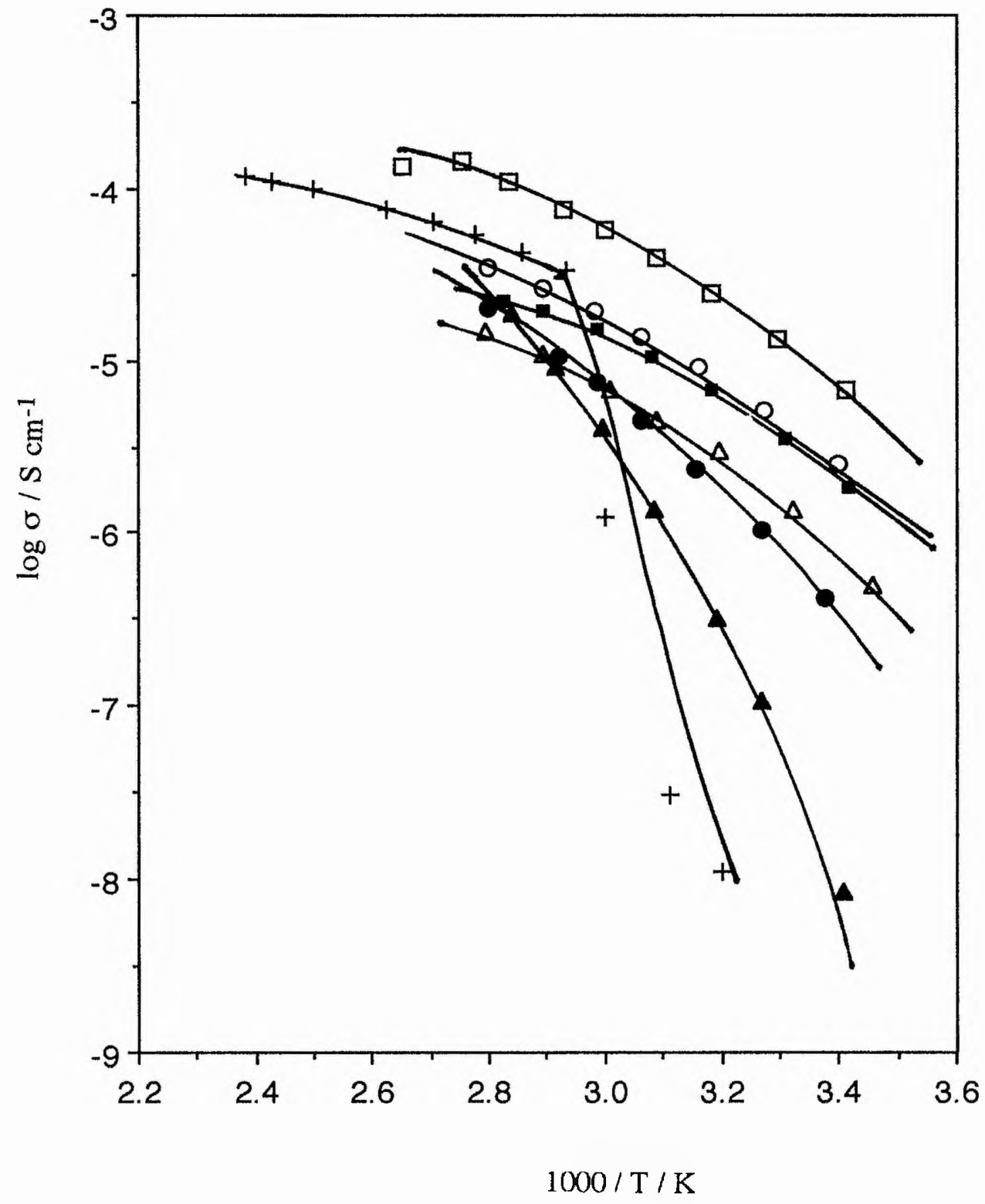

Figure 6-14 Conductivity variation with temperature for $\mathrm{PMEO}-\mathrm{Zn}\left(\mathrm{CF}_{3} \mathrm{SO}_{3}\right)_{2}$

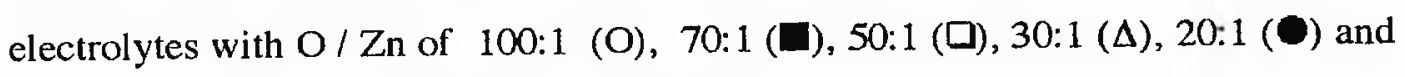
10:1 (A), (+) for PEO-Zn( $\left(\mathrm{CF}_{3} \mathrm{SO}_{3}\right)_{2}$ with EO/Zn of 50:1. 


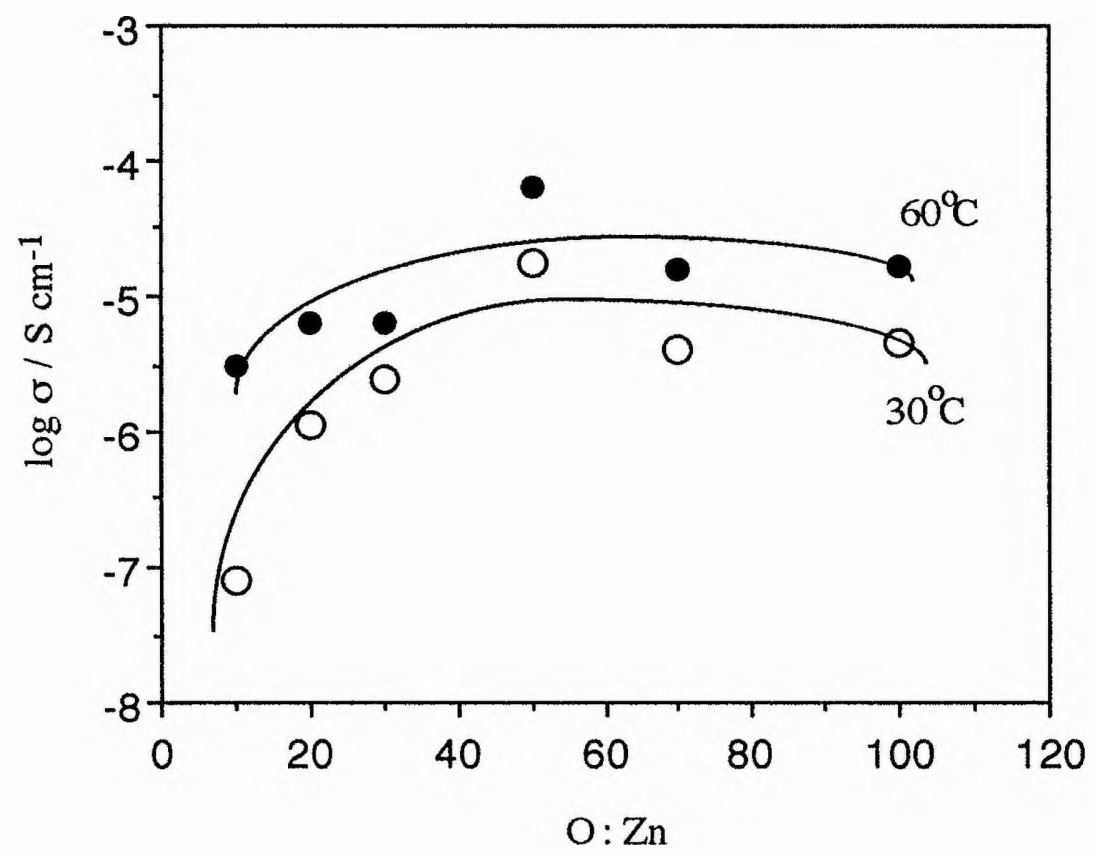

Figure 6-15 Variation in conductivity with salt concentration for $\mathrm{PMEO}-\mathrm{Zn}\left(\mathrm{CF}_{3} \mathrm{SO}_{3}\right)_{2}$ electrolyte. 


\section{CHAPTER 7}

\section{CONDUCTIVITY AND TRANSPORT PROPERTIES IN POLYMER ELECTROLYTES BASED ON CALCIUM AND MAGNESIUM SALTS}

Magnesium and calcium salt-based polymer electrolytes may be suitable for applications in all-solid-state batteries due to their high charge densities and light weights. Such materials have been studied since the early 1980's [1,2]. It has been found, however, that there are complications in the study of these materials, not only in the preparation since it is very difficult to get absolutely anhydrous electrolytes, but also in techniques for electrochemical studies since it is very easy to form resistive passivating layers on calcium and magnesium electrodes [3]. Understanding of these materials is therefore still poor.

In this chapter, polymer electrolytes based on $\mathrm{PEO}, \mathrm{PMEO}, \mathrm{Ca}\left(\mathrm{CF}_{3} \mathrm{SO}_{3}\right)_{2}$ and $\mathrm{Mg}\left(\mathrm{CF}_{3} \mathrm{SO}_{3}\right)_{2}$ have been investigated. Study of the electrode-electrolyte interfaces has confirmed the passivation of metal electrodes and has led to improvement of electrode performance. The mobilities of calcium and magnesium species in polymer electrolytes have been investigated using the dc polarisation method. In addition, the conductivity and phase behaviour of the polymer electrolytes have been studied by ac impedance, thermal analysis and $\mathrm{X}$-ray diffraction techniques. 


\section{7-1 Electrode-Electrolyte Interfaces}

In this section, electrode-electrolyte interfaces in the polymer electrolytes based on $\mathrm{PEO}(400)$ dimethyl ether and $\mathrm{Ca}\left(\mathrm{CF}_{3} \mathrm{SO}_{3}\right)_{2}$ or $\mathrm{Mg}\left(\mathrm{CF}_{3} \mathrm{SO}_{3}\right)_{2}$ salts are briefly investigated. The results are required for the study of the mobilities of calcium and magnesium species in the polymer electrolytes using electrochemical methods.

\section{7-1-1 Electrochemical Reaction on $\mathrm{Ca}$ and $\mathrm{Mg}$ Electrodes}

Two electrode cells where the metal electrodes are basically non-blocking towards $\mathrm{M}^{\mathrm{n}+} / \mathrm{M}$ are of ten used to study the transport properties in PEO-MX $\mathbf{n}$ polymer electrolytes. For instance, as discussed in Chapter 6, the zinc cell: $\mathrm{Zn} \mathrm{/} \mathrm{PEO-}$ $\mathrm{Zn}\left(\mathrm{CF}_{3} \mathrm{SO}_{3}\right)_{2} / \mathrm{Zn}$ has been used to investigate the transport properties of zinc species in $\mathrm{PEO}-\mathrm{Zn}\left(\mathrm{CF}_{3} \mathrm{SO}_{3}\right)_{2}$ polymer electrolytes.

In order to investigate the behaviour of calcium and magnesium electrodes, a three electrode cell, with calcium or magnesium metal as the reference and working electrodes and a mercury film electrode as the counter electrode, was used. Figure 7-1 shows the cyclic voltammogram of the calcium electrode in such a cell. For the anodic process, the potential for observing significant current was about $0.4 \mathrm{~V}$. After the first cycle, the overpotential was significantly reduced. The anodic current was also observed to increase after the first cycle (Figure 7-2). For the cathodic process however, the overpotential was very large, and the cathodic current very small, indicating that while calcium can be stripped under a modest anodic potential, it is very difficult to plate calcium ions onto a calcium cathode. 
The behaviour of the magnesium system is not much different from the calcium system (Figure 7-3). The overpotential of the anodic process, about $0.1 \mathrm{~V}$, was somewhat smaller than that in the calcium system. Again after the first anodic cycle, the overpotential was also significantly reduced (Figure 7-4). The cathodic current, however, was even less than that in the calcium system, indicating that magnesium is even more difficult to plate onto a magnesium electrode.

It is well-known that there is usually a very resistive passivating film on calcium or magnesium electrodes in organic [4] or solid electrolytes [5]. This is due to the fact that alkaline earth metals are very active, and can react with oxygen, nitrogen and moisture to form oxides, nitrides and hydroxide compounds. These products are difficult to dissolve in the solvent and may be very resistive. In addition to the above reactions, there are other processes involving the anions of the electrolytes, which may lead to the formation of a passivating layer. For example, it has been reported that these electrodes could be oxidised by the perchlorate anions in $\mathrm{Ca}\left(\mathrm{ClO}_{4}\right)_{2}$ or $\mathrm{Mg}\left(\mathrm{ClO}_{4}\right)_{2}$ electrolytes; also for anions with halogen elements, halides may be formed, eg. in $\mathrm{Ca}\left(\mathrm{CF}_{3} \mathrm{SO}_{3}\right)_{2}$ and $\mathrm{Mg}\left(\mathrm{CF}_{3} \mathrm{SO}_{3}\right)_{2}$ electrolytes, $\mathrm{CaF}_{2}$ or $\mathrm{MgF}_{2}$ were found to be formed $[5,6]$. Because the ionic conductivities of these products are also very low, for example the conductivity of the pure $\mathrm{CaF}_{2}$ is in the order $10^{-8} \mathrm{Scm}^{-1}$ at about $120^{\circ} \mathrm{C} \mathrm{[5]} \mathrm{-} \mathrm{much}$ lower than $\mathrm{LiF}$ on a lithium electrode, the electrochemical reactions on electrodes covered by a passivating films may consequently be blocked. In organic electrolytes with common inorganic calcium or magnesium salts, the passivating films may dissolve under an anodic polarisation potential [7]. The passivation of calcium and magnesium electrodes in $\mathrm{PEO}(400)$ dimethyl ether- $\mathrm{Ca}\left(\mathrm{CF}_{3} \mathrm{SO}_{3}\right)_{2}$ and $\mathrm{PEO}(400)$ dimethyl ether$\mathrm{Mg}\left(\mathrm{CF}_{3} \mathrm{SO}_{3}\right)_{2}$ electrolytes, may be similar to that occurring in conventional organic electrolytes. 


\section{7-1-2 Electrochemical Reactions on Mercury Film Electrodes}

In order to reduce the effect of the passivating layers on the electrochemical reactions on alkaline earth metal anodes and cathodes in electrochemical cells, two methods are often employed. The first is to use specific electrolytes. For instance, it was found that magnesium could be deposited onto magnesium electrode in electrolytes formed from organomagnesium halides [6]. The second is to use modified electrodes. For instance, $\mathrm{Ca}_{1-\mathrm{x}} \mathrm{Yb}_{\mathrm{x}}$ alloy has been used as an anode to replace pure calcium metal in fluoride solid-state batteries [5]. Passivation on the alloy anode was found to be significantly reduced in comparison with that on a pure calcium metal anode. Because many metals can dissolve in mercury to form an amalgam, mercury film electrodes are often employed as electrodes in the potentiometric stripping analysis (PSA) [8]. It has been found that the mercury film electrodes could be used to probe the behaviour of alkaline earth ions in ethanol solution [9]. This may indicate that calcium and magnesium may deposit on mercury film electrodes. In order to find a suitable nonblocking cathode towards $\mathrm{Ca}^{2}+/ \mathrm{Ca}$ or $\mathrm{Mg}^{2+} / \mathrm{Mg}$ in the polymer electrolytes based on $\mathrm{Ca}\left(\mathrm{CF}_{3} \mathrm{SO}_{3}\right)_{2}$ or $\mathrm{Mg}\left(\mathrm{CF}_{3} \mathrm{SO}_{3}\right)_{2}$, a mercury film electrode on a gold substrate was therefore examined.

Figure 7-5 shows the cyclic voltammogram of $\mathrm{Ca}^{2}+/ \mathrm{Hg}(\mathrm{Ca})$ in a three electrode cell with a mercury film electrode as the working electrode and calcium metal as the reference and the counter electrodes. The cathodic current was much larger when using the mercury electrode than when using the pure calcium electrode (Figure 7-1). This indicates that calcium can deposit more easily on the mercury film electrode. Further, a peak appeared in the anodic process, indicating that calcium can also be stripped from the mercury film electrode. Magnesium could also be deposited on a mercury film electrode quite easily (Figure 7-6), but the cathodic current was not so high as that in the calcium system. The cathodic process seemed somehow complicated in comparison with the calcium system. Part of the reason for this may be attributed to the strong 
interaction between magnesium cations and the oxygen atoms on the polymer chains. As discussed later, $\mathrm{PEO}-\mathrm{Mg}\left(\mathrm{CF}_{3} \mathrm{SO}_{3}\right)_{2}$ electrolytes are different from $\mathrm{PEO}-\mathrm{Ca}\left(\mathrm{CF}_{3} \mathrm{SO}_{3}\right)_{2}$ electrolytes. The bonds between magnesium ions and oxygen atoms in PEO are less labile than those in calcium system, due to the stronger acidity of $\mathrm{Mg}^{2+}[10]$. Magnesium ions are thus more difficult to release from the coordinated sites, and this may consequently give rise to slower electrode reactions. The anodic process, however, was much different from that in the calcium system, since the deposited magnesium in the mercury could not be stripped in the potential range studied. This behaviour is not fully understood.

In these cyclic voltammograms, it is interesting to note that the cathodic current can be observed at somewhat higher potential (positive potential in the cyclic voltammogram) than that of the $\mathrm{Ca}^{2+/ C a}$ or $\mathrm{Mg}^{2+} / \mathrm{Mg}$ reference. When a metal is deposited on a foreign substrate, it is often found that the difference of the surface state between the deposited atoms and the given metal leads to change of the potential for deposition of metal. For example, "underpotential deposition" [11] is well known. For mercury film electrodes, in addition to the change due to the difference in surface state of the electrodes, because of the formation of an amalgam, the potential for deposition may also be changed due to the difference of activity between the amalgam and the metal. The influence of the latter can be discussed as follows. The potential of $\mathrm{Ca}^{2}+/ \mathrm{Hg}(\mathrm{Ca})$ can be expressed as:

$$
E_{\mathrm{Ca}^{2+} / \mathrm{Ca}(\mathrm{Hg})}=\mathrm{E}_{\mathrm{Ca}^{2+} / \mathrm{Ca}}^{\mathrm{o}}+\frac{\mathrm{RT}}{2 \mathrm{~F}} \ln \frac{{ }^{\mathrm{a}} \mathrm{Ca}^{2+}}{\mathrm{a} \mathrm{Ca}(\mathrm{Hg})}
$$

while the potential of $\mathrm{Ca}^{2+} / \mathrm{Ca}$ is:

$$
\mathrm{E}_{\mathrm{Ca}^{2+} / \mathrm{Ca}}=\mathrm{E}_{\mathrm{Ca}^{2+} / \mathrm{Ca}}^{0}+\frac{\mathrm{RT}}{2 \mathrm{~F}} \ln \mathrm{a}_{\mathrm{Ca}^{2+}}
$$


where $\mathrm{E}^{\mathrm{O}} \mathrm{Ca}^{2+/ \mathrm{Ca}}$ is the standard potential, ${ }^{a} \mathrm{Ca}^{2+}$ is the activity of calcium ions in the electrolyte, ${ }^{a_{\mathrm{Ca}}(\mathrm{Hg})}$ is the activity of $\mathrm{Ca}$ in the mercury, $\mathrm{R}, \mathrm{T}$ and $\mathrm{F}$ have their conventional meanings. Because calcium is dilute in the mercury, i.e. $\mathrm{a}_{\mathrm{Ca}(\mathrm{Hg})}$ is much smaller than unity, $\mathrm{E}_{\mathrm{Ca}^{2+/} \mathrm{Ca}(\mathrm{Hg})}$ will be larger than $\mathrm{E}_{\mathrm{Ca}^{2+} \mathrm{Ca}}$, i.e. the deposition potential of calcium on the mercury film electrode is more positive than that on the pure calcium metal. Thus, a relatively positive potential for reduction of calcium or magnesium ions on a mercury film electrode can be expected.

Because the concentration of calcium or magnesium in the mercury film electrode increases with the time and the polarisation potentials for cathodic process, $\mathrm{E}_{\mathrm{Ca}^{2+} / \mathrm{Ca}(\mathrm{Hg})}$ or $\mathrm{E}_{\mathrm{Mg}^{2+} / \mathrm{Mg}(\mathrm{Hg})}$ thus will decrease (i.e. become more negative) with the time. Figure7-7 shows that the potentials for starting to observe the cathodic current decrease with the time having a value of $\sim 0.4 \mathrm{~V}$ for the first cathodic process cycle, $\sim 0.3 \mathrm{~V}$ for the second cycle and $\sim 0.2 \mathrm{~V}$ for the third cycle.

\section{7-1-3 The Electrode-Electrolyte Interfaces Under De Polarisation}

In order to further investigate the electrode-electrolyte interfaces, ac impedance analysis of $\mathrm{Ca}$ or $\mathrm{Mg}$ metal electrode-electrolyte and mercury electrode-electrolyte interfaces was carried out using a three electrode cell with $\mathrm{Ca}$ or $\mathrm{Mg}$ metal as reference electrode, mercury film electrode as counter or working electrode and PEO(400) dimethyl ether- $\mathrm{M}\left(\mathrm{CF}_{3} \mathrm{SO}_{3}\right)_{2}$ as electrolyte (hereafter $\mathrm{M}$ represents $\mathrm{Ca}$ or $\mathrm{Mg}$ ). The ac impedance spectra are shown in Figure 7-8 for calcium and Figure 7-9 for magnesium. When no de potential was applied to the working electrode, or the applied de potential was small, the ac impedance spectrum showed only a semicircle in the high frequency range corresponding to the bulk electrolyte, and a spike in the low frequency range which may be attributed to the electrode-electrolyte interface. This is a blocking electrode behaviour, indicating that the passivating layer on the electrode has blocked the 
electrode reaction. When a dc potential higher than the overpotential in the cyclic voltammogram was applied, two semicircle were then observed. That at high frequency again corresponds to the bulk electrolyte, while that with a capacitance of $7 \mu \mathrm{F} \mathrm{cm}-2$, which is of the order of magnitude for an electrical double layer capacitance [12], corresponds to the electrode-electrolyte interface. This indicates that the influence of the passivating layer on the electrode can be significantly reduced under this potential, leading to a relatively small resistance for $\mathrm{M}^{2+} / \mathrm{M}$ reaction at the electrode, which is consistent with the results of cyclic voltammetry. After stopping application of the dc potential, the metal electrode behaved like a blocking electrode once again. This may be explained by the instant reformation of the passivating layer.

Figure 7-10 and Figure 7-11 show the ac impedance spectra of mercury film electrodes in the calcium and magnesium electrolytes respectively. When a dc potential of $-50 \mathrm{mV}$ was applied, two semicircles were observed for both calcium and magnesium systems. The electrode-electrolyte interfacial capacitances obtained from the ac impedance spectra were about $19 \mu \mathrm{F} \mathrm{cm}^{-2}$ and $23 \mu \mathrm{F} \mathrm{cm}^{-2}$ for calcium and magnesium system respectively, which were again typical for an electrical double layer at a mercury electrode-electrolyte interface. This result also indicates that the mercury electrodes are non-blocking towards $\mathrm{M}^{2+} \mathrm{M}$.

It can therefore be concluded that $\mathrm{Ca}$ or $\mathrm{Mg}$ metal electrodes can allow $\mathrm{M} \longrightarrow \mathrm{M}^{2+}+2 \mathrm{e}$ reaction to take place easily under anodic potential of $0.4 \mathrm{~V}$ and $0.1 \mathrm{~V}$ respectively, and that mercury amalgam film electrodes are non-blocking towards $\mathrm{M}^{2+} / \mathrm{M}$ in electrolytes based on $\mathrm{PEO}$ and $\mathrm{M}\left(\mathrm{CF}_{3} \mathrm{SO}_{3}\right)_{2}$. 


\section{7-2 Dc Polarisation of $\mathrm{M} / \mathrm{PEO}-\mathrm{M}\left(\mathrm{CF}_{3} \mathrm{SO}_{3}\right)_{2} / \mathrm{Hg}(\mathrm{M})$ Two Electrode Cells}

It was noted in the above discussion that if a two electrode cell with calcium or magnesium metal as anode and cathode is polarised, the current flow will be blocked by the passivating layer on the electrodes, even if calcium or magnesium species are mobile in the polymer electrolytes. This suggests that some previous conclusions about the mobilities of calcium and magnesium species in polymer electrolytes based on the dc polarisation of such cells may be doubtful. Figure 7-12 shows the steady state current obtained by dc polarisation of such cells with $\mathrm{PEO}(400)$ dimethyl ether- $\mathrm{Ca}\left(\mathrm{CF}_{3} \mathrm{SO}_{3}\right)_{2}$ and $\mathrm{Mg}\left(\mathrm{CF}_{3} \mathrm{SO}_{3}\right)_{2}$ electrolytes using an applied voltage of $1 \mathrm{~V}$ at room temperature. The current decayed very fast, and the limiting current was about $150 \mathrm{nA}$ for both systems, which was comparable with the background current of the cell.

\section{7-2-1 De Polarisation of Ca / PEO-Ca(CF $\left.{ }_{3} \mathrm{SO}_{3}\right)_{2} / \mathrm{Hg}$ (Ca) Cells}

A two electrode cell with calcium metal as the anode and a mercury film electrode with gold substrate as the cathode was studied. As a result of the investigation reported in the above section, a $0.5 \mathrm{~V}$ dc potential was applied to ensure that the electrodes allow the $\mathrm{Ca}^{2+} / \mathrm{Ca}$ electrode reaction to take place easily. Thus, a $0.5 \mathrm{~V}$ applied voltage was used for the de polarisation of the two electrode cell.

Figure 7-13 shows the typical ac impedance spectra of the cell: $\mathrm{Ca} / \mathrm{PEO}(400)$ dimethyl ether $-\mathrm{Ca}\left(\mathrm{CF}_{3} \mathrm{SO}_{3}\right)_{2}(\mathrm{EO} / \mathrm{Ca}=20) / \mathrm{Hg}(\mathrm{Ca})$ measured during polarisation (under $0.5 \mathrm{~V} \mathrm{dc}$ potential for an initial 5 minutes) and after polarisation (measured immediately after stopping polarisation) at $45^{\circ} \mathrm{C}$. When the de polarisation potential was applied, as expected, the passivating layer on the calcium anode began to be reduced, and the ac impedance spectrum of the cell showed a semicircle corresponding to the electrode-electrolyte interface. This was consistent with the observations in the studies 
of cyclic voltammetry and ac impedance of the three electrode cell (Section 7-1). After the polarisation was stopped, a semicircle in the ac impedance corresponding to the electrode-electrolyte interfaces was still observed. But, unlike to the situation for the dc polarisation of the $\mathrm{Li} / \mathrm{PMEO}_{\mathrm{LiPF}}$ / Li cell (see Chapter 5), the interfacial resistance increased rather than decreased. This could be attributed to the rapid formation of a passivating layer on the calcium anode as discussed above. In addition, a straight line with an angle of about $48^{\circ}$ to the $x$-axis appears in the complex plane in very low frequency range. This is similar to the Warburg impedance arising from diffusioncontrolled transport of the electroactive species either within the electrode or the electrolyte bulk, e.g. in an intercalation electrode system [13]. In the present case, the low frequency straight line may be attributed to the diffusion controlled process caused by the diffusion of calcium atoms in the mercury film electrode.

The plot of polarisation current against polarisation time for the cell: $\mathrm{Ca} /$ $\mathrm{PEO}(400)$ dimethyl ether $-\mathrm{Ca}\left(\mathrm{CF}_{3} \mathrm{SO}_{3}\right)_{2}(\mathrm{EO} / \mathrm{Ca}=20) / \mathrm{Hg}(\mathrm{Ca})$ under an applied voltage of $0.5 \mathrm{~V}$ at room temperature is shown in Figure 7-14. The current after 20 hours is about $7.5 \mu \mathrm{A}$, which is relatively high. It is of interest to note that the I-t curve is different in form from those of polymer electrolytes based on the high molecular weight PEO. This may be due to the relatively low viscosity of $\mathrm{PEO}(400)$ dimethyl ether - $\mathrm{Ca}\left(\mathrm{CF}_{3} \mathrm{SO}_{3}\right)_{2}$ electrolyte which makes the motion of polymer chains easy, and thus may lead to some convection in the electrolyte in the cell.

Figure 7-15 shows a typical ac impedance spectrum of the cell Ca / PMEO$\mathrm{Ca}\left(\mathrm{CF}_{3} \mathrm{SO}_{3}\right)_{2}(\mathrm{EO} / \mathrm{Ca}=20) / \mathrm{Hg}(\mathrm{Ca})$ measured during polarisation (under an initial polarisation of $0.5 \mathrm{~V}$ potential for 5 minutes) at $80^{\circ} \mathrm{C}$. The spectrum measured during dc polarisation is similar to that in $\mathrm{PEO}(400)$ dimethyl ether- $\mathrm{Ca}\left(\mathrm{CF}_{3} \mathrm{SO}_{3}\right)_{2}$ system, except that the interfacial resistance is somewhat larger than in the latter. Therefore, the electrodes are also non-blocking towards $\mathrm{Ca}^{2+} / \mathrm{Ca}$ in this system. The spectrum 
measured after dc polarisation shows a large interfacial resistance. This may be explained by assuming that the passivating layer on calcium electrode forms almost instantly in this cell. A similar de polarisation for $\mathrm{PEO}-\mathrm{Ca}\left(\mathrm{CF}_{3} \mathrm{SO}_{3}\right)_{2}(\mathrm{EO} / \mathrm{Ca}=20)$ electrolyte in which the molecular weight of PEO was $4,000,000$ has been also carried out at $80^{\circ} \mathrm{C}$. The ac impedance of the cell with $\mathrm{PEO}-\mathrm{Ca}\left(\mathrm{CF}_{3} \mathrm{SO}_{3}\right)_{2}$ is not much different from that of the cell with PMEO-Ca(CF $\left.3 \mathrm{SO}_{3}\right)_{2}$ electrolyte (Figure 7-15).

Plots of the polarisation current against the polarisation time for cells with $\mathrm{PMEO}-\mathrm{Ca}\left(\mathrm{CF}_{3} \mathrm{SO}_{3}\right)_{2}$ and $\mathrm{PEO}-\mathrm{Ca}\left(\mathrm{CF}_{3} \mathrm{SO}_{3}\right)_{2}$ electrolytes are shown in Figure 7-14. These curves are similar to the typical polarisation behaviour of, for example, the $\mathrm{Li} /$ PMEO-LiPF $/$ Li cell (Chapter 5). The most important feature of these curves is that the limiting currents are significant for the cells with PMEO-Ca(CF $\left.3 \mathrm{SO}_{3}\right)_{2}$ and PEO$\mathrm{Ca}\left(\mathrm{CF}_{3} \mathrm{SO}_{3}\right)_{2}$ electrolytes. The limiting current for the former is $1.39 \mu \mathrm{A}$ and for the latter is $1.16 \mu \mathrm{A}$.

In order to correct the limiting current for the background, the cell: $\mathrm{Ca} /$ salt-free $\mathrm{PMEO} / \mathrm{Hg}(\mathrm{Ca})$ was polarised with a dc potential of $0.5 \mathrm{~V}$ at $80^{\circ} \mathrm{C}$. The polarisation current decreased from about $600 \mathrm{nA}$ to about 140nA immediately (Figure 7-16a). This indicates that the significant limiting current for the cell with $\mathrm{PMEO}-\mathrm{Ca}\left(\mathrm{CF}_{3} \mathrm{SO}_{3}\right)_{2}$ or $\mathrm{PEO}-\mathrm{Ca}\left(\mathrm{CF}_{3} \mathrm{SO}_{3}\right)_{2}$ electrolyte does not arise from the transport of impurities in the salt free polymer. In an additional experiment, $\mathrm{PMEO}-\mathrm{Ca}\left(\mathrm{CF}_{3} \mathrm{SO}_{3}\right)_{2}$ electrolyte was sandwiched between two gold electrodes and polarised at $80^{\circ} \mathrm{C}$ with a dc potential of $1.5 \mathrm{~V}$ (Figure 7-16b). The polarisation current decreased from about $50 \mu \mathrm{A}$ to about 3.5 $\mu \mathrm{A}$ in the first minute, and then further decreased to about $300 \mathrm{nA}$ after polarisation for three hours. Because a gold electrode is blocking both to $\mathrm{Ca}(\mathrm{II})$ species and $\mathrm{CF}_{3} \mathrm{SO}_{3}$ ions in this potential range, we cannot expect any limiting current from the electrochemical reactions for calcium (II) species or anions. The polarisation current is thus expected to decrease very fast, due to the polarisation of the electrolyte in the 
electric field. The residual current of about $300 \mathrm{nA}$ may again arise from impurities. Because the polarisation potential of $1.5 \mathrm{~V}$ is much higher than that for the polarisation of $\mathrm{PMEO}-\mathrm{Ca}\left(\mathrm{CF}_{3} \mathrm{SO}_{3}\right)_{2}$ or $\mathrm{PEO}-\mathrm{Ca}\left(\mathrm{CF}_{3} \mathrm{SO}_{3}\right)_{2}$ electrolyte, it is reasonable to believe that the limiting current arising from impurities in the electrolyte is not higher than 300 nA.

Since in the cell with $\mathrm{PMEO}-\mathrm{Ca}\left(\mathrm{CF}_{3} \mathrm{SO}_{3}\right)_{2}$ or $\mathrm{PEO}-\mathrm{Ca}\left(\mathrm{CF}_{3} \mathrm{SO}_{3}\right)_{2}$ electrolyte and calcium metal as the anode and mercury film electrode as the cathode, $\mathrm{CF}_{3} \mathrm{SO}_{3}{ }^{-}$ cannot undergo any electrochemical reactions on the anode under the de potential applied, the limiting current must arise from the transport of calcium(II) species in the electrolyte. The existence of a significant limiting current therefore suggests that calcium species in $\mathrm{PMEO}-\mathrm{Ca}\left(\mathrm{CF}_{3} \mathrm{SO}_{3}\right)_{2}$ and $\mathrm{PEO}-\mathrm{Ca}\left(\mathrm{CF}_{3} \mathrm{SO}_{3}\right)_{2}$ are mobile rather than immobile as reported previously [14].

Ion transport number, $\mathrm{t}_{+}$or $\mathrm{t}_{-}$, and limiting current fraction, $\mathrm{F}_{+}$, are often used to characterise the mobilities of ions in polymer electrolytes as discussed in Chapter 6. For dc polarisation of a two electrode cell, the potential acting across the electrolyte can be expressed as: $\Delta \phi=\Delta \mathrm{V}-\Delta \mathrm{E}$, where $\Delta \mathrm{V}$ is the corrected applied potential, and $\Delta \mathrm{E}$ is the Nernst potential difference of the two electrodes. For a two electrode cell with identical electrodes, $\Delta E=0$ at initial state, and $\Delta E=(R T / 2 F) \ln \left(a_{a} / a_{c}\right)$ at steady state, where $a_{a}$ and $a_{c}$ are the activities of salt on the anode and the cathode arising from the concentration gradient across the electrolyte. For a two electrode cell with nonidentical electrodes, however, there is a Nernst potential difference $\Delta \mathrm{E}$ between the two electrodes in the initial state. For the $\mathrm{Ca} / \mathrm{PEO}-\mathrm{Ca}\left(\mathrm{CF}_{3} \mathrm{SO}_{3}\right)_{2} / \mathrm{Hg}(\mathrm{Ca})$ cell, the equilibrium electrode potentials for calcium metal and mercury film electrodes are expressed in equation (7-1-1) and (7-1-2). In the initial state, $a_{a}=a_{c}$, the potential difference across the electrolyte is: 


$$
\Delta \phi=-\frac{\mathrm{RT}}{2 \mathrm{~F}} \ln \mathrm{a}_{\mathrm{Ca}(\mathrm{Hg})}
$$

where $\mathrm{a}_{\mathrm{Ca}(\mathrm{Hg})}$ is the activity of calcium in the mercury. The potential difference across the electrolyte at steady state is:

$$
\Delta \phi=\Delta V-\Delta E=\Delta V-\frac{R T}{2 F} \ln a_{C a(H g)}-\frac{R T}{2 F} \ln \left(\frac{a_{a}}{a_{c}}\right)
$$

Because $a_{\mathrm{Ca}(\mathrm{Hg})}$ is less than 1 , the actual potential difference across the electrolyte at initial state is greater than zero. With polarisation, the concentration of calcium in the mercury electrolyte increases, and the potential difference across the electrolyte thus decreases. The absolute value of the equilibrium potential difference between the two electrodes for the cell: $\mathrm{Ca} / \mathrm{PEO}(400)$ dimethyl ether $-\mathrm{Ca}\left(\mathrm{CF}_{3} \mathrm{SO}_{3}\right)_{2}(\mathrm{EO} / \mathrm{Ca}=20) /$ $\mathrm{Hg}(\mathrm{Ca})$ measured by potentiometer was reduced by about $60 \mathrm{mV}$ after dc polarisation for 20 hours with $0.5 \mathrm{~V}$ potential. In the light of this and possible non-Ohmic behaviour arising from the relatively large value of polarisation potential, it is seen to be very difficult to determine the actual potential difference across the electrolyte in Ca / PEO$\mathrm{Ca}\left(\mathrm{CF}_{3} \mathrm{SO}_{3}\right)_{2} / \mathrm{Hg}(\mathrm{Ca})$ cell. It was therefore concluded that no significance could readily be given to the value of limiting current fractions for such non-symmetric cells.

\section{7-2-2 Dc Polarisation of $\mathrm{Mg} / \mathrm{PEO}-\mathrm{Mg}\left(\mathrm{CF}_{3} \mathrm{SO}_{3}\right)_{2} / \mathrm{Hg}(\mathrm{Mg})$ Cells}

As in the calcium case, a mercury film electrode with gold substrate was used in the two electrode magnesium cell. As shown in the cyclic voltammogram, a passivating layer was again found on the magnesium metal electrode, which could be reduced by anodic polarisation using a potential of around $0.1 \mathrm{~V}$. The mercury film electrode allowed the magnesium to deposit quite easily.

Figure 7-17 shows the ac impedance spectrum of the cell: $\mathrm{Mg} / \mathrm{PEO}(400)$ dimethyl ether $-\mathrm{Mg}\left(\mathrm{CF}_{3} \mathrm{SO}_{3}\right)_{2}(\mathrm{EO} / \mathrm{Mg}=20) / \mathrm{Hg}(\mathrm{Mg})$ measured after dc polarisation at 
$0.1 \mathrm{~V}$ for 5 minutes at $80^{\circ} \mathrm{C}$. Similar to the cell with PEO(400) dimethyl ether$\mathrm{Ca}\left(\mathrm{CF}_{3} \mathrm{SO}_{3}\right)_{2}$ electrolyte, it showed a semicircle corresponding to the electrodeelectrolyte interface. The ac impedance spectrum of the same cell after dc polarisation at $0.1 \mathrm{~V}$ for 20 hours is shown in Figure 7-17. The semicircle corresponding to the electrode-electrolyte interface is still observable, but the interfacial resistance is enlarged. This may again arise from the instant formation of a passivating layer on the anode. In addition to the low frequency semicircle, a straight line with the angle of about $40^{\circ}$ relative to the $\mathrm{x}$-axis was observed in the lower frequency range. This was similar to the behaviour observed in the calcium system, and may be caused by the diffusion of magnesium atoms in the mercury film electrode. From analysis of these ac impedance spectra and the results of cyclic voltammograms shown in the above section (Section $7-1$ ), it can be concluded that both the anode and the cathode are non-blocking towards the $\mathrm{Mg}^{2+} / \mathrm{Mg}$ electrochemical reactions under an applied potential of $0.1 \mathrm{~V}$. Hence, this two electrode cell can be used to study de polarisation of $\mathrm{PEO}-\mathrm{Mg}\left(\mathrm{CF}_{3} \mathrm{SO}_{3}\right)_{2}$ electrolytes.

A series of polymer electrolytes, with the ratio of $\mathrm{EO} / \mathrm{Mg}$ of $20: 1$, based on $\mathrm{Mg}\left(\mathrm{CF}_{3} \mathrm{SO}_{3}\right)_{2}$ salt, methoxy end-capped PEO with molecular weight of 400,1000 , 1450,2000 and 3350, and ordinary PEO with molecular weight of 4000,6000,10000, 20000 and 4000000 were polarised with an applied voltage of $0.1 \mathrm{~V}$ at $80^{\circ} \mathrm{C}$ using the above two electrode cells. Typical ac impedance spectra during dc polarisation (measured after the initial 5 minutes) are shown by Figure 7-18 for the cell with $\mathrm{PEO}(1000)-\mathrm{Mg}\left(\mathrm{CF}_{3} \mathrm{SO}_{3}\right)_{2}$ electrolyte, Figure 7-19 for the cell with $\mathrm{PEO}(2000)-$ $\mathrm{Mg}\left(\mathrm{CF}_{3} \mathrm{SO}_{3}\right)_{2}$ electrolyte, Figure 7-20 for the cell with $\mathrm{PEO}(3350)-\mathrm{Mg}\left(\mathrm{CF}_{3} \mathrm{SO}_{3}\right)_{2}$ electrolyte, Figure 7-21 for the cell with $\mathrm{PEO}(4000)-\mathrm{Mg}\left(\mathrm{CF}_{3} \mathrm{SO}_{3}\right)_{2}$ electrolyte, Figure 7-22 for the cell with $\mathrm{PEO}(6000)-\mathrm{Mg}\left(\mathrm{CF}_{3} \mathrm{SO}_{3}\right)_{2}$ electrolyte and Figure 7-23 for the cell with $\mathrm{PEO}\left(4 \times 10^{6}\right)-\mathrm{Mg}\left(\mathrm{CF}_{3} \mathrm{SO}_{3}\right)_{2}$ electrolyte. The ac impedance spectra of the cells with polymer electrolytes based on PEO with molecular weight below 3350 are not 
significantly different and all show semicircles corresponding to the electrode-electrolyte interface. They are, however, very different from those based on higher molecular weight $\mathrm{PEO}$ in which the semicircles corresponding to the electrode-electrolyte interface are very large.

Typical plots for limiting current against the polarisation time are shown in Figure 7-24. The limiting currents for polymer electrolytes based on lower molecular weight $\mathrm{PEO}$ are quite significant, around 1-2 $\mu \mathrm{A}$. When the molecular weight of $\mathrm{PEO}$ is high, however, the limiting current is very low, around 100-300nA.

In order to subtract the background current, salt free polymers were polarised in two electrode cells under the same conditions. The limiting currents for polymer electrolytes were then corrected by subtracting the current following the polarisation of the salt free polymer to give net limiting currents. Values of the latter for polymer electrolytes based on different molecular weight PEO are listed in Table 7-1. For the electrolytes based on relatively low molecular weight PEO $(<3350)$, the net limiting current is significant, e.g $1.7 \mu \mathrm{A}$ for $\mathrm{PEO}(400)$ dimethyl ether- $\mathrm{Mg}\left(\mathrm{CF}_{3} \mathrm{SO}_{3}\right)_{2}$ electrolyte. For those based on high molecular weight PEO (> 3350), the net limiting current was low, around 200-100 nA, eg. the net limiting current of the cell with PEO $\left(4 \times 10^{6}\right)-\mathrm{Mg}\left(\mathrm{CF}_{3} \mathrm{SO}_{3}\right)_{2}$ electrolyte was about $100 \mathrm{nA}$. For the electrolyte based on PEO with the molecular weight of 3350 , the net limiting current is about $350 \mathrm{nA}$, much lower than those based on low molecular weight PEO, but higher than those based on high molecular weight PEO.

The limiting current from the dc polarisation of a two electrode cell may be controlled by two processes: (i) the kinetics of the electrochemical reactions on the electrodes and (ii) the diffusion and migration of electroactive substances. If the electrochemical reactions on the electrodes are very slow, the limiting current flow is 
controlled by the kinetics of the electrode reactions. On the other hand, if the kinetics of the electrode are fast, the limiting current flow is then controlled by the diffusion and migration of electroactive species. As shown above, both the anode and the cathode can permit $\mathrm{Mg}^{2+/} \mathrm{Mg}$ electrochemical reactions to take place under the polarisation potential used in the two electrode cells, so that the electrode reactions are not the controlling step. Therefore, the existence of significant net limiting current in the cells with the polymer electrolytes based on PEO(400), PEO(1000), PEO(1450) and PEO(2000) implies that magnesium species in the electrolytes are mobile. The very low net limiting current in the cells with the polymer electrolytes based on PEO with molecular weight above 3350 indicates that the mobilities of magnesium species in these electrolytes are very small. In $\mathrm{PEO}\left(4 \times 10^{6}\right)-\mathrm{Mg}\left(\mathrm{CF}_{3} \mathrm{SO}_{3}\right)_{2}$ electrolyte the magnesium species may be taken as effectively immobile due to very low net limiting current. The moderate net limiting current for the cell with the electrolyte based on PEO(3350) may imply that there are still significant mobilities for the magnesium species, but that these are much smaller than those in low molecular weight PEO.

If the mobilities of the electroactive species are very small, there may be no significant number of electroactive species to be transported to the electrode area in the time scale studied. Consequently, no significant electrode reactions can take place due to severe concentration polarisation. For polymer electrolytes with magnesium species, as shown above, because both the anode and the cathode can allow the electrochemical reactions to take place in the two electrode cell under dc polarisation, if the electroactive species are mobile, small interfacial resistance should be expected. For the polymer electrolytes based on PEO with the molecular weight below 3350 , because the ac impedance spectra show small interfacial resistances (smaller than $20 \mathrm{k} \Omega$ ), it may imply that the magnesium species in these electrolytes are relatively mobile. The interfacial resistances for polymer electrolytes based on high molecular weight PEO $(>4000)$ are very large, larger than $100 \mathrm{k} \Omega$. This may imply that the mobilities of magnesium species 
are very small in these polymer electrolytes. For electrolyte based on PEO(4 x 106), the interfacial resistance is so large that it shows a blocking electrode feature in the ac impedance spectrum. The interfacial resistance for the cell with electrolyte based on $\mathrm{PEO}(3350)$ is about $30 \mathrm{k} \Omega$, which is much smaller than those with the high molecular weight $\mathrm{PEO}$, implying that the magnesium species are not so immobile as in the case with the high molecular weight PEO. This is consistent to the conclusion obtained by the analysis of the net limiting current.

As discussed in the calcium case, the different potentials of the anode and cathode do not allow us to deduce the actual potential difference across the electrolyte, and it is therefore not useful to record the limiting current fractions.

\section{7-3 Explanations of the Mobilities of Calcium and Magnesium Species in the Polymer Electrolytes}

The above experimental results have shown that, in polymer electrolytes with high molecular weight $\mathrm{PEO}$, calcium species are relatively mobile, but magnesium species are immobile. In this section, an explanation is offered, in the light of discussion of the interactions between the cations and the EO units in the PEO chains.

\section{7-3-1 Interaction Between Cations And Polymer Host: the Hard-Soft Acid-Base Principle}

The hard-soft acid-base (HSAB) principle [15] has been widely used to explain and predict the stability of complexes formed between Lewis acids and bases. For the following complexes, the formation reaction:

$$
A: B^{\prime}+A^{\prime}: B=A: B+A^{\prime}: B^{\prime}
$$


is assumed as a reaction between acids $\mathrm{A}, \mathrm{A}^{\prime}$ and bases $\mathrm{B}, \mathrm{B}^{\prime}$. The reaction direction and the stability of products can be predicted according to the hardness and softness of the acids and bases.

Hard acids or bases are those atoms, molecules or ions which are highly electronegative, have low polarizability and are hard to oxidize. They tend to hold electrons. In contrast to the hard acids or bases, those atoms, molecules or ions which have low electronegativity, are highly polarizable and easy to oxidize are termed soft acids or bases. They tend to lose electrons. It has been found that hard acids prefer to react with hard bases, and the soft acids prefer to react with soft bases, to give stable complexes.

The formation of polymer electrolytes requires that there are certain interactions between the metal ions and the polar atoms. If these interactions are too weak, the loss of salt lattice energy due to dissolution cannot be compensated and consequently the salt cannot dissolve in the polymer solvent. Poly(ethylene oxide) is a hard base, due to the high electronegativity and low polarizability of oxygen atoms. Magnesium and calcium cations, because they have high positive charge density and low polarizability, are classified to hard acids [15]. It is therefore expected that the complexes formed by dissolving magnesium and calcium salts in PEO will be stable. In the other word, the interaction between the oxygen atoms in the polymer chains and magnesium or calcium cations is strong.

\section{7-3-2 Kinetics of Exchange of Bound Water Molecules in Aqueous}

\section{Solutions}

For polymer electrolytes based on the high molecular weight host, because the polymer chains cannot undergo long range motion due to entanglement of the polymer chains, the transport of cations or cation constituents is believed to be controlled by 
short range motion of the polymer chains, i.e. segmental motion [16]. Therefore, an essential pre-requisite for significant long range cation (or cation constituent) conduction in these polymer electrolytes is a high lability of the bonds between the metal ions and polar atoms in the polymer chains. Although the HSAB principle can predict the stability of complexes, it cannot predict their kinetic behaviour. As discussed above, the HSAB principle has predicted that the interaction between magnesium or calcium ions and the oxygen atoms in PEO chains will be strong. This may explain the lower mobilities for magnesium and calcium species compared with those of lithium or mercury species in the polymer electrolytes, but it cannot offer a reasonable explanation of the difference between polymer electrolytes based on magnesium and calcium salts. The ionic conduction of polymer electrolytes involves the formation and decomposition of the bonds between metal ions and the polar atoms in the polymer host, similar to the exchange of ligands for a complex. If the exchange rate is very slow, no significant ion transport can be observed in the high molecular weight polymer electrolytes. Therefore, the lability of the metal ion-polymer bonds is a key factor in determining whether a metal ion species is mobile or not. Unfortunately, data on the rate for exchange of coordinated segments in polymer electrolytes are not available. However, the rates for exchange of bound water molecules in aqueous solutions are available [17]. Because the oxygen atoms in $\mathrm{PEO}$ and $\mathrm{H}_{2} \mathrm{O}$ have very similar donicities (For PEO, DN is estimated to be 20, while DN of $\mathrm{H}_{2} \mathrm{O}$ is 16.4 [18]), the exchange rates in $\mathrm{PEO}$ can be expected to follow a similar trend to those in water.

The rates of exchange of bound water molecules for different metal ions in aqueous solutions are plotted in Figure 7-25. The exchange rates obviously depend on the characteristics of the metal ions. According to the measured exchange rate, metal ions can be classified into three categories. The first category includes those metal ions with high water exchange rate $\left(>10^{7} \mathrm{~s}^{-1}\right)$. The second category includes metal ions with low water exchange rate $\left(10^{5}-10^{7} \mathrm{~s}^{-1}\right)$. And the third category includes metal ions 
with very low water exchange rate $\left(<10^{5} \mathrm{~s}^{-1}\right)$. The ion radius and charge are two important factors affecting the water exchange rates. For example, the monovalent alkali metal ions are in the first category, the first row transition metal ions belong to the second category, and the trivalent $\mathrm{Al}^{3+}$ is in the third category.

It is interesting to note that while the water exchange rate of $\mathrm{Ca}^{2+}$ is in the first category $\left(\sim 4 \times 10^{8} \mathrm{~s}^{-1}\right)$, that of $\mathrm{Mg}^{2+}$ is in the second category $\left(\sim 10^{5} \mathrm{~s}^{-1}\right)$, about three orders of magnitude lower than that of $\mathrm{Ca}^{2+}$. This indicates that, although $\mathrm{Ca}^{2+}$ and $\mathrm{Mg}^{2+}$ are hard acids, expected to form quite stable complexes with water molecules, the formation and decomposition rates are very different. This may be attributed to the influence of the size of ions on the kinetics of exchange and in particular on the energy level of the transition state. (The ionic radius of $\mathrm{Ca}^{2+}$ and $\mathrm{Mg}^{2+}$ are $0.099 \mathrm{~nm}$ and $0.065 \mathrm{~nm}$ [19] respectively.) The larger radius of $\mathrm{Ca}^{2+}$ leads to the lower surface charge density and a higher water exchange rate. It may therefore be inferred that a low exchange rate and strong interaction between magnesium ions and oxygen atoms in PEO leads to very low mobility of magnesium species in the polymer electrolyte. In the time scale for ionic conduction, they can be taken as immobile. For polymer electrolytes based on PEO and calcium salts, due to the high exchange rate, the calcium species may be expected to be mobile.

\section{7-4 Conductivity and Phases in $\mathrm{PEO}-\mathrm{Ca}\left(\mathrm{CF}_{3} \mathrm{SO}_{3}\right)_{2}$ and PEO- $\mathrm{Mg}\left(\mathrm{CF}_{3} \mathrm{SO}_{3}\right)_{2}$ Electrolytes}

\section{7-4-1 Phase Behaviour}

The DSC trace of PEO-Ca( $\left.\mathrm{CF}_{3} \mathrm{SO}_{3}\right)_{2}$ of $\mathrm{EO} / \mathrm{Ca}=20$ is shown in Figure 7-26a. There are two endothermic peaks in the electrolyte. The first peak is at $70.0^{\circ} \mathrm{C}$, which is very close to the meiting point of pure $\mathrm{PEO}$ and may be attributed to the melting of 
crystalline PEO. The second peak is at about $278.6^{\circ} \mathrm{C}$ and may be attributed to the precipitation of salt in the electrolyte [20]. The latter process has been observed in polymer electrolytes based on certain sodium and lithium salts [21], and is explained by assuming that the solvation of those salts in PEO is a process with a negative entropy. The DSC shows no thermal effect in the range of temperature of $30-150^{\circ} \mathrm{C}$ (Figure 7-26b), indicating that the $\mathrm{PMEO}-\mathrm{Ca}\left(\mathrm{CF}_{3} \mathrm{SO}_{3}\right)_{2}$ electrolyte with an EO/Ca ratio of 20:1 was completely amorphous.

The DSC trace of PEO-Mg(CF $\left.\mathrm{SO}_{3}\right)_{2}$ electrolyte with the EO/Mg ratio of $20: 1$ shows only one endothermic peak which is at $70^{\circ} \mathrm{C}$ and a very small peak at about $200^{\circ} \mathrm{C}$ (Figure $7-27$ ). This differs from a previous report [2]. X-ray powder diffraction patterns are shown in Figure 7-28a. For the 20:1 sample, at room temperature, the diffraction pattern of crystalline PEO is observed, while at a temperature above the melting point of PEO, there is no diffraction pattern indicating that the system was totally amorphous. When the temperature was high, eg. above $200^{\circ} \mathrm{C}$, the diffraction pattern of $\mathrm{Mg}\left(\mathrm{CF}_{3} \mathrm{SO}_{3}\right)_{2}$ salt was found (Figure 7-28b). This may imply that in polymer electrolytes based on $\mathrm{Mg}\left(\mathrm{CF}_{3} \mathrm{SO}_{3}\right)_{2}$, similar to those based $\mathrm{Ca}\left(\mathrm{CF}_{3} \mathrm{SO}_{3}\right)_{2}$, salt precipitates at high temperature. In the DSC trace, the small endothermic peak from 190 $-200^{\circ} \mathrm{C}$ may be attributed to this effect.

$\mathrm{X}$-ray diffraction patterns of electrolytes with $\mathrm{EO} / \mathrm{Mg}=4$ and $\mathrm{EO} / \mathrm{Mg}=10$ are shown in Figure 7-29 and Figure 7-30. At the room temperature, crystalline PEO and $\mathrm{Mg}\left(\mathrm{CF}_{3} \mathrm{SO}_{3}\right)_{2}$ salt patterns are observed. At a temperature above the melting point of $\mathrm{PEO}$, there is only the salt pattern left. In comparison with that of the sample of EO/Mg $=20$, it may be concluded that there may be no crystalline complexes formed between magnesium ions and polymer host. This is similar to the observation in $\mathrm{P}(\mathrm{EO}){ }_{16} \mathrm{MgCl}_{2}$ system [22] and is much different from the electrolytes based on calcium or alkali metal salts. 
It has been reported that crystalline complexes were found in polymer electrolytes based on semi-crystalline $\mathrm{PEO}$ and many metal salts, eg. $\mathrm{PEO}-\mathrm{LiCF}_{3} \mathrm{SO}_{3}$ [23], $\mathrm{PEO}-\mathrm{Zn}\left(\mathrm{CF}_{3} \mathrm{SO}_{3}\right)_{2}$ (Chapter 6) and $\mathrm{PEO}-\mathrm{PbI}_{2}$ [22]. The analysis of $\mathrm{X}$-ray diff raction data for $\mathrm{PEO}-\mathrm{NaClO}_{4}$ electrolyte showed that the crystalline complexes are formed by coordinating the cations within the PEO helixes [24]. In these electrolytes, the ionic radii of cations cover a large range, from $0.060 \mathrm{~nm}$ for $\mathrm{Li}^{+}, 0.074 \mathrm{~nm}$ for $\mathrm{Zn}^{2+}$ to $0.120 \mathrm{~nm}$ for $\mathrm{Pb}^{2}+[19]$. If the sizes of cations in these electrolytes control the formation of crystalline complexes, magnesium cations should be accommodated in the $\mathrm{PEO}$ helixes to form crystalline complexes, because the ionic radius of $\mathrm{Mg}^{2+}(0.065 \mathrm{~nm})$ is close to that of $\mathrm{Li}^{+}$. This may indicate that the size of a ion may not be a key factor for the formation of crystalline complexes in polymer electrolytes based on semi-crystalline PEO. The situation of crystalline phases in polymer electrolytes is complicated, especially for electrolytes based on transition metal salts, because this may be influenced by electrolyte preparation methods, moisture level in the sample and so on. It has been reported that for the polymer electrolytes based on $\mathrm{Co}^{2+}[25,26], \mathrm{Mn}^{2+}[25]$ and $\mathrm{Ni}^{2+}[20,25]$ salts many fewer crystalline complexes have been observed. These ions, as in the case of $\mathrm{Mg}^{2+}$, have small ionic radii and show slow metal ion water exchange rates (about $10^{6}-10^{4} \mathrm{~s}^{-1}$ ), leading to very small metal ion mobilities in polymer electrolytes. In contrast, most polymer electrolytes based on salts with fast cation-water exchange rate $\left(>10^{7} \mathrm{~s}^{-1}\right)$ have clearly shown the formation of crystalline polymer-salt complexes, eg. PEO-Ca(CFy $\left.\mathrm{SO}_{3}\right)_{2}, \mathrm{PEO}-\mathrm{Cu}\left(\mathrm{CF}_{3} \mathrm{SO}_{3}\right)_{2}$ [27], $\mathrm{PEO}-\mathrm{PbI}_{2}$ [22] and $\mathrm{PEO}$ $\mathrm{CdBr}_{2}[25]$ in addition to those mentioned above. This may lead to the assumption that the lability of metal ion - polymer bonds has something to do with the formation of crystalline complexes in these polymer electrolytes. A preliminary explanation of this may be offered as follows: the configuration of crystalline PEO may not always be in favour of accommodating these cations to form a stable complex, because the configuration of the helix is relatively fixed in crystalline PEO and deformation of this 
configuration is limited. When cations enter the helixes, in order to form stable complexes, they may adjust their own position by rapid exchange of coordination bonds. If the deformation of the configuration of crystalline PEO is not too large, the ordered structure will be kept, leading to the formation of crystalline complexes. When magnesium cations enter the helixes of the crystalline PEO, because the interaction between magnesium cations and oxygen atoms is rather strong due to the small radius and high positive charge of magnesium cations, and the magnesium-oxygen bonds are much less labile, it is difficult for them to adjust their position. The only way to form complexes for them is thus by major deformation of the configuration of crystalline PEO, leading to relative absence of crystalline complexes. Under this circumstance, magnesium cations may be seen as only entering the amorphous component rather than the crystalline component of the polymer.

\section{7-4-2 Conductivity}

The temperature dependence of the conductivity of $\mathrm{PMEO}-\mathrm{Ca}\left(\mathrm{CF}_{3} \mathrm{SO}_{3}\right)_{2}$ and $\mathrm{PEO}-\mathrm{Ca}\left(\mathrm{CF}_{3} \mathrm{SO}_{3}\right)_{2}$ electrolytes are plotted in Figure 7-31. For PMEO-Ca( $\left(\mathrm{CF}_{3} \mathrm{SO}_{3}\right)_{2}$ electrolytes, VTF behaviour is shown. This is consistent with the DSC observations. For $\mathrm{PEO}-\mathrm{Ca}\left(\mathrm{CF}_{3} \mathrm{SO}_{3}\right)_{2}$, there is a knee at about $65^{\circ} \mathrm{C}$, which corresponds to the endothermic peak observed in DSC arising from the melting of crystalline PEO at that temperature. Above the melting temperature of PEO, the conductivities of PEO$\mathrm{Ca}\left(\mathrm{CF}_{3} \mathrm{SO}_{3}\right)_{2}$ and $\mathrm{PMEO}-\mathrm{Ca}\left(\mathrm{CF}_{3} \mathrm{SO}_{3}\right)_{2}$ with a ratio of $\mathrm{EO} / \mathrm{Ca}$ of 20 are similar. This is also consistent with the DSC result in which $\mathrm{PEO}-\mathrm{Ca}\left(\mathrm{CF}_{3} \mathrm{SO}_{3}\right)_{2}$ is amorphous above the melting temperature of PEO.

The conductivity of the sample of $\mathrm{EO} / \mathrm{Ca}=100$ is the highest in the concentration range of $20: 1$ to $100: 1$. It is interesting to note that the conductivities of the samples with the ratio of $\mathrm{EO} / \mathrm{Ca}=70,50$ and 30 are almost all the same. This is different from the case of polymer electrolytes based on alkali metals. In $\mathrm{PMEO}^{-\mathrm{LiClO}_{4}}$ 
electrolytes, as shown in Chapter 5, when the salt concentration increases, the concentration of ions increases making the conductivity increase; on the other hand when the salt concentration is very high, transient crosslinking effect is dominant causing the conductivity to decrease. In the calcium system, the invariant conductivity from $\mathrm{EO} / \mathrm{Ca}=70$ to $\mathrm{EO} / \mathrm{Ca}=30$ may imply that the influence on the conductivity of increasing the concentration of ions is compensated by the effect of increasing the transient crosslinking.

The temperature dependence of conductivity of $\mathrm{PEO}-\mathrm{Mg}\left(\mathrm{CF}_{3} \mathrm{SO}_{3}\right)_{2}$ electrolytes with an EO/Mg ratio of 20 is shown in Figure 7-32. The conductivity of $\mathrm{PEO}\left(4 \times 10^{6}\right)$ $\mathrm{Mg}\left(\mathrm{CF}_{3} \mathrm{SO}_{3}\right)_{2}$ electrolyte above the melting temperature of $\mathrm{PEO}$ is similar to that of $\mathrm{PEO}\left(4 \times 10^{6}\right)-\mathrm{Ca}\left(\mathrm{CF}_{3} \mathrm{SO}_{3}\right)_{2}$ with $\mathrm{EO} / \mathrm{Ca}$ of 20 , and is very close to previously reported data [2] and that of $\mathrm{P}(\mathrm{EO})_{9} \mathrm{LiCF}_{3} \mathrm{SO}_{3}$ electrolyte [28]. This is consistent with the conclusion that magnesium species are immobile in the electrolyte. As discussed above, because the mobility of calcium is not so high as lithium in high molecular weight PEO, the conductivity thus mainly arises from motion of the anions. The conductivity of PEO$\mathrm{Ca}\left(\mathrm{CF}_{3} \mathrm{SO}_{3}\right)_{2}$ will therefore not differ much from that of $\mathrm{PEO}-\mathrm{Mg}\left(\mathrm{CF}_{3} \mathrm{SO}_{3}\right)_{2}$.

For the electrolytes $\mathrm{PEO}-\mathrm{Mg}\left(\mathrm{CF}_{3} \mathrm{SO}_{3}\right)_{2}$ based on different molecular weights of $\mathrm{PEO}$, the knee point in the plot of $\log \sigma$ versus $1000 / \mathrm{T}$ increases with increasing molecular weight of PEO at the low end of the range studied. These knee points are the same as the melting points of PEO observed in DSC (Table 7-2). When the temperature is below the knee point, the conductivity decreases with increasing molecular weight of PEO. This may arise from the increase in the fraction of amorphous PEO with decreasing molecular weight. When the temperature is above the melting temperature, however, the conductivity shows little molecular weight dependence. At a temperature higher than the melting point, the electrolyte becomes totally amorphous. Because the anions are much mobile than cations, and the conductivity arises from the motion of 
both anions and cations, the conductivities should be similar, so long as the segmental motion of PEO is not particularly molecular weight dependent. Thus, this is not only consistent with the conclusion derived from the dc polarisation experiment, but also supports the assertion of that the mobility of anions is not significantly molecular weight dependent.

\section{7-5 Conclusions}

(1) There is a very resistive passivating layer on both calcium and magnesium metal electrodes. This passivating layer can be reduced by anodic polarisation under certain potentials, but still blocks the deposition of calcium and magnesium.

(2) By choosing mercury film electrode as cathode for the calcium and magnesium cells, calcium and magnesium can be deposited.

(3) Dc polarisation results show that calcium species are mobile in $\mathrm{PEO}-\mathrm{Ca}\left(\mathrm{CF}_{3} \mathrm{SO}_{3}\right)_{2}$ electrolytes based on the high molecular weight PEO. For PEO-Mg( $\left.\mathrm{CF}_{3} \mathrm{SO}_{3}\right)_{2}$ electrolytes based on the high molecular weight PEO however, the results show that magnesium species are immobile. For electrolytes based on very low molecular weight PEO, magnesium species are mobile. It is concluded that a crucial criterion for the mobility of metal ions in polymer electrolytes based on high molecular weight polymer host is the lability of metal-polymer host bond.

(4) No crystalline PEO-salt complexes were found to form in $\mathrm{PEO}-\mathrm{Mg}\left(\mathrm{CF}_{3} \mathrm{SO}_{3}\right)_{2}$ electrolytes. This may be a consequence of the low lability of metal ion-polymer bond. At higher temperature, eg. above $200^{\circ} \mathrm{C}$, salt precipitation was observed. 
(5) The conductivity of PEO- $\mathrm{Mg}\left(\mathrm{CF}_{3} \mathrm{SO}_{3}\right)_{2}$ electrolytes is barely molecular weight dependent at temperatures above the melting point of $\mathrm{PEO}$. $\mathrm{PMEO}-\mathrm{Ca}\left(\mathrm{CF}_{3} \mathrm{SO}_{3}\right)_{2}$ electrolytes are totally amorphous and the conductivity is not salt concentration dependent in the range of $\mathrm{EO} / \mathrm{Ca}=70$ to $\mathrm{EO} / \mathrm{Ca}=30$. Conductivities of both PEO$\mathrm{Mg}\left(\mathrm{CF}_{3} \mathrm{SO}_{3}\right)_{2}$ and $\mathrm{PEO}-\mathrm{Ca}\left(\mathrm{CF}_{3} \mathrm{SO}_{3}\right)_{2}$ with $\mathrm{EO} / \mathrm{Mg}$ or EO/Ca ratio of 20 are very close to that of $\mathrm{PEO}-\mathrm{LiCF}_{3} \mathrm{SO}_{3}$ with EO/Li ratio of 9 . 


\section{References}

[1] L. L. Yang, R. Huq and G. C. Farrington, Solid St. Ionics, 18 / 19, 291 (1986).

[2] A. Gilmour, M. Z. A. Munshi, B. B. Owens and W. H. Smyrl, in Materials and Processes for Lithium Batteries, ed. K. M. Abraham and B. B. Owens, Electrochem. Soc. $89-4,1989$, page 358 .

[3] G. C. Farrington and R. G. Linford, in Polymer Electrolyte Reviews 2, ed. J. R. MacCallum and C. A. Vincent, Elsevier, London, 1989, page 255.

[4] E. Peled, J. Electrochem. Soc. 126, 2047 (1979).

[5] J. Schoonman and A. Wolfert, J. Electrochem. Soc. 128, 1522 (1981).

[6] T. D. Gregory, R. J. Hoffman and R. C. Winterton, J. Electrochem. Soc. 137, 775 (1990).

[7] D. Aurbach, R. Skaletsky and Y. Gofer, J. Electrochem. Soc. 138, 3536 (1991),

[8] W. R. Heineman, H. B. Mark, Jr. and J. A. Wise, in Laboratory Techniques in Electroanalytical Chemistry, ed. P. T. Kissinger and W. R. Heineman, Marcel Dekker Inc. New York, 1984, page 499.

[9] J. F. Coetzee, A. Hussan and T. R. Petrick, J. Anal. Chem. 55, 120 (1983).

[10] M. Armand, Discuss. Farad. Soc. 88, 65 (1989); also see: General Discussion, Discuss. Farad. Soc. 88, 87 (1989). 
[11] A. J. Bard and L. R. Faulkner, Electrochemical Methods: Fundamentals and Applications, Wiley, New York, 1980.

[12] P. Payre, in Advances in Electrochemistry and Electrochemical Engineering, Vol.7, ed. P. Delahay and C. W. Tobias, Wiley-Interscience, New York, 1970.

[13] C. Ho, I. D. Raistrick and R. A. Huggins, J. Electrochem. Soc. 127, 343 (1980).

[14] F. M. Gray, C. A. Vincent, P. G. Bruce and J. Nowinski, in Second International Symposium on Polymer Electrolytes, ed. B. Scrosati, Elsevier, London, 1990, page 299.

[15] R. G. Pearson, J. Chem. Ed. 45, 581 (1968).

[16] M. A. Ratner, in Polymer Electrolyte Reviews 1, ed. J. R. MacCallum and C. A. Vincent, Elservier, London, 1987, page 173.

[17] M. Eigen, Pure Appl. Chem. 6, 97 (1963).

[18] M. Armand, in Polymer Electrolyte Reviews 1, ed. J. R. MacCallum and C. A. Vincent, Elscier, London, 1987, page 1.

[19] J. G. Stark and H. G. Wallace, Chemistry Data Book SI ed. John Murray Ltd. London, 1980.

[20] M. A. Mehta, PhD thesis, University of St. Andrews, 1992. 
[21] D. Teeters and R. Frech, Solid St. Ionics, 18 / 19, 271 (1986).

[22] A. Wendsjo and H. Yang, in Second International Symposium on Polymer Electrolytes, ed. B. Scrosati, Elsevier, London, 1990, page 225.

[23] F. M. Gray, Solid Polymer Electrolytes: Fundamentals and Technological Applications, VCH Publishers, New York, 1991.

[24] P. Lightfoot, M. A. Mehta and P. G. Bruce, J. Mater. Chem., 2, 379 (1992).

[25] R. Huq and G. C. Farrington, Solid St. Ionics, 28-30, 990 (1988).

[26] B.V. R. Chowdari, R. Huq and G. C. Farrington, Solid St. Ionics, 57, 49 (1992).

[27] S. Passerini, R. Curini and B. Scrosati, Appl. Phys. A 49, 425 (1989).

[28] L. Yang, A. Zhang, B. Qiu, J. Yin and Q. Liu, Solid St. Ionics, 28-30, 1029 (1988). 
Table 7-1. Limiting current of dc polarisation of $\mathrm{Mg} / \mathrm{PEO}\left(\mathrm{M}_{w}\right)-\mathrm{Mg}\left(\mathrm{CF}_{3} \mathrm{SO}_{3}\right)_{2} /$ $\mathrm{Hg}(\mathrm{Mg})$ under $0.1 \mathrm{~V}$ applied potential at $80^{\circ} \mathrm{C}$

\begin{tabular}{llc}
\hline $\mathrm{M}_{\mathrm{w}}$ & $\mathrm{I}_{\mathrm{s}} / \mu \mathrm{A}$ & net limiting $\mathrm{I}_{\mathrm{s}} / \mu \mathrm{A}$ \\
\hline 400 & 1.76 & 1.61 \\
1000 & 1.34 & 1.26 \\
1450 & 1.22 & 1.20 \\
2000 & 1.25 & 1.05 \\
3350 & 0.58 & 0.35 \\
4000 & 0.40 & 0.20 \\
6000 & 0.35 & 0.30 \\
8000 & 0.37 & 0.25 \\
$1 \times 10^{4}$ & 0.25 & 0.15 \\
$2 \times 10^{4}$ & 0.30 & 0.20 \\
$4 \times 10^{6}$ & 0.25 & 0.15 \\
\hline
\end{tabular}

Table 7-2. Melting temperatures and temperatures of the knee points in the log ovs. $1000 / \mathrm{T}$ curves for $\mathrm{PEO}\left(\mathrm{M}_{\mathrm{w}}\right)-\mathrm{Mg}\left(\mathrm{CF}_{3} \mathrm{SO}_{3}\right)_{2}(20: 1)$ electrolytes

\begin{tabular}{lccccc}
\hline $\mathrm{M}_{\mathrm{w}}$ & 1450 & 3350 & 6000 & $2 \times 10^{4}$ & $4 \times 10^{6}$ \\
\hline melting point $/{ }^{\circ} \mathrm{C}$ & 47.6 & 52.6 & 53.0 & 57.0 & 75.2 \\
\hline knee point $/{ }^{\circ} \mathrm{C}$ & 48 & 50 & 57 & 57 & 72 \\
\hline
\end{tabular}

a: measured by DSC. 

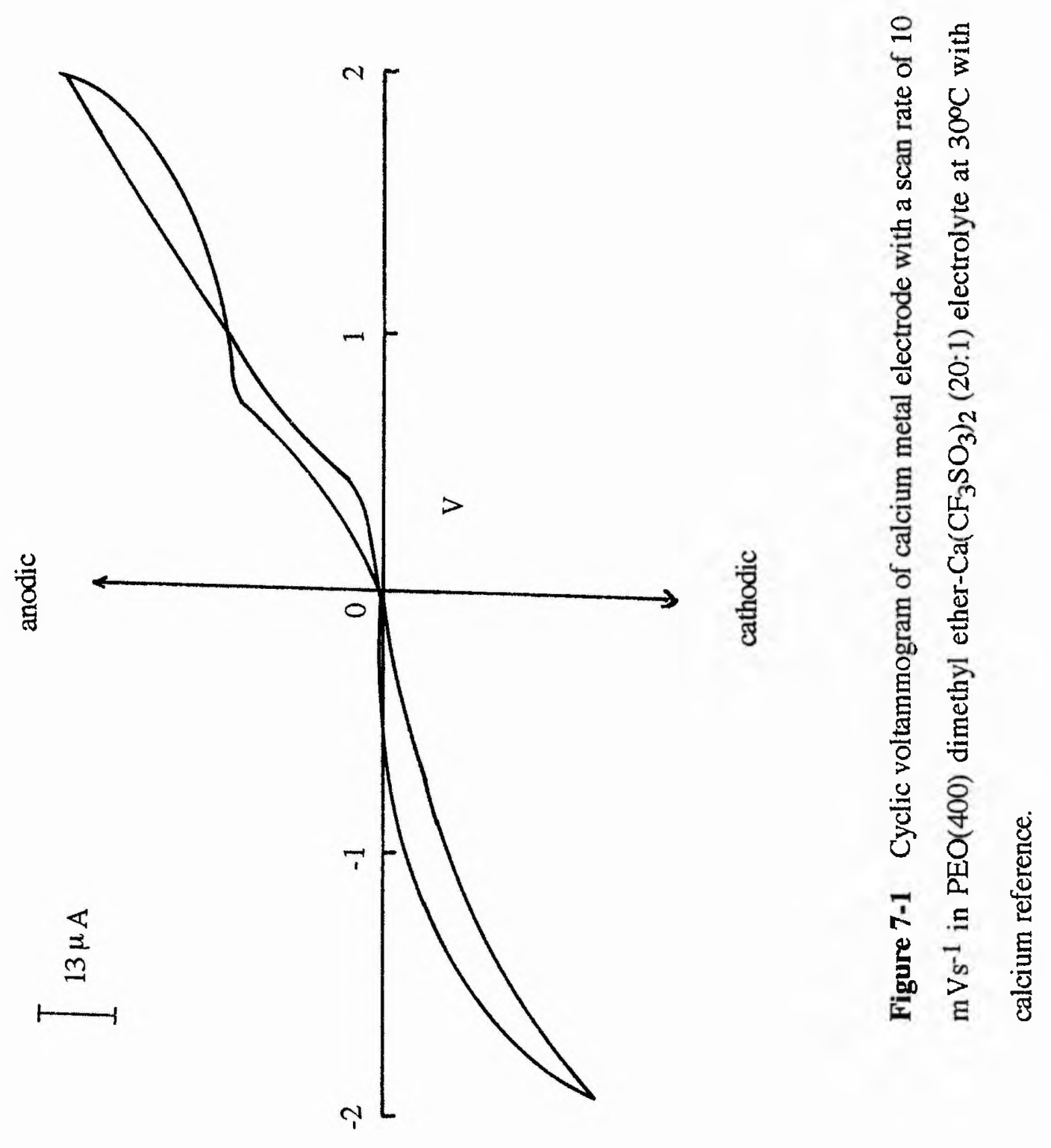


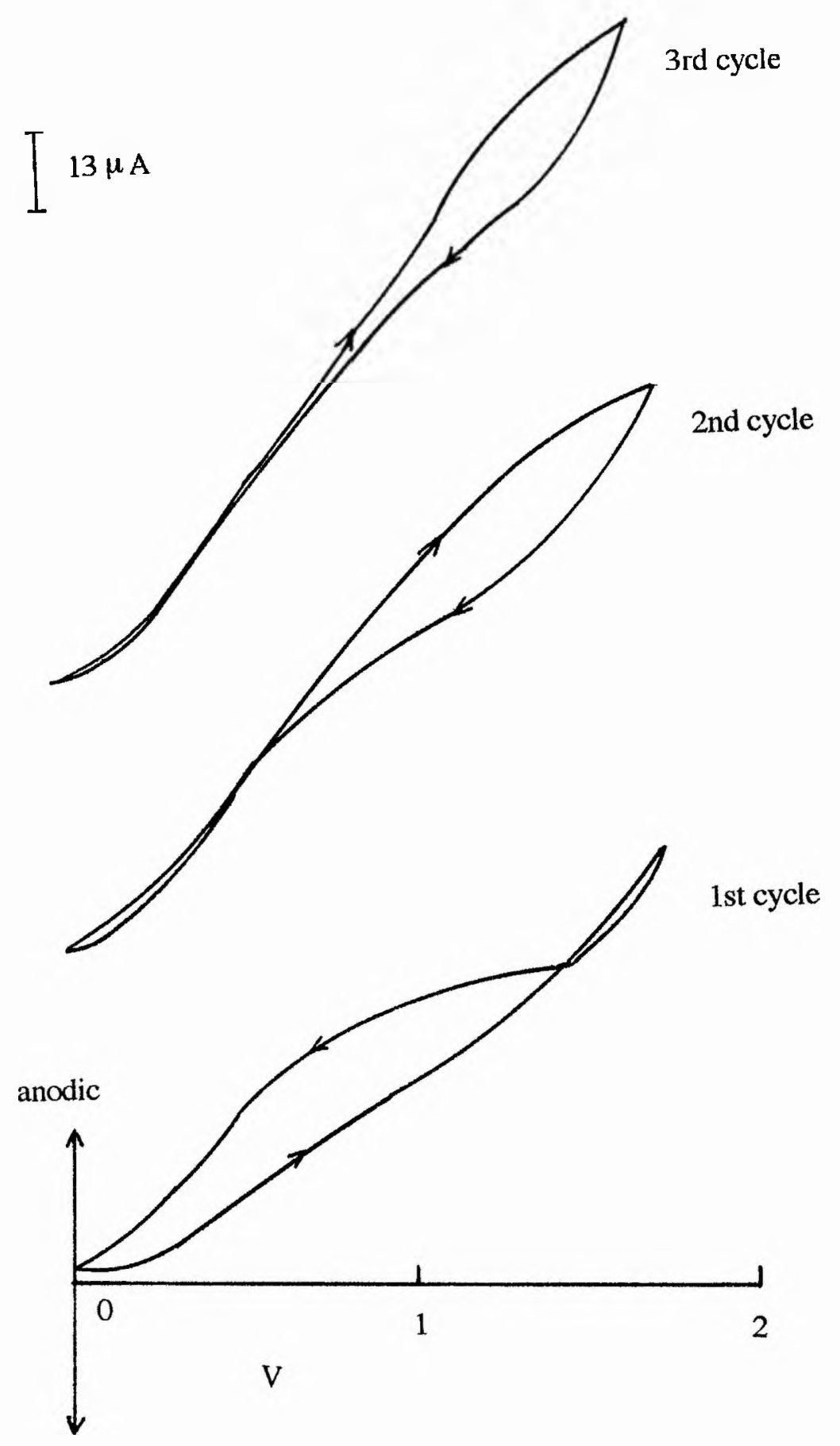

cathodic

Figure 7-2 Cyclic voltammogram of calcium metal electrode with a scan rate of 10 $\mathrm{mVs}^{-1}$ in $\mathrm{PEO}(400)$ dimethyl ether- $\mathrm{Ca}\left(\mathrm{CF}_{3} \mathrm{SO}_{3}\right)_{2}(20: 1)$ electrolyte at $30^{\circ} \mathrm{C}$ with calcium reference for three anodic cycles. 


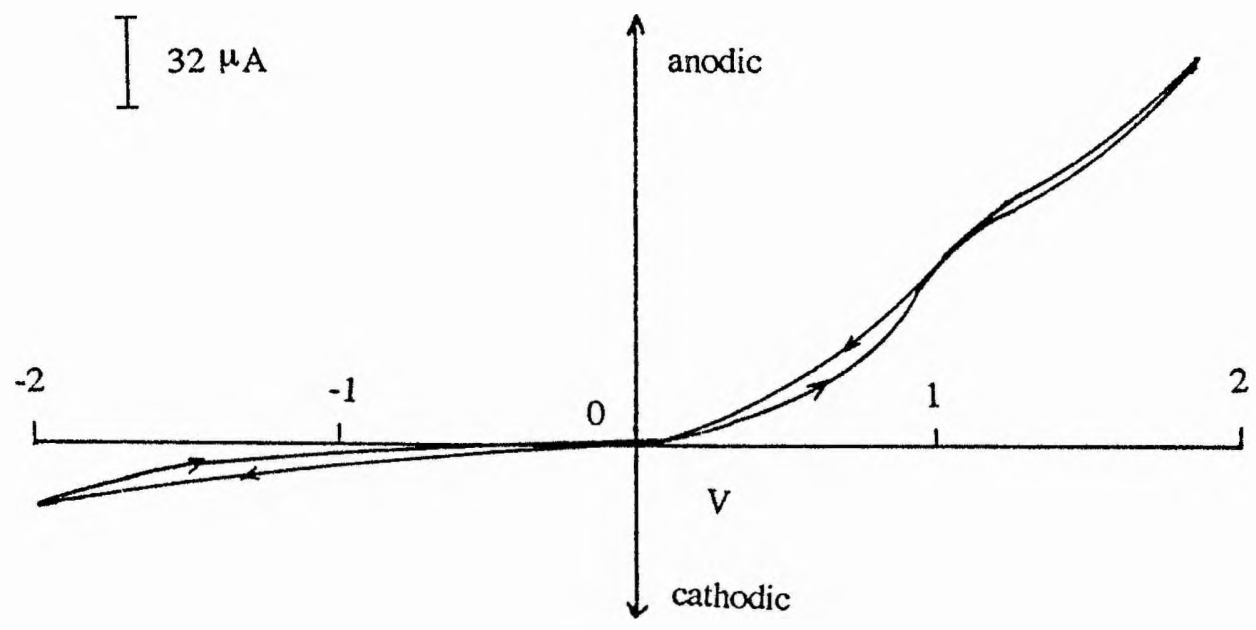

Figure 7-3 Cyclic voltammogram of magnesium metal electrode with a scan rate of $10 \mathrm{mVs}^{-1}$ in $\mathrm{PEO}(400)$ dimethyl ether- $\mathrm{Mg}\left(\mathrm{CF}_{3} \mathrm{SO}_{3}\right)_{2}(20: 1)$ electrolyte at $30^{\circ} \mathrm{C}$ with magnesium reference.

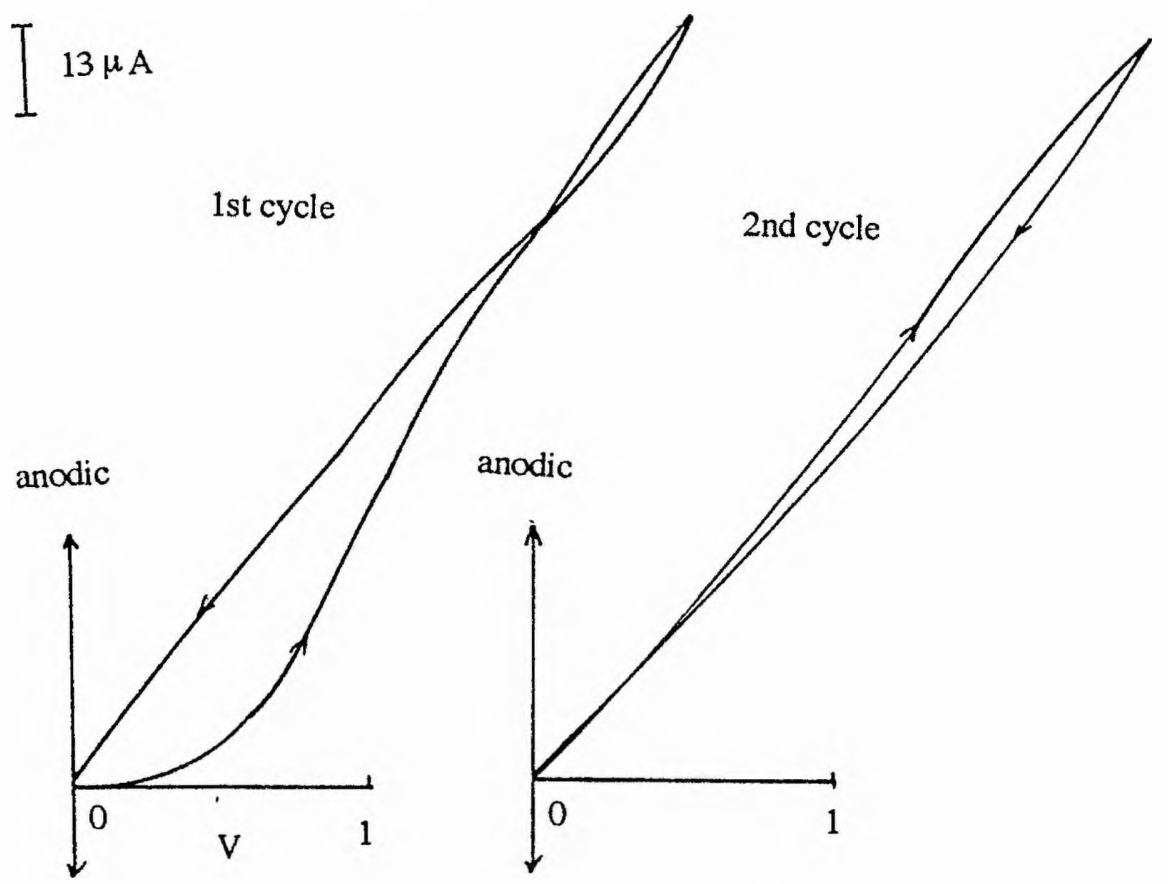

Figure 7-4 Cyclic voltammogram of magnesium metal electrode with a scan rate of $10 \mathrm{mVs}^{-1}$ in $\mathrm{PEO}(400)$ dimethyl ether $-\mathrm{Mg}\left(\mathrm{CF}_{3} \mathrm{SO}_{3}\right)_{2}(20: 1)$ electrolyte at $30^{\circ} \mathrm{C}$ with $\mathrm{Mg}$ reference for two anodic cycles. 


\section{anodic}

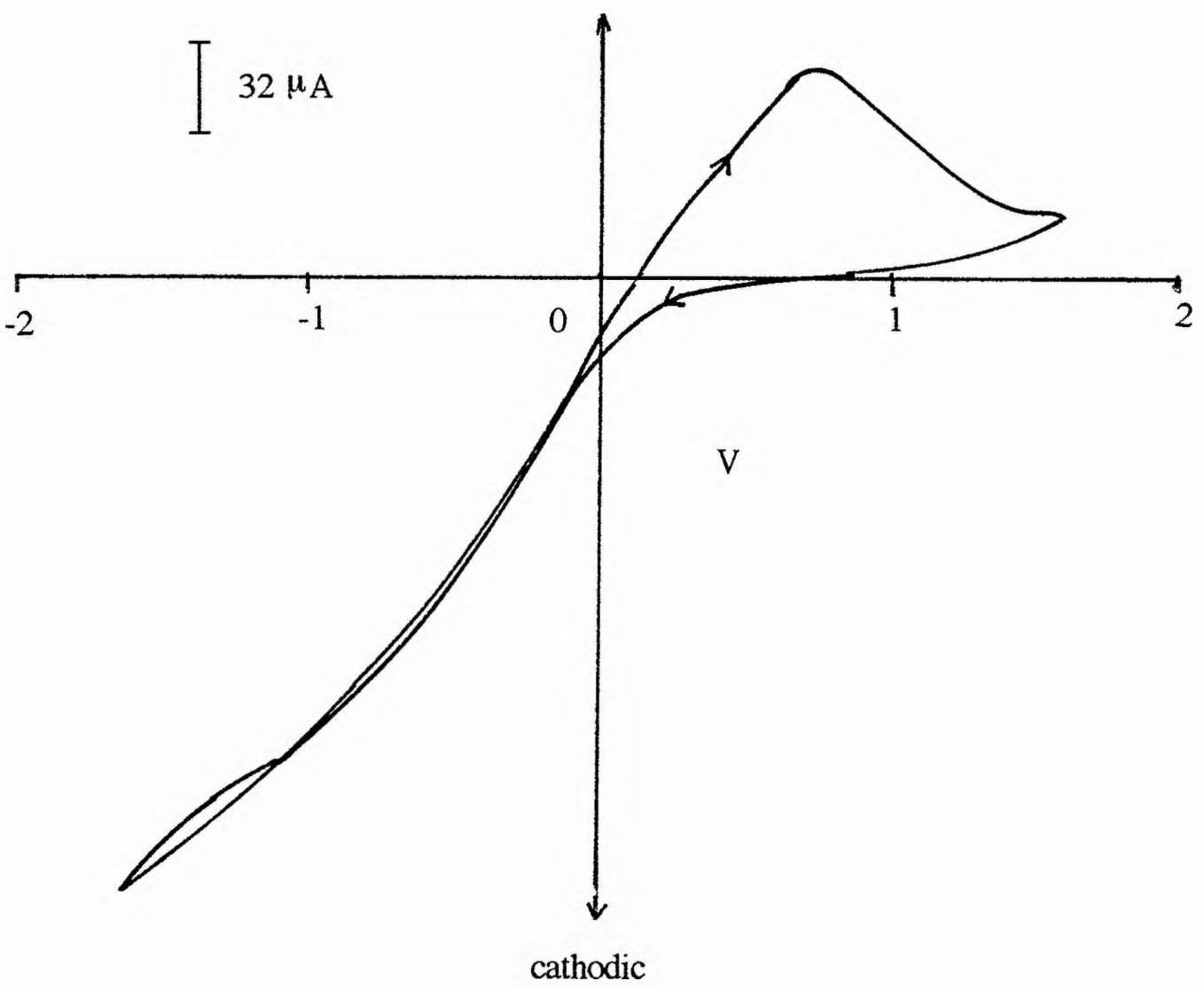

Figure 7-5 Cyclic voltammogram of mercury film electrode with a scan rate of 10 $\mathrm{mVs}^{-1}$ in $\mathrm{PEO}(400)$ dimethyl ether- $\mathrm{Ca}\left(\mathrm{CF}_{3} \mathrm{SO}_{3}\right)_{2}(20: 1)$ electrolyte at $30^{\circ} \mathrm{C}$ with calcium reference. 


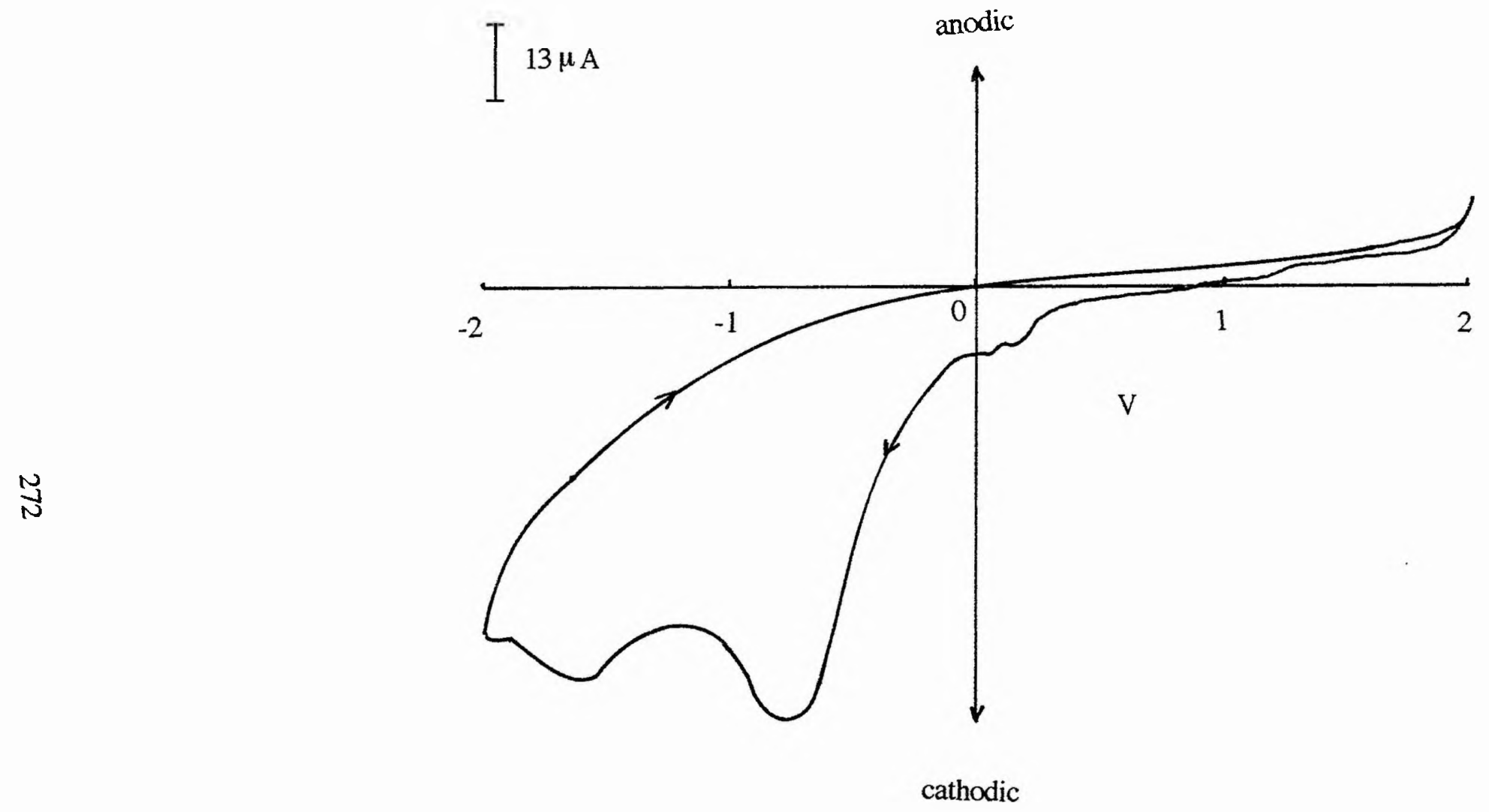

Figure 7-6 Cyclic voltammogram of mercury film electrode with a scan rate of 10 $\mathrm{mVs}^{-1}$ in $\mathrm{PEO}(400)$ dimethyl ether- $\mathrm{Mg}\left(\mathrm{CF}_{3} \mathrm{SO}_{3}\right)_{2}(20: 1)$ electrolyte at $30^{\circ} \mathrm{C}$ with magnesium reference. 


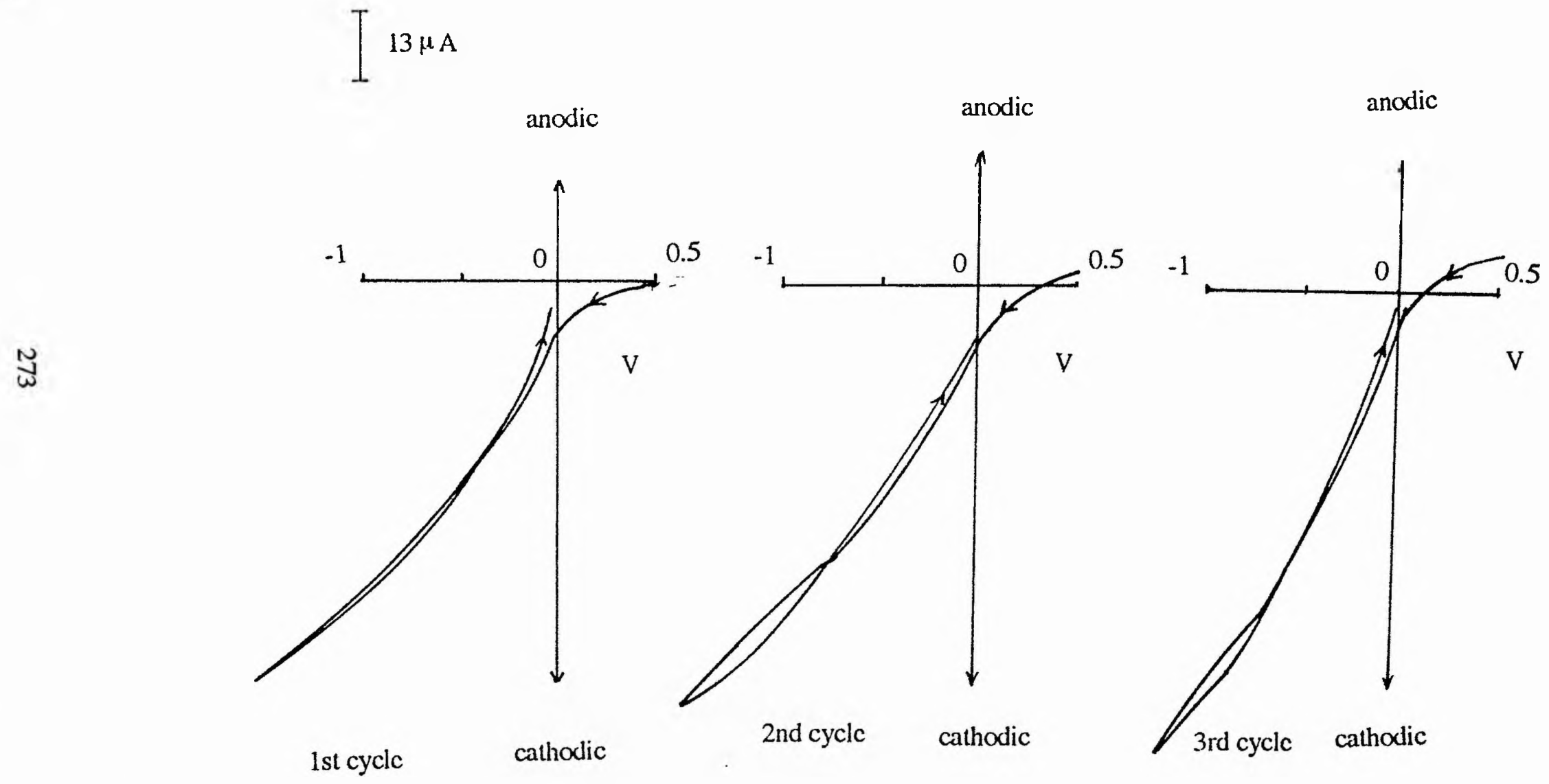

Figure 7-7 Cyclic voltammogram of mercury film clectrode with a scan rate of 10 $\mathrm{mVs}^{-1}$ in $\mathrm{PEO}(400)$ dimethyl ether- $\mathrm{Ca}\left(\mathrm{CF}_{3} \mathrm{SO}_{3}\right)_{2}(20: 1)$ electrolyte at $30^{\circ} \mathrm{C}$ with calcium reference for threc cathodic cycles. 


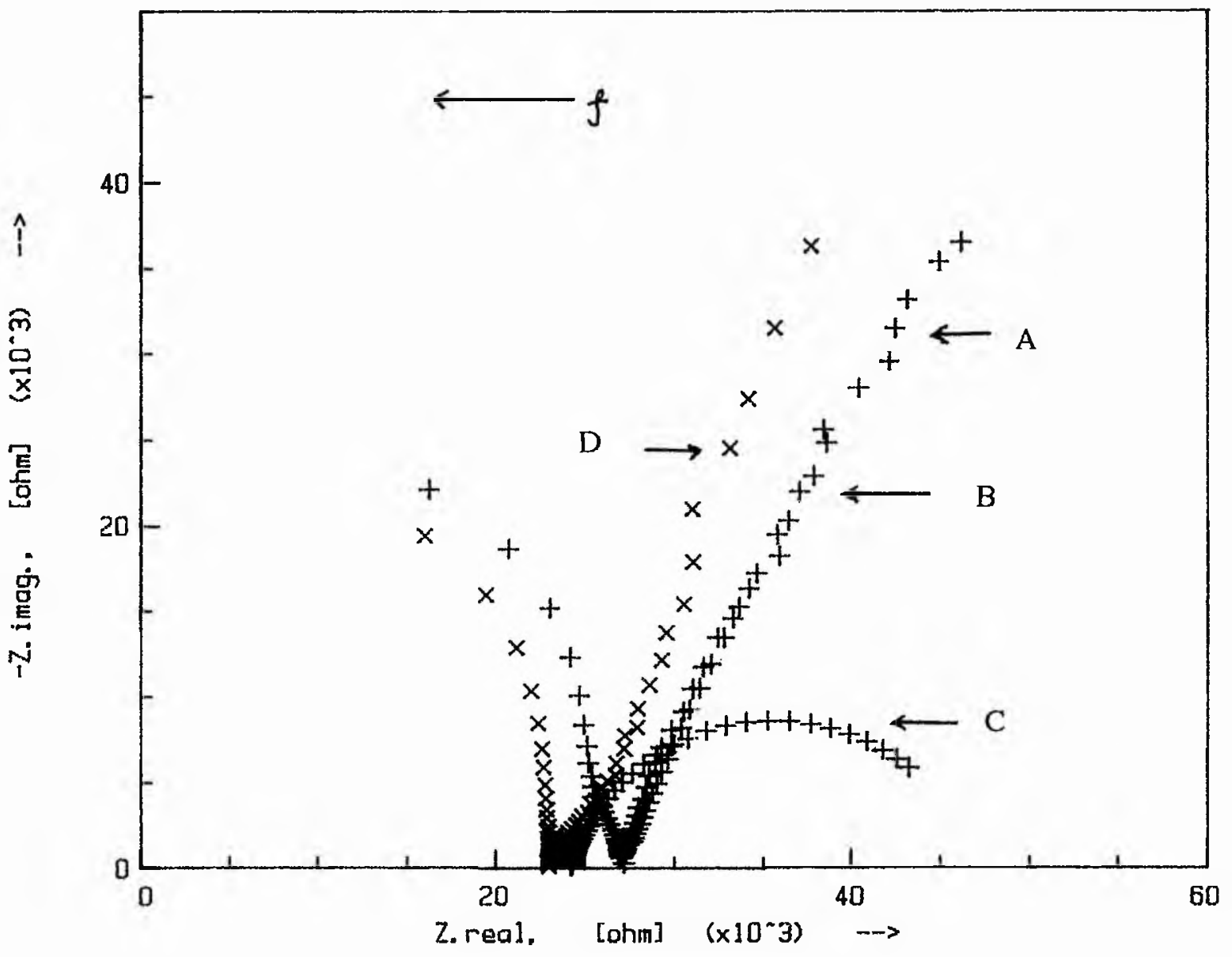

Figure 7-8 Ac impedance spectra of calcium electrode under anodic polarisation in $\operatorname{PEO}(400)$ dimethyl ether- $\mathrm{Ca}\left(\mathrm{CF}_{3} \mathrm{SO}_{3}\right)_{2}(20: 1)$ electrolyte at $30^{\circ} \mathrm{C}$ with calcium reference, (A) without application of the polarisation potential, (B) under $0.2 \mathrm{~V}$, (C) under $0.5 \mathrm{~V}$ and (D) after stopping applicarion of the polarisation potential. 


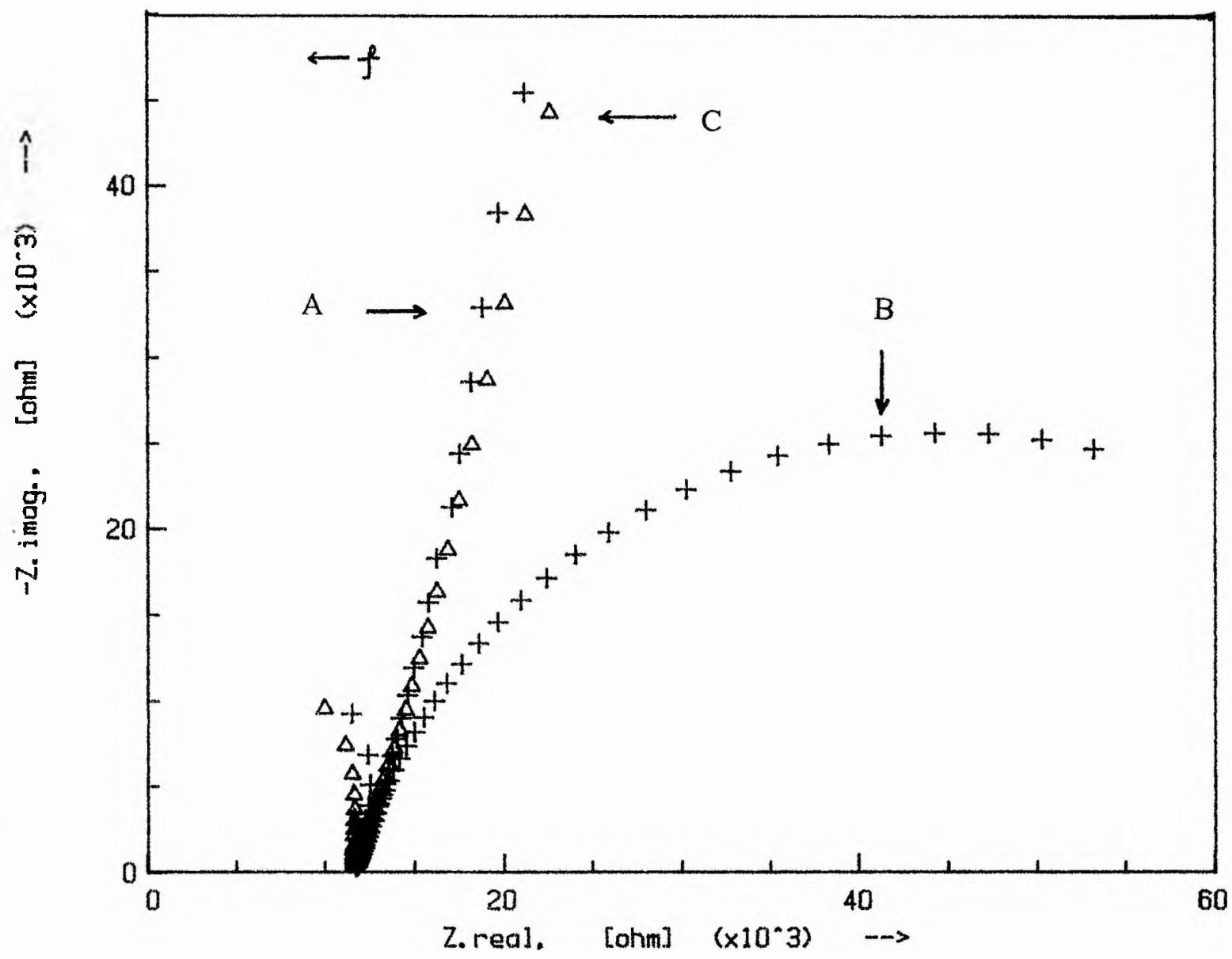

Figure 7-9 Ac impedance spectra of magnesium electrode under anodic polarisation in $\mathrm{PEO}(400)$ dimethyl ether- $\mathrm{Mg}\left(\mathrm{CF}_{3} \mathrm{SO}_{3}\right)_{2}(20: 1)$ electrolyte at $30^{\circ} \mathrm{C}$ with magnesium reference, (A) without application of the polarisation potential, (B) under $0.5 \mathrm{~V}$ and (C) after stopping application of the polarisation potential. 


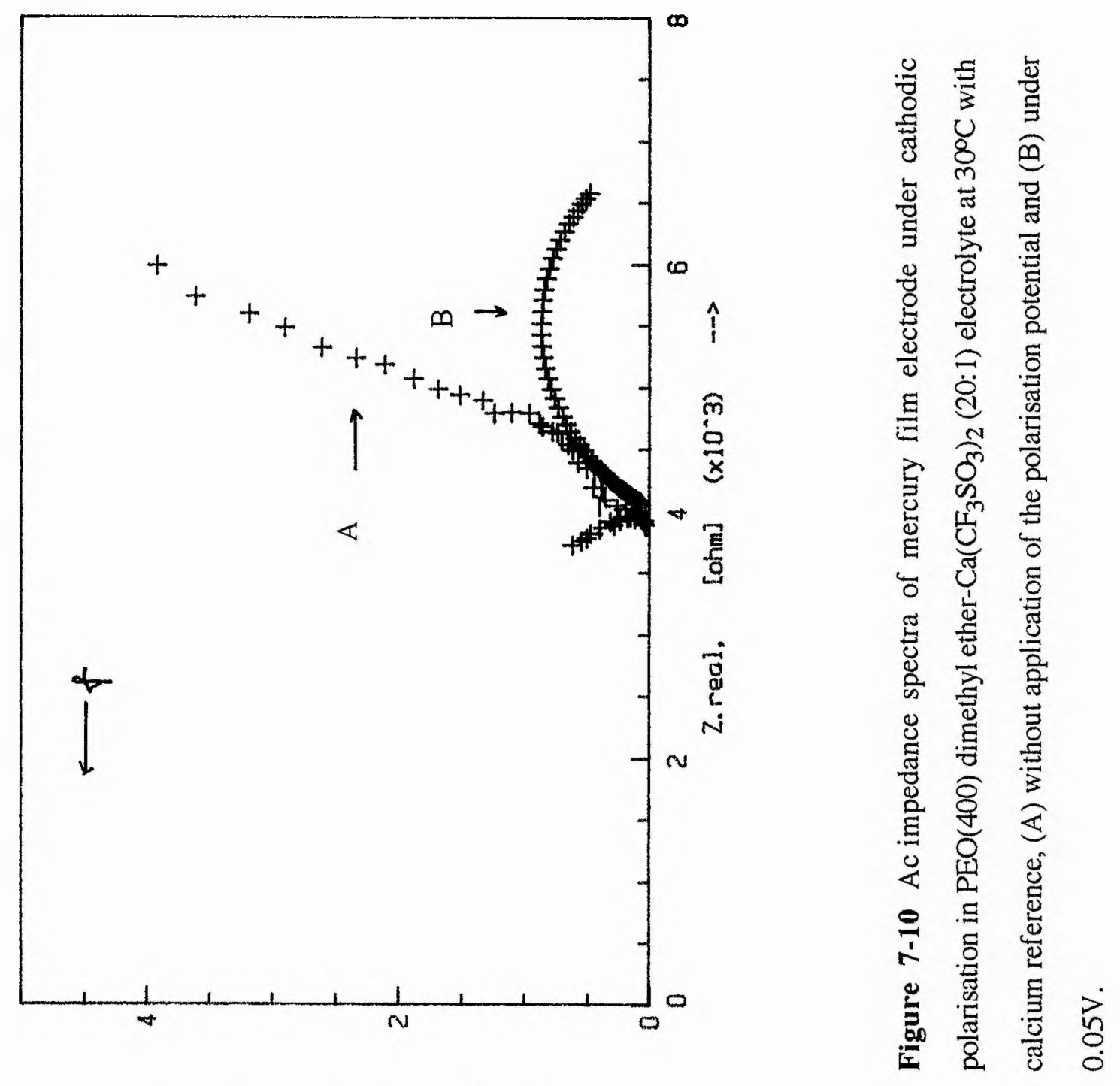

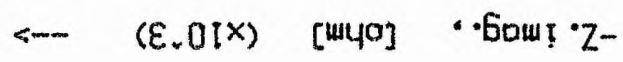




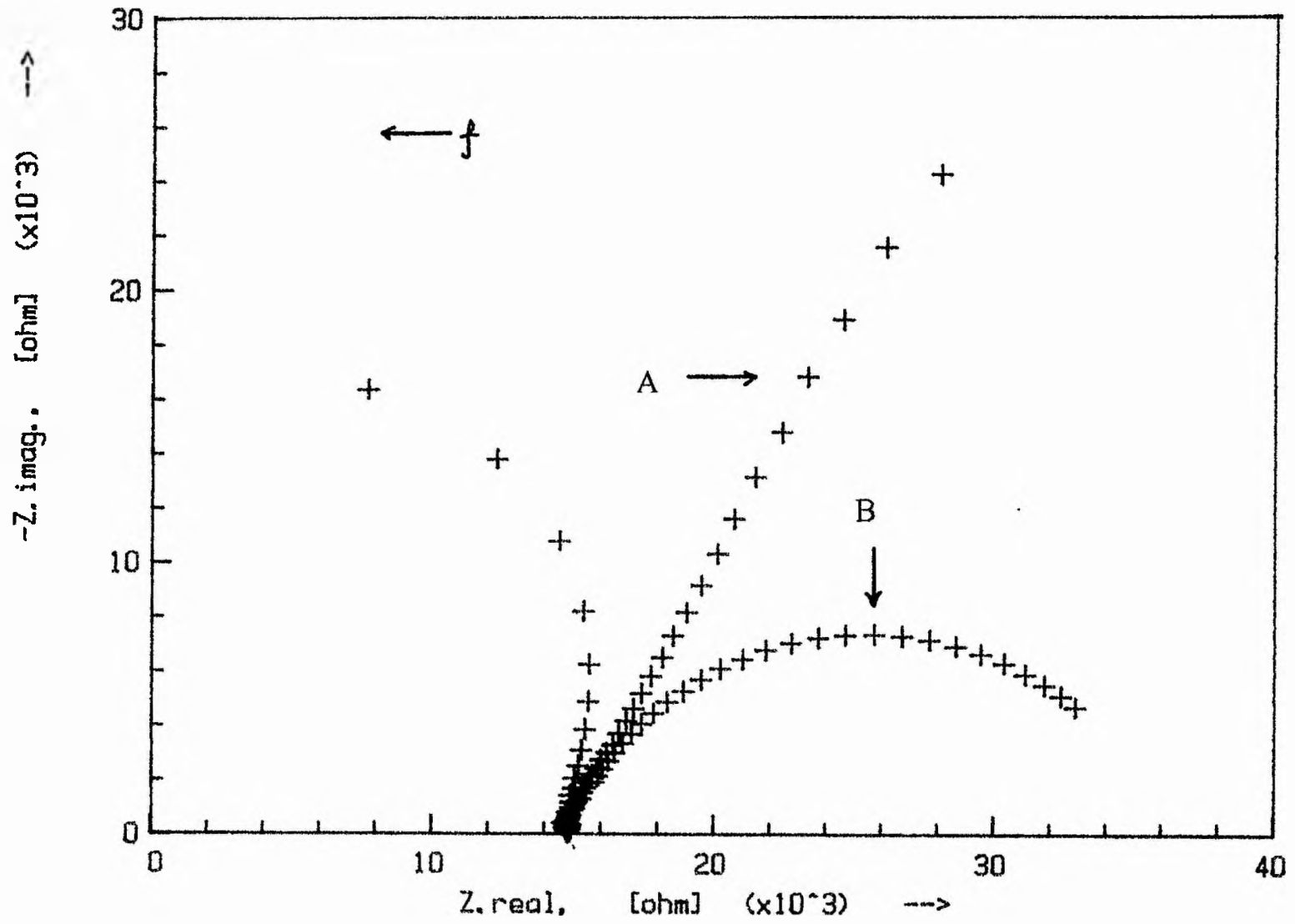

Figure 7-11 Ac impedance spectra of mercury film electrode under cathodic polarisation in $\mathrm{PEO}(400)$ dimethyl ether $-\mathrm{Mg}\left(\mathrm{CF}_{3} \mathrm{SO}_{3}\right)_{2}(20: 1)$ electrolyte at $30^{\circ} \mathrm{C}$ with magnesium reference, (A) without application of the polarisation potential and (B) under $0.05 \mathrm{~V}$. 


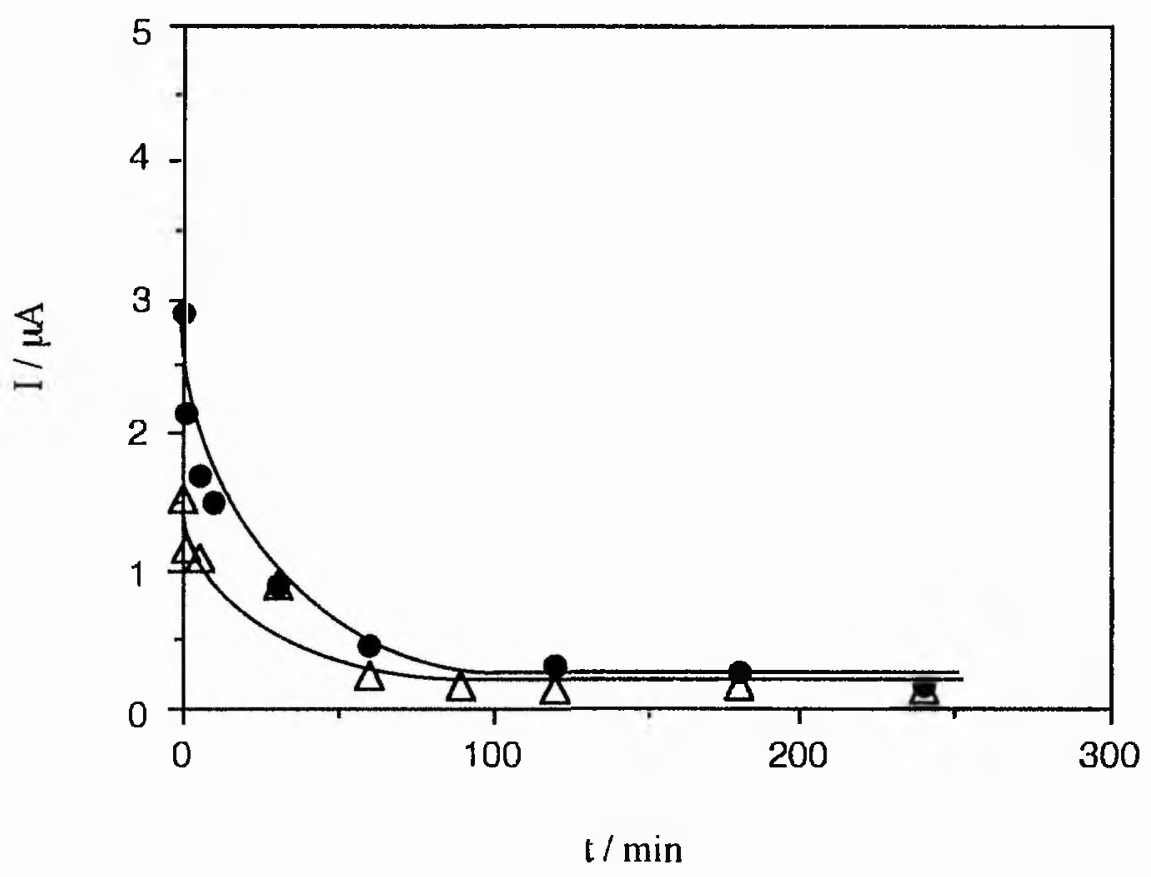

Figure 7-12 I-t curves for dc polarisation of $\mathrm{M} / \mathrm{PEO}(400)$ dimethyl ether$\mathrm{M}\left(\mathrm{CF}_{3} \mathrm{SO}_{3}\right)_{2} / \mathrm{M}$ cell with $1 \mathrm{~V}$ applied voltage $(\Delta) \mathrm{Ca}$ cell and $(\bullet) \mathrm{Mg}$ cell. 


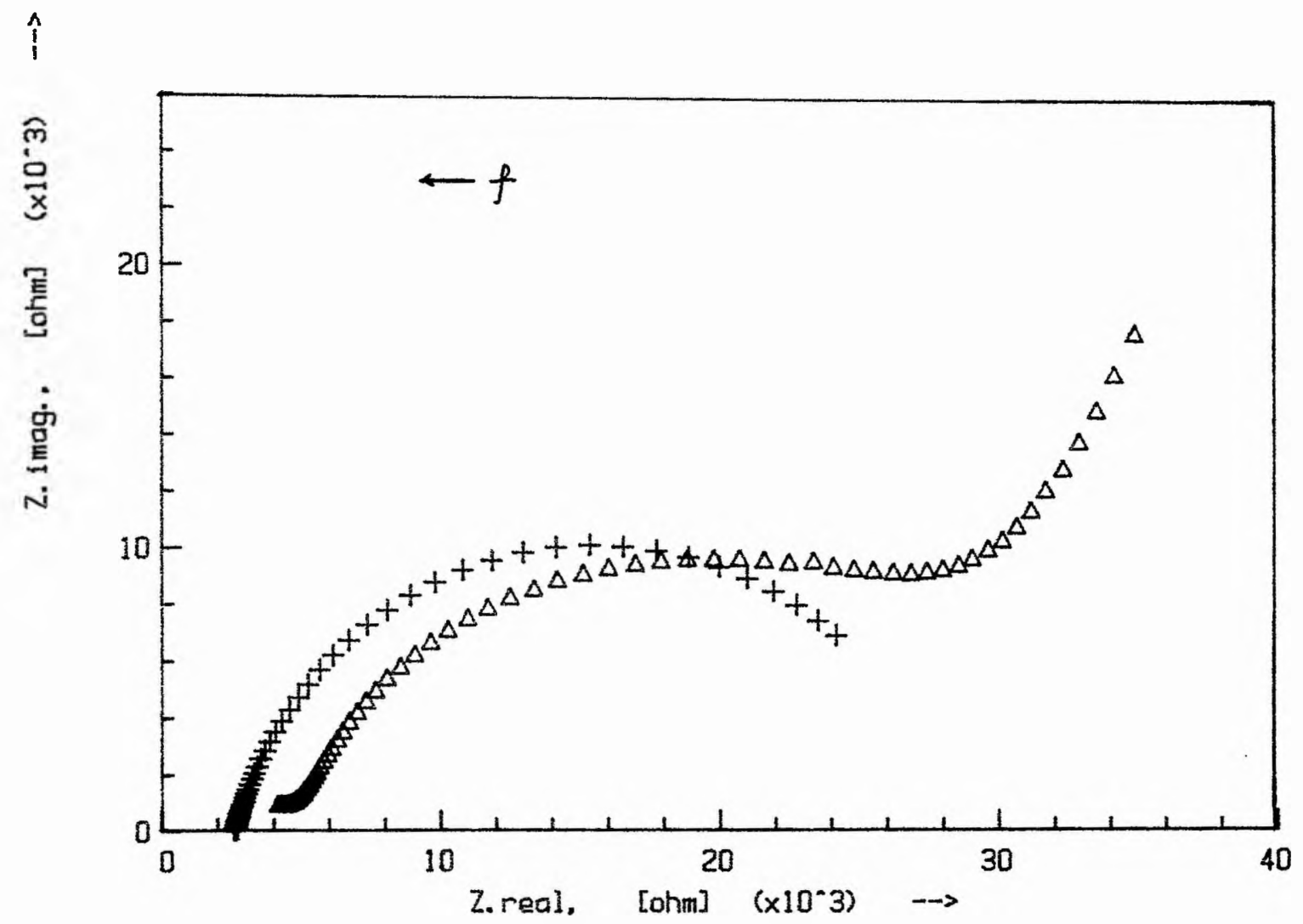

Figure 7-13 Ac impedance spectra of $\mathrm{Ca} / \mathrm{PEO}(400)$ dimethyl ether- $\mathrm{Ca}\left(\mathrm{CF}_{3} \mathrm{SO}_{3}\right)_{2}$ $(20: 1) / \mathrm{Hg}(\mathrm{Ca})$ cell $(+)$ measured after polarised for 5 minutes under $0.5 \mathrm{~V}$ applied vol tage at $40^{\circ} \mathrm{C}$ and $(\Delta)$ measured after stopping polarisation. 


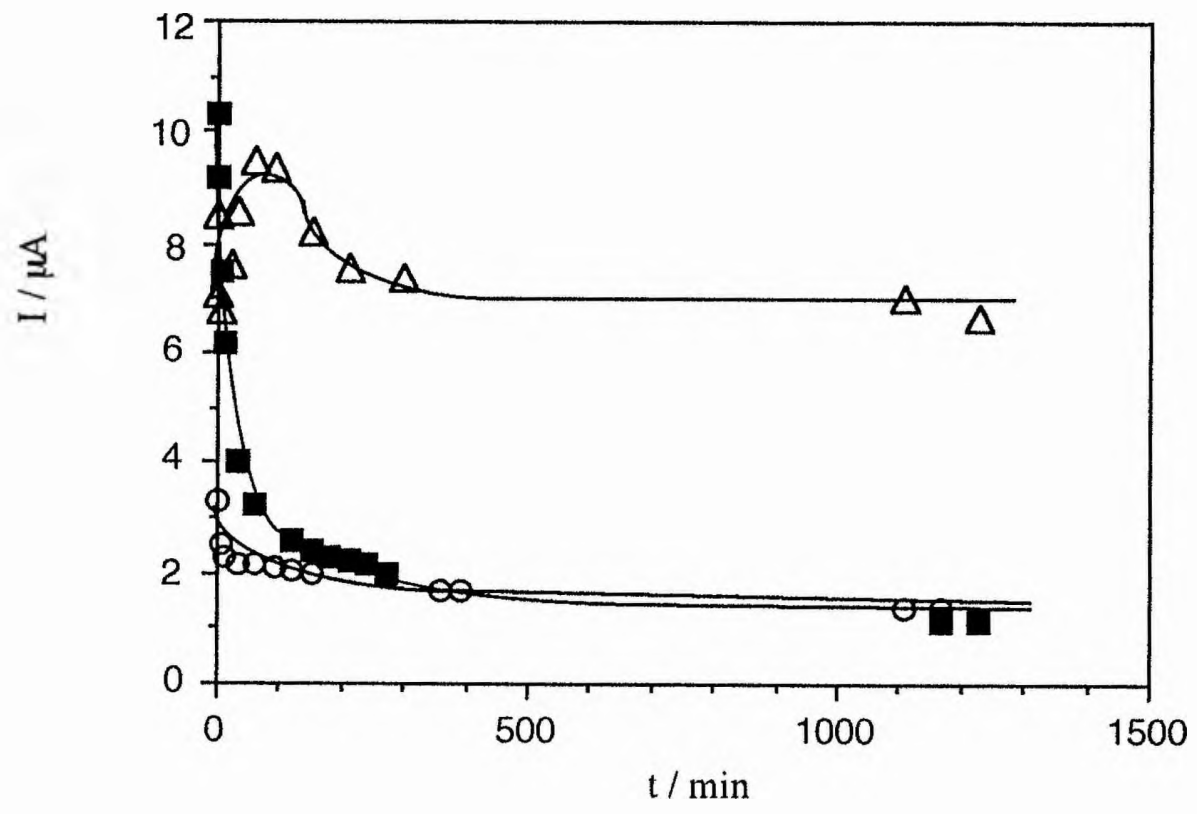

Figure 7-14 Plot of polarisation current against time for dc poarisation of $\mathrm{Ca} / \mathrm{PEO}$ $\mathrm{Ca}\left(\mathrm{CF}_{3} \mathrm{SO}_{3}\right)_{2}(20: 1) / \mathrm{Hg}(\mathrm{Ca})$ cell with $0.5 \mathrm{~V}$ applied voltage: $(\Delta) \mathrm{PEO}(400)$ dimethyl ether at $40^{\circ} \mathrm{C}$, (O) PMEO and (W) PEO with $\mathrm{M}_{\mathrm{W}}$ of $4 \times 10^{6}$ at $80^{\circ} \mathrm{C}$. 


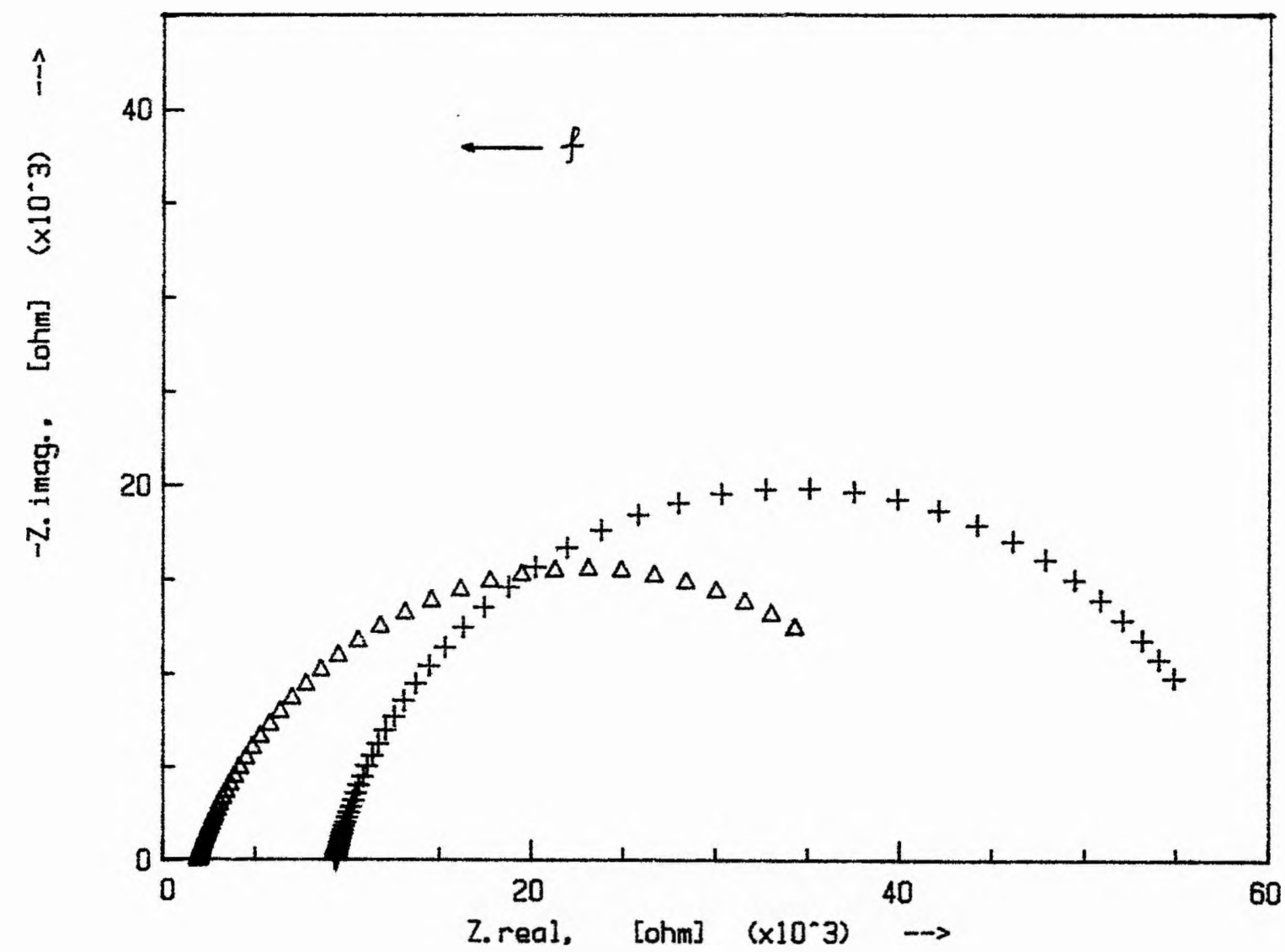

Figure 7-15 $\mathrm{Ac}$ impedance spectra of $\mathrm{Ca} / \mathrm{PMEO}-\mathrm{Ca}\left(\mathrm{CF}_{3} \mathrm{SO}_{3}\right)_{2}(20: 1) / \mathrm{Hg}(\mathrm{Ca})$ cell (+) and $\mathrm{Ca} / \mathrm{PEO}-\mathrm{Ca}\left(\mathrm{CF}_{3} \mathrm{SO}_{3}\right)_{2}(20: 1) / \mathrm{Hg}(\mathrm{Ca})$ cell $(\Delta)$ measured after polarised for 5 minutes under $0.5 \mathrm{~V}$ applied voltage at $80^{\circ} \mathrm{C}$. 


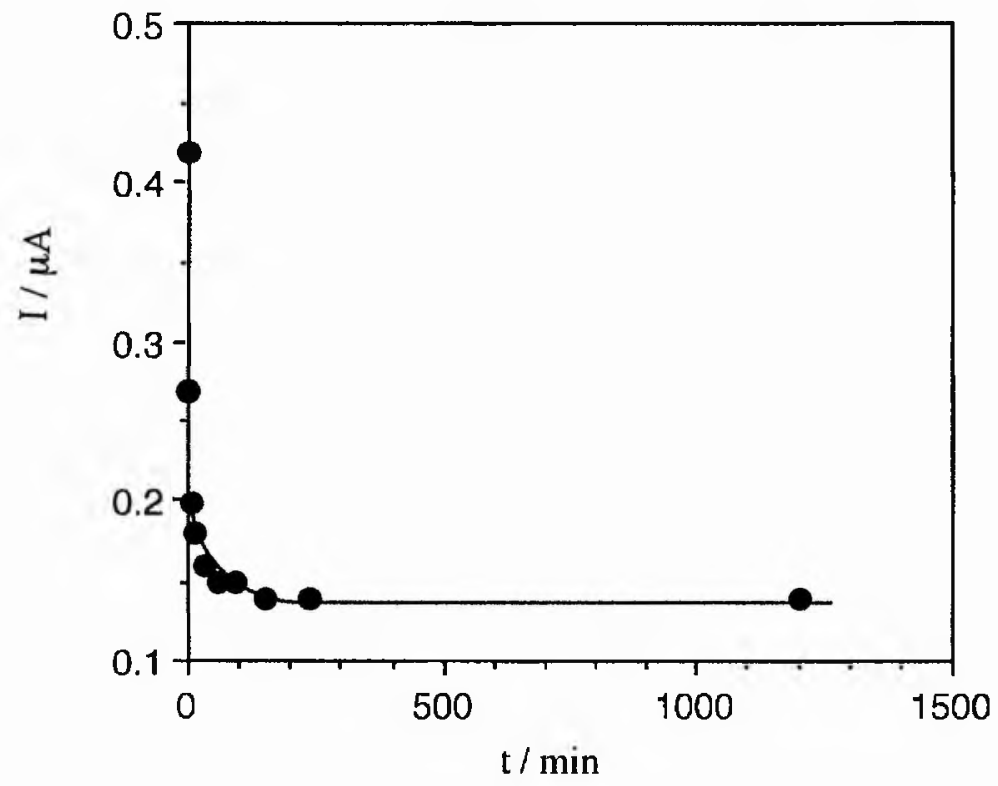

Figure 7-16a I-t curve for dc polarisation of $\mathrm{Ca} /$ salt-free $\mathrm{PMEO} / \mathrm{Hg}$ (Ca) cell with $0.5 \mathrm{~V}$ applied voltage at $80^{\circ} \mathrm{C}$.

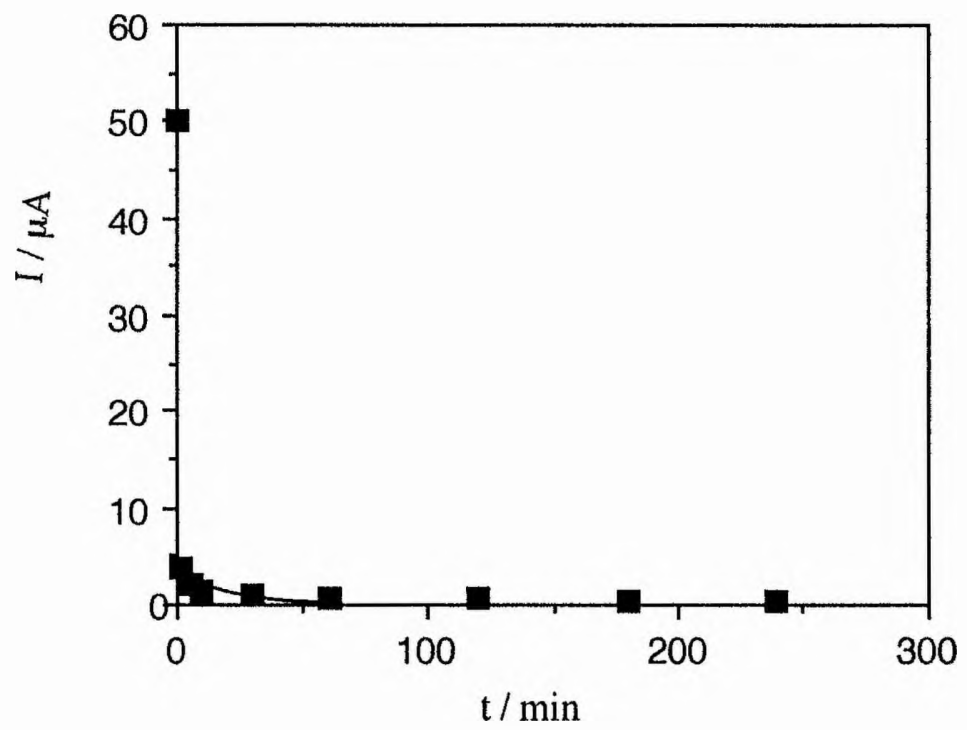

Figure 16b I-t curve for de polarisation of $\mathrm{Au} / \mathrm{PMEO}-\mathrm{Ca}\left(\mathrm{CF}_{3} \mathrm{SO}_{3}\right)_{2}(20: 1) / \mathrm{Au}$ cell with $1.5 \mathrm{~V}$ applied voltage at $80^{\circ} \mathrm{C}$. 


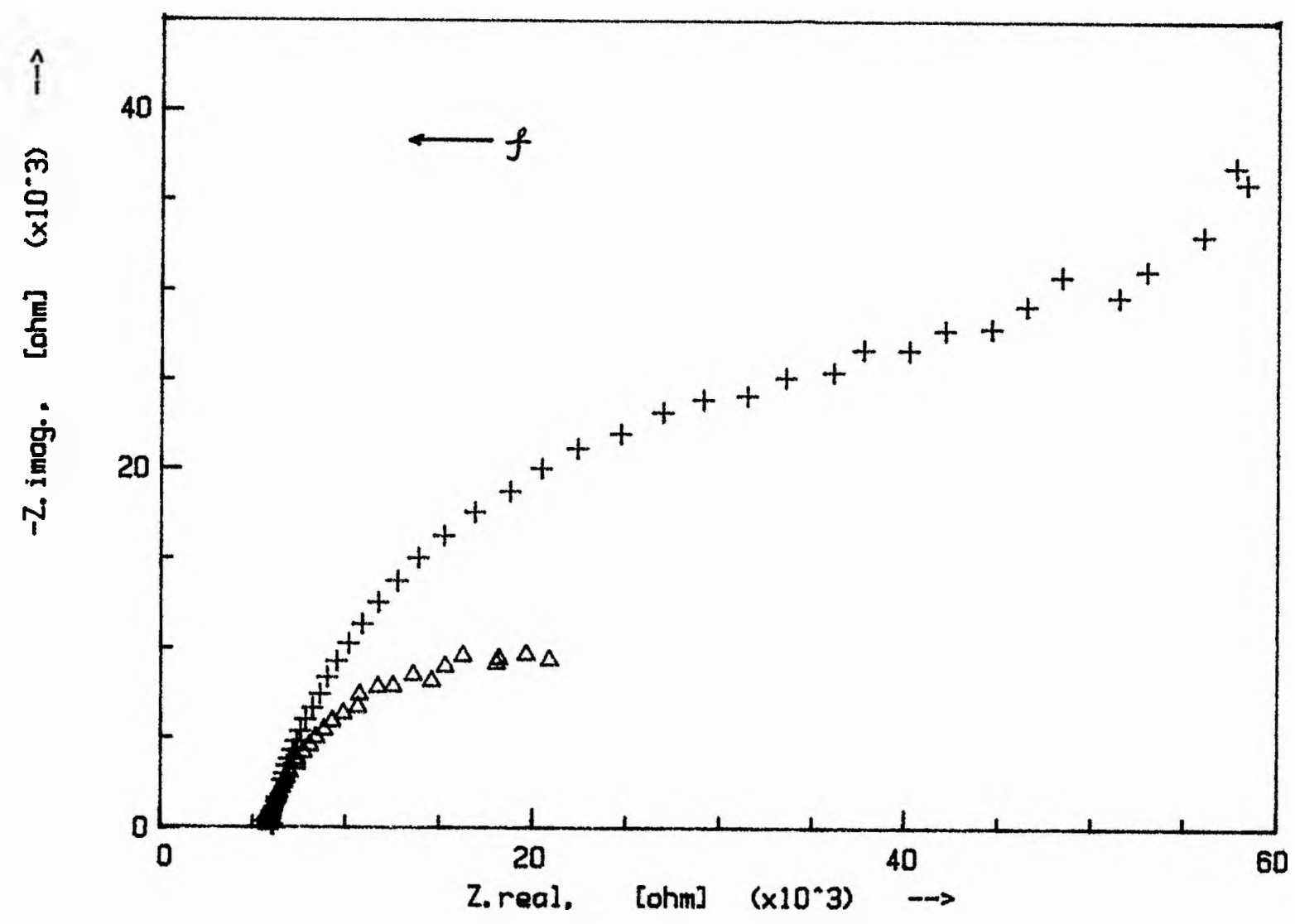

Figure 7-17 Ac impedance spectra of $\mathrm{Mg} / \mathrm{PEO}(400)$ dimethyl ether- $\mathrm{Mg}\left(\mathrm{CF}_{3} \mathrm{SO}_{3}\right)_{2}$ (20:1) / Hg (Mg) cell $(\Delta)$ measured after polarised for 5 minutes under $0.1 \mathrm{~V}$ applied vol tage at $80^{\circ} \mathrm{C}$ and (+) measured after stopping polarisation. 


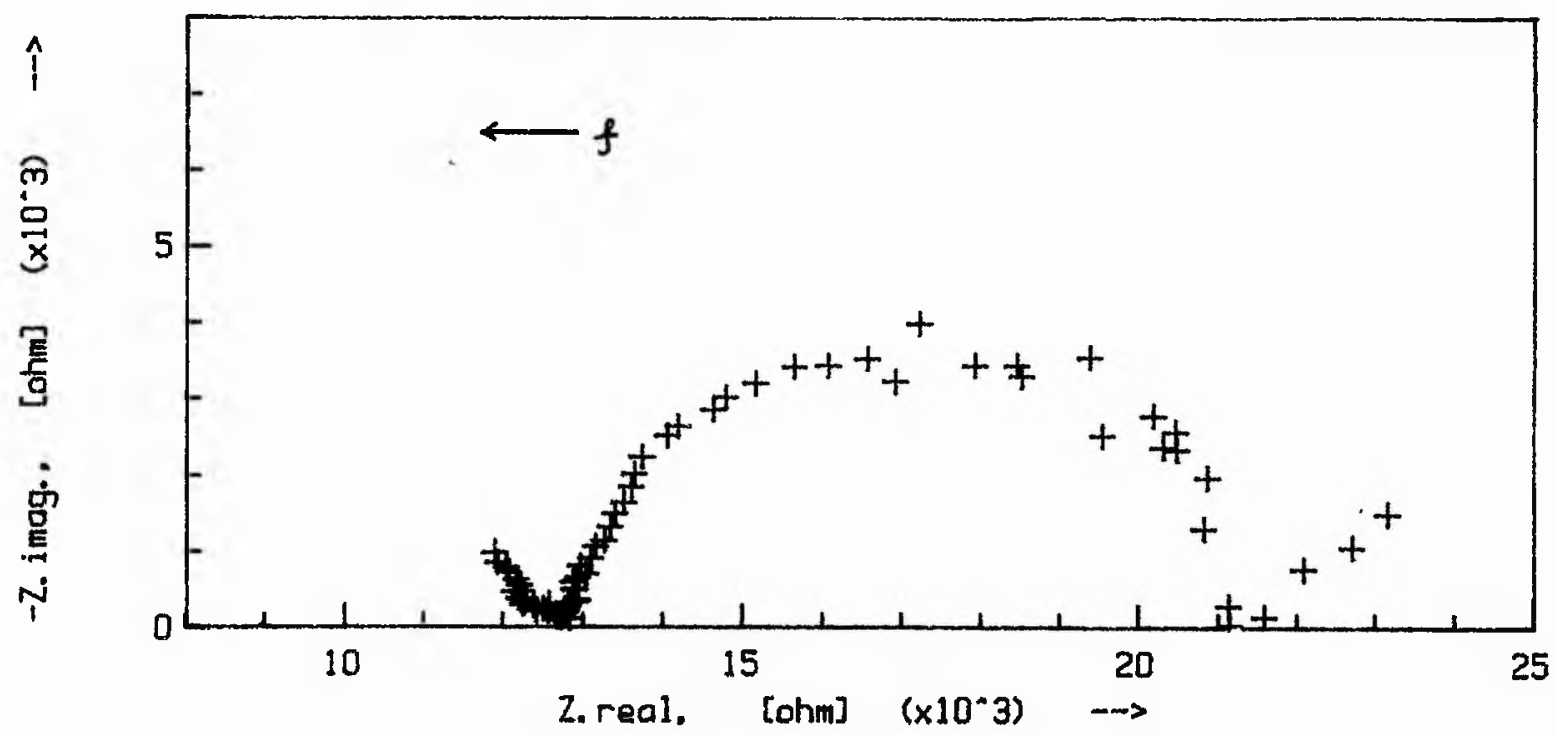

Figure 7-18 Ac impedance spectra of $\mathrm{Mg} / \mathrm{PEO}(1000)$ dimethyl ether$\mathrm{Mg}\left(\mathrm{CF}_{3} \mathrm{SO}_{3}\right)_{2}(20: 1) / \mathrm{Hg}(\mathrm{Mg})$ cell measured after polarised for 5 minutes under 0.1 $\mathrm{V}$ applied voltage at $80^{\circ} \mathrm{C}$.

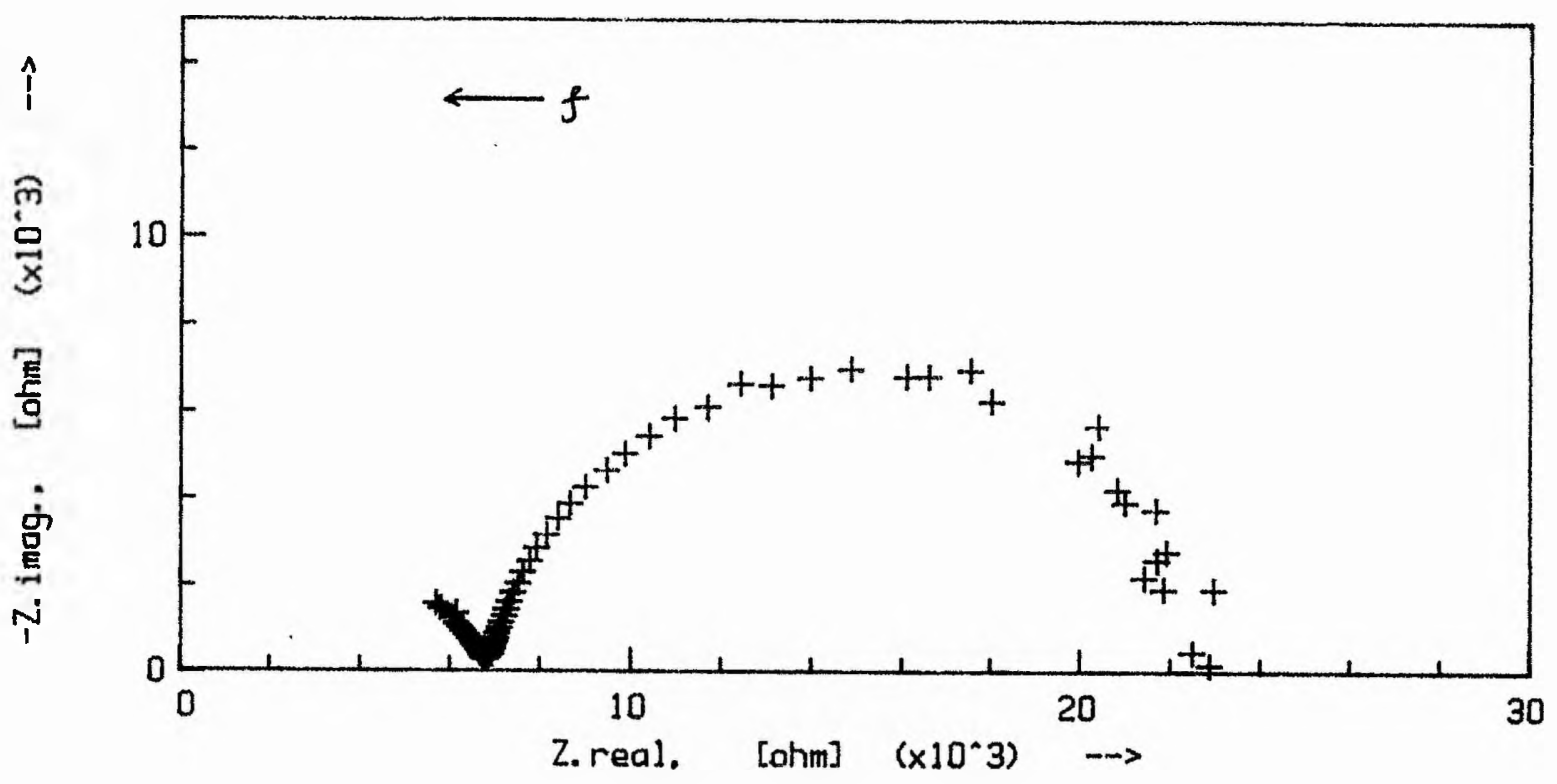

Figure 7-19 Ac impedance spectra of $\mathrm{Mg} / \mathrm{PEO}(2000)$ dimethyl ether$\mathrm{Mg}\left(\mathrm{CF}_{3} \mathrm{SO}_{3}\right)_{2}$ (20:1)/ $\mathrm{Hg}(\mathrm{Mg})$ cell measured after polarised for 5 minutes under 0.1 $\mathrm{V}$ applied voltage at $80^{\circ} \mathrm{C}$. 


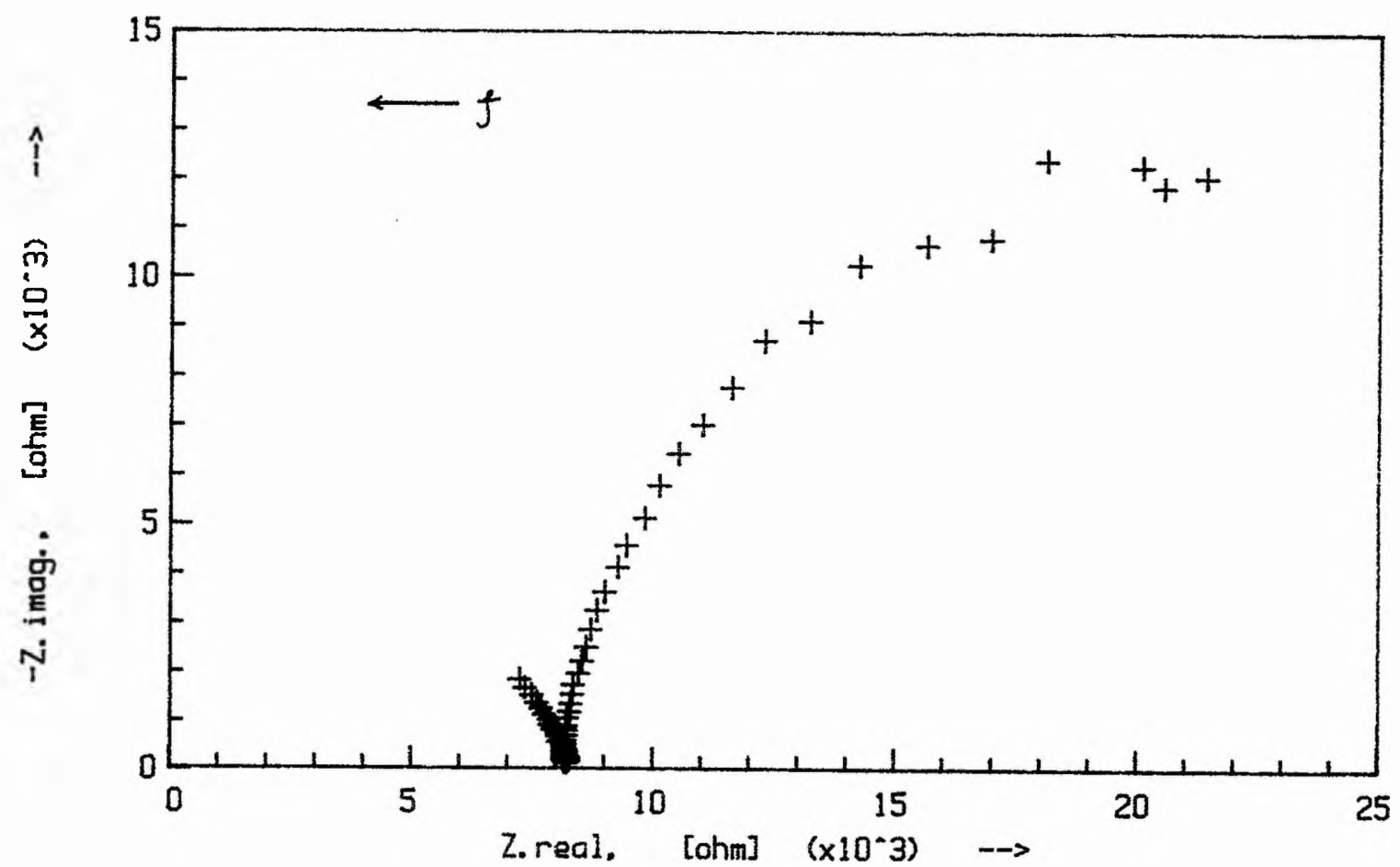

Figure 7-20 Ac impedance spectra of $\mathrm{Mg} /$ methylated $\mathrm{PEO}(3350)-\mathrm{Mg}\left(\mathrm{CF}_{3} \mathrm{SO}_{3}\right)_{2}$ (20:1) / $\mathrm{Hg}(\mathrm{Mg})$ cell measured after polarised for 5 minutes under $0.1 \mathrm{~V}$ applied voltage at $80^{\circ} \mathrm{C}$. 


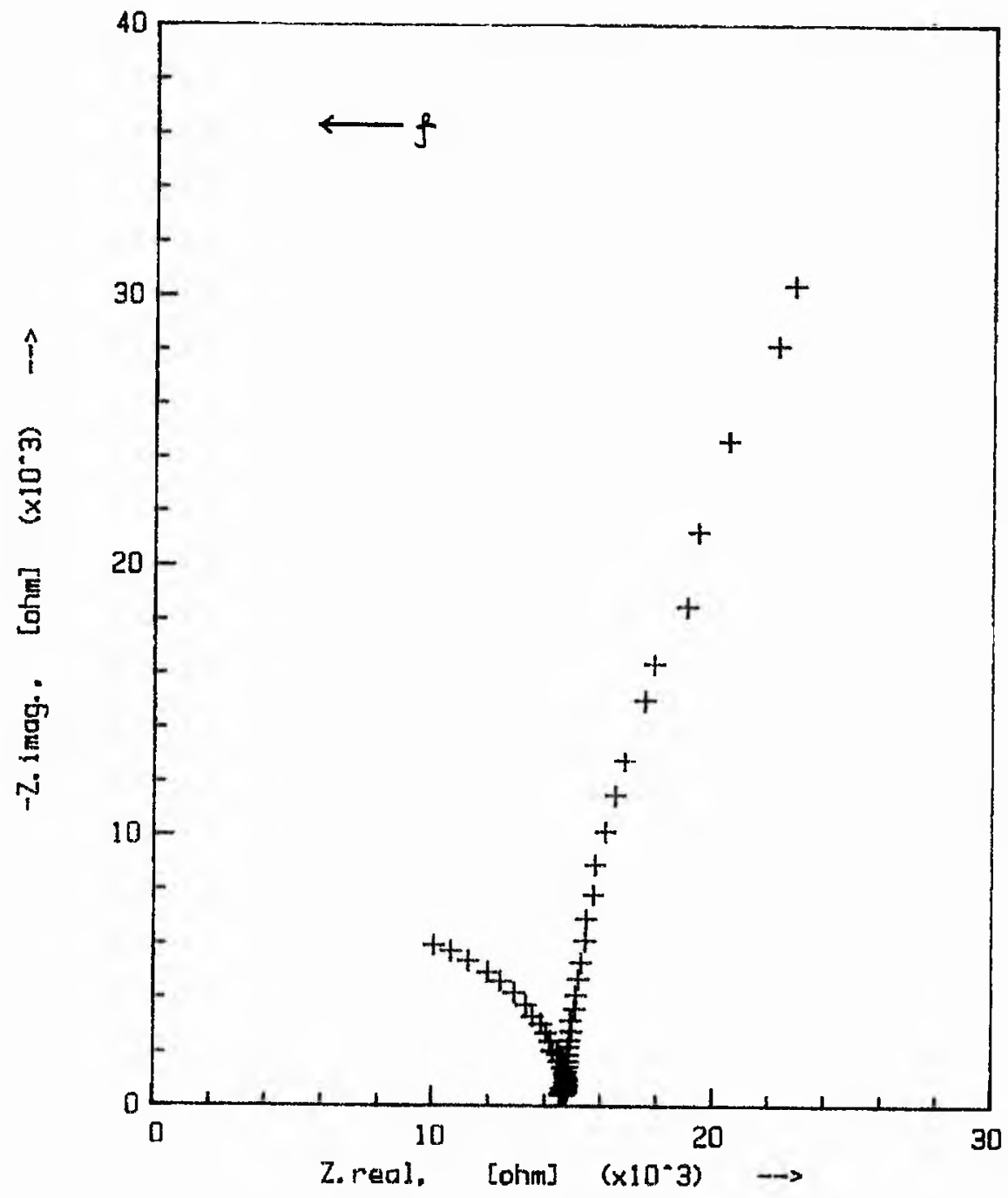

Figure 7-21 Ac impedance spectra of $\mathrm{Mg} / \mathrm{PEO} 4000-\mathrm{Mg}\left(\mathrm{CF}_{3} \mathrm{SO}_{3}\right)_{2}(20: 1) / \mathrm{Hg}$ $(\mathrm{Mg})$ cell measured after polarised for 5 minutes under $0.1 \mathrm{~V}$ applied voltage at $80^{\circ} \mathrm{C}$. 


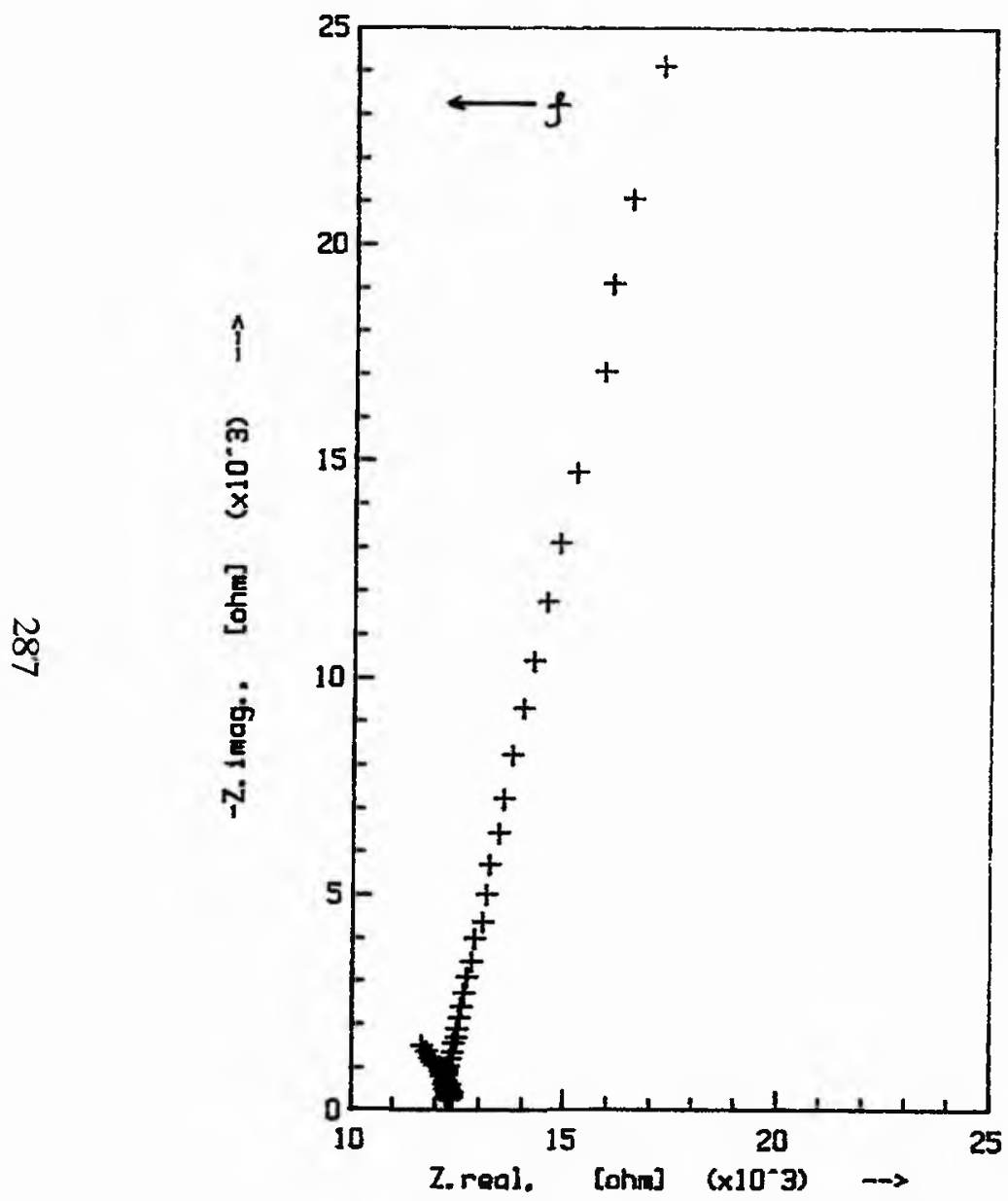

Figure 7-22 Ac impedance spectra of $\mathrm{Mg} / \mathrm{PEO} 0000-$ $\mathrm{Mg}\left(\mathrm{CF}_{3} \mathrm{SO}_{3}\right)_{2}(20: 1) / \mathrm{Hg}(\mathrm{Mg})$ cell measured after polarised for 5 minutes under $0.1 \mathrm{~V}$ applied voltage at $80^{\circ} \mathrm{C}$. 


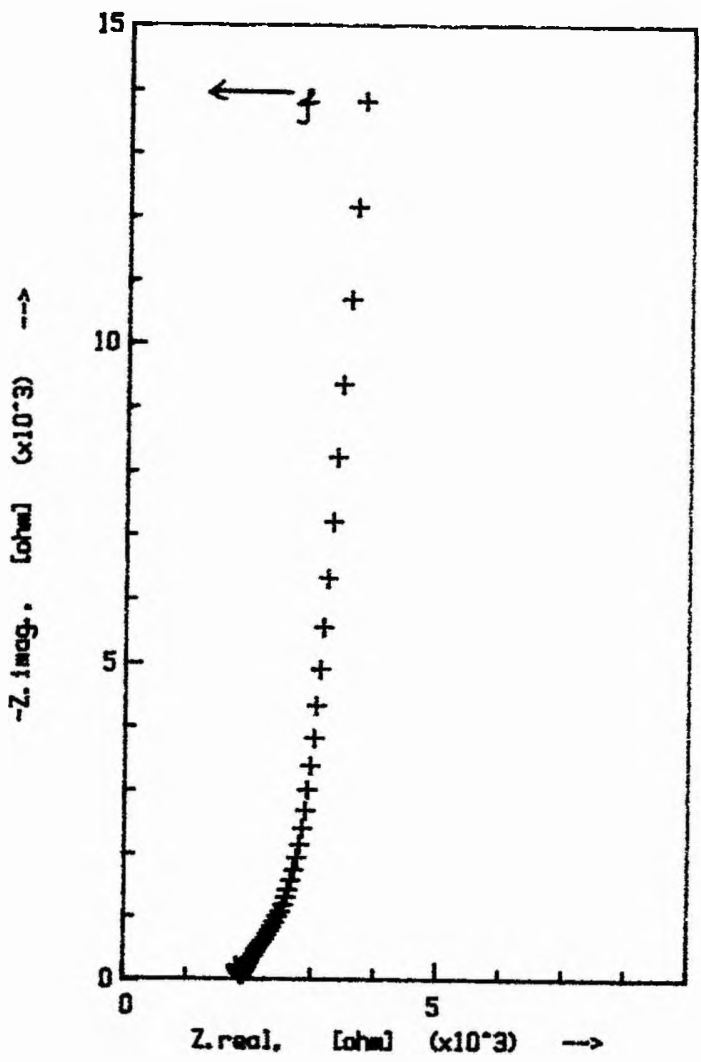

Figure 7.23 Ac impedance spectra of $\mathrm{Mg} / \mathrm{PEO} 4 \times 10^{6}$ $\mathrm{Mg}\left(\mathrm{CF}_{3} \mathrm{SO}_{3}\right)_{2}(20: 1) / \mathrm{Hg}(\mathrm{Mg})$ cell measured after polarised for 5 minutes under $0.1 \mathrm{~V}$ applied voltage at $80^{\circ} \mathrm{C}$. 


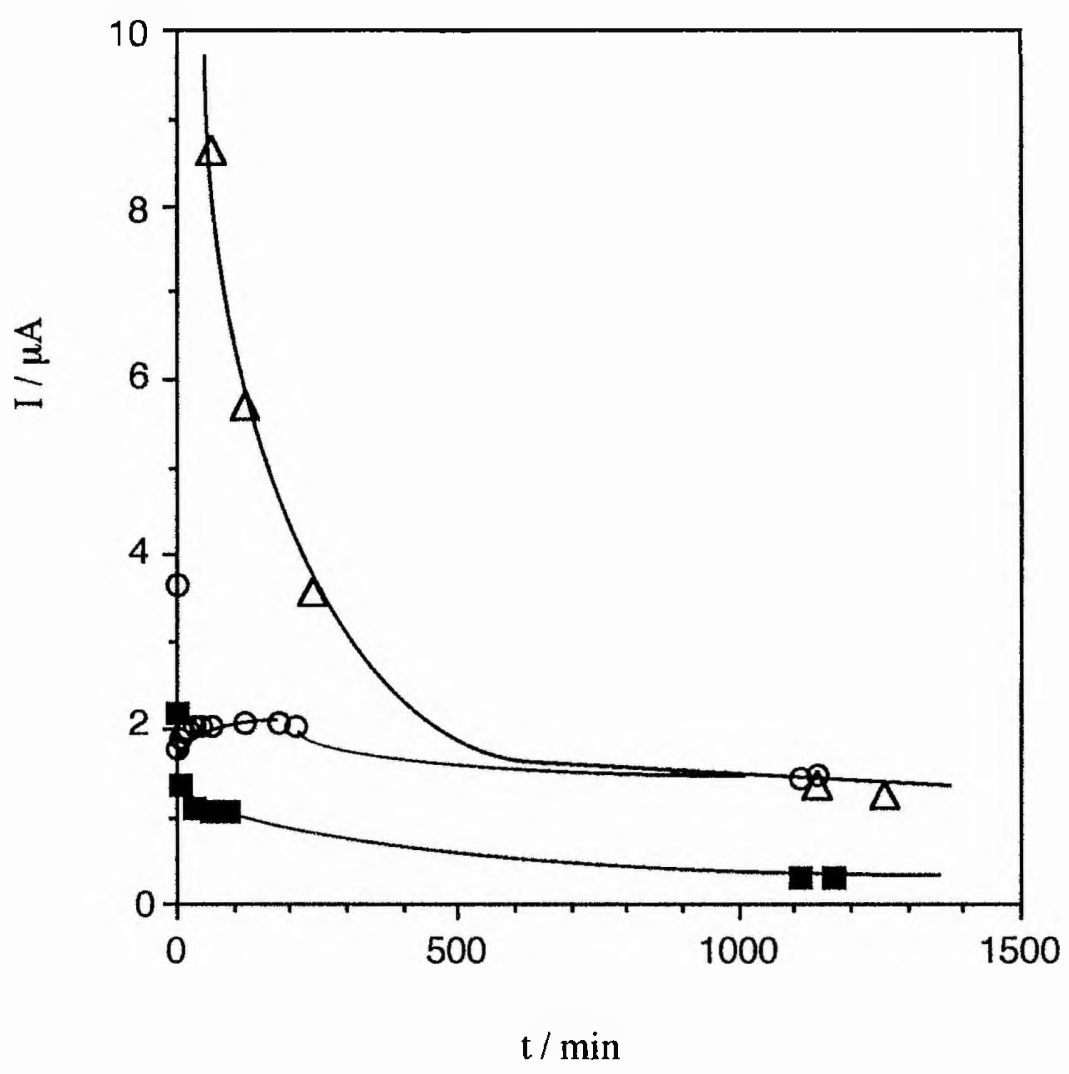

Figure 7-24 I-t curves for de polarisation for $\mathrm{Mg} / \mathrm{PEO}-\mathrm{Mg}\left(\mathrm{CF}_{3} \mathrm{SO}_{3}\right)_{2} / \mathrm{Hg}(\mathrm{Mg})$ cell with $0.1 \mathrm{~V}$ applied voltage, the polymers are: $(\mathrm{O}) \mathrm{PEO}(400)$ dimethyl ether, $(\Delta)$ PEO(2000) dimethyl ether and ( $\square$ PEO $4 \times 10^{6}$. 


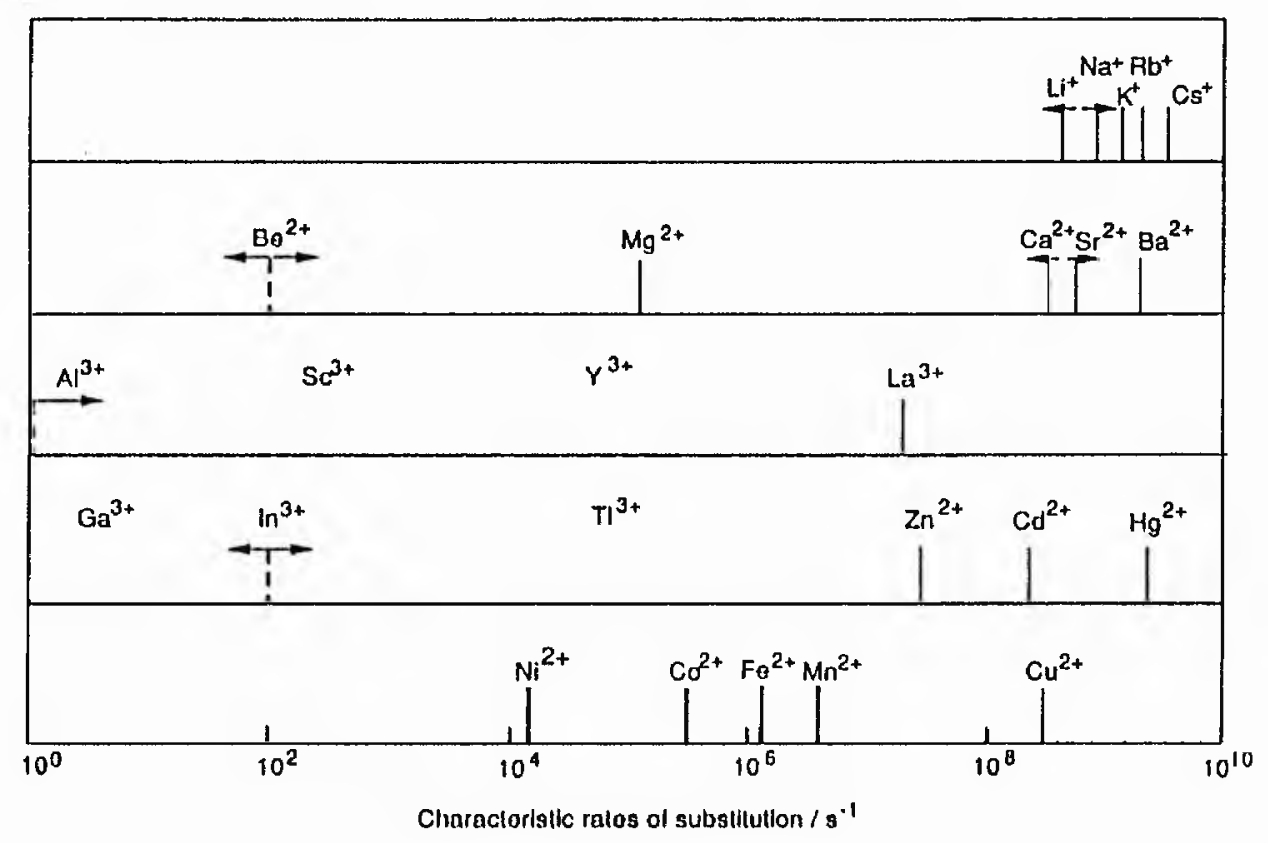

Figure 7-25 Characteristic rate constants for $\mathrm{H}_{2} \mathrm{O}$ substitution in the inner coordination aphere of metal ions. (data from ref. [17]). 


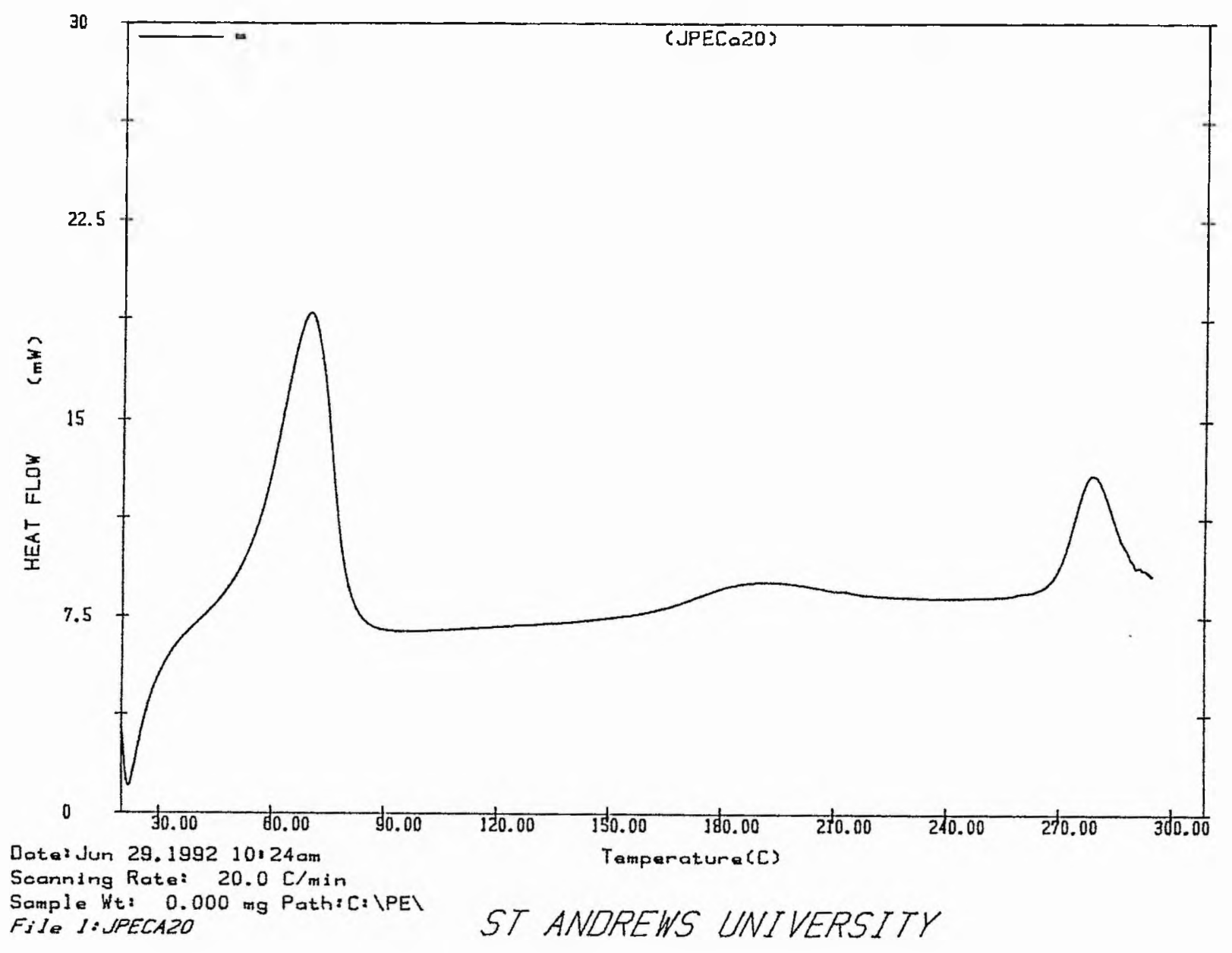

Figure 7-26a DSC trace of PEO-Ca $\left(\mathrm{CF}_{3} \mathrm{SO}_{3}\right)_{2}$ (20:1) electrolyte with scan rate of $20^{\circ} \mathrm{C} \mathrm{min}^{-1}$. 


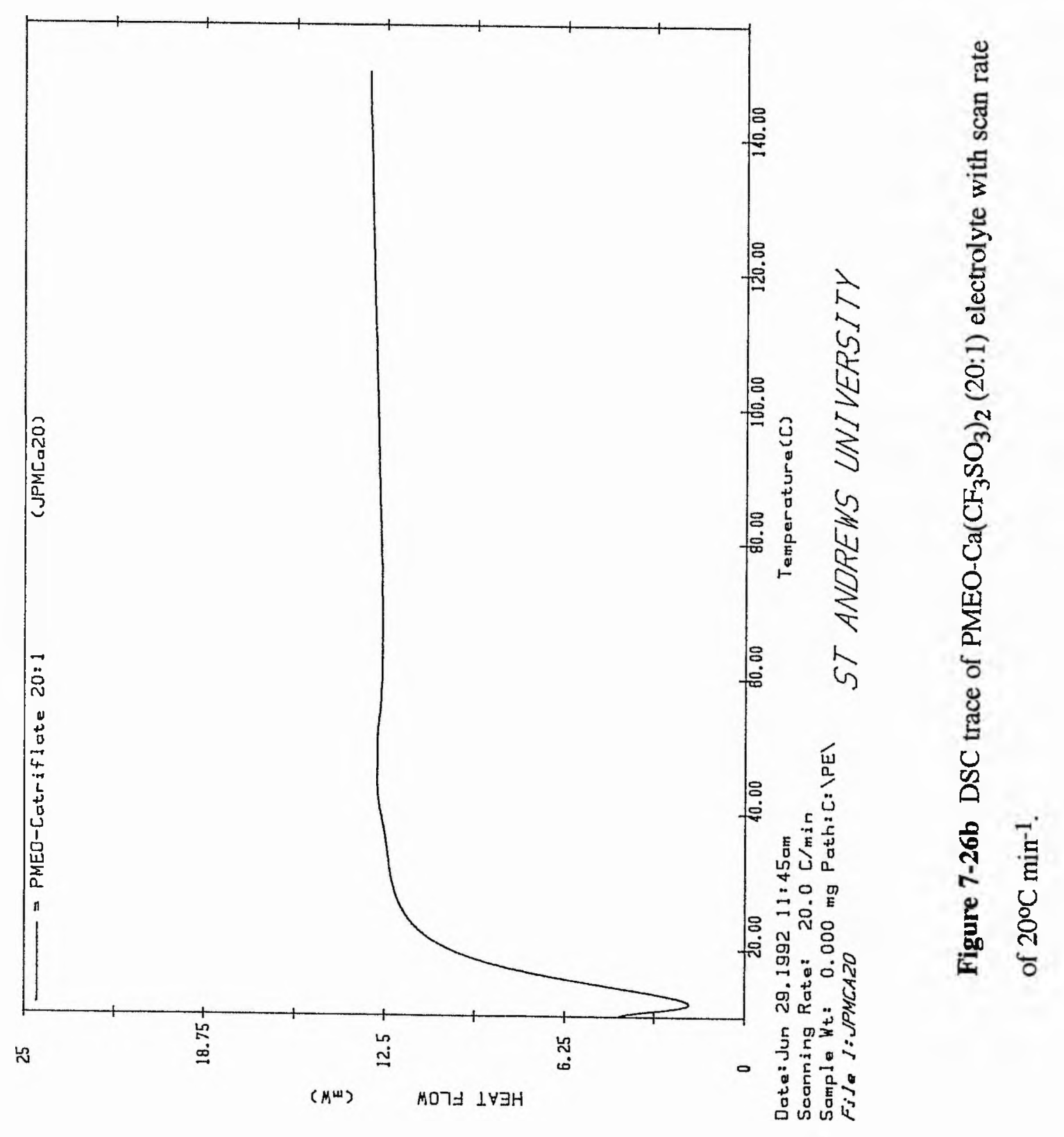




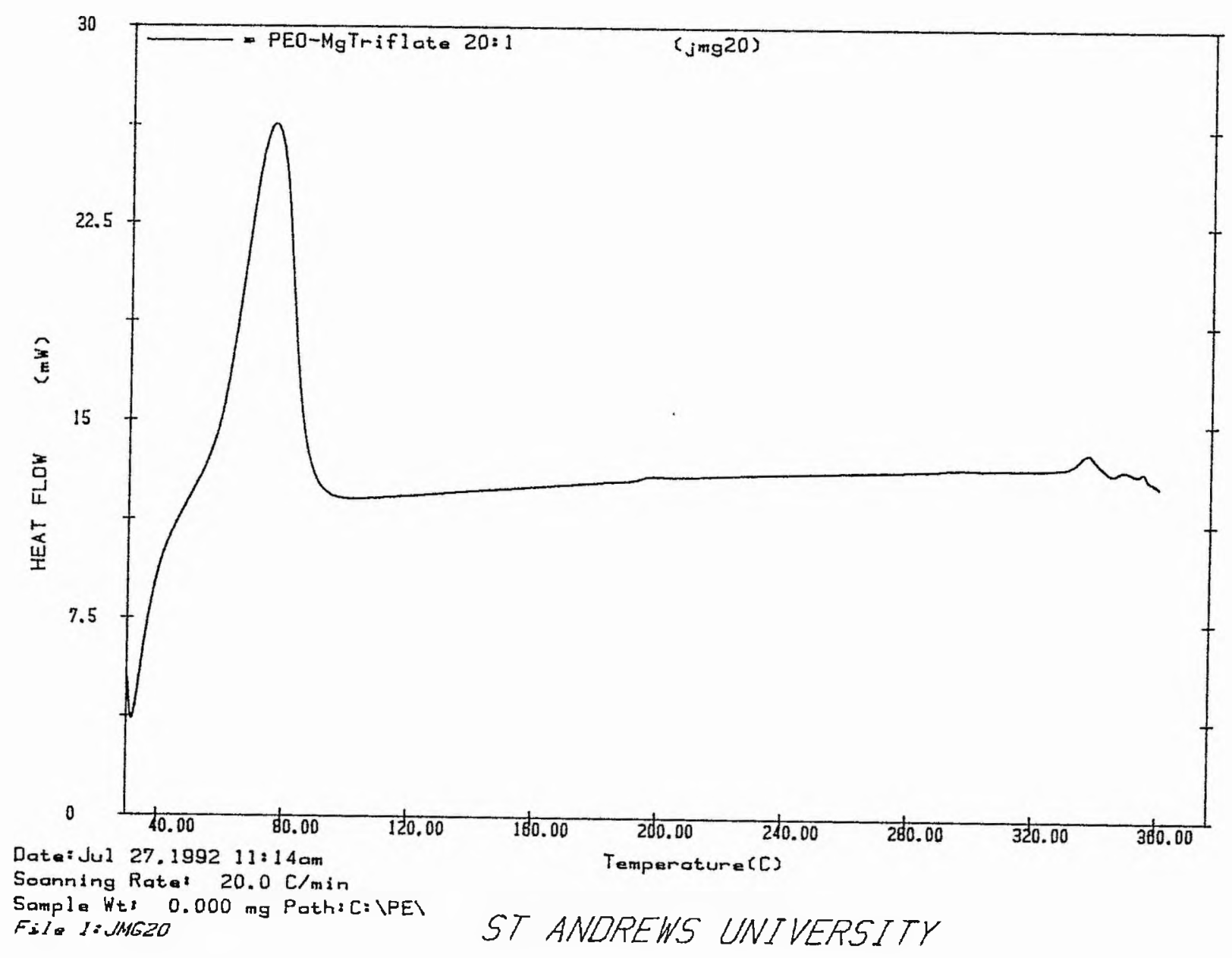

Figure 7-27 DSC trace of $\mathrm{PEO}-\mathrm{Mg}\left(\mathrm{CF}_{3} \mathrm{SO}_{3}\right)_{2}(20: 1)$ electrolyte with scan rate of $20^{\circ} \mathrm{C} \min ^{-1}$ 


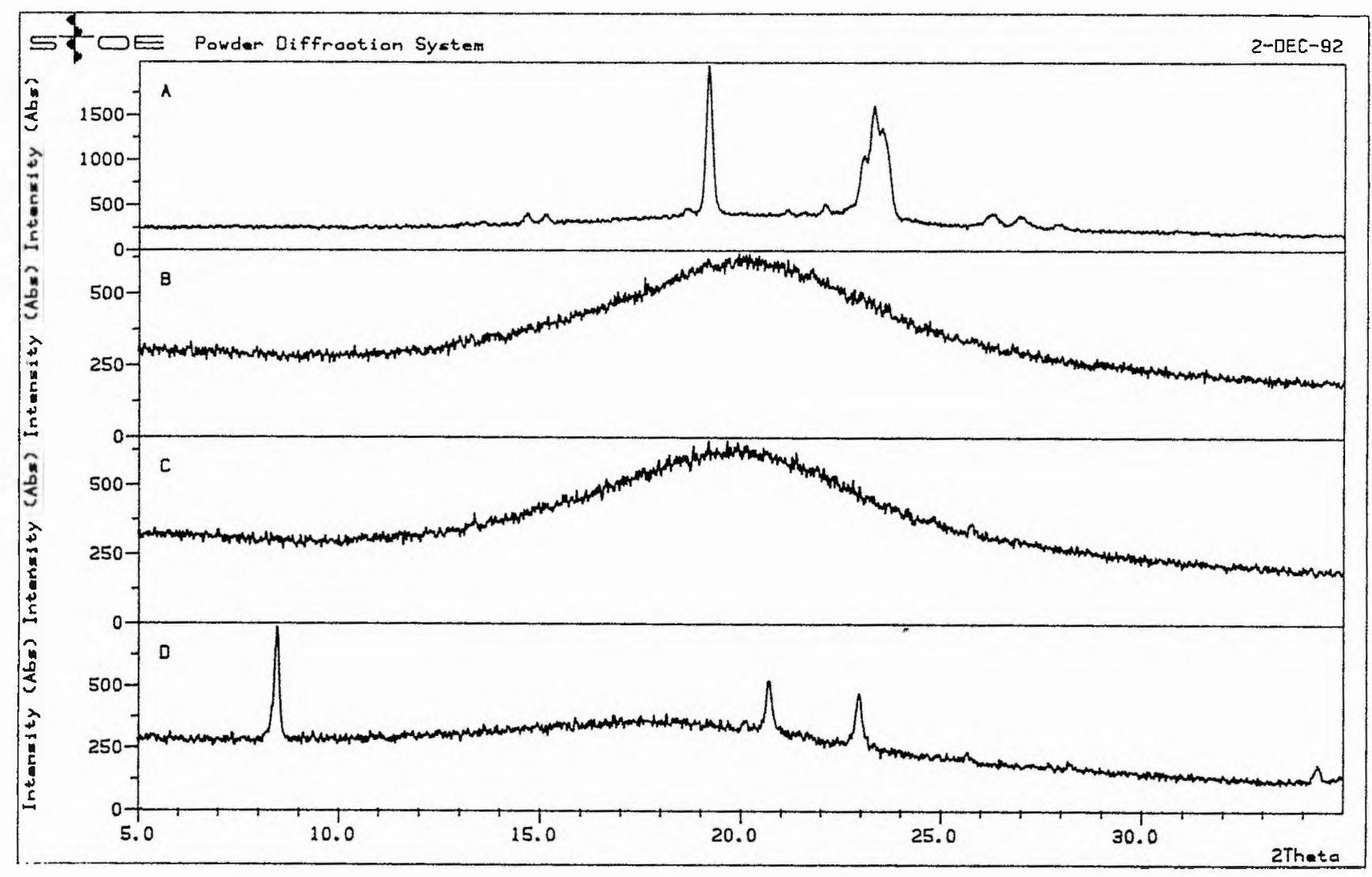

Figure 7-28a X-ray diffractogram of $\mathrm{PEO} 4 \times 10^{6}-\mathrm{Mg}\left(\mathrm{CF}_{3} \mathrm{SO}_{3}\right)_{2}$ (20:1) electrolyte at $28^{\circ} \mathrm{C}(\mathrm{A}), 65^{\circ} \mathrm{C}(\mathrm{B}), 90^{\circ} \mathrm{C}(\mathrm{C})$ and $300^{\circ} \mathrm{C}(\mathrm{D})$. 


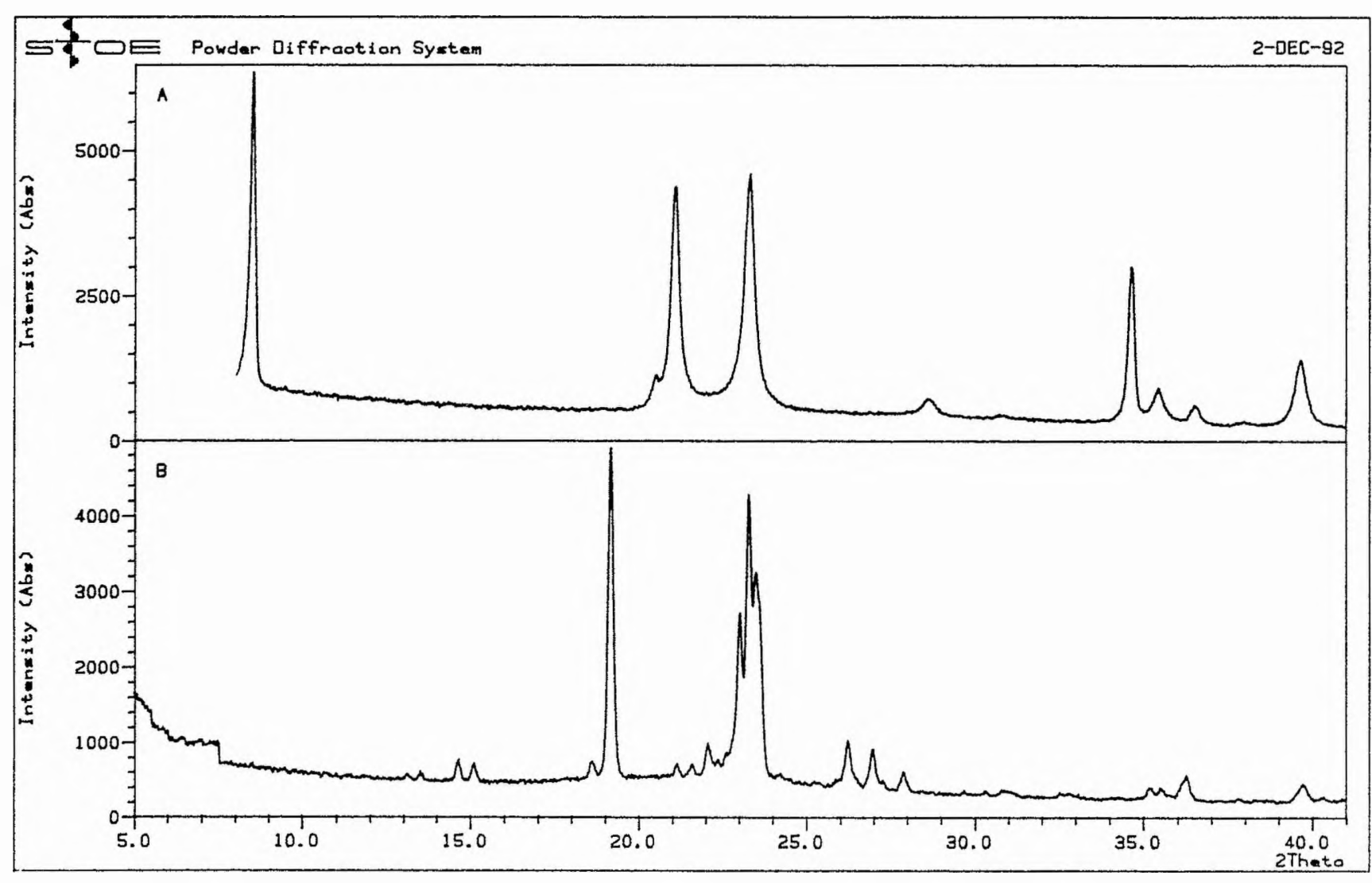

Figure 7-28b X-ray diffractogram of $\mathrm{Mg}\left(\mathrm{CF}_{3} \mathrm{SO}_{3}\right)_{2}$ salt $(\mathrm{A})$ and $\mathrm{PEO} 4 \times 10^{6}(\mathrm{~B})$. 


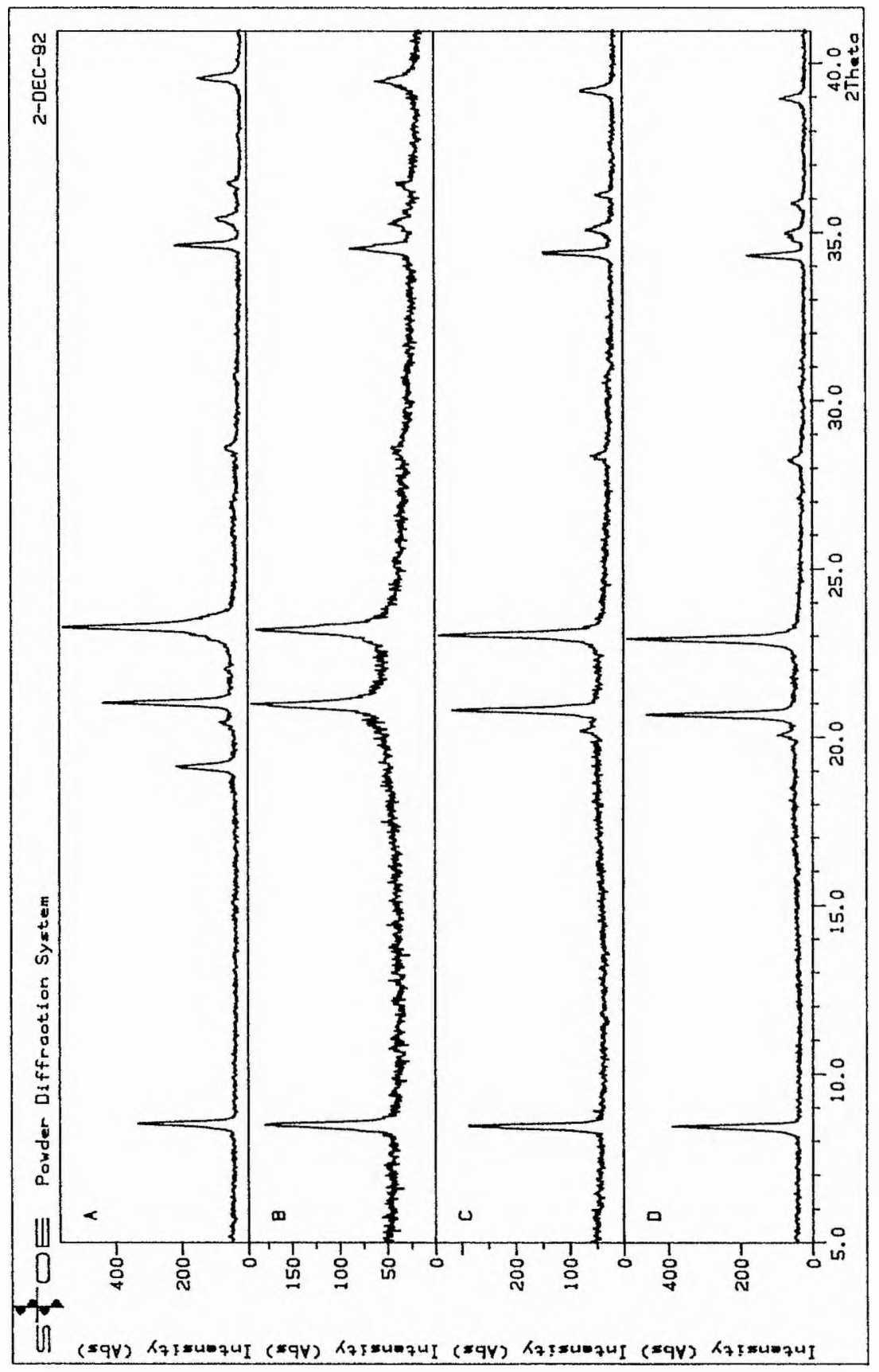

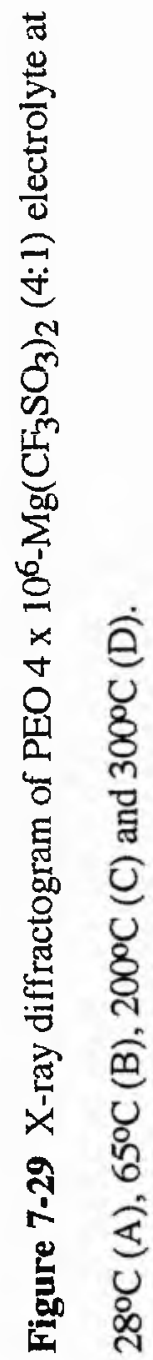




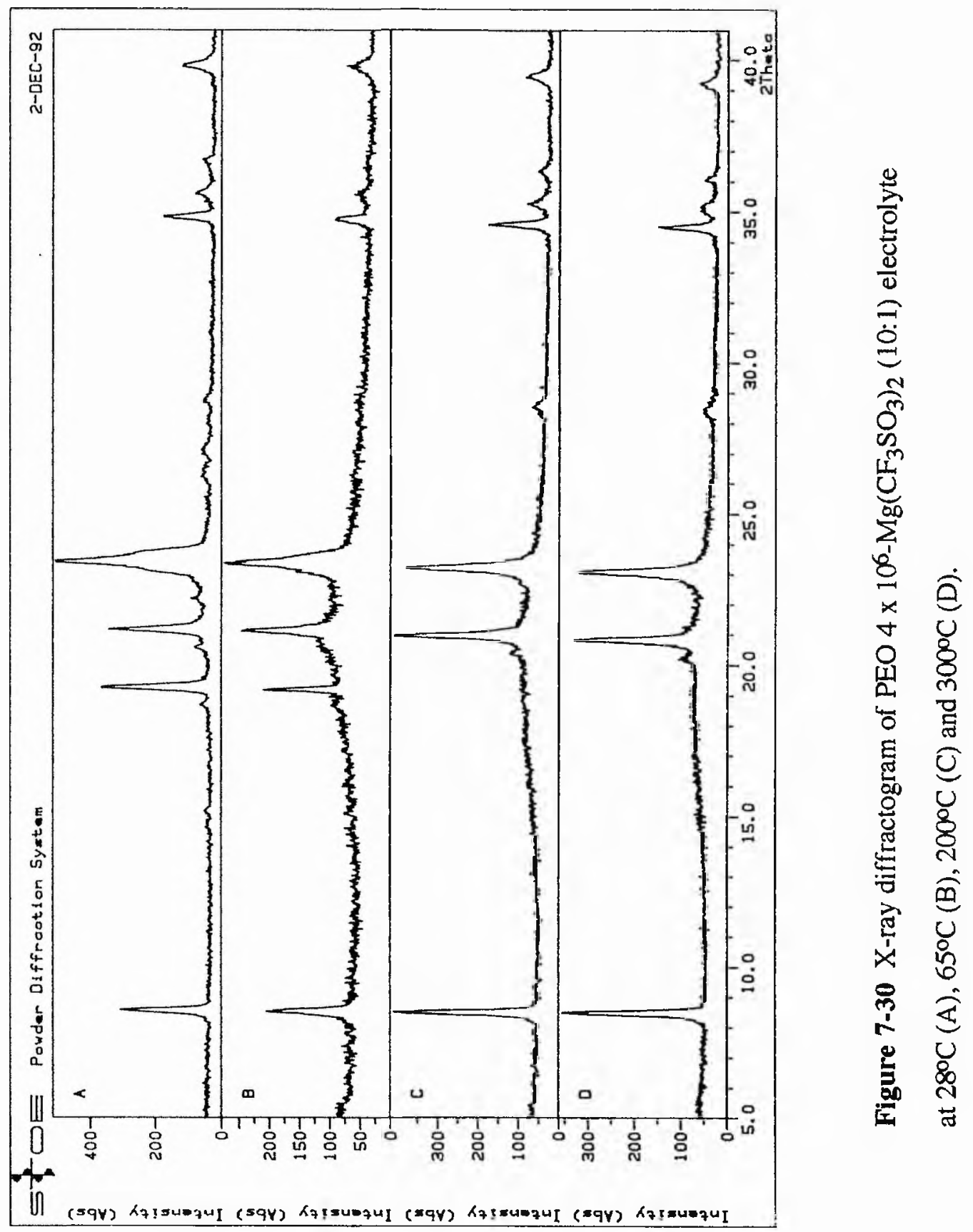




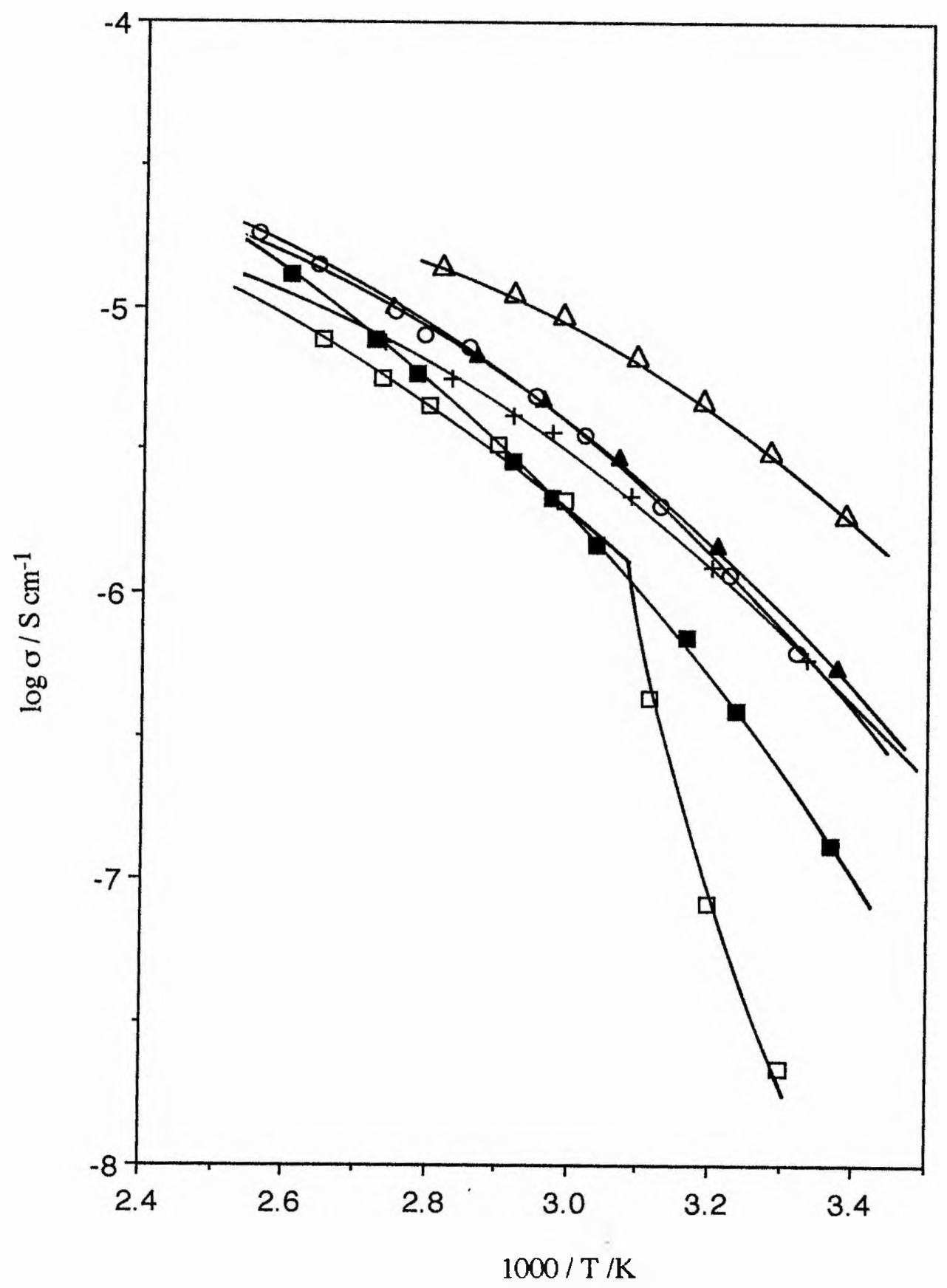

Figure 7-31 Temperature dependence of conductivity for $\mathrm{PMEO}-\mathrm{Ca}\left(\mathrm{CF}_{3} \mathrm{SO}_{3}\right)_{2}$

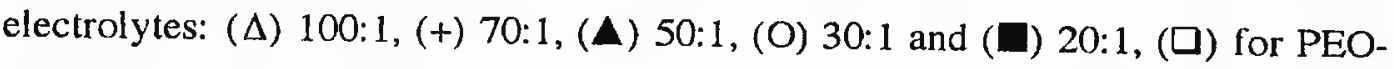
$\mathrm{Ca}\left(\mathrm{CF}_{3} \mathrm{SO}_{3}\right)_{2}$ 20:1 electrolyte. 


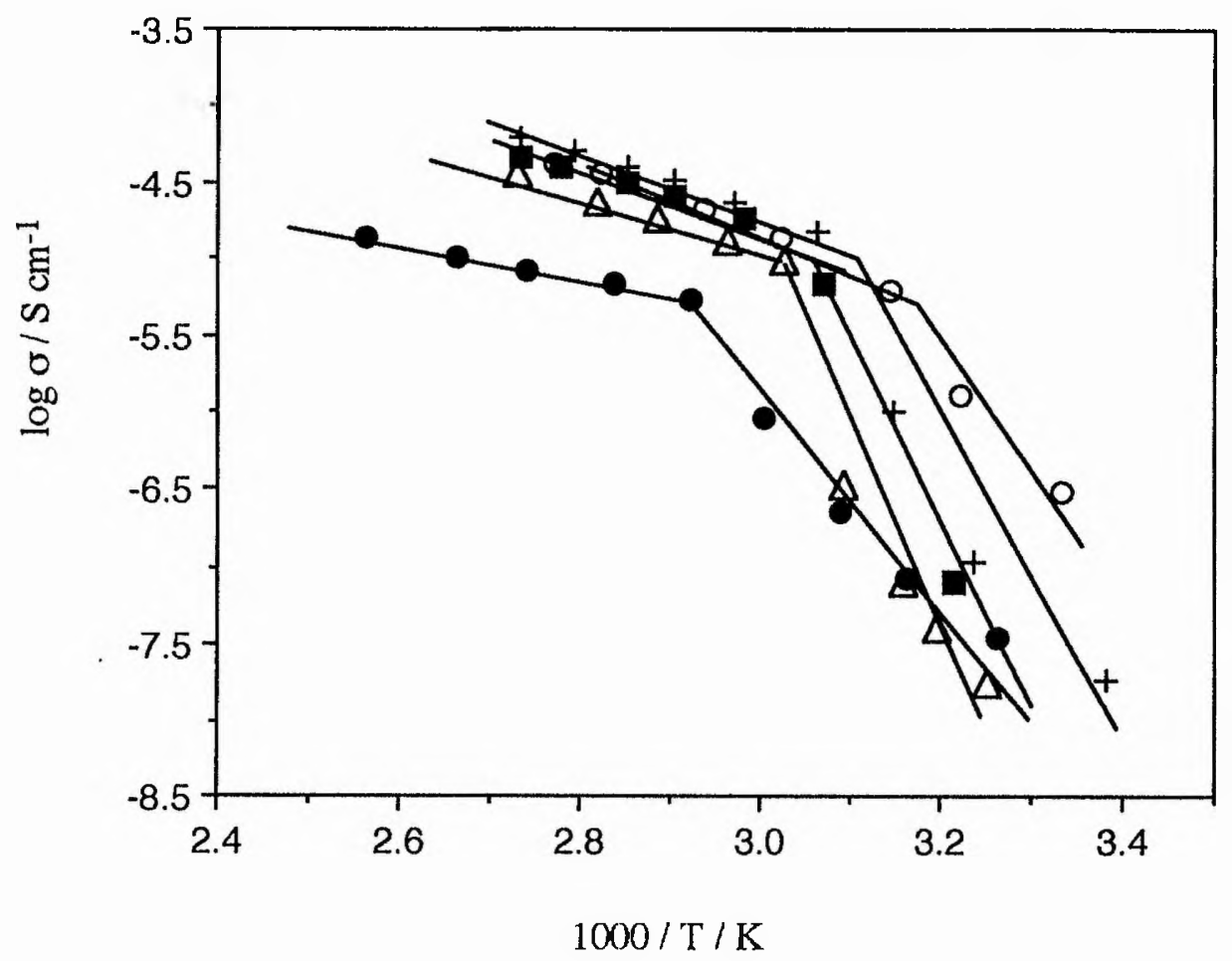

Figure 7-32 Temperature dependence of conductivities for polymer electrolytes based on $\mathrm{Mg}\left(\mathrm{CF}_{3} \mathrm{SO}_{3}\right)_{2}$ and methylated PEO $1450(\mathrm{O})$, methylated PEO 3350 (+), PEO 6000 $(\square)$, PEO $2 \times 10^{4}(\Delta)$ and PEO $4 \times 10^{6}(\mathbf{O})$. 


\section{CHAPTER 8}

\section{THE INFLUENCE OF THE MOLECULAR WEIGHT OF POLYMER ON THE IONIC CONDUCTION IN POLYMER ELECTROLYTES}

In conventional electrolyte solutions, it is well-known that ions can move together with their solvation layer. For polymer electrolytes based on high molecular weight polymer hosts, the solvent, polymer chains, can no longer move, since the polymer segments can only undergo the short-range motion rather than the long-range motion, due to the entanglements between polymer chains $[1,2]$. However, when the molecular weight of polymer is low, do the coordinated polymer chains move along with cations ? If the answer is yes, then what influence does the diffusion of the centre of gravity of polymer chains have on the ionic conduction? Also what is the range of the molecular weight of the polymer host to make the polymer electrolytes change in behaviour from liquid-like to solid-like? In some investigations for various reasons, liquid polymer electrolytes have been used to replace the solid high molecular weight polymer electrolytes in studies of the general mechanism of ionic conduction. Are these valid experiments? These questions are important both to the fundamental research and to the applications of polymer electrolytes. In this chapter, we endeavour to answer these questions by investigating theoretically and experimentally the influence of the diffusion of the centre of gravity of polymer chains on ionic conduction. 


\section{8-1 Dynamics of Linear Polymer Solutions and Melts}

The dynamic properties of simple liquids or solutions, low molecular weight polymer liquids or melts and high molecular weight polymer melts are all very different, which might be described by different models and mechanisms. For low molecular liquids, self-diffusion coefficients are generally considered in terms of hydrodynamic theory. Simple linear polymers of relatively low molecular weight behave differently from such molecular liquids: their dynamics can be described by the Rouse model. Above a critical value of molecular weight, chain entanglement becomes significant and a new model of polymer dynamics must be considered. At short times, a high molecular weight amorphous linear polymer behaves as a rubber since the "knots" formed by two polymer strands do not have time to become untied; at longer times Brownian motion results in local disentanglement allowing chains to slide past one another. This situation has been considered in the reptation model of de Gennes. In this section, these models are briefly presented and discussed.

\section{8-1-1 Dynamics of Simple Liquids or Solutions}

For a simple liquid, it is assumed [3] that flow involves a process in which the molecules move from one equilibrium position to the next by surmounting an energy barrier. The coefficient of viscosity of a liquid is defined by Newton's equation (8-1-1).

$$
f=\eta \frac{\mathrm{ds}}{\mathrm{dt}}
$$

where $f$ is shearing stress, $\eta$ the coefficient of viscosity, $s$ is the shear strain and ds/dt the rate of shear. 
According to the Eyring kinetic statistical theory, the activation energy for hopping of a liquid molecule from one equilibrium position to the next and the viscosity of a liquid can be related by the following equation [4]:

$$
\eta=\frac{h}{V} \exp \frac{\Delta E}{R T}
$$

where $\Delta \mathrm{E}$ is the activation energy, $\mathrm{V}$ is the volume of the flowing unit and $\mathrm{h}$ is Planck's constant.

The diffusion coefficient of particles in solution which can be regarded as spherical is described by the Stokes-Einstein equation:

$$
D=\frac{k T}{6 \pi \eta r}
$$

where $\mathrm{k}$ is Boltzman's constant, $\mathrm{T}$ is the absolute temperature, $\eta$ is the viscosity of the solution and $\mathrm{r}$ is the radius of the particles.

\section{8-1-2 Dynamics of Unentangled Polymer Liquids and Melts - the Rouse} Model

The dynamics of low molecular weight polymer liquids and melts may be described by a model which was proposed by Rouse in 1953 [5] and improved by Zimm [6]. Originally, this model was used to described the linear viscoelastic properties of dilute solutions of coiling polymers in low molecular weight solvents.

For a linear polymer dissolved in a solvent to form a dilute solution, it is assumed that the polymer chain consists of $\mathrm{N}$ identical segments joining $\mathrm{N}+1$ identical beads with complete flexibility at each bead. The separation of the ends of each segment 
is assumed to obey, to a first approximation, a Gaussian probability function, $w\left(l_{x}, l_{y}, l_{z}\right)$, for the distribution of the components of the length $l$ of the segment:

$$
w\left(l_{x}, l_{y}, l_{z}\right)=a \exp \left(-3 l_{x}{ }^{2}-3 l_{y}{ }^{2}-3 l_{z}{ }^{2}\right) / 2 b^{2}
$$

where $a$ and $b$ are constants and $b^{2}$ is the mean of $\mathrm{l}^{2}$. It is supposed that the interaction between the chain and its surroundings takes place only through the beads. This interaction at the $j$ th bead consists of a force with components $F_{x j}, F_{y j}, F_{z j}$ exerted on the surroundings by the bead. This force is assumed to be proportional to the velocity of the bead through the fluid:

$$
\begin{aligned}
& F_{x j}=\xi\left(\frac{d x_{j}}{d t}-v_{x j}^{\prime}\right) \\
& F_{y j}=\xi\left(\frac{d y_{j}}{d t}-v_{y j}^{\prime}\right) \\
& F_{z j}=\xi\left(\frac{d z_{j}}{d t}-v_{z j}^{\prime}\right)
\end{aligned}
$$

where $\xi$ is a friction coefficient, and $v_{\mathrm{xj}}, v_{\mathrm{yj}}$ ' and $v_{\mathrm{zj}}{ }^{\prime}$ are the velocity components that the fluid would have at the position of $j$ if the bead were absent.

The chain segments exert force on the beads. The $\mathrm{x}$-component of the mean force exerted on $\mathrm{j}$ by the segment from $\mathrm{j}-1$ to $\mathrm{j}$ is:

$$
-\left(3 k T / b^{2}\right)\left(x_{j}-x_{j-1}\right)
$$

In addition to the mechanical forces, from the theory of Brownian motion, the beads move as if an additional force is present with components: 
$-\mathrm{k}^{\prime} \Gamma \mathrm{d} \ln \Psi / \mathrm{dx}_{\mathrm{j}} ; \quad-\mathrm{kTd} \ln \Psi / \mathrm{dy}_{\mathrm{j}} ; \quad-\mathrm{kTd} \ln \Psi / \mathrm{dz}_{\mathrm{j}}$

where $\Psi\left(\mathrm{x}_{0}, \mathrm{y}_{0},--\mathrm{z}_{\mathrm{N}}\right)$ is a distribution function of all the coordinates.

Thus, the average forces for the $\mathrm{x}$-components are:

$$
\begin{aligned}
& F x_{0}=-k T d \ln \Psi / d x_{0}-\left(3 k T / b^{2}\right)\left(x_{o}-x_{1}\right) \\
& F x_{j}=-k T d \ln \Psi / d x_{j}-\left(3 k T / b^{2}\right)\left(-x_{j-1}+2 x_{j}-x_{j+1}\right) ; \quad(0<j<N) \\
& F x_{N}=-k T d \ln \Psi / d x_{N}-\left(3 k T / b^{2}\right)\left(x_{N}-x_{N-1}\right)
\end{aligned}
$$

It is assumed that the effect of a force on the motion of a fluid is given by the Kirkwood - Riseman [7] approximate form of the Oseen interaction formula:

$$
v_{x j}^{\prime}=v_{x j}+\sum_{j \neq p} T_{j p} F_{p}
$$

where $T_{j p}$ is the interaction coefficient. Combining equations (8-1-5), (8-1-6) and (8-17), the velocity of bead in the $\mathrm{x}$-direction is expressed as:

$$
\begin{aligned}
\frac{d x_{j}}{d t}= & v_{x j}-D \frac{d \ln \Psi}{d x_{j}}-\sigma\left(-x_{j-1}+2 x_{j}-x_{j+1}\right) \\
& -\xi \sum_{j \neq p} T_{j p}\left[D \frac{d \ln \Psi}{d x_{p}}+\sigma\left(-x_{p-1}+2 x_{p}-x_{p+1}\right)\right], \quad 0<j<N
\end{aligned}
$$


where $\mathrm{D}=\mathrm{kT} / \xi$ and $\sigma=3 \mathrm{kT} / \mathrm{b}^{2} \xi$. The expressions for the $\mathrm{y}$ and $\mathrm{z}$ components are of the same form. Thus, the differential equation of diffusion can be derived as follows (in matrix form).

$$
\begin{aligned}
\frac{\mathrm{d} \mathbf{x}}{\mathrm{dt}} & =\mathbf{v}_{\mathbf{x}}+(1 / \xi) \mathbf{H} \cdot \mathbf{F}_{\mathbf{x}} \\
& =\mathbf{v}_{\mathbf{x}}-\mathrm{D} \mathbf{H} \cdot\left(\frac{\mathbf{d}}{\mathbf{d x}}\right) \ln \Psi-\sigma \mathbf{H} \cdot \mathbf{A} \cdot \mathbf{x}
\end{aligned}
$$

where $\mathbf{x}$ and $(\mathbf{d} / \mathbf{d} \mathbf{x})$ is a $(\mathrm{N}+1)$ dimensional column matrix,

$$
\mathbf{x}=\left[\begin{array}{c}
\mathrm{x}_{\mathrm{o}} \\
\mathrm{x}_{1} \\
\cdot \\
\mathrm{x}_{\mathrm{N}}
\end{array}\right]
$$

$$
\frac{d}{d x}=\left[\begin{array}{c}
\frac{d}{d x_{0}} \\
\frac{d}{d x_{1}} \\
\cdot \\
\frac{d}{d x_{N}}
\end{array}\right]
$$

A and $\mathbf{H}$ are matrices of order $\mathrm{N}+1$. $\mathbf{H}$ has elements $H_{\mathrm{jp}}$ as follows:

$$
H_{\mathrm{ij}}=1 ; \quad \mathrm{H}_{\mathrm{jp}}=\xi \mathrm{T}_{\mathrm{jp}} \quad \mathrm{j} \neq \mathrm{p}
$$

By solving the above differential equation, the intrinsic viscosity of the solution and the diffusion coefficient of the polymer chain can be derived [6].

$$
[\eta]=N_{a v} N^{2} b^{2} \xi / 36 \mathrm{M} \eta ; \quad \text { for the free-draining case }{ }^{*}
$$

\footnotetext{
${ }^{*}$ Free-draining flow means that the beads are relatively far from one another, so that the disturbances of the flow caused by the individual beads can be considered not to interact.
} 
where $N_{a v}$ is the Avogadro constant, $\eta$ is the viscosity of solvent, $M$ is the molecular weight of polymer, $\mathrm{N}$ is the number of segments in the polymer, and $\xi$ is the friction coefficient.

From equation (8-1-11), this theory predicts that the diffusion coefficient D of polymer chains should be inversely proportional to the molecular weight of polymer. In equation $(8-1-10)$, because $b^{2}, \eta$ and $\xi$ are constants, the viscosity is directly proportional to the molecular weight of polymer.

In most cases of low molecular weight polymer liquids and melts, it has indeed been found $[8,9]$ that the diffusion coefficient of polymer chains is inversely proportional to the molecular weight of polymer, while the viscosity shows a low power dependence in molecular weight, i.e., $\eta \sim \mathrm{M}^{\alpha}$, in which $\alpha$ is of ten slightly larger than unity. For example [9], when the molecular weight of polyethylene is lower than 5,000 , the product $\mathrm{D} \eta$ is almost constant at $175^{\circ} \mathrm{C}$ for all molecular weights examined.

Therefore, although the Rouse model was originally developed for the dilute solution of linear polymers, it can also be applied quite successfully in most cases to unentangled polymer liquids and melts, i.e. the molecular weight of the polymer below the critical value for establishing entanglement.

\section{8-1-3 Dynamics of Entangled Polymer Melts - Reptation Theory}

The reptation theory for entangled polymer melts was proposed by de Gennes [10]. It is assumed that entanglement of polymer chains constrains their motion: at any instant every chain is considered to be confined within a "tube" within which it reptates (Figure 8-1a). 
Consider a freely jointed chain of $\mathrm{N}$ segments which is trapped inside a threedimensional network $\mathrm{G}$ which can be regarded as a thin wall tube. The chain cannot get out of the tube through its wall, but, it can move along the tube by reptation.

It is assumed that the motion of defects (they are taken as some stored part of the chain, as shown in Figure 8-1b) along the chain is so fast in comparison with the motion of the length of chain itself that one can postulate that the only allowed motions in the tube correspond to the migration of defects along the chain on a very short time scale. If the curvilinear interval between two successive segments is $a$, the number of joints is $n$, the density of defects is $\rho$, the stored length of chain by defects with density of $\rho$ is $b$. When an external force $f$ is applied on the segments, the force actually applied to each defect is $\varphi_{n}$, the defect current $J_{n}$ which is equal to the number of defects passing through point $\mathrm{n}$ per unit time is expressed as:

$$
\mathrm{J}_{\mathrm{n}}=\mathrm{D}_{\text {def }}\left[-\frac{1}{a} \frac{\mathrm{d} \rho}{\mathrm{dn}}+\frac{\rho \varphi_{\mathrm{n}}}{\mathrm{kT}}\right]
$$

where $\mathrm{k}$ is Boltzmann's constant, $\mathrm{T}$ is the absolute temperature, and $\mathrm{D}_{\text {def }}$ is the diffusion coefficient of defects along the chain. $D_{\text {def }}$ is independent of the molecular weight of polymer because the diffusion of defects is very much faster than that of the chain.

The time $T_{d}$ required for equilibration of the defect gas can be deduced from equation (8-1-12).

$$
T_{\mathrm{d}}=\frac{\mathrm{N}^{2} a^{2}}{\pi^{2} \mathrm{D}_{\mathrm{def}}}
$$


When the defects move along the chain, the chain itself progresses in the tube. The velocity of the motion of $n$th segment in the tube is related to the defect current $J_{n}$ by

$$
\frac{\mathrm{d} \mathbf{r}_{\mathrm{n}}}{\mathrm{dt}}=\mathbf{b} \mathrm{J}_{\mathrm{n}}
$$

where $\mathbf{r}_{\mathbf{n}}$ is the position of the nth segment, and $\mathbf{b}$ is vector of magnitude $b$.

During $\mathrm{T}_{\mathrm{r}}$, the time required for complete renewal of chain conformation (much longer than the equilibration time of the defects, $T_{d}$ ), motion of the whole chain (i.e. its centre of gravity) is undertaken. In other words, the chain begins to move out from its initial tube.

Consider a chain $\mathbf{P}$ in reptation under a constant weak force $\mathbf{F}$ which is applied along a certain direction ( e.g. z-direction). The force actually exerted on a segment is: $f_{n}=F / N$, and the force applied to a defect is:

$$
\varphi_{11}=\frac{\mathbf{b} \cdot \mathbf{F}}{\mathrm{N} a}
$$

The steady state conduction current $J$ of the defect gas is written as:

$$
\begin{aligned}
J & =\frac{\bar{\rho} \mu}{N} \int_{0}^{N} \mathrm{dn} \varphi_{n} \\
& =\frac{\bar{\rho} \mu}{N^{2} a} \int_{0}^{N} \mathbf{b} \cdot \mathbf{F} d n
\end{aligned}
$$

By noting that $a \mathrm{dn}=\mathrm{dl}$, where $\mathrm{l}$ is the line element of the chain, and $\int \mathrm{d} \mathbf{l}=\mathbf{P}$, the conduction current is converted to: 


$$
\mathrm{J}=\frac{\bar{\rho} \mu \mathrm{b}}{\mathrm{N}^{2} a^{2}} \mathbf{F} \cdot \mathbf{P}
$$

where $\mu=D_{\text {def }} / \mathrm{kT}$, is the defect mobility, and $\bar{\rho}$ is the equilibrium value of the defect density. The velocity of motion of the centre of gravity of the chain is the average of the velocity of each segment over the whole chain, expressed as follows:

$$
\frac{\mathrm{dg}}{\mathrm{dt}}=\mathrm{N}^{-1} \int_{0}^{\mathrm{N}} \frac{\mathrm{d} \mathbf{r}_{\mathrm{n}}}{\mathrm{dt}} \mathrm{dn}
$$

where $\mathrm{dg} / \mathrm{dt}$ is the velocity of motion of the centre of gravity of the chain, and $\mathrm{dr} / \mathbf{n} / \mathrm{dt}$ is the velocity of the nth segment. By substituting (8-1-14) into (8-1-18) and combining equation (8-1-17), (8-1-18) is converted to:

$$
\frac{\mathrm{dg}}{\mathrm{dt}}=\frac{\mathrm{Jb}}{\mathrm{N} a} \mathbf{P}
$$

From equation (8-1-17) and (8-1-19), by averaging $\mathbf{F}$ and $\mathbf{P}$ over all directions, the only non-vanishing velocity component is:

$$
\left(\frac{\mathrm{dg}}{\mathrm{dt}}\right)_{\mathrm{z}}=\frac{\mu \bar{\rho} b^{2}}{\mathrm{~N}^{2} a} \mathrm{~F}_{\mathrm{z}}
$$

Therefore, the diffusion coefficient of the centre of gravity of the chain is:

$$
\mathrm{D}_{\mathrm{g}}=\frac{\mathrm{kT} \boldsymbol{\mu} \overline{\boldsymbol{\rho}} \mathrm{b}^{2}}{\mathrm{~N}^{2} a}
$$

When the time scale is shorter than $T_{r}$ but much longer than $T_{d}$, i.e. $T_{d} \ll t<$ $T_{r}$, overall diffusion of the polymer chain occurs. In other words, the time is long 
enough to allow the chain to move within its initial tube, but not to move out of this tube. The diffusion coefficient of the chain reptating in the tube can be derived from equation (8-1-14).

Combining equations (8-1-13), (8-1-14) and (8-1-15), and with the condition of the steady state current of the defect gas, the velocity of the chain for diffusion along its fixed curvilinear path can be expressed as:

$$
\frac{\mathrm{dc}_{\mathrm{L}}}{\mathrm{dt}}=\frac{\mathrm{D}_{\mathrm{def}} \overline{\boldsymbol{\rho}}}{\mathrm{kTN} a} \int_{0}^{\mathrm{N}} \mathbf{b} \cdot \mathbf{b} \cdot \mathbf{F} \mathrm{dn}
$$

Averaging over all directions, only the z-component remains, i.e.

$$
\frac{\mathrm{dc}_{\mathrm{L}}}{\mathrm{dt}}=\frac{\bar{\rho} \mu \mathrm{b}^{2}}{\mathrm{~N} a} \mathrm{~F}
$$

The curvilinear diffusion coefficient of the chain is:

$$
D_{c}=\frac{k T \bar{\rho} \mu b^{2}}{N a}
$$

where all parameters have the same meanings as above.

It is clear that the time $T_{r}$ is related to $D_{c}$ and the length of the tube $\mathrm{Na}$, i.e, to the extent of entanglement. When $D_{c}$ is large, the time for moving out of the tube should be short and there is a similar consequence for a short fixed tube. $T_{\mathbf{r}}$ is expressed as:

$$
T_{r}=\frac{N^{2} a^{2}}{\pi^{2} D_{c}}=\frac{N^{3} a^{3}}{\pi^{2} \bar{\rho} b^{2} D_{\text {def }}}
$$


The viscosity of the polymer can be expressed as [9]:

$$
\eta=\frac{5}{8} \rho_{P} N_{a v} \frac{<S^{2}>}{M}\left(\frac{M}{M_{e}}\right)^{2} N \xi
$$

where $\rho_{p}$ is the density of polymer, $M_{e}$ is the molecular weight between entanglements, which can be looked on as constant for polymers whose molecular weight are well above the critical value for entanglement, and $\xi$ is a friction coefficient which can be taken to be the reciprocal of the mobility of defects $\mu$. Also $<S^{2}>/ M$, the mean-square radius of gyration divided by the molecular weight, can also be assumed to be constant, because $\left\langle\mathrm{S}^{2}\right\rangle=\mathrm{N} a^{2} / 6$, i.e. $\left\langle\mathrm{S}^{2}>\right.$ is directly proportional to the molecular weight of polymer, where $\mathrm{N}$, the number of segments, and $a$, the length of segment, are fixed. Therefore $\eta$ is proportional to $\mathrm{M}^{3}$. This is a fundamental conclusion of reptation theory.

In summary, reptation theory predicted that $D_{g} \propto M^{-2}, D_{c} \propto M^{-1}, \eta \propto M^{3}$, $\mathrm{T}_{\mathrm{d}} \propto \mathrm{M}^{2}$ and $\mathrm{T}_{\mathrm{r}} \propto \mathrm{M}^{3}$.

\section{8-2 Dynamics of Non-linear Polymer Solutions and Melts}

\section{8-2-1 Dynamics of Polymer Liquids and Melts With Branched Chains}

For a non-linear Gaussian chain with $f$ branches of $n_{b}$ segments in a dilute solution, and using the same assumptions as in the linear chain system, the diffusion coefficient of the polymer can be derived as [11]:

$$
\mathrm{D}=(\mathrm{kT} / \xi) \mathrm{N}^{-1}
$$


where $\xi$ is a friction coefficient, and $\mathrm{N}$ is the total number of segments of the polymer, i.e., $N=n_{b} f$, for $n_{b}$, the number of segments in each branch, and $f$, the number of branches in the polymer.

When there are entanglements in the non-linear polymer, a modified reptation model must be employed [12]. Consider a symmetric star-shaped polymer with $f$ arms and $n_{b}$ segments for each arm, moving in non-crossable tubes. The mean number of entanglement length $N$ or $N_{b}$ (the former is for whole and the latter is for the arm) is used to express the length of the chain or arm, i.e. $N=n / n_{c} ; N_{b}=n_{b} / n_{c}$, where $n_{c}$ is the critical number of segments for entangled behaviour.

It is assumed that translational diffusion can only take place when an arm retracts, without crossing any of the obstacles (Figure 8-2). This allows the centre of the segments to move a tube diameter $\chi$. The probability of an arm folding back on itself is:

$$
P_{1}\left(N_{b}\right) \propto \exp \left(-\alpha N_{b}\right)
$$

where $\alpha$ is a constant. The rate of such steps is proportional to this probability. Thus, the time for the arm relaxation can be expressed as:

$$
\tau_{\mathrm{s} 1}\left(\mathrm{n}_{\mathrm{b}}\right)=\tau_{\mathrm{rep}}\left(\mathrm{n}_{\mathrm{b}}\right) / \mathrm{P}_{1}\left(\mathrm{~N}_{\mathrm{b}}\right) \approx\left(\tau_{\mathrm{o}} / \mathrm{n}_{\mathrm{c}}\right) \mathrm{n}_{\mathrm{b}}{ }^{3} \exp \left(\mathrm{c} \mathrm{N}_{\mathrm{b}}\right)
$$

where $\tau_{\mathrm{rep}}\left(\mathrm{n}_{\mathrm{b}}\right)=\mathrm{n}_{\mathrm{b}}{ }^{3}\left(\tau_{\mathrm{o}} / \mathrm{n}_{\mathrm{c}}\right)$ is the corresponding reptation time for a linear chain with $\mathrm{n}_{b}$ segments, and $\tau_{\mathrm{o}}$ is a microscopic jump time which can be written as:

$$
\tau_{\mathrm{o}}=\mathrm{l}^{2} / 2 \mathrm{~B}_{\mathrm{o}} \mathrm{kT}
$$

for 1 the length of segments and $B_{0}$ the mobility of segments. 
Since the centre of gravity of chain moves by an independent stochastic step of size $\chi$ over the time $\tau_{s}$, the corresponding translational diffusion coefficient of the centre of gravity $D_{s}$ is:

$$
D_{s} \propto \chi^{2} / \tau_{s}=\frac{n_{c} \chi^{2}}{\tau_{0} n_{b}^{3}} \exp \left(-\alpha N_{b}\right) \propto \frac{D_{\text {rep }}\left(n_{b}\right)}{N_{b}} \exp \left(-\alpha N_{b}\right) \text { for } f=3
$$

where $D_{\text {rep }}\left(n_{b}\right)$ is the reptation diffusion coefficient for a linear chain with $n_{b}$ segments. For $\mathrm{f} \geq 3$ cases, equation (8-2-4) is in the form:

$$
D_{s}=\frac{D_{\text {rep }}\left(n_{b}\right)}{N_{b}} \exp \left[-\alpha(f-2) N_{b}\right]
$$

This implies that the existence of branches (arms) will make the diffusion coefficient of polymer chain to decrease with the molecular weight and number of arms in an exponential manner.

\section{8-2-2 Dynamies of Branched Polymer in Linear Polymer Melts}

The dynamics of a branched polymer in a linear polymer melt is different from those of homopolymer systems, because the constraints result not only from the branched polymer itself, but also from the linear polymer.

First of all, consider a linear polymer $\mathrm{H}$ with $\mathrm{n}$ segments in a linear polymer melt $\mathrm{J}$ with p segments which is chemically identical to polymer $\mathrm{H}$. One can imagine that the $\mathrm{H}$ chain reptates in a tube. Whenever any neighbouring $J$ molecule reptates away, a real topological change in the tube may result, and the tube contour may change by a small amount. This kind change would influence the motion of the chain $\mathrm{H}$.

Thus the translational diffusion coefficient of polymer $\mathrm{H}$ includes the contributions of reptation of the chain itself and the renewal of the tube, i.e., 


$$
\mathrm{D}_{\mathrm{T}}(\mathrm{H})=\mathrm{D}_{\mathrm{rep}}(\mathrm{H})+\mathrm{D}_{\mathrm{tb}}(\mathrm{H})
$$

where $D_{\text {rep }}(H)$ is the diffusion coefficient of the $H$ chain reptation, which has been discussed above, and is defined by equation (8-1-21), and $D_{t b}(H)$ is the tube renewal diffusion coefficient, which has been discussed by Klein [12].

When the molecular weight of polymer $J$ is not much different from that of polymer $H$, i.e. $n / p$ is not very large, $D_{t b}(H)$ takes the form:

$$
D_{t b}(H)=\left(n n_{c}^{2 / p^{5 / 2}}\right) D_{r e p}(H)
$$

When $n / p$ is quite large, $D_{t d}(H)$ takes the form:

$$
\mathrm{D}_{\mathrm{tb}}(\mathrm{H})=\left(\mathrm{n}^{2 / 3} / \mathrm{p}^{3}\right) \mathrm{p}_{\mathrm{c}} \mathrm{D}_{\mathrm{rep}}(\mathrm{H})
$$

where $p_{c}$ is the critical number of segments for establishing entanglement.

If $\mathbf{n} / \mathrm{p}$ is very large, the chain $\mathrm{H}$ behaves like a hydrodynamic sphere of radius $\mathrm{R}_{(\mathrm{H})}$ moving within a continuous medium. In this case, the diffusion coefficient of the chain reverts to a Stokes-Einstein form:

$$
\mathrm{D}_{\mathrm{T}}(\mathrm{H})=\frac{\mathrm{kT}}{6 \pi \eta_{(\mathrm{J})} \mathrm{R}_{(\mathrm{H})}}
$$

where $\eta_{(J)}$ is the viscosity of polymer melt $\mathrm{J}$.

When $\mathrm{n}=\mathrm{p}$, i.e., for a homopolymer melt, from equation (8-2-7), the behaviour of the chain $\mathrm{H}$ is dominated by its own reptation, as discussed in section 8-1-3. When $n<n^{*}=p^{5 / 2} n_{c}^{-2}$, the dynamic behaviour of the chain $H$ is also dominated 
by its reptation. When $\mathrm{n} \gg \mathrm{n}^{*}$, however, the dynamics of the chain $\mathrm{H}$ is dominated by the renewal of the tube according to equation (8-2-7).

In equation (8-2-9), the hydrodynamic sphere radius $R_{(H)}$ is proportional to the root-mean-square radius $\left\langle\mathrm{S}_{(\mathrm{H})}{ }^{2}{ }^{1 / 2}\right.$, and $\left\langle\mathrm{S}_{(\mathrm{H})}{ }^{2}\right\rangle$ is proportional to the number of segments of the polymer. Thus, if the molecular weight of polymer is very large, $R_{(H)}$ will be also very large, and consequently the diffusion coefficient will be very small.

For a branched polymer (say a star polymer with an equal number $n_{b}$ of segments in each of $f$ arms), each arm can be imagined reptating in a tube (Figure 8-2). The motion of the centre of gravity of the chain results from the retraction of an arm from its initial tube. If this star polymer $\mathrm{SN}$ is in a linear polymer melt $\mathrm{J}$, the translational diffusion coefficient of the centre will be the sum of reptation and tuberenewal diffusion coefficients, i.e.

$$
\mathrm{D}_{\mathrm{T}}(\mathrm{SN})=\mathrm{D}_{\mathrm{rep}}(\mathrm{SN})+\mathrm{D}_{\mathrm{tb}}(\mathrm{SN})
$$

where $D_{\text {rep }}(\mathrm{SN})$ is the diffusion coefficient of reptation which is expressed by equation (8-2-5), and $D_{t b}(S N)$ is the tube renewal diffusion coefficient. If the f-arm star polymer is looked on as consisting of $f$ linear polymer with $n_{b}$ segments, equation (8-2-7), (8-2$8)$ and (8-2-9) can be applied to the star polymer system, in which $n$ is replaced by $n_{b}$. Because $D_{\text {rep }}(S N)$ decreases with the molecular weight and number of arms $f$ in an exponential form, the influence of the tube renewal process on the diffusion of a star polymer is more important than in the linear polymer case.

Finally, we consider the case of a crosslinked polymer sphere $\mathrm{G}$ or microgel diffusing in a linear polymer melt $\mathrm{J}$. This case is more or less similar to that where the molecular weight of the chain $\mathrm{H}$ is much larger than that of melt polymer, and can be 
linked to a sphere diffusing in a solvent, as described by Stokes-Einstein equation (8-29). Thus the diffusion coefficient of crosslinked polymer microgel can be given by

$$
D_{G}=\frac{k T}{6 \pi \eta_{(J)} R_{e f f}}
$$

where $\eta_{(J)}$ is the viscosity of melt $J$, and $R_{\text {eff }}$ is the effective radius of the microgel. Thus, large microgels can no longer diffuse a significant distance, because of the very small value of $D_{G}$. It is also obvious from equation (8-2-11) that if the system does not contain any linear polymer melt, the microgel cannot diffuse at all.

\section{8-3 Dynamics of Polymer Chain Diffusion in Polymer Electrolytes}

\section{8-3-1 Models for the Diffusion of Polymer Chain in Polymer Electrolytes}

For solid polymer electrolytes based on high molecular weight polymers, the diffusion of the polymer chains is negligible, because as discussed above, the high molecular weight of polymer makes the diffusion coefficient of polymer chains very small. This implies that polymer chains can only undertake microscopic, i.e. segmental motion, rather than significant macroscopic diffusion. It is believed [1] that in solid polymer electrolytes, the ionic conduction results mainly from the segmental motion of the polymer, i.e. the rearrangement or reorientation of segments, which allows cations to move from one coordinate site to another. However, for low or medium molecular weight polymer electrolytes which are liquids or melts at relatively low temperatures, macroscopic diffusion of polymer chain may be possible, and this may influence the mechanism of ionic conduction. 
Suppose that in a particular polymer electrolyte melt or liquid, cations are tightly bound to the polymer chains, i.e., cationic conduction of the polymer electrolyte is completely controlled by the macroscopic diffusion of the polymer chains. The dynamics of these polymer electrolytes can be roughly classified as belonging to one of three classes (Figure 8-3), in terms of the manner in which the cations and polymer host chains are coordinated in the polymer electrolyte. Here, the term of "coordinated-chain" is employed to represent the unit formed by the coordination between polymer host chains and cations. The shape of the coordinated-chain may be linear or branched.

\section{(1) System with linear coordinated-chains}

Assume that there are only intrachain coordinations in the polymer electrolyte, and that all the coordinated-chains in the polymer electrolytes are linear and with the same length.

When the molecular weight is too low for significant entanglement, the dynamic behaviour of this chain can be described by the Rouse model. Therefore, the translational diffusion coefficient of the polymer chain-bound cations is:

$$
\mathrm{D}=(\mathrm{k} T / \xi) \mathrm{N}^{-1}
$$

or

$$
\mathrm{D}=(\mathrm{kT} / \xi) \mathrm{M}_{\mathrm{s}} / \mathrm{M}
$$

where $\xi$ is the friction coefficient, $M_{s}$ is the molecular weight of segments for the PEO based polymer electrolyte, $\mathrm{M}_{\mathrm{s}}$ is approximately equal to the molecular weight of an EO unit, and $\mathrm{M}$ is the molecular weight of the polymer.

When the molecular weight is above the critical value for entanglement, i.e, $M>$ $\mathrm{M}_{\mathrm{c}}$ (or $\mathrm{n}>\mathrm{n}_{\mathrm{c}}$ ), the chain will reptate within the confines of a tube. The dynamics of the 
translational diffusion of the centre of gravity of the chain is given by equation (8-1-21). This equation can be transformed to equation (8-3-2) when the number of segments is replaced by the molecular weight [9]:

$$
\mathrm{D}=\frac{4 \mathrm{kT}}{15 \xi} \frac{\mathrm{M}_{\mathrm{s}} \mathrm{M}_{\mathrm{e}}}{\mathrm{M}^{2}}
$$

where $\mathrm{M}_{\mathrm{e}}$ is the molecular weight of the chain between entanglements.

\section{(2) System with linear and branched coordinated-chains}

Suppose in the polymer electrolyte system, the main coordinate forms are intrachain coordinations forming equal length linear chains, and there are a few nonlabile interchain coordinations to form star-shaped chains with equal length of arms $\left(n_{b}\right)$. (Note: here, a cation can be taken to be the nucleus of a star-shaped unit.)

When the molecular weights of the polymer is less than the critical value for entanglement of the linear chains and the branched chains, the total translational diffusion coefficient of the chain-bound cations is:

$$
D=x D_{1}+y D_{s}
$$

where $D_{1}$ is the diffusion coefficient of linear chain, and $D_{s}$ is the diffusion coefficient of the star chain, and $x$ and $y$ are their molar fractions. $D_{1}$ and $D_{s}$ are expressed by equations (8-1-11) and (8-2-1) respectively. Thus in this case, the translational diffusion coefficient is inversely proportional to the molecular weight of the polymer once again.

When the molecular weight of polymer is high enough to establish entanglements for both the star and the linear chains i.e, $n>n_{c}$ and $n_{b}>n_{b c}\left(n_{c}\right.$ may be 
different from $n_{b c}$, they will reptate in tubes formed by the linear chains, because linear chains are the domain in this case. Thus, the total translational diffusion coefficient of these chains can be written as:

$$
\mathrm{D}=\mathrm{x} \mathrm{D}_{1}+\mathrm{y} \mathrm{D}_{\mathrm{s}}+\mathrm{y} \mathrm{D}_{\mathrm{tb}}
$$

where $x, y, D_{l}$ and $D_{s}$ have the same meanings as the above, and $D_{t d}$ is the tube renewal time in which the star reptates.

Because a linear chain reptates in a tube consisting of other linear chains, $D_{1}$ can be expressed by equation (8-3-2) without any modification. $D_{s}$ can be expressed by equation (8-2-5), and $\mathrm{D}_{\mathrm{tb}}$ can be expressed by equation (8-2-7) or (8-2-8).

In equation (8-3-4), the total translational diffusion of polymer results mainly from the diffusion of linear chains. This is because the diffusion coefficient of star chains is strongly reduced by the molecular weight and number of arms, and furthermore, it is assumed that in this model the proportion of the linear chain is much larger than that of star polymer, i.e., $x>y$. Therefore, in this case, the total translation diffusion coefficient of polymer chains with cations is inversely proportional to the square of the molecular weight of the polymer.

\section{(3) System dominated by branched coordinated-chains}

When interchain coordination is dominant, the diffusion of the polymer with cations is controlled by the motion of branched chains. If the shape of these chains is that of a star with equal length of arms, the translational diffusion coefficient of these chains is either inversely proportional to the molecular weight [equation (8-2-1)] or inversely proportional to the square of the molecular weight of polymer expressed by 
equation (8-3-2), depending on whether the molecular weight is lower or higher than the critical point for entanglement.

From equation (8-3-2) it can be concluded that the interchain coordination for high molecular weight polymer will reduce the diffusion coefficient very significantly. The higher the interchain coordination number $Q$ and/or molecular weight, the lower $D$. D decreases exponentially with increasing $Q$ or $\mathbf{n}_{\mathbf{b}}$.

If in the domain of the branched chains there are still a few linear coordinatedchains, these linear chains will diffuse in the branched chain domain. For the case where the molecular weight of polymer is lower than the critical values for entanglement of both linear and branched chains, the total translational diffusion coefficient $D$ is the average sum of $D_{1}$ defined by equation (8-2-1) and $D_{s}$ defined by (8-3-1), i.e. again $D$ is inversely proportional to the molecular weight.

When both types of chains are entangled, the linear chains can be assumed to reptate in the tube consisting of the branched chains, and the branched polymer chains reptate in tubes consisting of other branched chains. Therefore, the total translational diffusion coefficient can also be written as:

$$
D=x D_{s}+y D_{1}
$$

This is different from the case where the star chains diffuses in the domain of the linear chains (8-3-4), since the tube renewal process is very slow in comparison with the reptation of the linear chains, and can be neglected.

When the molecular weight of the polymer is very high and/or the interchain coordination number $Q$ is large, the diffusion coefficient of the star chains is so small that they can be considered immobile in comparison with the linear polymer, i.e. the 
linear chains reptate in a fixed tube consisting of these star chains. Therefore, the total translational diffusion coefficient of the polymer electrolyte chain is solely dominated by the reptation of the linear chain as described by equation (8-3-2).

Therefore, when the interchain coordinate is dominant giving rise to a star-shape unit, the cation diffusion coefficient will be much smaller than that for a linear chain unit in the high molecular weight system.

\section{8-3-2 Relaxation Modes of Polymer Chains and Ionic Conduction in Polymer Electrolytes}

We discussed above the influence of the molecular weight on the diffusion coefficient of tightly-bound cations. For more usual polymer electrolytes, cations are not tightly bound by polymer chains, i.e. the cations can easily be released from one polar atom and re-coordinated to another. On the one hand, when the molecular weight of polymer is significantly low, the diffusion of the polymer chains may have a significant effect on the ionic conduction in the polymer electrolyte. One the other hand, when the molecular weight of polymer is high, the contribution from the translational diffusion of polymer chain on the conduction is negligible. In this section, the relaxation modes in polymer electrolytes and their effects on the ionic conduction are discussed.

\section{(1) Relaxation modes in polymers}

In polymer liquids or melts, there are three principal types of relaxation mode [13], namely: (a) rearrangement and reorientation of polymer segments, with a relaxation time $\tau_{a}$, (b) curvilinear diffusion of polymer chains, with a relaxation time $\tau_{b}$, and (c) fluctuation of surroundings, with a relaxation time $\tau_{c}$ (Figure 8-4),

The rearrangement and reorientation of segments is a very fast process. This kind of motion can be described in terms of the crankshaft rotation of neighbouring 
segments, i.e. simultaneous rotation around parallel main chain bonds. This is dominated by the properties of polymer chains, such as rotation barrier of bond etc, rather than by the length of the chains. Thus, the relaxation time for segmental rearrangement and reorientation, $\tau_{a}$, is independent in the molecular weight of the polymer [13].

The curvilinear diffusion of polymer chains has been discussed above. For an entangled polymer chain, it can be assumed that the polymer chain reptates in a tube. When the time scale is short, the relationship between the curvilinear diffusion coefficient $\mathrm{D}_{c}$ and the molecular weight of polymer is expressed by equation (8-1-24). The correlation function for the reptation of polymer chain in the tube is written as $[10,14]$ :

$$
<\left[s_{n}(0)-s_{n}(t)\right]^{2}>=2 D_{c} t+\frac{2 C}{N} \sum_{p} \cos ^{2}\left(\frac{\pi p n}{N}\right) \tau_{p}\left[1-\exp \left(\frac{-t}{\tau_{p}}\right)\right]
$$

where $\mathrm{s}_{\mathrm{n}}(0), \mathrm{s}_{\mathrm{n}}(\mathrm{t})$ is the curvilinear abscissa along the tube, $\mathrm{C}$ a constant relating to $a$ and $b, p$ is a positive integer, and $\tau_{p}$ is the relaxation time which is expressed as:

$$
\tau_{\mathrm{p}}=\mathrm{T}_{\mathrm{d}} \mathrm{p}^{-2}
$$

Among those $\tau_{p}{ }^{\prime} s, \tau_{p=1}$ is the longest, corresponding to $T_{d}$. When the time scale is quite long, i.e. $t>T_{d}$, the first term in equation (8-3-6) becomes dominant. Thus,

$$
<\left[s_{n}(0)-s_{n}(t)\right]^{2}>=2 D_{c} t
$$

The characteristic time $\tau_{\mathrm{b}}$ for the chain moving a distance $\mathrm{I}$ can be derived from equation (8-3-8) by replacing $<\left[s_{n}(0)-s_{n}(t)\right]^{2}>$ by $1^{2}$. 


$$
\tau_{\mathrm{b}}=\frac{1^{2}}{2 \overline{\mathrm{L}}_{\mathrm{c}}}
$$

For unentangled polymer, i.e. $M<M_{c}$, which can be described by the Rouse model, the correlation function takes the same form as (8-3-6). Also, equation (8-3-8) can be used to correlate the relaxation time and diffusion coefficient. In this case, $D_{c}$ is replaced by the diffusion coefficient of the polymer, i.e. D as derived from equation (8-1-11). Because both $\mathrm{D}_{\mathrm{c}}$ for entangled polymer and for unentangled polymer are inversely proportional to the molecular weight of polymer, the relaxation time, $\tau_{b}$, for the diffusion of a distance 1 is directly proportional to the molecular weight of polymer.

When a polymer chain diffuses in a tube, the environmental microstructure of the chain will change, due to configurational fluctuation of the surroundings. The time for the environmental microstructure to reach equilibrium is much longer than that for an individual chain to diffuse a small distance 1 . For example, in reptation theory, $T_{\mathrm{r}}$ is very long and proportional to cube of the molecular weight of the polymer. Therefore, process (c) can be regarded as the diffusion of polymer chain for a distance of the length, L, of the tube (for entangled polymer) or of the diameter of the polymer coil (for unentangled polymer). By replacing $<\left[s_{n}(0)-s_{n}(t)\right]^{2}>$ by $L^{2}$ or $2<S^{2}>$ in equation (8-3-8), where $\mathrm{L}=\mathrm{Na}, \mathrm{N}$ the number of segments and $a$ the the length of segment, and $\left\langle\mathrm{S}^{2}>=\mathrm{N} a^{2 / 6},\left\langle\mathrm{~S}^{2}>\right.\right.$ the mean-square radius of gyration of the polymer coil, the characteristic time, $\boldsymbol{\tau}_{\mathbf{c}}$, for this process is:

$$
\begin{array}{lll} 
& \tau_{c} \propto \frac{L^{2}}{2 D_{c}}=\frac{N^{2} a^{2}}{2 D_{c}} & \text { for } M>M_{c} \\
\text { or } & \tau_{c} \propto \frac{\left\langle S^{2}>\right.}{D}=\frac{N a^{2}}{6 D} & \text { for } M<M_{c}
\end{array}
$$


Because $\mathrm{D}_{\mathrm{c}}$ or $\mathrm{D}$ (for unentangled polymer) is inversely proportional to the molecular weight $M$, the relaxation $\tau_{c}$ is proportional to square of $M$ for the $M<M_{c}$ case, and for $M>M_{c}$, the relaxation time is proportional to the cube of M. Obviously, process (c), the configurational fluctuation of surrounding of chain, is the slowest among these three relaxation modes.

(2) Relationship between the ionic conduction and relaxation modes in polymer electrolytes

In the dynamic bond percolation model proposed by Ratner and coworkers [1], the ionic conduction in a polymer electrolyte is controlled by the ion hopping rate $\mathrm{w}$, renewal time $\tau$ which is related to the segmental motion and $f$, the available sites. In the case where the polymer chain can undergo macroscopic diffusion, the "site percolation" arising from the macroscopic diffusion of the polymer chains must be considered, in addition to $w, \tau$ and $\mathrm{f}$.

The cationic conduction in solid polymer electrolytes basically arises from the segmental motion of polymer chains. This corresponds to the process. (a), rearrangement and reorientation of polymer segments. Even in polymer electrolytes based on low molecular weight polymer, this provides a significant contribution to ionic conduction. Because this process is independent of the molecular weight, the contribution of this process to the ionic conduction is the same in systems with different molecular weight. This is why the conductivities of some polymer electrolytes based on slightly crosslinked amorphous PEO segments are almost as high as those based on linear amorphous PEO chains [15].

For a polymer electrolyte based on low molecular weight polymer, ionic conduction arising from the "site percolation" may have to be considered. This involves the process (b), the curvilinear diffusion of polymer chains, and / or the process (c), the 
fluctuation of surroundings. Because the process (b) only involves the diffusion of some individual chains within the confines of their surroundings, e.g. in a tube in the reptation theory or viscous media in the Rouse model, without any interaction with these surroundings, its effect on the cations is limited, i.e. it only influences the cations with intrachain coordination.

For the process (c), because it is the slowest and the relaxation time is proportional to the cube of the molecular weight of polymer, it is negligible when the molecular weight is slightly above the critical value. However, when the molecular weight of polymer is very low, i.e., the relaxation time $\tau_{c}$ is relatively short, the contribution of this process to the overall ionic conduction may be significant. Because this process involves the change of surroundings of an individual chain, this implies that process (c) in fact allows a few entangled or transient crosslinked chains to move. Thus, this process can not only allow the cations with intrachain coordination to move along with the polymer, but also may allow the cations to exchange coordinate sites during moving with the coordinated or entangled polymer chains.

\section{8-4 Relationship Between the Molecular Weight of Polymer and the Cationic Conductivity in Polymer Electrolytes}

In the above sections, the relationship between the diffusion coefficient of polymer chains and the molecular weight of polymers and the relaxation modes of polymer chains in polymer electrolytes have been discussed. The different relaxation modes have been correlated to the motion of polymer chains. In the following section, the relationship between the molecular weight of polymers and cationic conductivity in polymer electrolytes will be described in the light of the above discussion. 


\section{8-4-1 Polymer Electrolytes with Cations Tightly Bound* by Polymer}

\section{Chains}

Consider a polymer electrolyte in which cations are all tightly coordinated with polar atoms in the polymer chains, such as PEO- $\mathrm{MgX}_{2}$ electrolyte, where $\mathrm{Mg}^{2+}$ cations interact strongly with oxygen atoms. For high molecular weight PEO this electrolyte behaves like an anionic conductor (as discussed below). For a tightly-bound system, any cationic conductances arise merely from motion of cationic species which are transported along with their coordinated polymer chains. Thus, the diffusion coefficient of such cationic species $D_{+}$is the same as that of polymer chains $D_{P}$, i.e.

$$
D_{+}=D_{P}
$$

Because, as discussed previously, mobility and the diffusion coefficient of polymer chain can be correlated by the Einstein equation, the cationic conductivity can be correlated to the diffusion coefficient of cationic species by the Nernst - Einstein equation:

$$
\sigma_{+}=\frac{n q^{2}}{k T} D_{+}=\frac{n q^{2}}{k T} D_{P}
$$

where $\mathrm{k}$ is the Boltzmann constant, $\mathrm{T}$ is the temperature, $\mathrm{n}$ the number of cationic species and $q$ is the charge on the cationic species.

When the molecular weight of polymer is lower than $M_{c}$, the critical value for entanglement, the diffusion coefficient of the polymer chain is inversely proportional to the molecular weight of polymer as described in Section 8-3:

\footnotetext{
* The term "polymer electrolytes with cations tightly bound by polymer chains" means that the bonds between polar atoms on the polymer chains and coordinated cations are relatively non-labile. In contrast, "polymer electrolytes with cations loosely bound by polymer chains" means that the bonds between polar atoms on the polymer chains and coordinated cations are labile. No inference is made concerning the value of the electrostatic or other energy of the ion-polymer interaction.
} 


$$
D_{P}=\frac{k T M_{s}}{\xi M}
$$

where $\xi$ is a friction coefficient, and $M_{s}$ is the molecular weight of polymer segments (for PEO, $\mathrm{M}_{\mathrm{s}}=44.05$ ) and $\mathrm{M}$ is the molecular weight of the polymer.

By substituting (8-4-3) into (8-4-2), the cationic conductivity is then correlated to the molecular weight of polymer, i.e.,

$$
\sigma_{+}=\frac{\mathrm{nq}^{2} \mathrm{M}_{\mathrm{s}}}{\xi \mathrm{M}}=\frac{\mathrm{A}}{\mathrm{M}}
$$

where $A=n q^{2} M_{s} / \xi$. For a specific polymer electrolytes with a particular salt concentration, $\mathrm{A}$ is a constant. Therefore, the cationic conductivity of polymer electrolytes with tightly bound cations is inversely proportional to the molecular weight of polymer in the molecular weight range corresponding to the regime described by the Rouse model.

Assuming strong electrolyte behaviour and no significant convection, the steady state cationic current for this type polymer electrolyte following dc polarisation of the symmetric cell: $M / \mathrm{PEO}-\mathrm{MX} / \mathrm{M}$ (where $\mathrm{M}$ represents a non-blocking electrode towards $\left.\mathrm{M}^{+} / \mathrm{M}\right)$, can be expressed as:

$$
\mathrm{I}_{+}=\Delta \phi \sigma_{+} \mathrm{K}=\frac{\Delta \phi \mathrm{AK}}{\mathrm{M}}
$$

where $\Delta \phi$ is the polarisation potential acting over the electrolyte at steady state and $\mathrm{K}$ is the cell constant, equal to the area of electrodes divided by the distance between them. 
$\Delta \phi$ can be expressed as: $\Delta \phi=\Delta V-\Delta E$, where $\Delta V$ is the applied potential difference corrected for any interfacial drop, and $\Delta \mathrm{E}$ is the potential difference between two electrodes at steady state caused by the salt concentration difference at the two electrodes (Nernst potential ). If all conditions for dc polarisation are the same, and $\Delta \mathrm{V}$ is small, the potential difference across the electrolyte at steady state is equal to half of the applied potential $\Delta V$. With the same applied potential difference $\Delta V$, the potential difference across the electrolyte $\Delta \phi$ will thus not differ with molecular weight. Therefore, the steady state cationic current will also be inversely proportional to the molecular weight of the polymer for molecular weight below the entanglement limit. When the the molecular weight is high, there should be no significant steady state cationic current.

If neutral species are dominant in this kind of electrolyte, there will be no significant ionic conduction in the high molecular weight case. In fact, there is no significance to consider this case.

\section{8-4-2 Polymer Electrolytes with Loosely Coordinated Cations}

(1) Polymer electrolytes with fully dissociated ions

Consider a polymer electrolyte in which cations are loosely coordinated to the polar atoms on polymer chains, i.e. cations can readily move from one coordinate site to another, and assume strong electrolyte behaviour.

When the molecular weight of the polymer is high enough, the motion of the cations is completely controlled by segmental motion, due to the difficulty of the macroscopic motion of polymer chains. The cationic conductivity can then be correlated with the cation diffusion coefficient by the Nernst-Einstein equation:

$$
\sigma_{+}^{\mathrm{H}}=\frac{\mathrm{nq}^{2}}{\mathrm{kT}} D_{+}^{\mathrm{H}}
$$


where $n, q, k$ and $T$ have the same meanings as before, and $\sigma_{+}^{H}$ is the cationic conductivity of polymer electrolyte based on high molecular weight polymer, and $D_{+}^{\mathrm{H}}$ is the diffusion coefficient of cations in such a host.

When the molecular weight of polymer is lower than the critical value for entanglement, the macroscopic motion of the polymer chains will also contribute to the transport of cations. Therefore, in the polymer electrolytes based on low molecular weight polymers there may exist two relatively independent processes simultaneously contributing to the motion of cations, namely segmental motion and motion of whole polymer chain.

$$
D_{+}^{L}=D_{+}^{S}+D_{+}^{p}
$$

where $D_{+}^{L}$ is the overall diffusion coefficient of cations, and $D_{+}^{S}$ and $D_{+}^{P}$ are diffusion coefficients of cations resulting from the segmental and whole chain motion respectively. The cationic conductivity, can thus be expressed as:

$$
\sigma_{+}^{L}=\frac{n^{2}}{k T}\left(D_{+}^{s}+D_{+}^{P}\right)
$$

Because the segmental motion is closely related to characteristics of bonds or segments of polymer chains, and depends only to a very limited extent on the molecular weight of polymer [13], the diffusion coefficient of cations arising from the contribution of segmental motion in the polymer electrolyte with low molecular weight polymer can be taken to be approximately equal to the diffusion coefficient of cations in the polymer electrolyte with high molecular weight polymer, i.e., $D_{+}^{S} \approx D_{+}^{H}$. Furthermore, since the carrier numbers can be assumed to be independent of the molecular weight of the polymer, the cationic conductivity of this kind of polymer electrolyte can be written as: 


$$
\sigma_{+}^{L}=\sigma_{+}^{H}+\frac{\mathrm{nq}^{2}}{\mathrm{kT}} D_{+}^{\mathrm{P}}
$$

Because $D_{+}^{P}$ results from the motion of cations moving along with a part of the coordinated polymer chains, $D_{+}^{P}$ can be taken to be the same to the diffusion coefficient of polymer chains, $D_{P}$. Thus, equation (8-4-9) is converted to:

$$
\sigma_{+}^{L}=\sigma_{+}^{H}+\frac{\mathrm{nq}^{2}}{\mathrm{kT}} D_{\mathrm{P}}
$$

For a polymer electrolyte based on a polymer with molecular weight $\mathrm{M}$, the difference between $\sigma_{+}^{\mathrm{I}}$ and $\sigma_{+}^{\mathrm{H}}$ is expressed, by substituting (8-4-3) into (8-4-10), as:

$$
\Delta \sigma_{+}=\sigma_{+}^{L}-\sigma_{+}^{H}=\frac{n^{2}}{k T} D_{p}=\frac{A}{M}
$$

where $M$ is the molecular weight of polymer, and $A=n q^{2} M_{s} / \xi$, in which $M_{s}$ is the molecular weight of segments and $\xi$ is a friction coefficient as before. Therefore, for polymer electrolytes with loosely coordinated cations, the difference between the cationic conductivities of polymer electrolytes with low and high molecular weight polymers is inversely proportional to the molecular weight of the former polymer, providing that its molecular weight falls within the range of the Rouse model.

The cationic current of this type of polymer electrolyte is related to the steady state current resulting from dc polarisation of the symmetric cell: $M / P E O-M X / M$, where $M$ represents a non-blocking electrode towards $M^{+} / M$ again, and assuming that there is no convection of electrolyte in the cell. The cationic current can be expressed as: 


$$
I_{+}^{L}=\Delta \phi \sigma_{+}^{L} K=I_{+}^{H}+\frac{\Delta \phi A K}{M}
$$

and

$$
\Delta \mathrm{I}=\mathrm{I}_{+}^{\mathrm{L}}-\mathrm{I}_{+}^{\mathrm{H}}=\frac{\Delta \phi \mathrm{AK}}{\mathrm{M}}
$$

where $\Delta \phi$ is the polarisation potential across the electrolyte at steady state, as discussed above, is invariant with the molecular weight, providing that $\Delta \mathrm{V}$ is constant and small. $\mathrm{I}_{+}^{\mathrm{H}}$ is cationic current for high molecular weight polymer electrolyte, and $\mathrm{M}$ is the molecular weight of a polymer within the range of the Rouse model. Hence, the difference between cationic steady state currents of polymer electrolytes for low and high molecular weight polymers is inversely proportional to the molecular weight of the low molecular weight polymer .

\section{(2) Polymer electrolytes with mobile neutral species}

In real polymer electrolytes, as discussed in Chapter 5, ion association occurs between anions and cations due to the low dielectric constant of the polymer which acts as solvent. Suppose that mobile neutral species, eg. mobile ion pairs in PEO-MX electrolytes, are dominant in the polymer electrolyte, and that the steady state current of the cell: $\mathrm{M} / \mathrm{PEO}-\mathrm{MX} / \mathrm{M}$ following dc polarisation is mainly controlled by the diffusion of these ion pairs (theory is discussed in Chapter 6, 6-2-1). The effective conductivity of such a polymer electrolyte based on high molecular weight polymer can be expressed approximately as follows :

$$
\sigma_{\text {eff }}^{\mathrm{H}}=\frac{n q^{2}}{k T}\left(D_{+}^{H}+D_{-}^{H}\right)
$$

where $D_{+}^{H}$ and $D_{-}^{H}$ are diffusion coefficients for cations and anions respectively. For polymer electrolytes based on low molecular weight polymer, because the diffusion of cations occurs as a result both of the motions of segments and of whole chains (i.e., 
$D_{+}^{L}=D_{+}^{S}+D_{+}^{P}$ ), the effective conductivity in such circumstances can be written as:

$$
\sigma_{\text {eff }}^{L}=\frac{n q^{2}}{k T}\left(D_{+}^{S}+D_{+}^{P}+D_{-}^{L}\right)
$$

where $D_{+}^{S}$ and $D_{+}^{P}$ are diffusion coefficients of cations resulting from segmental motion and the motion of whole polymer chain, and $D_{-}^{L}$ is the diffusion coefficient of anions.

As discussed above, $D_{+}^{S} \approx D_{+}^{H}$ (if the transport of the cations is controlled by an activation process in addition to the segmental motion, this process may be taken as a microscopic process as segmental motion, which can be also assumed to be independent of the molecular weight.), and $D_{+}^{P}=D_{p}$. Therefore, the difference between the effective conductivities of polymer electrolytes based on low and high molecular weight polymers where mobile neutral species are dominant is:

$$
\Delta \sigma_{\text {eff }}=\sigma_{\text {eff }}^{L}-\sigma_{\text {eff }}^{H}=\frac{n q^{2}}{k T}\left[D_{P}+\left(D_{-}^{L}-D_{-}^{H}\right)\right]
$$

The diffusion coefficient of ions in the conventional liquid electrolyte solution can be correlated to the viscosity of solution by Stokes-Einstein equation (8-1-3). In polymer electrolytes [1], the diffusion coefficient of anions can also be assumed to follow this equation, i.e.

$$
D=\frac{k T}{6 \pi \eta_{(r)} R}
$$

where $R$ is the radius of anions, and $\eta_{(r)}$ is the so-called "local viscosity" or "microscopic viscosity" of the polymer chains. According to the free volume model, the 
local viscosity or microscopic viscosity is related to the free volume fraction in the polymer. Thus the viscosity can be expressed as:

$$
\eta_{(\mathrm{r})} \propto \exp \left(-\gamma \frac{v^{*}}{v_{\mathrm{f}}}\right)
$$

where $y$ is a Lagrange parameter, and $v^{*}$ is the critical free volume, and $v_{f}$ is the average free volume. When the number of atoms in the backbone of a linear amorphous polymer is over $20^{*}$, the local viscosity or microscopic viscosity, $\eta_{(r)}$, has been found [16] to be independent of the length of the polymer chain at constant reduced temperature, $T=T_{g}+k$ ( $\mathrm{k}$ is a constant). From equation (8-4-17), it is reasonable to infer that the diffusion coefficient of anions in a polymer electrolyte is independent of the molecular weight of polymer. Thus, the difference of anion diffusion in low and high molecular weight polymers is assumed to be very small.

Neglecting ( $\left.D_{-}^{L}-D_{-}^{h}\right)$ in equation (8-4-16), therefore, the difference of effective conductivities between polymer electrolytes with low and high molecular polymer can be expressed as:

$$
\Delta \sigma_{\text {eff }}=\sigma_{\text {eff }}^{L}-\sigma_{\text {eff }}^{h} \cong \frac{n q^{2}}{k T} D_{P}
$$

Using the Rouse model (8-4-3), equation (8-4-19) is converted to:

$$
\Delta \sigma_{\text {eff }}=\frac{A}{M}
$$

\footnotetext{
* In a PEO chain, each EO unit has three backbone atoms, i.e., two carbon and one oxygen. Thus, 20 atoms correspond to about $7 \mathrm{EO}$ units, i.e. the molecular weight of $\mathrm{PEO}$ of about 300 , which is lower than what we are discussing here ( not less than 400 ).
} 
Thus, in the system where mobile neutral species are dominant, the difference of effective conductivities between polymer electrolytes with low and high molecular weight polymers is inversely proportional to the molecular weight of polymer in the molecular weight range corresponding to the Rouse model.

The steady state current following dc polarisation of the symmetric cell: M / PEO$\mathrm{MX} / \mathrm{M}$, where $\mathrm{M}$ represents a non-blocking electrode towards $\mathrm{M}^{+} / \mathrm{M}$, and assuming that there is no convection in the cell, can be expressed as:

$$
\begin{aligned}
& \mathrm{I}_{\mathrm{s}}^{\mathrm{L}}=\Delta \phi \sigma_{\text {eff }}^{\mathrm{L}} \mathrm{K} \\
& \mathrm{I}_{\mathrm{s}}^{\mathrm{H}}=\Delta \phi \sigma_{\text {eff }}^{\mathrm{H}} \mathrm{K}
\end{aligned}
$$

The difference between the steady state currents of the polymer electrolytes based on the low and high molecular weight polymer can be expressed as:

$$
\Delta \mathrm{I}_{\mathrm{s}}=\mathrm{I}_{\mathrm{s}}^{\mathrm{L}}-\mathrm{I}_{\mathrm{s}}^{\mathrm{H}}=\Delta \sigma_{\text {eff }} \Delta \phi \mathrm{K}=\frac{\Delta \phi \mathrm{AK}}{\mathrm{M}}
$$

Thus, for the polymer electrolytes where mobile neutral species are dominant, the difference between the steady state currents of polymer electrolytes based on low and high molecular weight polymers is inversely proportional to the molecular weight of the low molecular weight polymer, providing that $\Delta \phi$ does not significantly vary with changing the molecular weight. 


\section{8-5 Experimental Results and Discussion}

In this section, the experimental results concerning the influence of the molecular weight of polymer on the ionic conduction is discussed in the light of above discussion. Two polymer electrolytes based on PEO with different molecular weight and $\mathrm{Mg}\left(\mathrm{CF}_{3} \mathrm{SO}_{3}\right)_{2}$ and $\mathrm{LiCF}_{3} \mathrm{SO}_{3}$ salts have been studied by dc polarisation technique. The former behaves as an anionic conductor when the molecular weight of PEO is high as discussed in Chapter 7, and the latter, both cations and anions are mobile, is a well studied polymer electrolyte. In addition, the diffusion coefficient of ${ }^{7} \mathrm{Li}$ nuclei in polymer electrolytes based on the different molecular weight $\mathrm{PEO}$ and $\mathrm{LiCF}_{3} \mathrm{SO}_{3}$ salt has been investigated by pulsed field gradient NMR.

\section{8-5-1 Influence of the Molecular Weight of PEO on Ionic Conduction in PEO-Mg( $\left.\mathrm{CF}_{3} \mathrm{SO}_{3}\right)_{2}$ Electrolytes}

In order to study the influence of the diffusion of polymer chains on the motion of cations, polymer electrolytes based on PEO with different molecular weights ${ }^{*}$ and $\mathrm{Mg}\left(\mathrm{CF}_{3} \mathrm{SO}_{3}\right)_{2}(\mathrm{EO} / \mathrm{Mg}=20)$ have been investigated. Due to very strong Lewis acidity and very high charge density of $\mathrm{Mg}^{2+}$, the interaction between $\mathrm{Mg}^{2+}$ and oxygen atoms on the polymer chain is so strong that $\mathrm{Mg}^{2+}$ cannot be released from their coordinated oxygen atoms (as shown in Chapter 7 where magnesium species in $\mathrm{PEO}-\mathrm{Mg}\left(\mathrm{CF}_{3} \mathrm{SO}_{3}\right)_{2}$ electrolyte based on the high molecular weight PEO were shown to be immobile). Therefore, the cationic conduction arises only from the cations moving with their coordinated polymer chains.

The limiting current following dc polarisation of the cell: $\mathrm{Mg} / \mathrm{PEO}-$ $\mathrm{Mg}\left(\mathrm{CF}_{3} \mathrm{SO}_{3}\right)_{2} / \mathrm{Hg}(\mathrm{Mg})$, where $\mathrm{Mg}$ is the anode and $\mathrm{Hg}(\mathrm{Mg})$, a mercury film electrode

\footnotetext{
* where the ends of PEO with molecular weight of $400,1000,1450,2000$ and 3350 are methoxycapped.
} 
with gold substrate, is used as the cathode at $80^{\circ} \mathrm{C}$, is due to the motion of magnesium cationic species in the polymer electrolyte. Figure 8-5 shows the change of the limiting current for electrolytes with molecular weight of the PEO. The limiting current initially decreases steeply with increase in the molecular weight of PEO until the molecular weight $\mathrm{M}$ reaches 3200 , from $\sim 1.5 \mu \mathrm{A}$ for that based on $\mathrm{PEO}(400)$ dimethyl ether to $\sim 350 \mathrm{nA}$ for that based on methylated PEO with the molecular weight of 3350 . For the molecular weights of PEO just above 3350, the limiting current decreases further to an constant low value, $\sim 100 \mathrm{nA}$, and then remains at this low value right up to the molecular weights $4,000,000$.

For $\mathrm{PEO}-\mathrm{Mg}\left(\mathrm{CF}_{3} \mathrm{SO}_{3}\right)_{2}$ electrolytes, as with conventional polymers, when the molecular weight of the PEO is too low to establish entanglements, the diffusion of chain is relatively fast and the diffusion coefficient of polymer is inversely proportional to its molecular weight. Consequently, the PEO chain-bound cationic species also move relatively fast, giving rise to a relatively high cationic currents. The behaviour of the polymer electrolytes with the molecular weight of PEO less than 3200 corresponds to the unentangled polymer case. Theoretically, as discussed in the above section, the cationic current of this system should be inversely proportional to the molecular weight of PEO. However, because the properties of the cathode, the mercury film electrode, are different from the anode, magnesium metal electrode, in the two electrode cell for $\mathrm{dc}$ polarisation, Nernst potential difference exists between these two electrodes which is variable with the deposition of metal. Accordingly, as discussed in Chapter 7 , there is no significance to be deduced the cationic currents from the limiting currents.

When the molecular weight of $\mathrm{PEO}$ is high enough to establish significant entanglement, the motion of polymer chains is constrained, and the diffusion coefficient of the polymer chain becomes very small, and is inversely proportional to the square of the molecular weight. Thus, the motion of cationic species is also constrained, and the 
diffusion coefficient of PEO chain-bound cationic species is very small leading to a very small cationic current. The behaviour of the polymer electrolytes with molecular weight of PEO above 3200 corresponds to the entangled polymer case. For polymer electrolytes with molecular weight of PEO above 3350, the change of the limiting current may arise from the reptation of polymer chains in a tube consisting of those entanglements. When the molecular weight of PEO is very high, the diffusion coefficient of the polymer chains is too small to allow the cationic species to move significantly. As a result, one can hardly observe any cationic current, i.e. the electrolyte behaves like an anionic conductor. Although the limiting current is corrected (by subtracting the background current) with the salt free polymer, there is still some residual limiting current left for the electrolytes with the higher molecular weight PEO. This residual limiting current may be attributed to poly-distribution of the molecular weight of PEO or low molecular weight impurities. Because, as discussed above, if in the system, there are some polymer chains whose molecular weight is not so high as that of major polymer chains, an increase in the diffusion coefficient of the polymer will occur.

As a comparison, the viscosity $\eta$ data of PEO (quoted from the product data of Union Carbide Ltd.[17]) is plotted against the molecular weight of PEO, M (Figure 86). The viscosity increases steeply when the molecular weight is above 3200 . The plot of $\log \eta$ vs $\log M$ (Figure 8-7) shows that the slope of the straight line changes at $M \approx$ 3200. The the slope of the line for $M<3200$, is 1.07 , i.e. $\eta \propto M$, and the slope of the line for $M>3200$ is 3.05, i.e. $\eta \propto M^{3}$. This indicates that, when the molecular weight is below 3200 , the dynamics of the diffusion of the polymer chain can be described by the Rouse model, and when the molecular weight is above 3200 , the dynamics of the diffusion of the polymer chain can be described by de Gennes' reptation theory. 
It is interesting that critical molecular weights for entanglement of the salt free linear $\mathrm{PEO}$ and of polymer electrolytes based on linear $\mathrm{PEO}$ containing $\mathrm{Mg}\left(\mathrm{CF}_{3} \mathrm{SO}_{3}\right)_{2}$ salt are very similar. As discussed in 8-3-1, if in the case of the electrolyte the interchain coordinations are dominant, then the topology of the polymer chains in the electrolyte is different from the linear chains in salt free polymer, and the critical molecular weight of polymer for establishing entanglement should be different from the linear chains. However, if intrachain coordination is a significant form in the polymer electrolyte, the dominant coordinated-chains are still linear chains. The topology of these coordinated chains may thus not differ much from that of salt free polymer chains, leading to that the critical molecular weight for entanglement may be very close to that of the salt free PEO. This may therefore indicate that the linear coordinated-chain is an important shape of chains in the polymer electrolyte, i.e. the intrachain coordination between polymer and magnesium ions is significant.

\section{8-5-2 Influence of the Molecular Weight of PEO on Ionic Conduction in

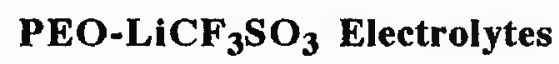

Polymer electrolytes based on PEO and $\mathrm{LiCF}_{3} \mathrm{SO}_{3}$ have been well studied in terms of conductivity, phases of complexes, ionic transport and ion association etc. [18]. For polymer electrolytes based on PEO and lithium salts, such as $\mathrm{LiCF}_{3} \mathrm{SO}_{3}, \mathrm{LiClO}_{4}$, it is generally believed [19] that lithium cations can easily be coordinated by oxygen atoms on PEO chains and may also be released quite easily. We have investigated the influence of the molecular weight of PEO on ionic conduction and ion diffusion in PEO$\mathrm{LiCF}_{3} \mathrm{SO}_{3}$ electrolytes by dc polarisation and pfg NMR.

\section{(1) De polarisation}

Polymer electrolytes based on $\mathrm{LiCF}_{3} \mathrm{SO}_{3}$ salt and PEO of different molecular weight $(\mathrm{EO} / \mathrm{Li}=20)$ have been examined by dc polarisation of the cell: $\mathrm{Li} / \mathrm{PEO}-$ $\mathrm{LiCF}_{3} \mathrm{SO}_{3} / \mathrm{Li}$ with a de potential of $10 \mathrm{mV}$ at $85^{\circ} \mathrm{C}$. A typical plot of the current 
against polarisation time is showed in Figure 8-8. It shows that the steady state can be achieved and kept for long time. This implies that the convection in the electrolyte is negligible. For the polymer electrolyte based on $\mathrm{PEO}(400)$ dimethyl ether, however, although steady state can be eventually achieved after polarisation for about 15 hours, the polarisation current increases after quickly decreasing during the initial 0.5 hour (Figure 8-9). This may be due to the relatively small viscosity of the electrolyte which may lead to the significant change of the electrode surface during the polarisation and even may give arise to some convection of electrolyte. Interpretation of the steady state current by de polarisation of $\mathrm{PEO}(400)$ dimethyl ether $-\mathrm{LiCF}_{3} \mathrm{SO}_{3}$ electrolyte is thus used conservatively.

The experimental results for dc polarisation of the two electrode lithium cells with electrolytes based on $\mathrm{LiCF}_{3} \mathrm{SO}_{3}$ and different molecular weight $\mathrm{PEO}$ are listed in Table 8-1. Because the varying interfacial voltage drop in different cells led to nonstandardized steady state currents $\mathrm{I}_{\mathrm{s}}$, a corrected steady state current, $\mathrm{I}_{\mathrm{s}}{ }^{*}$, was therefore used. $I_{s} *$ is the steady state current after interfacial correction and calculated on the basis of a fixed value of potential $\Delta V^{*}$ which is taken as $10 \mathrm{mV}$, i.e. $I_{s}{ }^{*}=I_{s}\left(\Delta V^{*} / \Delta V\right)$, where $\Delta \mathrm{V}$ is the corrected applied potential. Strictly, the potential difference across the electrolyte $\Delta \phi$ should be used to normalise $\mathrm{I}_{\mathrm{s}}{ }^{*}$. The relationship between $\Delta \phi$ and $\Delta \mathrm{V}$ is: $\Delta \phi=\Delta V-\Delta E$. In the present calculation, since $\Delta \phi$ could not be easily determined, especially in the electrolytes based on low molecular weight polymers, e.g. $\mathrm{PEO}(400)$ dimethyl ether, due to relatively low viscosities, $\Delta \phi$ was replaced by the applied potential $\Delta V$. In a fully dissociated electrolyte, because $\Delta \phi=\Delta E=1 / 2 \Delta V, \Delta \phi$ is invariant with change in the molecular weight of the polymer, i.e. the steady state concentration gradient is independent of the molecular weight. Although in some cases, $\Delta \phi$ and $\Delta E$ may change with changing electrolyte composition, it has been found [20] that in $\mathrm{PEO}-\mathrm{LiCF}_{3} \mathrm{SO}_{3}$ electrolyte they did not change significantly. In the present case, because all the electrolytes and experimental conditions were the same except for the 
molecular weight of PEO, the concentration gradient at steady state can be assumed not to vary significantly in the dc polarisation of these electrolytes with a fixed polarisation potential. Further, because the applied potential was small $(10 \mathrm{mV})$, the steady state concentration gradient should be small, i.e. $\Delta \mathrm{E}$ was small. The potential difference across the electrolyte may thus be related approximately to the applied potential by $\beta \Delta \phi=\Delta V$, where $\beta$ is a coefficient, taken as invariant with change of the molecular weight of the polymer, e.g. for fully dissociated system, $\beta=2$. Therefore, $\mathrm{I}_{\mathrm{s}}^{*}=\mathrm{I}_{\mathrm{S}}\left(\Delta \mathrm{V}^{*} / \Delta \mathrm{V}\right) \approx \mathrm{I}_{\mathrm{s}}\left(\Delta \phi^{*} / \Delta \phi\right)$, where $\Delta \phi^{*}$ is a standardized potential across the electrolyte, and the effective conductivity can be taken approximately as: $\sigma_{\text {eff }} \approx \beta \mathrm{K} \mathrm{I}_{\mathrm{s}}^{*} / \Delta \mathrm{V}^{*}$, where $\mathrm{K}$ is the cell constant. Figure 8-10 shows the relationship between the corrected steady state current $\mathrm{I}_{\mathrm{s}}{ }^{*}$ and the molecular weight of PEO.

When the molecular weight of PEO increases from 400 to 3350 , the corrected steady state current decreases steeply, from $\sim 28 \mu \mathrm{A}$ to $\sim 7 \mu \mathrm{A}$. When the molecular weight of PEO is over 3350, the corrected steady state current has a constant value of approximately $5 \mu \mathrm{A}$.

For $\mathrm{PEO}-\mathrm{LiCF}_{3} \mathrm{SO}_{3}$ electrolytes, there are basically three relaxation processes of polymer to be considered: segmental motion, diffusion of individual chains and fluctuation of surroundings. Because the segmental motion is almost independent of the molecular weight of polymer, the process for segmental motion is always significant in polymer electrolytes, and makes the motion of cations possible in systems with high molecular weight. If the relaxation time for segmental motion is fast enough in comparison with those for the chain diffusion and the fluctuation of surroundings, these latter two processes involving the macroscopic motion of polymer chains are not significant. Thus, the latter two processes are negligible when the molecular weight of polymer is high, because the relaxation time of the process for the chain diffusion is proportional to the molecular weight of polymer, and the relaxation time of the 
fluctuation of surroundings is proportional to the cube of molecular weight of polymer. Therefore, the almost constant steady state current of $\mathrm{PEO}-\mathrm{LiCF}_{3} \mathrm{SO}_{3}$ electrolytes based on the high molecular weight PEO is likely to be dominated by the process of segmental motion.

If the molecular weight of the polymer is low enough to make these relaxation times to be short, the processes for diffusion of individual chains and fluctuation of surroundings, may however also contribute to the motion of cations. When the molecular weight of PEO is lower than 3200 , the steady state currents of PEO$\mathrm{LiCF}_{3} \mathrm{SO}_{3}$ electrolytes decrease with the increase of the molecular weight of PEO, as in the case of the PEO-Mg(CF$\left.{ }_{3} \mathrm{SO}_{3}\right)_{2}$ electrolytes. This may also be attributed to the contribution of diffusion of polymer chains.

Unlike the situation discussed above for PEO- $\mathrm{Mg}\left(\mathrm{CF}_{3} \mathrm{SO}_{3}\right)_{2}$ electrolytes, there is no observable change of the steady state current of $\mathrm{PEO}-\mathrm{LiCF}_{3} \mathrm{SO}_{3}$ electrolytes when the molecular weight of PEO is just above 3200. This suggests that the relaxation time for chain diffusion is very long in comparison with that for segmental motion. Thus, when the molecular weight of PEO is higher than 3200 , the motion of cations and hence the ionic conduction of $\mathrm{PEO}-\mathrm{LiCF}_{3} \mathrm{SO}_{3}$ electrolytes are completely controlled by the segmental motion.

It has been reported [18] that ion association takes place in $\mathrm{PEO}-\mathrm{LiCF}_{3} \mathrm{SO}_{3}$ electrolytes, and that ion pairs are dominant. Thus, the steady state current from the dc polarisation may be largely controlled by the motion of ion pairs as discussed in Chapter 6.

Figure 8-11 shows logarithmic plots of the difference of effective conductivity $\Delta \sigma_{\text {eff }}$ between that with the molecular weight of PEO below 3200 and that with high 
molecular weight PEO against the molecular weight of PEO, M. It is of interest to find the slope of the straight line is very close to -1 . As discussed in section $8-4$, according to the Rouse model, the difference of cationic conductivity or cationic current is inversely proportional to the molecular weight of polymer for the completely dissociated polymer electrolytes. In the polymer electrolytes in which mobile neutral species are dominant, if the difference of diffusion coefficient of anions between those based low and high molecular weight PEO is too small to be considered, the difference of effective conductivity $\Delta \sigma_{\text {eff }}$ is also inversely proportional to the molecular weight of polymer.

The critical molecular weight of PEO for entanglement is very close to those of the salt free linear PEO and PEO-Mg( $\left(\mathrm{CF}_{3} \mathrm{SO}_{3}\right)_{2}$ electrolyte. This implies (a) significant existence of intrachain coordination and/or (b) the rapid exchange of coordination between lithium ions and oxygen atoms in comparison with the macroscopic motion of polymer chains for the polymer electrolyte with the molecular weight of polymer near 3200 , which enables the coordinated-chain to move like a linear chain.

It is also of interest that the current fractions $\mathrm{F}_{+}$of $\mathrm{PEO}-\mathrm{LiCF}_{3} \mathrm{SO}_{3}$ electrolytes remain constant with change of the molecular weight of PEO within the experimental error (Table 8-1). This is easy to understand if the mobile ion pairs dominate the system, because, as expressed by equations (8-4-14) and (8-4-15), the current fraction, ratio of the effective and total conductivities, becomes independent of the number and character of cations and anions. For a completely dissociated system, the current fraction will be complicated. For the system with low and high molecular weight polymers, the current fraction $\mathrm{F}_{+}^{\mathrm{L}}$ and $\mathrm{F}_{+}^{\mathrm{H}}$ can be expressed as:

$$
F_{+}^{L}=\frac{D_{+}^{S}+D_{p}}{D_{-}^{L}+D_{+}^{S}+D_{P}}
$$




$$
\text { and } F_{+}^{H}=\frac{D_{+}^{H}}{D_{-}^{H}+D_{+}^{H}}
$$

Because, as discussed in the above section, $D_{+}^{S} \cong D_{+}^{H}$ and $D_{-}^{L} \cong D_{-}^{H}, F_{+}^{L}$ is larger than $\mathrm{F}_{+}^{\mathrm{H}}$. Therefore, it is unlikely that free ions are dominant in these electrolytes.

(2) Pulsed field gradient NMR studv of $\mathrm{PEO}-\mathrm{LiCF}_{3} \mathrm{SO}_{3}$ electrolytes

${ }^{7} \mathrm{Li}$ pfg NMR can measure the diffusion coefficient of lithium species. Whatever these lithium species are, as discussed in Chapter 4, an averaged diffusion coefficient is given. Under these circumstances, when the diffusion of cations is replaced by the diffusion of lithium species, all equations and conclusions discussed in Section 8-4 can be applied, if these species are coordinated by the polymer chains.

${ }^{7} \mathrm{Li} \mathrm{pfg-NMR}$ was carried out for $\mathrm{PEO}-\mathrm{LiCF}_{3} \mathrm{SO}_{3}$ electrolytes based on different molecular weight $\mathrm{PEO}$ at $90^{\circ} \mathrm{C}$ and $70^{\circ} \mathrm{C}$ respectively, except that based on $\mathrm{PEO}$ with molecular weight of $4 \times 10^{6}$. Because the electrolytes based on PEO with a molecular weight of $4 \times 10^{6}$ have crystalline phases at a temperature below $150^{\circ} \mathrm{C}$, the direct measurement of the diffusion coefficient at $70^{\circ} \mathrm{C}$ and $90^{\circ} \mathrm{C}$ may be influenced by the crystalline phases. Thus, for this electrolyte, the diffusion coefficient of lithium was measured at temperatures higher than $150^{\circ} \mathrm{C}$. The values at lower temperature were extrapolated from the $\log \mathrm{D}$ versus 1000 / T plot. Figure 8-12 shows the plot of $\log \mathrm{D}$ versus $1000 / \mathrm{T}$, derived at higher temperature.

The change of the diffusion coefficient of lithium species with changing molecular weight of PEO at two different temperatures is shown in Figure 8-13. As the molecular weight increased up to about 3200 , the diffusion coefficient of lithium species decreases rapidly. After that value, the diffusion coefficient changes little. This observation is similar to that in the dc polarisation study, and the explanation is similar, 
i.e. when the molecular weight is lower than the critical value for establishing entanglement, the macroscopic diffusion of the polymer chain will contribute to the motion of the lithium species, but when the molecular weight is high, the motion of the lithium species is controlled by segmental motion, due to extremely small diffusion coefficient of the polymer chain.

The slope of the plot of the logarithm of the difference of the diffusion coefficient $\Delta \mathrm{D}\left(\Delta \mathrm{D}=\mathrm{D}_{\mathrm{L}}-\mathrm{D}_{\mathrm{H}}\right)$ of the lithium species with the low and high molecular weight polymer against $\log M$ is very close to -1 (Figure 8-11). This is also similar to the observation in the de polarisation study. This again indicates that the Rouse model is valid in the region of the molecular weight below 3200 .

\section{8-6 Conclusion}

The dynamics of polymer chains in polymer electrolytes can be described either by the Rouse model for low molecular weight polymer or by the reptation theory for high molecular weight and entangled polymer as in the case of conventional polymer liquids or melts. There are three principal types of relaxation processes, viz. segmental motion, diffusion of individual chains and fluctuation of the surroundings which are relevant to the ionic conduction of polymer electrolytes.

When the molecular weight of polymer is low enough, the diffusion of polymer chains and/or the fluctuation of surroundings contributes to the ionic conduction. The dc polarisation and ${ }^{7} \mathrm{Li}$ pfg NMR experiments have shown that, in PEO-Mg(CF $\left.\mathrm{SO}_{3}\right)_{2}$ and PEO-LiCF $\mathrm{SO}_{3}$ systems, when the molecular weight of PEO is lower than 3200 , the diffusion of polymer chain will contribute to the motion of lithium ions, and the diffusion coefficient of the chain can be described by the Rouse model; when the 
molecular weight of polymer is high enough, the contribution of whole chain diffusion to the ionic conduction is negligible, because the diffusion coefficient of the chain becomes too small. The ionic conduction in a polymer electrolyte based on high molecular weight polymer is basically controlled by the microscopic motion of polymer, i.e. segmental motion. For the polymer electrolytes studied, when the molecular weight of PEO is higher than 3200 , the ionic conduction is totally controlled by the microscopic motion of the polymer.

The critical molecular weights of PEO for establishing entanglement in all systems examined, i.e. salt free PEO, PEO- $\mathrm{Mg}\left(\mathrm{CF}_{3} \mathrm{SO}_{3}\right)_{2}$ and $\mathrm{PEO}-\mathrm{LiCF}_{3} \mathrm{SO}_{3}$ electrolytes are around 3200.

The coordinated chains in $\mathrm{PEO}-\mathrm{Mg}\left(\mathrm{CF}_{3} \mathrm{SO}_{3}\right)_{2}$ and $\mathrm{PEO}-\mathrm{LiCF}_{3} \mathrm{SO}_{3}$ electrolytes behave like linear chains. For PEO- $\mathrm{Mg}\left(\mathrm{CF}_{3} \mathrm{SO}_{3}\right)_{2}$ electrolytes, this indicates that intrachain coordination between cations and oxygen atoms on the polymer chains is significant. For $\mathrm{PEO}-\mathrm{LiCF}_{3} \mathrm{SO}_{3}$ electrolytes, this indicates that either the intrachain coordination is dominant or there are rapid exchanges between interchain coordination sites. 


\section{References}

[1] M. A. Ratner, in Polymer Electrolyte Reviews 1, ed. J. R. MacCallum and C. A. Vincent, Elsevier, London, 1987, page 173.

[2] J. D. Ferry, Viscoelastic Properties of Polymers, 2nd ed. Wiley, New York, 1970.

[3] H. Mark and A. V. Tobolsky, Physical Chemistry of High Polymeric Systems, 2nd ed. Interscience Publishers, New York, 1950.

[4] T. Erdy-Gruz, Transport phenomena in aqeous solutions, Adam Hilger Ltd, London, 1974.

[5] P. E. Rouse, Jr., J. Chem. Phys. 21, 1272 (1953).

[6] R. H. Zimm, J. Chem. Phys. 24, 269 (1956).

[7] J. G. Kirkwood, Rec. trav. chim. 68, 649 (1949).

[8] D. W. McCall , D. C. Douglass, J. Chem. Phys. 31, 860 (1959).

[9] D. S. Pearson, G. Ver Strate, E. von Meerwall and F. C. Schilling, Macromolecules, 20, 1133 (1987).

[10] P. G. de Gennes, J. Chem. Phys. 55, 572 (1971).

[11] B. H. Zimm and R. W. Kilb, J. Polym. Sci. 37, 19 (1959). 
[12] J. Klein, Macromolecules, 19, 105 (1986).

[13] R. Kimmich, Polymer, 18, 233 (1977).

[14] E. Dubios-Violette and P. G. de Gennes, Physics, 3, 181 (1967).

[15] H. Cheradame and J. F. Le Nest, in Polymer Electrolyte Reviews 1, ed. J. R. MacCallum and C. A. Vincent, Elsevier, London, 1987, page 103.

[16] G. C. Berry and T. G. Fox, Adv. Polym. Sci. 5, 261 (1968).

[17] Union Carbide Ltd: Typical physical properties of CARBOWAX ${ }^{\circledR}$ datasheet.

[18] J. R. MacCallum, A. S. Tomlin and C. A. Vincent, Eur. Polym. J. 22, 787 (1986).

[19] C. A. Vincent, Prog. Solid State Chem. 17, 145 (1987).

[20] M. T. Hardgrave, PhD thesis, University of St. Andrews, 1990. 
Table 8-1. Experimental results of dc polarisation of $\mathrm{Li} / \mathrm{PEO}\left(\mathrm{M}_{\mathrm{W}}\right)-\mathrm{LiCF}_{3} \mathrm{SO}_{3}(20: 1) / \mathrm{Li}$ cell at $85^{\circ} \mathrm{C}$

\begin{tabular}{|c|c|c|c|c|c|c|}
\hline $\mathrm{M}_{\mathrm{w}}$ & 400 & 1000 & 1450 & 2000 & 3350 & 6000 \\
\hline$\Delta V_{\text {app }} a / m V$ & 10 & 30 & 10 & 10 & 20 & 10 \\
\hline $\mathrm{R}_{\mathrm{io}} / \mathrm{k} \Omega$ & 0.040 & 12.7 & 0.26 & 0 & 0.10 & 0.22 \\
\hline $\mathrm{R}_{\mathrm{is}} / \mathrm{k} \Omega$ & 0.043 & 23.0 & 0.25 & 0 & 0.068 & 0.13 \\
\hline $\mathrm{R}_{\mathrm{b}} \mathrm{b} / \mathrm{k} \Omega$ & 0.20 & 0.34 & 0.36 & 0.36 & 0.65 & 0.63 \\
\hline$\sigma_{0} c / S^{-1}$ & $5.75 \times 10^{-4}$ & $3.42 \times 10^{-4}$ & $3.17 \times 10^{-4}$ & $3.19 \times 10^{-4}$ & $1.76 \times 10^{-4}$ & $1.82 \times 10^{-4}$ \\
\hline$\sigma^{*} \mathrm{~d} / \mathrm{Scm}^{-1}$ & $3.22 \times 10^{-4}$ & $1.55 \times 10^{-4}$ & $1.45 \times 10^{-4}$ & $1.14 \times 10^{-4}$ & $7.41 \times 10^{-5}$ & $7.98 \times 10^{-5}$ \\
\hline $\mathrm{I}_{0} / \mu \mathrm{A}$ & 41.7 & 2.28 & 16.32 & 23.53 & 27.13 & 11.70 \\
\hline $\mathrm{I}_{0} * \mathrm{e} / \mu \mathrm{A}$ & 50.0 & 21.80 & 28.35 & 23.53 & 15.69 & 15.76 \\
\hline $\mathrm{I}_{s} / \mu \mathrm{A}$ & 25.0 & 1.26 & 9.67 & 9.70 & 12.43 & 6.33 \\
\hline $\mathrm{I}_{\mathrm{s}} * \mathrm{~g} / \mu \mathrm{A}$ & 28.00 & 13.48 & 12.61 & 9.91 & 6.45 & 6.91 \\
\hline $\mathrm{F}_{+}$ & 0.56 & 0.45 & 0.46 & 0.36 & 0.42 & 0.44 \\
\hline
\end{tabular}

a: applicd voltage without interfacial correction;

b: bulk resistance measured at $t=0$;

c: $\sigma_{o}=K / R_{b}$, where $K$, the cell constant, is $0.115 \mathrm{~cm}^{-1}$ for all cells

except for that for electrolyte based on PEO $4 \times 10^{6}$, which is $0.0233 \mathrm{~cm}^{-1}$;

d: $\sigma^{*}=K I_{s} /\left(\Delta V_{\text {app }}-I_{s} R_{\text {is }}\right)=\beta^{-1} \sigma_{\text {eff }}$

e: $I_{0}{ }^{*}=I_{0} \Delta V^{*} /\left(\Delta V_{\text {app }}-I_{0} R_{\text {io }}\right), \Delta V^{*}=10 \mathrm{mV}$;

f: calculated without correction of the cell constant;

g: corrected steady state current, $\mathrm{I}_{\mathrm{s}}{ }^{*}=\mathrm{I}_{\mathrm{s}} \Delta \mathrm{V}^{*} /\left(\Delta \mathrm{V}_{\mathrm{app}}-\mathrm{I}_{\mathrm{s}} \mathrm{R}_{\mathrm{is}}\right)$. 


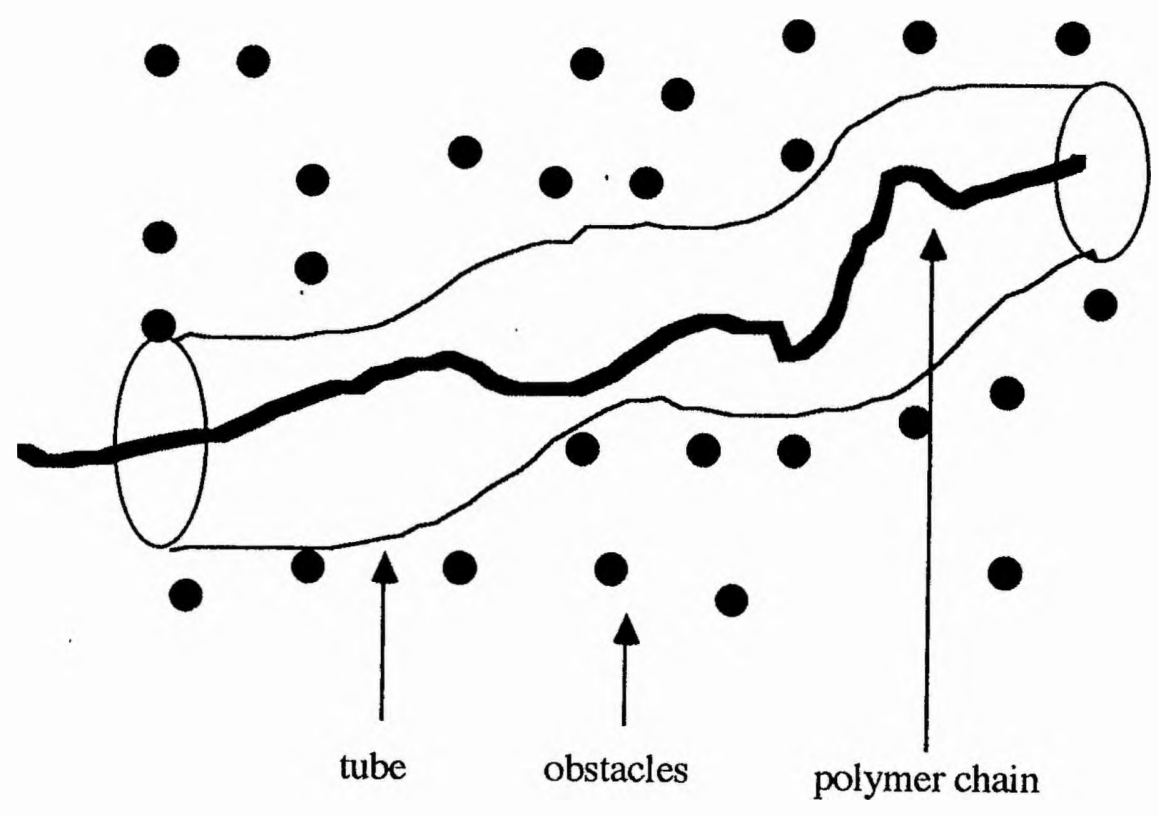

Figure 8-1a Schematic illustration of a polymer chain reptating in a tube formed by obstacles.

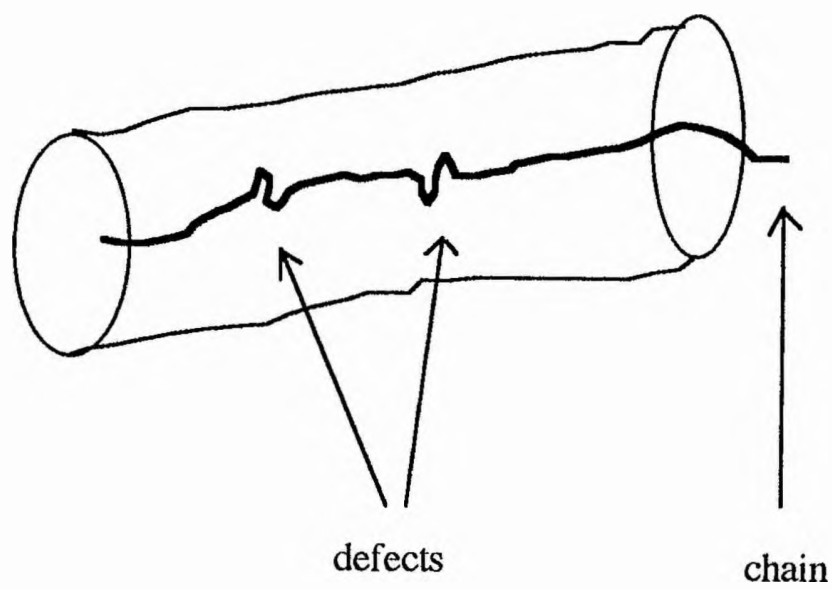

Figure 8-1b Schematic representation of defects in a chain in a tube. 
(a)

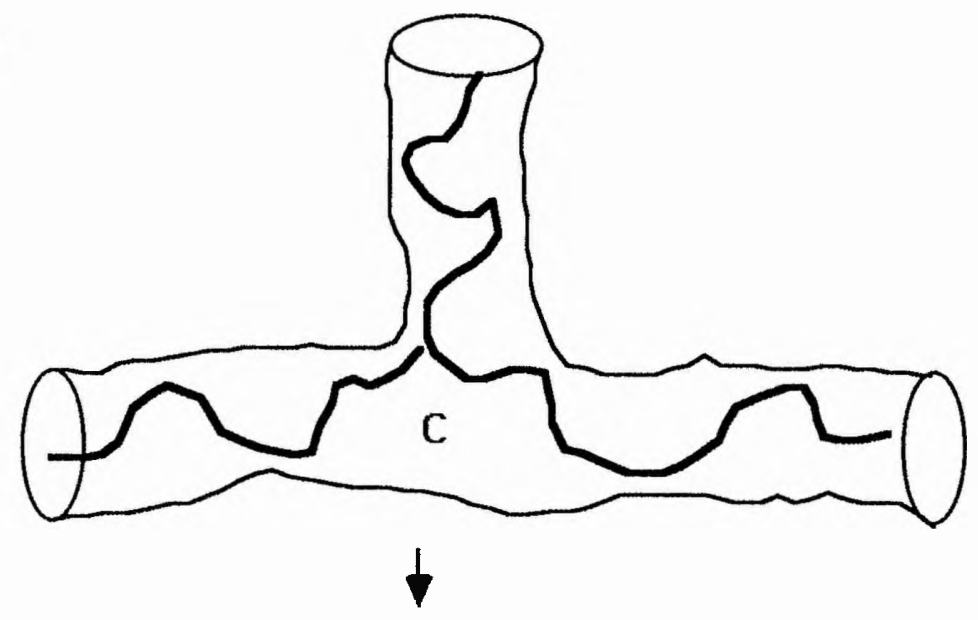

(b)

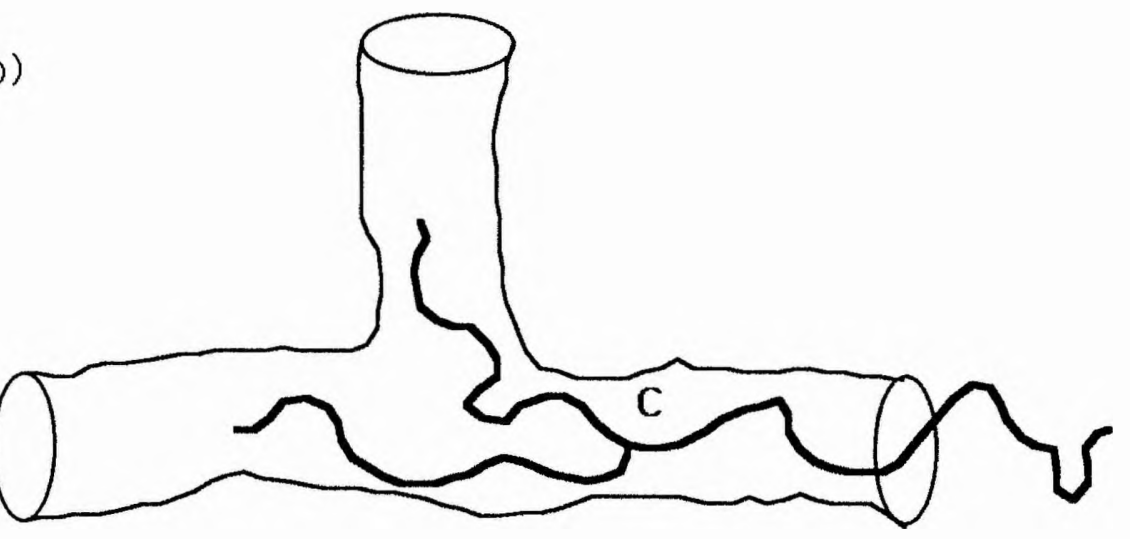

$\downarrow$

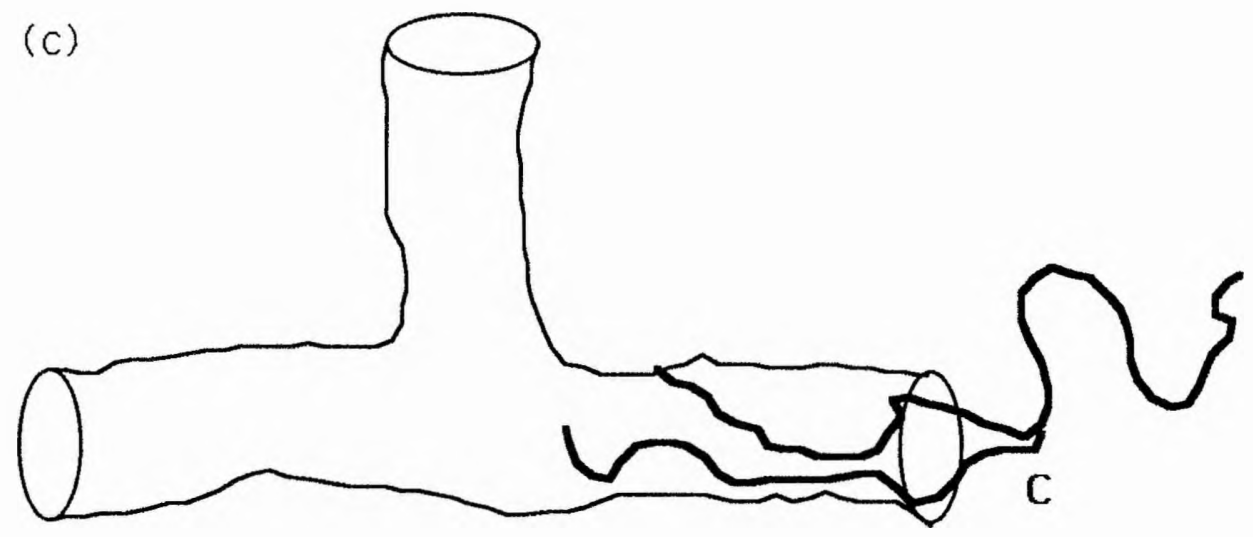

Figure 8-2 Schematic representation of a star-shaped polymer reptating in tubes: (a) at the initial position, (b) moving down one of the arm tubes, dragging other arms to move and (c) full retracted past the original position. 
A

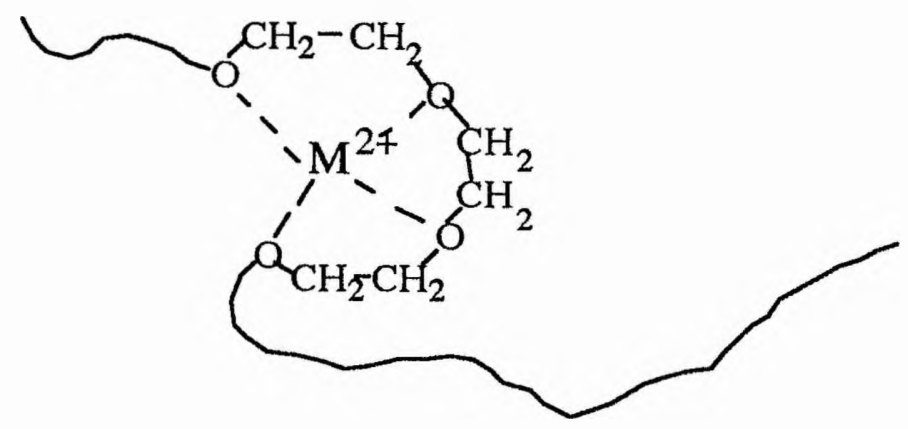

B

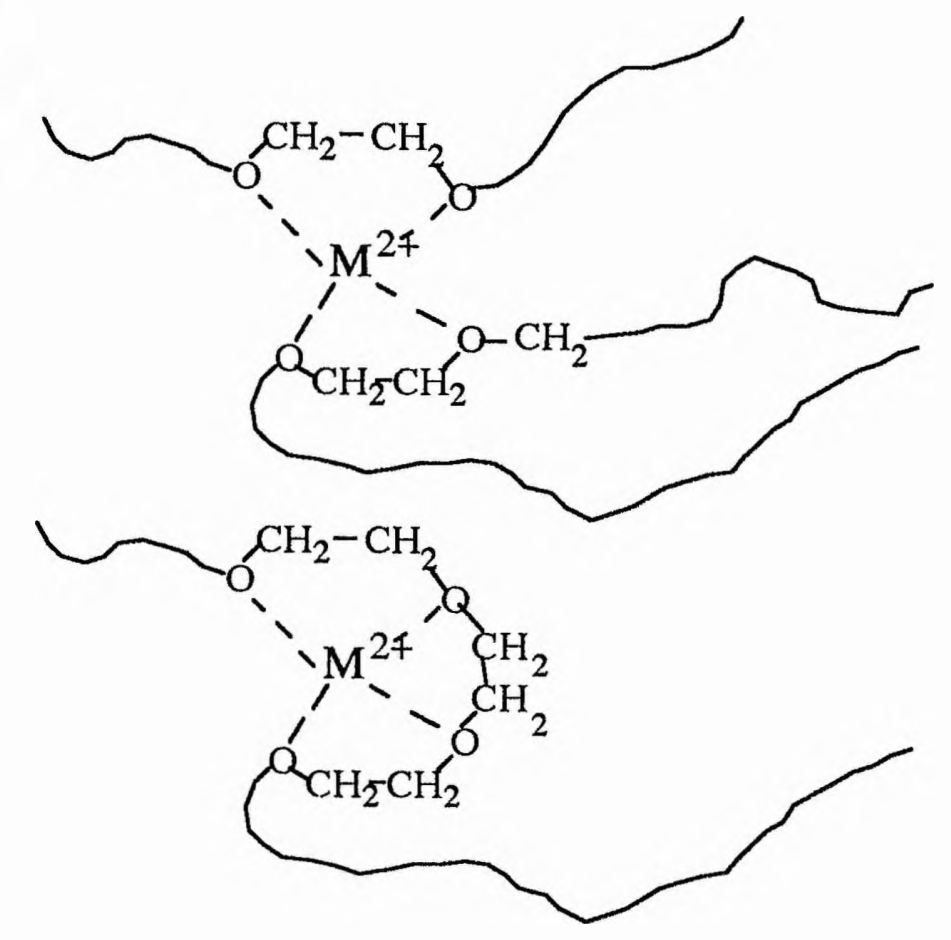

$\mathrm{C}$

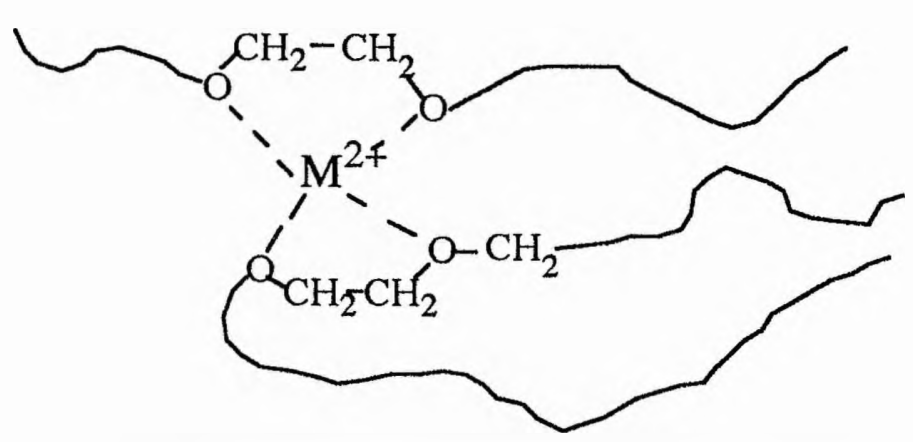

Figure 8-3 Coordination in a polymer electrolyte (A) intrachain coordination (B) intrachain and interchain coordination and $(C)$ interchain coordination. 


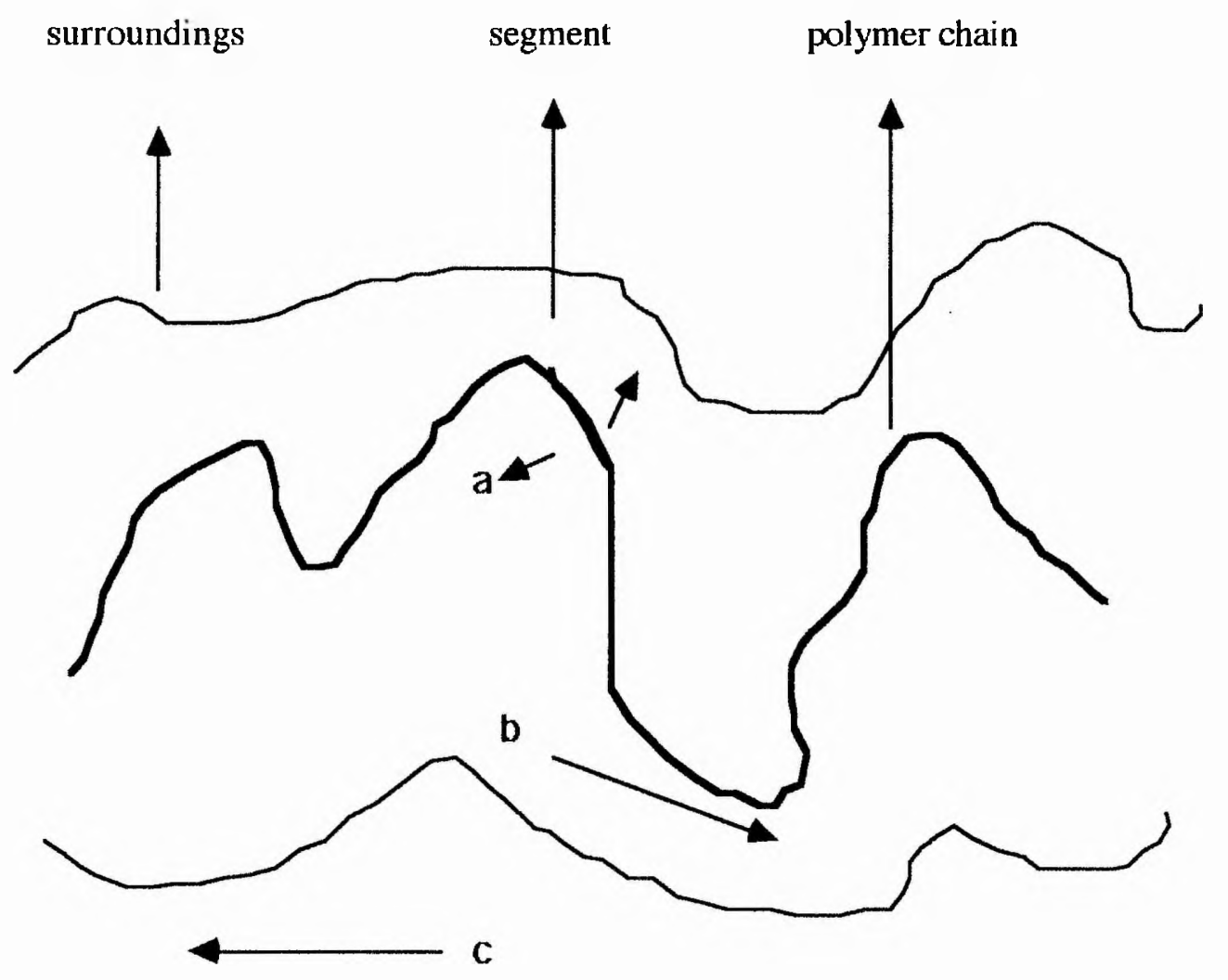

Figure 8-4 Schematic diagram of the three types of relaxational mode
a: reorientation and rearrangement of segments
b: diffusion of the individual polymer chain
c: configurational fluctuation of surroundings. 


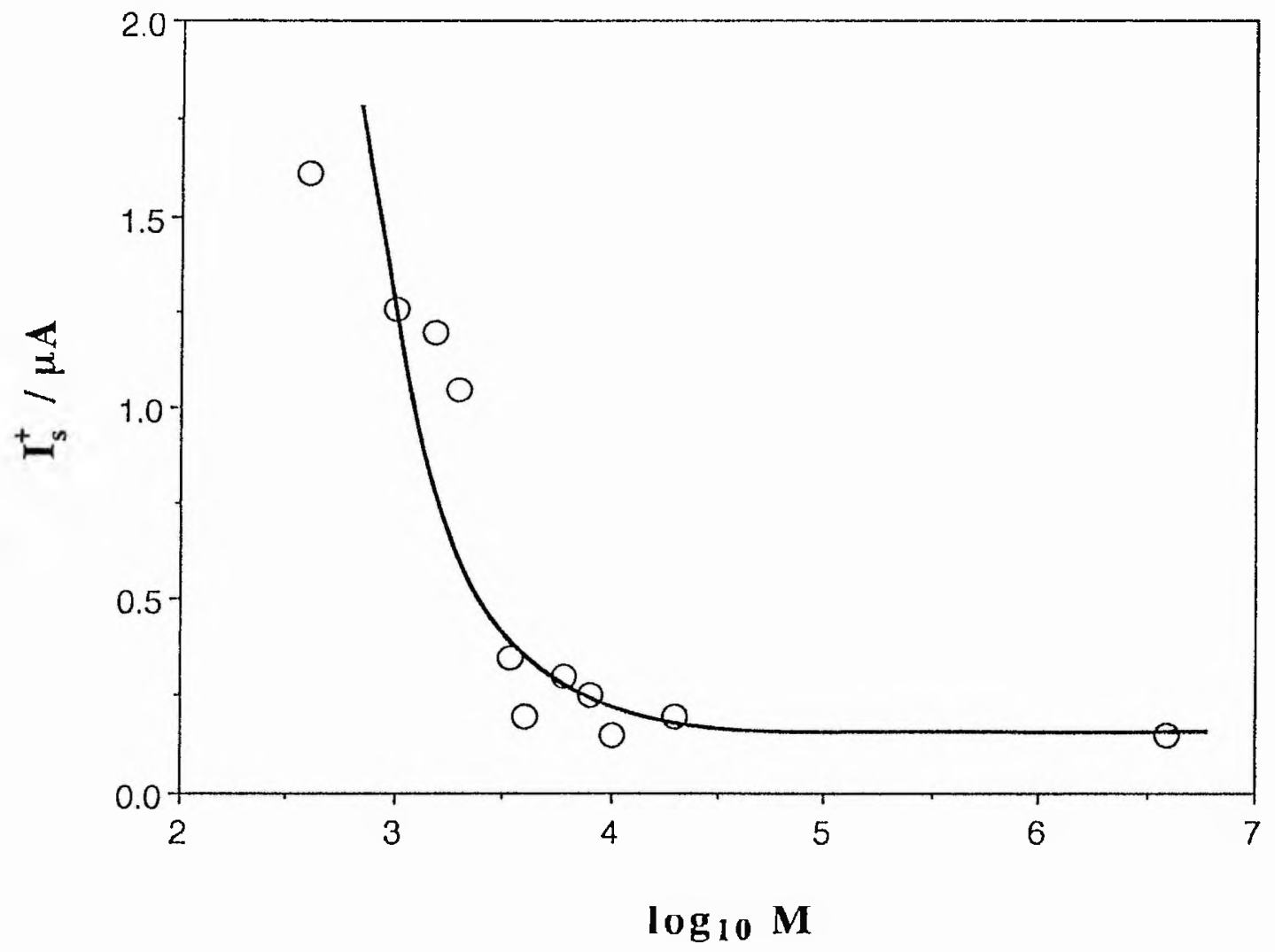

Figure 8-5 Cationic current versus $\log \mathrm{M}_{\mathrm{w}}$ of PEO for PEO-Mg( $\left.\mathrm{CF}_{3} \mathrm{SO}_{3}\right)_{2}$ (20:1) electrolytes at $80^{\circ} \mathrm{C}$. 


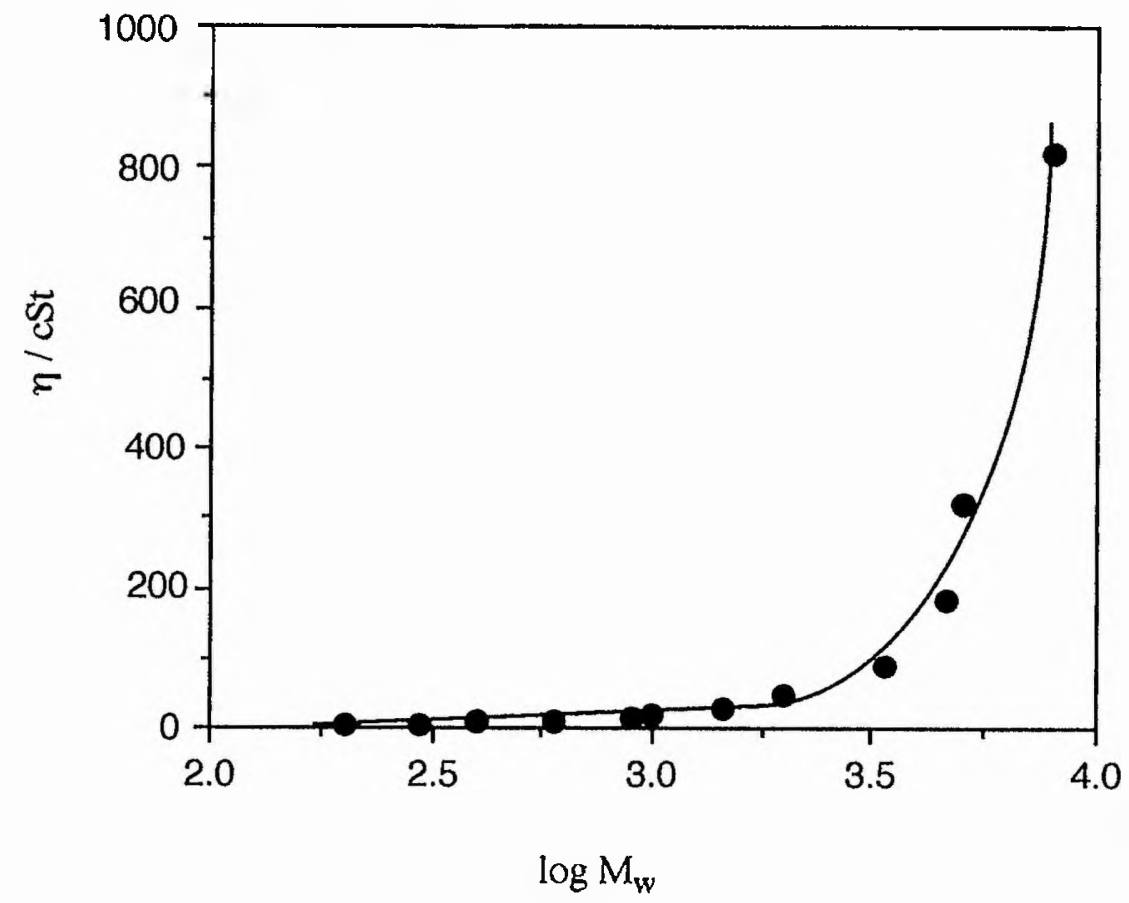

Figure 8-6 Molecular weight dependence of the viscosity for PEO at $100^{\circ} \mathrm{C}$, data from ref.[17]. 


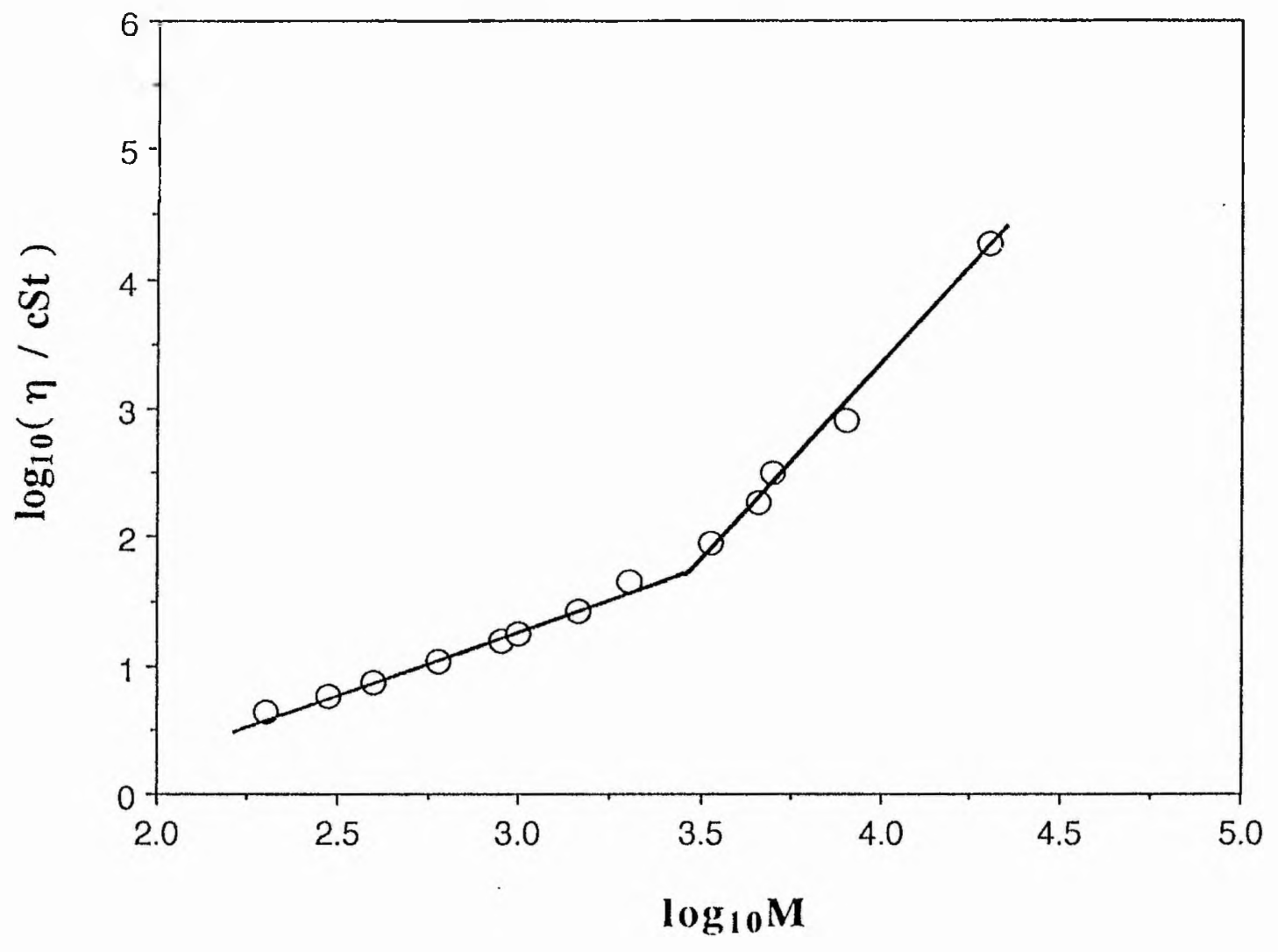

Figure 8-7 $\log \eta$ versus $\log M_{w}$ for $P E O$ at $100^{\circ} \mathrm{C}$, data from ref. [17]. 


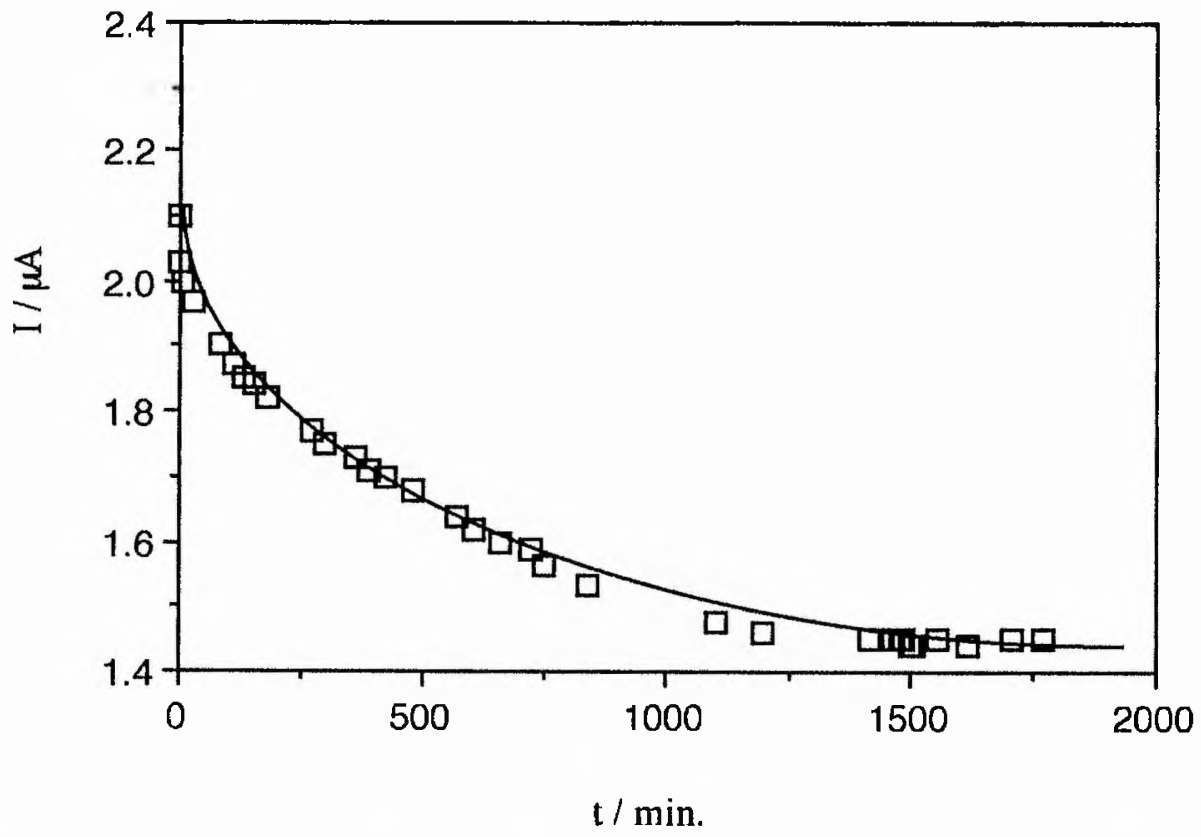

Figure 8-8 I-t curve for dc polarisation of $\mathrm{Li} / \mathrm{PEO}(1000)$ dimethyl ether- $\mathrm{LiCF}_{3} \mathrm{SO}_{3}$ (20:1) / Li cell at $85^{\circ} \mathrm{C}$ under $30 \mathrm{mV}$ applied voltage.

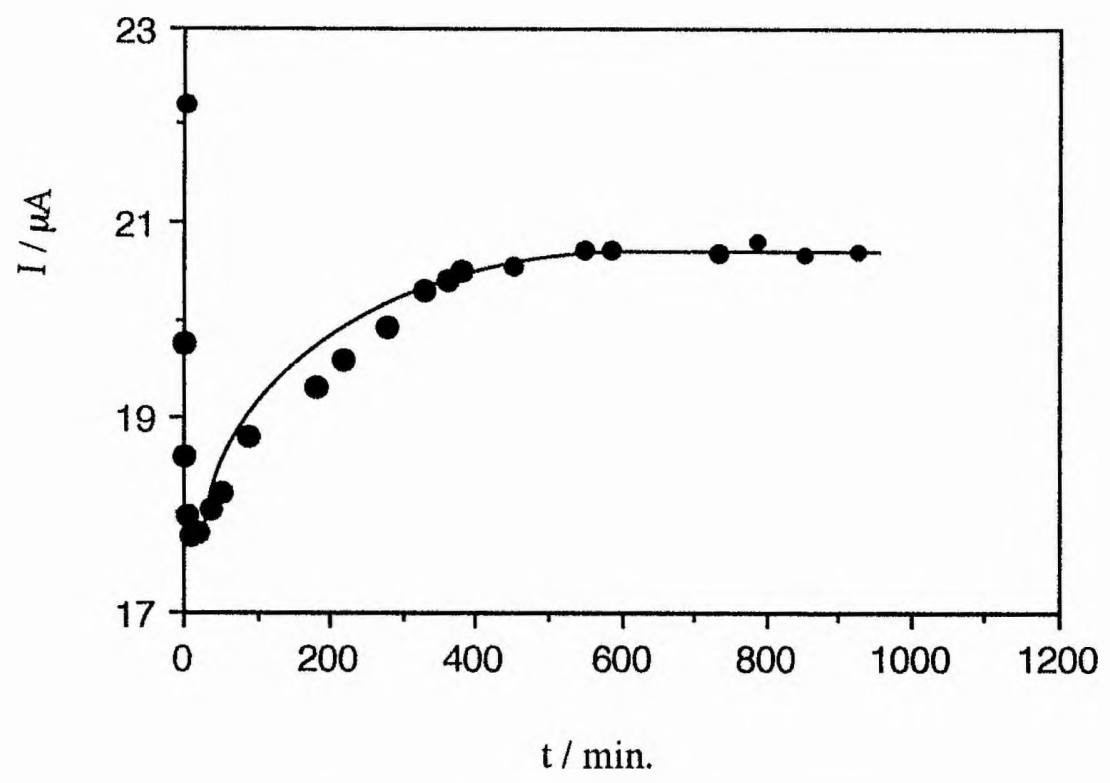

Figure 8-9 I-t curve for dc polarisation of $\mathrm{Li} / \mathrm{PEO}(400)$ dimethyl ether- $\mathrm{LiCF}_{3} \mathrm{SO}_{3}$ (20:1) / Li cell at $85^{\circ} \mathrm{C}$ under $10 \mathrm{mV}$ applied voltage. 


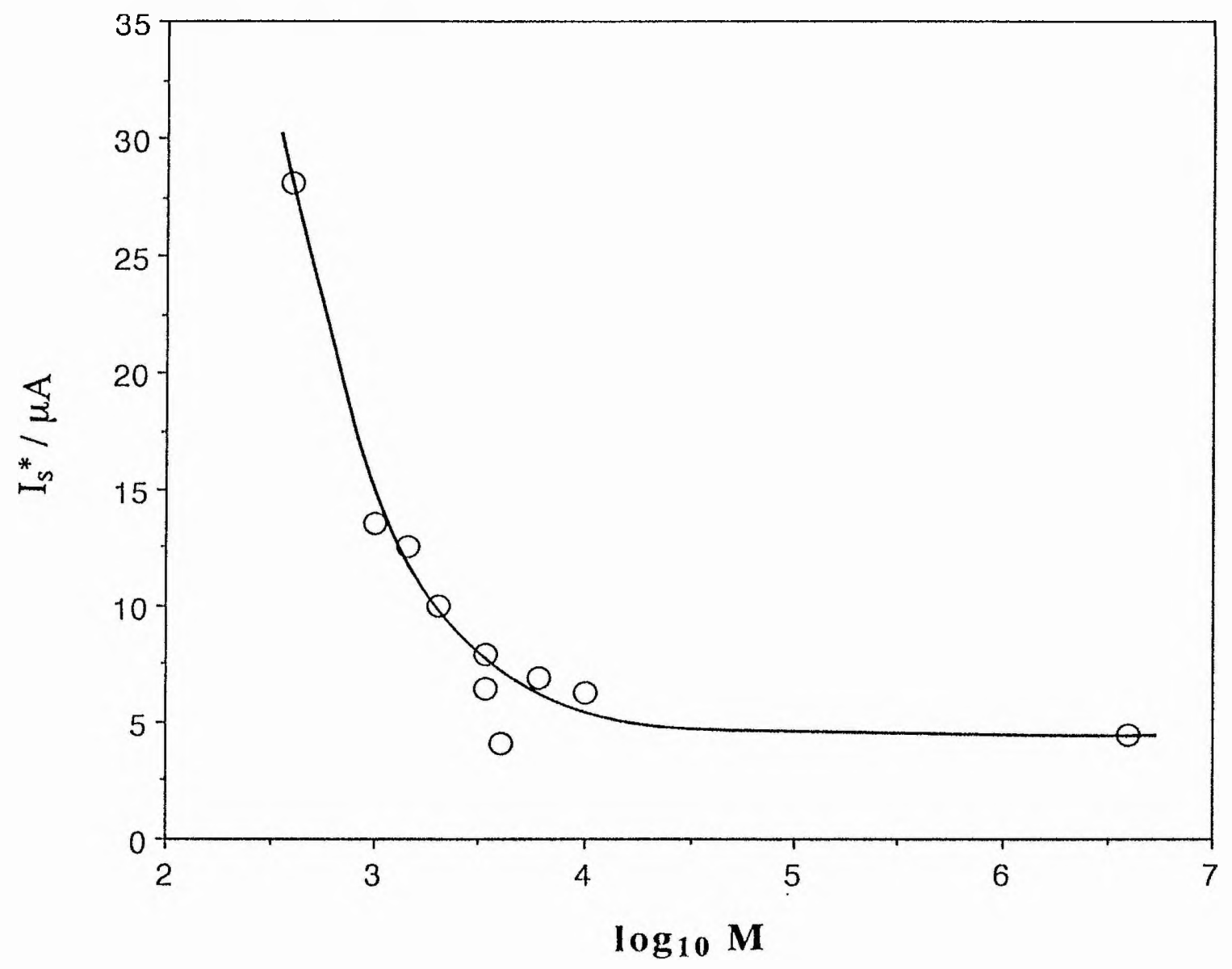

Figure 8-10 Corrected steady state current $\mathrm{I}_{\mathrm{s}}{ }^{*}$ versus $\log \mathrm{M}_{\mathrm{w}}$ of PEO for PEO$\mathrm{LiCF}_{3} \mathrm{SO}_{3}(20: 1)$ at $85^{\circ} \mathrm{C}$. 


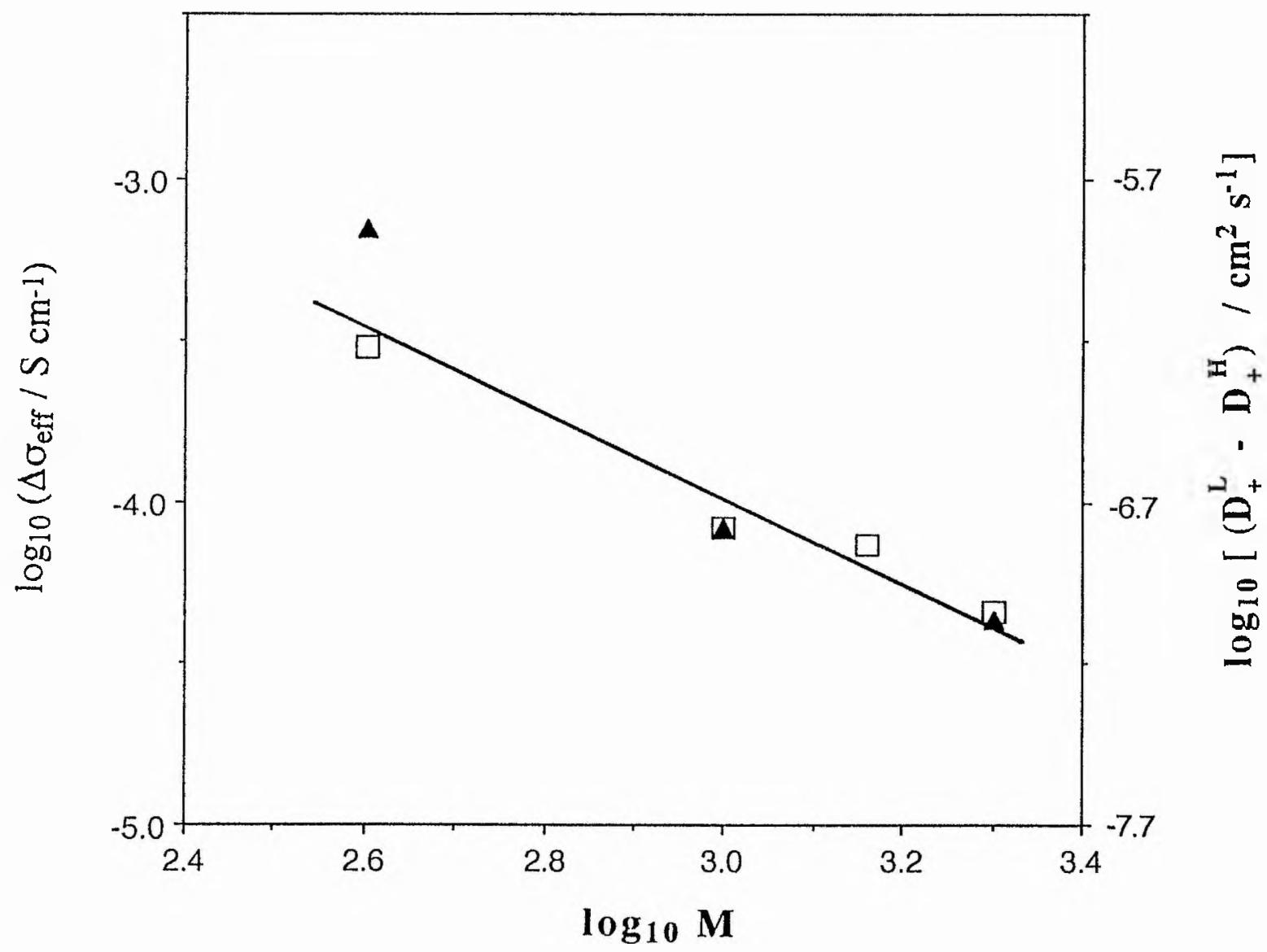

Figure 8-11 $\log \Delta \sigma_{\text {eff }}$ versus $\log \mathrm{M}_{w}$ of PEO at $85^{\circ} \mathrm{C}(\square)$ and $\log \left(\mathrm{D}_{+}^{\mathrm{L}}-\mathrm{D}_{+}^{\mathrm{H}}\right)$ of ${ }^{7} \mathrm{Li}$ versus $\log \mathrm{M}_{\mathrm{w}}$ of $\mathrm{PEO}$ at $90^{\circ} \mathrm{C}$ ( $\left.\mathrm{A}\right)$ for $\mathrm{PEO}-\mathrm{LiCF}_{3} \mathrm{SO}_{3}(20: 1)$ electrolytes. 


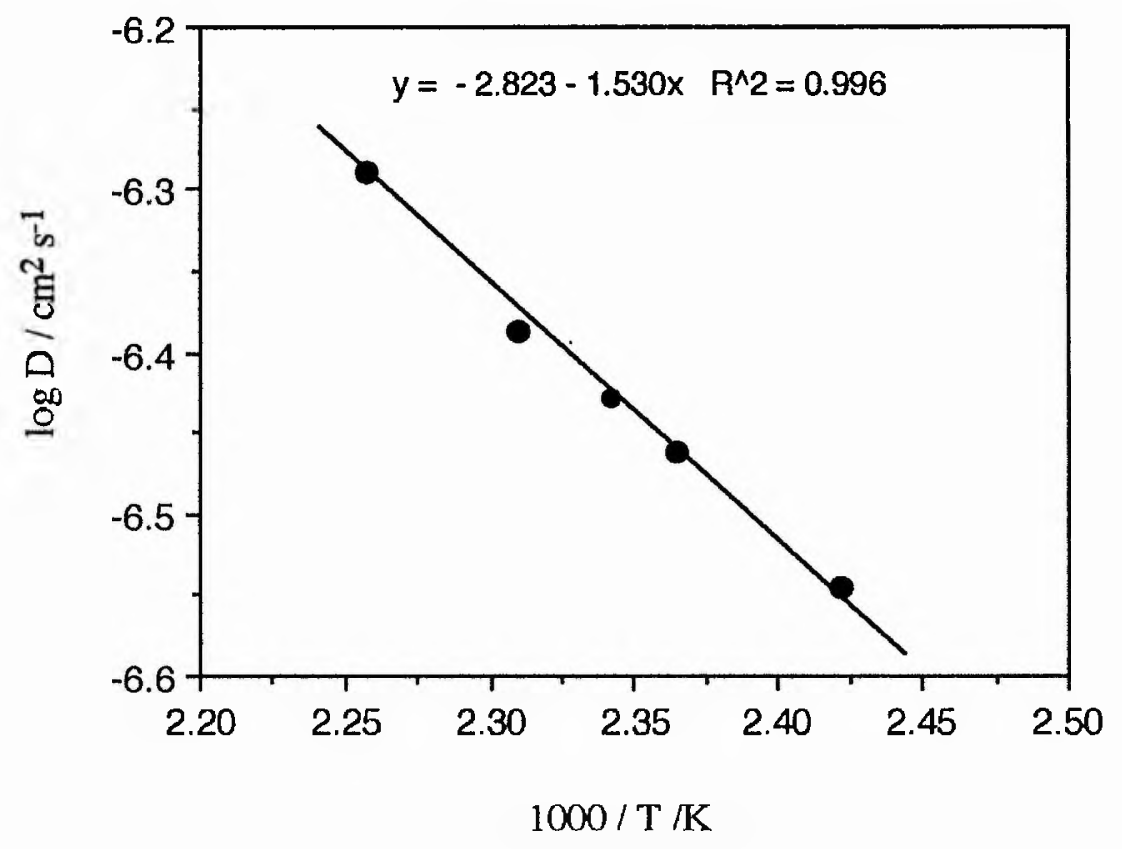

At $90^{\circ} \mathrm{C}, \mathrm{D}=9.16 \times 10^{-\overline{8}} \mathrm{~cm}^{2} \mathrm{~s}^{-1}$

At $70^{\circ} \mathrm{C}, \mathrm{D}=5.20 \times 10^{-8} \mathrm{~cm}^{2} \mathrm{~s}^{-1}$.

Figure 8-12 Temperature dependence of the ${ }^{7} \mathrm{Li}$ diffusion coefficient for PEO $4 \times 10^{6}-$ $\mathrm{LiCF}_{3} \mathrm{SO}_{3}$ (20:1) electrolyte. 


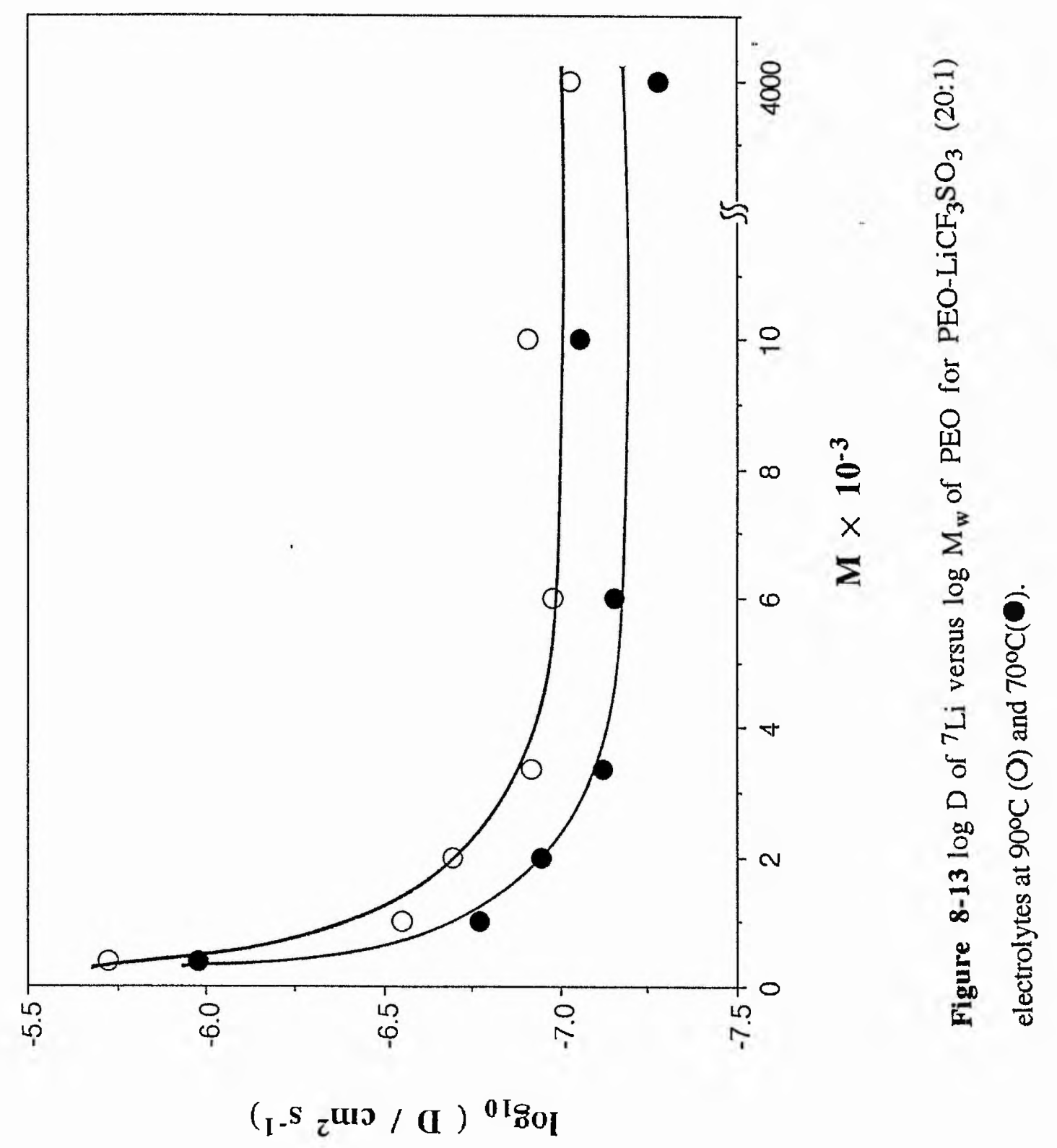




\section{CHAPTER 9}

\section{ION TRANSPORT IN POLYMER ELECTROLYTES : GENERAL CONCLUSION}

\section{9-1 Ion Association and Ion Transport in High Molecular Weight Polymer Electrolytes Based on Lithium Salts}

Investigation of polymer electrolytes based on PMEO and lithium salts, such as $\mathrm{LiClO}_{4}, \mathrm{LiCF}_{3} \mathrm{SO}_{3}, \mathrm{LiSCN}, \mathrm{LiBF}_{4}$ and $\mathrm{LiPF}_{6}$ by conductivity measurement, magicangle spinning ${ }^{7}$ Li NMR and pulsed field gradient NMR has shown:

(1) In these polymer electrolytes the ion pairing effect is the dominant in the very dilute concentration region. In the medium salt concentration region, the increase of the molar conductivity may arise mainly from the formation of triple ions. In the relatively high concentration region, the decrease of the molar conductivity may arise from the transient crosslinking effect of the salt.

(2) Ionic conduction of these polymer electrolytes based on high molecular weight polymer hosts is basically controlled by the segmental motion of the polymer and can be described by the VTF equation in the low and medium concentration region. In the relatively high concentration region an activated process is important for the ion transport, and the ionic conduction can be described by a modified VTF equation: 


$$
\sigma=\sigma_{0} \exp \left[\frac{-\mathrm{B}}{\mathrm{T}-\mathrm{T}_{\mathrm{o}}}-\frac{\Delta \mathrm{E}}{\mathrm{RT}}\right]
$$

where $\sigma_{o}, B$ and $T_{o}$ are constants, $\Delta \mathrm{E}$ is the activation energy.

(3) ${ }^{7} \mathrm{Li}$ mas NMR of $\mathrm{PMEO}-\mathrm{LiClO}_{4}$ electrolytes shows three-line pattern spectra. The postulated existence of "free ions", ion pairs" and ion clusters allows these spectra to be interpreted. In conductivity and mas NMR measurements, it is found that ion association in $\mathrm{PMEO}-\mathrm{LiClO}_{4}$ electrolyte increases with increasing temperature and salt concentration.

(4) The diffusion coefficient of the anion constituents measured by the pfg NMR experiment on PMEO-LiPF 6 electrolytes is almost an order of magnitude higher than that of the cation constituents. The results of the pfg NMR experiment support the theory that in the low salt concentration region, transport of cationic species is controlled by segmental motion of the polymer, but is controlled by an activated process in the relatively high salt concentration region. The transport of anionic species, however, is controlled mainly by the segmental motion of the polymer in the concentration range studied.

\section{9-2 Ion Transport in Polymer Electrolytes Based on Divalent Salts}

Investigation of polymer electrolytes based on $\mathrm{PEO}, \mathrm{PMEO}, \mathrm{Zn}\left(\mathrm{CF}_{3} \mathrm{SO}_{3}\right)_{2}$, $\mathrm{Ca}\left(\mathrm{CF}_{3} \mathrm{SO}_{3}\right)_{2}$ and $\mathrm{Mg}\left(\mathrm{CF}_{3} \mathrm{SO}_{3}\right)_{2}$ by dc polarisation, conductivity measurement and phase analysis has shown:

\footnotetext{
* "Free ions" may include solvent separated ion pairs; ion pairs in this context are contact ion pairs.
} 
(1) There are passivating layers on the zinc, calcium and magnesium electrodes in the two electrode cells, which can block current flow. The influence of these passivating layers on the dc polarisation of the two electrode cell can be reduced by electrochemical pre-treatment of the electrodes or by choosing mercury film electrodes.

(2) The ionic conducting behaviour of PMEO- $\mathrm{Zn}\left(\mathrm{CF}_{3} \mathrm{SO}_{3}\right)_{2}$ electrolytes is nonArrhenius. Dc polarisation of $\mathrm{Zn} / \mathrm{PMEO}-\mathrm{Zn}\left(\mathrm{CF}_{3} \mathrm{SO}_{3}\right)_{2} / \mathrm{Zn}$ cell shows that zinc species are mobile in the system. The limiting current fraction of $\mathrm{PMEO}-\mathrm{Zn}\left(\mathrm{CF}_{3} \mathrm{SO}_{3}\right)_{2}$ electrolyte is 0.3 at $30^{\circ} \mathrm{C}$. The anionic transference number determined by Hittorf method in $\mathrm{PEO}-\mathrm{Zn}\left(\mathrm{CF}_{3} \mathrm{SO}_{3}\right)_{2}$ electrolytes is 0.95 with a standard deviation of 0.038 based on the analysis of the anode compartment and 0.84 with a standard deviation of 0.062 based on the analysis of the cathode compartment. In the polymer electrolytes mobile uncharged triples, $\mathrm{Zn}\left(\mathrm{CF}_{3} \mathrm{SO}_{3}\right)_{2}$, may be important, and there may also exist some $\mathrm{Zn}\left(\mathrm{CF}_{3} \mathrm{SO}_{3}\right)^{+}$and $\mathrm{Zn}\left(\mathrm{CF}_{3} \mathrm{SO}_{3}\right)_{3}^{-}$species.

(3) The experimental results of dc polarisation of two electrode cells with the metal anode and the mercury cathode show that calcium species are mobile in PEO$\mathrm{Ca}\left(\mathrm{CF}_{3} \mathrm{SO}_{3}\right)_{2}$ electrolytes based on the high molecular weight PEO. For PEO$\mathrm{Mg}\left(\mathrm{CF}_{3} \mathrm{SO}_{3}\right)_{2}$ electrolytes based on the high molecular weight PEO, however, the results show that magnesium species are immobile. The crucial criterion for the mobility of metal ions may be the lability of metal-polymer host bond. No crystalline PEO-salt complexes were detected in $\mathrm{PEO}-\mathrm{Mg}\left(\mathrm{CF}_{3} \mathrm{SO}_{3}\right)_{2}$ electrolytes. 


\section{9-3 Influence of the Molecular Weight of Polymer on the Cationic Transport in Polymer Electrolytes}

The dynamics of polymer electrolytes are discussed. The molecular weight of the polymer has been correlated to the ionic conduction of polymer electrolytes. Experimental studies of $\mathrm{PEO}-\mathrm{Mg}\left(\mathrm{CF}_{3} \mathrm{SO}_{3}\right)_{2}$ and $\mathrm{PEO}-\mathrm{LiCF}_{3} \mathrm{SO}_{3}$ electrolytes by $\mathrm{dc}$ polarisation and ${ }^{7} \mathrm{Li} \mathrm{pfg} \mathrm{NMR} \mathrm{techniques} \mathrm{are} \mathrm{examined} \mathrm{in} \mathrm{terms} \mathrm{of} \mathrm{these} \mathrm{theoretical}$ analyses.

(1) The dynamics of polymer chains in polymer electrolytes can be described either by the Rouse model for low molecular weight polymer or by de Gennes' reptation theory for high molecular weight and entangled polymer as in the case of conventional polymer liquids or melts. There are three principal types of relaxation processes which are relevant to the ionic conduction of polymer electrolytes, viz. segmental motion, diffusion of individual chains and fluctuation of the surroundings. When the molecular weight of polymer is low enough, the diffusion of polymer chains and/or the fluctuation of surroundings contributes to the ionic conduction.

(2) The dc polarisation and ${ }^{7} \mathrm{Li}$ pfg NMR experiments have shown that, in PEO$\mathrm{Mg}\left(\mathrm{CF}_{3} \mathrm{SO}_{3}\right)_{2}$ and $\mathrm{PEO}-\mathrm{LiCF}_{3} \mathrm{SO}_{3}$ systems, when the molecular weight of $\mathrm{PEO}$ is lower than 3200 , the diffusion of polymer chain will contribute to the motion of lithium ions, and the diffusion coefficient of the chain can be described by the Rouse model; when the molecular weight of polymer is high enough, the contribution of whole chain diffusion to the ionic conduction is negligible. The ionic conduction in a polymer electrolyte based on high molecular weight polymer is apparently controlled by the microscopic motion of polymer, i.e. segmental motion. 
(3) The critical molecular weights of PEO for establishing entanglement in all systems examined, i.e. salt free $\mathrm{PEO}, \mathrm{PEO}-\mathrm{Mg}\left(\mathrm{CF}_{3} \mathrm{SO}_{3}\right)_{2}$ and $\mathrm{PEO}-\mathrm{LiCF}_{3} \mathrm{SO}_{3}$ electrolytes are around 3200. The coordinated chains in PEO- $\mathrm{Mg}\left(\mathrm{CF}_{3} \mathrm{SO}_{3}\right)_{2}$ and $\mathrm{PEO}-\mathrm{LiCF}_{3} \mathrm{SO}_{3}$ electrolytes behave like linear chains. For $\mathrm{PEO}-\mathrm{Mg}\left(\mathrm{CF}_{3} \mathrm{SO}_{3}\right)_{2}$ electrolytes, this indicates that intrachain coordination between cations and oxygen atoms on the polymer chains is significant. For $\mathrm{PEO}-\mathrm{LiCF}_{3} \mathrm{SO}_{3}$ electrolytes, this indicates that either the intrachain coordination is dominant or there is rapid exchange between interchain coordination sites. 